\title{
S3-Leitlinie
}

\section{Kolorektales Karzinom}

Langversion 2.1. - Januar 2019

AWMF-Registernummer: 021/007OL

\section{Leitlinie (Langversion)}


Das ist neu!

\section{Wesentliche Neuerungen durch die Aktualisierung der Leitlinie (Version 2.1, 2019)}

Es wurden zwei Empfehlungen im Kapitel 8.1.6.3: Dauer der adjuvanten Therapie ergänzt.

Die bisherigen Empfehlung 10.17 zur Nachsorge bei pT1-Low-Risk' wurde mit Verweis auf die aktuellere Empfehlung 6.15 gestrichen.

\section{Wesentliche Neuerungen durch die Aktualisierung der Leitlinie (Version 2, 2017)}

Es erfolgte die Aktualisierung von:

- Kapitel 6 Endoskopie: Durchführung und Polypenmanagement

- Kapitel 8: Adjuvante und neoadjuvante Therapie und

- Kapitel 9: Therapeutisches Vorgehen bei Metastasierung und in der palliativen Situation

Eine detaillierte Auflistung der vorgenommenen Änderungen kann Kapitel 12.4 entnommen werden.

Im Kapitel $\underline{6}$ wurde insbesondere die Empfehlung zur Kontrollkoloskopie nach Abtragung von sessilen serratierten Adenomen geändert (siehe Empfehlung 6.25.) sowie Empfehlungen zur Kontrollkoloskopie nach Abtragung von 1 oder 2 Adenomen $<1 \mathrm{~cm}$ ohne höhergradige intraepitheliale Neoplasie ergänzt (siehe Empfehlungen 6.18. und $\underline{6.19}$.).

Empfehlungen zum Tumorbudding wurden ebenfalls ergänzt (siehe Empfehlung $\underline{6.10 . u n d ~ 6.12 .) .}$

Im Kapitel $\underline{8}$ sind u. a. Empfehlungen zum zeitlichen Abstand einer adjuvanten Chemotherapienach nach der Operation eines Kolonkarzinoms (siehe Empfehlung 8.2.und 8.3. ), zur neoadjuvanten Therapie bei einem Rektumkarzinom (siehe Kapitel 8.2.1) und zur Frage einer adjuvante Chemotherapie nach neoadjuvanter Therapie des Rektumkarzinoms (siehe Empfehlung 8.33.) ergänzt bzw. aktualisiert worden.

Das Kapitel 9 komplett überarbeitet und neu strukturiert.

\footnotetext{
1 Nach lokaler Abtragung eines pT1-Low-Risk Karzinoms sollten lokale endoskopische Befundkontrollen nach 6, 24 und 60 Monaten erfolgen.
} 


\section{Inhaltsverzeichnis}

Wesentliche Neuerungen durch die Aktualisierung der Leitlinie (Version 2.1, 2019) ......................... 2

Wesentliche Neuerungen durch die Aktualisierung der Leitlinie (Version 2, 2017) ......................... 2

1. Informationen zu dieser Leitlinie ................................................................ 10

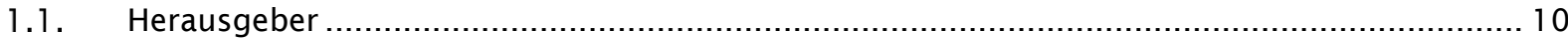

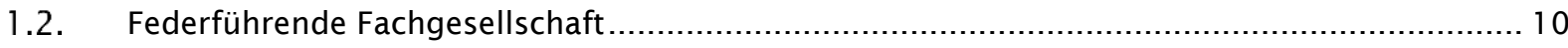

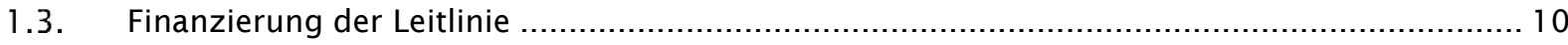

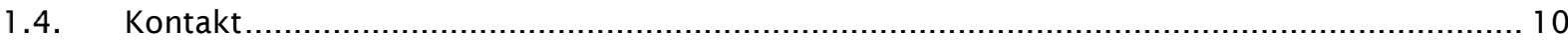

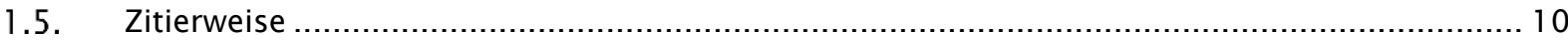

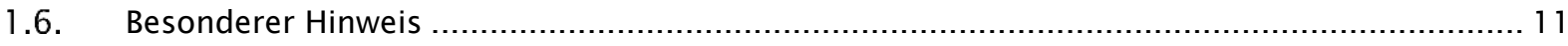

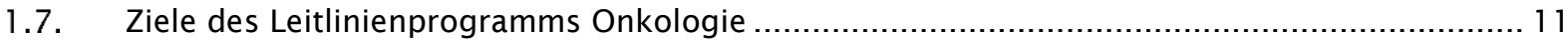

1.8. Verfügbare Dokumente zur Leitlinie und Implementierung …........................................... 12

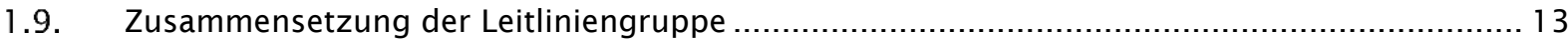

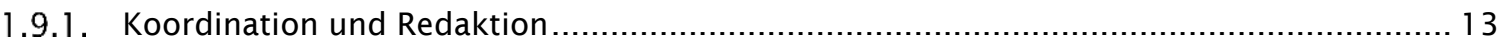

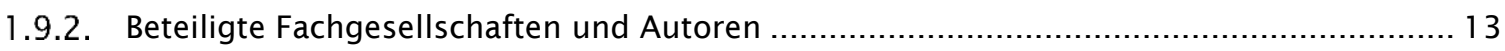

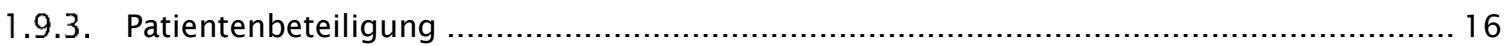

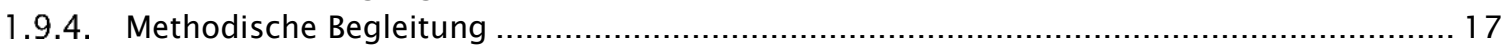

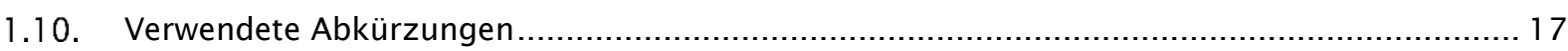

2. Einführung..................................................................................................................20

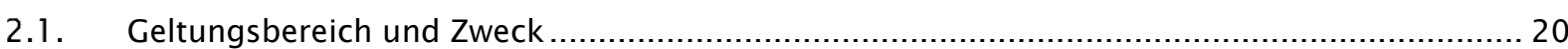

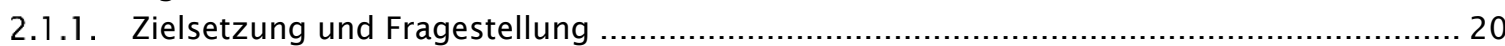

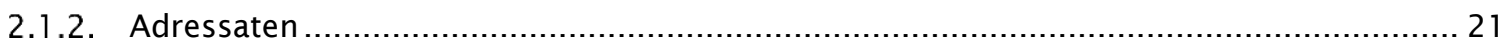

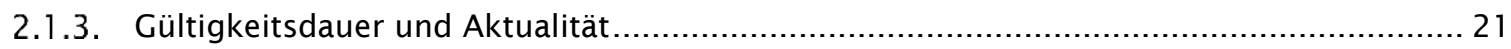

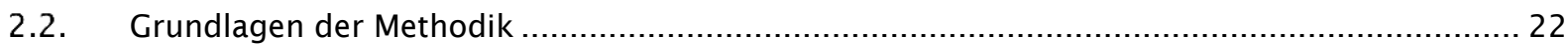

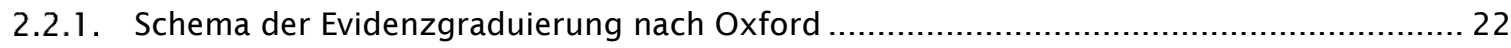

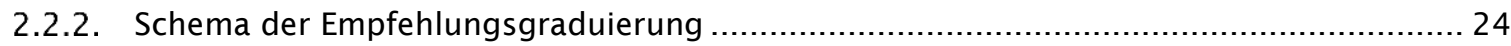

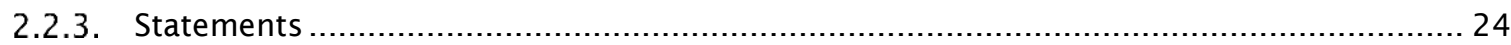

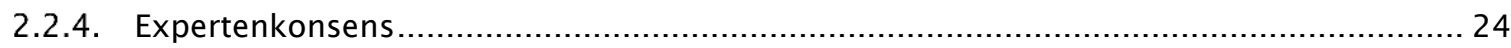

2.2.5. Unabhängigkeit und Darlegung möglicher Interessenkonflikte .................................... 25

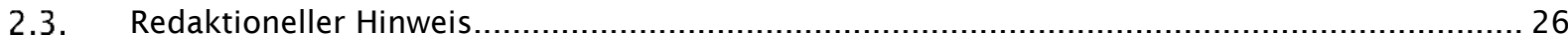

3. Prävention asymptomatische Bevölkerung.................................................27

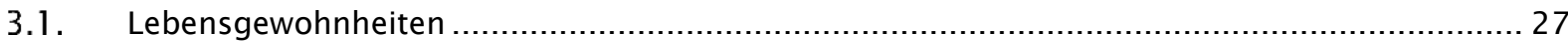

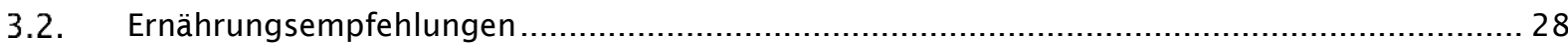

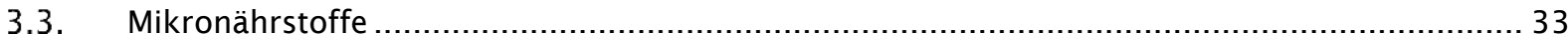

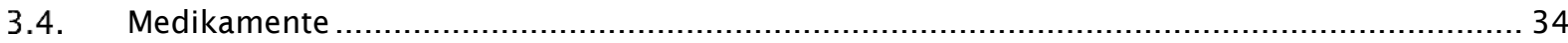

4. Vorsorge/ Früherkennung asymptomatische Bevölkerung .....................37 


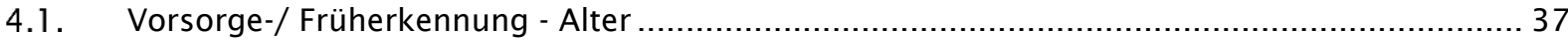

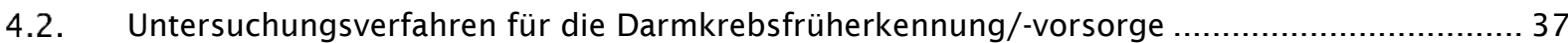

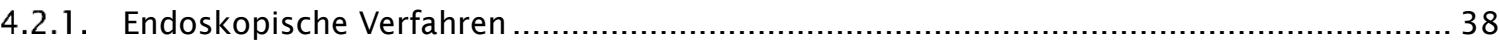

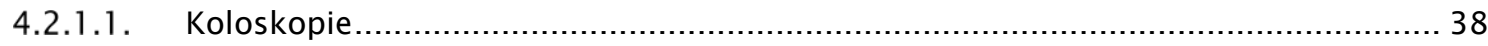

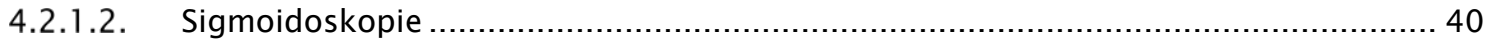

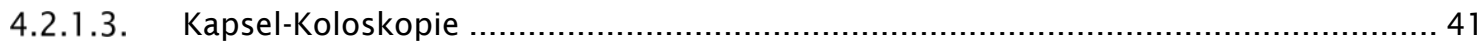

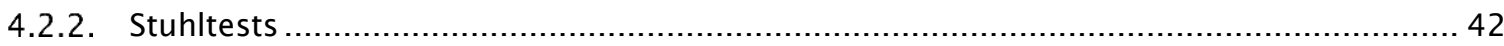

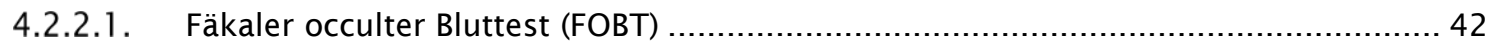

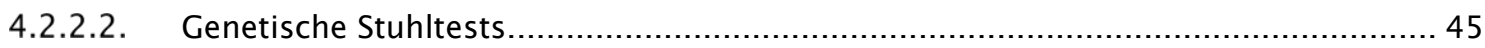

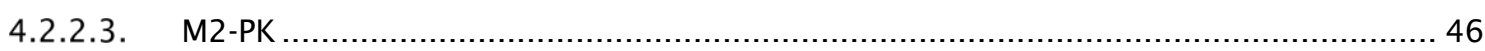

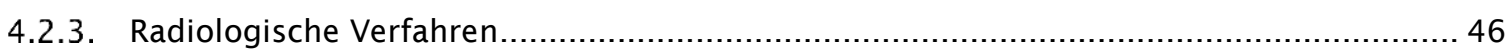

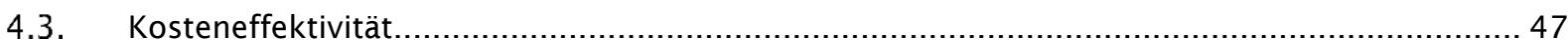

4.4. Empfehlungen anderer eingeschlossener Leitlinien ................................................ 47

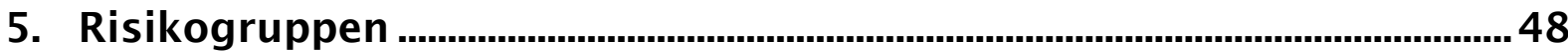

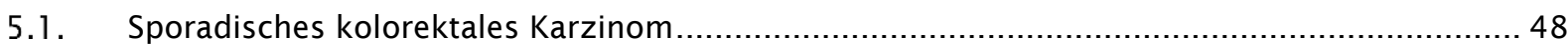

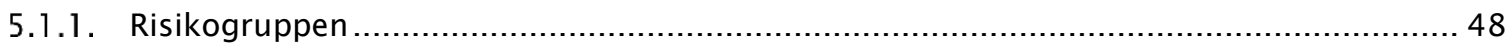

5.1.1.1. Verwandte von Patienten mit kolorektalem Karzinom ................................. 48

5.1.1.2. Verwandte von Patienten mit kolorektalem Adenom ....................................... 49

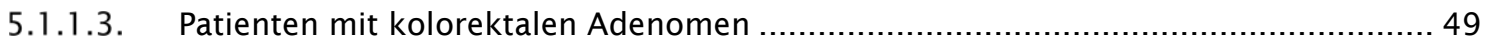

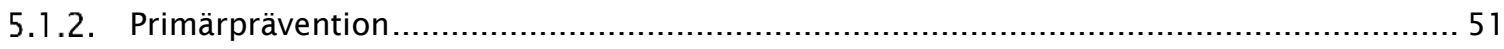

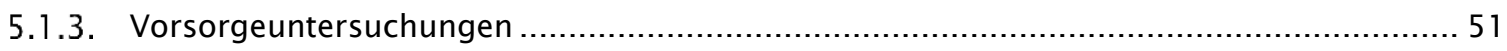

5.1.3.1. Verwandte ersten Grades von Patienten mit kolorektalem Karzinom...................... 51

5.1.3.2. Verwandte von Patienten mit kolorektalen Adenomen .................................... 53

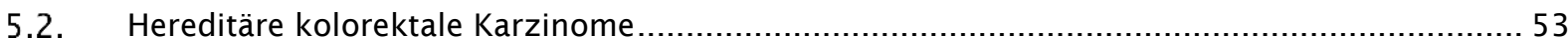

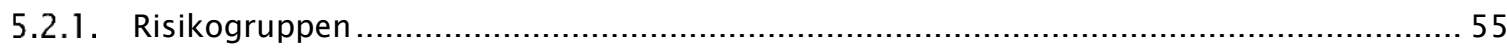

5.2.1.1. HNPCC (hereditäres kolorektales Karzinom ohne Polyposis)/ Lynch-Syndrom ..........55

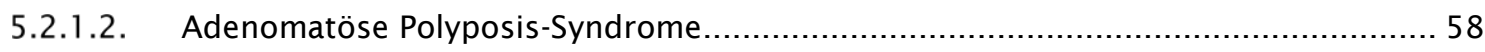

5.2.1.2.1. Patienten mit klassischer familiär adenomatöser Polyposis (FAP) 58

5.2.1.2.2. Patienten mit attenuierter familiärer adenomatöser Polyposis (AFAP) 59

5.2.1.2.3. Patienten mit MUTYH-assoziierter Polyposis (MAP) 59

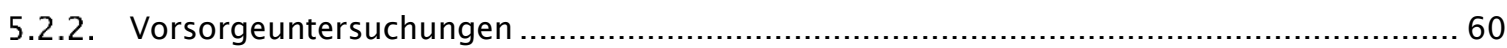

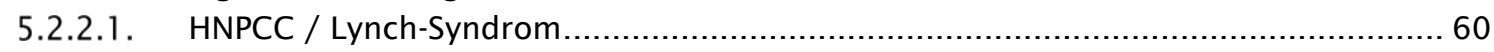

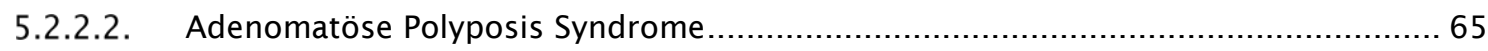

5.2.2.2.1. Patienten mit klassischer familiärer adenomatöser Polyposis (FAP) 65

5.2.2.2.2. Patienten mit attenuierter familiärer adenomatöser Polyposis 71

5.2.2.2.3. Patienten mit MUTYH-assoziierter Polyposis (MAP) 72

5.2.2.3. Nicht-adenomatöse Polyposis-Syndrome ................................................. 74

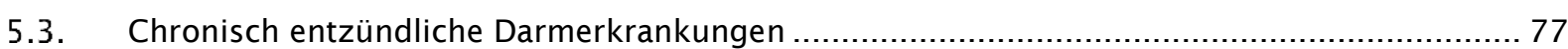

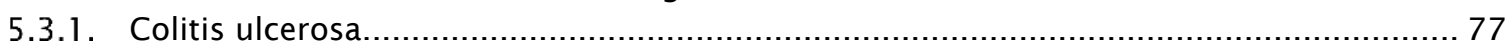

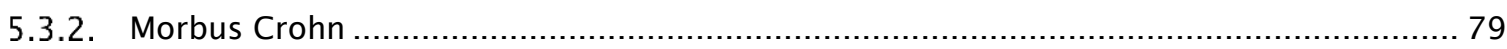

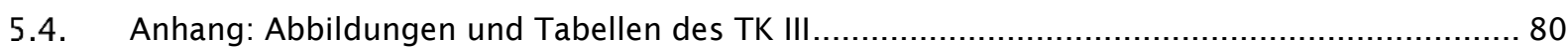

5.4.1. Algorithmus: Genetische Diagnostik und Vorsorge.......................................... 80

5.4.2. Testalgorithmus Immunhistochemie / MSI zur Abklärung Mismatch-Reparatur-Defekt .... 81

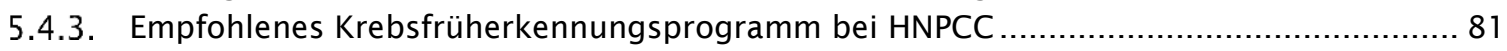

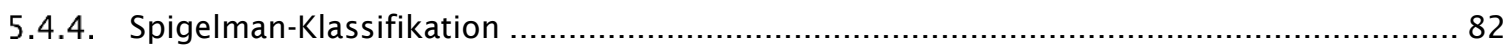


6. Endoskopie: Durchführung und Polypenmanagement 83

6.1. Stellenwert der Endoskopie in der Diagnostik von Polypen und kolorektalen Karzinomen....... 83

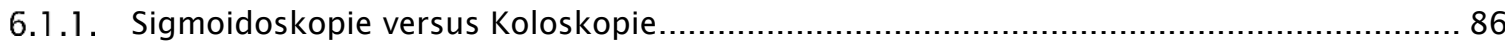

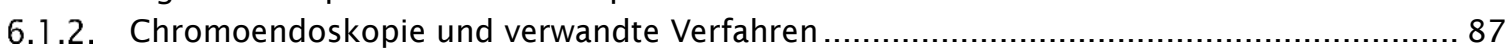

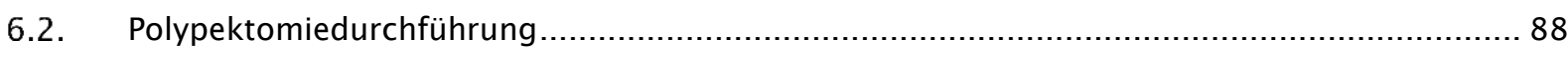

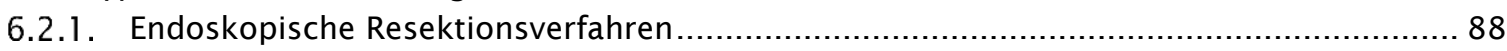

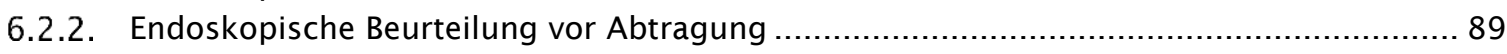

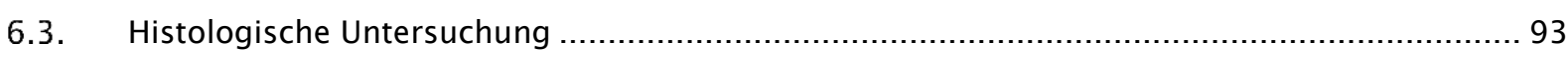

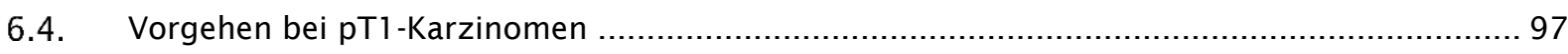

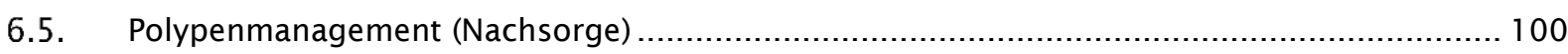

6.6. Medikamentöse Sekundärprävention bei Adenomen .......................................................... 104

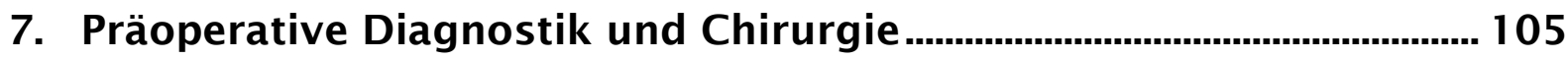

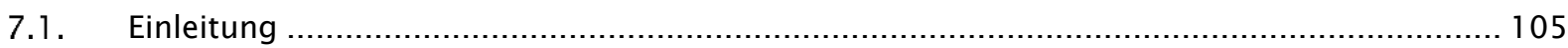

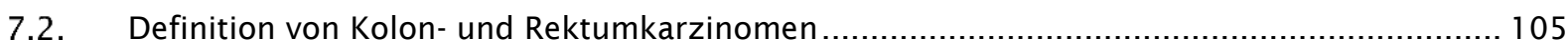

7.3. Definition Interdisziplinäre Tumorkonferenz …….................................................... 106

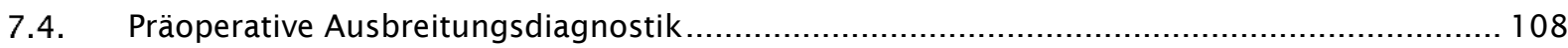

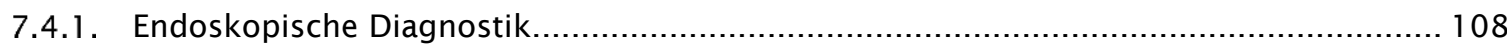

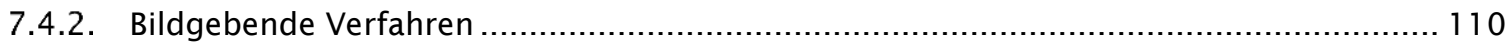

7.4.2.1. Bedeutung einzelner bildgebender Verfahren (außer PET) zur Abklärung von Fernmetastasen bei der Primärbehandlung des Kolorektalen Karzinoms................ 110

7.4.2.2. Stellenwert des präoperativen lokalen Stagings durch CT, (MRT) beim

Kolonkarzinom bzgl. lokaler Ausbreitung ......................................................... 112

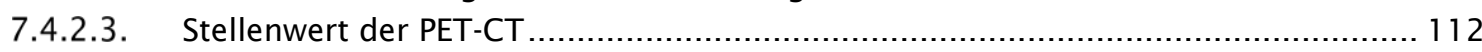

$\begin{array}{ll}\text { 7.4.2.3.1. bei der Primärdiagnostik des kolorektalen Karzinoms } & 112\end{array}$

$\begin{array}{ll}\text { 7.4.2.3.2. vor Resektion kolorektaler Lebermetastasen } & 113\end{array}$

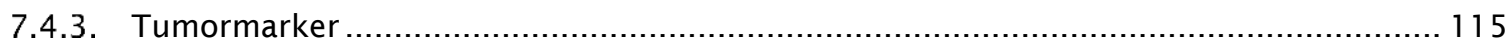

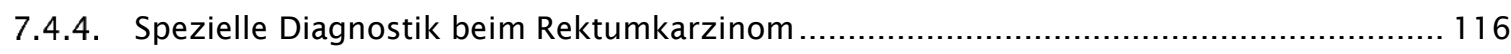

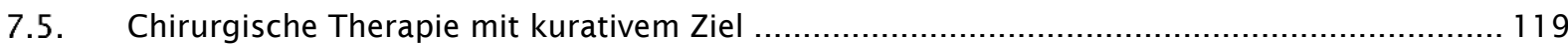

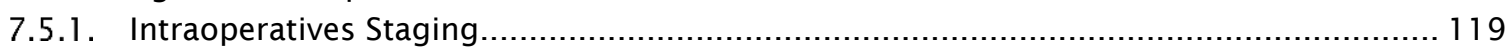

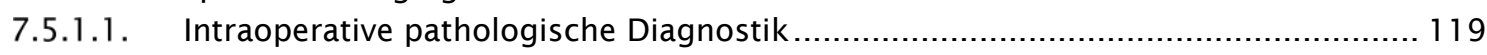

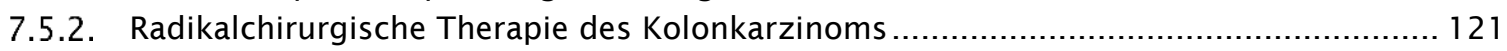

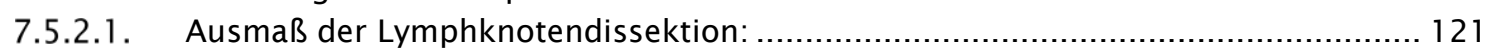

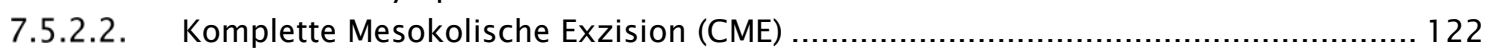

7.5.2.3. Lokalablative Verfahren bei Lebermetastasen ..................................................... 123

$\begin{array}{ll}\text { 7.5.2.3.1. Radiofrequenzablation (RFA) } & 123\end{array}$

$\begin{array}{lll}\text { 7.5.2.3.2. Selective Internal Radiation Therapy (SIRT) } & 124\end{array}$

$\begin{array}{ll}\text { 7.5.2.3.3. Laserinduzierte interstitielle Thermotherapie (LITT) } & 124\end{array}$

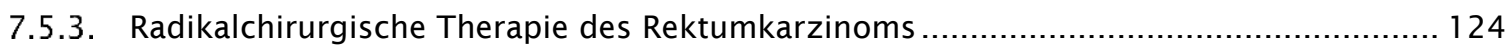

7.5.3.1. Allgemeine Onkologische Grundsätze........................................................ 125

7.5.3.2. Vorgehen bei Tumoren des oberen Rektumdrittels ........................................... 126

7.5.3.3. Vorgehen bei Tumoren des mittleren und unteren Rektumdrittels ........................ 126

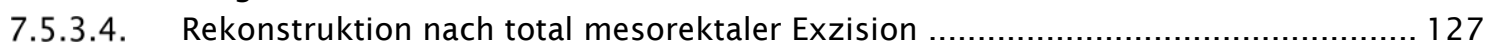

7.5.3.5. Entscheidung zum Sphinkter-Erhalt .......................................................... 129 
7.5.3.6. Vorgehen bei komplettem Response nach neoadjuvanter Therapie.................... 129

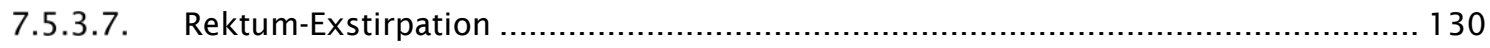

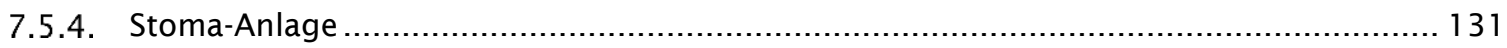

7.5.5. Lokale Operationsverfahren des Rektumkarzinoms ......................................... 132

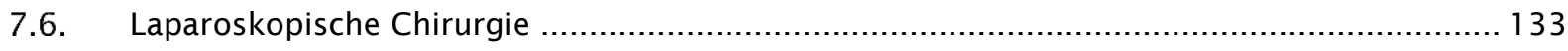

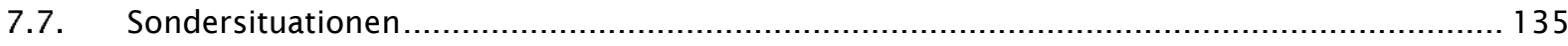

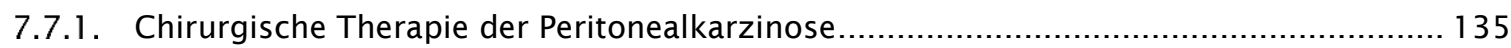

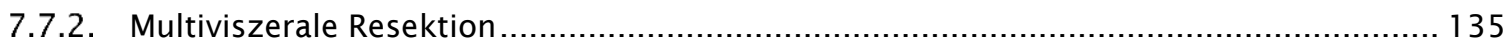

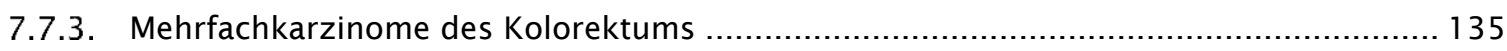

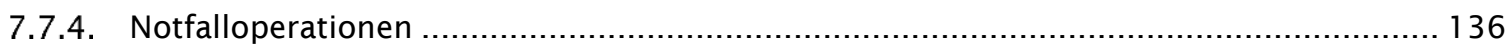

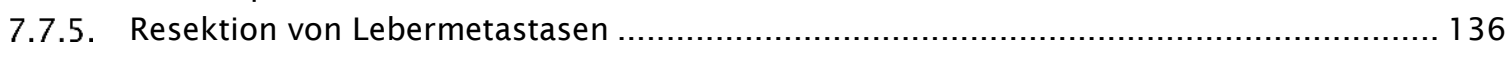

7.7.6. Ausgedehnte Fernmetastasierung und asymptomatischer Primärtumor .................... 137

7.7.7. Karzinome auf dem Boden eines hereditären kolorektalen Karzinoms ohne Polyposis

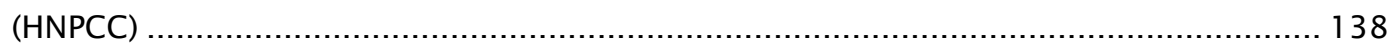

7.7.8. Karzinome auf dem Boden einer familiären adenomatösen Polyposis (FAP) ................. 139

7.7.9. Karzinome auf dem Boden einer Colitis ulcerosa .......................................... 139

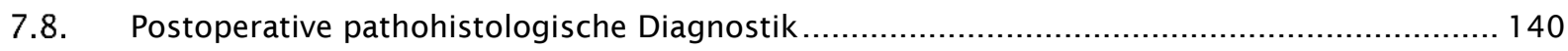

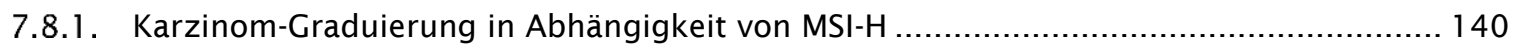

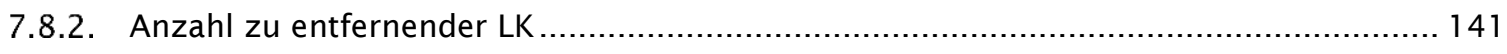

7.8.3. Bedeutung des Tumorabstands zur Resektionsfläche des Mesokolons beim

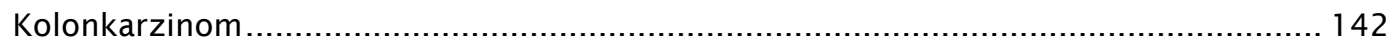

7.8.4. Bedeutung des Abstands vom zirkumferentiellen Resektionsrand (CRM-Klassifikation) beim Kolon- und Rektumkarzinom ............................................................... 143

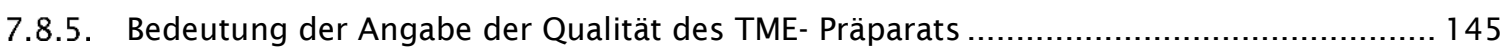

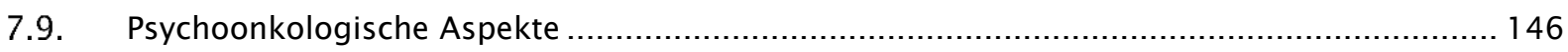

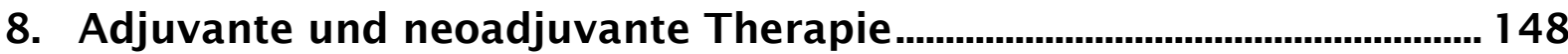

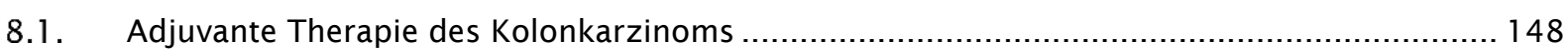

8.1.1. Indikation zur adjuvanten Behandlung bei Kolonkarzinomen ................................. 148

8.1.2. Altersbeschränkung für die Durchführung einer adjuvanten Chemotherapie ............... 148

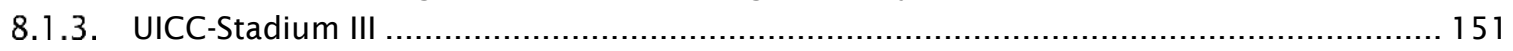

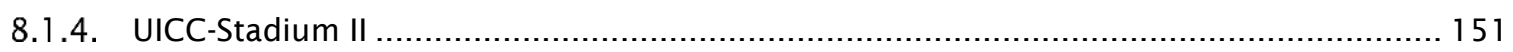

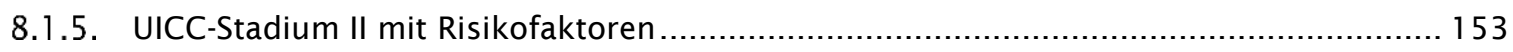

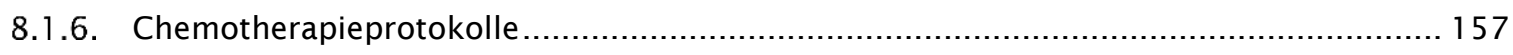

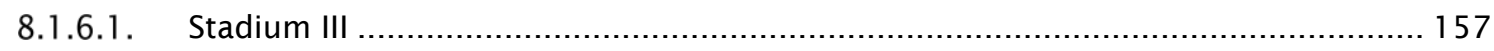

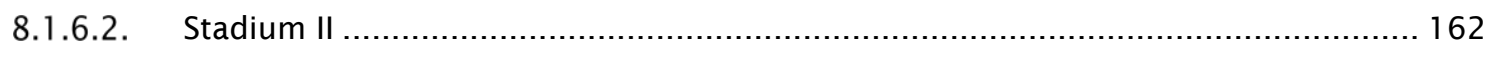

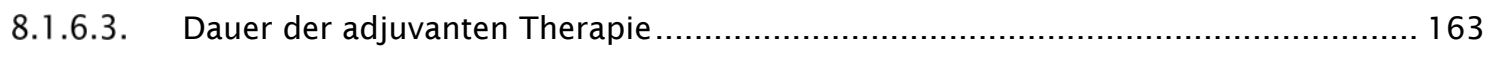

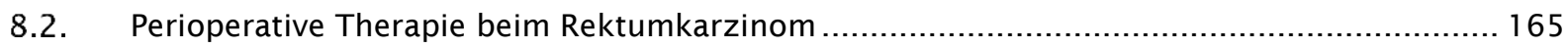

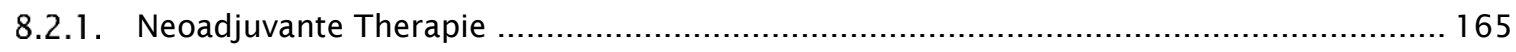

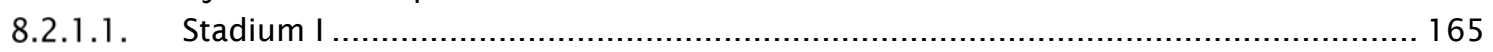

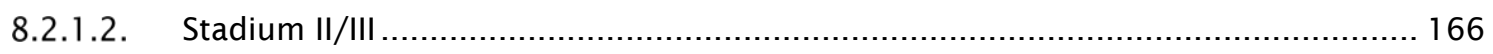

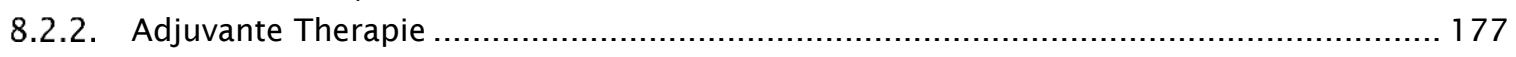

8.2.2.1. Adjuvante Therapie des Rektumkarzinoms bei primärer Operation (ohne

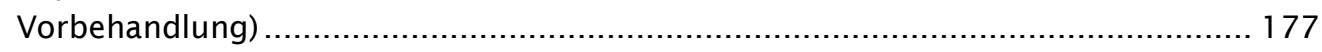

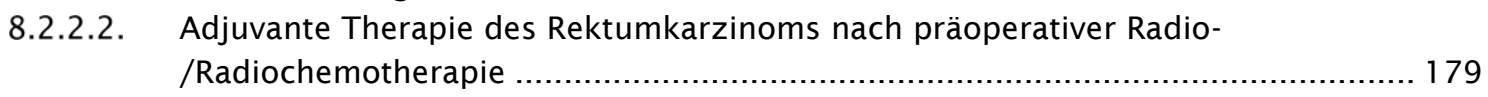




\section{Therapeutisches Vorgehen bei Metastasierung und in der

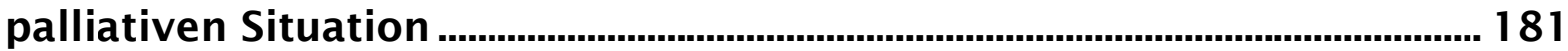

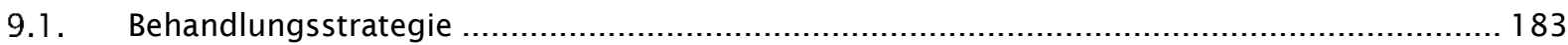

9.1.1. Zuordnung entsprechend des Allgemeinzustandes ........................................... 184

9.1.2. Zuordnung hinsichtlich der Krankheitsausdehnung ....................................... 184

9.1.3. Zuordnung hinsichtlich der Molekularbiologie des Tumors .................................. 184

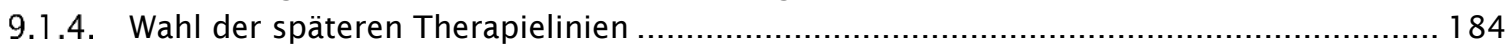

9.2. Initiale molekularbiologische Diagnostik vor Therapieeinleitung ............................... 185

9.2.1. (ALL) RAS- und BRAF-Diagnostik vor Beginn der Erstlinientherapie........................ 185

9.2.1.1.1. Bestimmung des RAS Mutationsstatus 186

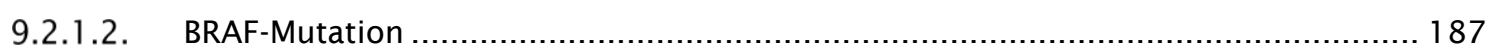

9.3. Pharmakogenetische Diagnostik vor Beginn der Erstlinientherapie ................................. 188

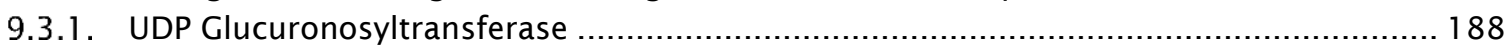

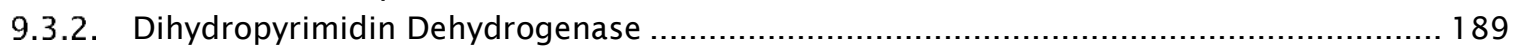

9.4. Diagnostik ohne unmittelbare Relevanz für die Erstlinientherapie................................ 189

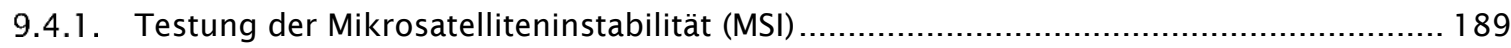

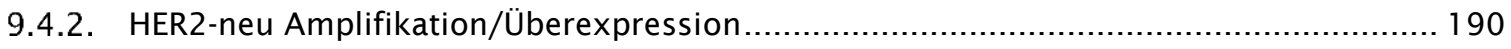

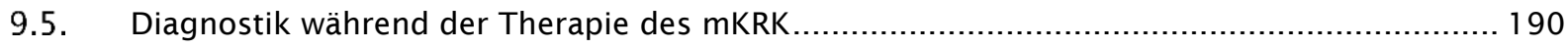

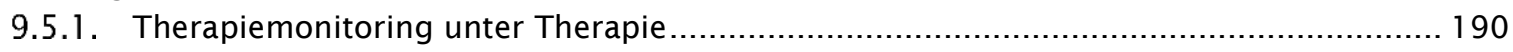

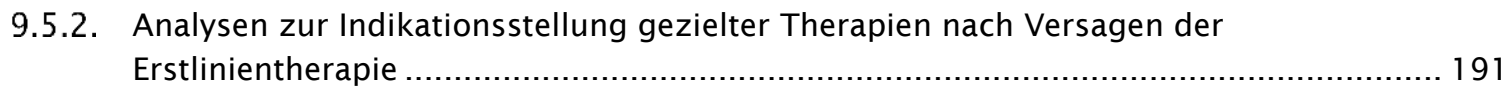

9.6. Behandlung von Patienten, die nicht für eine intensive Therapie in Frage kommen ............. 191

9.6.1. Primär resektable Erkrankung bei reduziertem Allgemeinzustand ........................... 191

9.6.2. Primär nicht resektable Erkrankung bei reduziertem Allgemeinzustand .................... 192

9.6.3. Schlechter Allgemeinzustand aufgrund der Tumorerkrankung ............................. 192

9.7. Behandlung von Patienten, die für eine intensive Therapie geeignet sind......................... 192

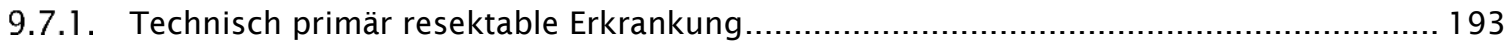

9.7.1.1. Primär resektable Erkrankung bei onkologisch günstiger Prognose ................... 193

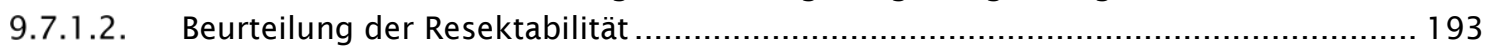

9.7.1.3. Primär resektable Erkrankung bei prognostisch ungünstigen Kriterien ................ 194

9.7.1.4. Optimaler Zeitpunkt der Resektion ......................................................... 194

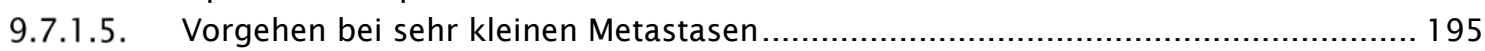

9.7.1.6. Neoadjuvante Therapie resektabler Lebermetastasen ...................................... 195

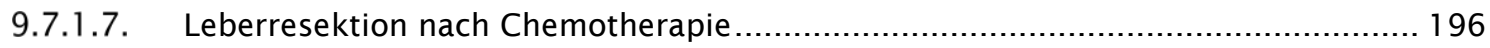

9.7.1.8. Adjuvante / additive Therapie nach Resektion von Lebermetastasen ................... 197

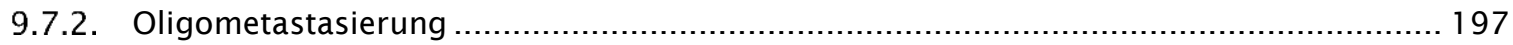

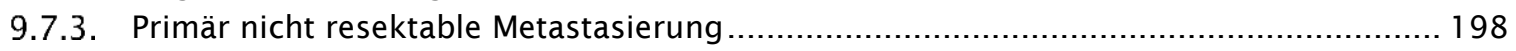

9.8. Wahl der systemischen Therapie in Abhängigkeit von der molekularpathologischen

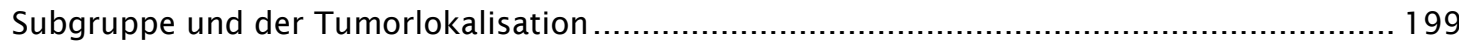

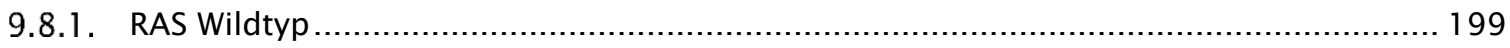

9.8.2. Relevanz der Tumorlokalisation in der Behandlung von RAS Wildtyp Tumoren .............. 200

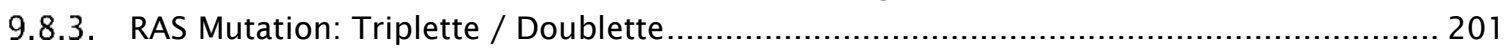

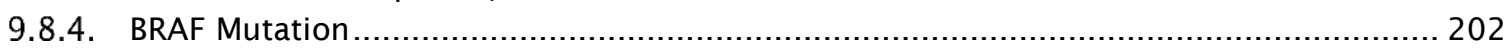

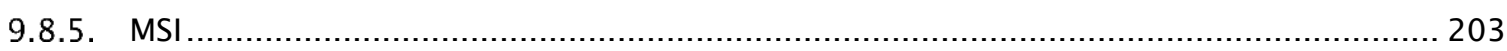

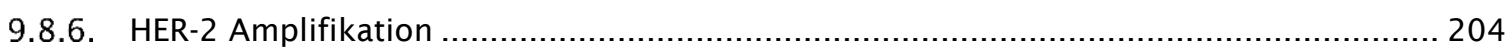

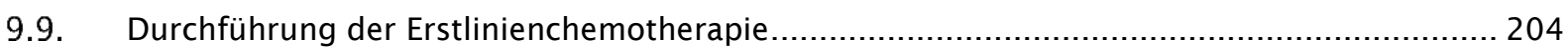

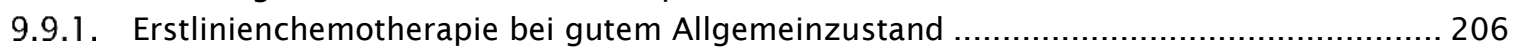


9.9.2. Erstlinienchemotherapie bei herabgesetztem Allgemeinzustand ab ECOG 2 _............. 206

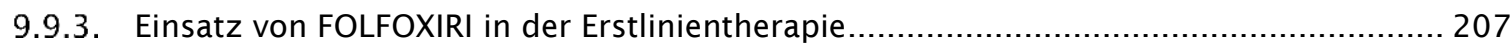

9.9.4. Kombination von Chemotherapie mit anti-EGFR Substanzen............................... 208

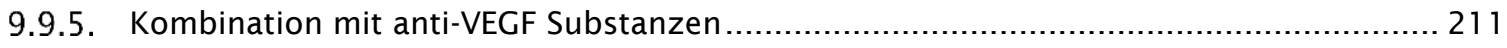

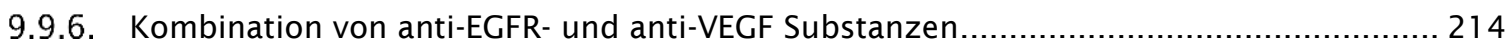

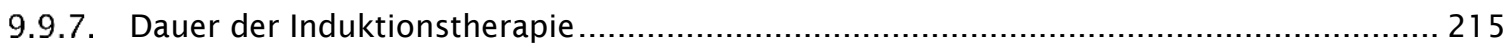

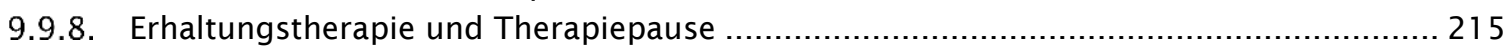

9.9.8.1. Erhaltungstherapie vs Fortführung der Induktionstherapie ............................ 216

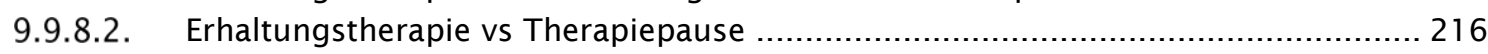

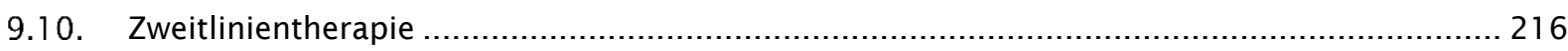

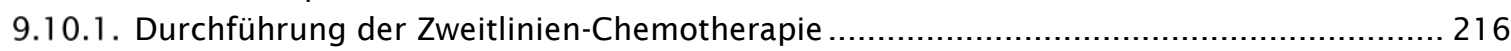

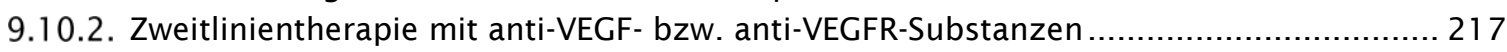

9.10.3. Zweitlinientherapie mit anti-EGFR Substanzen .................................................. 218

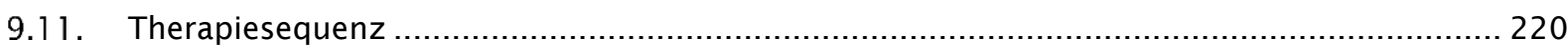

9.11.1.1. Fortführung der anti-VEGF Therapie in der Zweitlinienbehandlung.......................220

9.11.1.2. Fortführung der anti-EGFR Therapie in der Zweitlinienbehandlung.....................221

9.11.1.3. Sequenzieller Einsatz von anti-EGFR und anti-VEGF-Therapie .......................... 221

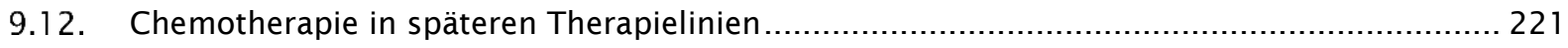

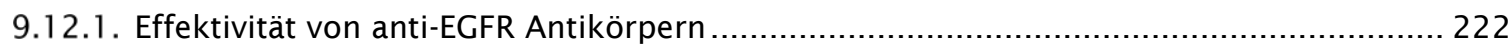

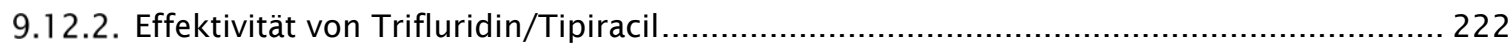

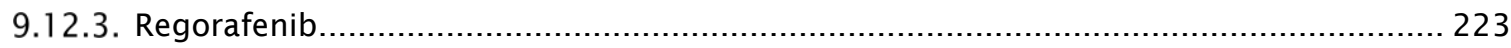

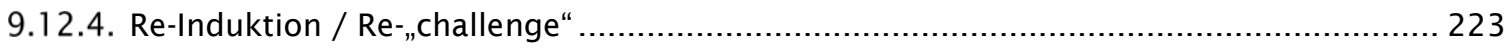

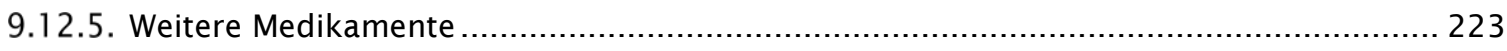

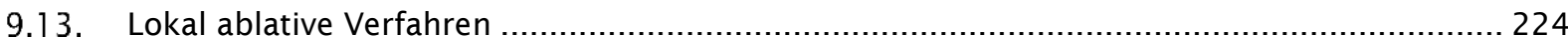

9.13.1. Lokal ablative Verfahren zur Behandlung von Lebermetastasen............................... 225

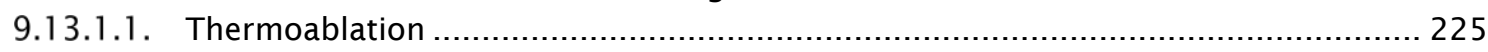

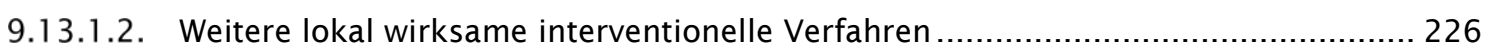

$\begin{array}{ll}\text { 9.13.1.2.1. SBRT } & 226\end{array}$

9.13.1.2.2. Brachytherapie 226

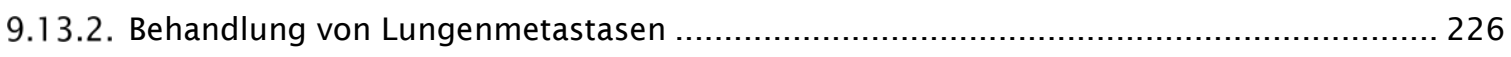

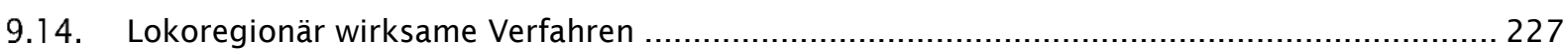

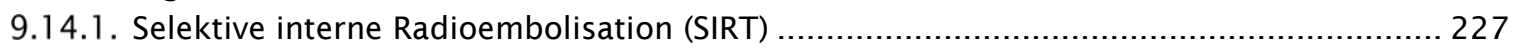

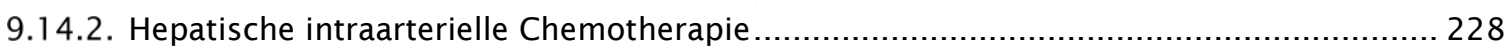

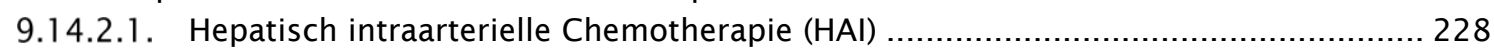

9.14.2.2. Einsatz von Irinotecan-beladenen Microbeads ........................................... 228

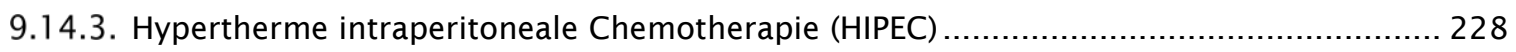

9.15. Therapiebegleitendes interprofessionelles Management von Symptomen, Nebenwirkungen und Toxizitäten

10. Nachsorge

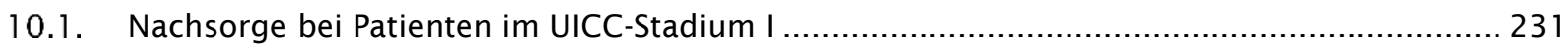

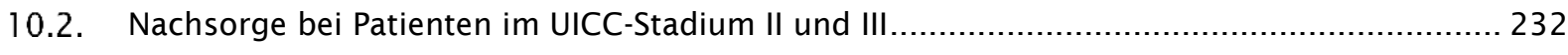

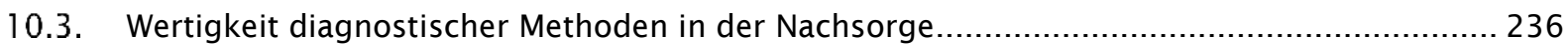

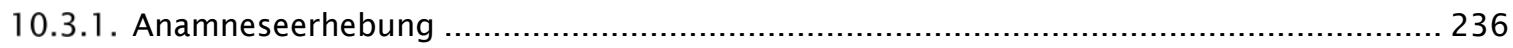

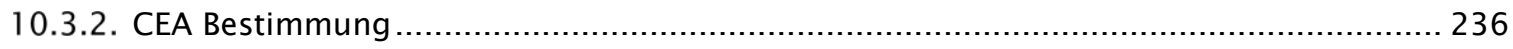

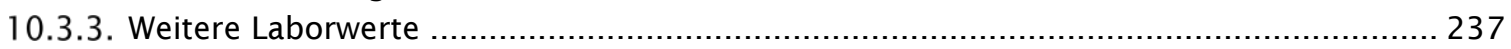


10.3.4. FOBT

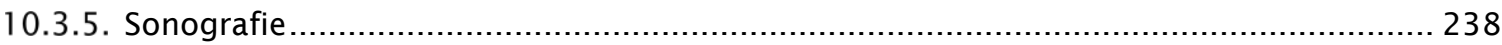

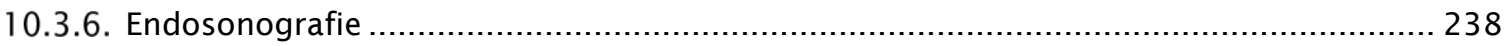

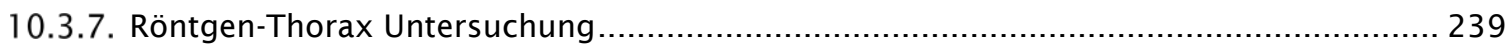

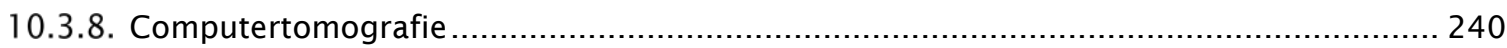

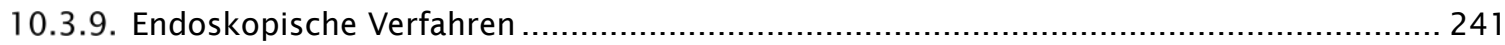

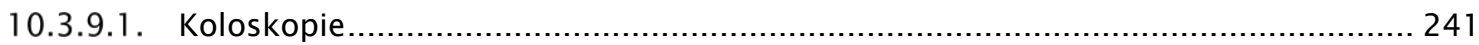

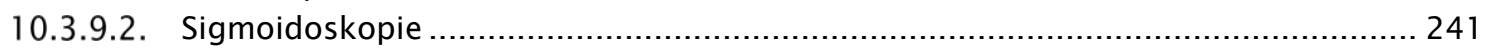

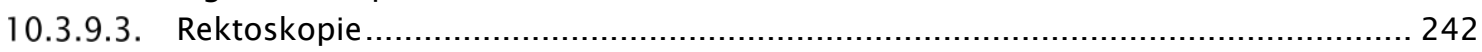

10.3.10. Kolonkontrasteinlauf, virtuelle Kolonografie und PET .................................. 243

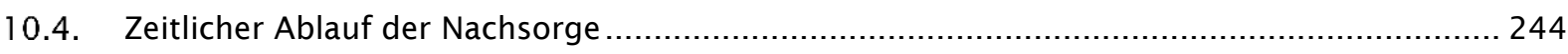

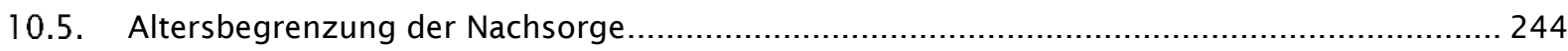

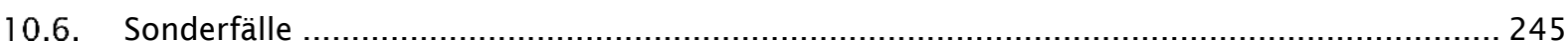

10.7. Rehabilitation nach Resektion eines kolorektalen Karzinoms .................................... 246

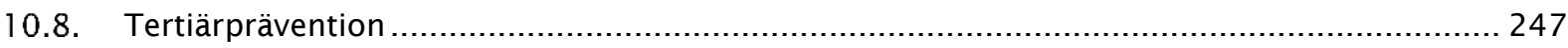

10.8.1. Versorgungskontinuität und Fortführung der gesundheitsförderlichen Aktivitäten nach

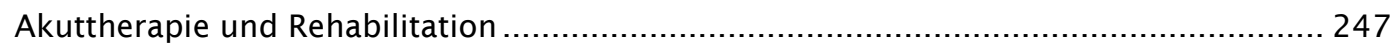

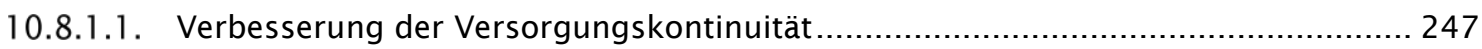

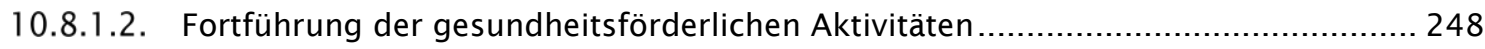

10.8.2. Tertiärprävention: Physikalische Aktivität, Ernährung sowie komplementäre und

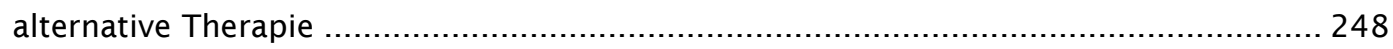

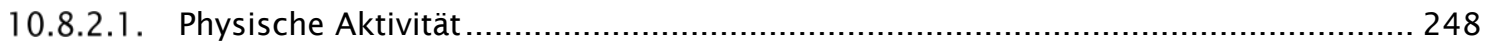

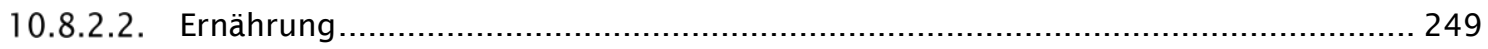

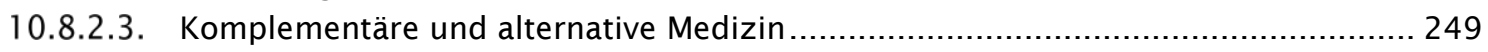

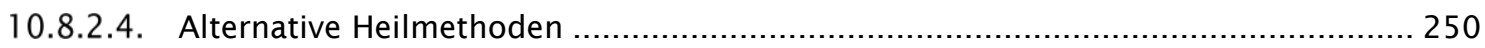

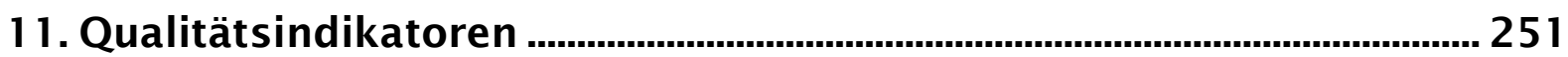

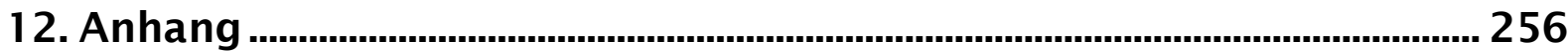

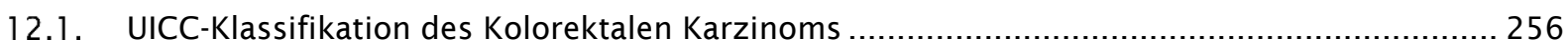

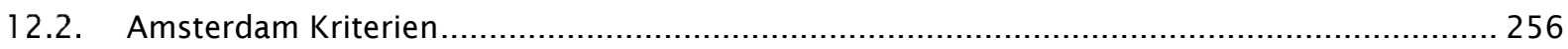

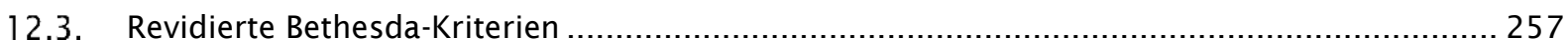

12.4. Änderungen der Empfehlungen durch Aktualisierung 2017 und 2018 (Versionen 2.0 und 2.1).

13. Abbildungsverzeichnis

14. Tabellenverzeichnis 


\section{Informationen zu dieser Leitlinie}

\subsection{Herausgeber}

Leitlinienprogramm Onkologie der Arbeitsgemeinschaft der Wissenschaftlichen Medizinischen Fachgesellschaften e. V. (AWMF), Deutschen Krebsgesellschaft e.V. und Deutschen Krebshilfe.

\subsection{Federführende Fachgesellschaft}

Deutsche Gesellschaft für Gastroenterologie, Verdauungs- und Stoffwechselkrankheiten (DGVS)

\section{DGVS}

Deutsche Gesellschaft für

Gastroenterologie,

Verdauungs- und

Stoffwechselkrankheiten

\subsection{Finanzierung der Leitlinie}

Diese Leitlinie wurde von der Deutschen Krebshilfe im Rahmen des Onkologischen Leitlinienprogramms gefördert.

1.4. Kontakt

Leitlinienprogramm Onkologie Office

c/o Deutsche Krebsgesellschaft e.V.

Kuno-Fischer Str. 8

14057 Berlin

leitlinienprogramm@krebsgesellschaft.de

www.leitlinienprogramm-onkologie.de

\subsection{Zitierweise}

Leitlinienprogramm Onkologie (Deutsche Krebsgesellschaft, Deutsche Krebshilfe, AWMF): S3-Leitlinie Kolorektales Karzinom, Langversion 2.1, 2019, AWMF Registrierungsnummer: 021/0070L, http://www.leitlinienprogrammonkologie.de/leitlinien/kolorektales-karzinom/ [abgerufen am: TT.MM.JJJ] 


\subsection{Besonderer Hinweis}

Die Medizin unterliegt einem fortwährenden Entwicklungsprozess, so dass alle Angaben, ins-besondere zu diagnostischen und therapeutischen Verfahren, immer nur dem Wissensstand zurzeit der Drucklegung der Leitlinie entsprechen können. Hinsichtlich der angegebenen Empfehlungen zur Therapie und der Auswahl sowie Dosierung von Medikamenten wurde die größtmögliche Sorgfalt beachtet. Gleichwohl werden die Benutzer aufgefordert, die Beipackzettel und Fachinformationen der Hersteller zur Kontrolle heranzuziehen und im Zweifelsfall einen Spezialisten zu konsultieren. Fragliche Unstimmigkeiten sollen bitte im allgemeinen Interesse der OL-Redaktion mitgeteilt werden.

Der Benutzer selbst bleibt verantwortlich für jede diagnostische und therapeutische Applikation, Medikation und Dosierung.

In dieser Leitlinie sind eingetragene Warenzeichen (geschützte Warennamen) nicht besonders kenntlich gemacht. Es kann also aus dem Fehlen eines entsprechenden Hinweises nicht geschlossen werden, dass es sich um einen freien Warennamen handelt.

Das Werk ist in allen seinen Teilen urheberrechtlich geschützt. Jede Verwertung außerhalb der Bestimmung des Urhebergesetzes ist ohne schriftliche Zustimmung des Leitlinienprogramms Onkologie (OL) unzulässig und strafbar. Kein Teil des Werkes darf in irgendeiner Form ohne schriftliche Genehmigung des OL reproduziert werden. Dies gilt insbesondere für Vervielfältigungen, Übersetzungen, Mikroverfilmungen und die Einspeicherung, Nutzung und Verwertung in elektronischen Systemen, Intranets und dem Internet.

\subsection{Ziele des Leitlinienprogramms Onkologie}

Die Arbeitsgemeinschaft der Wissenschaftlichen Medizinischen Fachgesellschaften e.V., die Deutsche Krebsgesellschaft e.V. und die Deutsche Krebshilfe haben sich mit dem Leitlinienprogramm Onkologie $(\mathrm{OL})$ das Ziel gesetzt, gemeinsam die Entwicklung und Fortschreibung und den Einsatz wissenschaftlich begründeter und praktikabler Leitlinien in der Onkologie zu fördern und zu unterstützen. Die Basis dieses Programms beruht auf den medizinisch-wissenschaftlichen Erkenntnissen der Fachgesellschaften und der DKG, dem Konsens der medizinischen Fachexperten, Anwender und Patienten sowie auf dem Regelwerk für die Leitlinienerstellung der AWMF und der fachlichen Unterstützung und Finanzierung durch die Deutsche Krebshilfe. Um den aktuellen Stand des medizinischen Wissens abzubilden und den medizinischen Fortschritt zu berücksichtigen, müssen Leitlinien regelmäßig überprüft und fortgeschrieben werden. Die Anwendung des AWMF-Regelwerks soll hierbei Grundlage zur Entwicklung qualitativ hochwertiger onkologischer Leitlinien sein. Da Leitlinien ein wichtiges Instrument der Qualitätssicherung und des Qualitäts-managements in der Onkologie darstellen, sollten sie gezielt und nachhaltig in den Versorgungsalltag eingebracht werden. So sind aktive Implementierungsmaßnahmen und auch Evaluationsprogramme ein wichtiger Bestandteil der Förderung des Leitlinienprogramms Onkologie. Ziel des Programms ist es, in Deutschland profes-sionelle und mittelfristig finanziell gesicherte Voraussetzungen für die Entwicklung und Bereitstellung hochwertiger Leitlinien zu schaffen. Denn diese hochwertigen Leitlinien dienen nicht nur dem strukturierten Wissenstransfer, sondern können auch in der Gestaltung der Strukturen des Gesundheitssystems ihren Platz finden. Zu erwähnen sind hier evidenzbasierte Leitlinien 
als Grundlage zum Erstellen und Aktualisieren von Disease Management Programmen oder die Verwendung von aus Leitlinien extrahierten Qualitätsindikatoren im Rahmen der Zertifizierung von Organtumor-zentren.

\subsection{Verfügbare Dokumente zur Leitlinie und Implementierung}

Bei diesem Dokument handelt es sich um die Langversion der S3-Leitlinie Kolorektales Karzinom, welche über die folgenden Seiten zugänglich ist:

- Leitlinienprogramm Onkologie (http://www.leitlinienprogrammonkologie.de/leitlinien/kolorektales-karzinom/)

- AWMF (http://www.awmf.org/leitlinien/detail/II/021-0070L.html)

- Beteiligte Fachgesellschaften (z. B. https://www.dgvs.de/wissenkompakt/leitlinien/leitlinien-der-dgvs/)

- Guidelines International Network (www.g-i-n.net)

Darüber hinaus wird die Langversion dieser Leitlinie in der Zeitschrift für Gastroenterologie veröffentlicht werden.

Neben der Langversion gibt es folgende ergänzende Dokumente zu dieser Leitlinie:

- Kurzversion

- Drei Laienversionen (Patientenleitlinien) zu den Themen Früherkennung, frühes Stadium und fortgeschrittenes Stadium (werden derzeit aktualisiert)

- Leitlinienreport

- Englische Version (wird derzeit aktualisiert)

- Separate Evidenzberichte bzw. Publikationen (Vorsorge, Früherkennung, präoperative Diagnistik, Therapeutisches Vorgehen bei Metastasierung und in der palliativen Situation: Analyse Einsatz von Angiogenesehemmern und antiEGFR-Antikörpern bei Patienten mit metastasiertem KRK)

Alle diese Dokumente werden ebenfalls auf den oben genannten Homepages abrufbar sein. 


\subsection{Zusammensetzung der Leitliniengruppe}

\subsubsection{Koordination und Redaktion}

Prof. Dr. Wolff Schmiegel (Bochum) und PD Dr. Christian Pox (Bremen)

Leitliniensekretariat: Jutta Thurn (Bochum)

\subsubsection{Beteiligte Fachgesellschaften und Autoren}

In Tabelle 1 sind die an der Ersterstellung und Aktualisierung beteiligten Fachgesellschaften und anderen Organisationen sowie die jeweils benannten Fachexperten/Fachexpertinnen aufgelistet. In Tabelle 2 sind die Mitglieder der jeweiligen Arbeitsgruppen aufgelistet.

Tabelle 1: Beteiligte Fachgesellschaften und Organisationen

\section{Beteiligte Fachgesellschaften und Organisationen}

Arbeitsgemeinschaft „Supportive Maßnahmen in der Onkologie, Rehabilitation und Sozialmedizin" in der DKG (ASORS)

Arbeitsgemeinschaft Deutscher Tumorzentren (ADT)

Arbeitsgemeinschaft für Psychoonkologie in der DKG (PSO)

Arbeitsgemeinschaft Internistische Onkologie in der DKG (AIO)

Arbeitsgemeinschaft Konferenz Onkologische Kranken- und Kinderkrankenpflege in der DKG (KOK)

Arbeitsgemeinschaft Onkologische Pathologie in der DKG (AOP)

Arbeitsgemeinschaft Onkologische Pharmazie in der DKG (OPH)

Arbeitsgemeinschaft Prävention und integrative Medizin in der Onkologie in der DKG (PRIO)

Arbeitsgemeinschaft Radiologische Onkologie in der DKG (ARO)

Arbeitsgmeinschaft Bildgebung und Radioonkologie in der DKG $(\mathrm{ABO})$

Berufsverband Niedergelassener Gastroenterologen

Deutschlands (bng)

Bundesverband der Niedergelassenen Hämatologen und Onkologen in Deutschland (BNHO)

\section{Mandatsträger/beteiligte} Experten

J. Körber*, R. Caspari (Vertr.) ***, H. Link*

H. Barlag $* * *$

P. Heußner

M. Geissler ${ }^{* * *}$, R.-D. Hofheinz ${ }^{* * *}$,

S. Stintzing***, V. Heinemann***

D. Arnold $* * *$ S. HegewischBecker ${ }^{* * *}$, C. - H. Köhne ${ }^{* * *}$

M. Landenberger*

G. Baretton*

M. Höckel $* * *$

J. Hübner**

H. A. Wolff****

J. Menke $* * *$

A. Theilmeier*, B. Bokemeyer ${ }^{* * *}$

M. J. Eckart**** 


\section{Beteiligte Fachgesellschaften und Organisationen}

Bundesverband Deutscher Pathologen (BDP)

Chirurgische Arbeitsgemeinschaft für Colo-Proktologie in der DGAV (CACP)

Chirurgische Arbeitsgemeinschaft für Minimal Invasive Chirurgie in der DGAV (CAMIC)

Chirurgische Arbeitsgemeinschaft für Onkologie in der DGAV (CAO-V ()

Chirurgische Arbeitsgemeinschaft Onkololgie in der DKG (CAO)

Deutsche Gesellschaft für Allgemein- und Viszeralchirurgie (DGAV)

Deutsche Gesellschaft für Allgemeinmedizin und Familienmedizin (DEGAM)

Deutsche Gesellschaft für Chirurgie (DGCH)

Deutsche Gesellschaft für Ernährungsmedizin (DGEM)

Deutsche Gesellschaft für Hämatologie und Onkologie (DGHO)

Deutsche Gesellschaft für Humangenetik (GfH)

Deutsche Gesellschaft für Innere Medizin (DGIM)

Deutsche Gesellschaft für interventionelle Radiologie und minimal-invasive Therapie (DeGIR)

Deutsche Gesellschaft für Klinische Chemie und Laboratoriumsmedizin (DGKL)

Deutsche Gesellschaft für Koloproktologie (DGK)

Deutsche Gesellschaft für Nuklearmedizin (DGN)

\section{Mandatsträger/beteiligte} Experten

C. Wittekind**

S. Post***

M. Walz**

H.-R. Raab***, H. Lang*, J. Weitz**, M. Sailer**

\section{T. Germer**}

A. Glitsch***, C. T. Germer***, W. Hohenberger**, M. Anthuber**, W. Bechstein ${ }^{* *}, \mathrm{~K}-\mathrm{W}$. Jauch**, K-H. Link ${ }^{* *}, H-R$. Raab**

J.-F. Chenot ${ }^{* * *}$, G. Egidi (Vertr) $)^{* * *}$

W. Hohenberger ${ }^{* * *}$, H.-R. Raab ${ }^{* * *}$

S. C. Bischoff*** J. Ockenga**, W. Scheppach**

M. Geissler ${ }^{* * *}$, R.-D. Hofheinz $z^{* * *}$, S. Stintzing $* * *$, V. Heinemann $* * *$, D. Arnold***, S. HegewischBecker ${ }^{* * *}$, C. - H. Köhne $* * *$, M. Heike ${ }^{* *}$, T. Höhler**

N. Rahner*** J. Epplen***

T. Seufferlein $* * *$ J.F. Riemann $* *$

P. L. Pereira***

S. Holdenrieder ${ }^{* * *}, M$. Neumaier $* * *$ C. Wagener $* *$

W. Hohenberger $* * *$

H. Amthauer ${ }^{* * *}, K$ K. Scheidhauer**, H. Ahmadzadehfar*** 


\section{Beteiligte Fachgesellschaften und Organisationen}

Deutsche Gesellschaft für Pathologie (DGP)

Deutsche Gesellschaft für Radioonkologie (DEGRO)

Deutsche Gesellschaft für Rehabilitationswissenschaften (DGRW)

Deutsche Gesellschaft Gastroenterologie, Verdauungs- und Stoffwechselkrankheiten (DGVS)

Deutsche Morbus Crohn/Colitis Ulcerosa Vereinigung (DCCV)

Deutsche Röntgengesellschaft (DRG)

Deutscher Hausärzteverband (HÄV)

Eingeladene Fachexperten (ohne Stimmrecht)

Felix-Burda-Stiftung

Institut für angewandte Qualitätsförderung und Forschung im Gesundheitswesen (AQUA)

Stiftung Lebensblicke

Vereinigung für Stomaträger und für Menschen mit Darmkrebs (Deutsche ILCO)

Zentralinstitut der Kassenärztlichen Versorgung in der BRD (ZI)

\section{Mandatsträger/beteiligte} Experten
A. Tannapfel*, A. Jung $* * *$,
T. Kirchner*, P. Schirmacher***,
G. Baretton*, C. Wittekind**
C. Rödel*, W. Budach ${ }^{* * * *}$,
H. Schmidberger ${ }^{* * *}$, R. Sauer**

\section{J. Körber****}

R. Kiesslich $* * *$, W. Schmitt***, F. Kolligs*, H. Neuhaus***, C. Pox*, T. Rösch***, J. Trojan****, R. Porschen*, G. Folprecht ${ }^{* * *}$, U. Graeven*, M. Ebert ${ }^{* * *}$, W. Schmiegel*, T. Seufferlein***, J.F. Riemann**, S. C. Bischoff**, J. Ockenga**, W. Scheppach**, A. Sieg***, K. Schulmann"**, B. Bokemeyer $* *$ U. Melle**, A. Reinacher-Schick**, A. Holstege ${ }^{* * *}$

C. Witte**

A. Schreyer***, T. J. Vogl*, C. Stroszczynski (Vertr)****, H-J. Brambs**, P. L. Pereira**

P. Engeser**

H.Brenner**, P. Lux ${ }^{* *}$

C. Maar**

S. Ludt**

J.F. Riemann***

M. Hass*

L. Altenhofen**

Zeitraum der Beteiligung

$*=2011-2017$ (Version 1 un d 2);

$* *=2011-2012($ Version 1)

$* * *=2013-2017$ (Version 2) 
Darüber hinaus wurde die Aktualisierung der Leitlinie 2017 in Zusammenarbeit mit der DGP (Deutsche Gesellschaft für Palliativmedizin) vorgenommen.

Tabelle 2: Mitglieder der Arbeitsgruppen

\section{Arbeitsgruppe}

Kapitel 3:

Prävention asymptomatische

Bevölkerung

Kapitel 4:

Früherkennung/ Vorsorge

asymptomatische

Bevölkerung

Kapitel 5:

Risikogruppen

Kapitel 6:

Endoskopie: Durchführung

und Polypenmanagement

Kapitel 7:

Präoperative Diagnostik und

Chirurgie

Kapitel 8:

Adjuvante und neoadjuvante

Therapie

Kapitel 9:

Therapeutisches Vorgehen bei Metastasierung und in

der palliativen Situation

Kapitel 10

Nachsorge

Qualitätsindikatoren

\section{Mitglieder der Arbeitsgruppe (AG-Leiter fett markiert)}

J.F. Riemann, S. C. Bischoff, F. Kolligs, J. Ockenga, W. Scheppach

C. Pox, A. Sieg, L. Altenhofen, H-J. Brambs, H. Brenner, P. Engeser, A. Theilmeier

N. Rahner, K. Schulmann, G. Baretton, B. Bokemeyer, J. Epplen, U. Melle, R. Porschen, J. Weitz, C. Witte

T. Rösch, W. Schmitt, G. Baretton, A. Glitsch, R. Kiesslich, F. Kolligs, H. Neuhaus, C. Pox, A. Schreyer, A. Tannapfel, A. Theilmeier, J. Trojan

W. Hohenberger, S. Post, M. Anthuber, W. Bechstein, U. Graeven, M. Hass, M. Heike, K-W. Jauch, T. Kirchner, H. Lang, K-H. Link, P. Pereira, H-R. Raab, A. Reinacher-Schick, C. Rödel, M. Sailer, R. Sauer, K. Scheidhauer, A. Tannapfel, T. Vogl, C. Wagener, M. Walz, C. Wittekind

C. Rödel, R. Porschen, W. Budach, G. Folprecht, M. Geissler, R.-D. Hofheinz, W. Hohenberger, S. Holdenrieder, J. Körber, J. Menke, H.-R. Raab, H. Schmidberger, S. Stintzing

V. Heinemann, U. Graeven, H. Amthauer, D. Arnold, R. Caspari, J.-F. Chenot, M. Ebert, M. J. Eckart, G. Egidi, C. T. Germer, M. Hass, S. Hegewisch-Becker, M. Höckel, A. Jung, T. Kirchner, C.-H. Köhne, M. Landenberger, H. Lang, H. Link, M. Neumaier, P. L. Pereira, P. Schirmacher, W. Schmiegel, T. Seufferlein, C. Stroszczynski, T. J. Vogl, H. A. Wolff

A. Holstege, P. Heußner, T. Höhler, J. Hübner, J. Körber, M. Landenberger, H. Link

S. Wesselmann, T. Langer, H. Ahmadzadehfar, D. Arnold, G. Baretton, H. Barlag, M. Ebert, M. Hass, V. Heinemann, W. Hohenberger, T. Kirchner, C.H. Köhne, F. Kolligs, M. Nothacker

\subsubsection{Patientenbeteiligung}

Die Leitlinie wurde unter direkter Beteiligung von Patientenvertretern erstellt. Frau Maria Hass (Deutsche ILCO) sowie C. Witte (DCCV) waren an der Aktualisierung der 
Leitlinie beteiligt und nahmen mit eigenem Stimmrecht an den Konsensuskonferenzen teil.

\subsubsection{Methodische Begleitung}

durch das Leitlinienprogramm Onkologie

- $\quad$ Prof. Dr. Ina Kopp (AWMF), Marburg (2011/2012)

- Dr. Monika Nothacker, MPH (AWMF), Berlin (2013-2018)

- Dr. Markus Follmann, MPH MSc (DKG), Berlin (2011-2018)

- $\quad$ Dipl. Soz.Wiss. Thomas Langer (DKG), Berlin (2013-2018)

Durch externe Auftragnehmer:

- Dr. Barbara Buchberger, MPH, Laura Krabbe, M.A., Dr. Beate Lux, MPH (Essener Forschungsinstitut für Medizinmanagement $\mathrm{GmbH}$ ): Evidenzbericht für Version 2, siehe Kapitel 1.8)

- Dr. med. Simone Wesselmann, MBA (Aktualisierung der Qualitätsindikatoren)

- Dr. Barbara Buchberger, Dr. Romy Heymann (Alfried Krupp von Bohlen und Halbach-Stiftungslehrstuhl für Medizinmanagement): Evidenzbericht für Version 1, siehe Kapitel 1.8)

- Dr. Michaela Eikermann, Christoph Mosch, Thomas Jaschinski, Monika Becker (Institut für Forschung in der Operativen Medizin [IFOM]): Evidenzbericht für Version 1, siehe Kapitel 1.8)

\subsection{Verwendete Abkürzungen}

\begin{tabular}{|l|l|}
\hline Abkürzung & Erläuterung \\
\hline AFAP & Attenuierte FAP \\
\hline ADR & Adenomdetektionsrate \\
\hline AHB & Anschlussheilbehandlung \\
\hline ASS & Acetylsalicylsäure \\
\hline AWMF & Arbeitsgemeinschaft der Wissenschaftlichen Medizinischen Fachgesellschaften \\
\hline BMI & Body Mass Index \\
\hline BSC & Best supportive Care \\
\hline CEA & Karzinoembryonales Antigen \\
\hline CT & Computer Tomographie \\
\hline CTC & CT-Kolonographie \\
\hline CU & Colitis Ulcerosa \\
\hline DGE & Deutsche Gesellschaft für Ernährung \\
\hline ESD & Endoskopische Mukosaresetkion \\
\hline
\end{tabular}




\begin{tabular}{|c|c|}
\hline Abkürzung & Erläuterung \\
\hline FAP & Familiäre Adenomatöse Polyposis \\
\hline FICE & Fujinon intelligent colour enhancement \\
\hline FOBT & Fäkaler Occulter Bluttest \\
\hline FS & Folinsäure \\
\hline HNPCC & Hereditäres Kolorektales Karzinom ohne Polyposis \\
\hline IEN & Intraepitheliale Neoplasie \\
\hline iFOBT/ FIT & Immunologischer FOBT \\
\hline IHC & Immunhistochemische Untersuchung \\
\hline KRK & Kolorektales Karzinom \\
\hline LITT & Laserinduzierte interstitielle Thermotherapie \\
\hline LL & Leitlinie \\
\hline MAP & MUTYH-assoziierte Polyposis \\
\hline MMR & Mismatch-repair Gen \\
\hline MSA & Mikrosatellitenanalyse \\
\hline MSCT & Mehrzeilen-CT \\
\hline MSI & Mikrosatelliteninstabilität \\
\hline MSI-H & Mikrosatelliteninstabilität hoch (high) \\
\hline MSI-L & Mikrosatelliteninstabilität gering (low) \\
\hline MSS & Mikrosatellitenstabilität \\
\hline $\mathrm{NBI}$ & Narrow Band Imaging \\
\hline ÖGD & Ösophagogastroduodenoskopie \\
\hline OL & Leitstelle Onkologie der DKG \\
\hline OR & Odds Ratio \\
\hline ORR & Overall response rate \\
\hline $\mathrm{PCl}$ & Peritoneal cancer index \\
\hline PET & Positron Emission Tomography \\
\hline PJS & Peutz-Jeghers-Syndrom \\
\hline
\end{tabular}




\begin{tabular}{|l|l|}
\hline Abkürzung & Erläuterung \\
\hline PSC & Primär sklerosierende Cholangitis \\
\hline RCT & Randomisierte kontrollierte Studie \\
\hline RFA & Radiofrequenzablation \\
\hline RR & Relatives Risiko \\
\hline RT & Radiotherapie \\
\hline SIRT & Selective Internal Radiation Therapy \\
\hline SR & Systematische Übersichtsarbeit \\
\hline SSA & Sessiles serratiertes Adenom \\
\hline TME & Totale Mesorektumexzision \\
\hline TSA & Traditionelles serratiertes Adenom \\
\hline WHO & Weltgesundheitsorganisation \\
\hline
\end{tabular}




\section{Einführung}

\subsection{Geltungsbereich und Zweck}

\subsubsection{Zielsetzung und Fragestellung}

Das Kolorektale Karzinom (KRK) ist mit etwa 64.000 Neuerkrankungen und ca. 26.000 Todesfällen pro Jahr in Deutschland einer der häufigsten malignen Tumoren. 1999 wurde erstmalig von der DGVS in Zusammenarbeit mit der Deutschen Krebsgesellschaft eine S3-Leitlinie für das KRK veröffentlicht, die flächendeckend eine standardisiert hochwertige Patientenversorgung auf dem Boden evidenzbasierter Medizin erreichen sollte. Inzwischen ist auch eine europäische Leitlinie zum Kolorektalen Karzinom veröffentlicht worden. Die Leitlinienkommission sah die Notwendigkeit einer Spezifizierung aufgrund der ungleichen Gesundheitssysteme und Versorgungsstandards in den europäischen Ländern. Diese hier vorliegende deutsche Leitlinie deckt gegenüber der europäischen Leitlinie weitere Teilbereiche ab und ist bestrebt, alle auf Deutschland bezogenen Fragestellungen zu berücksichtigen.

Die Leitlinie ist in acht Themenkomplexe (TK) gegliedert:

- $\quad$ TK I: Prävention asymptomatische Bevölkerung (siehe Kapitel 3)

- $\quad$ TK II: Früherkennung/ Vorsorge asymptomatische Bevölkerung (siehe Kapitel 4)

- $\quad$ TK III: Risikogruppen (siehe Kapitel 5)

- $\quad$ TKIV: Endoskopie: Durchführung und Polypenmanagement (siehe Kapitel 6)

- $\quad$ TK V: Präoperative Diagnostik und Chirurgie (siehe Kapitel 7)

- $\quad$ TK VI: Adjuvante und neoadjuvante Therapie (siehe Kapitel 8)

- $\quad$ TK VII: Therapeutisches Vorgehen bei Metastasierung und in der palliativen Situation (siehe Kapitel 9)

- TK VIII: Nachsorge (siehe Kapitel 10)

Um die Empfehlungen auf dem neuesten Stand wissenschaftlicher Erkenntnisse zu halten, wird die Leitlinie seitdem in enger Zusammenarbeit mit der AWMF regelmäßig aktualisiert (2004 komplett, 2008 die Themenkomplexe IV, VI und VII und 2011/2012 die Themenkomplexe I, II, III, V und VIII sowie einzelne Abschnitte aus IV, VI und VII). Die aktuelle Überarbeitung betrifft die Themenkomplexe IV, VI und VII.

Im Aktualierungsprozess 2017 legte die Leitliniegruppe fest, dass u.a. zu folgenden Fragen Stellung genommen werden sollte:

- Wann sollte nach Abtragung von sessilen serratierten Adenomen eine Kontrollkoloskopie erfolgen?

- Wann sollte nach Abtragung von 1 oder 2 Adenomen $<1 \mathrm{~cm}$ ohne höhergradige intraepitheliale Neoplasie eine Kontrollkoloskopie erfolgen?

- Bis zu welchem zeitlichen Abstand nach einer Operation eines Kolonkarzinoms sollte eine adjuvante Chemotherapie begonnen werden? 
- Welcher Patient mit einem Rektumkarzinom sollte neoadjuvant behandelt werden?

- Besteht eine Indikation für eine adjuvante Chemotherapie nach neoadjuvanter Therapie des Rektumkarzinoms?

- Wie ist der Nutzen einer adjuvanten Chemotherapie nach R0-Resektion von Lebermetastasen?

- Wie ist der Nutzen einer Kombinationstherapie mit einem EGFR-Antikörper bzw. mit einem VEGF-pathway Inhibitor in der Firstline-Therapie?

Entsprechend dieser Fragestellungen wurden alle Empfehlungen auf Aktualität überprüft und gegebenenfalls nach Literaturrecherchen überarbeitet.

\subsubsection{Adressaten}

Diese Leitlinie richtet sich vorrangig an Ärztinnen und Ärzte, die in der Prävention und Behandlung des KRK im ambulanten und stationären Sektor tätig sind.

Darüber hinaus soll sie Kooperationspartner der Ärzteschaft (Fachbereiche im Gesundheitswesen), Berufsverbänden, Patienten- und Selbsthilfeorganisationen, Qualitätssicherungseinrichtungen und -projekte auf Bundes- und Länderebene (z.B. KoQK, ADT, IQWiG, GEKID, IQTIG), gesundheitspolitische Einrichtungen und Entscheidungsträger auf Bundes- und Länderebene, Zertifizierungseinrichtungen (z.B. OnkoZert), Kostenträger, sowie die (Fach-) Öffentlichkeit zur Information über gute medizinische Vorgehensweise dienen.

\subsubsection{Gültigkeitsdauer und Aktualität}

Die S3-Leitlinie ist bis zur nächsten Aktualisierung gültig. Die Gültigkeitsdauer der Leitlinie wird auf 5 Jahre geschätzt. Vorgesehen sind regelmäßige Aktualisierungen der gesamten Leitlinie, bei dringendem Änderungsbedarf können einzelne Empfehlungen/Themen überarbeitet werden.

In den Empfehlungskästen ist jeweils das Datum der letzten Überarbeitung (2008, 2013 oder 2017) aufgeführt.

Kommentare und Hinweise für den Aktualisierungsprozess sind ausdrücklich erwünscht und können an das Leitliniensekretariat adressiert werden:

Leitlinienkoordination: PD Dr. Christian P. Pox

Leitliniensekretariat

Medizinische Klinik der Ruhr-Universität Bochum

Knappschaftskrankenhaus

In der Schornau 23-25

44892 Bochum

meduni-kkh@rub.de 


\subsection{Grundlagen der Methodik}

Das methodische Vorgehen richtete sich nach dem AWMF-Regelwerk (http://www.awmfleitlinien.de) und ist im Leitlinienreport zu dieser Leitlinie dargelegt.

\subsubsection{Schema der Evidenzgraduierung nach Oxford}

Zur Klassifikation des Verzerrungsrisikos der identifizierten Studien wurde in dieser Leitlinie das in Tabelle 3 aufgeführte System des Oxford Centre for Evidence-based Medicine in der Version von 2009 (verfügbar unter www.cebm.net) verwendet. Dieses System sieht die Klassifikation der Studien für verschiedene klinische Fragestellungen (Nutzen von Therapie, prognostische Aussagekraft, diagnostische Wertigkeit) vor.

Tabelle 3: Schema der Evidenzgraduierung nach Oxford (Version 2009)

\begin{tabular}{|c|c|c|c|c|c|}
\hline Level & $\begin{array}{l}\text { Therapy / } \\
\text { Prevention, } \\
\text { Aetiology / } \\
\text { Harm }\end{array}$ & Prognosis & Diagnosis & $\begin{array}{l}\text { Differential } \\
\text { diagnosis / } \\
\text { symptom } \\
\text { prevalence } \\
\text { study }\end{array}$ & $\begin{array}{l}\text { Economic and } \\
\text { decision analyses }\end{array}$ \\
\hline $1 \mathrm{a}$ & $\begin{array}{l}\text { SR (with } \\
\text { homogeneity) of } \\
\text { RCTs }\end{array}$ & $\begin{array}{l}\text { SR (with } \\
\text { homogeneity) } \\
\text { inception cohort } \\
\text { studies; CDR } \\
\text { validated in } \\
\text { different } \\
\text { populations }\end{array}$ & $\begin{array}{l}\text { SR (with } \\
\text { homogeneity) of } \\
\text { Level 1 diagnostic } \\
\text { studies; CDR with } \\
1 \text { b studies from } \\
\text { different clinical } \\
\text { centers }\end{array}$ & $\begin{array}{l}\text { SR (with } \\
\text { homogeneity) of } \\
\text { prospective cohort } \\
\text { studies }\end{array}$ & $\begin{array}{l}\text { SR (with homogeneity) of } \\
\text { Level leconomic studies }\end{array}$ \\
\hline $1 b$ & $\begin{array}{l}\text { Individual RCT } \\
\text { (with narrow } \\
\text { Confidence } \\
\text { Interval) }\end{array}$ & $\begin{array}{l}\text { Individual } \\
\text { inception cohort } \\
\text { study with }>80 \% \\
\text { follow-up; CDR } \\
\text { validated in a } \\
\text { single population }\end{array}$ & $\begin{array}{l}\text { Validating cohort } \\
\text { study with good } \\
\text { reference } \\
\text { standards; or CDR } \\
\text { tested within one } \\
\text { clinical centre }\end{array}$ & $\begin{array}{l}\text { Prospective cohort } \\
\text { study with good } \\
\text { follow-up }\end{array}$ & $\begin{array}{l}\text { Analysis based on clinically } \\
\text { sensible costs or } \\
\text { alternatives; systematic } \\
\text { review(s) of the evidence; } \\
\text { and including multi-way } \\
\text { sensitivity analyses }\end{array}$ \\
\hline $1 c$ & All or none§ & $\begin{array}{l}\text { All or none case- } \\
\text { series }\end{array}$ & $\begin{array}{l}\text { Absolute SpPins } \\
\text { and SnNouts" “ }\end{array}$ & $\begin{array}{l}\text { All or none case- } \\
\text { series }\end{array}$ & $\begin{array}{l}\text { Absolute better-value or } \\
\text { worse-value analyses " " " " }\end{array}$ \\
\hline $2 a$ & $\begin{array}{l}\text { SR (with } \\
\text { homogeneity) of } \\
\text { cohort studies }\end{array}$ & $\begin{array}{l}\text { SR (with } \\
\text { homogeneity) of } \\
\text { either } \\
\text { retrospective } \\
\text { cohort studies or } \\
\text { untreated control } \\
\text { groups in RCTs }\end{array}$ & $\begin{array}{l}\text { SR (with } \\
\text { homogeneity) of } \\
\text { Level >2 diagnostic } \\
\text { studies }\end{array}$ & $\begin{array}{l}\text { SR (with } \\
\text { homogeneity) of } \\
\text { Level } 2 \mathrm{~b} \text { and better } \\
\text { studies }\end{array}$ & $\begin{array}{l}\text { SR (with homogeneity) of } \\
\text { Level >2 economic studies }\end{array}$ \\
\hline $2 b$ & $\begin{array}{l}\text { Individual cohort } \\
\text { study (including } \\
\text { low quality RCT; } \\
\text { e.g., }<80 \% \text { follow- } \\
\text { up) }\end{array}$ & $\begin{array}{l}\text { Retrospective } \\
\text { cohort study or } \\
\text { follow-up of } \\
\text { untreated control } \\
\text { patients in an } \\
\text { RCT; Derivation } \\
\text { of CDR or } \\
\text { validated on } \\
\text { split-sample only }\end{array}$ & $\begin{array}{l}\text { Exploratory cohort } \\
\text { study with good } \\
\text { reference } \\
\text { standards; CDR } \\
\text { after derivation, or } \\
\text { validated only on } \\
\text { split-sample or } \\
\text { databases }\end{array}$ & $\begin{array}{l}\text { Retrospective } \\
\text { cohort study, or } \\
\text { poor follow-up }\end{array}$ & $\begin{array}{l}\text { Analysis based on clinically } \\
\text { sensible costs or } \\
\text { alternatives; limited } \\
\text { review(s) of the evidence, or } \\
\text { single studies; and } \\
\text { including multi-way } \\
\text { sensitivity analyses }\end{array}$ \\
\hline $2 c$ & $\begin{array}{l}\text { "Outcomes" } \\
\text { Research; } \\
\text { Ecological studies }\end{array}$ & $\begin{array}{l}\text { "Outcomes" } \\
\text { Research }\end{array}$ & & Ecological studies & Audit or outcomes research \\
\hline
\end{tabular}




\begin{tabular}{|c|c|c|c|c|c|}
\hline Level & $\begin{array}{l}\text { Therapy / } \\
\text { Prevention, } \\
\text { Aetiology / } \\
\text { Harm }\end{array}$ & Prognosis & Diagnosis & $\begin{array}{l}\text { Differential } \\
\text { diagnosis / } \\
\text { symptom } \\
\text { prevalence } \\
\text { study }\end{array}$ & $\begin{array}{l}\text { Economic and } \\
\text { decision analyses }\end{array}$ \\
\hline $3 a$ & $\begin{array}{l}\text { SR (with } \\
\text { homogeneity) of } \\
\text { case-control } \\
\text { studies }\end{array}$ & & $\begin{array}{l}\text { SR (with } \\
\text { homogeneity) of } \\
3 \mathrm{~b} \text { and better } \\
\text { studies }\end{array}$ & $\begin{array}{l}\text { SR (with } \\
\text { homogeneity) of } 3 b \\
\text { and better studies }\end{array}$ & $\begin{array}{l}\text { SR (with homogeneity) of } 3 \mathrm{~b} \\
\text { and better studies }\end{array}$ \\
\hline $3 b$ & $\begin{array}{l}\text { Individual Case- } \\
\text { Control Study }\end{array}$ & & $\begin{array}{l}\text { Non-consecutive } \\
\text { study; or without } \\
\text { consistently } \\
\text { applied reference } \\
\text { standards }\end{array}$ & $\begin{array}{l}\text { Non-consecutive } \\
\text { cohort study; or } \\
\text { very limited } \\
\text { population }\end{array}$ & $\begin{array}{l}\text { Analysis based on limited } \\
\text { alternatives or costs, poor } \\
\text { quality estimates of data, } \\
\text { but including sensitivity } \\
\text { analyses incorporating } \\
\text { clinically sensible variations }\end{array}$ \\
\hline 4 & $\begin{array}{l}\text { Case-series (and } \\
\text { poor quality } \\
\text { cohort and case- } \\
\text { control studies) }\end{array}$ & $\begin{array}{l}\text { Case-series (and } \\
\text { poor quality } \\
\text { prognostic } \\
\text { cohort studies) }\end{array}$ & $\begin{array}{l}\text { Case-control study, } \\
\text { poor or non- } \\
\text { independent } \\
\text { reference standard }\end{array}$ & $\begin{array}{l}\text { Case-series or } \\
\text { superseded } \\
\text { reference } \\
\text { standards }\end{array}$ & $\begin{array}{l}\text { Analysis with no sensitivity } \\
\text { analysis }\end{array}$ \\
\hline 5 & $\begin{array}{l}\text { Expert opinion } \\
\text { without explicit } \\
\text { critical appraisal, } \\
\text { or based on } \\
\text { physiology, } \\
\text { bench research } \\
\text { or "first } \\
\text { principles" }\end{array}$ & $\begin{array}{l}\text { Expert opinion } \\
\text { without explicit } \\
\text { critical appraisal, } \\
\text { or based on } \\
\text { physiology, } \\
\text { bench research } \\
\text { or "first } \\
\text { principles" }\end{array}$ & $\begin{array}{l}\text { Expert opinion } \\
\text { without explicit } \\
\text { critical appraisal, } \\
\text { or based on } \\
\text { physiology, bench } \\
\text { research or "first } \\
\text { principles" }\end{array}$ & $\begin{array}{l}\text { Expert opinion } \\
\text { without explicit } \\
\text { critical appraisal, } \\
\text { or based on } \\
\text { physiology, bench } \\
\text { research or "first } \\
\text { principles" }\end{array}$ & $\begin{array}{l}\text { Expert opinion without } \\
\text { explicit critical appraisal, or } \\
\text { based on physiology, bench } \\
\text { research or "first principles" }\end{array}$ \\
\hline
\end{tabular}

* By homogeneity we mean a systematic review that is free of worrisome variations (heterogeneity) in the directions and degrees of results between individual studies. Not all systematic reviews with statistically significant heterogeneity need be worrisome, and not all worrisome heterogeneity need be statistically significant. As noted above, studies displaying worrisome heterogeneity should be tagged with a "-" at the end of their designated level.

" Clinical Decision Rule. (These are algorithms or scoring systems that lead to a prognostic estimation or a diagnostic category.)

"i See note above for advice on how to understand, rate and use trials or other studies with wide confidence intervals.

$\S$ Met when all patients died before the Rx became available, but some now survive on it; or when some patients died before the Rx became available, but none now die on it.

$\S \S$ By poor quality cohort study we mean one that failed to clearly define comparison groups and/or failed to measure exposures and outcomes in the same (preferably blinded), objective way in both exposed and non-exposed individuals and/or failed to identify or appropriately control known confounders and/or failed to carry out a sufficiently long and complete follow-up of patients. By poor quality case-control study we mean one that failed to clearly define comparison groups and/or failed to measure exposures and outcomes in the same (preferably blinded), objective way in both cases and controls and/or failed to identify or appropriately control known confounders

$\S \S \S$ Split-sample validation is achieved by collecting all the information in a single tranche, then artificially dividing this into "derivation" and "validation" samples.

" "An "Absolute SpPin" is a diagnostic finding whose Specificity is so high that a Positive result rules-in the diagnosis. An "Absolute SnNout" is a diagnostic finding whose Sensitivity is so high that a Negative result rules-out the diagnosis.

" $\mathrm{i}$ " Good, better, bad and worse refer to the comparisons between treatments in terms of their clinical risks and benefits.

" " " Good reference standards are independent of the test, and applied blindly or objectively to applied to all patients. Poor reference standards are haphazardly applied, but still independent of the test. Use of a non-independent reference standard (where the 'test' is included in the 'reference', or where the 'testing' affects the 'reference') implies a level 4 study.

" " " " Better-value treatments are clearly as good but cheaper, or better at the same or reduced cost. Worse-value treatments are as good and more expensive, or worse and the equally or more expensive.

** Validating studies test the quality of a specific diagnostic test, based on prior evidence. An exploratory study collects information and trawls the data (e.g. using a regression analysis) to find which factors are 'significant'.

**** By poor quality prognostic cohort study we mean one in which sampling was biased in favour of patients who already had the target outcome, or the measurement of outcomes was accomplished in $<80 \%$ of study patients, or outcomes were determined in an unblinded, non-objective way, or there was no correction for confounding factors.

$* * * *$ Good follow-up in a differential diagnosis study is $>80 \%$, with adequate time for alternative diagnoses to emerge (for example 1-6 months acute, 1 - 5 years chronic 


\subsubsection{Schema der Empfehlungsgraduierung}

Die Methodik des Leitlinienprogramms Onkologie sieht - entsprechend dem AWMFRegelwerk - eine Vergabe von Empfehlungsgraden durch die Leitlinienautoren im Rahmen eines formalen Konsensusverfahrens vor. Dementsprechend wurden durch moderierte, nominale Gruppenprozesse bzw. strukturierte Konsensuskonferenzen durchgeführt [1]. Im Rahmen dieser Prozesse wurden die Empfehlungen von den stimmberechtigten Mandatsträgern formal abgestimmt. Die Ergebnisse der jeweiligen Abstimmungen (Konsensstärke) sind entsprechend den Kategorien in Tabelle 5 den Empfehlungen zugeordnet.

In der Leitlinie werden zu allen evidenzbasierten Statements (siehe Kapitel 2.2.3) und Empfehlungen das Evidenzlevel (siehe Kapitel 2.2.1) der zugrunde liegenden Studien sowie bei Empfehlungen zusätzlich die Stärke der Empfehlung (Empfehlungsgrad) ausgewiesen. Hinsichtlich der Stärke der Empfehlung werden in dieser Leitlinie drei Empfehlungsgrade unterschieden (siehe Tabelle 4), die sich auch in der Formulierung der Empfehlungen jeweils widerspiegeln.

Tabelle 4: Schema der Empfehlungsgraduierung

\begin{tabular}{|l|l|l|}
\hline Empfehlungsgrad & Beschreibung & Ausdrucksweise \\
\hline A & Starke Empfehlung & soll/soll nicht \\
\hline B & Empfehlung & sollte/sollte nicht \\
\hline 0 & Empfehlung offen & kann/kann verzichtet werden \\
\hline
\end{tabular}

Tabelle 5: Klassifikation der Konsensusstärke

\begin{tabular}{|l|l|}
\hline Konsensusstärke & Prozentuale Übereinstimmung \\
\hline Starker Konsens & Zustimmung von $>95 \%$ der Teilnehmer \\
\hline Konsens & Zustimmung von $>75-95 \%$ der Teilnehmer \\
\hline Mehrheitliche Zustimmung & Zustimmung von $>50-75 \%$ der Teilnehmer \\
\hline Kein Konsens & Zustimmung von $<50 \%$ der Teilnehmer \\
\hline
\end{tabular}

\subsubsection{Statements}

Als Statements werden Darlegungen oder Erläuterungen von spezifischen Sach-verhalten oder Fragestellungen ohne unmittelbare Handlungsaufforderung bezeichnet. Sie werden entsprechend der Vorgehensweise bei den Empfehlungen im Rahmen eines formalen Konsensusverfahrens verabschiedet und können entweder auf Studien-ergebnissen oder auf Expertenmeinungen beruhen.

\subsubsection{Expertenkonsens}

Als Expertenkonsens werden Empfehlungen bezeichnet, zu denen keine Recherche nach Literatur durchgeführt wurde. In der Regel adressieren diese Empfehlungen 
Vorgehensweisen der guten klinischen Praxis, zu denen keine wissenschaftlichen Studien notwendig sind bzw. erwartet werden können. Für die Graduierung des Expertenkonsens wurden keine Symbole verwendet, die Stärke der Empfehlung ergibt sich aus der verwendeten Formulierung (soll/sollte/kann) entsprechend der Abstufung in Tabelle 4.

\subsubsection{Unabhängigkeit und Darlegung möglicher Interessenkonflikte}

Die Erstellung und Aktualisierung der Leitlinie erfolgte in redaktioneller Unabhängigkeit von der finanzierenden Organisation, der Deutschen Krebshilfe. Für die ausschließlich ehrenamtliche Arbeit der Mandatsträger und Experten, ohne die die S3-Leitlinie nicht zu realisieren gewesen wäre, ist ihnen zu danken.

Alle Mitglieder der Leitliniengruppe legten eine schriftliche Erklärung zu eventuell bestehenden Interessenkonflikten ab. Die offengelegten Sachverhalte, die auf Interessenkonflikte hinweisen, können im Leitlinienreport zur Leitlinie eingesehen werden (http://www.leitlinienprogramm-onkologie.de/leitlinien/kolorektaleskarzinom/).

Die Relevanz von Interessenkonflikten für die Leitlinie wurde bei mehreren Veranstaltungen (Kick-Off-Treffen und Konsensustreffen) und per E-Mail wiederholt diskutiert. Bei der Aktualisierung 2010-2013 (Version 1) erfolgte die Sichtung und Bewertung der offengelegten Sachverhalte durch die Koordinatoren. Für den Aktualisierungsprozess 2015-2017 (Version 2) sichtete Prof. Kolligs als Interessenskonfliktbeauftragter der Leitliniengruppe die offengelegten Sachverhalte.

Auf Vorschlag von Prof. Kolligs entschied die Leitliniengruppe, keine Einschränkungen bzgl. der Abstimmung oder Beratung bei einzelnen Personen vorzunehmen, da angesichts des methodischen Vorgehens und der multidisziplinären Zusammensetzung der Leitliniengruppe kein Risiko für eine unangemessene Verzerrung der Leitlinienempfehlungen (Interessenkonflikt) gesehen wurde.

Beim Aktualisierungsprozess 2010-2013 hatte sich Prof. Schmiegel aufgrund eines möglichen Interessenskonfliktes bei den Abstimmungen zum FOBT/iFOBT, genetischer Stuhltests und M2-PK enthalten.

Die Gefahr der Beeinflussung durch Interessenkonflikte wurde u.a. reduziert, indem für die Recherche, Auswahl und Bewertung der Literatur politisch besonders brisanter Themen externe Institute beauftragt worden sind. Die formale Konsensbildung und die interdisziplinäre Erstellung sind weitere Instrumente, die Einflussnahme der Industrie zu minimieren. 


\subsection{Redaktioneller Hinweis}

\section{Geschlechtsneutrale Formulierung}

Ausschließlich zum Zweck der besseren Lesbarkeit wird auf die geschlechtsspezifische Schreibweise verzichtet. Alle personenbezogenen Bezeichnungen in diesem Dokument sind somit geschlechtsneutral zu verstehen.

\section{Partizipative Entscheidungsfindung}

Alle Empfehlungen der Leitlinie sind als Empfehlungen zu verstehen, die im Sinne einer partizipativen Entscheidungsfindung zwischen Arzt und Patient und ggf. der Angehörigen getroffen werden und umzusetzen sind. 


\section{Prävention asymptomatische Bevölkerung}

\subsection{Lebensgewohnheiten}

\begin{tabular}{|l|l|}
\hline $\begin{array}{l}\text { 3.1. } \\
\text { Empfehlungsgrad } \\
\mathbf{B}\end{array}$ & $\begin{array}{l}\text { Zur Risikoreduktion eines kolorektalen Karzinoms sollten regelmäßig körperliche } \\
\text { Aktivitäten durchgeführt werden. }\end{array}$ \\
\hline $\begin{array}{l}\text { Level of Evidence } \\
\mathbf{2} \mathbf{a}\end{array}$ & \begin{tabular}{l} 
Evidenz aus Aktualisierungsrecherche': $[2-13]$ \\
\hline
\end{tabular} \\
\hline
\end{tabular}

3.2 .

Empfehlungsgrad

B

Level of Evidence

$2 \mathrm{a}$

\section{Evidenzbasierte Empfehlung}

Zur Risikoreduktion eines kolorektalen Karzinoms sollte eine Gewichtsreduktion bei übergewichtigen Personen angestrebt werden.

Evidenz aus Aktualisierungsrecherche: [2, 9, 14-19]

starker Konsens

\section{3.}

Empfehlungsgrad

A

Level of Evidence

$2 \mathrm{a}$

\section{Evidenzbasierte Empfehlung}

Die Bevölkerung soll zum Verzicht auf Tabakrauchen angehalten werden.

Evidenz aus Aktualisierungsrecherche: $[2,11,20-26]$

starker Konsens

\section{Hintergrund}

Personen mit höherem körperlichen Aktivitätsgrad haben in Querschnittsuntersuchungen und prospektiven Kohortenstudien weniger Kolonpolypen (Adenome) und ein 
um bis zu 30\% geringeres Karzinomrisiko. Bereits 30 bis 60 Minuten tägliche moderate körperliche Aktivität gehen mit einem verringerten Karzinomrisiko einher [2-13].

Es findet sich eine positive Assoziation zwischen dem Auftreten von Kolonpolypen (Adenomen) und kolorektalen Karzinomen und einem höheren BMI, als auch einer Zunahme des Bauchumfangs. Dieser Effekt ist ab einem BMI von $>25 \mathrm{~kg} / \mathrm{m}^{2}$ nachweisbar, nimmt linear mit dem BMI zu und ist stärker bei Männern als bei Frauen ausgeprägt. Bei übergewichtigen Personen insbesondere bei stammbetonter Adipositas war das Risiko für ein Kolonkarzinom bis zu zweifach erhöht [19]. Es ist unklar, ob die Risikoerhöhung durch das Übergewicht, veränderte Hormonspiegel, die erhöhte Kalorienaufnahme oder durch die fehlende körperliche Aktivität bedingt ist [2, 9, 14-19].

Rauchen ist mit einem zweifach erhöhten Risiko für Kolonadenome und erhöhtem Risiko für kolorektale Karzinome assoziiert [2, 11, 20-26].

\subsection{Ernährungsempfehlungen}

3.4 .

Level of Evidence

2b

\section{Evidenzbasiertes Statement}

Eine spezifische Diätempfehlung zur Reduktion des KRK-Risikos kann derzeit nicht gegeben werden.

Evidenz aus Aktualisierungsrecherche: [27-33]

Konsens
3.5.

EK

\section{Konsensbasierte Empfehlung}

Es sollten die allgemeinen Ernährungsempfehlungen der DGE befolgt werden.

Konsens

\section{Hintergrund}

In einer ausführlichen Literaturübersicht aus dem Jahre 2010 wurde ein Zusammen-hang zwischen einer "gesunden" Ernährungsweise bzw. einer „ungesünderen“ Ernährungsweise und dem kolorektalem Karzinom als wahrscheinlich angesehen. Eine "gesunde" Ernährungsweise wurde von den Autoren gekennzeichnet durch einen hohen Konsum von Obst und Gemüse sowie einer verminderten Aufnahme von rotem und verarbeitetem Fleisch, wohingegen eine „ungesündere“ Ernährungsweise durch eine hohe Aufnahme von rotem und verarbeitetem Fleisch, Kartoffeln und raffinierter Stärke charakterisiert wurde [27]. In Originalpublikationen der letzten Jahre wurde wiederholt eine Assoziation zwischen Ernährungsfaktoren und der Ausbildung eines KRK beobachtet, die mit einer Evidenzstärke zwischen $2 \mathrm{~b}$ und $4 \mathrm{zu}$ bewerten sind [28-31]. Allerdings gibt es auch Studien, in denen keine Korrelation zwischen Ernährungsfaktoren und KRK beobachtet wurde [32, 33]. Dabei handelt es sich um Assoziationen, nicht um Interventionsstudien. Ob solche Zusammenhänge eine spezifische Diätempfehlung zur KRK Prävention rechtfertigen, wurde bislang nicht untersucht. Deshalb kann derzeit trotz der skizzierten Zusammenhänge keine spezifische Diätempfehlung 
abgegeben werden. Es wird stattdessen empfohlen, dass zur Risikoreduktion eines Karzinoms die aktuellen Ernährungsempfehlungen der DGE berücksichtigt werden. Die Assoziationen zwischen der Aufnahme bestimmter Nahrungsstoffe und dem KRK Risiko wird im Folgenden genauer dargestellt. In diesem Zusammenhang ist auch hervorzuheben, dass eine Ernährungsweise, die keine Gewichtszunahme bewirkt, anzuraten ist (siehe Kapitel 3.1).

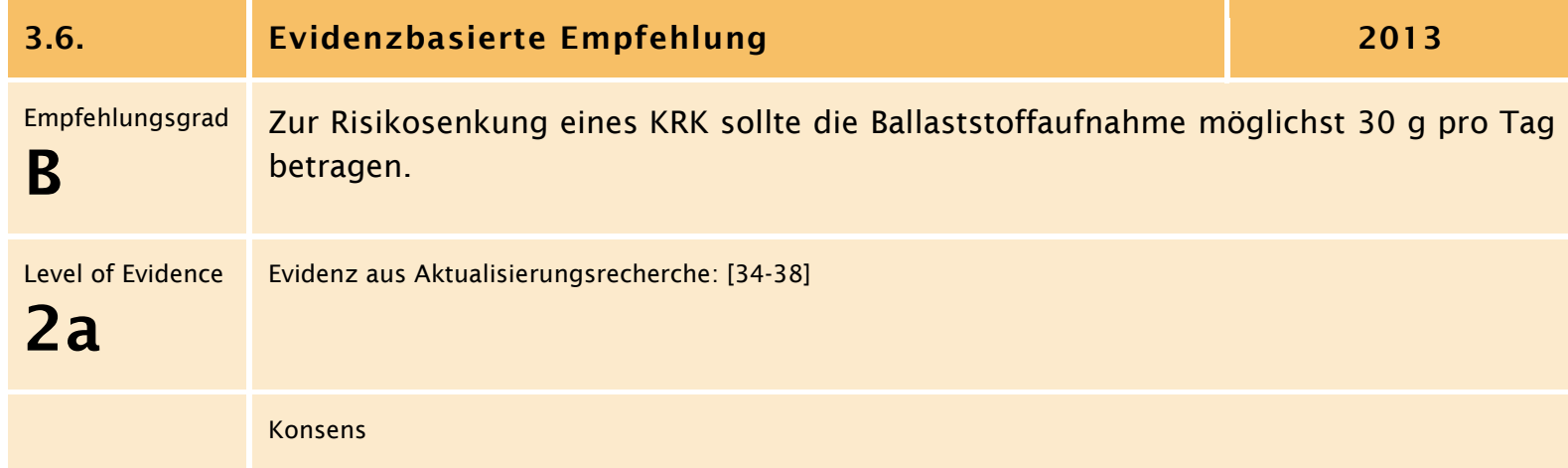

\section{Hintergrund}

Trotz kontroverser Datenlage ist die Evidenz ausreichend, um eine ballaststoffreiche Ernährung von $30 \mathrm{~g} / \mathrm{Tag}$ zu empfehlen [34-38]. Eine aktuelle britische Studie, in der Daten von sieben Kohortenstudien zusammengefasst wurden, wies nach, dass eine inverse Korrelation zwischen Ballaststoffaufnahme und Karzinomrisiko besteht. Der Vergleich des täglichen Ballaststoffkonsums von 10 und $24 \mathrm{~g}$ zeigte in dieser Arbeit, dass ein höherer Konsum mit einem um 30 \% reduzierten Kolonkarzinomrisiko assoziiert ist [34]. In einer anderen Studie, in der 13 prospektive Kohortenstudien zusammengefasst wurden, zeigten sich ähnliche Ergebnisse. Obwohl das pooling project of prospective studies of diet and cancer noch größere Spannbreiten zwischen der niedrigsten und höchsten Quintile der Ballaststoffaufnahme aufwies, wurde eine signifikante inverse Korrelation zwischen dem Ballaststoffkonsum und dem Krebsrisiko nach alterskorrigierter Analyse, jedoch nicht nach Adjustierung für weiterer ernährungsbedingte Risikofaktoren, beobachtet [37]. Die nur eingeschränkt positiven Daten könnten darauf zurückzuführen sein, dass lediglich zu Studienbeginn eine Erfassung der Ballaststoffaufnahme erfolgte, die möglicherweise inkorrekt den langfristigen Konsum widerspiegelt. Trotz der eingeschränkten Ergebnisse sind die verbleibenden Aussagen sehr robust, weil sie auf großen Personenkollektiven basieren. Deshalb wurde der Empfehlungsgrad B festgelegt.

\section{7.}

Empfehlungsgrad

B

Level of Evidence

$2 a$

\section{Evidenzbasierte Empfehlung}

Zur Reduktion des Risikos eines KRK sollte der Alkoholkonsum limitiert werden.

Konsens 


\section{Hintergrund}

Es besteht eine positive Korrelation zwischen einem hohen Alkoholkonsum und der Entstehung eines KRK [39-42] insbesondere bei Menschen mit geringer Folsäureund/oder Methioninaufnahme [40]. Abstinenzler und Personen mit einem geringen Alkoholkonsum weisen ein signifikant geringeres Karzinomrisiko auf [39-42]. Eine Metaanalyse von 14 prospektiven Kohortenstudien zeigte, dass bereits eine Alkoholaufnahme von $100 \mathrm{~g}$ wöchentlich mit einem 15 \%igen Anstieg sowohl des Kolonals auch des Rektumkarzinom-Risikos assoziiert ist [42]. Das Risiko korreliert mit der Menge des aufgenommenen Alkohols und nicht mit der Art des alkoholischen Getränks [40].

\begin{tabular}{|l|l|}
\hline $\begin{array}{l}\text { 3.8. } \\
\text { Evidenzbasierte Empfehlung } \\
\mathbf{B}\end{array}$ & $\begin{array}{l}\text { Rotes bzw. verarbeitetes Fleisch sollte nur in geringen Mengen (nicht täglich) } \\
\text { konsumiert werden. }\end{array}$ \\
\hline $\begin{array}{l}\text { Level of Evidence } \\
\mathbf{2} \mathbf{A}\end{array}$ & \begin{tabular}{l} 
Evidenz aus Aktualisierungsrecherche: [38,43-47] \\
\hline
\end{tabular} \\
\hline & Konsens \\
\hline
\end{tabular}

\section{Hintergrund}

Ein hoher Konsum von rotem Fleisch (Rind, Kalb, Schwein und Lamm) und ver-arbeitetem Fleisch ist mit einem erhöhten Risiko für ein KRK assoziiert [38, 43-47]. Zwischen dem Verzehr von Geflügel und/oder -produkten besteht keine positive Korrelation [46]. Die positive Assoziation wird am ehesten durch die Verarbeitung und die Zubereitungsart verursacht, wie Daten des Prostate, Lung, Colorectal, and Ovarian cancer trials zeigen. Die regelmäßige Aufnahme von insbesondere durchgegartem rotem Fleisch, Speck (Bacon) und Würstchen korrelierte mit einer signifikanten Erhöhung des Risikos zur Ausbildung eines KRK [47].

\section{9.}

Level of Evidence

\section{$2 \mathrm{a}$}

\section{Evidenzbasiertes Statement}

Zu erhöhtem Fischkonsum kann keine Empfehlung abgegeben werden.

Evidenz aus Aktualisierungsrecherche: [43, 45, 46, 48-50]

Konsens

\section{Hintergrund}

In einer Metaanalyse von Geelen und Kollegen, die 19 Kohortenstudien zusammen-fasst, wurde der Einfluss des Fischverzehrs auf das KRK Risiko untersucht. Der Vergleich des höchsten wöchentlichen Fischkonsums mit dem geringsten zeigte, dass ein höherer Konsum mit einem um 12\% reduzierten Krebsrisiko assoziiert ist. Die Korrelation war umso ausgeprägter je höher die Differenz zwischen der größten und geringsten Fischaufnahme war [48]. Die Datenlage insgesamt ist allerdings wider-sprüchlich, was wahrscheinlich darauf zurückzuführen ist, dass in den verschiedenen Studien 
unterschiedlich hohe Fischverzehrsmengen miteinander verglichen wurden $[43,45,46$, 48-50] Trotz der Vermutung, dass vermehrter Fischkonsum das KRK Risiko leicht senken kann, wird aufgrund der nicht eindeutigen Datenlage derzeit keine Empfehlung zum erhöhten Fischkonsum abgegeben.

3.10 .

\section{EK}

\section{Konsensbasiertes Statement}

Ein Zusammenhang zwischen der Aufnahme von Kaffee/Tee und der Reduktion des Risikos für ein KRK ist nicht belegt, deshalb gibt es keine Empfehlung zu Kaffee- oder Teekonsum.

Starker Konsens

\section{Hintergrund}

Zu diesem Thema liegen drei Metaanalysen vor, die keine Korrelation zwischen dem Konsum von Kaffee und/oder Tee und dem KRK Risiko beobachtet haben [51-53].

\subsection{1 .}

\section{EK}

\section{Konsensbasierte Empfehlung}

Trotz der nicht eindeutigen Datenlage zur Prävention des KRK sollten Obst und Gemüse vermehrt konsumiert werden (5 Portionen am Tag).

Konsens

\section{Hintergrund}

In einer Fallkontrollstudie und einer Kohortenstudie wurde eine inverse Korrelation zwischen einem vermehrten Obst- und Gemüsekonsum und einer Reduzierung des KRK Risikos beobachtet $[54,55]$. Allerdings wurde in einer Metaanalyse gezeigt, dass ein erhöhter Obst- und Gemüseverzehr lediglich mit einem um 6-9 \% reduzierten Kolonkarzinomrisiko assoziiert ist. Eine stärkere inverse Korrelation wurde beim distalen Kolonkarzinom beobachtet [56]. Unklar ist jedoch, welche Bestandteile (Ballaststoffe, sekundäre Pflanzenstoffe) diesen protektiven Effekt haben. Trotz nicht eindeutiger Datenlage hinsichtlich Reduktion des KRK Risikos wird eine Förderung des Obst- und Gemüsekonsums als wünschenswert angesehen, da ein regelmäßiger Obst- und Gemüseverzehr wahrscheinlich das allgemeine Krankheitsrisiko senkt.

\subsection{2 .}

Es gibt keinen Zusammenhang zwischen Nahrungszubereitung oder Nahrungsfettkomponenten und KRK-Risiko.

Konsens

\section{Hintergrund}

Es wurde wiederholt diskutiert, ob die Nahrungszubereitung bzw. der durch Nahrungszubereitungsmethoden möglicherweise erhöhte Anteil an potentiell schädlichen Fettsäuren, wie z.B. Transfettsäuren, das KRK Risiko erhöhen kann. Die Literaturdaten dazu sind spärlich und uneinheitlich, so dass angenommen werden muss, dass es keinen 
klaren Zusammenhang gibt. Dies wurde in einer neueren prospektiven, populationsbasierten Kohortenstudie in den USA untersucht. In dieser Studie an über 35.000 Frauen konnte bestätigt werden, dass Transfettsäuren das KRK Risiko nicht erhöhen [57].

Darüber hinaus gibt es keine spezifischen Empfehlungen zum Fettkonsum hinsichtlich Reduktion des KRK Risikos. Zu dieser Thematik liegen mehrere Studien vor, die keinen Zusammenhang zwischen dem Fettkonsum und der Ausbildung eines KRK gefunden haben. Ein Effekt von Kofaktoren, wie z.B. Zufuhr von rotem Fleisch oder Zubereitungsart, kann nicht hinreichend abgetrennt werden [31, 32, 38, 48, 58-60].

\begin{tabular}{|l|l|}
$\begin{array}{l}\text { 3.13. } \\
\text { Evidenzbasiertes Statement }\end{array}$ & 2013 \\
\hline $\mathbf{2 6}$ & $\begin{array}{l}\text { Es gibt keinen Zusammenhang zwischen der Aufnahme von Acrylamid und KRK- } \\
\text { Risiko. }\end{array}$ \\
\hline & de Novo: [61-64] \\
\hline & Starker Konsens \\
\hline
\end{tabular}

\section{Hintergrund}

In einer in Schweden durchgeführten prospektiven, populationsbasierten Kohortenstudie an mehr als 45.000 Männern konnte mittels Food Frequency Questionnaire (FFQ) gezeigt werden, das es keinen Zusammenhang zwischen Acrylamid in der Nahrung und KRK Risiko gibt [61]. Diese Studie bestätigt frühere Untersuchungen, nach denen bei Männern wie Frauen kein Zusammenhang zwischen Acrylamid und KRK-Enstehung besteht [62-64]. 


\subsection{Mikronährstoffe}

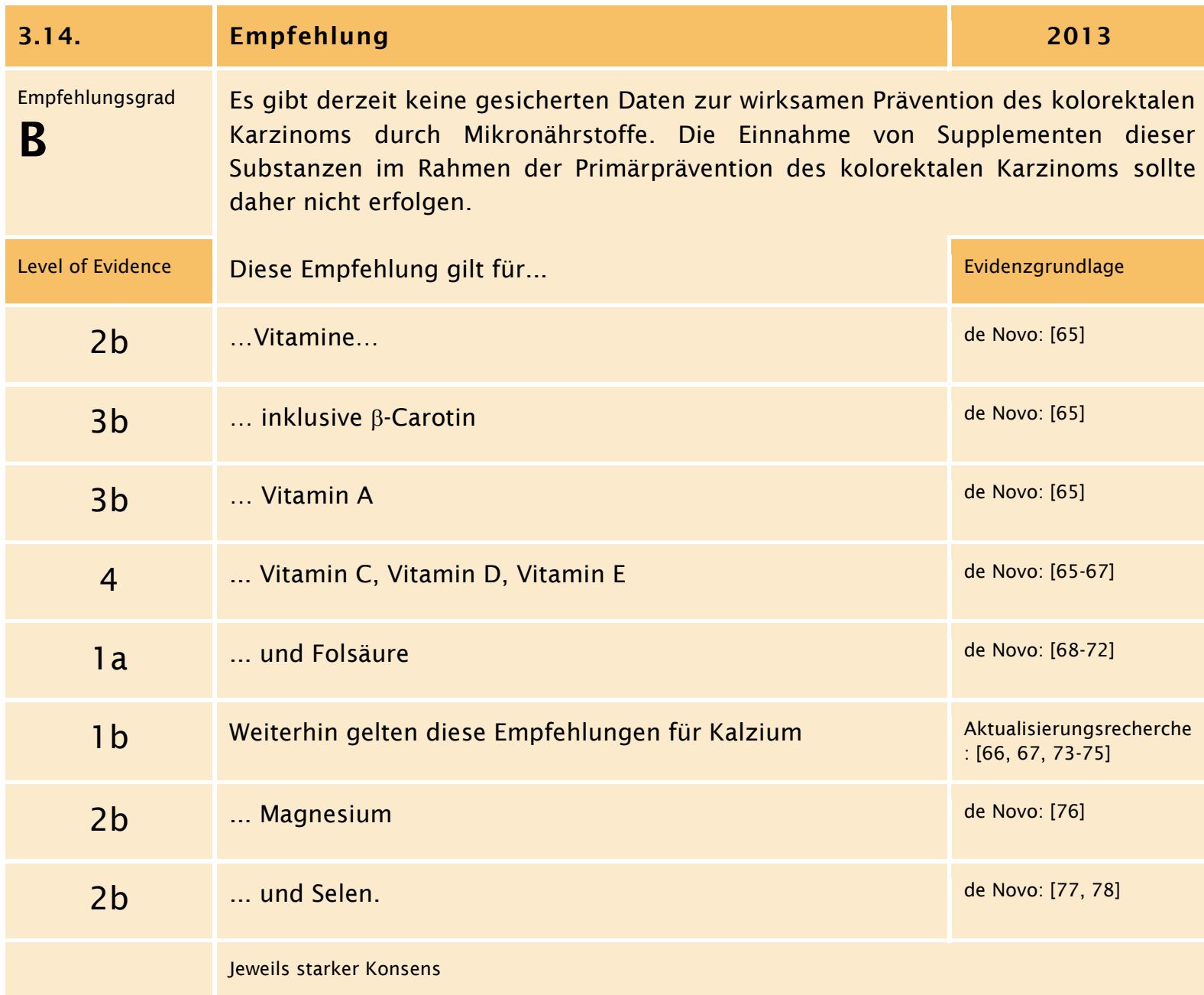

\section{Hintergrund}

Die folgenden Ausführungen beziehen sich auf Supplemente von Mikronährstoffen, teilweise in pharmakologischer, d.h. durch Aufnahme entsprechender Lebensmittel (Obst, Gemüse, Milchprodukte), oftmals nicht erreichbarer Dosierung.

Für Kalzium konnte ein moderater, klinisch nicht relevanter Hemmeffekt auf die Rekurrenz von Kolonadenomen nachgewiesen werden [73-75]. Hingegen ist die Datenlage für eine risikosenkende Wirkung von Kalzium oder Vitamin D, allein oder in Kombination, hinsichtlich des KRK selbst nicht überzeugend [66, 67].

Es gibt keine Evidenz für die Annahme, dass Beta-Carotin, Vitamin A oder Vitamin E das Risiko des kolorektalen Karzinoms vermindern könnten. Im Gegenteil konnte in einer Metaanalyse [65] gezeigt werden, dass die Supplementierung der vorgenannten Vitamine, allein oder in Kombination gegeben, mit einer erhöhten generellen Mortalität assoziiert ist.

Es ist nicht eindeutig belegt, dass die Einnahme von hohen Dosen an Vitamin C das Risiko für das KRK vermindert. 
Ein das KRK-Risiko senkender Effekt von Folsäure konnte bislang nicht zweifelsfrei nachgewiesen werden [68]. Studien zur Rekurrenz von Kolonadenomen führten zu divergenten Ergebnissen [69-72]

Eine Interventionsstudie mit einem Selen-Supplement und dem Hauptzielkriterium „KRKInzidenz" wurde bislang nicht durchgeführt. Die Korrelation niedriger SelenKonzentrationen im Serum mit einem erhöhten Adenomrisiko ist nicht ausreichend, um eine Empfehlung zur Selensupplementierung auszusprechen [77, 78].

\subsection{Medikamente}

3.15.

Empfehlungsgrad

A

Level of Evidence

$3 b$

\section{Evidenzbasierte Empfehlung}

COX-2 Hemmer sollen in der asymptomatischen Bevölkerung nicht zur Prophylaxe des kolorektalen Karzinoms eingenommen werden.

de Novo: [79-82]

Konsens

\section{3}

\subsection{6.}

Empfehlungsgrad

B

Level of Evidence

$2 b$

3.17.

Empfehlungsgrad

A

Level of Evidence

$2 \mathrm{a}$

\section{Evidenzbasierte Empfehlung}

2013

Statine sollten nicht zur Primärprophylaxe des KRK eingesetzt werden.

de Novo: [83]

Starker Konsens

013




\begin{tabular}{|l|l|}
\hline $\begin{array}{l}\text { 3.18. } \\
\text { Empfehlungsgrad }\end{array}$ & $\begin{array}{l}\text { Eine Hormontherapie }{ }^{3} \text { zur Risikoreduktion eines kolorektalen Karzinoms bei Frauen } \\
\text { soll nicht gegeben werden. }\end{array}$ \\
\hline $\begin{array}{l}\text { Level of Evidence } \\
\mathbf{1}\end{array}$ & Leitlinienadaptation: $[88,89]$ \\
\hline & \\
\hline
\end{tabular}

\section{Hintergrund}

Für die Einnahme von Cyclooxygenase-2 (COX-2) Inhibitoren zur Primärprävention des kolorektalen Karzinoms liegt eine positive Fall-Kontroll-Studie, aber keine randomisierte Studie vor [79]. Drei randomisierte Studien zur Sekundärprävention mit Celecoxib bzw. Rofecoxib nach Polypektomie zeigen übereinstimmend, dass COX-2-Inhibitoren das Rezidivrisiko eines kolorektalen Adenoms signifikant senken [80-82]. In allen drei Studien zeigte sich jedoch eine deutlich erhöhte kardiovaskuläre Morbidität.

Eine aktuelle Metaanalyse von Fallkontroll-, Kohorten- und randomisierten Studien belegt einen statistisch signifikanten, aber nur geringen Effekt von Statinen in der Primärprävention des kolorektalen Karzinoms [83]. In einer Phase III Studie zur Sekundärprävention von Kolonadenomen senkte Ursodesoxycholsäure signifikant nur das Risiko für Adenome mit hochgradigen Dysplasien, aber nicht für Adenome insgesamt [90]. Prospektive Studien zur Primärprävention von Adenomen mittels Ursodesoxycholsäure liegen nicht vor.

Eine Metaanalyse von 2 großen randomisierten Studien mit zusammen über 7.500 Teilnehmern zeigt, dass die Einnahme von 300 mg oder mehr Acetylsalicylsäure pro Tag für 5 Jahre mit einer Latenz von 10 und mehr Jahren das Risiko, an einem kolorektalen Karzinom zu erkranken, senkt [85]. Eine andere Metaanalyse, die insgesamt 8 randomisierte Studien mit zusammen 25.570 Teilnehmern analysiert hat, weist nach, dass die tägliche Einnahme von mindestens 75 mg ASS mit einer Latenz von 10 Jahren die Mortalität am kolorektalen Karzinom senkt [86]. Für die Einnahme nicht-steroidaler Antirheumatika (NSAR) wird in Kohorten- und Fallkontrollstudien eine Erniedrigung der Inzidenz des kolorektalen Karzinoms beschrieben. Diese Befunde wurden allerdings noch nicht durch randomisierte Studien bestätigt [85]. Aufgrund der gehäuften Inzidenz von gastrointestinalen Blutungen unter ASS [87] und der fehlenden Bewertung der Nutzen-/Risikorelation folgt die Leitliniengruppe der Leitlinie der U.S. Preventive Services Task Force zum Einsatz von ASS oder NSAR zur Primärprävention des kolorektalen Karzinoms aus dem Jahr 2007, die empfiehlt, ASS und NSAR nicht zur Primärprävention des kolorektalen Karzinoms einzusetzen [84].

Eine Hormontherapie kann das kolorektale Karzinomrisiko reduzieren [89]. Aufgrund der erhöhten Inzidenz von unerwünschten Wirkungen, insbesondere venöser Thromboembolien, kann die Hormontherapie bei postmenopausalen Frauen aber nicht zur Primärprävention des kolorektalen Karzinoms empfohlen werden. Hier folgt die Leitliniengruppe der Leitlinie der U.S. Preventive Services Task Force zum Einsatz von Hormontherapie bei postmenopausalen Frauen [88] und der Leitlinie Hormontherapie in

\footnotetext{
${ }^{3}$ Da der Begriff „Hormonersatztherapie“ irreführend ist, wird in der Leitlinie nur der Begriff „Hormontherapie“ verwendet
} 
der Peri- und Postmenopause der Deutschen Gesellschaft für Gynäkologie und Geburtshilfe [91]. 


\title{
4. Vorsorge/ Früherkennung asymptomatische Bevölkerung
}

\author{
Asymptomatische Bevölkerung - Definition: \\ Personen, die keiner Risikogruppe für das Auftreten eines kolorektalen Karzinoms \\ angehören.
}

\subsection{Vorsorge-/ Früherkennung - Alter}

\begin{tabular}{|l|l|}
\hline 4.1. & Konsensbasierte Empfehlung \\
\hline EK & $\begin{array}{l}\text { Mit der Darmkrebs- Vorsorge/ -Früherkennung für die asymptomatische Bevölkerung } \\
\text { sollte ab dem Alter von 50 Jahren begonnen werden. Eine obere Altersbegrenzung für } \\
\text { die Darmkrebs-Vorsorge/-Früherkennung kann bei steigender Lebenserwartung nicht } \\
\text { gegeben werden. Hier ist eine individuelle Entscheidung unter Berücksichtigung der } \\
\text { Begleiterkrankungen angezeigt. }\end{array}$ \\
& Starker Konsens
\end{tabular}

\section{Hintergrund}

Die KRK-Inzidenz steigt ab einem Alter von 50 Jahren deutlich an [92, 93]. In einer prospektiven Koloskopiestudie zeigte sich eine deutlich niedrigere Nachweisrate fortgeschrittener Adenome bei 40- bis 49jährigen (3,5\%) [94]. Von großer Bedeutung ist die Identifikation von Personen mit erhöhtem KRK-Risiko, für die gesonderte Empfehlungen gelten (siehe Kapitel 5)

Zur Altersbegrenzung der Darmkrebsvorsorge existieren keine prospektiven Studien. In den FOBT-Studien wurden nur Personen bis 75 Jahren eingeschlossen. Die US Preventive Task Force rät von einer Vorsorge/ Früherkennung von Personen über 85 Jahren ab und hält fest, dass eine Vorsorge/ Früherkennung bei Personen zwischen 76 und 85 Jahren generell nicht durchgeführt werden sollte, in individuellen Fällen jedoch überlegt werden könnte [95]. Die Inzidenz fortgeschrittener Neoplasien nimmt mit dem Alter zu [96]. Endoskopische Untersuchungen scheinen auch bei älteren Patienten sicher durchführbar zu sein [97], jedoch nahm in einer Kohortenstudie die Komplikationsrate mit dem Alter zu [98]. In einer Studie war die relative 5-Jahres-überlebensrate nach kurativer Operation eines kolorektalen Karzinoms für Patienten über 74 Jahre vergleichbar mit der von Patienten zwischen 50 und 74 Jahren [99]. Die Sinnhaftigkeit der Durchführung einer KRK-Früherkennung/-vorsorge sollte daher individuell in Abhängigkeit des „biologischen Alters" und unter Berücksichtigung vorhandener Begleiterkrankungen überprüft werden. Zum Nutzen-/ Risikoverhältnis der Darmkrebs- Vorsorge/ -Früherkennung in verschiedenen Altersgruppen existiert keine ausreichende Datenlage.

\subsection{Untersuchungsverfahren für die Darmkrebsfrüherkennung/-vorsorge}

Bei den zur Verfügung stehenden Verfahren muss zwischen solchen, die vorwiegend Karzinome nachweisen (FOBT, genetische Stuhltests, M2-PK) und solchen, die zusätzlich in der Lage sind Adenome nachzuweisen (Koloskopie, Sigmoidoskopie, CT- 
Kolonographie, Kapselendoskopie) unterschieden werden. Folgende Verfahren werden besprochen:

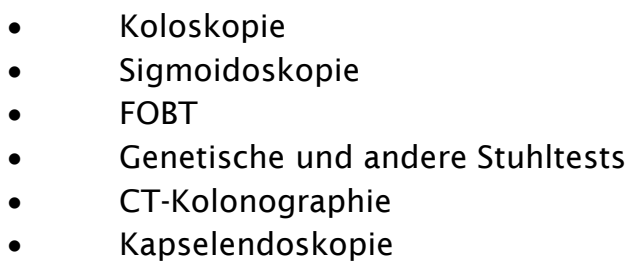

\subsubsection{Endoskopische Verfahren}

Von allen Maßnahmen zur Früherkennung kolorektaler Neoplasien besitzt die Koloskopie die höchste Sensitivität und Spezifität (Goldstandard). Endoskopische Maßnahmen sind als einzige diagnostisch und therapeutisch und haben den Vorteil, dass durch sie auch nichtblutende Karzinome und Adenome mit hoher Sensitivität nachgewiesen werden können. Durch die Abtragung von Adenomen kann zudem die Entstehung von Karzinomen effektiv verhindert (Unterbrechung der AdenomKarzinomsequenz) $[100,101]$ und wie kürzlich gezeigt wurde, auch die KRK-bedingte Mortalität gesenkt werden [102].

In einzelnen randomisierten Studien konnte zudem gezeigt werden, dass trotz geringerer Teilnahmerate im Vergleich zum FOBT durch sowohl Sigmoidoskopie [103] als auch Koloskopie [104] in einer intention to screen Analyse mehr fortgeschrittene Neoplasien detektiert werden, was vor allem durch die deutlich höhere Sensitivität für fortgeschrittene Adenome bedingt war.

\subsubsection{Koloskopie}

\begin{tabular}{|l|l|}
$\begin{array}{l}\text { 4.2. } \\
\text { Empfehlungsgrad }\end{array}$ & $\begin{array}{l}\text { Evidenzbasierte Empfehlung } \\
\text { Bie komplette qualitätsgesicherte Koloskopie besitzt die höchste Sensitivität und } \\
\text { Spezifität für das Auffinden von Karzinomen und Adenomen und sollte daher als } \\
\text { Standardverfahren für die KRK-Vorsorge/-Früherkennung eingesetzt werden. Bei } \\
\text { unauffälligem Befund sollte die Koloskopie nach } 10 \text { Jahren wiederholt werden. Zur } \\
\text { Durchführung wird auf die Krebsfrüherkennungsrichtlinie } 4 \text { verwiesen, die digitale } \\
\text { rektale Untersuchung ist hierbei obligat. Bei Personen, die an der Koloskopie- } \\
\text { Vorsorge/-Früherkennung entsprechend dieser Richtlinie teilnehmen, erübrigt sich } \\
\text { das FOBT-Vorsorge/Früherkennungsverfahren. }\end{array}$ \\
\hline $\begin{array}{l}\text { Level of Evidence } \\
\mathbf{3 b}\end{array}$ & \begin{tabular}{l} 
De Novo: [105-121] \\
\hline
\end{tabular} \\
\hline
\end{tabular}

\section{Hintergrund}

Für den Einsatz der Koloskopie in der Früherkennung bzw. Vorsorge kolorektaler Karzinome existieren anders als für den FOBT und die Sigmoidoskopie keine Ergebnisse aus randomisierten Studien. Derartige Studien sind in Europa und den USA begonnen worden, Ergebnisse werden jedoch erst in frühestens 15 Jahren vorliegen. Dennoch wird

\footnotetext{
${ }^{4}$ Siehe: https://www.g-ba.de/informationen/richtlinien/17/
} 
der Einsatz der Koloskopie durch indirekte Evidenz unterstützt. In der externen Literatursuche wurde eine Arbeit aus Deutschland identifiziert, in der Patienten mit kolorektalen Karzinomen, die im Rahmen einer Vorsorgekoloskopie entdeckt worden waren, eine bessere Prognose aufwiesen als Karzinompatienten, die aufgrund von Beschwerden koloskopiert worden waren [106]. In einer weiteren Arbeit aus den USA wies eine Kohorte von 715 Personen nach Vorsorgekoloskopie eine signifikante Senkung von KRK-bedingter Mortalität und Inzidenz im Vergleich zu einem Vergleichskollektiv auf [107].

In großen Kohorten u.a. aus Deutschland konnte gezeigt werden, dass durch die Koloskopie eine hohe Anzahl an Karzinomen im frühen Stadium sowie Adenomen im gesamten Dickdarm entdeckt werden kann [105]. In Deutschland befinden sich etwa 1/3 der im Rahmen der Vorsorgekoloskopie detektierten Karzinome proximal des Colon descendens [105]. In anderen Studien wiesen 46 bis 52\% der Patienten mit proximalen Neoplasien keine zusätzlichen distalen Adenome auf [108, 109]. Bei diesen Patienten wäre eine Diagnose der Neoplasien mittels Sigmoidoskopie unmöglich.

Die Ergebnisse der Fall-Kontrollstudien sowie der randomisierten UK-Studie zur Sigmoidoskopie, die jeweils eine Senkung der Karzinominzidenz und -mortalität zeigen konnten, sollten auf die Koloskopie übertragbar sein [110-113], wobei der Effekt im proximalen Kolon geringer zu sein scheint als im distalen Kolon [114-116]. Auch der protektive Effekt der FOBT-Studien beruht letztendlich auf der Abklärung positiver Tests mittels Koloskopie.

Die Komplikationsrate der Untersuchung in Deutschland war in einer Studie auf freiwilliger Basis sehr gering [1 17]. Diese Ergebnisse konnten kürzlich bestätigt werden [105]. Es ist jedoch von einer Untererfassung der Komplikationen auszugehen, da Spätkomplikationen nur inkomplett erfasst werden. Tandemuntersuchungen haben gezeigt, dass größere Adenome nur selten (0-6\%) übersehen werden [1 18 ].

Es wird davon ausgegangen, dass eine unauffällige Koloskopie nach 10 Jahren wiederholt werden sollte. So fanden sich 5,5 Jahre nach einer unauffälligen Koloskopie keine Karzinome und weniger als 1\% fortgeschrittene Neoplasien [119]. FallKontrollstudien legen nahe, dass das Risiko auch noch mehr als zehn Jahre nach einer unauffälligen Koloskopie sehr niedrig ist $[114,120]$.

Von entscheidender Bedeutung ist, dass die Koloskopie mit höchstmöglicher Qualität durchgeführt wird. Für die Durchführung der Koloskopie existieren in Deutschland klare Richtlinien [122]. 


\subsubsection{Sigmoidoskopie}

\begin{tabular}{|c|c|}
\hline 4.3. & Evidenzbasierte Empfehlung \\
\hline $\begin{array}{l}\text { Empfehlungsgrad } \\
\text { B }\end{array}$ & $\begin{array}{l}\text { Eine qualitätsgesicherte Sigmoidoskopie sollte Personen, die die Koloskopie als } \\
\text { Vorsorge-/ Früherkennungsmaßnahme ablehnen, angeboten werden. }\end{array}$ \\
\hline Level of Evidence & De Novo:[123] \\
\hline & Starker Konsens \\
\hline 4.4. & Evidenzbasierte Empfehlung \\
\hline $\begin{array}{l}\text { Empfehlungsgrad } \\
\text { B }\end{array}$ & $\begin{array}{l}\text { Zur möglichen Detektion proximaler Karzinome sollte zusätzlich zur Sigmoidoskopie } \\
\text { eine jährliche FOBT-Durchführung erfolgen. }\end{array}$ \\
\hline Level of Evidence & De Novo:[1 10, $113,124-131]$ \\
\hline & Starker Konsens \\
\hline
\end{tabular}

\section{Hintergrund}

Die Effektivität der Sigmoidoskopie als Früherkennungs-/Vorsorge-Methode für das KRK ist gesichert. In einer randomisierten Studie aus England wurde im Vergleich zu keinem Screening durch eine einmalige Sigmoidoskopie nach einer Nachbeobachtungs-zeit von 11,2 Jahren eine Senkung der KRK-bedingten Mortalität um 43\% und eine Senkung der Inzidenz kolorektaler Karzinome um 33\%, die distaler Karzinome um 50\% beobachtet [123].

Es ist jedoch zu bedenken, dass im Rahmen einer Sigmoidoskopie nicht alle Darmabschnitte eingesehen werden können. Entsprechend wurde in der Sigmoidoskopiestudie die Inzidenz proximaler Karzinome nicht beeinflusst. Hier ist die Koloskopie der Sigmoidoskopie überlegen.

Der protektive Effekt einer Sigmoidoskopie für distale Neoplasien scheint 6 bis 10 Jahre [113, 124], in einer Studie sogar 16 Jahre anzuhalten [125]. In einer Untersuchung an 9.417 Personen, die 3 Jahre nach unauffälliger Sigmoidoskopie einer erneuten Sigmoidoskopie unterzogen wurden, fanden sich jedoch bei 0,8\% der Patienten im distalen Kolon ein fortgeschrittenes Adenom oder Karzinom [126]. In einer weiteren Studie mit 2.146 Teilnehmern mit unauffälliger Sigmoidoskopie wurde ein Kontrollintervall von 3 und 5 Jahren verglichen [127]. Die Rate an fortgeschrittenen Neoplasien unterschied sich nicht signifikant (0,9\% vs. 1.1\%). Entsprechend wird derzeit eine Wiederholung der Untersuchung bei unauffälligem Befund nach 5 Jahren empfohlen.

Da durch die Sigmoidoskopie isoliert proximal gelegene Tumoren nicht entdeckt werden können, ist weiterhin ein jährlicher FOBT sinnvoll. Dieser sollte vor einer Sigmoidoskopie durchgeführt werden, da bei positivem Test eine Koloskopie erforderlich ist und die Sigmoidoskopie entfallen kann. Der zusätzliche Nutzen der Kombination im Sinne einer 
Mortalitätssenkung ist jedoch nicht gesichert. Eine prospektive nicht-randomisierte Studie fand zwar eine niedrigere KRK-bedingte Mortalität für die Kombination, das Ergebnis verfehlte jedoch grenzwertig die Signifikanz und die Compliance war ausgesprochen niedrig [128]. In mehreren Studien war jedoch eine Kombination aus Sigmoidoskopie und einmaligem FOBT der alleinigen Sigmoidoskopie nicht signifikant überlegen $[129,130]$. In der aktuellsten Studie aus Japan wurden durch die Kombination aus Sigmoidoskopie und FIT absolut 10\% mehr fortgeschrittene Neoplasien entdeckt [131].

$\mathrm{Zu}$ bedenken ist, dass die Sigmoidoskopie derzeit in Deutschland nicht im Leistungskatalog der Krankenkassen enthalten ist und somit nicht abgerechnet werden kann. Ferner sind derzeit anders als für die Vorsorgekoloskopie keine qualitätssichernden Maßnahmen zur Durchführung der Sigmoidoskopie etabliert. In England war eine Voraussetzung bei den Untersuchern für die Teilnahme an der Sigmoidoskopiestudie eine Mindestzahl von 50 angeleiteten und 100 eigenständigen Sigmoidoskopien [110]. Jede Untersuchung wurde per Video dokumentiert und die erreichte Eindringtiefe, die Qualität der Darmvorbereitung und die Ergebnisse dokumentiert.

\subsubsection{Kapsel-Koloskopie}

\begin{tabular}{|l|l|}
$\begin{array}{l}\text { 4.5. } \\
\text { Empfehlungsgrad } \\
\mathbf{B}\end{array}$ & $\begin{array}{l}\text { Die Kapsel-Koloskopie sollte nicht für die Darmkrebs-Vorsorge/-Früherkennung in der } \\
\text { asymptomatischen Bevölkerung eingesetzt werden. }\end{array}$ \\
\hline $\begin{array}{l}\text { Level of Evidence } \\
\mathbf{4}\end{array}$ & \begin{tabular}{l} 
De Novo: [132-138] \\
\hline
\end{tabular} \\
\hline
\end{tabular}

\section{Hintergrund}

Zur Kapsel-Koloskopie konnte in der Literatursuche keine Studie zum Einsatz in der Früherkennung kolorektaler Karzinome identifiziert werden. Es existiert eine Reihe von Fallserien zur Sensitivität und Spezifität kolorektaler Neoplasien mit der ersten Kapselgeneration [132-136]. Für die zweite Kapselgeneration (PCC2) mit verbesserten technischen Eigenschaften wurden Sensitivitäten für Polypen größer 6 mm von 84-89\% beschrieben [137, 138]. Es handelt sich jedoch um kleine Kohorten mit vorselektionierten Patienten, so dass derzeit der Einsatz in der Früherkennung kolorektaler Karzinome in der Allgemeinbevölkerung nicht empfohlen werden kann. 


\subsubsection{Stuhltests ${ }^{5}$}

\subsubsection{Fäkaler occulter Bluttest (FOBT)}

\begin{tabular}{|l|l|l|}
\hline $\begin{array}{l}\text { 4.6. } \\
\text { Konsesbasierte Empfehlung/ Statement }\end{array}$ & 2013 \\
\hline $\begin{array}{l}\text { Bei Personen mit durchschnittlichem Darmkrebsrisiko, die keine Koloskopie } \\
\text { wünschen, sollte ein FOBT jährlich durchgeführt werden. }\end{array}$ & 2008 \\
\hline $\mathbf{4 . 7 .}$ & Starker Konsens \\
\hline $\begin{array}{l}\text { Level of Evidence } \\
\mathbf{1}\end{array}$ & $\begin{array}{l}\text { Ein positives Testergebnis macht die endoskopische Untersuchung des gesamten } \\
\text { Dickdarmes erforderlich. }\end{array}$ \\
\hline
\end{tabular}

\begin{tabular}{|l|l|}
$\begin{array}{l}\text { 4.8. } \\
\text { Level of Evidence }\end{array}$ & $\begin{array}{l}\text { Der jährliche FOBT ist bezüglich einer Senkung der KRK-bedingten Mortalität der } \\
\mathbf{1} \mathbf{D}\end{array}$ \\
\hline & zweijährlichen Untersuchung überlegen. \\
\hline & De Novo: [139] \\
\hline & Starker Konsens \\
\hline
\end{tabular}

\begin{tabular}{|l|l|}
$\begin{array}{l}\text { 4.9. } \\
\text { Level of Evidence }\end{array}$ & $\begin{array}{l}\text { Bei Personen, die an der Koloskopie- Vorsorge/-Früherkennung teilnehmen, erübrigt } \\
\text { sich ein FOBT und auch andere Maßnahmen. }\end{array}$ \\
\hline $\begin{array}{l}\mathbf{1} \\
\mathbf{2}\end{array}$ & \begin{tabular}{l} 
Starker Konsens \\
\hline
\end{tabular}
\end{tabular}

\footnotetext{
${ }^{5}$ Prof. Schmiegel hat sich aufgrund eines möglichen Interessenskonfliktes bei den Abstimmungen zum FOBT/iFOBT, genetischer Stuhltests und M2-PK enthalten (siehe Leitlinienreport).
} 


\begin{tabular}{|l|l|l|}
$\begin{array}{l}\text { 4.10. } \\
\text { Evidenzbasierte Empfehlung }\end{array}$ & 2013 \\
\hline $\begin{array}{l}\text { Empfehlungsgrad } \\
\mathbf{0}\end{array}$ & $\begin{array}{l}\text { Immunologische FOBT (iFOBT) mit nachgewiesen hoher Spezifität }>90 \% \text { und } \\
\text { Sensitivität können alternativ zum Guaiak-Test eingesetzt werden. }\end{array}$ \\
\hline $\begin{array}{l}\text { Level of Evidence } \\
\mathbf{3}\end{array}$ & \begin{tabular}{l} 
De Novo: $[103,140-149]$ \\
\hline
\end{tabular} \\
\hline
\end{tabular}

\section{Hintergrund}

Grundlage für die Stuhltestung auf okkultes Blut ist die Tatsache, dass kolorektale Karzinome häufiger bluten als die normale Darmmukosa. Herkömmliche FOBT verwenden mit Guaiakharz imprägniertes Filterpapier, das sich in Anwesenheit von im Stuhl enthaltenem Hämoglobin nach Zugabe von Wasserstoffperoxid blau färbt. In Deutschland stehen aktuell drei Guaiaktests zur Verfügung: Hämoccult ${ }^{\circledR}$, HemoCare ${ }^{\circledR}$ und HemoFEC ${ }^{\circledR}$. Der sensitivste gFOBT, der Hämoccult Sensa ${ }^{\circledR}$ ist in Deutschland nicht verfügbar. Da viele Karzinome intermittierend bluten [150], führt die wiederholte Testung zu einer zuverlässigeren Erkennung von KRK [151, 152]. Das in den Studien eingesetzte Verfahren beinhaltet, aus drei aufeinander folgenden Stuhlgängen je zwei Proben pro Stuhl auf zwei Testfelder aufzutragen und auf okkultes Blut zu testen [153].

Zur Effektivität des FOBT als Früherkennungsmethode für kolorektale Karzinome liegen die Ergebnisse von 4 großen randomisierten Studien vor [139, 154, 155]. In der aktuellsten Meta-Analyse dieser Studien konnte eine Senkung der KRK-bedingten Mortalität um 25\% für diejenigen gezeigt werden, die sich mindestens einmal einem FOBT unterzogen hatten (relatives Risiko 0,75, 95\%Cl 0,66 - 0.84) [156]. In drei der vier Studien wurde der gFOBT alle zwei Jahre durchgeführt. In einer Studie mit ein- und zweijähriger Testung war die jährliche der zweijährigen Testung in Bezug auf die Reduktion der Mortalität überlegen [139].

Sensitivität und Spezifität des Tests hängen entscheidend von der Art der Testdurchführung und der Patienteninstruktion ab. Eine Rehydrierung der Testbriefchen vor Entwicklung steigert die Sensitivität des Screenings, verringert jedoch die Spezifität deutlich (in einer Studie von 97,6 auf 90,2\%, in einer weiteren von 97 auf 85,4\% [139, 157]) und wird daher nicht empfohlen. Es gibt Hinweise dafür, dass die Instruktion des Patienten vor der Testdurchführung in Bezug auf Ernährung und interferierende Medikamente die Zahl der falsch positiven Testergebnisse und somit auch die Zahl der erforderlichen Koloskopien reduzieren kann [158-160]. Es erscheint daher sinnvoll, den Patienten über Faktoren, die das Testergebnis beeinflussen könnten, aufzuklären. Der Einfluss von Pflanzenperoxidasen kann alternativ durch eine Testentwicklung 3 Tage nach Durchführung vermieden werden [161]. Die Notwendigkeit einer Ernährungsempfehlung für den Hämoccult ${ }^{\circledR}$ wird allerdings durch eine Meta Analyse in Frage gestellt [162].

Bereits bei positivem Testergebnis auf okkultes fäkales Blut von einem der Testfelder ist keine Kontrolle, sondern eine komplette endoskopische Darstellung des Dickdarmes nach digitaler rektaler Untersuchung erforderlich. Leider wurden selbst unter Studienbedingungen teilweise unter $90 \%$ aller Personen mit einem positivem FOBT koloskopiert [163], in einer Studie sogar nur 64 \% [164]. 
Der Effekt des FOBT beruht auf einer Diagnose kolorektaler Karzinome in einem früheren prognosegünstigeren Stadium. Vorteile des FOBT sind die leichte Durchführbarkeit sowie die geringen Kosten. Nachteilig ist eine mäßige Sensitivität für Karzinome und eine geringe Sensitivität für Adenome. In einer randomisierten Studie konnte zwar eine Senkung der Inzidenz kolorektaler Karzinome gezeigt werden, es muss jedoch bedacht werden, dass im Rahmen dieser Studie über 30 \% der Teilnehmer koloskopiert wurden [165].

Immunologische Tests weisen spezifisch menschliches Hämoglobin nach. Insofern ist während der Testdurchführung keine Änderung der Ernährung erforderlich. Auch besteht anders als bei den gFOBT bei einigen der Tests die Möglichkeit einer automatisierten Auswertung und Änderung des Hämoglobingrenzwerts, bei dem ein Test als positiv bewertet wird. Die Tests werden entweder als immunologische FOBT (iFOBT) oder als fäkale immonochemische Tests (FIT) bezeichnet und stellen derzeit in Deutschland keine Kassenleistung dar. Es existieren keine Studien wie für den gFOBT, die eine Senkung der KRK-bedingten Mortalität zum Endpunkt hätten. Jedoch gibt es eine Reihe randomisierter Studien, in denen einzelne iFOBT direkt mit gewissen gFOBT verglichen werden. In der im Rahmen der Literatursuche identifizierten Meta-Analyse dieser Studien waren einzelne iFOBT $\left({ }^{O C C}\right.$-Sensor $\left.{ }^{\circledR}\right)$ dem Hämoccult ${ }^{\circledR}$ Test in der Detektionsrate fortgeschrittener Neoplasien signifikant überlegen mit einer pooled odds ratio (OR) von 2.12 (95\% Cl 1.66-2.71) [140]. In zwei Studien, in denen der gFOBT HemoFEC ${ }^{\circledR}$ bzw. Hämoccult Sensa ${ }^{\circledR}$ mit einem iFOBT $\left(\right.$ Inform ${ }^{\circledR}$ bzw. FlexSure ${ }^{\circledR}$ ) verglichen wurde, fand sich hingegen kein signifikanter Unterschied [140]. Insbesondere in den beiden größten randomisierten Studien aus den Niederlanden [103, 141] fand sich jedoch ein signifikanter Unterschied, die für eine Überlegenheit des eingesetzten iFOBT (OC-Sensor) gegenüber dem Hämoccult ${ }^{\circledR}$ Test sprechen.

Die Sensitivität und Spezifität von in Deutschland verfügbaren iFOBT variiert deutlich, so dass der generelle Einsatz aller iFOBT nicht empfohlen werden kann [142]. Es erscheint vielmehr erforderlich, dass für jeden iFOBT, der für die Krebsfrüherkennung eingesetzt werden soll, individuell eine ausreichend hohe Sensitivität und vor allem Spezifität nachgewiesen wird. Für die Spezifität in Bezug auf das Vorhandensein neoplastischer Veränderungen wird allgemein die untere akzeptable Grenze bei $90 \%$ angesehen. Die Ergebnisse von Screening-Studien legen nahe, dass, bei entsprechen-der Einstellung des Grenzwerts, mit den iFOBTs eine dem gFOBT vergleichbar hohe Spezifität von $>90 \%$ bei zugleich sehr viel höherer Sensitivität erreicht werden kann [143, 149]. Diskutiert wird derzeit noch der optimale Hämoglobin-Gehalt, bei dem ein iFOBT als positiv bewertet werden sollte. In den beiden niederländischen Arbeiten betrug der Grenzwert $100 \mathrm{ng} / \mathrm{ml}$. In den eingeschlossenen Studien wurde jeweils eine Stuhlprobe mittels des iFOBT untersucht. Es gibt Daten die zeigen, dass die Testung von mehreren Stuhlproben die Sensitivität erhöht [145-147]. Eine Untersuchung aus den Niederlanden konnte jedoch zeigen, dass durch eine Absenkung des Grenzwerts ein ähnlicher Effekt erzielt werden kann [148].

Insgesamt zeigt die Datenlage, dass die iFOBT, für die eine entsprechende Datengrundlage vorhanden ist, einen sinnvollen Ersatz für den gFOBT darstellen. 


\subsubsection{Genetische Stuhltests}

\begin{tabular}{|c|c|}
\hline 4.11. & Evidenzbasierte Empfehlung \\
\hline $\begin{array}{l}\text { Empfehlungsgrad } \\
\text { B }\end{array}$ & $\begin{array}{l}\text { Stuhluntersuchungen auf DNA-Veränderungen sollten nicht für die Darmkrebs- } \\
\text { Vorsorge/-Früherkennung in der asymptomatischen Bevölkerung eingesetzt werden. }\end{array}$ \\
\hline Level of Evidence & De Novo: [166-171] \\
\hline & Starker Konsens \\
\hline
\end{tabular}

\section{Hintergrund}

Die Entstehung kolorektaler Karzinome über die Zwischenstufe der Adenome geht in vielen Fällen mit charakteristischen genetischen Veränderungen einher. Eine Isolierung und Untersuchung von DNA aus Kolonepithelzellen im Stuhl ist möglich [166-169]. Dieses Testverfahren hat den Vorteil, dass theoretisch auch nicht blutende Läsionen entdeckt werden könnten. In einer Studie von Imperiale mit nahezu 5.500 asymptomatischen Personen wurde eine Stuhlprobe von 2.500 Teilnehmern auf insgesamt 23 genetische Veränderungen untersucht und mit dem gFOBT verglichen [170]. Die Sensitivität des genetischen Tests war höher als der des gFOBT, betrug aber lediglich $50 \%$ für Karzinome und $15 \%$ für fortgeschrittene Adenome bei Kosten von mehreren Hundert US Dollar pro Test und aufwändiger Testdurchführung. In der Literatursuche wurde lediglich eine weitere Studie identifziert [171]. In dieser prospektiven Studie wurden bei 3.764 asymptomatischen Personen zwischen 50 und 80 Jahren ein gFOBT sowie eine Koloskopie durchgeführt. Von 2.497 dieser Teilnehmer wurde eine Stuhlprobe mit einem DNA-Panel I mit denselben Markern wie in der Studie von Imperiale und von 217 mit einem DNA-Panel II mit nur noch 3 Mutationen inklusive Methylierungsmarker Vimentin untersucht. Die Sensitivität für relevante Neoplasien betrug 20\% für DNA-Panel I und $40 \%$ für DNA-Panel II. Die Sensitivität für den Hämoccult ${ }^{\circledR}$-Test betrug $11 \%$, für den Hämoccult-Sensa ${ }^{\circledR} 21 \%$. Die Spezifität für das DNAPanel II konnte nicht ermittelt werden. Insgesamt war Panel I dem einen gFOBT (Hämoccult ${ }^{\circledR}$ ) überlegen und dem anderen gFOBT (HämoccultSensa ${ }^{\circledR}$ ) gleichwertig. Panel II schien beiden gFOBT überlegen, wurde jedoch nur bei einem kleinen Teil der Teilnehmer untersucht.

Zusammenfassend sind die Daten bzw. Ergebnisse unter Berücksichtigung des Aufwands und der Kosten für einzelne Tests nicht ausreichend, so dass der Einsatz aktuell nicht erfolgen sollte. 


\subsubsection{M2-PK}

4.12.

Empfehlungsgrad

B

Level of Evidence

4

\section{Evidenzbasierte Empfehlung}

Der M2-PK Stuhltest sollte nicht für die Darmkrebs-Vorsorge/-Früherkennung in der asymptomatischen Bevölkerung eingesetzt werden.

De Novo: $[172,173]$

Starker Konsens

\section{Hintergrund}

Die Mehrzahl der Studien erfolgte an vorselektierten Patientenkollektiven und erlaubt somit keine Aussage zur Wertigkeit des Tests für die Vorsorge/ Früherkennung in der asymptomatischen Bevölkerung. In der extern durchgeführten Literatursuche wurden zwei Studien [172, 173] zur Untersuchung einer Vorsorge-/Früherkennungspopulation identifiziert. In einer Studie wurde der M2-PK Test mit der Koloskopie bei 1082 asymptomatischen Personen verglichen. Die Sensitvität für fortgeschrittene Adenome betrug $21,7 \%$ bei einer Spezifität von $82 \%$. In einer weiteren Studie mit 1.079 Teilnehmern wurde der M2-PK Test mit verschiedenen FOBT verglichen. Die Sensitivität für fortgeschrittene Neoplasien betrug 27,3\% (im Vergleich zu 7,3-20,0\% für die FOBT) bei einer Spezifität von $86,2 \%$ (FOBT 92,9-94,0\%). Der positive prädiktive Wert für fortgeschrittene Neoplasien betrug $11,5 \%$ und war niedriger als alle getesteten FOBT. Insgesamt ist die Datenlage nicht ausreichend, um den Test für die Vorsorge/ Früherkennung in der asymptomatischen Bevölkerung empfehlen zu können.

\subsubsection{Radiologische Verfahren}

\subsection{3.}

Empfehlungsgrad

B

Level of Evidence

3b

\section{Evidenzbasierte Empfehlung}

Die CT-Kolonographie und die MR-Kolonographie sollten nicht für die DarmkrebsVorsorge/-Früherkennung in der asymptomatischen Bevölkerung eingesetzt werden. Bei inkompletter Koloskopie (z. B. Adhäsionen) und fortbestehendem Wunsch des Patienten auf komplette Kolonbeurteilung sollte eine CT- oder MR-Kolonographie erfolgen.

De Novo:[174-176]

Starker Konsens

\section{Hintergrund}

Für den Einsatz der MR-Kolonographie existieren nur einzelne kleine Studien, so dass die Datenlage nicht ausreicht, um einen Einsatz für die Vorsorge/Früherkennung zu befürworten. Für die CT-Kolonographie (CTC) ist die Datenlage umfangreicher. In den beiden in der Literatursuche identifizierten aktuellsten Meta-Analysen für den Vergleich 
der CTC und der Koloskopie als Vorsorge-Früherkennungsmaßnahme in der asymptomatischen Bevölkerung zeigte sich eine hohe Sensitivität von $100 \%$ für die Detektion von Karzinomen und von 87,9\% für die Detektion von Adenomen $\geq 10 \mathrm{~mm}$. Die Sensitivität für kleinere Adenome war geringer [174, 175]. Weiterhin fällt zwischen den verschiedenen Studien eine nicht unerhebliche Heterogenität auf. Es bleibt unklar, ob die Ergebnisse der Studien, die an erfahrenen Zentren durchgeführt wurden, auf die klinische Praxis übertragbar sind. Weiterhin ist die Bedeutung extrakolonischer Befunde unklar. Die Untersuchung geht mit einer Strahlenexposition einher, so dass sich der Einsatz bei vorhandenen Alternativmethoden in Deutschland als FrüherkennungsNorsorgemethode aufgrund der Strahlenschutzverordnung verbietet (StrlSchV §80). Das genaue Neoplasierisiko durch eine CTC unter Verwendung neuerer Geräte mit verringerter Strahlendosis ist unklar. Unklar ist weiterhin, ab welcher Polypengröße eine Koloskopie durchgeführt werden sollte und in welchem Intervall Patienten mit unauffälliger CTC oder kleineren Polypen kontrolliert werden sollten [176].

Patienten, bei denen eine Koloskopie aus technischen Gründen nur inkomplett durchgeführt werden konnte, sollte eine Wiederholung der Koloskopie z.B. unter stationären Bedingungen oder die CTC als Alternative zur Beurteilung des restlichen Kolons angeboten werden (siehe Kapitel 6.1.)

\subsection{Kosteneffektivität}

\subsection{4.}

EK

\section{Konsensbasiertes Statement}

Sowohl FOBT als auch Sigmoidoskopie, Koloskopie und die Kombination aus Sigmoidoskopie und FOBT gelten als kosteneffektiv (im Vergleich zu Vorsorge-/ Früherkennungsverfahren anderer Zielkrankheiten).

Konsens

\section{Hintergrund}

Prospektive Studien zur Kosteneffektiviät der verschiedenen KRK-Vorsorgeverfahren existieren nicht. Mathematische Modellrechnungen legen nahe, dass Koloskopie, Sigmoidoskopie und FOBT kosteneffektiv sind [141, 177-187].

\subsection{Empfehlungen anderer eingeschlossener Leitlinien}

Anhand der DELBI-Kriterien wurden 2 Leitlinien zur KRK-Vorsorge/-Früherkennung eingeschlossen. Zum einen die Empfehlungen der US Preventive Task Force aus dem Jahr 2008 [95] und zum anderen die Asian Pacific consensus recommendations aus dem Jahr 2007 [188]. Von der US-Preventive Task Force werden die folgenden Methoden empfohlen: ein sensitiver FOBT (aufgeführt Hämoccult sensa ${ }^{\circledR}$ und IFOBT/FIT) jährlich, eine Sigmoidoskopie alle 5 Jahre zusammen mit einem FOBT alle 3 Jahre oder eine Koloskopie alle 10 Jahre. Der Einsatz der CT-Kolonographie und genetischer Stuhltests wird nicht empfohlen, die Kapselendoskopie und der M2-PK-Test werden nicht aufgeführt. Zu erwähnen ist, dass der Hämoccult sensa ${ }^{\circledR}$ in Deutschland nicht verfügbar ist.

In der Asian Pacific Leitlinie werden der FOBT (gFOBT und iFOBT) alle 1-2 Jahre, die Sigmoidoskopie alle 5 Jahre und die Koloskopie alle 10 Jahre empfohlen. Die CTKolonographie wird nicht empfohlen, genetische Stuhltests, Kapselendoskopie und M2PK-Test sind nicht aufgeführt. 
5. Risikogruppen

Personen, die aufgrund einer besonderen Prädisposition ein erhöhtes Risiko für die Entwicklung eines kolorektalen Karzinoms im Vergleich mit der Normalbevölkerung aufweisen, gehören in der Regel zu einer von drei definierten Risikogruppen:

- $\quad$ Personen mit einem familiär gesteigerten Risiko für ein kolorektales Karzinom, dessen genetische Grundlagen z. Zt. noch nicht umfassend bekannt sind

- $\quad$ nachgewiesene oder mögliche Anlageträger für ein hereditäres kolorektales Karzinom

- $\quad$ Risikopersonen auf dem Boden einer chronisch entzündlichen Darmerkrankung

\subsection{Sporadisches kolorektales Karzinom}

\subsubsection{Risikogruppen}

\subsubsection{Verwandte von Patienten mit kolorektalem Karzinom}

\begin{tabular}{|l|l|}
$\mathbf{5 . 1 .}$ & Evidenzbasiertes Statement \\
\hline $\begin{array}{l}\text { Level of Evidence } \\
\mathbf{2 a}\end{array}$ & $\begin{array}{l}\text { Verwandte ersten Grades von Patienten mit einem kolorektalen Karzinom haben ein } \\
\text { erhöhtes Risiko, ebenfalls an einem kolorektalen Karzinom zu erkranken. }\end{array}$ \\
\hline & Evidenz aus Aktualisierungsrecherche: [189-203] \\
\hline & Konsens \\
\hline
\end{tabular}

\begin{tabular}{|l|}
\hline $\mathbf{5 . 2}$. \\
\hline Level of Evidence \\
$\mathbf{2 b}$ \\
\hline
\end{tabular}

\section{Evidenzbasiertes Statement}

2013

Verwandte zweiten Grades haben ein gering erhöhtes Risiko, an einem kolorektalen Karzinom zu erkranken.

Evidenz aus Aktualisierungsrecherche: [189, 190, 193, 194, 204, 205]

Konsens

\section{Hintergrund}

Etwa 20-30\% der KRK-Fälle treten „familiär gehäuft“ auf, d. h. es kann ein gehäuftes Vorkommen der Krebserkrankung innerhalb einer Familie beobachtet werden, ohne dass jedoch konkrete genetische Ursachen identifiziert werden können. In diesem Fall wird der Begriff „Familiäres kolorektales Karzinom“ verwendet [189]. Für Verwandte ersten Grades (Eltern, Geschwister, Kinder) eines Patienten mit einem KRK ist das mittlere Risiko 2- bis 3-fach erhöht. Eine weitere, 3- bis 4-fache Risikosteigerung besteht, wenn bei dem Indexpatienten das KRK vor dem 60. Lebensjahr aufgetreten ist und/oder mehr als ein 
Verwandter ersten Grades von einem KRK betroffen ist [190-202]. In dieser Gruppe befinden sich allerdings auch bislang unentdeckte hereditäre KRK (z. B. HNPCC; s. u.). Das Risiko ist für das Kolon- im Vergleich zum Rektumkarzinom höher (relatives Risiko 2,4 vs. 1,9). Für erstgradig Verwandte von betroffenen Patienten kann das KRK-Risiko weiter aufgeteilt werden. So ist das Risiko für die Geschwister etwa 2,5-fach höher als für die Kinder. Ist der Indexpatient nach dem 60. Lebensjahr erkrankt, ist das KRK-Risiko für die erstgradig Verwandten nur noch gering erhöht [191, 203].

Verwandte zweiten Grades (Großeltern, Geschwister der Eltern, Enkel) von Patienten mit kolorektalen Karzinomen haben ein leicht erhöhtes Karzinomrisiko ( $R R$ 1,5); dieses ist aber derzeit nur unzureichend untersucht und bisher nicht in der Praxis verifiziert [190, 193, 194, 204, 205]. Für Verwandte dritten Grades von Patienten mit KRK ist kein erhöhtes Karzinomrisiko anzunehmen.

\subsubsection{Verwandte von Patienten mit kolorektalem Adenom}

\begin{tabular}{|l|l|}
\hline $\mathbf{5 . 3 .}$ & Evidenzbasiertes Statement \\
\hline $\begin{array}{l}\text { Level of Evidence } \\
\mathbf{2 0}\end{array}$ & $\begin{array}{l}\text { Verwandte ersten Grades von Patienten, bei denen ein kolorektales Adenom vor dem } \\
\text { 50. Lebensjahr nachgewiesen wurde, haben ein erhöhtes Risiko, an einem } \\
\text { kolorektalen Karzinom zu erkranken. }\end{array}$ \\
\hline Evidenz aus Aktualisierungsrecherche: [191, 194, 206-209] \\
\hline Konsens
\end{tabular}

\section{Hintergrund}

Das Risiko dieser Verwandten, ein KRK zu entwickeln, ist im Mittel etwa 2-fach gegenüber der Allgemeinbevölkerung gesteigert [191, 194, 206-209]; es besteht ein 80\% höheres Risiko bei Eltern und Geschwistern von Adenom-Patienten im Vergleich mit deren Lebenspartnern [206]. Auch hier ist die Risikohöhe vom Alter des Indexpatienten abhängig: Ist dieser jünger als 60 Jahre, ist das mittlere Risiko nur leicht erhöht, ist er jünger als 50 Jahre, ist das Risiko ca. 4,4-fach erhöht [207]. Ist der Indexpatient älter als 60 Jahre, ist das kolorektale Karzinomrisiko nicht mehr statistisch signifikant erhöht.

Aufgrund der Datenlage gibt es keine Evidenz, dass Verwandte von Patienten, bei denen ein hyperplastischer Polyp nachgewiesen wurde, ein erhöhtes Risiko haben, an einem kolorektalen Karzinom zu erkranken. Eine Ausnahme ist das sehr seltene hyperplastische Polyposis Syndrom.

\subsubsection{Patienten mit kolorektalen Adenomen}

\begin{tabular}{|c|c|c|}
\hline 5.4. & Evidenzbasiertes Statement & 2008 \\
\hline \multirow[t]{3}{*}{ Level of Evidence } & $\begin{array}{l}\text { Jedes histologisch nachgewiesene Adenom stellt ein erhöhtes } \\
\text { kolorektales Karzinom dar. Dies gilt insbesondere für: } \\
\text { multiple }(\geq 3) \text { Adenome } \\
\text { große }(>1 \mathrm{~cm}) \text { Adenome }\end{array}$ & Risiko für ein \\
\hline & {$[100,101,210,211]$} & \\
\hline & Konsens & \\
\hline
\end{tabular}




\section{Hintergrund}

Generell führt die Abtragung kleiner, singulärer Adenome im Vergleich zur Normalbevölkerung zu einem um bis zu 90\% verminderten Risiko, ein metachrones KRK zu entwickeln [100, 101, 210, 211]. Dieses reflektiert den Vorsorgewert der Koloskopie im Rahmen der Adenom-Karzinom-Sequenz. Kontrolluntersuchungen dienen insbesondere der Entdeckung übersehener oder metachron aufgetretener Adenome.

Adenome $>1 \mathrm{~cm}$ sind mit einem etwa 4-fach erhöhten Karzinomrisiko assoziiert [194, 21 1-218]. Auch bei multiplen Adenomen ist das Risiko, ein metachrones Karzinom zu entwickeln, deutlich (4- bis 6-fach) gesteigert [194, $211,213,214,216,217]$. Hierbei dürfte das erhöhte Risiko einerseits auf einer stärkeren individuellen Disposition, andererseits auf einer höheren Prävalenz übersehener Polypen bei der initialen Koloskopie beruhen: beim koloskopischen Nachweis von $\geq 3$ Polypen besteht eine signifikant größere Wahrscheinlichkeit, dass weitere Polypen übersehen wurden [118, 219].

\section{5 .}

\section{Konsensbasierte Empfehlung}

2013

Ein erhöhtes familiäres Darmkrebsrisiko wird durch die ärztliche Anamnese erfasst. Jedoch besteht keine verpflichtende Erfassung der Familienanamnese und ggf. deren Wiederholung im Intervall. Die Verwendung von standardisierten Fragebögen kann geeignet sein, die Erfassung von Personen mit erhöhtem Risiko zu verbessern.

Starker Konsens

\section{Hintergrund}

Die Risikofeststellung erfolgt in der Regel über das ärztliche Anamnesegespräch. Eine Möglichkeit zur Integration der Risikofeststellung in die medizinische Betreuung bestünde in der Verwendung eines standardisierten Fragebogens in der hausärztlichen Praxis im Alter von 35 Jahren im Rahmen der zu diesem Zeitpunkt angebotenen Checkup-Untersuchung.

Durch eine Integration in die Praxis-Software als Memo kann diese Befragung nachgeholt werden, sofern der Patient zu dem o.g. Zeitpunkt nicht in der Praxis vorstellig wird. Ein entsprechendes Konzept wird gegenwärtig im Gemeinsamen Bundesausschuss geprüft.

Weitere sinnvolle Stellen sind gynäkologische Praxen, da hier regelmäßige Vorstellungen im Rahmen der gynäkologischen Krebsfrüherkennung empfohlen werden, sowie Vorstellungen in gastroenterologischen Praxen.

Darüber hinaus steht eine Reihe von Fragebögen, die teilweise im Internet frei zugänglich sind, zur Verfügung:

- $\quad$ http://www.onkozert.de/hinweise_zertifizierung_genetische_beratung.htm

- $\quad$ http://www.netzwerk-gegen-darmkrebs.de/index.php?id=103

- $\quad$ http://www.felix-burda-stiftung.de/darmkrebs/frageboegen/index.php?f=2

- $\quad$ http://www.lebensblicke.de/darmkrebs/

- $\quad$ http://www.ilco.de/darmkrebs/erblicher-darmkrebs.html

- $\quad$ http://www.krebsrisikotest.de/ 
Von den genannten Fragebögen ist der vom „Netzwerk gegen Darmkrebs“ verwendete Fragebogen evaluiert worden [220]. Zu den anderen verwendeten Fragebögen liegen bisher keine Daten vor.

\subsubsection{Primärprävention}

\begin{tabular}{|l|}
\hline 5.6. \\
\hline Level of Evidence \\
\hline b \\
\hline
\end{tabular}

Evidenzbasiertes Statement

Eine gesonderte Empfehlung zur Primärprävention (diätetische Maßnahmen, Chemoprävention) im Vergleich zur Normalbevölkerung kann aufgrund der widersprüchlichen Daten für die genannten Risikogruppen nicht gegeben werden.

Evidenz aus Aktualisierungsrecherche: [221-224]

Starker Konsens

\section{Hintergrund}

Generell können die für die Normalpopulation genannten Empfehlungen (siehe Kapitel 3) auch für die Angehörigen der Risikogruppen übernommen werden; für spezielle Maßnahmen fehlen gesicherte Daten [221-224].

\subsubsection{Vorsorgeuntersuchungen}

\subsubsection{Verwandte ersten Grades von Patienten mit kolorektalem Karzinom}

\begin{tabular}{l|l|l} 
5.7. Konsensbasierte Empfehlung & 2013
\end{tabular}

\section{EK Verwandte ersten Grades von Patienten mit kolorektalem Karzinom sollten in einem} Lebensalter, das 10 Jahre vor dem Alterszeitpunkt des Auftretens des Karzinoms beim Indexpatienten liegt, erstmals komplett koloskopiert werden, spätestens im Alter von 40-45 Jahren. Die Koloskopie sollte bei polypenfreiem Darm in der initialen Koloskopie mindestens alle 10 Jahre wiederholt werden.

Starker Konsens

\section{Hintergrund}

Das Risiko eines Verwandten ersten Grades eines Patienten mit kolorektalem Karzinom, ebenfalls an einem kolorektalen Karzinom zu erkranken, ist, insbesondere bei einem Manifestationsalter unter 50 Jahren beim Indexpatienten, erhöht [194, 196, 203, 205, 225-230].

Die Empfehlung lehnt sich an die amerikanische Leitlinienempfehlung an [231]. Dort wird eine Empfehlung zur Koloskopie im Alter von 40 Jahren ausgesprochen, wenn ein erstgradig Verwandter vor dem 60. Lebensjahr an einem KRK erkrankt ist oder zwei oder mehr erstgradig Verwandte an einem KRK (unabhängig vom Erkrankungsalter) erkrankt sind.

Die Frage des maximalen Untersuchungsintervalls ist bisher nicht eindeutig geklärt; es gilt aber als wahrscheinlich, dass ein Intervall von 10 Jahren in der Regel ausreichen dürfte, dieses aber nicht überschritten werden sollte. In der amerikanischen Leitlinie wird ein 5-Jahres-Intervall empfohlen. 
5.8.

\section{EK}

5.9.

\section{Konsensbasierte Empfehlung}

2013

\section{EK}

Bei erstgradig Verwandten von Patienten mit kolorektalem Karzinom aus Familien, in denen die Bethesda-Kriterien, nicht aber die Amsterdam-Kriterien erfüllt sind, sollte in kürzeren Intervallen koloskopiert werden:

Wenn kein Tumorgewebe zur Untersuchung auf HNPCC-typische Auffälligkeiten zur Verfügung steht, sollte das Intervall 3 Jahre nicht überschreiten.

Konsens

\section{Empfehlung}

Wenn das Tumorgewebe eine Mikrosatellitenstabilität (MSS) oder eine niedriggradige Mikorsatelliteninstabilität (MSI-L) zeigt, sollte das Intervall 3-5 Jahre betragen.

Konsens

\section{Hintergrund}

Bei jungen Indexpatienten in der Verwandtschaft sollte die Diagnose eines HNPCCSyndroms (siehe Abschnitt 5.2.1.1) in Erwägung gezogen werden und eine Mikrosatellitenanalyse und/oder immunhistochemische Untersuchung der MismatchReparatur-Proteine durchgeführt werden. In der klinischen Praxis finden sich immer wieder Familien, in denen eine Häufung kolorektaler Karzinome vorliegt, jedoch ohne, dass die klinischen Diagnosekriterien für HNPCC (Amsterdam II-Kriterien) erfüllt sind. Sollte in diesen Familien kein Tumorgewebe mehr verfügbar sein oder dieses eine Mikrosatellitenstabilität (MSS) zeigen, kann das Vorliegen einer bislang unbekannten erblich bedingten gastrointestinalen Tumordisposition nicht sicher ausgeschlossen werden. Ein Untersuchungsintervall von höchstens 10 Jahren erscheint in dieser Konstellation nicht engmaschig genug, auch wenn diese Fragestellung bisher nicht eindeutig geklärt ist; ein Intervall von 3 bis 5 Jahren dürfte dem familiären Risiko angemessen sein.

Bei Patienten aus Familien, welche die Amsterdam-Kriterien erfüllen, eine Mikrosatelliteninstabilität aber ausgeschlossen werden konnte, wurde bisher analog zu den Familien mit nachgewiesener MSI eine jährliche Vorsorge empfohlen. Dies ist aufgrund 
der Datenlage nicht erforderlich. Intervalle von 3 bis 5 Jahren sind angemessen [232, 233]. Eine Überwachung hinsichtlich extrakolonischer Tumoren ist bei diesem Personenkreis außerhalb der allgemeinen Krebsfrüherkennungsmaßnahmen nicht erforderlich.

\subsubsection{Verwandte von Patienten mit kolorektalen Adenomen}

\begin{tabular}{l|l} 
5.11. & Konsensbasierte Empfehlung \\
\hline EK & $\begin{array}{l}\text { Verwandte ersten Grades von Indexpatienten, bei denen Adenome vor dem } 50 . \\
\text { Lebensjahr nachgewiesen wurden, sollten } 10 \text { Jahre vor dem Lebensalter zum } \\
\text { Zeitpunkt des Nachweises des Adenoms koloskopiert werden. Die Koloskopie sollte } \\
\text { bei polypenfreiem Darm in der initialen Koloskopie mindestens alle } 10 \text { Jahre } \\
\text { wiederholt werden. Bei Nachweis von Polypen gelten die Empfehlungen von Kapitel } 0 .\end{array}$ \\
& Starker Konsens
\end{tabular}

\section{Hintergrund}

Die Empfehlung basiert auf dem in Abschnitt 5.1.1 dargelegten erhöhten Risiko dieser Population [93, 196, 205, 207].

\subsection{Hereditäre kolorektale Karzinome}

\subsection{2 .}

\section{EK}

\section{Konsensbasierte Empfehlung}

2013 des Darmkrebses vorliegt oder, dass eine gesunde Person ein hohes Risiko für eine erbliche Form von Darmkrebs hat, sollte der Patient in einem interdisziplinären Zentrum mit ausgewiesener Expertise auf dem Gebiet des erblichen Darmkrebses vorgestellt werden.

Konsens

\section{Hintergrund}

Monogen erbliche kolorektale Karzinome sind selten (weniger als $5 \%$ aller kolorektalen Karzinome). Die Diagnose hat erhebliche Konsequenzen für die Patienten und ihre Verwandten. Im Verdachtsfall sollte ein erfahrenes Zentrum für familiären Darmkrebs oder ein äquivalentes Angebot mit entsprechender Expertise kontaktiert werden. Eine molekulargenetische Diagnostik bei erkrankten Patienten dient der Diagnosesicherung und ermöglicht die prädiktive Testung von Familienangehörigen. Eine entsprechende Keimbahnmutationssuche soll nach den Richtlinien zur Diagnostik der genetischen Disposition für Krebserkrankungen der Bundesärztekammer und dem Gendiagnostikgesetz (GenDG) erfolgen [121]. Beispielhaft ist der Algorithmus für das HNPCC-/LynchSyndrom in Abbildung 1 dargestellt. Er gilt in analoger Weise für die anderen erblichen Syndrome mit erhöhtem Darmkrebsrisiko. Neben einem erhöhten Risiko für kolorektale Karzinome haben alle Patienten und Risikopersonen dieser Gruppe ein zusätzlich erhöhtes Risiko extrakolischer Neoplasien. Aufgrund des meist autosomal-dominanten Erbgangs haben erstgradig Verwandte von Betroffenen ein 50\%iges Risiko, diese genetische Disposition ebenfalls geerbt zu haben. Eine prädiktive genetische Testung bei diesen gesunden Risikopersonen muss dem GenDG entsprechend an eine genetische 
Beratung gebunden sein und kann in der Regel nur erfolgen, wenn eine zweifelsfrei pathogene Keimbahnmutation bei einem erkrankten Familienmitglied nachgewiesen wurde (siehe Abbildung 1) [121].

\begin{tabular}{|l|l|}
\hline 5.13. & Konsensbasierte Empfehlung \\
\hline EK & $\begin{array}{l}\text { Bereits erkrankte Personen, Anlageträger und Risikopersonen für monogen erbliche } \\
\text { Erkrankungen mit erhöhtem Risiko für kolorektale Karzinome sollen auf Möglichkeit } \\
\text { und Nutzen einer psychosozialen Beratung und Betreuung hingewiesen werden. }\end{array}$ \\
\hline Starker Konsens
\end{tabular}

\section{Hintergrund}

Die Diagnose eines manifesten erblichen Tumorsyndroms, das Wissen um ein deutlich erhöhtes Erkrankungsrisiko oder der definitive Nachweis der Anlageträgerschaft können bei den Betroffenen und ihren Angehörigen mit einer Vielzahl psychosozialer Stressfaktoren einhergehen. Entsprechende Untersuchungen wurden insbesondere bei der FAP durchgeführt und schlossen neben Erwachsenen und Jugendlichen auch Kinder und deren Eltern ein [234-236]. Zu den relevante Stressoren gehören eine veränderte Körperwahrnehmung, Ängste in Bezug auf chirurgische Eingriffe, Früherkennungsuntersuchungen und eine zukünftige Krebsentwicklung, befürchtete berufliche Einschränkungen, das Kommunizieren des Krankheitsbildes im sozialen Umfeld, Unsicherheit in Bezug auf reproduktive Entscheidungen sowie das Verarbeiten früh an Krebs verstorbener Familienangehöriger und damit zusammenhängender innerfamiliärer Konflikte.

Die prädiktive Testung Minderjähriger geht zusätzlich mit spezifischen Herausforderungen wie fehlender eigener Entscheidungsfähigkeit und eingeschränktem Verständnis von Sinn und Konsequenzen der Testung einher. Ergänzend zur klinischen und humangenetischen Beratung kann die psychosoziale Beratung die Patienten und Risikopersonen im Prozess der Entscheidungsfindung für bzw. gegen eine prädiktive genetische Diagnostik unterstützen und für die Verarbeitung von Testergebnissen hilfreich sein. 


\subsubsection{Risikogruppen}

5.2.1.1. HNPCC (hereditäres kolorektales Karzinom ohne Polyposis)/ LynchSyndrom

\begin{tabular}{|l|l|}
\hline $\mathbf{5 . 1 4 .}$ & Konsensbasiertes Statement \\
\hline EK & $\begin{array}{l}\text { Personen aus Familien, die die Amsterdam-Kriterien erfüllen oder eines der Bethesda- } \\
\text { Kriterien mit Nachweis einer Mikrosatelliteninstabilität (MSI) und deren Verwandte, die } \\
\text { aufgrund des Erbgangs als Mutationsträger in Betracht kommen, sind Risikopersonen } \\
\text { für HNPCC. }\end{array}$ \\
\hline Starker Konsens
\end{tabular}

\section{Hintergrund}

Das HNPCC-Syndrom wird nach anamnestischen Kriterien definiert (Amsterdam-I- und II-Kriterien, siehe Kapitel 12.2). Um weitere Risikopersonen zu identifizieren, werden auch die revidierten Bethesda-Kriterien herangezogen (Bethesda-Kriterien, siehe Kapitel 12.3. In der Literatur wird favorisiert, Anlageträger einer pathogenen Keimbahnmutation in einem der MMR-Gene als Personen mit Lynch-Syndrom zu bezeichnen. Demgegenüber konnte bei Patienten mit einem HNPCC keine pathogene Keimbahnmutation nachgewiesen werden. Zur Vereinfachung wird nachfolgend nur der Begriff HNPCC verwendet.

Mutationsträger haben ein sehr hohes Risiko, ein kolorektales Karzinom (50-70\%) oder ein Endometriumkarzinom (20-60\%) zu entwickeln. Dies gilt vermindert auch für weitere Neoplasien wie Ovarial-, Magen- und Dünndarmkarzinome sowie Urothel-karzinome des Nierenbeckens und Harnleiters.

5.15.

Die Feststellung, ob bei einem Patienten die Bethesda- oder Amsterdam-Kriterien für HNPCC erfüllt sind, soll anamnestisch durch die behandelnden Ärzte erfolgen.

Starker Konsens

\section{Hintergrund}

Im Gegensatz zur FAP ist HNPCC aufgrund eines fehlenden auffälligen Phänotyps klinisch nicht einfach zu identifizieren. Aus diesem Grunde wurden Kriterien definiert, die es sinnvoll erscheinen lassen, zunächst eine Untersuchung des Tumorgewebes und anschließend ggf. eine Mutationssuche durchzuführen. Die Verdachtsdiagnose HNPCC kann klinisch gestellt werden, wenn in der Familie des Patienten die so genannten Amsterdam-I-Kriterien erfüllt sind [237]. Beim HNPCC treten neben KRK häufig auch Endometrium- und Urothelkarzinome aber auch Dünndarmkarzinome auf. Die Amsterdam-II-Kriterien beziehen diese extrakolischen Manifestationen in die Diagnosestellung ein [179]. Diese Kriterien stellen unter klinischen Gesichtspunkten eine Operationalisierung dar. Da viele Familien heute klein sind, fehlen allerdings oft die Voraussetzungen, um die Kriterien überhaupt zu erfüllen. Daher kann eine unauffällige Familienanamnese - insbesondere in kleinen Familien - kein Argument gegen HNPCC 
sein. Die weniger spezifischen Bethesda-Kriterien begründen den Verdacht HNPCC auch in kleineren Familien und in Einzelfällen (Anlage 12.3) [238].

Das Risiko von HNPCC-Anlageträgern, mindestens ein HNPCC-assoziiertes Karzinom zu entwickeln, wird mit $80-90 \%$ angegeben, wobei das KRK die mit Abstand häufigste Tumorentität darstellt. KRK treten im Rahmen eines HNPCC im Mittel im 44. Lebensjahr auf, vor dem 25. Lebensjahr sind kolorektale Karzinome bei HNPCC sehr selten. Das kumulative Lebenszeitrisiko eines HNPCC-Anlageträgers für ein KRK beträgt ca. 60-70\%, wobei das Risiko für Männer ca. 10\% höher ist als das für Frauen.

Das Endometriumkarzinom ist nach dem KRK der zweithäufigste Tumor bei HNPCC. Das Lebenszeitrisiko für weibliche Anlageträgerinnen, ein Endometriumkarzinom zu entwickeln, beträgt $40-60 \%$ bei einem medianen Erkrankungsalter zwischen 46 und 48 Jahren. Karzinome des Ovars treten bei ungefähr 10-15\% aller Anlageträgerinnen auf. Magenkarzinome kommen bei 2 bis $13 \%$ der HNPCC-Patienten vor und treten durchschnittlich im Alter von 51-56 Jahren auf. Erkrankungen vor dem 40. Lebensjahr sind selten. Vorwiegend handelt es sich dabei um Karzinome vom intestinalen Typ. Für Deutschland wurde ein kumulatives Lebenszeitrisiko von $6.8 \%$ bis zum 70 . Lebensjahr ermittelt [239].

Das kumulative Lebenszeitrisiko für Dünndarmkarzinome im Rahmen eines HNPCC beträgt $4-8 \% \quad[239,240]$. Bei $35-50 \%$ der Fälle ist das HNPCC-assoziierte Dünndarmkarzinom im Duodenum lokalisiert [241]. Erkrankungen vor dem 30. Lebensjahr sind selten. Karzinome der oberen ableitenden Harnwege (Ureter/ Nierenbecken) treten häufig als Zweit- oder Drittkarzinome auf. Das mittlere Erkrankungsalter für diese Tumoren wird mit 50-63 Jahren angegeben. Das Lebenszeitrisiko wird mit 1-12\% angegeben. In einigen Familien wurde eine Häufung von Urothelkarzinomen beobachtet. In einer aktuellen niederländischen Studie [242] wird ein relatives Risiko für Urothelkarzinome des gesamten Urogenitaltraktes (einschließlich der Blasen-karzinome) von 4.2 für männliche und von 2.2 für weibliche Träger(innen) einer pathogenen Keimbahnmutation in einem der MMR-Gene im Vergleich zur nieder-ländischen Allgemeinbevölkerung angegeben. Bislang noch unveröffentlichte Ergebnisse des Deutschen HNPCC-Konsortiums bestätigen diese Angaben.

Das Lebenszeitrisiko für biliäre Tumoren ist bei HNPCC erhöht, aber doch insgesamt relativ niedrig. Pankreaskarzinome werden im Rahmen eines HNPCC selten aber signifikant häufiger als in der Allgemeinbevölkerung beobachtet (Relatives Risiko 8,6; Lebenszeitrisiko 3,7\%) [243, 244].

Für Hirntumoren besteht bei HNPCC ein leicht erhöhtes Risiko; histologisch handelt es sich dabei überwiegend um Astrozytome und Glioblastome. Das mediane Erkrankungsalter wird mit 40-54 Jahren angegeben [245-247]. Beim Muir-Torre-Syndrom handelt es sich um eine seltene phänotypische Variante des HNPCC, bei der neben den bereits genannten HNPCC-Tumoren vor allem Talgdrüsenadenome oder -karzinome auftreten [248]. 


\begin{tabular}{l} 
5.16. \\
Konsensbasierte Empfehlung \\
\hline Eine weiterführende (molekular-) pathologische Untersuchung hinsichtlich HNPCC soll \\
bei mindestens einem erfüllten revidierten Bethesda-Kriterium durchgeführt werden. \\
Hierfür kann entweder an dem Tumorgewebe primär eine qualitäts-gesicherte \\
Untersuchung der immunhistochemischen Expression der DNA-Mismatch-Reparatur- \\
Proteine MLH1, MSH2, MSH6 und PMS2 oder eine Untersuchung auf Mikrosatelliten- \\
Instabilität erfolgen. Um ein HNPCC sicher auszuschließen, soll bei unauffälliger \\
immunhistochemischer Expression der DNA-Mismatch-Reparatur-Proteine zusätzlich \\
eine Untersuchung auf Mikrosatellitenstabilität angeschlossen werden. Auf diese \\
kann nur verzichtet werden, wenn immunhistochemisch ein Verlust eindeutig \\
nachgewiesen wurde. \\
Starker Konsens
\end{tabular}

\section{Hintergrund}

Ein Algorithmus zur Testung auf einen Mismatch-Reparatur-Defekt im Tumorgewebe ist in Abbildung 2 dargestellt. Eine Mikrosatelliteninstabilität lässt sich bei etwa $80 \%$ der Tumorgewebe von Patienten, die die Amsterdam I/II-Kriterien erfüllen, nachweisen. Dieses Phänomen geht auf den zugrundeliegenden Defekt eines DNA-Reparaturenzyms zurück, durch den bei der Zellteilung entstehende Basenfehlpaarungen nicht mehr korrigiert werden können. Solche Fehlpaarungen treten besonders leicht an Stellen repetitiver kurzer DNA-Fragmente auf (sog. Mikrosatelliten). In Reparatur-defizienten HNPCC-Tumoren findet sich deshalb typischerweise an vielen Stellen des Genoms ein vom Wildtyp der normalen Zellen abweichendes Mikrosatellitenmuster, was zur Bezeichnung "Mikrosatelliteninstabilität" geführt hat. Bei Patienten aus Familien, in denen die Amsterdam-Kriterien erfüllt sind und deren Tumorgewebe eine Mikrosatellitenstabilität (MSS) zeigt, sollte nach Möglichkeit ein unabhängiger zweiter Tumor aus der Familie untersucht werden.

Bei Patienten, deren Familien die Bethesda-Kriterien erfüllen, ergibt sich bei etwa 30\% der Patienten eine Mikrosatelliteninstabilität und somit ein konkreter Hinweis auf HNPCC. Die klassischen Bethesda-Kriterien wurden 2004 revidiert (Anlage 12.3) [249]. Die Sensitivität der Mikrosatellitenanalyse in HNPCC-assoziierten Tumoren beträgt 79$93 \%$, die der immunhistochemischen Untersuchung (inkl. MSH6 und PMS2) liegt vergleichbar bei $94 \%$ [250]. Die Immunhistochemie (IHC) ist im Vergleich zur Mikrosatellitenanalyse (MSA) kostengünstiger, schneller durchzuführen und liefert durch den Ausfall eines DNA-Reparaturproteins zusätzlich den Hinweis, in welchem der vier bekannten Mismatch-Reparatur (MMR)-Gene sich die krankheitsverursachende Keimbahnmutation befindet. Sofern die IHC ein eindeutiges Ergebnis ergibt, kann auf eine MSA verzichtet werden. Andernfalls sollte eine MSA angeschlossen werden. 


\begin{abstract}
5.17.

\section{EK}

Bei Patienten mit hoch mikrosatelliteninstabilen Tumoren und einem Ausfall des MLH1-Proteins in der immunhistochemischen Untersuchung soll eine Analyse hinsichtlich einer somatischen BRAF-Mutation p.Val600Glu durchgeführt werden, um ein HNPCC auszuschließen.

Konsens

\section{Hintergrund}

Ungefähr 15\% der sporadischen KRK zeigen im Tumorgewebe HNPCC-typische Veränderungen im Sinne einer MSI-H und eines Ausfalls des MLH1-Proteins in der immunhistochemischen Untersuchung (IHC). Ursächlich hierfür ist in der Regel eine somatische Methylierung des MLH1-Promotors. Die Methylierung geht wiederum mit der somatischen Mutation p.Val600Glu im BRAF-Gen einher [251-254]. Bei Tumoren mit MSI$\mathrm{H}$ und einem Ausfall des MLH1-Proteins in der IHC sollte daher zusätzlich eine BRAFAnalyse angeschlossen werden. Durch diese Untersuchung ist es möglich HNPCCassoziierte von sporadischen KRK zu unterscheiden, da HNPCC-assoziierte KRK keine BRAF-Mutation aufweisen. Für Verwandte ersten Grades eines Patienten mit einem sporadischen MSI-H KRK ist das Risiko ein KRK zu entwickeln leicht erhöht (standardisierte Inzidenz Ratio 1,60) [255]. Es sollten daher die Vorsorgeempfehlungen analog zu den Fällen mit positiver Familienanamnese durchgeführt werden. Möglicherweise ist für Patienten mit einem BRAF-positiven KRK das Risiko für andere Tumoren erhöht (Magen, Ovar) [255].
\end{abstract}

\title{
5.2.1.2. Adenomatöse Polyposis-Syndrome
}

5.2.1.2.1. Patienten mit klassischer familiär adenomatöser Polyposis (FAP)

\begin{tabular}{|l|l|}
\hline $\mathbf{5 . 1 8 .}$ & Evidenzbasiertes Statement \\
\hline $\begin{array}{l}\text { Level of Evidence } \\
\mathbf{2 a}\end{array}$ & $\begin{array}{l}\text { Patienten mit unbehandelter FAP entwickeln nahezu ausnahmslos ein kolorektales } \\
\text { Karzinom. }\end{array}$ \\
\hline & Quelle: [256]
\end{tabular}

\section{Hintergrund}

Eine klassische (typische) FAP ist durch das Vorhandensein von mehr als 100 kolorektalen Adenomen charakterisiert. Die Polypenbildung beginnt meist in der zweiten Lebensdekade. Aufgrund der Vielzahl der Adenome beträgt das Karzinom-risiko nahezu $100 \%$.

Die Mehrzahl der Patienten entwickelt darüber hinaus weitere extrakolische intestinale Manifestationen. Klinisch am bedeutsamsten sind Duodenal- bzw. Papillenadenome, die in etwa 75\% aller Patienten auftreten und als Präkanzerose anzusehen sind (siehe unten). Weitaus weniger häufig sind Magenadenome mit einer Inzidenz von $<10 \%$ der Patienten mit FAP. Drüsenkörperzysten des Magens, die bei mindestens einem Drittel der FAPPatienten auftreten, wird gegenwärtig keine potentielle präneoplastische Potenz zugeschrieben. 
Weitere extraintestinale Manifestationen sind abdominale und extraabdominale Desmoidtumoren, Schilddrüsenkarzinome, maligne ZNS-Tumoren (meist Medulloblastome), Hepatoblastome, sowie harmlose, jedoch oft diagnostisch wegweisende Osteome, Epidermoidzysten oder Pigmentanomalien der Retina [256].

\subsection{Patienten mit attenuierter familiärer adenomatöser Polyposis (AFAP)}

\begin{tabular}{|l|l|c|}
\hline 5.19. & Konsensbasiertes Statement & 2013 \\
\hline $\begin{array}{l}\text { Level of Evidence } \\
\mathbf{2 a}\end{array}$ & $\begin{array}{l}\text { Die attenuierte FAP (AFAP) ist von dem Krankheitsbild der typischen familiären } \\
\text { adenomatösen Polyposis abzugrenzen. Auch hier besteht ein sehr hohes Risiko für } \\
\text { ein kolorektales Karzinom, wobei sich Polypen und Karzinome bei den Anlage-trägern } \\
\text { meist später und häufig im proximalen Kolon entwickeln. }\end{array}$ \\
\hline & Evidenz aus Aktualisierungsrecherche: [256-264]
\end{tabular}

\section{Hintergrund}

Die AFAP ist typischerweise durch weniger als 100 kolorektale Adenome und/oder ein etwa 10-15 Jahre späteres Auftreten von Adenomen und KRK als bei klassischer FAP charakterisiert; das Lebenszeitrisiko für die Entwicklung eines KRK ist aber ebenfalls sehr hoch. Extrakolische Manifestationen (z. B. Desmoide) können auftreten [256-261]. Die klinisch definierte AFAP ist aus genetischer Sicht eine heterogene Gruppe. Keimbahnmutationen im APC-Gen (5' und 3'-Ende des Gens) lassen sich in 15-30\% der Familien nachweisen. Die wichtigste Differentialdiagnose ist die MUTYH-assoziierte Polyposis (MAP) (siehe unten) [262]. Die klinische Abgrenzung gegenüber HNPCC kann im Einzelfall schwierig sein [263]. Daher ist die molekulargenetische Diagnostik (Mikrosatellitenanalyse, APC, MUTYH) unter Umständen bei der klinischen Differentialdiagnose einer attenuierten FAP sehr hilfreich [264]. Bei einem Großteil der Patienten mit der klinischen Diagnose einer AFAP gelingt derzeit kein Mutationsnachweis, sodass von Mutationen in weiteren bisher nicht identifizierten Genen ausgegangen werden muss [264].

\subsection{Patienten mit MUTYH-assoziierter Polyposis (MAP)}

\subsection{0.}

Level of Evidence

\section{$2 a$}

\section{Evidenzbasiertes Statement}

Die MUTYH-assoziierte Polyposis (MAP) ist die wichtigste Differentialdiagnose der FAP. Der Phänotyp entspricht meist dem einer AFAP; auch bei der MAP ist das Lebenszeitrisiko für ein KRK sehr hoch. Aufgrund des autosomal-rezessiven Erbgangs besteht aber ein nur geringes Erkrankungsrisiko bei Kindern eines Patienten und heterozygoten Anlageträgern. Die Diagnosestellung ist in der Regel nur durch die molekulargenetische Untersuchung möglich.

Evidenz aus Aktualisierungsrecherche: [265-272]

\section{Hintergrund}

Die durch biallele Keimbahnmutationen im MUTYH-Gen verursachte, autosomal-rezessiv erbliche MAP ist die wichtigste Differentialdiagnose der APC-assoziierten FAP [265]; sie wird bei $15-20 \%$ der APC-mutationsnegativen kolorektalen Adenomatosen diagnostiziert [266, 267]. 
Der kolorektale Phänotyp der MAP ähnelt dem der AFAP: meist treten zwischen 20 und einigen hundert Adenomen auf, das mittlere Diagnosealter ist 45 Jahre (Streuung 12-68 Jahre) [268]. Unbehandelt beträgt das KRK-Lebenszeitrisiko etwa 70-80 \% [269]. Das phänotypische Spektrum der MAP ist noch nicht abschließend geklärt: einige große populationsbasierte Studien an KRK-Patienten zeigten, dass bis zu ein Drittel der biallelen MUTYH-Mutationsträger ein KRK ohne kolorektale Polypen entwickelt [270]. Daneben wurden bei bis zu 50\% der MAP-Patienten hyperplastische Polypen beschrieben [271].

Bei etwa 20\% der Patienten besteht eine Duodenalpolyposis, das Lebenszeitrisiko für ein Duodenalkarzinom beträgt etwa 4\%. Extraintestinale Malignome treten insgesamt signifikant häufiger als in der Allgemeinbevölkerung auf (Odds Ratio 1,9) und zeigen eine gewisse Überlappung zum HNPCC; es besteht allerdings kein dominierender Tumor. Typische FAP-assoziierte extraintestinale Tumoren wie Osteome, Desmoide und CHRPE treten nicht auf [272].

\subsubsection{Vorsorgeuntersuchungen}

\subsubsection{HNPCC / Lynch-Syndrom}

\begin{tabular}{|c|c|}
\hline 5.21. & Evidenzbasierte Empfehlung \\
\hline $\begin{array}{l}\text { Empfehlungsgrad } \\
\text { B }\end{array}$ & $\begin{array}{l}\text { Risikopersonen für HNPCC ist mit Erreichen der Einwilligungsfähigkeit (in der Regel } \\
\text { ab dem } 18 \text {. Lebensjahr), jedoch vor dem } 25 \text {. Lebensjahr eine genetische Beratung zu } \\
\text { empfehlen. Sobald die krankheitsverursachende Mutation in der betreffenden Familie } \\
\text { bekannt ist, sollten Risikopersonen auf die Möglichkeit einer prädiktiven Testung } \\
\text { hingewiesen werden. }\end{array}$ \\
\hline Level of Evidence & Evidenz aus Aktualisierungsrecherche:[273, 274] \\
\hline & Starker Konsens \\
\hline
\end{tabular}

\begin{tabular}{l|l|}
$\begin{array}{l}\text { 5.22. } \\
\text { Level of Evidence }\end{array}$ & $\begin{array}{l}\text { Wenn die krankheitsverursachende Mutation bei einer Risikoperson ausgeschlossen } \\
\text { W C }\end{array}$ \\
\hline & Wurde, gelten die allgemeinen Krebsvorsorgemaßnahmen. \\
\hline & Starker Konsens
\end{tabular}

\section{Hintergrund}

Anlageträger für ein HNPCC haben Mutationen in den Mismatch-Repair-Genen. Bislang konnten Keimbahnmutationen in 5 verschiedenen Genen nachgewiesen werden: MSH2, MLH1, MSH6, PMS2 und EPCAM. Fast 86\% der bislang identifizierten Mutationen liegen in den Genen MSH2 und MLH1 [273], etwa 10\% im MSH6-Gen und 2\% im PMS2-Gen. Mutationen im EPCAM-Gen werden bei ca. 2\% der Familien gefunden [274]. Vor einer prädiktiven genetischen Untersuchung muss entsprechend des GenDG eine humangenetische Beratung erfolgen. Eine prädiktive Testung ist in der Regel nur möglich, wenn 
in der Familie bei einem bereits erkrankten Mitglied eine zweifelsfrei pathogene Mutation nachgewiesen wurde. Der Nachweis von Polymorphismen oder Mutationen mit unklarer pathogenetischer Bedeutung eignet sich nicht als Grundlage für eine prädiktive genetische Diagnostik.

\begin{tabular}{|c|c|}
\hline 5.23. & Konsensbasierte Empfehlung \\
\hline EK & $\begin{array}{l}\text { HNPCC-Patienten und Risikopersonen sollen in der Regel ab dem } 25 \text {. Lebensjahr } \\
\text { jährlich koloskopiert werden. }\end{array}$ \\
\hline & Konsens \\
\hline
\end{tabular}

\section{Hintergrund}

HNPCC-Patienten haben ein deutlich erhöhtes Karzinomrisiko. Einen Überblick über die empfohlenen Krebsfrüherkennungsuntersuchungen gibt Tabelle 6 . Kolonkarzinome bei HNPCC-Patienten treten im Median im 44. Lebensjahr auf. Die Erkrankungswahrscheinlichkeit steigt ab dem 30. Lebensjahr deutlich an. Bei sehr früher Manifestation kolorektaler Karzinome in der Familie sollte abweichend von der o.g. Empfehlung die erste Koloskopie 5 Jahre vor dem jüngsten Manifestationsalter in der Familie erfolgen. Mehr als 50\% der HNPCC-assoziierten Karzinome befinden sich im rechtsseitigen Kolon [246]. Aus diesem Grunde reicht eine Rektoskopie bzw. Rektosigmoidoskopie als Vorsorgeuntersuchung nicht aus. Eine prospektive Studie konnte eine signifikante Reduktion der Mortalität und auch Inzidenz von KRK um mehr als jeweils $60 \%$ bei 3jährlichen Untersuchungsintervallen nachweisen [275]. Aufgrund einer beschleunigten Tumorprogression mit Intervallkarzinomen in etwa 4\% aller Patienten bei 2- bis 3jährlichen Untersuchungsabständen wird ein jährliches Intervall empfohlen [275-277] (Tabelle 6). In der prospektiven Studie des Deutschen HNPCC-Konsortiums mit 1-jährlichem Intervall weisen die unter Überwachung asymptomatisch detektierten CRC eine signifikant günstigere Stadienverteilung auf [232].

Die Stadienverteilung und damit auch Prognose HNPCC-assoziierter kolorektaler Karzinome, die im Rahmen eines Vorsorgeprogrammes entdeckt werden, ist signifikant günstiger verglichen zu Karzinomen, die aufgrund von Symptomen der Erkrankung diagnostiziert worden sind [278]. Die Koloskopie kann als Chromoendoskopie erfolgen. In prospektiven Studien wurde durch eine Chromoendoskopie eine signifikante Steigerung der Adenomdetektionsrate gesehen [279-281]. Es ist aber gegenwärtig unklar, ob dadurch die Intervallkarzinomrate oder Mortalität günstig beeinflusst wird.

\begin{tabular}{|l|l|}
$\begin{array}{l}\text { 5.24. } \\
\text { Empfehlungsgrad }\end{array}$ & $\begin{array}{l}\text { Evidenzbasierte Empfehlung } \\
\text { Beiblichen HNPCC-Patienten und Risikopersonen sollte ab dem 25. Lebensjahr } \\
\text { zusätzlich zur jährlichen gynäkologischen Untersuchung ein transvaginaler } \\
\text { Ultraschall im Hinblick auf Endometrium- und Ovarialkarzinome durchgeführt } \\
\text { werden. }\end{array}$ \\
\hline $\begin{array}{l}\text { Level of Evidence } \\
\mathbf{4}\end{array}$ & \begin{tabular}{l} 
Quellen: $[245,282-285]$ \\
\hline
\end{tabular} \\
\hline
\end{tabular}




\begin{tabular}{|l|l|}
\hline 5.25. & Konsensbasierte Empfehlung \\
\hline EK & $\begin{array}{l}\text { Bei weiblichen HNPCC-Patienten und Risikopersonen sollte ab dem } \\
\text { zusätzlich jährlich eine Endometriumbiospsie durchgeführt werden. }\end{array}$ \\
\hline Konsens
\end{tabular}

\section{Hintergrund}

Diese Empfehlungen ergeben sich aus dem natürlichen Verlauf des HNPCC (siehe oben). Bei Anlageträgerinnen beträgt das Risiko bis zum 70 . Lebensjahr ein Endometriumkarzinom zu entwickeln 40 bis $60 \%$, für ein Ovarialkarzinom ungefähr 10 15\% [245, 282]. Die bisher publizierten Studien hinsichtlich der Effektivität der Früherkennung des Endometriumkarzinoms bei HNPCC-Patientinnen zeigten deutliche Hinweise, dass der transvaginale Ultraschall (TVU) als Screening-Untersuchung zur Früherkennung des Endometriumkarzinoms insbesondere prä- und perimenopausal ungeeignet ist [283-285]. Da die Endometriumbiopsie mit der Pipelle-Methode in Ergänzung zum TVU aus den Literaturdaten als die sinnvollste Alternative hervorgeht und diese auch bereits in internationalen Empfehlungen für HNPCC-Patientinnen propagiert wurde [286], ist die Empfehlung zur jährlichen Endometriumbiopsie ab dem 35. Lebensjahr sinnvoll (siehe Tabelle 7). Bei Patienten mit abgeschlossener Familienplanung sollte die Möglichkeit einer prophylaktischen Hysterektomie und ggf. Adnektomie diskutiert werden (siehe Empfehlung 5.30.).

\begin{tabular}{|l|l|}
\hline $\mathbf{5 . 2 6 .}$ & Konsensbasierte Empfehlung \\
\hline EK & $\begin{array}{l}\text { Bei HNPCC-Patienten und Risikopersonen mit HNPCC sollte ab dem } \\
\text { zusätzlich regelmäßig eine ÖGD durchgeführt werden. }\end{array}$ \\
\hline Konsens
\end{tabular}

\section{Hintergrund}

HNPCC-assoziierte Magenkarzinome werden im Median im 54 . Lebensjahr diagnostiziert, sie treten in nur 2\% vor dem 35. Lebensjahr auf. Für Deutschland wurde ein kumulatives Lebenszeitrisiko von $6,8 \%$ bis zum 70 . Lebensjahr ermittelt [239]. Eine familiäre Häufung (mindestens 2 Betroffene mit Magenkarzinomen) ließ sich in nur 26\% der MLH1 - und MSH2-Mutationsträger nachweisen [287]. Daher erscheint eine ÖGD für alle Anlageträger und Risikopersonen ab dem 35. Lebensjahr sinnvoll (siehe Tabelle 6). Der Beginn der ÖGD mit 35 Jahren wird auch vorgeschlagen, da das Dünndarmkarzinomrisiko das Magenkrebsrisiko übersteigt und Erkrankungen ab dem 30. Lebensjahr auftreten. Das kumulative Lebenszeitrisiko für Dünndarmkarzinome im Rahmen eines HNPCC beträgt 4-8\% [239, 240]. Dabei sind 35-50\% der Dünndarmkarzinome im Duodenum lokalisiert [241]. Die Empfehlung der S3-Leitlinie zum Magenkarzinom wird aufgrund der Datenlage künftig zu präzisieren sein. Zur Frage des Untersuchungsintervalls gibt es keine Daten; in Analogie zur beschleunigten Tumorprogression kolorektaler Karznome auf dem Boden eines hereditären MMRDefektes wird ein jährliches Intervall als sinnvoll erachtet.

Wegen des erhöhten Risikos für Urothelkarzinome und hepatobiliäre Karzinome wurde früher eine jährliche Oberbauchsonographie empfohlen. Der Nutzen ist aber nicht belegt und vom kurativen Potential fraglich. Sie wird daher nicht länger allgemein empfohlen. 
Der Nutzen einer urinzytologischen Untersuchung ist nicht belegt und wird daher seit 2004 nicht mehr allgemein empfohlen.

\begin{tabular}{|c|c|}
\hline 5.27. & Evidenzbasierte Empfehlung \\
\hline $\begin{array}{l}\text { Empfehlungsgrad } \\
\text { B }\end{array}$ & Eine medikamentöse Prävention bei HNPCC sollte nicht durchgeführt werden. \\
\hline Level of Evidence & Evidenz aus Aktualisierungsrecherche:[288, 289] \\
\hline & Konsens \\
\hline
\end{tabular}

\section{Hintergrund}

Zur Chemoprävention bei HNPCC liegen bisher nur Daten einer prospektiv randomisierten Studie vor. Die CAPP2-Studie hat in einem 2x2-Design den Einsatz von $600 \mathrm{mg}$ Acetylsalicylsäure und von nicht resorbierbarer Stärke geprüft. Die primäre Analyse der definierten Endpunkte ergab keinen Nachweis eines signifikanten Effektes von ASS [288]. Nach einem längeren Follow-up von 55,7 Monaten fand sich für die Subgruppe von HNPCC-Patienten, die 600 mg ASS für mindestens 2 Jahre einge-nommen hatten eine signifikante Senkung der Inzidenz kolorektaler Karzinome (Hazard ratio 0,4 1 (95\%Cl 0,19-0,86), $\mathrm{p}=0,02))$ sowie eine nicht signifikante Senkung von anderen HNPCCassoziierten Karzinomen (Hazard ratio 0,47 (95\% Cl 0,21-1,06), $p=0,07)$ [289]. Die untersuchte ASS Dosis erscheint mit 600 mg hoch mit entsprechend zu befürchtender erhöhter Nebenwirkungsrate. Die Effektivität niedrigerer ASS-Dosen ist derzeit für HNPCC-Patienten nicht geklärt und soll in einer Nachfolgestudie (CAPP-3) untersucht werden. Es sollte angestrebt werden, möglichst viele HNPCC-Patienten in diese Studie einzuschließen. Bis die Ergebnisse dieser Studie vorliegen, sollte keine allgemeine medikamentöse Prävention mit ASS bei HNPCC-Patienten erfolgen.

\begin{tabular}{|l|l|}
\hline 5.28. & Konsensbasierte Empfehlung \\
\hline EK & $\begin{array}{l}\text { Eine prophylaktische Kolektomie bzw. Proktokolektomie bei HNPCC-Mutations- } \\
\text { trägern soll nicht durchgeführt werden. } \\
\text { Eine subtotale Kolektomie bei Karzinom sollte nicht generell durchgeführt, aber } \\
\text { individuell mit dem Patienten besprochen werden. }\end{array}$ \\
\hline Starker Konsens
\end{tabular}




\begin{tabular}{|c|c|}
\hline 5.29. & Evidenzbasierte Empfehlung \\
\hline Empfehlungsgrad & $\begin{array}{l}\text { Die koloskopische Überwachung des Patienten nach onkologischer Resektion soll } \\
\text { zusätzlich zur üblichen Nachsorge auch postoperativ nach dem gleichen Muster wie } \\
\text { vor der Operation fortgesetzt werden. }\end{array}$ \\
\hline Level of Evidence & Evidenz aus Aktualisierungsrecherche:[275, 276, 290-293] \\
\hline & Starker Konsens \\
\hline
\end{tabular}

\section{Hintergrund}

Da durch regelmäßige Vorsorgeuntersuchungen Karzinome bei fast allen Patienten im Stadium UICC I/II oder sogar als prämaligne Adenome entdeckt werden [275, 276] und die Penetranz unvollständig ist, wird von einer prophylaktische Kolektomie bzw. Proktokolektomie abgeraten. Individuelle Konstellationen wie endoskopisch nicht abtragbare Adenome, regelhaft schwierige Koloskopien, nicht ausreichende Kolonvorbereitung trotz adäquater Abführmaßnahmen und fehlende Compliance können eine Empfehlung zu einer prophylaktischen Kolektomie rechtfertigen.

Bei Nachweis eines Karzinoms werden die Patienten nach tumorchirurgischen Gesichtspunkten im Sinne einer onkologischen Resektion operiert (siehe auch Kapitel 7). Das Risiko eines kolorektalen Karzinoms im verbliebenen Dickdarm und das Risiko von extrakolischen Neoplasien bleibt jedoch erhöht, so dass diese Patienten einer intensiven postoperativen Nachsorge zugeführt werden müssen. In dieser sollte die Tumornachsorge für sporadische KRK mit dem HNPCC-spezifischen Früherkennungsprogramm für KRK und extrakolische Tumoren kombiniert werden. Ob eine erweiterte prophylaktische Resektion zur Prophylaxe metachroner KRK einer engmaschigen Überwachung überlegen ist, ist derzeit unklar. Die bisherigen Daten aus retrospektiven Fallserien sind unzureichend, und aufgrund nationaler Unterschiede im Bezug auf das Screening-Intervall nicht auf Deutschland übertragbar [290-293].

\begin{tabular}{|l|l|}
\hline 5.30. & Konsensbasierte Empfehlung \\
\hline EK & $\begin{array}{l}\text { Mit Patientinnen mit Lynch- und HNPCC-Syndrom sollte mit 40 Jahren, bzw. fünf Jahre } \\
\text { vor dem frühesten Erkrankungsalter in der Familie, eine prophylaktische } \\
\text { Hysterektomie und ggf. eine Ovarektomie besprochen werden. }\end{array}$ \\
\hline Konsens
\end{tabular}

\section{Hintergrund}

Dieses Vorgehen basiert auf der Expertenmeinung der Arbeitsgemeinschaft Gynäkologische Onkologie (AGO) der Deutschen Krebsgesellschaft. Der Eingriff sollte möglichst erst nach Abschluss der Familienplanung erfolgen. Eine retrospektive Studie konnte einen deutlichen Rückgang in der Inzidenz der Endometrium- und Ovarialkarzinome bei diesen Patientinnen zeigen [294]. Nach einer prophylaktischen Ovarektomie sollte eine Hormontherapie (HRT) durchgeführt werden. Nach einer Hysterektomie kann die HRT auch nur mit Östrogenen erfolgen, wodurch die Nebenwirkungen der Therapie gesenkt werden. 


\subsubsection{Adenomatöse Polyposis Syndrome}

\subsection{Patienten mit klassischer familiärer adenomatöser Polyposis (FAP)}

\subsection{1 .}

Empfehlungsgrad

B

Level of Evidence

4

5.32.

Empfehlungsgrad

A

Level of Evidence

$1 \mathrm{c}$

\section{Evidenzbasierte Empfehlung}

Verwandte eines FAP-Patienten, die aufgrund des autosomal-dominanten Erbgangs als Mutationsträger in Betracht kommen, werden als Risikopersonen bezeichnet. Bei diesen sollte ab dem 10. Lebensjahr im Anschluss an eine humangenetische Beratung der Familie eine prädiktive genetische Diagnostik empfohlen werden, soweit die zugrunde liegende APC-Keimbahnmutation in der Familie identifiziert werden konnte.

Quellen: [121, 295]

Starker Konsens

\section{Evidenzbasierte Empfehlung}

2008

Wurde die in der Familie identifizierte Mutation bei Risikopersonen (Kinder, Geschwister oder Eltern eines FAP-Patienten) ausgeschlossen, ist eine gesonderte Vorsorge nicht mehr notwendig.

Keine Angabe

Starker Konsens

\subsection{3 .}

Empfehlungsgrad

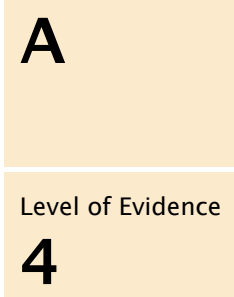

\section{Evidenzbasierte Empfehlung}

Risikopersonen, bei denen die Mutation bestätigt oder nicht ausgeschlossen werden konnte, sollen ab dem 10. Lebensjahr jährlich rekto-sigmoidoskopiert werden. Bei Nachweis von Adenomen soll eine komplette Koloskopie erfolgen und bis zur Proktokolektomie jährlich wiederholt werden (s.u.).

Quellen: [121, 194, 295, 296]

Starker Konsens

\section{Hintergrund}

Die humangenetische Beratung erfolgt bei nicht-einwilligungsfähigen Personen gemeinsam mit den Erziehungsberechtigten. Die Einleitung der humangenetischen Diagnostik vor dem 10. Lebensjahr ist selten notwendig, da kolorektale Karzinome bei FAP-Anlageträgern vor dem 15. Lebensjahr sehr selten sind [295]. Die molekulargenetische Untersuchung erfolgt in der Regel mittels direktem Mutationsnachweis im 
APC-Gen, in seltenen Fällen mit aussagekräftiger familiärer Konstellation kann die Anlageträgerschaft auch indirekt durch Kopplungsanalyse nachgewiesen werden. Eine sichere prädiktive Testung kann nur bei zuvor identifizierter pathogener Keimbahnmutation bei einem betroffenen Familienmitglied erfolgen und muss in eine humangenetische Beratung eingebettet sein [121] [GenDG]. Ein Mutationsnachweis gelingt bei ca. $70-80 \%$ der Patienten. Als weitere Methode zur Identifizierung von Genträgern kann eine Augenhintergrundspiegelung durchgeführt werden. Die Augenhintergrundspiegelung zur Identifizierung einer kongenitalen Hypertrophie des retinalen Pigmentepithels (CHRPE) hat durch die prädiktive DNA-Testung jedoch an Bedeutung verloren.

Bei der klassischen FAP werden immer auch Polypen im Rektum und Sigma beobachtet. Sind Rektumpolypen nachgewiesen worden, so können weiter proximal weitere Adenome oder sogar Karzinome vorhanden sein. In diesem Falle soll kurzfristig eine komplette Koloskopie durchgeführt werden, die je nach Befund in mindestens jährlichen Abständen wiederholt werden sollte. In Familien, in denen eine genetische Testung nicht durchgeführt wurde oder nicht aussagekräftig war, ist allen Risiko-personen die endoskopische Vorsorge ab dem 10. Lebensjahr zu empfehlen [194, 295, 296]. Bei bestimmten Mutationen, früher Karzinommanifestation in der Familie oder assoziierten Symptomen ist der Beginn der Vorsorge bereits zu einem früheren Zeitpunkt in Erwägung zu ziehen.

\subsection{4.}

Empfehlungsgrad

B

Level of Evidence

$1 \mathrm{c}$

\section{Evidenzbasierte Empfehlung}

Patienten mit klassischer FAP sollten prophylaktisch und unabhängig vom Ergebnis der molekulargenetischen Testung - wann immer möglich kontinenzerhaltend proktokolektomiert werden, wenn vertretbar erst nach Abschluss der Pubertät.

Evidenz aus Aktualisierungsrecherche: [264, 297-302]

Starker Konsens

\subsection{5 .}

\section{EK}

Nach einer Proktokolektomie soll regelmäßig eine Pouchoskopie erfolgen. Bei Patienten mit erhaltenem Rektumstumpf soll regelmäßig eine Rektoskopie durchgeführt werden. Das Intervall hängt vom Untersuchungsergebnis (Anzahl, Größe und Histologie nachgewiesener Neoplasien) ab und sollte 12 Monate nicht überschreiten.

Starker Konsens

\section{Hintergrund}

Die rechtzeitige Proktokolektomie ist zur Verhinderung des kolorektalen Karzinoms entscheidend [297-301]. Die Relevanz des molekulargenetischen Befundes für therapeutische Entscheidungen ist begrenzt, da der Nachweis einer Mutation nur selten eine individuelle Abschätzung des Krankheitsverlaufs erlaubt und bei einem nennenswerten 
Teil der klinisch sicher erkrankten Personen keine ursächliche Mutation identifiziert werden kann. Der Polyposis-Patient muss unabhängig vom Ergebnis der Mutations-suche adäquat behandelt werden, notwendige chirurgische Maßnahmen sollten deshalb auch bei fehlendem Mutationsnachweis und ggf. noch vor Abschluss der Mutationssuche durchgeführt werden [264]. Als Orientierung ist ein Operations-zeitpunkt zwischen Abschluss der Pubertät und dem 20. Lebensjahr für eine prophylaktische Proktokolektomie anzustreben. Der OP-Zeitpunkt muss sich jedoch individuell nach Alter, Anamnese und endoskopischem/histologischem Befund (Zahl der Polypen und Dysplasiegrad) richten [300-302]. Nach der Beobachtung zum natürlichen Verlauf der FAP entsteht das Karzinom im Median im 36. Lebensjahr [303]. Mit dem Patienten sollte das mögliche Belassen eines Rektumstumpfes diskutiert werden (ileorektale Anastomose, IRA). Hierbei ist zu beachten, dass nach Kolektomie mit Belassung des Rektums das Risiko der Entstehung eines Rektumstumpfkarzinoms ca. 13\% nach 25 Jahren beträgt [304], während die Langzeitprognose nach IPAA (ileo-pouchanaler Anastomose) bessere Langzeitergebnisse hinsichtlich der Karzinom-prophylaxe aufweist [305-308]. Aus diesem Grunde wird bei der klassischen FAP die Proktokolektomie mit IPAA empfohlen. Die Operation sollte in entsprechend erfahrenen Zentren durchgeführt werden. Die Durchführung einer Proktokolektomie mit endständigem Ileostoma kann heute größtenteils vermieden werden.

Da viele Patienten Polypen im Bereich des Pouches nahe der ileoanalen Anastomose entwickeln, die maligne entarten können, wird postoperativ eine jährliche Pouchoskopie empfohlen. Sofern keine Proktokolektomie erfolgte, ist die engmaschige Kontrolle des Rektumstumpfes in maximal 12-monatlichen Intervallen erforderlich, ggf. mit Abtragung neu auftretender Polypen.

\begin{tabular}{|c|c|}
\hline 5.36. & Konsensbasierte Empfehlung \\
\hline & $\begin{array}{l}\text { Eine ÖGD und Duodenoskopie (Seitblickoptik) mit besonderer Inspektion der } \\
\text { Papillenregion sollte spätestens ab dem 25-30. Lebensjahr durchgeführt werden. Bei } \\
\text { unauffälligem Befund wird ein 3-Jahres-Intervall empfohlen. Das Intervall sollte in } \\
\text { Abhängigkeit vom Schweregrad vorhandener Adenome (Spigelman-Klassifikation) auf } \\
\text { bis zu einem Jahr verkürzt werden. } \\
\text { Bei Nachweis von Duodenal-/Papillenadenomen ist die Indikation zur endoskopischen } \\
\text { Polypektomie zu überprüfen. } \\
\text { Bei schwergradiger Duodenalpolyposis (Spigelman IV) und invasivem nicht-fern- } \\
\text { metastasiertem Karzinom besteht eine Indikation zur operativen Resektion. }\end{array}$ \\
\hline & Konsens \\
\hline
\end{tabular}

\section{Hintergrund}

Das Lebenszeitrisiko Duodenalpolypen zu entwickeln beträgt für FAP-Patienten zwischen 80 und $90 \%$ [234, 235]. Weniger als 10\% der Patienten weisen Magen-adenome auf, über 50\% Drüsenkörperzysten des Magens. Magenkarzinome scheinen hingegen nicht signifikant häufiger als in der Normalbevölkerung zu sein [309]. Hinsichtlich der Ausprägung der Duodenal-Polyposis sollte die Spigelman-Klassifikation (siehe Tabelle 7) herangezogen werden [310]. Das durchschnittliche Alter der Patienten mit schwerer Duodenalpolyposis liegt bei 43 (Range 24-65) Jahren [236]. Insgesamt scheint das Wachstumsverhalten der Duodenaladenome langsamer als das von kolorektalen Adenomen zu sein [311, 312] und hängt mehr vom zunehmenden Alter (>40 Jahre zunehmend) als vom initialen Stadium ab [313]. Der Mutationsort (Codon 279-1309) 
korreliert mit dem Schweregrad der Polyposis im Duodenum aber wohl nicht mit der Wahrscheinlichkeit, dass sich hochgradige Dysplasien entwickeln [314, 315]. Das Lebenszeitrisiko für ein Duodenalkarzinom beträgt für Patienten mit FAP zwischen 3 und 4\% [316, 317] und ist somit bis zu 300-fach höher als in der Normalbevölkerung [318]. Das Risiko, dass bereits ein invasives Karzinom vorliegt, richtet sich nach der Ausprägung der Duodenalpolyposis. So liegt das Risiko für ein invasives Karzinom bei Spigelman II und III bei jeweils 2\%, demgegenüber bei Spigelman IV bei 36\% [319].

Sinn einer endoskopischen Überwachung kann nicht die Entfernung sämtlicher Polypen sein, sondern muss auf die Entdeckung von relevanten Neoplasien (high-grade intraepitheliale Neoplasie, villöse oder tubulovillöse Adenome) ausgerichtet sein. Eine endoskopische Sanierung bis hin zur Polypenfreiheit ist in der Regel nicht sinnvoll. Alle Polypen $>1 \mathrm{~cm}$ sollten, sofern technisch möglich, abgetragen werden. Bei kleineren Polypen sollten die größten Polypen abgetragen werden, um durch die histologische Untersuchung einen Spigelman-Score festlegen zu können.

Für FAP-Patienten mit leichtgradiger Duodenalpolyposis (Spigelman I und II ) scheint ein Untersuchungsintervall von 3 Jahren ausreichend [311]. Aufgrund des hohen Karzinomrisikos im Spigelman-Stadium IV muss hier ein chirurgisches Vorgehen empfohlen werden, wobei der pankreaserhaltenden Duodenektomie, auch aufgrund der geringeren Morbiditätsrate gegenüber der Pankreatikoduodenektomie der Vorzug zu geben ist [320, 321]. Die operative Duodenotomie und Polypektomie kann wegen der hohen Rezidivrate nicht empfohlen werden [322, 323]. Prinzipiell lässt sich auch nach extensivem chirurgischem Vorgehen das Wiederauftreten von Adenomen nicht verhindern [322]. Auch ist derzeit nicht klar, ob die regelmäßige Vorsorge-untersuchung des Duodenums lebensverlängernd ist [316]. Zum Vorgehen bei FAP-assoziierten Papillenadenomen gibt es unterschiedliche Vorgehensweisen. Insgesamt existieren zu dieser Problematik bei FAP nur sehr wenige Publikationen. Während z.T. bei jedem Patienten bei jedem Papillenadenom eine Papillektomie favorisiert wird [324, 325], favorisieren andere Gruppen bei kleinen Adenomen eine Überwachungsstrategie und eine Papillektomie nur bei Progress (Größe, Histologie) oder eingetretenen bzw. drohenden Komplikationen (z.B. Cholestase, Pankreatitis) [311, 312]. Zusammenfassend erscheint das folgende Überwachungsprogramm sinnvoll, wobei es jeweils der individuellen Situation angepasst werden sollte: Spigelman I und II: Kontrolle mindestens alle 3 Jahre, gegebenenfalls Polypektomie, Spigelman III: Kontrolle jährlich, gegebenenfalls Polypektomie, Spigelman IV: Operation.

\begin{tabular}{|l|l|}
\hline 5.37. & Konsensbasierte Empfehlung \\
\hline EK & $\begin{array}{l}\text { Bei vorliegender Therapieindikation (Symptomatik, Progredienz) sollten Desmoide bei } \\
\text { FAP in erster Linie mit einer Kombinationstherapie aus Sulindac und Tamoxifen } \\
\text { behandelt werden. Das Vorgehen bei progredienten Desmoiden unter dieser } \\
\text { medikamentösen Therapie soll interdisziplinär abgestimmt werden. Als Optionen } \\
\text { kommen Chemotherapie, Chirurgie und Radiatio in Betracht. }\end{array}$ \\
\hline Konsens
\end{tabular}

\section{Hintergrund}

Desmoidtumoren treten bei 10 bis 30\% aller Patienten mit einer FAP auf. Das Risiko für die Entwicklung eines Desmoidtumors ist für Frauen höher als für Männer. Neben einer erkennbaren Geno-/Phänotyp-Korrelation (APC-Mutation Kodon >1300) [326-328] wird 
ein chirurgisches Trauma als Triggerfaktor angesehen. Etwa 50\% der Desmoide treten intraabdominell und hier vor allem mesenterial auf und verursachen durch das lokal infiltrative Wachstum oft erhebliche Probleme. Aus diesem Grunde ist insbesondere bei Patienten mit einer positiven Familienanamnese oder einer distalen APC-Mutation vor einer Proktokolektomie auf das Vorhandensein von Desmoiden zu achten und die Indikation für eine Proktokolektomie möglichst spät zu stellen. Ein regelmäßiges Screening auf Desmoide wird bei beschwerdefreien Patienten nicht empfohlen. Präoperativ kann ein Screening auf Desmoide mit einem geeigneten bildgebenden Verfahren durchgeführt werden. Asymptomatische und nicht-größenprogrediente Desmoidtumoren bedürfen oft keiner Therapie. In einem systematischen Review publizierter klinischer Studien zur medikamentösen, nicht-zytotoxischen Therapie sind eine Therapie mit Sulindac (300 mg) oder Tamoxifen (40-120 mg) oder eine Kombinationstherapie am besten hinsichtlich ihrer Wirksamkeit belegt [329]. Auch zu Raloxifen liegen vergleichbare Daten aus einer kleinen Fallserie vor [330]. Progrediente Tumoren unter Sulindac oder antihormoneller Therapie sollten mit einer Chemo-therapie (Doxorubicin und Dacarbazine oder Methotrexat und Vinblastin) oder einer Strahlentherapie behandelt werden [331-333]. Ergebnisse zur Resektion sind kontrovers [334]. Vielfach wird insbesondere bei intraabdominellen Desmoiden über inkomplette Resektionen und hohe Rezidivraten berichtet [335-338].

Bei Bauchwanddesmoiden scheint in vielen Fällen ein chirurgisches Vorgehen mit Erreichen einer R0-Resektion und Rezidivfreiheit möglich zu sein [336].

5.38 .

Konsensbasierte Empfehlung

2013

EK

Eine jährliche Sonographie der Schilddrüse kann ab dem 15. Lebensjahr bei weiblichen FAP-Patienten durchgeführt werden.

Konsens

\section{Hintergrund}

Das Lebenszeitrisiko für Patienten mit einer FAP, ein Schilddrüsenkarzinom zu entwickeln, beträgt 1 bis $12 \%$. Ungefähr $95 \%$ aller berichteten Schilddrüsenkarzinome sind bei Frauen aufgetreten; das Risiko ist daher vor allem für weibliche Anlageträger erhöht. Die Diagnose erfolgt im Mittel zwischen dem 24. und 33. Lebensjahr. Erkrankungen vor dem 15. Lebensjahr sind selten [339]. Karzinome treten oft multifokal und zum Teil bilateral auf. Histologisch liegt in der Regel die kribiforme Variante eines papillären Schilddrüsenkarzinoms vor.

Zwei prospektive [340, 341] und eine retrospektive Studie [342] haben den Stellenwert eines einzeitigen Ultraschall-Screenings untersucht. Benigne Schilddrüsen-Knoten wurden dabei in 20 bis $79 \%$ entdeckt. Schilddrüsenkarzinome wurden mit einer Prävalenz von 2,6 bis 7,6\% diagnostiziert. In der größten Studie wurde keines der Karzinome durch die Anamnese oder Palpation der Schilddrüse entdeckt, sondern alle über die Sonographie [340]. Daher kann ein Ultraschall-Screening für weibliche FAPPatienten ab dem 15. Lebensjahr durchgeführt werden. Untersuchungen zum geeigneten Untersuchungsintervall liegen bisher nicht vor. Die Prognose FAP-assoziierter Schilddrüsenkarzinome ist gut; Todesfälle sind aber beschrieben [339, 343, 344]. Inwieweit durch das Screening eine Mortalitätssenkung erreicht werden kann, ist unklar. Möglicherweise kann die Häufigkeit der Notwendigkeit einer adjuvanten Radiotherapie gesenkt werden, wenn vermehrt Mikrokarzinome detektiert werden. 
Das Hepatoblastom ist eine sehr seltene Manifestation einer FAP. Weniger als $0,5 \%$ aller Kinder von FAP-Patienten entwickeln fast ausschließlich vor dem 10. Lebensjahr ein Hepatoblastom [345]. Dabei scheint das Risiko für Jungen höher zu sein. In einem Teil der Fälle gibt es eine positive Familienanamnese [346]. Aufgrund der Seltenheit und der unklaren Datenlage, ob durch ein Screening die Prognose von Hepato-blastompatienten verbessert werden kann, wird ein Screening nicht generell empfohlen [347, 348].

\begin{tabular}{|l|l|l|}
\hline $\mathbf{5 . 3 9 .}$ & Evidenzbasierte Empfehlung & 2013 \\
\hline $\begin{array}{l}\text { Empfehlungsgrad } \\
\mathbf{B}\end{array}$ & $\begin{array}{l}\text { Eine medikamentöse Behandlung von Adenomen im unteren und oberen Gastro- } \\
\text { intestinaltrakt sollte nicht generell empfohlen werden. }\end{array}$ \\
\hline $\begin{array}{l}\text { Level of Evidence } \\
\mathbf{2} \mathbf{b}\end{array}$ & \begin{tabular}{l} 
Evidenz aus Aktualisierungsrecherche:[349-357] \\
\hline
\end{tabular} \\
\hline & Starker Konsens \\
\hline
\end{tabular}

\section{Hintergrund}

Sulindac reduziert die Anzahl kolorektaler Adenome um mehr als 50\% [349-353]. Es verhindert jedoch nicht die Polypenneubildung [354]. Weiterhin wurden unter SulindacTherapie Karzinome im Rektumstumpf beobachtet. Sulindac ist in Deutschland nicht zugelassen. Eine Chemoprävention mit Sulindac bei FAP kann in Einzelfällen als additive Behandlung nach subtotaler Kolektomie durchgeführt werden, um die rektale Polypenlast zu reduzieren. Eine endoskopische Überwachung ist jedoch zwingend.

Der selektive COX2-Hemmer Celecoxib, der zu einer Reduktion rektaler Adenome führt [355], wurde zur Chemoprävention bei FAP als Ergänzung zu chirurgischen Maßnahmen und weiteren endoskopischen Kontrollen zugelassen. Celecoxib in hohen Dosierungen von 400-800mg reduziert die kolorektale Polypenzahl um 28\% [355] und beeinflusst auch eine Duodenalpolyposis [356]. Es ist allerdings ungeklärt, ob die Einnahme auch das Risiko der Karzinomentstehung bei diesen Patienten senkt. COX-2-Hemmer sind mit einer erhöhten kardiovaskulären Ereignisrate assoziiert [357]. Der Stellenwert der COX2-Inhibitoren ist aufgrund der kardiovaskulären Nebenwirkungen derzeit unklar und diese sollten gegenwärtig nur in ausgewählten Fällen bei strenger Indikationsstellung (Nutzen-Risiko-Abwägung) verwendet werden. Das für die FAP zugelassene Präparat mit dem Wirkstoff Celecoxib wurde im April 2011 vom Hersteller aufgrund mangelnder Rekrutierung einer von der europäischen Arzneimittelbehörde (EMA) geforderten PostZulassungsstudie vom Markt genommen. Die Verwendung von COX-2-Inhibitoren könnte bei selektionierten Patienten zur Verzögerung einer Kolektomie, bei subtotaler Kolektomie zur Reduzierung der Rektumpolypenlast, bei schwerer Duodenalpolyposis erhöhtem Operationsrisiko oder erhöhtem Perforations- und Blutungsrisiko bei Polypektomie in Einzelfällen gerechtfertigt werden. 


\subsection{Patienten mit attenuierter familiärer adenomatöser Polyposis}

\begin{tabular}{|l|l|}
\hline $\begin{array}{l}\mathbf{5 . 4 0 .} \\
\text { Empfehlungsgrad }\end{array}$ & $\begin{array}{l}\text { Evidenzbasierte Empfehlung } \\
\mathbf{B}\end{array}$ \\
$\begin{array}{l}\text { Und histologischem Befund therapiert werden. Bei endoskopisch nicht beherrsch-barer } \\
\text { Polyposis ist eine Kolektomie indiziert. Patienten, die nicht kolektomiert sind, sollten } \\
\text { zeitlebens jedes Jahr koloskopiert werden. }\end{array}$ \\
\hline $\begin{array}{l}\text { Level of Evidence } \\
\mathbf{4}\end{array}$ & \begin{tabular}{l} 
Quellen: $[257,258,260,261,358-361]$ \\
\hline
\end{tabular} \\
\hline
\end{tabular}

\begin{tabular}{|l|l|}
\hline $\mathbf{5 . 4 1 .}$ & Evidenzbasierte Empfehlung \\
\hline $\begin{array}{l}\text { Empfehlungsgrad } \\
\mathbf{C}\end{array}$ & $\begin{array}{l}\text { Risikopersonen aus Familien mit attenuierter FAP sollten im Rahmen der } \\
\text { Vorsorgeuntersuchung im Alter von 15 Jahren erstmals koloskopiert werden. Finden } \\
\text { sich keine Polypen, sollten diese Personen ab dem 20. Lebensjahr jährlich } \\
\text { koloskopiert werden. }\end{array}$ \\
\hline $\begin{array}{l}\text { Level of Evidence } \\
\mathbf{4}\end{array}$ & \begin{tabular}{l} 
Quellen: [359-361] \\
\hline
\end{tabular} \\
\hline
\end{tabular}

\section{Hintergrund}

Bei Patienten mit einer attenuierten FAP treten Polypen oft deutlich später und seltener auf als bei der klassischen FAP. Die Diagnose eines KRK in der Adoleszenz wurde kasuistisch berichtet [358]. Die Polypen sind häufig im rechtsseitigen Kolon lokalisiert. Aus diesem Grunde muss eine komplette Koloskopie zur Früherkennung durchgeführt werden [257, 258, 260, 261]. Da die klinische Ausprägung stark variieren kann, ist die Therapieentscheidung individuell abzuwägen. Bei Patienten mit bestehender Operationsindikation, aber weniger als 5 Rektumpolypen, ist unter Umständen eine ileorektale Anastomose mit Belassen eines Rektumstumpfes vertretbar. Da extrakolische Manifestationen genau wie bei der klassischen FAP auftreten können [359-361], gelten diesbezüglich die Empfehlungen für die klassische FAP. Bis zu welchem Alter Vorsorgeuntersuchungen bei Risikopersonen mit unauffälligem endoskopischem Befund fortgeführt werden sollen, ist aufgrund der Datenlage derzeit unklar. 
5.2.2.2.3. Patienten mit MUTYH-assoziierter Polyposis (MAP)

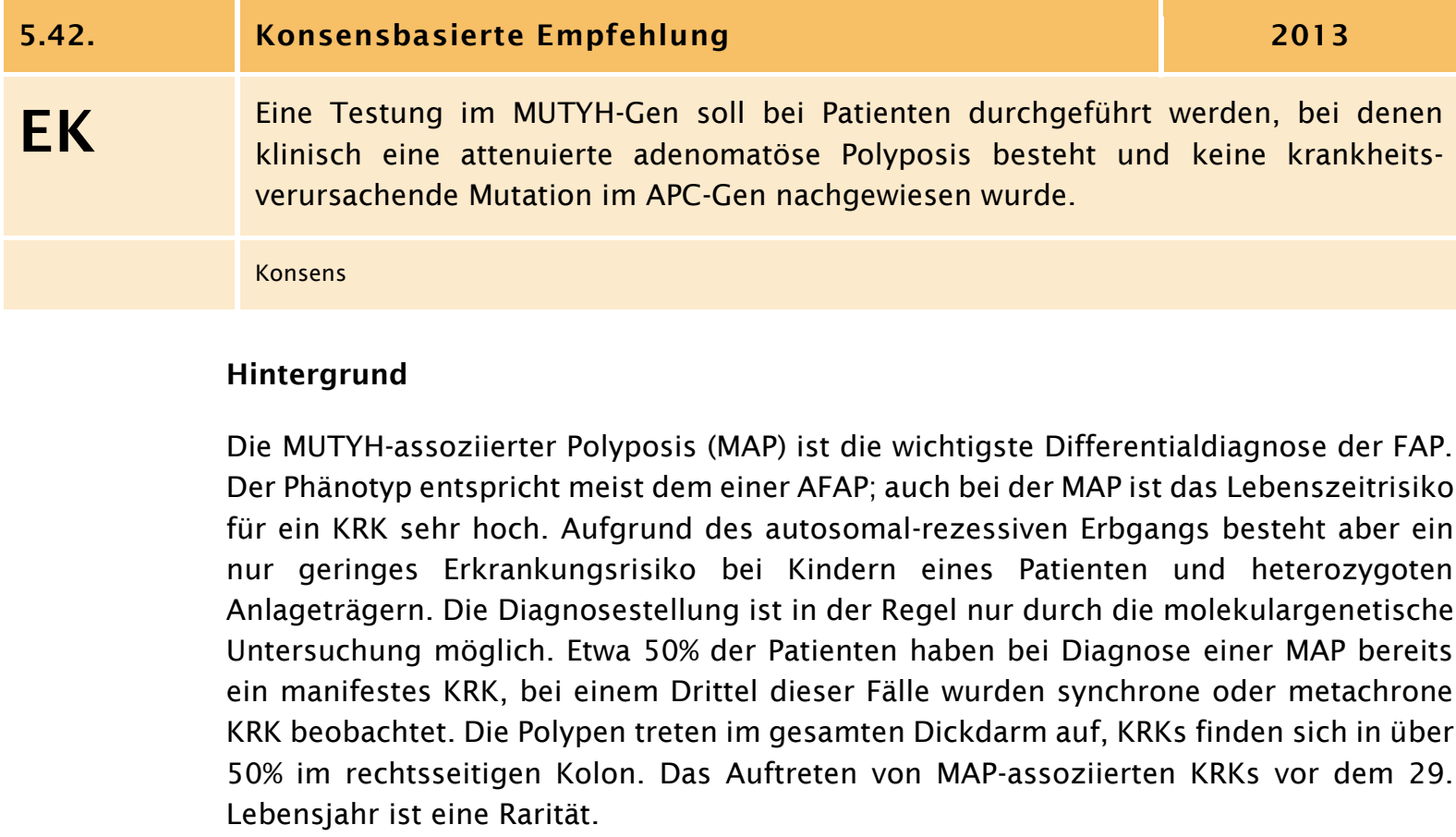

5.43

\section{EK}

EK

5.45 .

\section{EK}

\section{Konsensbasierte Empfehlung}

Geschwister eines MAP-Patienten haben aufgrund des autosomal-rezessiven Erbgangs ein Erkrankungsrisiko von 25\% und werden als Risikopersonen bezeichnet. Diesen sollte ab dem 18.-20. Lebensjahr im Anschluss an eine humangenetische Beratung eine prädiktive genetische Diagnostik empfohlen werden.

Starker Konsens

\section{3}

\section{Konsensbasierte Empfehlung}

2013

Werden beide MUTYH-Mutationen des Indexpatienten bei seinen Geschwistern ausgeschlossen, ist eine gesonderte Vorsorge bei diesen nicht mehr notwendig.

Starker Konsens 


\section{Hintergrund}

MAP ist die einzige bisher bekannte autosomal-rezessiv vererbte Erkrankung mit erhöhtem KRK-Risiko. Ob heterozygote Anlageträger ein erhöhtes KRK-Risiko tragen, wird kontrovers diskutiert; einige neuere populations- und familienbasierte Untersuchungen an großen Kollektiven fanden Hinweise für ein moderat erhöhtes Risiko im fortgeschrittenen Alter (RR 1,5-2,1) [270, 362, 363]. Das höchste Erkrankungsrisiko für ein KRK (RR 2,1) bestand bei erstgradig verwandten heterozygoten Anlageträgern eines MAP-Patienten, das Diagnosealter betrug hier im Mittel 70 Jahre (Streuung 58-82). Erkrankungsrisiko und -zeitpunkt sind somit vergleichbar dem von erstgradig Verwandten eines Patienten mit sporadischen KRK.

Aufgrund einer Heterozygotenfrequenz von etwa 1-1,5\% in der kaukasischen Allgemeinbevölkerung haben die obligat heterozygoten Kinder eines MAP-Patienten bei nicht-konsanguinen Partnerschaften ein nur geringes Erkrankungsrisiko für eine MAP $(<0,5 \%)$ [265]. Wenn zur Einschätzung des Erkrankungsrisikos eine prädiktive Testung des Kindes gewünscht wird, muss beim Kind oder dem nicht erkrankten Elternteil eine komplette Mutationssuche im MUTYH-Gen erfolgen, um eine ggf. vom gesunden Elternteil vererbte zweite MUTYH-Keimbahnmutation zu identifizieren. Dem Nutzen eines aussagekräftigen genetischen Befundes steht hier allerdings die (seltene) Identifizierung funktionell unklarer genetischer Varianten gegenüber, über deren pathogene Relevanz und damit klinische Konsequenzen im Einzelfall (derzeit) keine Aussage möglich ist. Da das Vorhandensein einer zweiten MUTYH-Mutation bei Kindern eines MAP-Patienten aufgrund der $z u$ vermutenden unvollständigen Mutationsdetektionsrate nicht gänzlich ausgeschlossen werden kann, besteht bei heterozygot getesteten Kindern eines MAP-Patienten ein - vermutlich sehr geringes - Restrisiko, an einer MAP zu erkranken. Eine komplette Koloskopie im etwa 30. und 40. Lebensjahr kann deshalb in Erwägung gezogen werden.

5.46 .

Konsensbasierte Empfehlung

2013

EK

Asymptomatische biallele MUTYH-Mutationsträger sollten im Alter von 18-20 Jahren erstmals koloskopiert werden. Finden sich keine Polypen, sollten diese Patienten weiterhin überwacht werden.

Ein Patient mit einer MAP sollte in Abhängigkeit von Alter, Polypenzahl und histologischem Befund therapiert werden. Bei endoskopisch nicht beherrschbarer Polyposis ist eine Kolektomie indiziert. Patienten, die nicht kolektomiert sind, sollen zeitlebens jedes Jahr koloskopiert werden.

Eine ÖGD und Duodenoskopie (Seitblickoptik) mit besonderer Inspektion der Papillenregion soll ab dem 25.-30. Lebensjahr mindestens alle drei Jahre durchgeführt werden.

Spezifische Vorsorgeuntersuchungen für extraintestinale Manifestationen sind bei MAP-Patienten nicht gerechtfertigt.

Eine Empfehlung zur medikamentösen Behandlung von Adenomen im oberen und unteren Gastrointestinaltrakt kann aufgrund fehlender Daten nicht gegeben werden.

Konsens 


\section{Hintergrund}

Der kolorektale Phänotyp der MAP ähnelt dem der APC-assoziierten AFAP. Bei Patienten mit einer MAP werden Polypen und KRKs meist erst zwischen dem vierten und siebten Lebensjahrzehnt symptomatisch. Etwa 50\% der Patienten haben bei Diagnose einer MAP bereits ein manifestes KRK, bei einem Drittel dieser Fälle wurden synchrone oder metachrone KRK beobachtet [364]. Die Polypen treten im gesamten Dickdarm auf, KRKs finden sich in über $50 \%$ im rechtsseitigen Kolon und in über 20\% im Rektosigmoid [266]. Aus diesem Grunde muss eine komplette Koloskopie zur Früherkennung durchgeführt werden [257, 258, 260, 261]. Das Auftreten von MAP-assoziierten KRKs vor dem 29. Lebensjahr ist eine Rarität. Da die klinische Ausprägung stark variieren kann, ist die Therapieentscheidung individuell abzuwägen. Bei Patienten mit bestehender Operationsindikation und wenigen Rektumpolypen ist unter Umständen eine ileorektale Anastomose mit Belassen eines Rektumstumpfes vertretbar [365].

Obwohl eine Duodenalpolyposis bei MAP-Patienten seltener (17\%) als bei FAP-Patienten beobachtet wird, erscheint das Risiko von etwa 4\% für die Entwicklung eines Duodenalkarzinoms vergleichbar hoch zu sein [272]. Bei der MAP treten Duodenalkarzinome vereinzelt auch ohne vorbestehende duodenale Adenome auf [366]. Ob für MAP-Patienten deshalb möglicherweise andere Früherkennungsstrategien als für (A)FAPPatienten erforderlich sind, lässt sich derzeit noch nicht abschließend beurteilen. Extraintestinale Malignome treten bei MAP-Patienten insgesamt signifikant häufiger (RR $1,9 \%$ als in der Allgemeinbevölkerung auf und zeigen eine gewisse Überlappung zum HNPCC. In der diesbezüglich bisher einzigen systematischen Studie an 276 Patienten wurde eine gering bis moderate, aber statistisch signifikant erhöhte Inzidenz für Ovarial, Blasen- und Hautkarzinome sowie ein Trend zur Risikoerhöhung für Mammakarzinome beobachtet [272]. Es fand sich allerdings kein dominierender extraintestinaler Tumor und keine Verschiebung zu einem frühen Auftreten (medianes Diagnosealter der vier Malignome zwischen 51 und 61 Jahren). Desmoide wurden nicht beobachtet.

\subsubsection{Nicht-adenomatöse Polyposis-Syndrome}

\subsection{7}

Level of Evidence

$2 \mathrm{a}$
Evidenzbasiertes Statement

2013

Hierzu zählen insbesondere die hamartomatösen Polyposis-Syndrome (Peutz-Jeghers Syndrom, Familiäre Juvenile Polyposis, Cowden-Syndrom), das Hyper-plastische Polyposis-Syndrom und die Erbliche gemischte Polyposis. Diese Erkrankungen sind zum Teil sehr selten (ihr Anteil an allen KRK beträgt unter einem Promille). Anlageträger haben ein erhöhtes Risiko sowohl für kolorektale Karzinome als auch für andere syndromspezische intestinale und extraintestinale Tumoren (Magen, Mamma, etc.).

Evidenz aus Aktualisierungsrecherche: [264, 367-397]

\section{Hintergrund}

Die Differentialdiagnose der nicht-adenomatösen Polyposis-Syndrome kann in Einzelfällen sehr schwierig sein und erfordert das interdisziplinäre Zusammenwirken von Gastroenterologen, Chirurgen, Pathologen, Humangenetikern, Radiologen und anderen klinischen Fachdisziplinen (v.a. Gynäkologie, Urologie). Die Diagnose und klinische Betreuung der Patienten sollte daher in Abstimmung und Zusammenarbeit mit Zentren erfolgen, die Erfahrung mit diesen Syndromen haben [264, 367]. Die hamartomatösen 
Polyposis-Syndrome folgen einem autosomal-dominanten Erbgang. Kinder (und ggf. auch Geschwister) einer erkrankten Person haben somit ein 50\%iges Risiko, die zugrunde liegende genetische Veränderung geerbt $z u$ haben und im Laufe des Lebens zu erkranken.

Das Peutz-Jeghers-Syndrom (PJS) ist eine autosomal-dominant erbliche Erkrankung, die charakterisiert ist durch das Auftreten von hamartomatösen Polypen des Gastrointestinaltraktes und mukokutanen Melaninpigmentierungen, die vor allem perioral auffällig sind. Letztere blassen aber oft im Laufe des Lebens ab und sind nicht spezifisch. Peutz-Jeghers-Polypen treten insbesondere im Dünndarm auf und zeigen eine charakteristische Histologie. Ursächlich sind Keimbahnmutationen des STK11/LKB1Gens. Ein Mutationsnachweis gelingt bei Patienten, die die klinisch-diagnostischen Kriterien erfüllen, in über 90\% [368]. Das Manifestationsalter ist sehr variabel, einige Patienten entwickeln schon in den ersten Lebensjahren Symptome. $\mathrm{Zu}$ den Komplikationen im Kindesalter zählen das akute Abdomen durch Invaginationen oder einen obstruktiven lleus sowie die chronische Blutung mit sekundärer Anämie. Bis zu $30 \%$ der Patienten sind im Alter von 10 Jahren bereits einmal laparotomiert worden [369]. Beim PJS muss für eine Reihe von intestinalen und extraintestinalen Tumoren von einem deutlich erhöhten Erkrankungsrisiko ausgegangen werden [370-376]. Neben dem KRK ist insbesondere das Risiko für Karzinome der Mamma, des Magens, des Dünndarms, des Pankreas, der Hoden, des Ovars und Uterus erhöht [378, 379] Das kumulative Lebenszeitrisiko für einen malignen Tumor wird mit etwa $85-90 \%$ angegeben. Für Tumoren im gesamten Gastrointestinaltrakt besteht ein kumulatives Lebenszeitrisiko von 57\%, das KRK-Risiko allein beläuft sich auf $35-39 \%$ und stellt damit das zweithäufigste Karzinom bei PJS dar. Die untere Grenze des 95\% Konfidenzintervalles beträgt 30 Jahre. Das Tumorrisiko steigt dabei nach dem 50. Lebensjahr rasch an [377379]. Das Lebenszeitrisiko für gynäkologische Tumoren wird mit 13-18\% angegeben $[378,379]$. Ovarialtumoren bei PJS sind in der Regel SCTAT und nicht-epithelialen Ursprunges und werden zum Teil bereits auch bei kleinen Mädchen diagnostiziert (mittleres Alter 28 Jahre, 4-57 Jahre). Cervixkarzinome treten mit einem Lebenszeitrisiko von $9 \%$ auf und entsprechen histologisch in mehr als drei Viertel der Fällen einem Adenoma malignum [378].Das mittlere Erkrankungsalter beträgt 34 Jahre (23-54 Jahre). Das Risiko für Endometriumkarzinome wird mit etwa 10\% angegeben.

Der Verdacht auf eine Familiäre Juvenile Polyposis (FJP) besteht bei der Diagnose von fünf oder mehr juvenilen Polypen im Kolon, Nachweis von extrakolischen juvenilen Polypen oder bei Nachweis eines juvenilen Polypen bei entsprechender positiver Familienanamnese. Die korrekte Diagnose juveniler Polypen kann aufgrund morphologischer Ähnlichkeiten mit hyperplastischen Polypen sowie lymphozytären Infiltraten und dysplastischen Anteilen schwierig sein: in einem nennenswerten Teil der genetisch gesicherten Fälle wird die juvenile Polyposis initial als Colitis ulcerosa oder hyperplastische Polyposis fehlgedeutet [380, 381]. Im Zweifelsfall sollte deshalb die Zweitbegutachtung der histologischen Präparate durch einen gastroenterologisch versierten Pathologen angestrebt werden.

Die Erkrankung kann sich bereits im frühen Kindesalter durch eine chronische gastrointestinale Blutung oder exsudative Enteropathie mit begleitender Entwicklungsverzögerung bemerkbar machen. Ursächlich sind Keimbahnmutationen im SMAD4- oder BMPR1A-Gen. Das Lebenszeitrisiko für die Entwicklung eines KRKs beträgt bis zu $68 \%$. Es bestehen klare Genotyp-Phänotyp-Beziehungen: Patienten mit einer SMAD4Keimbahnmutation haben ein erhöhtes Risiko für die Entwicklung von Magenpolypen und Magenkrebs sowie für eine hereditäre hämorrhagische Teleangiektasie (Morbus Osler-Rendu-Weber) [381, 382]. Daneben ist möglicherweise das Risiko für 
Pankreaskarzinome erhöht [383-387]. Bei sehr schweren frühmanifesten Verläufen ist an die seltene juvenile Polyposis des Kleinkindesalters zu denken [388].

Die endoskopisch-histologische Abgrenzung einer juvenilen Polyposis vom auf PTENMutationen beruhenden Cowden-Syndrom oder dem vermutlich nicht erblichen Cronkhite-Canada-Syndrom kann Probleme bereiten und erfolgt in der Regel durch das im Vordergrund stehende extraintestinale Tumorspektrum und die Molekulargenetik. Beim Cowden-Syndrom [389] ist insbesondere das Risiko für Mamma- und Schilddrüsenkarzinome erhöht. Das Risiko für kolorektale Karzinome scheint nach neueren Daten mit einem Lebenszeitrisiko von 9\% ebenfalls erhöht zu sein [390]. Weiterhin wurden erhöhte Risiken für Karzinome des Endometriums und der Nieren sowie für Melanome beschrieben. Das Bannayan-Riley-Ruvalcaba-Syndrom wird als Variante des Cowden-Syndroms betrachtet. Beide Syndrome sind mit Keimbahnmutationen des PTENGens assoziiert und werden unter der Bezeichnung PTEN-Hamartom-Tumor-Syndrom (PHTS) zusammengefasst [391, 392].

Bei der Erblichen gemischten Polyposis (hereditary mixed polyposis syndrome, HMPS) und dem Hyperplastischen Polyposis Syndrom (HPS) handelt es sich um schlecht definierte Entitäten, über deren genetische Grundlagen noch wenig bekannt ist. Beide Syndrome wurden mit einem - zum Teil deutlich - erhöhten KRK-Risiko in Verbindung gebracht [393-397]; sie sind jedoch selten und bisher sowohl klinisch wie genetisch schlecht charakterisiert, so dass die Erkenntnisse in Bezug auf Tumorrisiken nur bedingt aussagekräftig sind. Bei einigen Patienten mit HMPS wurden Mutationen im PTEN- oder BMPR1A-Gen nachgewiesen, diese Fälle sollten als (atypische) Varianten des CowdenSyndroms bzw. der FJP betrachtet und entsprechend behandelt werden.

\begin{tabular}{|l|l|}
\hline $\mathbf{5 . 4 8 .}$ & Konsensbasierte Empfehlung \\
\hline EK & $\begin{array}{l}\text { Generelle Überwachungsempfehlungen können wegen der spärlichen Datenlage nicht } \\
\text { gegeben werden. Die Überwachung der Patienten und Risikopersonen sollte in } \\
\text { Zusammenarbeit mit einem ausgewiesenen Zentrum durchgeführt werden. }\end{array}$ \\
\hline Konsens
\end{tabular}

\section{Hintergrund}

Valide Vorsorgeempfehlungen können wegen der spärlichen Datenlage nicht gegeben werden. Für die häufigeren Syndrome wurden von einzelnen Experten spezifische Früherkennungsprogramme vorgeschlagen. Eine publizierte Leitlinie zum Peutz-JeghersSyndrom hat methodische Defizite und weist für die meisten Empfehlungen nur geringe Evidenzen auf [398]. Die Patienten und Risikopersonen sollten deshalb in enger Abstimmung mit erfahrenen Zentren betreut werden. Für einige Syndrome wurden von Experten spezifische altersabhängige Früherkennungsprogramme vorgeschlagen (Übersicht in [264]), die Empfehlungen weichen allerdings zum Teil deutlich voneinander $\mathrm{ab}$ und die Wirksamkeit ist bei den sehr seltenen Syndromen aufgrund geringer Fallzahlen nur schwer zu validieren. 


\subsection{Chronisch entzündliche Darmerkrankungen}

\subsubsection{Colitis ulcerosa}

\begin{tabular}{l|l} 
5.49. & Konsensbasierte Statement \\
\hline EK & $\begin{array}{l}\text { Das kolorektale Karzinomrisiko ist bei Patienten mit Colitis ulcerosa im Vergleich zur } \\
\text { Normalbevölkerung erhöht. Entsprechende Empfehlungen sind in der S3-Leitlinie zur } \\
\text { Diagnostik und Therapie der Colitis ulcerosa genannt. }\end{array}$
\end{tabular}

Starker Konsens

Überwachungskoloskopie, zeitliche Strategie (Auszug aus der S3-LL CU [399])

\begin{tabular}{|c|c|}
\hline 5.50. & Evidenzbasierte Empfehlung \\
\hline Empfehlungsgrad & $\begin{array}{l}\text { Da die colitisassoziierte Kolonkarzinommortalität durch eine endoskopische } \\
\text { Überwachung gesenkt werden kann, sollen regelmäßige Überwachungskoloskopien } \\
\text { erfolgen. }\end{array}$ \\
\hline \multirow{2}{*}{$\begin{array}{l}\text { Evidenzgrad }^{6} \\
3 \mathbf{a}\end{array}$} & {$[400,401]$} \\
\hline & Konsens \\
\hline 5.51 & Evidenzbasierte Empfehlung \\
\hline Empfehlungsgrad & $\begin{array}{l}\text { Zur Festlegung der Überwachungsstrategie soll bei allen CU-Patienten unabhängig } \\
\text { von der Krankheitsaktivität eine Kontrollkoloskopie zur Erfassung des Befallsmusters } \\
\text { spätestens } 8 \text { Jahre nach Beginn der Symptomatik erfolgen. }\end{array}$ \\
\hline \multirow{2}{*}{$\begin{array}{l}\text { Evidenzgrad } \\
4\end{array}$} & [402] \\
\hline & Konsens \\
\hline
\end{tabular}




\begin{tabular}{|l|l|}
$\begin{array}{l}\text { 5.52. } \\
\text { Empfehlungsgrad }\end{array}$ & $\begin{array}{l}\text { Die Überwachungskoloskopien sollten dann bei ausgedehnter CU ab dem 8. Jahr und } \\
\text { bei linksseitiger oder distaler CU ab dem 15. Jahr nach Erstmanifestation } 1-2 \text { jährlich } \\
\text { erfolgen. }\end{array}$ \\
\hline $\begin{array}{l}\text { Evidenzgrad } \\
\mathbf{4}\end{array}$ & {$[403,404]$} \\
\hline & Konsens \\
\hline
\end{tabular}

\subsection{3.}

Empfehlungsgrad

A

Evidenzgrad

$3 a$

\section{Evidenzbasierte Empfehlung}

Wenn gleichzeitig eine PSC besteht, sollen die Überwachungskoloskopien unabhängig von der Krankheitsaktivität und Ausdehnung der CU ab dem Zeitpunkt der PSC-Diagnosestellung jährlich erfolgen.

$[405,406]$

Konsens

\subsection{4 .}

Empfehlungsgrad

A

Evidenzgrad

4

\section{Evidenzbasierte Empfehlung}

Nach subtotaler Kolektomie sollen in Analogie die gleichen endoskopischen Überwachungsstrategien wie bei einer CU ohne Resektion erfolgen.

[407]

Starker Konsens

\section{Erläuterung}

Eine Metaanalyse von Collins aus dem Jahr 2006 fasst die direkte und indirekte Evidenz zu Überwachungskoloskopien bei Colitis ulcerosa zusammen. Die drei identifizierten Fallkontrollstudien konnten keine statistisch signifikante Reduktion des Risikos, an einem kolorektalen Karzinom zu erkranken, zeigen. Dabei ist anzumerken, dass es sich um kleine Studien mit teilweise aus heutiger Sicht inadäquatem Koloskopie-standard handelte. Dahingegen konnte die Metaanalyse deutliche indirekte Evidenz identifizieren, dass Überwachungskoloskopien sehr wahrscheinlich das Risiko, an einem Colitisassoziierten Kolonkarzinom zu versterben, vermindern können, und gleichzeitig kosteneffektiv sind [400]. Dies basiert darauf, dass Colitis-assoziierte Kolonkarzinome früher erkannt werden, obwohl dennoch zwischen Überwachungs-untersuchungen Intervallkarzinome auftreten können [401]. Das Karzinomrisiko steigt mit der Krankheitsdauer und der Krankheitsausdehnung an, weshalb bei Patienten mit einer 
Pancolitis die regelmäßige Überwachung früher als bei Patienten mit einer distalen Colitis beginnen sollte. Da sich aus einer initial als distale Colitis beschriebenen Entzündung auch ohne klinische Hinweise eine Pancolitis entwickeln kann, sollte eine Kontrollkoloskopie innerhalb von 8 Jahren nach den ersten Krankheitssymptomen durchgeführt werden, um die Ausdehnung zu überprüfen und danach das Überwachungsvorgehen festlegen zu können. Eine niederländische Studie weist darauf hin, dass bereits bis zu 22\% der Patienten vor dem Start der bisher empfohlenen Überwachungskoloskopien Colitis-assoziierte Kolonkarzinome entwickel-ten [402]. Wenn Patienten mit PSC herausgerechnet wurden - die ab Diagnosestellung überwacht werden sollten - reduziert sich die Rate der „entgangenen” Karzinome auf ca. 15\%.

Das Überwachungsintervall zwischen den Untersuchungen sollte höchstens 2 Jahre betragen, weil in diesem Zeitraum bereits Intervallkarzinome auftreten können [403, 404]. Da bei einer Proktitis das Karzinomrisiko allenfalls minimal erhöht ist, muss - bei Fehlen sonstiger Risikofaktoren - keine regelhafte Überwachung erfolgen. Da das Karzinomrisiko bei Patienten mit CU und einer PSC fünffach erhöht ist [406] und Karzinome gehäuft rechtsseitig auftreten [405], sollte bei diesen Patienten eine Überwachung ab Diagnosestellung unabhängig vom Befall jährlich erfolgen.

Nach subtotaler Kolektomie können im verbliebenen Darm, wie auch nach restaurativer Proktokolektomie im Pouch bzw. je nach Operationstechnik im Bereich der verbliebenen Kolonmukosa distal der Anastomose, Karzinome auftreten [407], sodass eine regelhafte Überwachung des verbliebenen Kolons bzw. des Pouches erfolgen sollte.

\subsubsection{Morbus Crohn}

Das kolorektale Karzinomrisiko bei Patienten mit Morbus Crohn scheint, insbesondere bei Kolonbefall, im Vergleich zur Normalbevölkerung erhöht zu sein. Der Nutzen eines Überwachungsprogramms mit lleokoloskopie zur Früherkennung eines Karzinoms ist bei der Crohn Colitis ungeklärt. Die Datenlage wird in der S3-Leitlinie zur Diagnostik und Therapie des Morbus Crohn diskutiert [408]. 


\subsection{Anhang: Abbildungen und Tabellen des TK III}

\subsubsection{Algorithmus: Genetische Diagnostik und Vorsorge}

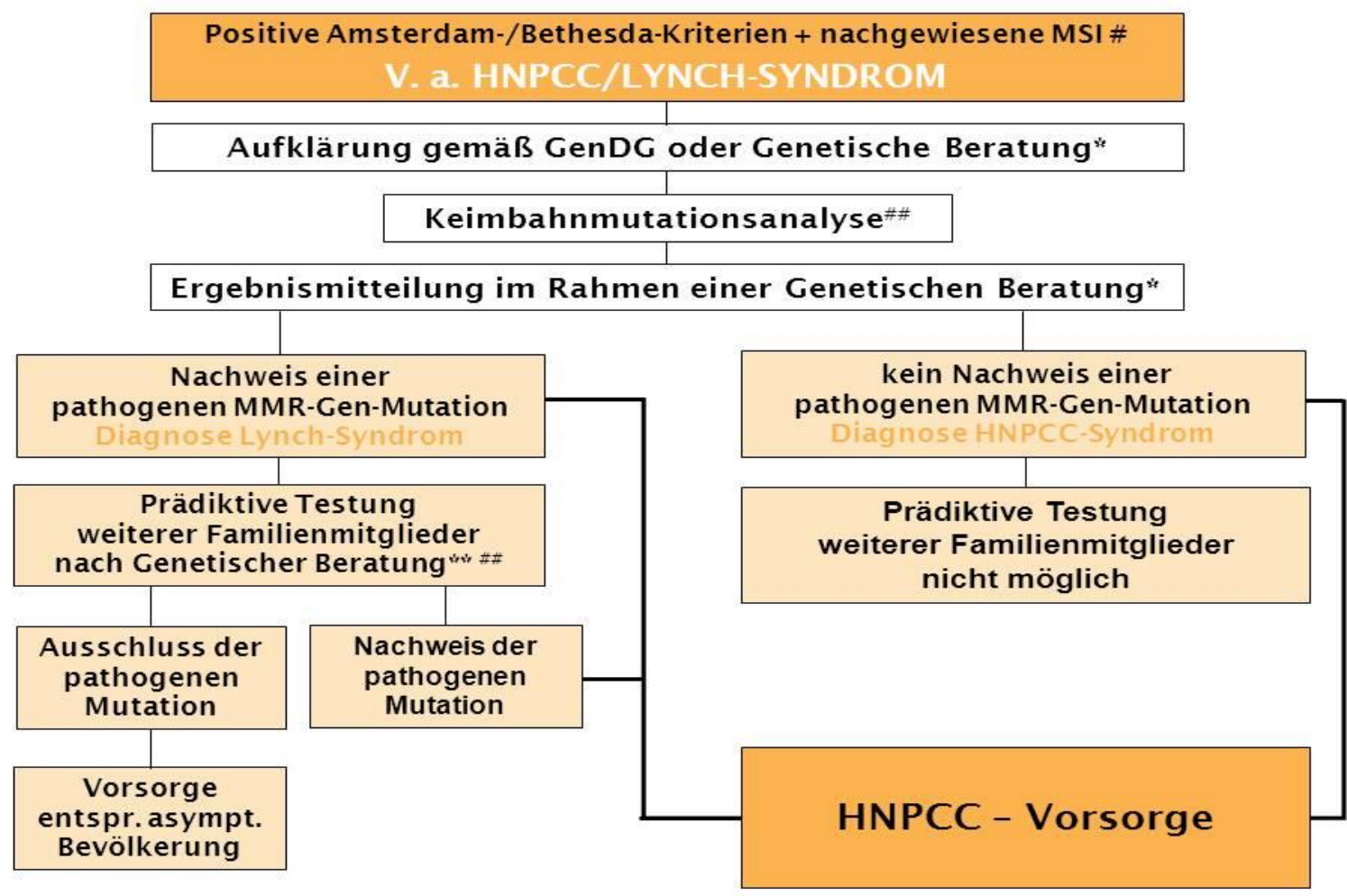

* Eine diagnostische Keimbahnuntersuchung erfordert gemäß GenDG eine Aufklärung und Dokumentation der Gesprächsinhalte durch den veranlassenden Arzt. Alternativ kann eine genetische Beratung erfolgen. Die Ergebnismitteilung muss gemäß GenDG im Rahmen einer genetischen Beratung erfolgen.

**: Eine prädiktive genetische Keimbahndiagnostik bei asymptomatischen Individuen darf gemäß GenDG nur nach einer genetischen Beratung erfolgen. Die Ergebnismitteilung muß gemäß GenDG ebenfalls im Rahmen einer genetischen Beratung erfolgen.

\# Bei hochgradigem V.a. HNPCC/Lynch-Syndrom (z.B. positive Amsterdam-Kriterien) und Nichtvorhandensein von Tumorgewebe kann auch direkt eine Mutationsanalyse erfolgen.

\#\# Sofern eine Keimbahndiagnostik vom Patienten nicht gewünscht wird, ist unabhängig davon in jedem Fall die HNPCC-Vorsorge zu empfehlen.

Abbildung 1: Algorithmus zum Ablauf der genetischen Diagnostik bei Patienten mit V.a. ein hereditäres Tumordispositions-Syndrom am Beispiel des HNPCC-/Lynch-Syndroms. Zum Nachweis der MSI bei V.a. HNPCC-/Lynch-Syndroms wird auf Abbildung 2 verwiesen. 


\title{
5.4.2. Testalgorithmus Immunhistochemie / MSI zur Abklärung Mismatch-Reparatur-Defekt
}

\author{
Positive Amsterdam-/ revidierte Bethesda- \\ Kriterien
}

\begin{tabular}{|c|}
\hline $\begin{array}{c}\text { Immunhistochemie Tumorgewebe } \\
\text { (Biopsie/Resektat) }\end{array}$ \\
\hline
\end{tabular}

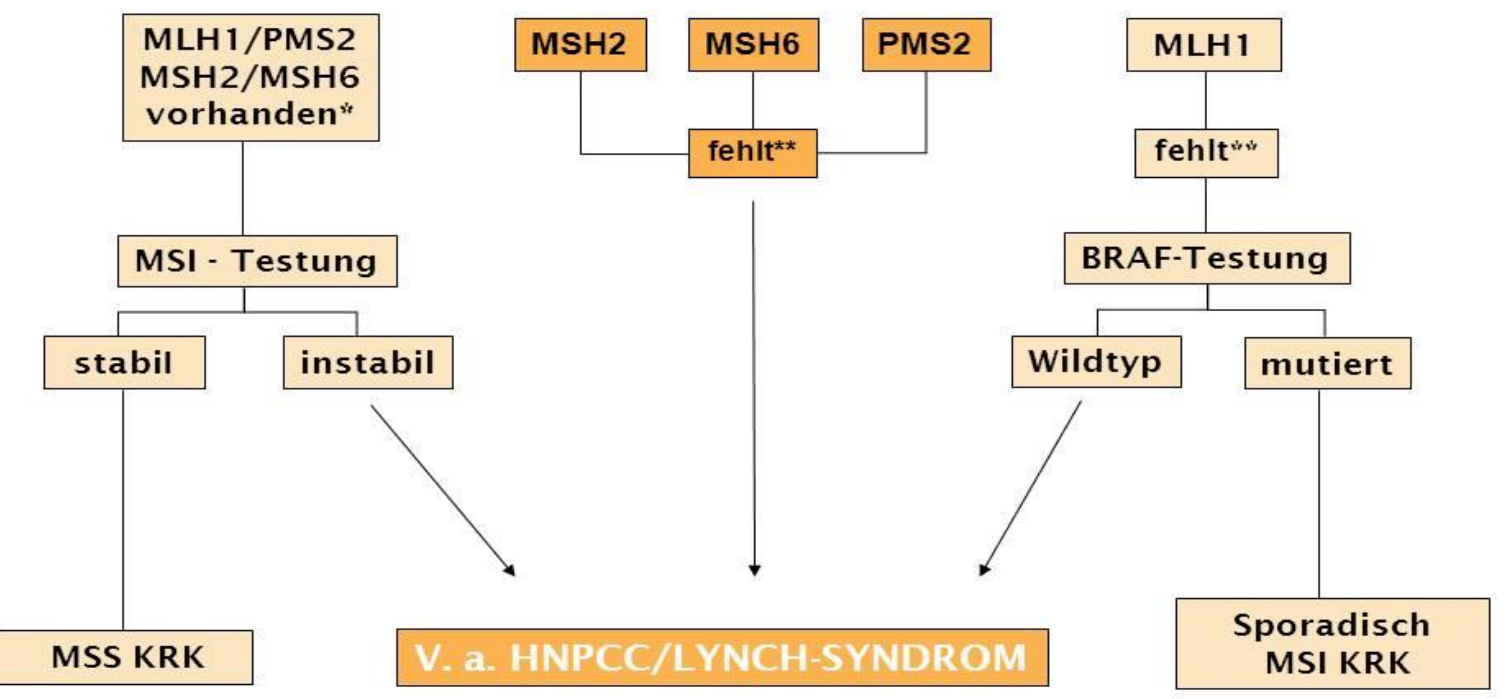

* in jeweils $>10 \%$ der Tumorzellen nukleär positiv; ** in $<10 \%$ der Tumorzellen nukleär positiv

Abbildung 2: Algorithmus zum Ablauf der molekularpathologischen Abklärung eines MismatchReparaturdefektes bei klinischem V.a. HNPCC-/Lynch-Syndrom. Zur sich ggf. anschließenden genetischen Diagnostik wird auf Abbildung 1 verwiesen.

\subsubsection{Empfohlenes Krebsfrüherkennungsprogramm bei HNPCC}

Tabelle 6: Empfohlenes Krebsfrüherkennungsprogramm bei HNPCC

\begin{tabular}{|l|l|l|}
\hline Altersangabe & Untersuchung & Intervall \\
\hline $\begin{array}{l}\text { Ab dem 25. } \\
\text { Lebensjahr }\end{array}$ & Körperliche Untersuchung & jährlich \\
\hline & Koloskopie & jährlich \\
\hline $\begin{array}{l}\text { Gynäkologische Untersuchung einschl. } \\
\text { transvaginaler Sonographie (bei Frauen) }\end{array}$ & jährlich \\
\hline $\begin{array}{l}\text { Ab dem 35. } \\
\text { Lebensjahr }\end{array}$ & ÖGD & regelmäßig \\
\hline & Endometriumbiopsie (bei Frauen) & jährlich \\
\hline
\end{tabular}




\subsubsection{Spigelman-Klassifikation}

Tabelle 7: Klassifikation der Ausprägung der Duodenalpolyposis nach der SpigelmanKlassifikation (modifiziert nach [310])

\begin{tabular}{|c|c|c|c|}
\hline & \multicolumn{3}{|c|}{ Punktezahl } \\
\hline & 1 & 2 & 3 \\
\hline Anzahl der Polypen & $1-4$ & $5-20$ & $>20$ \\
\hline Polypengröße (mm) & $1-4$ & $5-10$ & $>10$ \\
\hline Histologie & tubulär & tubulär villös & villös \\
\hline Intraepitheliale Neoplasie & niedriggradig & - & hochgradig \\
\hline $\begin{array}{l}\text { Stadium 0: } 0 \text { Punkte } \\
\text { Stadium I: 1-4 Punkte } \\
\text { Stadium II: 5-6 Punkte } \\
\text { Stadium III: 7-8 Punkte } \\
\text { Stadium IV: 9-12 Punkte }\end{array}$ & & & \\
\hline
\end{tabular}




\section{Endoskopie: Durchführung und Polypenmanagement}

\subsection{Stellenwert der Endoskopie in der Diagnostik von Polypen und kolorektalen Karzinomen}

\begin{tabular}{|l|l|}
\hline $\begin{array}{l}\text { 6.1. } \\
\text { Level of Evidence }\end{array}$ & $\begin{array}{l}\text { Evidenzbasiertes Statement } \\
\text { Die komplette Koloskopie stellt das Standardverfahren zur Detektion kolorektaler } \\
\text { Polypen und Karzinome dar. Sie besitzt die höchste Sensitivität und Spezifität für das } \\
\text { Auffinden eines KRK und von kolorektalen Polypen. Die Effektivität der Koloskopie } \\
\text { hängt entscheidend von der Qualität der Untersuchung ab. Diese ist technik- und } \\
\text { untersucherabhängig. }\end{array}$ \\
\hline & Quellen: [174] \\
\hline & Starker Konsens \\
\hline
\end{tabular}

\section{2.}

Empfehlungsgrad

0

A

Level of Evidence

4

6.3.

Empfehlungsgrad

B

Level of Evidence

$3 b$

\section{Evidenzbasierte Empfehlung}

2017

Bei inkompletter Koloskopie aufgrund eines stenosierenden Tumors kann präoperativ zusätzlich eine CT-Kolonographie erfolgen.

Postoperativ soll eine komplette Koloskopie erfolgen.

Quellen: [409-412]

Starker Konsens

\section{Evidenzbasierte Empfehlung}

2017

Bei inkompletter Koloskopie aufgrund anderer Ursachen (z. B. Adhäsionen) sollte eine CT-Kolonographie erfolgen.

Quellen: $[409,410,413]$

Starker Konsens 


\section{Hintergrund}

Die Koloskopie ist das zuverlässigste Verfahren zur Detektion kolorektaler Karzinome und Polypen, wenn sie mit hoher Qualität durchgeführt wird. Wichtige Qualitätsmerkmale beinhalten die Spiegelung bis zum Coecum, die optimale Darmvorbereitung mit wenig oder keinen verbliebenen Stuhlresten sowie die sorgfältige Inspektion der Darmschleimhaut beim Rückzug. Der wichtigste Surrogatparameter für den Outcome der (Vorsorge)Koloskopie ist die sog. Adenomdetektionsrate (ADR) [414, 415].

In der jüngsten Zeit sind mehrere Qualitätsleitlinien aus Europa und den USA erschienen, die sich teilweise in unterschiedlicher Weise bezüglich der Qualitätsmerkmale der Koloskopie festlegen [416-420]; die prozedurenspezifischen Hauptqualitätsparameter sind nachfolgend aufgelistet; hiervon sind Empfehlungen, die allgemein für alle Endoskopien gelten (Sedierung, Komplikationen, Gerätedesinfektion etc) nicht betroffen, auch wenn die praktische Umsetzung einzelner Parameter (Komplikations-Erfassung und -Audit, Prozedurenzahl) interessanten Diskussionsstoff böte.

Tabelle 8:Übersicht leitlinienbasierte prozedurenspezifischen Qualitätsparameter der Koloskopie

\begin{tabular}{|c|c|c|c|c|c|}
\hline & DGVS[416] & ESGE[4 17] & EU[4 18$]$ & UK[419] & US[420]*** \\
\hline Zoekalrate & $>90 \%$ & $>90 \%$ & $>90 \%$ & $>90 \%$ & ja \\
\hline Rückzugszeit & $>6 \mathrm{~min}$ & $>6 \min$ & ja & $>6 \min$ & $>6 \min$ \\
\hline ADR & $>20 \%$ & individuell & individuell & $>15 \% * *$ & ja \\
\hline Sauberkeit Kolon & $>90 \%$ gut & $>90 \%$ gut & ja & $>90 \%$ & nein \\
\hline Polypektomie & $\begin{array}{l}\text { Vollständ- } \\
\text { igkeit } \\
\text { (endoskop.) }\end{array}$ & detailliert* & vage & detailliert* & $\mathrm{Ja}$ \\
\hline Intervallläsionen & nein & ja & vage & empfohlen & nein \\
\hline F-up-Adhärenz & nein & nein & $>90 \%$ & nein & nein \\
\hline
\end{tabular}

Die Adenomdetektionsrate (ADR) ist als Hauptoutcome-Parameter der (Vorsorge)Koloskopie gesetzt und zeigt eine nahezu lineare negative Korrelation der ADR mit den Intervallkarzinomen auf. Jedoch sind mehrere methodische Mängel in den Studien zu finden (zur ausführlichen Diskussion siehe S2K-Leitlinie der DGVS zu Qualiätsanforderungen der gastrointestinalen Endoskopie [416]). Wie hoch die Adenomdetektionsrate, die nur für die Vorsorge-Koloskopie evaluiert ist, sein soll, wird unterschiedlich gesehen. Bei Frauen wird eine ADR von 20\%, bei Männern von 30\% gefordert! ([421]).

Die Rückzugszeit wird ebenfalls häufig als Qualitätsparameter genannt; diesbezüglich konnte in vielen, aber nicht in allen Studien demonstriert werden, dass die Polypen- 
detektionsrate mit der Rückzugszeit nach Erreichen des Coecums korreliert, wobei der Grenzwert bei 6 min lag [422-425]. Allerdings konnte in anderen Studien auch gezeigt werden, daß bei einer weiteren Erhöhung der Rückzugszeit $>6$ min die Adenomrate nicht weiter ansteigt [426, 427] und daß der genannte Cut-off von $6 \mathrm{~min}$ in größeren Datenbank-Analysen nicht mit der ADR korreliert [428].

Bei pathologischen Befunden im Rahmen der Koloskopie ist eine Zuordnung nach endoskopisch-antatomischen Strukturen und nach Diaphanoskopie ungenügend, eine Angabe in $\mathrm{cm}$ Gerätelänge $\mathrm{ab}$ ano sollte nur im Rektum und unteren Sigma erfolgen. Bei unklarem oder OP-würdigem Befund sollte eine Markierung mittels Clip (nur bei zeitnaher OP) oder Tusche (in Läsionsnähe, nicht in die Läsion) erfolgen, um eine Wiederauffindung zu ermöglichen (ggf. auch Röntgen-Durchleuchtung während der Koloskopie).

Allerdings hat die Koloskopie auch Limitationen. So werden Karzinome und (relevante) Adenome übersehen. Diese sogenannten Intervallkarzinome sind großteils auf übersehene Läsionen zurückzuführen. Weitere Faktoren sind die inkomplette Polypektomie (und fehlende Vorstellung des Patienten zur zeitnahen koloskopischen Kontrolle), sowie schnell wachsende sogenannte De novo-Tumore ([429], [430], [431]). Ältere retrospektiven Studien mit Database matching sprechen von 4-6\% übersehenen Karzinomen [432-434]. In Studien zur Polypennachsorge traten in einer kanadischen Studie in einem Zeitraum von 3 Jahren bis zu 1\% sog. "missed cancers" bzw. Intervallkarzinome nach Koloskopie auf, vor allem rechtsseitig [430]; diese Seitendifferenz wurde auch in einer deutschen Studie bestätigt, allerdings in erheblich geringerem Ausmaß [1 16]. In den beiden bereits zitierten großen Studien aus Polen und USA lag innerhalb von 52 bzw. 35-39 Monaten die Rate von Intervallkarzinomen nach Koloskopie bei $0.09 \%$ [415] bzw. 0.22\% [414], also ganz erheblich niedriger. Auch scheint in der Hochburg der Intervallkarzinome Kanada die Rate der Intervallkarzinome von 1996 bis 2010 um die Hälfte zurückzugehen (und lag hier dann am Ende bei 0.04\% pro Follow-up-Jahr [435].

Alternativen und Ergänzungen zur Koloskopie: Strahlenanwendung ist zu ScreeningZwecken, d.h. bei Gesunden, in Deutschland nur ausnahmsweise (MammakarzinomScreening) zugelassen. Unter anderem deswegen wird die CT-Kolonographie hier nicht eingesetzt. Theoretisch sind unvollständige Koloskopien und die Patientenverweigerung einer (diagnostischen) Koloskopie mögliche Indikationen zu radiologischen Verfahren. Sensitivität und Spezifität der CT-Kolonographie sind in den letzten Jahren seit Erstellung der letzten Leitlinie angestiegen. In Übersichten der letzten Jahre lag die CT-Kolonographie entweder um 10-20\% zurück (größenabhängig) für alle Neoplasien im Screening-Setting [174], erreichte eine gute (88\%) Treffsicherheit für Adenome $>5 \mathrm{~mm}$ bei Stuhltest-positiven Screening-Kandidaten bei allerdings nur 75\% Spezifität [436], und zeigte eine sehr hohe Treffsicherheit für Karzinome in einer radiologischen Übersicht bei allen Indikationen [413]. Was die Patientenpräferenz für CT versus Koloskopie betrifft, hängen die Ergebnisse offenbar vorwiegend vom Journal der Veröffentlichung (Radiologie vs. Gastroenterologie/Innere) und vom Ausmaß der Darmvorbereitung beim CT ab [437]. Insgesamt gibt es - noch weniger für die MRTKolographie [438] - aber keine Outcome-Studien im Bereich der Vorsorge, was den Einsatz hier zumindest vorerst nicht empfehlen läßt. Die Röntgendoppelkontrastuntersuchung des Dickdarms ist heute obsolet.

Diagnostische Probleme bei der CT-Kolonographie können Stuhlreste und eine schlechte Entfaltung des Darmlumens bereiten. Die Detektion flacher, eingesenkter und kleiner 
Polypen ist schwieriger als die prominenter Polypen. Aufgrund mangelnder Standardisierung sind die Ergebnisse derzeit stark zentrenabhängig.

Aus den genannten Gründen ist bei einem positiven fäkalen Occultbluttest (FOBT) oder zur Abklärung eines Tumorverdachtes die komplette Koloskopie der Goldstandard. Es besteht hierbei die Möglichkeit sowohl der Biopsieentnahme zur histologischen Diagnostik als auch der Polypektomie als gleichzeitige therapeutische Intervention.

Bei Patienten mit stenosierenden Tumoren oder inkompletter Koloskopie aus anderen Gründen konnten in Fallserien mittels CT- [409-412] oder MR-Kolonographie [439, 440] proximale Tumoren oder Polypen nachgewiesen werden, sodaß gemeinsame Leitlinien von ESGE und ESGAR von 2014 hier den Einsatz der CT-Kolographie empfehlen [441]. Allerdings gibt es inzwischen Evidenz für die Kolon-Kapselendoskopie bei inkompletter Koloskopie (natürlich nicht bei Stenosen), die in einer 2015 veröffentlichten kleineren randomisierten Studie $(n=100)$ hier doppelt soviele Adenome $\geq 6 \mathrm{~mm}$ wie die CTKolonographie fand (24.5\% vs. 12.2\%) [442]. Die Kolonkapsel wurde als primäre Methode in mehreren methodisch teilweise limitierten Studien evaluiert und zeigte hier größenabhängig eine ähnliche Detektionsrate wie die CT-Kolonographie; die Zahl der Karzinome reichte nicht für eine valide Analyse aus [443]. Als Vorsorge-Methode erreichte die Kapsel-Koloskopie ebenfalls gute Werte für Adenome $\geq 6 \mathrm{~mm}$ (Sens $81 \%$, Spez. 93\%), eines von vier Karzinomen wurde aber übersehen und es wurden $21 \%$ der initial eingeschlossenen Patienten von der finalen Analyse ausgeschlossen [444]. Aufgrund dieser Limitationen ist die Kolonkapsel derzeit nicht als primäre ScreeningMethode zu empfehlen.

Die letzte Übersicht von 2016 über Koloskopie-Komplikationen bei der VorsorgeKoloskopie aus 21 großen populationsbasierten Studien berichtet über eine gepoolte Rate an Perforationen, Nachblutungen sowie Mortalität von jeweils $0.05 \%, 0.3 \%$ und $0.003 \%$, nach Polypektomie lagen Perforationen bei $0.08 \%$ und Nachblutungen bei knapp $1 \%$ der Untersuchungen. Die Komplikationsrate war bei Screening/NachsorgeKoloskopie niedriger als bei der diagnostischen Koloskopie [445].

\subsubsection{Sigmoidoskopie versus Koloskopie}

6.4.

Empfehlungsgrad

A

Level of Evidence

2b
Evidenzbasierte Empfehlung

2017

Bei positivem FOBT/FIT-Test, bei Tumorverdacht oder sigmoidoskopischem Nachweis eines neoplastischen Polypen soll eine vollständige Koloskopie durchgeführt werden.

Quellen: [108, 129, 446-448]

Starker Konsens

\section{Hintergrund}

Zur Abklärung eines positiven FOBT-Testes bzw. eines Tumorverdachtes wird die komplette Koloskopie gefordert, da hiermit auch Adenome und Karzinome im rechten Hemikolon erkannt werden können. Relevante neoplastische Läsionen proximal des Sigmas findet man in 25-55\%. In Screening-Studien konnte gezeigt werden, dass bei 30 
bis $46 \%$ der Fälle mit proximalen fortgeschrittenen Neoplasien im rechten Hemikolon das Rektosigmoid adenomfrei ist [108, 129, 446-448].

Eine Sigmoidoskopie sollte nur im Ausnahmefall erfolgen, wenn eine vollständige Darmvorbereitung nicht möglich ist. Eine komplette Koloskopie ist in einem hohen Prozentsatz möglich und kann auch bei älteren Patienten in der Regel mit einer niedrigeren Rate an Nebenwirkungen durchgeführt werden [449-453]; bezüglich Risiken und Komorbiditäten müssen in dieser Gruppe allerdings auch auch die Belastung durch die Darmvorbereitung und die Sedierung abwägend in Betracht gezogen werden [454457]. Die Sigmoidoskopie hat für die Primärdiagnostik bei Tumorverdacht keine Berechtigung und in Deutschland wie auch in den meisten anderen europäischen Ländern [458] als Vorsorgemethode keine Bedeutung.

\subsubsection{Chromoendoskopie und verwandte Verfahren}

\begin{tabular}{|l|l|}
\hline $\begin{array}{l}\text { 6.5. } \\
\text { Empfehlungsgrad }\end{array}$ & $\begin{array}{l}\text { Evidenz Chromoendoskopie kann bei Patienten mit einer chronisch entzündlichen } \\
\text { Darmerkrankung und HNPCC zur besseren Erkennung von neoplastischen Läsionen } \\
\text { eingesetzt werden. }\end{array}$ \\
\hline $\begin{array}{l}\text { Level of Evidence } \\
\mathbf{1} \mathbf{0}\end{array}$ & \begin{tabular}{l} 
Quellen: [459-465] \\
\hline
\end{tabular} \\
\hline
\end{tabular}

\section{Hintergrund}

Neben der Chromoendoskopie mit Farbstoffen wie Methylenblau oder Indigokarmin sind in den letzten Jahren zahlreiche Bildbearbeitungsverfahren entstanden, die ein direktes Aufbringen von Farbstoff ersetzen wollen (Narrow Band imaging - NBI, Fujinon intelligent colour enhancement-FICE, iScan etc). Bei Patienten mit Colitis ulcerosa [459-463] oder HNPCC [464, 465] ist eine höhere Detektionsrate neoplastischer Läsionen durch Chromoendoskopie wahrscheinlich, allerdings ohne bewiesenen Effekt auf den GesamtOutcome [463]. Es ist bisher nicht geklärt, ob die Erkennung höherer Raten vorwiegend kleiner Veränderungen dem Patienten nutzen und den erhöhten Zeitaufwand der direkten Chromoendoskopie rechtfertigen.

Bezüglich der virtuellen Chromo-Endoskopie-Verfahren häufen sich die Metaanalysen [466-471]. NBI wird in keiner der Metaanalysen ein Effekt auf die ADR zugeschrieben, FICE wird in einer der Metaanalysen erfaßt, ebenfalls ohne Wirkung auf die ADR [467]. Eine weitere vor kurzem erschienene dreiarmige Studie mit NBI und FICE weist in dieselbe Richtung [472]. Für I-Scan zeigen zwei kleinere $(n=200$ und $n=67)$ rando-misierte Studien einen Vorteil [473, 474], der durch eine größere randomisierte Tandem-Studie $(n=389)$ nicht bestätigt werden konnte [475]. Interessanterweise wies die jüngste und umfangreichste Metaanalyse nur der konventionellen Chromo-endoskopie einen Effekt auf die ADR zu [467], und dies aufgrund von 9 solcher Studien, davon die meisten mit Verbesserung der Detektion von nur kleinen Adenomen, andere mit erheblichen methodischen Mängeln. 
Die Rolle der Chromoendoskopie-Verfahren liegt dagegen in einer besseren Abgrenzung flacher und eingesenkter Läsionen von der umgebenden gesunden Schleimhaut [476-481]. Die Chromoendoskopie kann daher vor der endoskopischen Abtragung flacher Adenome eingesetzt werden.

Insgesamt scheinen mechanische Verfahren in der Verbesserung der Adenomdetektion besser abzuschneiden als die Chromoendoskopie, allerdings wohl nicht die einfachen transparenten Abstandskappen, über die bereits 6 Metaanalysen vorliegen [467, 482485]. Neuere sog. Igelkappen (Endocuff) scheinen nach mehreren randomisierten Studien die Adenomrate zu erhöhen [486-489], was auch für auf das Endoskop aufgesetzte Ballone zuzutreffen scheint [490].

Eine Klassifikation des mukosalen Musters (Pit-Pattern) und der Mikroarchitektur der mukosalen und submukosalen Gefäße (,vessel-pattern“) sowie eine Beurteilung nach der Paris-Klassifikation [491] kann sinnvoll sein. Ziel der Zoomendoskopie ist es, anhand der „pit-pattern“ Klassifikation zwischen hyperplastischen und neoplastischen Läsionen zu unterscheiden, um ohne Histologie festzustellen, welche Läsionen endoskopisch abgetragen werden müssen. In letzter Zeit hat sich aber die Auflösung von HDEndoskopen mit oder ohne Bildnachbearbeitung in einer Weise verbessert, daß eine Vergrößerungendoskopie nicht mehr obligat erscheint [492]. So wurden auch neue Klassifikationen vorgeschlagen z.B. die sog. NICE-Klassifikation [493], die auf der Gerätetechnik einer speziellen Firma beruht.

Es gibt zahlreiche Veröffentlichungen zu der Überlegung einer endoskopischen Polypendifferentialdiagnose („endoskopische Histologie“) [492] mit dem Ziel, kleinere abgetragene Polypen nicht mehr histologisch untersuchen zu lassen („Resect and Discard“ [494, 495], kurz DISCARD policy) -vorwiegend aus Kostengründen [496, 497]. Die Nachsorgeempfehlungen richten sich dann hauptsächlich nach der endoskopischen Differentialdiagnose zwischen Adenomen und hyperplastischen Läsionen, da keine Histologie mehr vorliegt.

Da dieses Vorgehen bisher nicht ausreichend validiert ist, ist die histologische Beurteilung abgetragener Polypen in Übereinstimmung mit der S2K-Leitlinie zur endoskopischen Qualität [416] weiterhin als Standard zu betrachten.

Die konfokale Lasermikroskopie bleibt derzeit noch wissenschaftlichen

Fragestellungen vorbehalten [498].

\subsection{Polypektomiedurchführung}

\subsubsection{Endoskopische Resektionsverfahren}

6.6.

Empfehlungsgrad

A

Level of Evidence

$1 \mathrm{c}$
Evidenzbasierte Empfehlung

Polypen sollen unter Angabe der Lokalisation entfernt und geborgen werden. Die Polypektomie kann bei multiplen Polypen ggf. mehrzeitig erfolgen.

Starker Konsens 
6.7.

\section{EK}

Um eine repräsentative histologische Aussage zu erhalten und zur definitiven Therapie, sollen Polypen $>5 \mathrm{~mm}$ vollständig durch Schlingenektomie entfernt werden.

Grundsätzlich sollen diagnostische Koloskopien nur dann durchgeführt werden, wenn in gleicher Sitzung die Möglichkeit zur Schlingenektomie besteht.

Starker Konsens

\subsubsection{Endoskopische Beurteilung vor Abtragung}

Ziel einer Koloskopie muss das Erreichen eines polypenfreien Darms (clean-colon) sein. Um Doppeluntersuchungen zu vermeiden, sollte eine Koloskopie grundsätzlich nur bei vorhandener Interventionsmöglichkeit erfolgen. Ist die Abtragung eines Befundes nicht möglich oder sinnvoll (Risikosituation bei ambulanter Durchführung, fehlende Expertise bei größeren Polypen), sollte der Patient in ein Zentrum eingewiesen werden. Eine Zangen-biopsie von größeren Polypen (> $5 \mathrm{~mm}$ ) ist nicht sinnvoll, wenn eine Abtragung technisch möglich ist. Die Zangenbiopsie ist außerdem unzuverlässig mit einer Unterschätzung der histopathologischen Diagnose in 10\% der biopsierten Polypen insgesamt und $60 \%$ bei fortgeschrittenen Neoplasien [499]. Darüber hinaus können ausgiebige Biopsien durch nachfolgende Vernarbung die komplette endoskopische Abtragung beim Zweiteingriff erschweren. Bei eindeutigen Malignitätskriterien mit primärer OP-Indikation sind Biopsieentnahmen jedoch obligat.

Voraussetzung und Limitierung für die endoskopische Resektion größerer Polypen ist die realistische Option einer kompletten Abtragung mit einem niedrigen Blutungs- und Perforationsrisiko. Die Erfahrung des Untersuchers und die Lage des Polypen können ebenfalls limitierende Faktoren darstellen. $Z u$ bedenken sind die mit der Größe ansteigende Karzinomrate neoplastischer Polypen (bis zu 15\% bei Polypen $>3 \mathrm{~cm}$ ), die Wuchsform, die Lokalisation, der Allgemeinzustand des Patienten, das erhöhte Blutungsrisiko sowie das erhöhte Perforationsrisiko im proximalen Kolon [500-505]. Die Polypektomie kann bei multiplen Polypen ggf. mehrzeitig erfolgen.

Bei größeren flachen Adenomen (sog. laterally spreading tumours bzw. adenomas, LST) wird der prädiktive Wert des sog. nicht-granulären Typs für die Prädiktion der Malignität hervorgehoben, die etwa im Verhältnis 15\% zu 1-3\% zu den granulären Wuchsformen steht [506-512] - siehe auch ausführliche Diskussion in den S2K-Leitlinie der DGVS zu Qualiätsanforderungen der gastrointestinalen Endoskopie [416]. Beim sog. Non-liftingZeichen [513, 514] ist umstritten, ob es ein zuverlässiges Zeichen für Malignität ist [513, 515], es kann aber zu einer technisch erschwerten und unvollständigen Resektion auch bei low-grade Adenomen führen [511].

Bei ausschließlich eingesenkten flachen Läsionen (IIc) die endoskopisch suspekt erscheinen, v.a. wenn sie sich nach Unterspritzung nicht abheben, sollte in der Regel eine primär chirurgische Therapie erwogen werden, da es sich hierbei meistens nicht mehr um sogenannte frühinvasive T1-Karzinome handelt und die komplette endoskopische Entfernung (R0) oft nicht möglich ist. In ausgewählten Fällen kommt auch 
die endoskopische Vollwandresektion (FTRD) in Betracht (siehe unten). Im übrigen wurde wiederholt gezeigt, daß die primäre Abschätzung der endoskopischen „Abtragbarkeit“ eines Polypen von der Erfahrung des Koloskopikers abhängt und die meisten „nicht abtragbaren" Polypen in Zentren doch erfolgreich abgetragen werden können [516-521].

\section{Hyperplastische Polypen}

Kleine ( $\leq 5 \mathrm{~mm}$ ) häufig multipel auftretende typische hyperplastische Polypen im Rektum können belassen werden, wenn sie endoskopisch eindeutig als solche zu identifizieren sind. Weiter proximal sollten hyperplastisch anmutende Polypen immer abgetragen werden, da sie mit zunehmender Größe oft als serratierte Adenome klassifiziert werden.

Die serratierte Polyposis (SPS), früher als hyperplastisches Polyposis-Syndrom bezeichnet, scheint eines der häufigsten kolorektalen Polyposis-Syndrome zu sein. Bisher wurde kein Gendefekt identifiziert. Die aktuelle WHO-Definition (26) für die Diagnose einer serratierten Polyposis (serratiertes Polyposis-Syndrom / SPS) besteht aus drei Kriterien.

1. Mindestens fünf histologisch bestätigte serratierte Polypen proximal des Sigmas, davon zwei $>1 \mathrm{~cm}$ oder

2. Jede Zahl serratierter Polypen proximal des Sigmas bei Patienten mit einem/einer erstgradig Verwandten mit hyperplastischer Polypose oder

3. mehr als 20 serratierte Polypen jeglicher Größe im Kolon verteilt.

Das serratierte Polyposis-Syndrom geht mit einem erhöhten Karzinomrisiko einher und erfordert regelmäßige Kontrollkoloskopien.

\section{Endoskopische Resektionstechniken}

Die vollständige Entfernung und Bergung (insbesondere bei Abtragung in Teilstücken bei sog. fraktionierter endoskopischer Mukosaresektion - EMR) eines Polypen ist immer zu fordern, denn in einem verbliebenen Polypenrest können noch eine hochgradige intraepitheliale Neoplasie oder ein Karzinom nachweisbar sein. Um eine Zuordnung zu ermöglichen, sollen die Polypen einzeln unter Angabe der Lokalisation für eine histologische Aufarbeitung geborgen werden. Bei mehreren kleinere und nicht suspekten Polypen in einem Segment ist eine gemeinsame Bergung dieser Polypen vertretbar. Hierbei müssen aber im Falle histologischer Überraschungsbefunde die onkologischen Resektionsgrenzen beachtet werden; eine Markierung des polypektomierten Kolonsegmentes ist sinnvoll, wenn chirurgische Resektionen erforderlich werden.

Folgende endoskopische Verfahren stehen zur Verfügung:

- $\quad$ Zangen- versus Schlingenabtragung kleiner Polypen bis $5 \mathrm{~mm}$

- $\quad$ Polypektomie mit der Schlinge bei Polypen $>5 \mathrm{~mm}$

- $\quad$ Endoskopische Mukosaresektion (EMR)

Die einzelnen Verfahren sind ausführlich in der S2K-Leitlinie der DGVS zu Qualiätsanforderungen der gastrointestinalen Endoskopie [416] diskutiert. Für kleine Polypen bis $5 \mathrm{~mm}$ wurde gezeigt, daß die Abtragung mit der Zange oft Adenomgewebe zurücklässt [522] und vermutlich abhängig von der Sorgfalt und der Biopsiezahl ist. Am besten ist die Kaltschlingenabtragung untersucht; sie ist wohl bei kleinen Polypen vorzuziehen [523-525]. In zwei kleineren (etwa 60 Polypen pro Arm) randomisierten, 
histologisch nachkontrollierten Studien betrug die Rate komplett abgetragener Adenome in der Kaltschlingengruppe 93,2 bzw. $96,6 \%$ vs. $75,9 \%$ bzw. $82,6 \%$ in der Zangen-abtragungsgruppe [526, 527].

Größere Polypen werden mit der Schlinge abgetragen, bei flachen Polypen meist nach vorheriger Unterpritzung; größere (flache) Polypen erfordern i.d.R. eine stückweise Abtragung (endoskopische Mukosaresektion/EMR). Die Größe des entfernten Polypen, der histologische Adenomtyp und der Schweregrad der intraepithelialen Neoplasie bestimmen die Höhe des Risikos für Lokalrezidive und metachroner Polypen. Grundsätzlich sollte aus methodischen Gründen unterschieden werden zwischen Restadenomgewebe (bei der ersten meist kurzfristigen Kontrolle nach Resektion diagnostiziert) und Adenomrezidiven (d.h. ein Wiederauftreten nach - je nach Definition - ein oder zwei negativen endoskopisch-bioptischen Kontrollen). Bei Polypen $>2 \mathrm{~cm}$ beträgt nach neueren Studien die Rest/Rezidivadenomrate bis zu 20\% und mehr [528533]. Diese Rezidive können jedoch in der Regel endoskopisch erneut behandelt werden, sodaß insgesamt sehr hohe Erfolgsraten von >95\% zu erwarten sind.

Um die histologische Beurteilung des Resektats zu verbessern sowie die Rezidivrate zu senken, wird seit einigen Jahren die en-bloc-Resektion größerer flacher Polypen durch endoskopische Submukosadissektion (ESD) propagiert. Diesen Vorteilen stehen jedoch eine deutlich höhere Komplexität, meist deutlich längere Eingriffszeit und höhere Komplikationsraten dieses Verfahrens im Vergleich zur EMR gegenüber. Zudem bringt die Abtragung in einem Stück bei benignen Läsionen wie Colonadenomen prinzipiell weniger Vorteile als im onkologischen Setting von (Früh)karzinomen.

Die Literatur zum Thema Colon-ESD ist durch eine starke Dominanz fernöstlicher, v.a. japanischer Studien und die dortige histologische Vermischung von „Mukosakarzinomen" (=high-grade Dysplasie bzw. Tis-Tumore) und Submukosakarzinomen erschwert [534]. Vergleichende retrospektive und daher nicht randomisierte Studien der ESD mit der EMR gibt es ebenfalls nur aus Fernost [535-541]. Westliche Ergebnisse der KolonESD zeigen zumeist [542] eine deutlich niedrigere R0-Rate mit teilweise erheblichen (allerdings meist endoskopisch zu behandelnden) Komplikationen [543-545] [546]. Bei den Submukosa-Karzinomen, die in diesen westlichen Studien (ausschließlich Fälle im Rektum!) dankenswerterweise meist separat analysiert werden, erfolgte bei der überwiegenden Mehrzahl der Patienten allerdings postendoskopisch doch eine sekundäre Operation. Die Daten zeigen ein Selektions- bzw. Indikationsproblem auf: Auch in der jüngsten Publikation [546] mussten über 50\% der T1-Karzinomfälle onkologisch nachreseziert werden, weil keine Low-risk-Situation vorlag. Es ist nach wie vor nicht geklärt, welches Polypenkarzinom „ex ante“ von der ESD profitiert. Die Technik ist zwar als onkologisch beste one-piece-Behandlungsmethode von kolorektalen Frühkarzinomen anzusehen, aber aus oben genannten Gründen derzeit für wenige Spezialfälle in Zentren vorbehalten und nicht für einen breiten Einsatz im westlichen Colon geeignet. Für die Entfernung von benignen Polypen ist die ESD nicht notwendig. Die EMR bleibt die Standardmethode zur Entfernung benigner kolorektaler Polypen.

Abgetragene flache und sessile Polypen sollten zur Identifikation durch Aufspießen mit einer Stecknadel oder durch Farbstoff markiert werden. Die Fixierung auf einer Korkplatte ist bei diesen flachen Polypen für die histologische Aufarbeitung hilfreich.

Bei möglicher oder zu erwartender Operationspflichtigkeit ist eine präoperative Markierung des Polypenareals mit Clip oder Tusche obligat (Ausnahme: Coecum und distales Rektum), allerdings unbedingt etwas proximal und/oder distal und nicht in die Läsion. Eine Alternative ist die intraoperative Koloskopie zur Lokalisation des Polypen bzw. dessen Abtragungsstelle. Eine Markierung der Polypektomiestelle wie dargelegt 
kann auch bei schwieriger Lokalisation als Redetektionshilfe (erschwerte Wiederauffindbarkeit bei Überwachungsuntersuchungen) nach endoskopischer Intervention erfolgen.

Alternative Verfahren der Polypenentfernung (offene oder laparoskopische Resektion, Rendezvousverfahren, TEM, transanale Abtragung) sind im Einzelfall in Erwägung zu ziehen. Abgetragene flache und sessile Polypen sollten zur Identifikation durch Aufspießen mit einer Stecknadel oder durch Farbstoff markiert werden. Die Fixierung auf einer Korkplatte ist bei diesen flachen Polypen ebenfalls obligat.

Die endoskopische Vollwandresektion (Full-Thickness-Resection) stellt ein Reserveverfahren dar ([547]). Hierbei wird das abzutragende Areal in einen Hohlzylinder, welcher auf die Endoskopspitze aufgesetzt wird, mittels eines Greifers eingezogen und im Prinzip simultan eine Schlingenabtragung und ein Verschluss des Kolonwand-defektes durch einen großen Clip durchgeführt. Mögliche Indikationsgebiete sind: Restadenomgewebe, Adenomrezidive, submuköse nicht-neo-plastische Polypen etc.. Ein weiteres Anwendungsgebiet können Low-risk-Frühkarzinome entweder in primärer Intention oder (nach Erhalt der EMR-Histologie) in sekundärer Intention zur Gewährleistung einer R0-Resektion sein (siehe Absatz 6.4). Die Limitationen sind: Größe $\geq 3$ $\mathrm{cm}$, fehlende Mobilisierbarkeit in den Zylinder, keine adäquate endoskopische Erreichbarkeit mit dem Equipment, Fehlbedienungen der Technik. Die vorliegenden Daten reichen zu einer abschließenden Bewertung derzeit nicht aus. Auch über die Komplikationsraten ist wenig bekannt. Die Behandlungsmethode ist deshalb für klinische Einzelfälle in Zentren möglich. Eine Empfehlung zur allgemeinen breiten Anwendung kann (noch) nicht abgegeben werden.

\section{Komplikationen}

Bezüglich publizierter Komplikationsraten der Koloskopie sei auf eine Übersicht der ASGE [548] und einen spezifisch auf die Vorsorge-Koloskopie bezogen Review [549] verwiesen. Die S2K-Leitlinie Qualitätsanforderungen der gastrointestinalen Endoskopie [416] gibt einen Überblick über die wichtigsten deutschen [98, 550-557] und internationalen größeren Serien [558-567]. In einer großen deutschen prospektiven Studie waren unabhängige Faktoren bezüglich des Perforationsrisikos Polypengrößen über 1 $\mathrm{cm}$ sowie die Lokalisation im rechten Kolon, bzgl. des Blutungsrisikos lediglich die Polypengröße $>1 \mathrm{~cm}$ [557]. Das Risiko schwerer Blutungen (Transfusionspflichtigkeit, Operationsnotwendigkeit, rezidivierende Nachblutung) betrug 0,9\%; das Perforationsrisiko im rechten Kolon 1,2\%, im linken Kolon 0,4\%.

Bezüglich Prophylaxe-Maßnahmen gegenüber einer (Nach)Blutung nach Endoresektion sowie bezüglich Polypektomie unter Thrombozytenaggregationshemmung wird ebenfalls auf die S2K-Leitlinie Qualitätsanforderungen der gastrointestinalen Endoskopie [416] verwiesen. 


\subsection{Histologische Untersuchung}

\begin{tabular}{|l|l|}
\hline 6.8. & Konsensbasierte Empfehlung \\
\hline EK & 2017 \\
Die histologische Untersuchung jedes Polypen ist obligat. Die histologische \\
Befundung der Polypen soll entsprechend der WHO-Kriterien [568] mit einer Aussage \\
zur Vollständigkeit der Abtragung erfolgen. Konventionelle Adenome werden \\
klassifiziert nach histologischem Wachstumstyp (tubulär, tubulovillös und villös) und \\
dem Grad der intraepithelialen Neoplasie (niedrig- und hochgradige intraepitheliale \\
Neoplasie); serratierte Läsionen werden unterteilt in hyperplastische Polypen, sessile \\
serratierte Adenome, mixed Polypen (mit Angabe des IEN-Grades) und traditionelle \\
serratierte Adenome (mit Angabe des IEN-Grades) [569, 570].
\end{tabular}

\section{HIntergrund}

In den letzten Jahren wurde aufgrund molekulargenetischer Befunde evident, dass neben der „klassischen Adenom-Karzinom-Sequenz" weitere Pfade der Karzinomentwicklung bestehen. Zum einen der sog. „serratierte Karzinogeneseweg“, als dessen Vorläuferläsion die relativ neue Entität des sessilen serratierten Adenoms (SSA) betrachtet wird, zum anderen ein „Mischtyp“, der molekulare Charakteristika der beiden anderen Karzinogenesewege vereint und dessen Vorläuferläsionen das traditionelle serratierte Adenom (TSA) oder villöse Adenome sein können Die primäre Schlüsselmutation für die alternative serratierte Route liegt im B-raf-Gen mit Störung der Apoptose, gefolgt von Seneszenz mit Promotor (CpG)-Methylierungen und konsekutiven Genausfällen (z.B. hMLH1, MGMT, p16) und der Entwicklung einer meist hochgradigen Mikrosatelliteninstabilität (MSI-H) ([571], [572]).

Da der serratierte Weg der kolorektalen Karzinomentstehung möglicherweise schneller verläuft, sind Kenntnis und Diagnose der Vorläuferläsionen von entscheidender Bedeutung. Die neuen Entitäten sessil serratiertes Adenom (SSA) und traditionell serratiertes Adenom (TSA) sind erst seit 2010 definiert ([573]). Hyperplastische Polypen (HP) gehören formal zu den nicht-neoplastischen Läsionen, sind also „unschuldig“. Aufgrund der jetzt bekannten Daten und der relativ ähnlichen, aber nicht gleichen Morphologie von hyperplastischen Polypen und sessilen serratierten Adenomen ist aber zu vermuten, dass früher SSA als hyperplastische Polypen fehlklassifiziert wurden. Bei Läsionen, die $>0,5 \mathrm{~cm}$ sind, liegt die „Falschklassifizierungsrate“ bei etwa $30 \%$ ([571]).

\section{Sessile serratierte Adenome (SSA)}

Sessile serratierte Adenome sind typischerweise $>5 \mathrm{~mm}$, liegen im rechtsseitigen Kolon und sind flach erhaben, ragen also nicht polypös in das Darmlumen vor. Endoskopisch sind SSA relativ schwierig zu erkennen, grenzen sich durch eine aufgelagerte Schleimschicht von der Umgebung ab (sog. Schleimkappe). Aufgrund der Morphologie und der Lokalisation könnten sie eine wesentliche Ursache der sog. Intervallkarzinome darstellen. Inzwischen ist unbestritten, dass SSA eine Vorläuferläsion der „serratierten Karzinogenese" darstellen. Die differenzialdiagnostische Abgrenzung zwischen HP und SSA stützt sich auf das typische Gesamtbild der SSA mit L- und T-förmigen Verzweigungen an der Kryptenbasis, einer Serratierung bis zur Kryptenbasis, dem 
Vorliegen dilatierter, basal oft „eckiger" Krypten und gelegentlichem Vorkommen von unter die Lamina muscularis mucosae reichenden „invertierten Krypten“.

\section{Traditionelle serratierte Adenome (TSA)}

Traditionelle serratierte Adenome sind im Gegensatz zu den SSA polypoid in das Darmlumen vorragende Läsionen. Sie verbinden die sägezahnartige Architektur hyperplastischer Polypen mit der IEN der klassischen Adenome. Sie machen etwa $1 \%$ aller kolorektalen Adenome aus und sind ganz überwiegend im linksseitigen Kolon und im Rektum lokalisiert. TSA zeichnen sich molekular durch eine hohe Frequenz von K-rasMutationen aus.

\section{„Gemischter Polyp“}

Unter dem Begriff "gemischter Polyp“ wird eine heterogene Gruppe Läsionen subsummiert, die Anteile serratierter Adenome, hyperplastischer Polypen und tubulären, tubulo-villösen oder villösen Adenomen enthalten kann.

\section{Risikobewertung serratierter Läsionen}

Während die Zeit der malignen Transformation serratierter Läsionen nicht bekannt ist, scheint jedoch das Vorhandensein serratierter Läsionen ein erhöhtes Risiko für die Entstehung kolorektaler Neoplasien anzuzeigen. In Analogie zu Tumoren des Ösophagus und der Lunge sind serratierte Läsionen Ausdruck einer Feldkanzerisierung des Darms - sie zeigen an, dass der Betroffene prädisponiert ist, (prä)-neopastische Läsionen mit karzinogener Portenz an multiplen Stellen des Darms zu entwickeln.

Zahlreiche Studien konnten nachweisen, dass serratierte "Polypen" mit syn- und metachronen fortgeschrittenen kolorektalen Neoplasien assoziiert sind im Sinne von Indikatorläsionen. In einer Metaanalyse ([574]) werden neun Studien mit 34.480 Patienten zusammengefasst. Serratierte Polypen wurden in $15,6 \%$ der Patienten nachgewiesen. Bei Nachweis eines serratierten Polypen hatte der Patient ein 2,05-fach erhöhtes Risiko für eine fortgeschrittene Neoplasie. Der Nachweis proximal lokalisierter serratierter Polypen war mit einer 2,77-fachen Risikoerhöhung assoziiert. Handelte es sich um große serratierte Polypen $(>1 \mathrm{~cm})$ stieg das Risiko auf das 4-fache. Damit erscheinen serratierte Läsionen als Indikatorläsion, die ein erhöhtes Risiko für fortgeschrittene kolorektale Neoplasien anzeigen. Besonders Patienten mit großen serratierten Adenomen im proximalen Hemikolon sind gefährdet, fort-geschrittene kolorektale Neoplasien zu entwickeln.

Über den langfristigen Verlauf serratierter Läsionen existieren lediglich einige Daten, die eine abschließende Bewertung nicht erlauben. In einer populationsbasierten randomisierten Studie ([575]) wurde das Risiko der Entstehung kolorektaler Karzinome in Abhängigkeit von Adenomen im follow-up untersucht. Das Risiko für kolorektale Karzinome war 2,5-fach erhöht bei Patienten mit einem großen serratierten Polypen, 2fach erhöht für Patienten mit einem fortgeschrittenen (konventionellen) Adenom und 0,6 \% für Patienten mit einem nicht fortgeschrittenen Adenom. Die Autoren schlussfolgern, dass ein großes serratiertes Adenom als unabhängiger Risikofaktor für ein kolorektales Karzinom gelten kann, auch wenn man dieses Risiko durch Histologie, Größe und Multiplizität begleitender Adenome korrigiert.

Eine retrospektive nationale populationsbasierte Fallkontrollstudie von [576] an insgesamt 272.342 Koloskopien konnte zeigen, dass SSAs mit zytologischen Markern einer Dysplasie mit der Entwicklung eines Adenokarzinoms assoziiert sind. Frauen mit 
SSAs zeigten ein höheres Risiko für kolorektale Karzinome als Männer: ein SSA erhöhte das Risiko für ein kolorektales Karzinom bei Frauen um den Faktor fünf, bei Männern um den Faktor zwei. Patienten mit einem SSA proximal der linken Flexur wiesen das größte Risiko für die Entstehung eines kolorektalen Karzinoms auf.

Zusammenfassend kann aktuell davon ausgegangen werden, dass Patienten mit großen serratierten Adenomen ein erhöhtes Risiko der kolorektalen Karzinom-entstehung aufweisen, vergleichbar mit dem Risiko für fortgeschrittene konventionelle Adenome.

Für die Nachsorgeempfehlungen nach Polypektomie kommt der Histologie der abgetragenen Läsionen eine entscheidende Bedeutung zu. Entsprechend ist die histologische Untersuchung aller abgetragenen Läsionen obligat. Das von einzelnen Experten vorgeschlagene Konzept der Abtragung und Verwerfung von kleinen Polypen (Resect and Discard) wird deshalb abgelehnt ([577]).

6.9.

Empfehlungsgrad

A

Level of Evidence

$3 a$

\section{Evidenzbasierte Empfehlung}

Bei Karzinomnachweis soll der histologische Befund folgende Merkmale enthalten:

- das Ausmaß der Tiefeninfiltration (pT-Kategorie), bei sessilen Polypen die smInvasionsmessung in $\mu \mathrm{m}$,

- den histologischen Differenzierungsgrad (Grading),

- Vorhandensein oder Fehlen von Lymphgefäßinvasion (L-Klassifikation),

- und die Beurteilung der Resektionsränder (R-Klassifikation) im Hinblick auf die lokale Entfernung im Gesunden (zur Tiefe und zur Seite).

Quelle: [578]

Starker Konsens
6.10.

\section{Konsensbasierte Empfehlung}

2017

\section{EK}

Das Ausmaß des Tumorbuddings kann als zusätzlicher Parameter gewertet werden.

Starker Konsens 
6.11.

Empfehlungsgrad

A

Level of Evidence

$3 a$

\section{Evidenzbasierte Empfehlung}

In Hinblick auf weitere therapeutische Konsequenzen bei komplett entfernten pT1Karzinomen soll eine zusammenfassende Klassifikation in "Low-risk" (G1, G2 und keine Lymphgefäßeinbrüche (L0)) oder „High-risk“ (G3, G4, und/oder Lymphgefäßeinbrüche (L1)) erfolgen.

Konsens

6.12.

\section{Hintergrund}

Die Notwendigkeit einer Angabe über den Abstand der Entfernung im Gesunden bei pT1 Karzinomen ist strittig.

\section{Tumor-Grading und -budding}

Die Festlegung des Tumordifferenzierungsgrades erfolgt nach den Regeln der WHO (WHO 2010). Diese besagen, dass der Tumordifferenzierungsgrad den am schlechtesten differenzierten Tumoranteilen entspricht. Die Invasionsfront soll nicht berücksichtigt werden. Allerdings ist aus der täglichen Praxis bekannt, dass gerade die Invasionsfront eine Eigenschaft aufweist, die als Tumor-Budding bezeichnet wird. Es handelt sich um einzelne Tumorzellen oder auch Tumorzellaggregate an der Invasions-front, die zumeist schlecht differenziert sind und stammzellähnliche Eigenschaften aufweisen. Diese Zellen unterscheiden sich auch genetisch von denen des Tumor-zentrums. Bisher gab es allerdings keine einheitliche Definition, wie Tumor-Budding zu bewerten oder zu graduieren sei. Jetzt soll einem Vorschlag japanischer Autoren gefolgt werden: TumorBudding wird demnach definiert als histologischer Nachweis von Tumorzell-Clustern (fünf oder weniger Zellen) entdifferenzierter oder isolierter Tumorzellen an der Invasionsfront. Dabei soll die Invasionsfront mit 200-facher Vergrößerung mikroskopiert werden und die Grade 1 mit 0-4; 2 mit 5-9 und 3 mit >9 Buddings oder Tumorzell-Cluster bestimmt werden. Ein Tumor-Budding Grad 2 oder 3, d.h. $>4$ Buddings oder Cluster an der Invasionsfront waren in mehreren Studien ein zusätzlicher Parameter für ein erhöhtes Risiko von Lymphknotenmetastasen ([579], [580], [581]).

Da die Analysen zum Tumorbudding vorwiegend aus Japan stammen und die Angabe des Tumorbuddings bisher in Deutschland kein Standard ist, wurde der Empfehlungsgrad zur Berücksichtigung des Tumorbuddings in der Risikobewertung von T1Karzinomen abgeschwächt auf ein „kann“.

Eine Checkliste zur standardisierten histopathologischen Befundung kolorektaler Polypen sollte verwendet werden ([578]). 


\subsection{Vorgehen bei pT1-Karzinomen}

\begin{tabular}{|l|l|}
$\begin{array}{l}\text { 6.13. } \\
\text { Empfehlungsgrad }\end{array}$ & $\begin{array}{l}\text { Evidenzbasierte Empfehlung } \\
\text { prgibt die histologische Untersuchung eines endoskopisch R0-entfernten Polypen ein } \\
\text { sich um eine Low-risk Situation bei histologisch karzinomfreier Polypen-basis (R0) } \\
\text { handelt. In der High-risk Situation soll die radikale chirurgische Behandlung erfolgen, } \\
\text { auch wenn die Läsion komplett entfernt wurde. }\end{array}$ \\
\hline $\begin{array}{l}\text { Level of Evidence } \\
\mathbf{3} \mathbf{a}\end{array}$ & \begin{tabular}{l} 
Quellen: [582-584] \\
\hline
\end{tabular} \\
\hline
\end{tabular}

\subsection{4.}

Empfehlungsgrad

A

Level of Evidence

$3 a$

\section{Evidenzbasiertes Empfehlung}

Bei inkompletter Abtragung eines Low-risk pT1-Karzinoms soll eine komplette endoskopische oder lokale chirurgische Entfernung erfolgen. Wenn eine R0-Situation nicht erreichbar ist oder Zweifel am Vorliegen einer pT1-Situation bestehen, soll die onkologisch-chirurgische Resektion erfolgen.

Quellen: [582-584]

Starker Konsens

\section{Hintergrund}

pT1-Karzinome unterscheiden sich je nach Situation erheblich in ihrer Prognose. Drehund Angelpunkt einer Risikostratifizierung in eine Low- und eine High-risk-Gruppe stellt die Wahrscheinlichkeit einer Lymphknotenmetastasierung dar. Die Gesamtgruppe der T1-Karzinome hat eine Lymphknotenmetastasierungsrate $(\mathrm{N}+)$ von 0-20\% [582-584]. Für die Abschätzung der Metastasierungsrate gibt es eine Reihe von Prognosekriterien [585588]; hierbei wird das Lymphknotenmetastasierungsrisiko in der Low-risk-Situation bei etwa $1 \%$ oder darunter angesetzt [582, 583, 589], [585, 586, 590-594], [595].

\section{Folgende Faktoren sind für die Risikostratefizierung von pT1-Karzinomen von} Bedeutung:

- Grading: G1 und G2 gelten als low risk-Kriterien (G1 gut differenziert, G2 mäßig differenziert, G3 schlecht differenziert, G4 undifferenziert)

- Invasion in Lymph- (L-Klassifikation) oder Blutgefäße (V-Klassifikation): Abwesenheit von Invasionen (LO V0) bestimmen die Low risk Kategorie. Der Nachweis einer Veneninvasion (V-Klassifikation) sollte erwähnt werden, der Stellenwert für eine lokale Therapie ist jedoch nicht sicher belegt.

- Tumorzellbudding, d.h. die Anwesenheit von einzelnen, „versprengten“ Tumorzellen an der Invasionsfront des Karzinoms. (siehe Empfehlung_6_12) 
- Submucosainvasion, am Operations- bzw. Polypektomiepräparat ausgemessen (vor allem bei sessilen/flachen Läsionen). Hierbei hat sich bei Operationspräparaten bewährt, die Submukosaschicht in drei Drittel aufzuteilen. Am Präparat eines sessilen Polypen, das mittels endoskopischer Polypektomie gewonnen wurde, ist aber nur die Messung der Submukosainvasionsstrecke in $\mu \mathrm{m}$ sinnvoll, da die Submukosa als Gesamtschicht nicht zuverlässig zur Verfügung steht bzw. keine Muskularis propria miterfasst ist. Die sogenannten frühinvasiven Formen $(\mathrm{sm} 1=$ Submukosainvasion $\leq 1000 \mu \mathrm{m}$ ) haben mit $0-6 \%$ ein geringes $\mathrm{N}+$-Risiko [491, 582585]. Bei sm3-Karzinomen hingegen beträgt das Lymphknotenmetastasierungsrisiko etwa $20 \%$ [491, 596].

Cave: Die Messung der Submukosainfiltration am gestielten Polypen in $\mu \mathrm{m}$ ist nicht sinnvoll bzw. irreführend, weil die Submukosadicke von der Stiellänge abhängig ist. Die Einteilung nach der Haggitt-Klassifikation [597, 598], ist in der Praxis schwierig vorzunehmen. Mit Ausnahme einer fortgeschrittenen Stielinvasion (> $3000 \mu \mathrm{m}$ [599] wird ein T1-Karzinom im gestielten Polypen als sml klassifiziert.

- In multivariaten Analysen kommt der Lymphangioinvasion die größte Bedeutung als Risikofaktor für das Vorliegen von Lymphknotenmetastasen zu. Ein L+-Status ist mit einer 20\%igen N+-Rate vergesellschaftet ([600]). Bei L0 und G2 scheint die Submukosa-Invasionstiefe zwischen $1000 \mu \mathrm{m}$ und $2000 \mu \mathrm{m}$ die Lymphknotenmetastasierungsrate $(\mathrm{N}+)$ lediglich von $1 \%$ auf $2 \%$ zu erhöhen ([601], [602]).

\section{Ergänzende wichtige Anmerkungen zum Resektions- (R-)Status:}

Obligate Voraussetzung für eine Nachbeobachtung ohne onkologische Resektion bei endoskopisch/lokal abgetragenen low-risk Tl-Karzinomen ist die sichere Beurteilung der vollständigen Abtragung (R0). Gestielte Läsionen sollten möglichst in einem Stück abgetragen werden. Bei sessilen/flachen Läsionen und einer En-bloc-Entfernung ist histopathologisch die Beurteilung der seitlichen und basalen Ränder, bei einer piecemeal-Abtragung nur die der basalen Ränder zuverlässig histologisch möglich. Kontrovers wird ein Sicherheitsabstand von $1 \mathrm{~mm}$ zur Basis diskutiert [585]; hierzu gibt es in der endoskopischen Literatur keine guten Angaben. In der low-risk-Situation und gesichertem R0-Status (s.u.) ist eine nachfolgende radikal-chirurgische Resektion nach onkologischen Kriterien nicht erforderlich.

In der Regel erfolgt die endoskopische Krebstherapie im Rahmen einer Polypektomie, ohne dass vorher die Krebsdiagnose bekannt ist. Eine Entfernung in Piecemeal-Technik erscheint hier ausreichend [603]. Hierbei erfolgt die Beurteilung der R-Situation zur Seite endoskopisch-makroskopisch, die Beurteilung zur Tiefe histologisch (basal R0). Allerdings ist dann eine zeitnahe (2-6 Monate) endoskopisch-bioptische Überprüfung der lokalen RO-Situation erforderlich.

In ausgewählten Fällen kann die endoskopische Vollwandresektion (FTRD) bei Low-riskTumoren helfen, bei inkompletter Resektion eine kurative R0-Situation zu erreichen in dem die Abtragungsstelle endoskopisch nachreseziert wird (siehe auch Abschnitt 6.2.1 Endoskopische Resektionsverfahren).

In jedem Fall einer definitiven Rx-basalen oder R1-basalen oder unklarem vertikalem Resektionsrand ist bei operablen Patienten eine chirurgische Resektion, in der Regel als onkologische Resektion, erforderlich!

Randomisierte Studien zum Vergleich endoskopischer und chirurgischer Verfahren bei T1-Karzinomen liegen nicht vor. Retrospektive Studien zeigen, dass nach endoskopischer Therapie von Low-risk T1-Karzinomen in etwa 3\% mit Rezidiven in Form 
von Lokalrezidiven oder Fernmetastasen zu rechnen ist ([604]; [595]). Mit dem individuellen Patienten ist deshalb die onkologische Situation zu besprechen und OPRisiken der onkologischen Resektion gegenüber den Risiken der alleinigen endoskopischen Lokaltherapie darzustellen und abzuwägen.

Vorsicht ist geboten, wenn vor allem bei sessilen Läsionen prätherapeutisch bereits eine Karzinomdiagnose bioptisch gesichert wurde. Häufig handelt es sich dann schon nicht mehr um eine endoskopisch therapierbare Situation. Endoskopische Warn-kriterien sind: Ulzerationen, Einsenkungen, Kontaktblutungen und fehlendes Lifting-sign beim Unterspritzen der Läsion. Solche bekannten malignen Läsionen sollen endoskopisch nur in Zentren mit ausreichender Expertise in der primären Einschätzung wie auch in den endoskopischen Resektionstechniken abgetragen werden.

Bei bekanntem oder vermutetem T1-Karzinom ist eine endoskopische Entfernung als en bloc Resektion aus onkologischen Gründen optimal. Die Methode der Wahl bei der Lokalisation im Rektum ist die ESD (siehe 6.2.1 Endoskopische Resektionsverfahren).

\subsection{5.}

Empfehlungsgrad

A

Level of Evidence

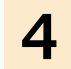

\section{Evidenzbasierte Empfehlung}

Die endoskopische lokale Nachsorge soll nach kompletter Entfernung (R0) von Low risk (pT1, low grade (G1, G2, L0)) Karzinomen nach einem halben Jahr erfolgen.

Eine komplette Koloskopie soll nach 3 Jahren erfolgen.

Starker Konsens

\section{Hintergrund}

Die oben genannten Empfehlungen dienen zur Erkennung von Lokalrezidiven. Eine komplette Koloskopie sollte zur frühzeitigen Erkennung und Sanierung von Rezidiven entsprechend den Empfehlungen der Adenomnachsorge erfolgen. 


\subsection{Polypenmanagement (Nachsorge)}

\begin{tabular}{|l|l|}
$\begin{array}{l}\text { 6.16. } \\
\text { Evidenzbasierte Empfehlung }\end{array}$ \\
\hline $\begin{array}{l}\text { Empfehlungsgrad } \\
\mathbf{B}\end{array}$ & $\begin{array}{l}\text { Nach Abtragung kleiner einzelner nicht neoplastischer Polypen sollte keine } \\
\text { endoskopische Nachsorge erfolgen. }\end{array}$ \\
\hline $\begin{array}{l}\text { Level of Evidence } \\
\mathbf{3}\end{array}$ & Quellen: [605-607] \\
\hline
\end{tabular}

\section{Hintergrund}

Bei Patienten mit kleinen $(<1 \mathrm{~cm})$ hyperplastischen Polypen und negativer Familienanamnese besteht offenbar kein erhöhtes Risiko für das Auftreten eines kolorektalen Karzinoms. Hier gelten die allgemeinen Regeln zur KRK-Prävention, das heißt eine Kontrollkoloskopie nach 10 Jahren [605-607]. Ausnahmen sind nichtneoplastische Polyposiserkrankungen (hyperplastische, juvenile, Peutz-Jeghers und SSA-Polypose) mit erhöhtem Risiko einer malignen Entartung [608].

\subsection{7.}

Empfehlungsgrad

A

Level of Evidence

2b

\section{Evidenzbasierte Empfehlung}

Der Zeitpunkt der Kontrollkoloskopie nach kompletter Abtragung neoplastischer Polypen (Adenome) soll von Anzahl, Größe und Histologie der entfernten Adenome abhängig gemacht werden.

Quelle: [609]

Starker Konsens

\subsection{8.}

Empfehlungsgrad

B

Level of Evidence

$3 b$

\section{Evidenzbasierte Empfehlung}

Bei Patienten mit 1 oder 2 Adenomen $<1 \mathrm{~cm}$ ohne höhergradige intraepitheliale Neoplasie sollte eine Kontrollkoloskopie nach 5-10 Jahren erfolgen.

Quellen: $[211,610,611]$

Starker Konsens 


\begin{tabular}{|l|l|}
$\begin{array}{l}\text { 6.19. } \\
\text { Empfehlungsgrad }\end{array}$ & $\begin{array}{l}\text { Werden bei dieser Kontrollkoloskopie wiederum keine oder nur 1 - } 2 \text { Adenome < } 10 \\
\text { mm ohne überwiegend villöse Histologie oder HGIEN gefunden, sollte die nächste } \\
\text { Kontrollkoloskopie nach 10 Jahren erfolgen. }\end{array}$ \\
\hline $\begin{array}{l}\text { Level of Evidence } \\
\mathbf{3} \mathbf{b}\end{array}$ & \begin{tabular}{l} 
Quellen: $[211,610,611]$ \\
\hline
\end{tabular} \\
\hline
\end{tabular}

6.20.

Empfehlungsgrad

B

Level of Evidence

$3 b$

\subsection{1.}

Empfehlungsgrad

B

Level of Evidence

$1 \mathrm{~b}$

6.22 .

Empfehlungsgrad

B

Level of Evidence

5

\section{Evidenzbasierte Empfehlung}

2017

Bei Patienten mit 3 - 4 Adenomen oder einem Adenom $\geq 1 \mathrm{~cm}$ oder einem Adenom mit überwiegend villöser Histologie oder HGIEN sollte die erste Kontrollkoloskopie nach 3 Jahren erfolgen.

Quellen: [612]

Konsens

\section{Evidenzbasierte Empfehlung}

Bei Patienten mit Adenom mit hochgradiger intraepithelialer Neoplasie und histologisch bestätigter vollständiger Abtragung sollte eine Kontrollkoloskopie nach 3 Jahren erfolgen.

Quellen: [612]

Starker Konsens

2017
Bei histologisch nicht bestätigter vollständiger Abtragung von Adenomen $>5 \mathrm{~mm}$ sollte auch bei makroskopisch kompletter Abtragung eine Kontrolle nach 6 Monaten erfolgen.

Expertenmeinung

Konsens 
6.23.

Empfehlungsgrad

B

Level of Evidence

5

6.24.

Empfehlungsgrad

A

Level of Evidence

$3 b$

6.25.

Empfehlungsgrad

B

Level of Evidence

3b

\section{Evidenzbasierte Empfehlung}

2017

Bei Nachweis von $\geq 5$ Adenomen jeder Größe sollte das Kontrollintervall $<3$ Jahre betragen.

Expertenmeinung

Konsens

\section{Evidenzbasierte Empfehlung}

2017

Nach Abtragung großer Adenome in Piecemeal-Technik soll eine kurzfristige Kontrolle der Abtragungsstelle nach 2-6 Monaten erfolgen.

Quelle: [613-617]

Konsens

\section{Evidenzbasierte Empfehlung}

2017

Nach kompletter Abtragung eines traditionellen serratierten Adenoms oder eines sessilen serratierten Adenoms sollte die Nachsorge analog zu klassischen Adenomen erfolgen.

Quellen: [576, 618, 619]

Konsens

\section{Hintergrund}

Nach Abtragung von Adenomen werden bei etwa 50\% der Patienten im Verlauf erneut Adenome detektiert ([609]). Kontrolluntersuchungen nach Polypektomie machen etwa $20 \%$ der Koloskopien aus und stellen somit einen wichtigen Kostenfaktor dar ([620]). Die Zielstruktur der Nachsorge nach Polypektomie sind zum einen Karzinome zum anderen fortgeschrittene Adenome. Bei Kontrollkoloskopien innerhalb von 3 Jahren nach Abtragung von Adenomen werden bei 0,7 bis $0,9 \%$ Karzinome sogenannte IntervallKarzinome entdeckt [430]. 
Tabelle 9: Nachsorgeintervalle nach Polypektomie

\begin{tabular}{|c|c|}
\hline Ausgangssituation & Intervall Kontrollkoloskopie \\
\hline $\begin{array}{l}1 \text { oder } 2 \text { kleine tubuläre Adenome }(<1 \mathrm{~cm}) \text { ohne } \\
\text { villöse Komponente oder hochgradige } \\
\text { intraepitheliale Neoplasie }\end{array}$ & 5 - 10 Jahre \\
\hline $\begin{array}{l}3 \text { oder } 4 \text { Adenome oder } \geq 1 \text { Adenom } \geq 1 \mathrm{~cm} \text { oder } \\
\text { villöse Komponente oder hochgradige } \\
\text { intraepitheliale Neoplasie }\end{array}$ & 3 Jahre \\
\hline$\geq 5$ Adenome & $<3$ Jahre \\
\hline Serratierte Adenome & wie bei klassischen Adenomen \\
\hline Abtragung in piece-meal Technik & $\begin{array}{l}\text { Kontrolle der Abtragungsstelle nach } 2 \text { - } 6 \\
\text { Monaten }\end{array}$ \\
\hline
\end{tabular}

Diese setzen sich zusammen aus übersehenen Läsionen (miss rate), inkompletten Polypektomien sowie dem Auftreten schnell wachsender Tumore [429, 431, 433, 621]. Die Festlegung der Untersuchungsintervalle nach Polypektomie hängen vom individuellen Risiko des Patienten ab. Das Risiko hängt entscheidend von der Anzahl, der Größe und Histologie der entfernten Adenome ab [609]. Patienten mit 1 oder 2 kleinen tubulären Adenomen haben kein wesentlich erhöhtes Karzinomrisiko [211], [610], [611]. Eine Kontrollkoloskopie nach 5 bis 10 Jahren erscheint daher ausreichend zu sein. Werden bei dieser Kontrollkoloskopie erneut nur 1 oder 2 kleine tubuläre Adenome gefunden besteht kein wesentlich erhöhtes Risiko, so dass die nächste Kontrollkoloskopie dann erst nach 10 Jahren durchgeführt werden sollte. Nach Abtragung von 3 oder 4 Adenomen oder mindestens 1 Adenom $\geq 10 \mathrm{~mm}$ oder mit überwiegend villöser Histologie (nicht tubulovillöse Adenome!) oder hochgradiger intraepithelialer Neoplasie sollte die Kontrollkoloskopie aufgrund des erhöhten Risikos einer fortgeschrittenen Neoplasie nach 3 Jahren erfolgen. Grundlage für diese Empfehlung sind die Daten der National Polyp Study, in der eine Untersuchung nach 3 Jahren eine ähnliche Rate an fortgeschrittenen Neoplasien ergab wie nach 1 Jahr [612]. Patienten mit 5 oder mehr Adenomen haben ein deutlich erhöhtes Risiko für eine fortgeschrittene Neoplasie von 24,9\% in einer gepoolten Analyse [609] vor allem wenn 1 der Adenome $\geq 10 \mathrm{~mm}$ ist [622]. Daher erscheint in diesen Fällen ein kürzeres Untersuchungsintervall angezeigt.

Das Nachsorgeintervall der 2. Kontrollkoloskopie hängt von den Befunden der Indexkoloskopie und der 1. Kontrollkoloskopie ab. Hierzu liegen keine Daten randomisierter Studien, sondern nur retrospektive Analysen vor. Ein möglicher Algorithmus ist in der vorstehenden Tabelle aufgeführt. Zu bedenken ist, dass Patienten mit Nachweis von fortgeschrittenen Adenomen in der Indexkoloskopie auch bei Nachweis von 1 oder 2 tubulären Adenomen in der ersten Kontrollkoloskopie ein erhöhtes Risiko für fortgeschrittene Neoplasien beibehalten ([623]) ([624]), ([625]), ([626]), so dass in diesem Fall eine erneute Kontrolle nach 3 Jahren sinnvoll erscheint. Es liegen weiterhin keine Studien vor, die randomisiert verschiedene Nachsorgeintervalle nach Abtragung serratierter Adenome untersuchen. In der Literatursuche wurden lediglich FallKontrollstudien und Fallserien identifiziert. In diesen wurde eine ähnliche Rate an fortgeschrittenen Adenomen in der Kontrollkoloskopie gefunden wie nach Abtragung 
von Adenomen ([576]), ([618]), ([619]). Es erscheint daher sinnvoll, dass nach Abtragung von serratierten Adenomen die gleichen Nachsorgeempfehlungen gelten sollten wie für nicht-serratierte Adenome (Für weitere Einzelheiten siehe Abschnitt 6.3).

Generell gilt, dass eine inkomplette endoskopische Abtragung von Adenomen mit einem erhöhten Intervallkarzinomrisiko einhergeht ([627]). Eine pathologisch bestätigte komplette Abtragung ist daher anzustreben. Entsprechend erscheint es sinnvoll nach Abtragung von Adenomen $>5 \mathrm{~mm}$ bei denen die Vollständigkeit der Abtragung histologisch nicht bestätigt werden kann, eine Kontrolle der Abtragungsstelle durchzuführen. Auch wenn keine vergleichenden Daten vorliegen wird analog zur Abtragung nach Piecemeal-Technik empfohlen, dass die Kontrolle nach etwa 6 Monaten erfolgen sollte. Bei kleineren Adenomen kann bei Zangenabtragung die Beurteilung der Vollständigkeit der Abtragung für den Pathologen schwierig bis unmöglich sein. Auch ist die klinische Relevanz kleiner Adenome unklar. Daher ist in diesen Fällen die endoskopische Beurteilung der Vollständigkeit der Abtragung entscheidend und eine erneute Kontrolle der Abtragungsstelle entbehrlich.

Nach Abtragung flacher oder sessiler Adenome in Piecemeal-Technik ist insbesondere bei größeren Adenomen die Rezidivrate deutlich erhöht (9-28\%) [613-617]. Der Einsatz von Argon Plasma Koagulation zur Entfernung von Restgewebe zur Sicherstellung einer kompletten Abtragung kann hilfreich sein [614, 617]. Allerdings fehlt dann die histologische Beurteilung. Die Sondergruppe von Patienten mit Z.n. Abtragung flacher oder sessiler Adenome in Piecemeal-Technik sollte wegen der erhöhten Lokal-rezidivrate eine Kontroll-Endoskopie der Abtragungsstelle nach 2-6 Monaten erhalten ([628]).

Bezüglich der Empfehlungen zur Nachsorge bei HNPCC, FAP und CED-Patienten siehe Abschnitt 10.6.

\subsection{Medikamentöse Sekundärprävention bei Adenomen}

\begin{tabular}{|l|l|l|}
\hline $\mathbf{6 . 2 6 .}$ & Evidenzbasierte Empfehlung & \multicolumn{2}{|c|}{2008} \\
\hline $\mathbf{B}$ & $\begin{array}{l}\text { Eine medikamentöse Sekundärprophylaxe nach Polypektomie sollte außerhalb von } \\
\text { Studien nicht durchgeführt werden. }\end{array}$ \\
\hline $\mathbf{1} \mathbf{b}$ & Quellen: [80-82, 629-631] \\
\hline & Starker Konsens \\
\hline
\end{tabular}

\section{Hintergrund}

Obwohl in mehreren prospektiv randomisierten Untersuchungen mit hoher Evidenzstärke ( $1 \mathrm{~b})$ ein geringer präventiver Effekt nach Einnahme von niedrigdosiertem ASS gefunden wurde [629, 630], kann aufgrund des geringen Effektes (Senkung der Rezidivadenomrate um max. 35\%) und der medikamentös bedingten Risiken [632] derzeit eine Einnahme zur Senkung des Rezidivrisikos von Adenomen nicht empfohlen werden. Gleiches gilt für die COX-2 Hemmer, für die eine Senkung der Adenomrezidivrate um 24 bis $45 \%$ erreicht wurde [80-82], die jedoch mit einer signifikant erhöhten Rate kardiovaskulärer Nebenwirkungen einhergingen [633, 634], die den potentiellen Nutzen aufwiegen [635]. Auch die Senkung der Adenomrezidivrate um 12\% durch Kalzium erscheint zu gering, um die längerfristige Einnahme für diese Indikation rechtfertigen zu können [631]. 
7. Präoperative Diagnostik und Chirurgie

7.1. Einleitung

Im Folgenden werden allgemeine Gesichtspunkte der Diagnose und Therapie, soweit sie Kolon- und Rektumkarzinome gemeinsam betreffen, für beide Entitäten zusammenfassend aufgezeigt; spezielle diagnostische und therapeutische Aspekte werden gesondert aufgeführt.

Die Therapie kolorektaler Karzinome sollte grundsätzlich auf der Basis einer histologischen Untersuchung geplant werden. Als Karzinome gelten Veränderungen, bei denen atypische epitheliale Formationen in der Submukosa infiltrieren (pTl oder mehr). Nicht einbezogen sind sog. Mukosakarzinome oder sog. intraepitheliale Karzinome (pTis), bei denen keine Metastasierung erfolgt und die durch lokale Abtragungen im Gesunden ausreichend behandelt werden.

\subsection{Definition von Kolon- und Rektumkarzinomen}

Die Grenze zwischen Kolon und Rektum wird unterschiedlich definiert. Die intraoperative Beurteilung anhand des Endes der Taeniae oder der peritonealen Umschlagsfalte ist individuell unterschiedlich und von Alter, Geschlecht und anderen Faktoren abhängig. Die präoperative Messung der Höhenangabe des Tumors mit dem flexiblen Endoskop ist unzuverlässig. Zuverlässiger sind die Höhenangaben mit dem starren Rektoskop. Die Anokutanlinie dient als distaler Messpunkt.

Nach dem internationalen Dokumentationssystem [636, 637] gelten als Rektumkarzinome Tumoren, deren aboraler Rand bei der Messung mit dem starren Rektoskop $16 \mathrm{~cm}$ oder weniger von der Anokutanlinie entfernt ist. Nach der UICC 2003 werden die Rektumkarzinome entsprechend ihrem Abstand von der Anokutanlinie in Karzinome des oberen Rektumdrittels $(12-16 \mathrm{~cm})$, des mittleren Rektumdrittels $(6-<12 \mathrm{~cm})$ und des unteren Rektumdrittels $(<6 \mathrm{~cm})$ unterteilt [638].

Dem gegenüber gelten in den USA $[639,640]$ als Kolonkarzinome Tumoren, die mehr als $12 \mathrm{~cm}$ und als Rektumkarzinome Tumoren, die $12 \mathrm{~cm}$ und weniger von der Linea anocutanea entfernt sind. Begründet wird dies mit der deutlich höheren Lokal-rezidivrate bei Tumoren unterhalb von $12 \mathrm{~cm}[641]$. 


\subsection{Definition Interdisziplinäre Tumorkonferenz}

\begin{tabular}{|l|l|}
\hline 7.1. & Konsensbasierte Empfehlung \\
\hline EK & $\begin{array}{l}\text { Alle Patienten mit KRK sollen nach Abschluss der Primärtherapie (z.B. Operation, } \\
\text { Chemotherapie) in einer interdisziplinären Tumorkonferenz vorgestellt werden. } \\
\text { Bereits prätherapeutisch sollen Patienten in folgenden Konstellationen vorgestellt } \\
\text { werden: } \\
\text { - jedes Rektumkarzinom } \\
\text { - jedes Kolonkarzinom im Stadium IV } \\
\text { - metachrone Fernmetastasen } \\
\text { - Lokalrezidive } \\
\text { - vor jeder lokal ablativen Maßnahme, z.B. RFA/LITT/SIRT } \\
\text { Konsens }\end{array}$ \\
\hline
\end{tabular}

\section{Hintergrund}

Um der Komplexität der Therapie des kolorektalen Karzinoms gerecht zu werden, ist eine Besprechung der Patienten in einer interdisziplinären Tumorkonferenz erforderlich. Mitglieder der Konferenz auf Facharztebene sollten u.a. sein: ein Gastroenterologe, ein Hämato-/Onkologe, ein Viszeralchirurg, ein Strahlentherapeut, ein Radiologe und ein Pathologe. Zur Beurteilung der primären oder sekundären Resektabilität von Lebermetastasen sollte ein erfahrener Leberchirurg hinzugezogen werden; ist dieser vor Ort nicht verfügbar, sollte eine externe konsiliarische Zweitmeinung eingeholt werden.

In bestimmten Fällen ist eine Vorstellung in der Tumorkonferenz vor Einleitung einer Therapie erforderlich. So ist z.B. bei Patienten mit Rektumkarzinom zu klären, ob eine neoadjuvante Therapie erfolgen sollte. Durch eine Vorstellung in der Tumorkonferenz und interdisziplinärer Festlegung des Therapiekonzepts konnte in einer Studie die Rate an befallenem circumferentiellen Rand im OP-Präparat deutlich gesenkt werden [642]. Liegen Fernmetastasen vor ist zu klären, ob ein rein palliatives Konzept einzuschlagen ist, oder ob durch eine primäre oder sekundäre Resektion von Metastasen (v.a. Lebermetastasen) eine Heilung möglich ist. Mit einer gehäuften Vorstellung von Patienten im Stadium UICC IV in der Tumorkonferenz nahm die Rate an Metastasenchirurgie zu [643].

Auch Patienten mit metachronen Fernmetastasen oder Lokalrezidiven im Verlauf sind zur Festlegung des weiteren Konzepts primär vorzustellen. Patienten bei denen ein lokalablatives Verfahren geplant ist, sollen ebenfalls vorgestellt werden, um alternative Therapiemöglichkeiten zu besprechen.

Im Falle von Kolonkarzinomen ohne Fernmetastasen erfolgt in der Regel primär eine onkologische Resektion des Karzinoms. Hier ist eine präoperative Vorstellung nicht erforderlich.

In allen Fällen ist eine Vorstellung der Patienten nach Abschluss der Therapie erforderlich, z.B. nach Operation eines kolorektalen Karzinoms u.a. um dann die Indikation zur Durchführung einer adjuvanten Therapie zu besprechen. Hierdurch konnte in einer Studie aus Großbritannien die Rate an adjuvanter Chemotherapie und das Überleben der Patienten signifkant gesteigert werden [644]. 
Aber auch Patienten mit Fernmetastasen, bei denen primär eine Chemotherapie eingeleitet wurde, sollen im Verlauf erneut in der Tumorkonferenz (unter Hinzuziehung erfahrener Leber- bzw. Lungenchirurgen) vorgestellt werden, um eine mögliche sekundäre Resektabilität der Fernmetastasen zu klären. 


\subsection{Präoperative Ausbreitungsdiagnostik}

Abbildung 3: Staging beim kolorektalen Karzinom

\begin{tabular}{l|l|l|}
\hline Untersuchung & KolonCa & RektumCa \\
\hline Komplette Koloskopie & $\mathrm{X}$ & $\mathrm{X}$ \\
\hline CEA & $\mathrm{X}$ & $\mathrm{X}$ \\
\hline Abdomensonographie & $\mathrm{X}$ & $\mathrm{X}$ \\
\hline Rö-Thorax & $\mathrm{X}$ & $\mathrm{X}$ \\
\hline Starre Rektoskopie & $\mathrm{X}$ \\
\hline MR (CT)-Becken mit Angabe Abstand des Tumors zur mesorektalen Faszie & $\mathrm{X}$ \\
\hline Rektale Endosonographie bei lokal begrenztem Tumor & $\mathrm{X}$
\end{tabular}

\subsubsection{Endoskopische Diagnostik}

\begin{tabular}{|c|c|c|c|}
\hline 7.2. & Evidenzbasierte Empfehlung & \multicolumn{2}{|c|}{2008} \\
\hline Empfehlungsgrad & $\begin{array}{l}\text { Folgende Untersuchungen sollten obligater Bestandteil } \\
\text { der präoperativen Ausbreitungsdiagnostik beim kolo- } \\
\text { rektalen Karzinom sein: }\end{array}$ & $\begin{array}{l}\text { Level of } \\
\text { Evidence }\end{array}$ & Evidenzgrundlage \\
\hline B & - digital-rektale Untersuchung & 5 & [645-647] \\
\hline A & - komplette Koloskopie mit Biopsie & 4 & [645-647] \\
\hline A & $\begin{array}{l}\text { - im Falle einer nicht passierbaren Stenose } \\
\text { Koloskopie 3-6 Monate postoperativ }\end{array}$ & $3 \mathbf{b}$ & {$[645-647]$} \\
\hline
\end{tabular}

\section{Hintergrund}

Die digital-rektale Untersuchung erlaubt eine orientierende Beurteilung der Sphinkterfunktion sowie der Tiefeninfiltration bei tiefsitzenden Rektumkarzinomen und lässt damit eine gewisse Abschätzung des Sphinktererhaltes zu.

Vor der Therapie eines Patienten mit einem kolorektalen Karzinom muss eine Koloskopie mit Biopsie vorliegen. Da in bis zu 5\% der kolorektalen Karzinome synchrone Tumoren zu erwarten sind, die der intraoperativen Beurteilung entgehen könnten, ist eine Koloskopie des gesamten Kolons vorzunehmen [645-647]. Ist aus technischen Gründen eine komplette Koloskopie nicht möglich, kann ein alternatives radiologisches Verfahren eingesetzt werden. (siehe Kapitel 6.1). 
Stellenwert der Virtuellen Koloskopie (bei stenosierenden Tumoren und inkompletter Koloskopie)

\begin{tabular}{|l|l|l|}
\hline $\begin{array}{l}\text { 7.3. } \\
\text { Empfehlungsgrad }\end{array}$ & $\begin{array}{l}\text { Evidenzbasierte Empfehlung } \\
\mathbf{0}\end{array}$ & $\begin{array}{l}\text { Busätzlich eine CT- oder MR-Kolonografie erfolgen. Postoperativ soll eine komplette } \\
\text { Koloskopie erfolgen. }\end{array}$ \\
\hline $\begin{array}{l}\text { Level of Evidence } \\
\mathbf{4}\end{array}$ & \begin{tabular}{l} 
Quellen: [41 1] \\
\hline Starker Konsens
\end{tabular} \\
\hline $\mathbf{7 . 4 .}$ & \begin{tabular}{l} 
Evidenzbasierte Empfehlung \\
\hline Empfehlungsgrad
\end{tabular} & $\begin{array}{l}\text { Bei inkompletter Koloskopie infolge anderer Ursachen (z. B. Adhäsionen) sollte eine } \\
\text { CT- oder MR-Kolonografie erfolgen. }\end{array}$ \\
\hline Level of Evidence & Quellen: [411] \\
\hline $\mathbf{4}$ & \\
\hline
\end{tabular}

\section{Hintergrund}

Die virtuelle Kolonografie stellt hierfür ein vielversprechendes Verfahren [411]. Ist eine komplette Koloskopie aufgrund eines stenosierenden Prozesses nicht möglich, sollte eine Koloskopie ca. 3 - 6 Monate nach Resektion erfolgen. Ein präoperativer Kolonkontrasteinlauf ist von der Wertigkeit her eingeschränkt und bei Stenosen mit der Gefahr einer lleusinduktion verbunden und wird daher nicht empfohlen. 


\subsubsection{Bildgebende Verfahren}

\subsubsection{Bedeutung einzelner bildgebender Verfahren (außer PET) zur Abklärung von Fernmetastasen bei der Primärbehandlung des Kolorektalen Karzinoms}

7.5 . Konsensbasierte Empfehlung

2013

\section{EK}

Als Basisuntersuchungen des präoperativen Stagings des Kolorektalen Karzinoms sollen die Ultraschalluntersuchung des Abdomens und die konventionelle Röntgenaufnahme des Thorax in 2 Ebenen erfolgen.

Im Falle eines unklaren Befundes oder des Verdachtes auf Fernmetastasen oder Infiltration von Nachbarorganen oder umgebende Strukturen soll ein Mehrzeilen-CT des Abdomens und Beckens bzw. im Falle des Verdachtes auf Lungenmetastasen ein CT des Thorax durchgeführt werden.

Konsens

\section{Hintergrund}

Ziel einer prätherapeutischen Bildgebung vor Therapieeinleitung ist zum einen, das Vorliegen von Fernmetastasen zu klären. So liegen zum Zeitpunkt der Erstdiagnose eines Kolonkarzinoms bei 25\% der Patienten Fernmetastasen vor: in 13\% auf ein Organ beschränkt (Mla), in $12 \%$ in mehr als einem Organ oder im Peritoneum (M1b). Lebermetastasen finden sich in 19\%, Lungenmetastasen in 3\%, Peritonealmetastasen in 9\%. Weitere Fernmetastasen in nichtregionären Lymphknoten (2\%), der Haut (2\%), des Ovars (1\%), der Knochen (<1\%) oder anderer Lokalisationen (2\%) sind selten.

Für das Rektumkarzinom beträgt die Häufigkeit von Fernmetastasen bei Erstdiagnose 18\%: in 12\% auf ein Organ beschränkt (Mla), in 6\% in mehr als einem Organ oder im Peritoneum (M1b). Fernmetastasen in der Leber finden sich bei 15\%, Lungenmetastasen in $4 \%$. Weitere Fernmetastasen finden sich bei $3 \% \mathrm{im}$ Peritoneum, bei $2 \%$ in nichtregionären Lymphknoten. Fernmetastasen in der Haut, Knochen, Gehirn, Ovar oder anderen Lokalisationen finden sich jeweils in weniger als 1\%. [Daten aus Klinischem Krebsregister der Chirurgischen Universitätsklinik Erlangen-Nürnberg].

Zur Klärung von Lebermetastasen soll primär die Abdomen-Sonographie eingesetzt werden (Sensitivität 63-86\%, Spezifität 98\% [648-650]. Bei verdächtigen Befunden oder unzureichender Beurteilbarkeit der Leber in der Sonographie oder klinischen Verdacht auf das Vorliegen von Lebermetastasen ist ein Mehrzeilen-CT des Abdomens durchzuführen (Sensitivität 75-83\%, Spezifität 95-98\% [648, 651]. Das Mehrzeilen-CT ist auch am besten geeignet für die Zuordnung der Metastasen zu den Lebervenen, den Hilusstrukturen sowie der Vena cava, die zur Beurteilung der Resektabilitiät von Lebermetastasen erforderlich ist (siehe Kapitel 7.7.5). Das Ausmaß der Lebermetastasierung wird am besten durch die Magnetresonanztomographie erfasst (Sensivitität und Spezifität: MRT 80-88\% und 93-97\%, CT 74-84\% und 95-96\%). [648, 651].

Die kontrastmittelverstärkte Sonographie der Leber ist CT und MRT annähernd gleichwertig (Sensitivität 83-86\%, Spezifität 94-98\%), setzt jedoch adäquate Qualitätsstandards (technische Ausrüstung und Erfahrung des Untersuchers) voraus [649, 650, 652]. 
Da das Mehrschicht-CT gleichzeitig eine Aussage zur lokalen Tumorausdehnung erlaubt (siehe unten) besteht die Tendenz, statt bzw. in Ergänzung zu einem Sono-Abdomen primär ein Abdomen-CT durchzuführen. In Studien ergab sich bei Patienten mit Kolonkarzinomen allerdings durch einen routinemäßigen Einsatz eines präoperativen Abdomen-CTs lediglich in wenigen Fällen eine Änderung des weiteren Vorgehens [653, 654]. 


\subsubsection{Stellenwert des präoperativen lokalen Stagings durch CT, (MRT) beim Kolonkarzinom bzgl. lokaler Ausbreitung}

\begin{tabular}{|l|l|}
\hline 7.6. & Konsensbasierte Statement \\
\hline EK & $\begin{array}{l}\text { Mit einem präoperativen Staging-CT kann unterschieden werden zwischen Tumoren, } \\
\text { die auf die Darmwand beschränkt sind und denen, die sie überschreiten. Die } \\
\text { Identifikation des Nodalstatus gelingt jedoch signifikant schlechter. Die besten } \\
\text { Ergebnisse bietet das Mehrzeilen-CT (MSCT). }\end{array}$ \\
\hline Konsens
\end{tabular}

\section{Hintergrund}

Eine neoadjuvante Therapie beim nicht ausgedehnt metastasierten Kolonkarzinom wird derzeit nur in Ausnahmefällen (z. B. distales Sigmakarzinom mit sehr ausgedehnter regionärer lymphogener Metastasierung oder Tiefeninfiltration bis an die absehbaren Resektionsränder heranreichend) in Erwägung gezogen. Allerdings wird zunehmend diskutiert durch eine entsprechende präoperative Bildgebung den Therapiealgorithmus von Patienten zu optimieren, z.B Selektion für eine laparoskopische Resektion oder Zuweisung von Patienten mit absehbarer multiviszeraler Resektion an erfahrene Zentren.

Die Aussagekraft der präoperativen Ultraschalluntersuchung des Abdomens ist diesbezüglich unzureichend. Erhebungen zur Genauigkeit des Kernspintomogrammes zum lokalen Staging des Kolonkarzinomes liegen nicht vor. Das moderne MehrschichtCT (MSCT) erreicht eine hohe Sensitivität (86\%) und Spezifität (78\%) bzgl. der lokalen Tumorausdehnung, aber eine deutlich geringere Sensitivität (70\%) bei gleicher Spezifität (78\%) bzgl. der Detektion lokaler Lymphknotenmetastasen [655].

\subsubsection{Stellenwert der PET-CT}

\subsubsection{1. bei der Primärdiagnostik des kolorektalen Karzinoms}

\begin{tabular}{|l|l|}
\hline 7.7. & Evidenzbasiertes Statement \\
\hline $\begin{array}{l}\text { Level of Evidence } \\
\mathbf{2 6}\end{array}$ & $\begin{array}{l}\text { Die PET/PET-CT hat keinen Stellenwert in der Ausbreitungsdiagnostik bei Erstdiagnose } \\
\text { eines kolorektalen Karzinoms. }\end{array}$ \\
\hline & De Novo: [656-671] \\
\hline & Starker Konsens \\
\hline
\end{tabular}

\section{Hintergrund}

In der Literatursuche wurden 15 prospektive und 5 retrospektive Kohortenstudien und Fallserien identifiziert. In 13 Studien waren Patienten mit Kolon- und Rektum-karzinomen eingeschlossen, in 7 Studien nur Rektumkarzinompatienten. In 14 Studien wurden alle Patienten eingeschlossen, in 2 retrospektiven Studien nur Pat. mit lokal fortgeschrittenen Rektumkarzinomen, in einer Studie nur Patienten mit CEA-Erhöhung oder unklarer CTBildgebung. In den eingeschlossenen Studien wurde in 10 Fällen eine PET, in 6 Fällen eine PET-CT, in 4 Fällen eine PET-CT mit CT-Kolonographie durchgeführt. Verglichen wurde die PET mit CT +/- anderen Modalitäten in 14 Studien, mit einem MRT in 3 Studien; 
in 4 Studien wurde kein Vergleich vorgenommen. Referenz war in 18 Fällen die Histologie und der klinische Verlauf, in zwei Fällen das klinische Staging anhand der vorgenommenen Untersuchungen.

Die Sensititität der PET für Fernmetastasen war hoch (75-100\%) und in einigen Fällen den Vergleichsmethoden überlegen [656, 661], in anderen Fällen nicht überlegen [657-659, 664]. Unklar ist die Qualität der Vergleichsmethoden. In neueren Studien mit Multidetector-Spiral-CT erscheint kein wesentlicher Unterschied nachweisbar [657, 658].

Die Sensitivität für Lymphknotenmetastasen war vorwiegend niedrig (29-85\%), bei 2 retrospektiven Rektumkarzinomstudien 44 und $85 \%$ und nicht besser als Vergleichsstudien (CT bzw. MRT) [656-664].

In den Studien führte der Einsatz der PET bzw. PET-CT in 2-27\% zu einer Änderung des therapeutischen Vorgehens [657, 658, 661, 664-671].

Insgesamt zeigen die Daten keinen eindeutigen zusätzlichen Nutzen für die PET in der primären Diagnostik des kolorektalen Karzinoms.

\subsubsection{2. vor Resektion kolorektaler Lebermetastasen}

\section{8 .}

Empfehlungsgrad

0

Level of Evidence

2b

\section{Evidenzbasierte Empfehlung}

Eine PET-CT kann bei Patienten mit resektablen Lebermetastasen eines kolorektalen Karzinoms mit dem Ziel der Vermeidung einer unnötigen Laparotomie durchgeführt werden.

De Novo: $[672,673]$

Konsens

\section{9.}

Empfehlungsgrad

A

Level of Evidence

2b

\section{Evidenzbasierte Empfehlung}

2013

Eine PET-CT soll nicht innerhalb von 4 Wochen nach Gabe einer systemischen Chemotherapie oder Antikörpertherapie durchgeführt werden, da die Sensitivität deutlich reduziert ist.

De Novo: [674-676]

Starker Konsens

\section{Hintergrund}

Zur Frage des Nutzens einer PET/PET-CT bei der Rezidivdiagnostik und dem Rezidivstaging bei Patienten mit kolorektalem Karzinom war im August 2011 vom IQWIG (Institut für Qualität und Wirtschaftlichkeit im Gesundheitswesen) ein Vorbericht vorgelegt worden [677]. Neben der Bewertung des Patienten-relevanten Nutzens sollte eine systematische Bewertung der prognostischen und diagnostischen Güte der PET/PET-CT durchgeführt werden. Die Literaturrecherche umfasste den Zeitraum bis 
August 2009. Aktuellere Publikationen wurden im Text erwähnt. Den hier getroffenen Leitlinienempfehlungen liegen die Evidenzbewertungen des IQWIG Berichts zugrunde. Der Empfehlungsgrad wurde unter zusätzlicher Einbeziehung der klinischen Bewertung des Verfahrens erarbeitet. Ferner wurden in einer neueren Literaturrecherche von August 2009 - Dezember 2011 ein RCT in Abstraktform [673], zwei systematische Reviews [678];[651], eine prospektive Fallkontrollstudie [674] und eine retrospektive Fallserie [676] identifiziert, die in die Bewertung ebenfalls mit eingegangen sind (Einzelheiten siehe Evidenzbericht). Die bisherige Empfehlung mit einem Grad B für die PET/PET-CT Untersuchung vor einer Resektion von kolorektalen Lebermetastasen bei einem FONG Score $>2$ der letzten Leitlinienaktualisierung 2008 wurde geändert, da die Studie, die zu dieser Empfehlung führte, bis heute nicht als Vollmanuskript publiziert ist [679].

Zur Frage des Patienten-relevanten Nutzens der PET/PET-CT ist bislang eine RCT als Vollpublikation und 1 RCT in Abstraktform publiziert. Bei Patienten vor Resektion von Lebermetastasen eines KRK hat eine ergänzende PET/PET-CT keinen Einfluss auf das krankheitsfreie oder Gesamtüberleben des Patienten. Ob die ergänzende PET-CT Untersuchung überflüssige Laparotomien als klinisch relevanten Endpunkt vermeiden kann, ist nicht vollständig geklärt. Die hier konsentierte Empfehlung stützt sich vor allem auf die voll publizierte Studie von Ruers [672], die jedoch methodische Schwächen aufweist. In dieser Studie wurden 150 Patienten mit kolorektalen Lebermetastasen, die zur Resektion vorgesehen waren, in zwei Arme randomisiert, CT oder CT plus 18F-FDG PET. Das primäre Studienziel wurde in der Publikation angegeben als die Rate an überflüssigen Laparotomien, die durch die PET Untersuchung eingespart werden kann. Dieser Endpunkt ist für den Patienten relevant. Die Studie fand keinen signifikanten Unterschied im Überleben im PET-Arm, jedoch eine signifikante Verringerung der Zahl „überflüssiger Laparotomien“ im PET-Arm. So war im Kontrollarm die Rate an überflüssigen Laparotomien 45\%, im PET-Arm 28\%. Dies entsprach einer Risikoreduktion von $38 \%$ mit sehr großem Konfidenzintervall $(95 \% \mathrm{Cl}, 4-60 \%, \mathrm{p}=0.042)$. Die Autoren schlussfolgern, dass man mit einer zusätzlichen PET vor Lebermetastasenresektion einem von sechs Patienten die Laparotomie ersparen könnte. Die sekundären Endpunkte DFS und OS waren wie folgt: DFS: $35,5 \%$ versus $29,8 \%$ (p-Wert $=0,194$ ); OS: $61,3 \%$ versus $65,8 \%$ ( $p$-Wert $=0,378$ ). Die Studie wurde von der Konsensuskonferenz im Evidenzlevel herabgestuft (siehe auch IQWIG-Bericht, Herabstufung von Ib auf II), da der bei der Studienplanung genannte primäre Studienendpunkt von dem in der Publikation angegebenen abwich (ursprünglicher Endpunkt: Rate der Patienten, die nach 9 Monaten krankheitsfrei sind).

Eine weitere, bislang auf dem ASCO Jahresmeeting 2011 in Abstraktform vorgestellte multizentrische randomisierte Studie untersuchte ebenfalls diese Fragestellung [673]. Endpunkt dieser Studie war die Änderung im Patientenmanagement nach PET-Diagnostik (nicht durchgeführte OP wegen zusätzlicher Befunde oder Ausweitung der OP im Vergleich zur Intention ohne/vor der PET-Diagnostik) in einem 2:1 randomisierten Design bei Patienten mit KRK, die für eine Leberresektion bei Lebermetastasen geeignet erschienen. Es wurden 404 Patienten randomisiert (270 Patienten im PET/CT-Arm, 134 Patienten ohne PET). Man fand keinen Unterschied hinsichtlich der Managementänderung zwischen beiden Armen. Der Endpunkt wurde somit nicht erreicht. Allerdings hatten - soweit dies aus der Präsentation zu erheben war - ca. 70\% der Patienten vor der PET Diagnostik eine Chemotherapie erhalten, was die Sensitivität der Untersuchungstechnik deutlich reduziert (s.u.). Kritisch anzumerken gilt ferner, dass der Endpunkt „Änderung im Patientenmanagement“ im Gegensatz zu „Verringerung überflüssiger Operationen" nicht als Patienten-relevant gilt.

Eine Reihe von Untersuchungen weisen darauf hin, dass sich die Sensivität einer PET deutlich reduziert, falls es innerhalb von 4 Wochen nach einer Chemotherapie 
durchgeführt wird (Evidenzlevel Ila-III). Daher wird eine PET in diesem Zeitraum nicht empfohlen, da zu viele falsch negative Fälle auftreten. Diese Fragestellung hat der IQWIG Bericht nicht bewertet. Eine größere Fall-Kontroll-Studie, die die Sensitivität einer PET nach Chemotherapie nicht-randomisiert prüfte, wurde 2010 publiziert [674]. Die Studie fand einen negativ prädiktiven Wert von nur $13.3 \%$ und einen positiven prädiktiven Wert von $94 \%$ mit einer Spezifität von $22,2 \%$ bei einer Accuracy von $85 \%$, falls die PET innerhalb von vier Wochen nach Chemotherapieende durchgeführt wurde. Die Autoren schlussfolgern, dass eine diagnostische PET Untersuchung kurz nach einer Chemotherapiegabe nicht sinnvoll sei. In einer retrospektiven Untersuchung aus Australien wurden PET-Ergebnisse von Patienten mit Lebermetastasen vor Leberresektion ausgewertet [680]. Auch diese Studie war klein und heterogen. 21 Patienten wurden präoperativ systemisch therapiert, 53 nicht. Korrekte Ergebnisse mittels PET wurden für 29\% nach Chemotherapie und 53\% in der Nicht-ChemotherapieGruppe erzielt. Unterschätzte Befunde ergaben sich zu 52\% in der ChemotherapieGruppe, nur zu 34\% in der Nicht-Chemotherapie-Gruppe. Diese Studie gibt ebenfalls Hinweise darauf, dass eine PET Untersuchung kurz nach einer Chemotherapie nicht sinnvoll ist. Eine weitere prospektive [675] und eine retrospektive Untersuchung [676] kamen zu ähnlichen Ergebnissen.

\subsubsection{Tumormarker}

\begin{tabular}{|l|l|}
\hline 7.10. & Konsensbasierte Empfehlung \\
\hline EK & Der CEA-Wert sollte präoperativ bestimmt werden. \\
\hline & \\
\hline & Starker Konsens \\
\hline
\end{tabular}

\begin{tabular}{|l|l|}
\hline 7.11. & Konsensbasiertes Statement \\
\hline EK & $\begin{array}{l}\text { CA 19-9 erhöht die Aussagefähigkeit bezüglich des Vorliegens eines Rezidivs im } \\
\text { Vergleich zu einer alleinigen CEA-Wert-Bestimmung nicht. }\end{array}$ \\
\hline Starker Konsens
\end{tabular}

\begin{tabular}{l|l|l|}
\hline 7.12. & Konsensbasiertes Statement & \\
\hline EK & $\begin{array}{l}\text { Die Bedeutung von CA } 125 \text { zur Diagnose von Ovarialmetastasen und als } \\
\text { Verlaufsparameter zur weiteren Behandlung einer nachgewiesenen Peritoneal- } \\
\text { karzinose ist derzeit unklar. }\end{array}$ \\
& Starker Konsens
\end{tabular}

\section{Hintergrund}

Bei etwa 30\% aller kolorektaler Karzinome ist der Tumormarker CEA zum Zeitpunkt der Erstdiagnose erhöht. [Daten aus Klinischem Krebsregister der Chirurgischen Universitätsklinik Erlangen-Nürnberg] 
Insbesondere in der Tumornachsorge ist dieser Tumormarker ein zuverlässiger Hinweis auf ein Rezidiv. Zudem ist es im Falle von Lebermetastasen ein unabhängiger Prognosefaktor.

Als weitere Tumormarker werden CA 19-9 und CA 125 diskutiert, wobei letzterer ein Marker der Peritonealkarzinose ist. [681-683].

Der Nachweis von zirkulierender DNA und sogenannter zirkulierender Tumorzellen im peripheren Blut wie im Knochenmark hat keinerlei Konsequenz.

\subsubsection{Spezielle Diagnostik beim Rektumkarzinom}

\begin{tabular}{|l|l|l|}
\hline 7.13. & Konsensbasierte Empfehlung & 2013 \\
\hline EK & $\begin{array}{l}\text { Beim Rektumkarzinom sollte die starre Rektoskopie mit Höhenangabe des } \\
\text { Tumorunterrandes obligater Bestandteil der präoperativen Diagnostik sein. }\end{array}$ \\
\hline Starker Konsens
\end{tabular}

\section{Hintergrund}

Die starre Rektoskopie ermöglicht eine genaue Bestimmung des Abstandes des distalen Tumorrandes von der Linea dentata und ist somit für die weitere Therapieentscheidung von wesentlicher Bedeutung.

\subsection{4 . \\ Empfehlungsgrad \\ B \\ Level of Evidence \\ 2b}

\section{Evidenzbasierte Empfehlung/ Statement}

Zum lokalen Staging eines Rektumkarzinoms sollte vorzugsweise eine MRT, im Falle eines mutmaßlichen T1-Karzinoms eine Endosonographie, durchgeführt werden.

De Novo:[684-692]

Starker Konsens

\section{3}

7.15 .

Evidenzbasiertes Statement

2013

Level of Evidence

3

De Novo:[684-692]

Starker Konsens 


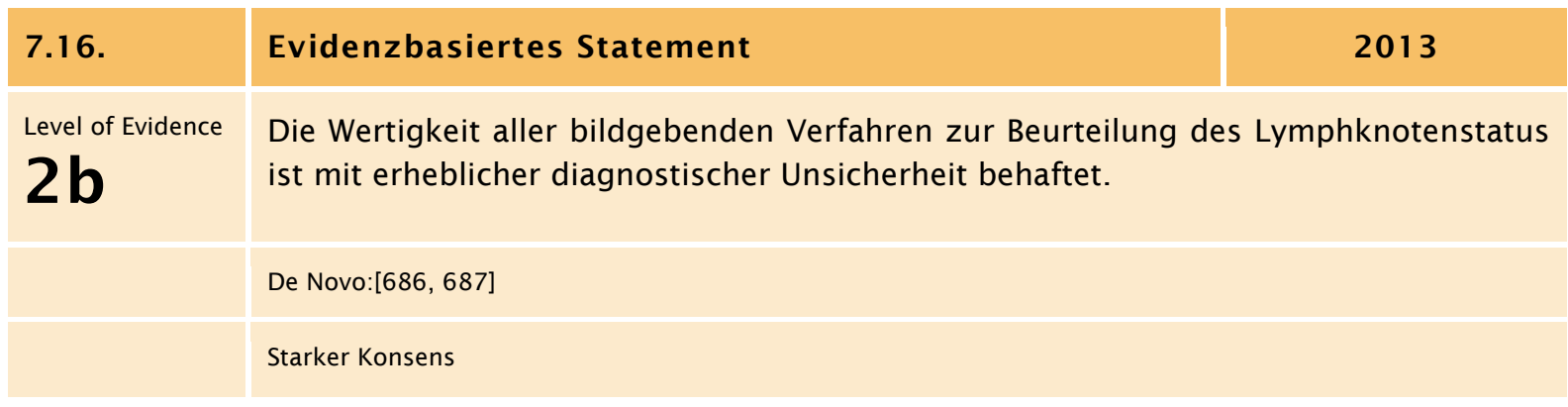

\subsection{7.} Konsensbasierte Empfehlung

\section{3}

EK

Die Befundbeschreibung soll eine Aussage über den Abstand zur mesorektalen Faszie beinhalten.

Starker Konsens

\section{Hintergrund}

Beim Rektumkarzinom kommt dem lokalen Staging eine entscheidende Bedeutung bei der weiteren Therapieplanung zu. Während bei einem low-risk T1-Karzinom eine lokale Abtragung ausreichend ist, ist bei high-risk T1 sowie T2-Karzinomen eine Resektion nach onkologischen Kriterien erforderlich. Bei Nachweis einer Tumorinfiltration in das Mesorektum (T3) wird in Deutschland eine neoadjuvante Therapie, bei Nachweis einer Infiltration in Nachbarorgane (T4) in Form einer neoadjvuanten Radiochemotherapie empfohlen. Bei T3-Karzinomen liegen Daten vor die zeigen, dass dem Ausmaß der Beteiligung des Mesorektums insbesondere der Abstand von der mesorektalen Faszie eine wichtige prognostische Bedeutung zukommt [684]. Diese Ebene stellt bei der TME die zirkumferentielle Resektionsgrenze (CRM) dar. Ist die mesorektale Faszie infiltriert bzw. reicht der Tumor bis $1 \mathrm{~mm}$ an die Faszie heran (CRM+) ist das Lokalrezidivrisiko deutlich erhöht [685]. Ein weiterer Prognosefaktor sind befallene Lymphknoten [684].

Bei der Literatursuche zur Wertigkeit verschiedener Verfahren im lokalen Staging von Rektumkarzinomen musste eine Reihe von Studien ausgeschlossen werden, da das Studienkollektiv auch Patienten nach erfolgter Radio- bzw. Radiochemotherapie enthielt. Für weitere Einzelheiten sei auf den Evidenzbericht verwiesen.

Die Genauigkeit der einzelnen diagnostischen Verfahren hängt von den technischen Voraussetzungen der Geräte (z.B. Multidetector Spiral-CT vs. 1-Schicht CT) und der lokalen Expertise ab. Bei höhergradigen Stenosen oder Tumoren im proximalen Rektum ist eine Endosonographie häufig technisch nicht durchführbar.

In einer Meta-Analyse, in der die Daten zur Endosonographie, zum MRT und zum CT bis 2002 analysiert wurden, wies die Endosonographie die höchste Genauigkeit bei T1Karzinomen auf [686]. Die hohe Sensitivität und Spezifität der Endosonographie konnte in einer neueren Meta-Analyse bestätigt werden [687]. Das MRT mit endorektaler Spule stellt eine mögliche Alternative zum EUS dar, ist aber mit höheren Kosten verbunden und wird von den Patienten als unangenehm empfunden und ist an sehr wenigen Standorten etabliert. Das CT ist für die Festlegung von T1-Karzinomen nicht geeignet. 
Bei der Differenzierung von T2 und T3-Karzinomen wies die Endosonographie wiederum eine höhere Sensitivität im Vergleich zu MRT und CT auf bei vergleichbarer Spezifität [686]. Bei T4-Karzinomen gab es in der Meta-Analyse keine wesentlichen Unterschiede zwischen den Untersuchungsmodalitäten. In einzelnen neueren Kohorten wurden für das MRT und vereinzelt das Spiral-CT für Tumoren mit Infiltration des Mesorektums (> T2) höhere Sensitivitäten gezeigt [688-692], wobei im unteren Drittel die Genauigkeit für das CT deutlich geringer war als in den oberen zwei Dritteln [693]. Für Fragestellungen, die eine Darstellung der mesorektalen Faszie und die Beziehung des Tumors zu ihr erfordern, weist das MRT aktuell die höchste Sensitivität auf [694]. Das Spiral-CT stellt eine mögliche Alternative dar [688], in der Endosonographie lässt sich die Faszie nicht darstellen.

Bei der Beurteilung der Lymphknoten sind Sensitivität (55-73\%) und Spezifität (74-78\%) aller Verfahren derzeit unbefriedigend [686, 687]. Dies ist u.a. dadurch bedingt, dass einerseits eine reaktive Lymphknotenvergrößerung auftritt, andererseits auch Lymphknoten von $5 \mathrm{~mm}$ und kleiner Metastasen enthalten können. Aus diesem Grund sollte die Indikation zu einer neoadjuvanten Therapie sehr zurückhaltend gestellt werden, wenn sie allein auf der Beschreibung suspekter Lymphknoten in der prätherapeutischen Bildgebung basiert.

Ferner ist zu bedenken, dass die Genauigkeit der einzelnen Verfahren wesentlich von der lokalen Expertise abhängt, was insbesondere auch für die Kernspintomographie gilt.

Unter Berücksichtigung insbesondere der Möglichkeit der Darstellung der mesorektalen Faszie wird von vielen Experten aktuell das MRT - mit Ausnahme von frühen Karzinomen - für das lokale Staging des Rektumkarzinoms bevorzugt.

Mit Ausnahme der Kurzzeitbestrahlung wird durch eine neoadjuvante Therapie die Genauigkeit der einzelnen diagnostischen Verfahren eingeschränkt (siehe Evidenzbericht).

\begin{tabular}{|l|r|}
\hline 7.18. & Empfehlung/ EK \\
\hline Empfehlungsgrad & Folgende Untersuchungen können im Einzelfall nützlich sein: \\
\hline $\mathbf{0}$ & $\bullet \quad$ Sphinktermanometrie \\
\hline EK & $\bullet \quad$ Gynäkologische Untersuchung \\
\hline EK & \begin{tabular}{l} 
- Zystoskopie \\
\hline
\end{tabular}
\end{tabular}

$\begin{array}{r}2008 \\ \hline \text { Level of Evidence }\end{array}$

Jeweils starker Konsens

\section{Hintergrund}

Die Sphinktermanometrie hat im Allgemeinen keinen Einfluss auf den Therapieentscheid bezüglich des Sphinktererhaltes über das Ergebnis der rektal-digitalen Untersuchung und der differenzierten Anamnese hinaus. In unklaren Fällen kann sie eine Entscheidung bezüglich des Sphinktererhaltes erleichtern.

Bei Verdacht auf eine Infiltration der Blase kann eine Zystoskopie hilfreich sein, bei Verdacht auf Infiltration von Vagina, Uterus oder Adenexe sollte eine gynäkologische 
Untersuchung erfolgen. Entgegen der früheren Leitlinie wird ein Urinsediment bei Rektum- oder Sigmakarzinomen nicht mehr empfohlen, da die Untersuchung zu unspezifisch ist.

\subsection{Chirurgische Therapie mit kurativem Ziel}

\subsubsection{Intraoperatives Staging}

\begin{tabular}{|l|l|}
\hline 7.19. & Konsensbasierte Empfehlung \\
\hline EK & $\begin{array}{l}\text { Eine intraoperative Inspektion und, bei offener Operation, die Palpation der Leber } \\
\text { sollte in jedem Fall, d.h. auch bei unauffälligem präoperativen Staging, erfolgen. }\end{array}$ \\
\hline $\begin{array}{l}\text { Bei suffizienter präoperativer Diagnostik rechtfertigt der diagnostische Zugewinn nicht } \\
\text { den Aufwand einer intraoperativen Sonographie zur Suche nach weiteren Metastasen. }\end{array}$ \\
\hline Konsens
\end{tabular}

\section{Hintergrund}

Aufgrund der zwischenzeitlichen Qualität von Kernspintomographie und ComputerTomographie werden bei einer Laparotomie in der Regel nur zusätzliche subseröse Lebermetastasen $(<2 \mathrm{~mm})$ durch die intraoperative Inspektion und Palpation entdeckt.

Allerdings liegt die Sensitivität und der positiv prädiktive Wert der intraoperativen Ultraschalluntersuchung mit Kontrastmittel sehr hoch (in einer einzigen Serie mit 24 Patienten bei je $100 \%$ [695].

\subsubsection{Intraoperative pathologische Diagnostik}

Allgemein ist die Indikation zur Schnellschnittuntersuchung nur bei sich unmittelbar ergebenden Konsequenzen zu stellen. Häufigste Indikation ist die Verifikation von Strukturen mit Verdacht auf Fernmetastasen, z.B. am Peritoneum, in der Leber oder in nicht regionären (z. B. paraaortalen) Lymphknoten.

Bei chirurgischen lokalen Exzisionen (Vollwandexzisionen) stellt sich die sehr wichtige Frage, ob ein zuvor bioptisch gesichertes Karzinom seitlich oder basal im Gesunden entfernt wurde.

Allerdings kann diese nicht mit hinreichender Sicherheit intraoperativ durch Schnellschnittuntersuchung geklärt werden.

Bei einem tiefsitzenden Rektumkarzinom kann nach zunächst vorgenommener tiefer anteriorer Resektion gelegentlich die Schnellschnittuntersuchung des aboralen Resektionsrandes für die Indikation zu einer Erweiterung zur Rektumexstirpation von Bedeutung sein.

Bei möglicher Segment- und tubulärer Resektion wegen großer, polypöser, insbesondere villöser Tumoren des Kolons, bei denen prätherapeutisch eine Karzinomdiagnose nicht gesichert werden konnte, ist eine Dignitätsbeurteilung im Schnellschnitt aus untersuchungstechnischen Gründen (Untersuchung multipler Gewebsblöcke!) häufig 
nicht möglich. Daher empfiehlt sich in diesen Situationen in der Regel die radikale Tumoroperation.

Bei Adhärenz eines Tumors an Nachbarorganen ist makroskopisch nicht sicher zu klären, ob es sich um eine Infiltration des Karzinoms in das Nachbarorgan oder nur um eine peritumoröse Entzündungsreaktion handelt. In solchen Fällen sollten Biopsien und Schnellschnittuntersuchungen strikt vermieden werden, da hierbei stets die Gefahr einer örtlichen Tumorzelldissemination besteht, was mit einer signifikanten Verringerung der Überlebenschancen einhergeht [696]. Dies begründet die En bloc-Resektion in allen Fällen von Tumoradhärenzen zu benachbarten Organen oder sonstigen Strukturen (siehe Abschnitt 7.7.2).

\begin{tabular}{|l|l|}
\hline 7.20. & Konsensbasierte Empfehlung \\
\hline EK & $\begin{array}{l}\text { Sofern durch die Bildgebung keine eindeutige diagnostische Zuordnung von unklaren } \\
\text { Leberläsionen getroffen werden kann, sollte eine histologische Sicherung erfolgen. }\end{array}$ \\
\hline Konsens
\end{tabular}

\section{Hintergrund}

Bei unklaren Läsionen der Leber (siehe unten) sollte, sofern sich hieraus unmittelbarer Handlungsbedarf ergibt, eine histologische Sicherung möglichst durch eine Nadelbiopsie durch gesundes Leberparenchym hindurch erfolgen. Inzisionsbiopsien sind strikt zu vermeiden. Bei kleineren Läsionen kann auch eine vollständige Exzision im Sinne einer Exzisionsbiopsie erfolgen.

7.21. EK

\section{Konsensbasiertes Statement}

Die Sentinel-Node-Biopsie (Wächterlymphknoten-Exzision) hat keinen Stellenwert beim kolorektalen Karzinom.

Konsens

\section{Hintergrund}

Bei malignen Melanomen und Mammakarzinomen wird die Sentinel-Node-Biopsie durchgeführt, um bei Patienten mit histologisch negativen Pförtnerlymphknoten eine weitergehende Dissektion mit Erhöhung der Morbititätsrate zu vermeiden.

Mit Einführung der laparoskopischen Operationstechniken wurde diskutiert, ob auch bei kolorektalen Karzinomen eingeschränkte Resektionsverfahren durchgeführt werden können, wenn Sentinel-Node-Biopsien negative Pförtnerlymphknoten erbringen.

Außerdem wurde die Frage gestellt, ob sich bei Ultrastaging (Immunhistochemische Aufarbeitung) des Pförtnerlymphknotens Änderungen im Tumorstadium ergeben könnten, mit entsprechender Notwendigkeit für eine adjuvante Therapie. [697, 698] 


\subsubsection{Radikalchirurgische Therapie des Kolonkarzinoms}

\subsubsection{Ausmaß der Lymphknotendissektion:}

Im Falle einer lymphogenen Metastasierung des Kolonkarzinoms erfolgt diese nach regelhaftem Metastasierungsmuster, nämlich zunächst longitudinal, zu beiden Seiten des Tumors in die parakolischen Lymphknoten, im weiteren zu den intermediären Lymphknoten entlang der radiären Arterien bis hin zu den zentralen Lymphknoten am Stamm der versorgenden Arterien. Hierbei erfolgt die parakolische Metastasierung nie über eine längere Distanz von mehr als $10 \mathrm{~cm}$ [699-701].

Aus der Durchtrennung der zentralen Arterien ergibt sich das Ausmaß der Darmresektion, wobei im Falle rechtsseitiger Karzinome Lymphknotenmetastasen am terminalen lleum nur sehr selten und dann nur bei sehr weit fortgeschrittenen Karzinomen vorkommen [702]. Deshalb ist eine Resektion des terminalen lleums von max. $10 \mathrm{~cm}$ bei der Hemikolektomie rechts ausreichend.

\section{Onkologische Grundsätze}

Bei der Kolonkarzinomchirurgie korreliert die Lymphknotenausbeute auch im Falle nodal negativer Karzinome (UICC II) mit der Prognose [703, 704].

\section{Vorgehen bei Karzinomen des Coecums und des Colon ascendens}

Karzinome in diesem Bereich metastasieren nach zentral über die Arteria ileocolica und die Arteria colica dextra. Entsprechend müssen beide Gefäße zentral durchtrennt werden. Allerdings liegt eine echte Arteria colica dextra mit Abgang aus der Arteria mesenterica superior nur in weniger als $15 \%$ aller Fälle vor [705]. Demnach werden bei nicht angelegtem Gefäß nach rechts ziehende Äste aus dem Hauptstamm der Arteria colica media zentral durchtrennt. Anteile des Omentum majus müssen nur bei direktem Tumorkontakt mitreseziert werden.

\section{Vorgehen bei Karzinomen der rechten Flexur und des rechten Colon transversum}

Bei der erweiterten Hemikolektomie rechts werden die Arteria ileocolica, die Arteria colica dextra (soweit vorhanden) und die Arteria colica media zentral ligiert. Entsprechend ergibt sich eine distale Resektionsgrenze im Bereich des linken Colon transversum. Da in dieser Tumorlokalisation auch eine lymphogene Metastasierung über das große Netz Richtung Magenantrum und weiter zum Pankreaskopf hin [700] stattfindet, werden neben der Skelletierung der großen Magenkurvatur und Resektion der Gastroepiploica-dextra-Arkade und damit der rechtsseitigen Omentumanteile auch die Lymphknoten über dem Pankreaskopf disseziert.

\section{Vorgehen bei Karzinomen des mittleren Colon transversum}

Diese Tumore metastasieren einerseits über die Arteria colica media nach zentral Richtung A. mesenterica superior, andererseits über die Arteria colica sinistra Richtung Arteria mesenterica inferior. Eine Transversumresektion schließt beide Colonflexuren mit ein, bei Metastasierung über das Omentum majus Richtung große Magenkurvatur muss korrespondierend zur Tumorlokalisation auch eine Omentumresektion unter Beachtung des Arkadenprinzips (Einbeziehung der Omentumarterie innerhalb einer Arkade zu beiden Seiten des Karzinoms von $10 \mathrm{~cm}$ ) sowie eine Skelettierung der großen Magenkurvatur mit Entfernung dieser Lymphknoten durchgeführt werden. 


\section{Vorgehen bei Karzinomen des distalen Colon transversum und der linken Colonflexur}

Die Metastasierung verläuft hier nach rechts über die Arteria colica media, nach links über die Arteria colica sinistra. Somit wird die Arteria colica media zentral, die Arteria colica sinistra am Abgang aus der Arteria mesenterica inferior abgesetzt. Ein Vorteil einer höheren Radikalität durch zentrale Absetzung der Arteria mesenterica inferior ist nicht belegt. Eine Kontinuitätswiederherstellung kann als Ascendo-Sigmoideo-stomie erfolgen. Wegen der Metastasierung über das große Netz Richtung große Magenkurvatur müssen entsprechend die linksseitigen Omentumanteile unter Dissektion der Arkade an der großen Magenkurvatur mit entfernt werden. Bei fortgeschrittenen Tumoren in diesem Bereich können auch Lymphknoten am linken Pankreasunterrand befallen sein, so dass auch diese vom Isthmus bis zum Pankreasschwanz disseziert werden.

\section{Vorgehen bei Karzinomen des Colon descendens}

Bei einer Metastasierung über die Arteria colica sinistra und den Arteriae sigmoideae nach zentral ist hier eine Hemikolektomie links mit zentraler Durchtrennung der Arteria mesenterica inferior erforderlich. Die distale Resektionsgrenze liegt dabei im oberen Rektumdrittel, die proximale im Flexurbereich links. Entsprechend müssen eventuell adhärente Omentumanteile mit reseziert werden.

\section{Vorgehen bei Karzinomen des Sigmas}

Diese Tumoren metastasieren über die Arteriae sigmoideae zum Stamm der Arteria mesenterica inferior. Die proximale Colondurchtrennung liegt dabei im Colon descendens mit zentraler Durchtrennung der Arteria mesenterica inferior; bei der distalen Darmdurchtrennung greifen auch die Richtlinien für das Rektumkarzinom im oberen Rektumdrittel, wobei hier mindestens ein Sicherheitsabstand nach distal von 5 cm zum Tumorunterrand eingehalten werden muss mit Durchtrennung des Mesorektums ohne „Coning“ (Ausdünnung proximalwärts).

Hintergrund: Bei 2 - 4 \% der Patienten liegen Lymphknotenmetastasen abgangsnah am Stamm der Arteria mesenterica inferior vor [706, 707].

\subsubsection{Komplette Mesokolische Exzision (CME)}

\begin{tabular}{l|l} 
7.22. & Konsensbasierte Empfehlung \\
\hline
\end{tabular}
EK Die chirurgische Therapie des Kolonkarzinoms sollte die komplette mesokolische
Exzision beinhalten.

Konsens

\section{Hintergrund}

Analog zum Mesorektum existiert in gleicher Weise ein Mesokolon, welches als beidseitige Hülle die Lymphknoten an den versorgenden Arterien umfasst.

Analog zur TME beim Rektumkarzinom wird die CME bei der Operation von Kolonkarzinomen angewandt, um bei der Präparation in vorgegebenen anatomischen Schichten mit zentraler Absetzung der versorgenden Gefäße eine maximale lokale Radikalität mit maximaler Lymphknotenausbeute zu erzielen, und zudem auf die Erhaltung der beiden mesokolischen Schichten („Faszien“) geachtet. 
Die komplette mesokolische Exzision beim Kolonkarzinom ist geeignet, eine maximale lokale Radikalität mit hoher Lymphknotenausbeute zu erreichen.

Sie führt zu qualitativ höherwertigen Präparaten [708, 709]. Höhere Komplikations-raten scheinen mit Erhöhung der Radikalität nicht verbunden zu sein [710]. Die bisherigen Daten deuten auf eine Verbesserung der Überlebensraten bei konsequenter Durchführung der CME hin [708].

Die morphometrische Untersuchung des Kolonpräparates kann zukünftig zur objektiven Bewertung von Kolonkarzinompräparaten herangezogen werden.

\subsubsection{Lokalablative Verfahren bei Lebermetastasen}

\subsection{Radiofrequenzablation (RFA)}

\begin{tabular}{|l|l|}
\hline $\begin{array}{l}\text { 7.23. } \\
\text { Empfehlungsgrad }\end{array}$ & $\begin{array}{l}\text { Evidenz RFA kann durchgeführt werden, wenn nicht resektable Lebermetastasen } \\
\text { vorliegen oder der Allgemeinzustand des Patienten eine Resektion nicht zulässt, } \\
\text { insbesondere nach vorangegangener Leberresektion. }\end{array}$ \\
\hline $\begin{array}{l}\text { Level of Evidence } \\
\mathbf{3} \mathbf{a}\end{array}$ & \begin{tabular}{l} 
De Novo: [711-713] \\
\hline
\end{tabular} \\
\hline
\end{tabular}

\section{Hintergrund}

Die derzeitige Evidenzlage zur Sicherheit und Effektivität der Radiofrequenzablation bei kolorektalen Lebermetastasen ist angemessen, um die Verwendung dieser Methode bei Patienten zu befürworten bei denen entweder nicht resektable Lebermetastasen vorliegen, deren Zustand eine Resektion nicht zulässt, oder bei denen zuvor eine Leberresektion durchgeführt worden ist. [711].

Die RFA kann auch primär in Kombination mit der chirurgischen Resektion durch-geführt werden.

Neuere Arbeiten legen die Vermutung nahe, dass bei solitären Lebermetastasen $<3 \mathrm{~cm}$ mittels RFA ähnlich gute Ergebnisse wie durch die Resektion erzielt werden können [712, 713]. Die bisherige Datenlage hierzu ist aber widersprüchlich und es fehlen weiterhin vergleichende kontrollierte-randomisierte Studien. 
7.5.2.3.2. Selective Internal Radiation Therapy (SIRT)

\begin{tabular}{|l|l|}
\hline $\begin{array}{l}\text { 7.24. } \\
\text { Evidenzbasierte Empfehlung }\end{array}$ & 2013 \\
\hline $\mathbf{B}$ & $\begin{array}{l}\text { Eine SIRT zur Behandlung von disseminierten Lebermetastasen bei KRK sollte nur bei } \\
\text { Patienten, für die keine andere Therapieoption infrage kommt, und dann nur } \\
\text { innerhalb klinischer Studien durchgeführt werden. }\end{array}$ \\
\hline $\begin{array}{l}\text { Level of Evidence } \\
\mathbf{2} \mathbf{a}\end{array}$ & \begin{tabular}{l} 
De Novo: $[714,715]$ \\
\hline
\end{tabular} \\
\hline
\end{tabular}

\section{Hintergrund}

Patienten mit fehlender oder allenfalls begrenzter extrahepatischer Metastasierung, die zudem systemtherapeutisch austherapiert sind, zeigen in einzelnen Studien mit SIRT (gleichbedeutend mit dem Begriff Radioembolisation) ein verlängertes medianes Überleben sowie ein längeres Intervall bis zum Progress von Lebermetastasen. Für eine endgültige Bewertung, insbesondere auf Überleben und Lebensqualität liegen zu wenige Daten vor. Deshalb sollten Patienten, die für eine SIRT in Frage kämen, nur innerhalb klinischer Studien behandelt werden [714, 715].

\subsection{Laserinduzierte interstitielle Thermotherapie (LITT)}

\begin{tabular}{|l|l|}
$\begin{array}{l}\text { 7.25. } \\
\text { Empfehlungsgrad }\end{array}$ & $\begin{array}{l}\text { Eine LITT zur Behandlung von Lebermetastasen bei KRK sollte nur innerhalb klinischer } \\
\text { B }\end{array}$ \\
\hline $\begin{array}{l}\text { Studien durchgeführt werden. } \\
\text { Level of Evidence }\end{array}$ & De Novo: $[716,717]$ \\
\hline
\end{tabular}

\section{Hintergrund}

In einzelnen Fallserien war die interstitielle Laserthermoablation eine sichere und wirksame Behandlung für Patienten mit inoperablen Lebermetastasen kolorektaler Karzinome [716, 717]. Daten zum Effizienzvergleich mit der perkutanen Radiofrequenzablation liegen nicht vor. Für eine endgültige Bewertung, insbesondere auf Überleben und Lebensqualität liegen zu wenige Daten vor. Deshalb sollten Patienten, die für eine LITT in Frage kämen, nur innerhalb klinischer Studien behandelt werden.

\subsubsection{Radikalchirurgische Therapie des Rektumkarzinoms}

Die kurative Therapie des Rektumkarzinoms erfordert in der Regel neben der Resektion des Primärtumors im Gesunden die partielle oder totale Entfernung des Mesorektums und damit des regionären Lymphabflussgebiets (sog. radikale Resektion nach internationalem Dokumentationssystem für das kolorektale Karzinom [636, 637]. Nur in streng selektionierten Fällen ist eine kurative Resektion durch lokale Maßnahmen 
möglich. Folgende Operationsverfahren sind bei Einhaltung der Kriterien der onkologischen Chirurgie als gleichwertig anzusehen, wobei die Indikationsstellung von der Tumorlokalisation, insbesondere der Beziehung zur Linea dentata und dem Levatorschenkel, der Tiefeninfiltration und der Sphinkterfunktion abhängig ist:

- die (tiefe) anteriore Rektumresektion,

- die abdomino-perineale Rektumexstirpation,

- $\quad$ die intersphinktere Rektumresektion (auch als abdominoperanale Rektumresektion bezeichnet). Diese Operation setzt besondere Erfahrungen voraus.

Zu beachten ist, dass auch bei der tiefen anterioren Rektumresektion sehr häufig zur Erreichung eines ausreichenden Sicherheitsabstandes nach aboral eine intersphinktäre Präparation erforderlich ist. Hiermit ist jedoch nicht das Operationsverfahren im Sinne der abdominoperanalen Präparation zu verwechseln.

Nach Möglichkeit sind kontinenzerhaltende Verfahren unter Abwägung der zu erwartenden späteren Lebensqualität zu bevorzugen. Bei schlechter Sphinkterfunktion sollte an Stelle einer tiefen Resektion die Anlage einer permanenten Kolostomie bevorzugt werden, die je nach Sicherheitsabstand vom Beckenboden als Rektumexstirpation oder Beckenboden-erhaltend ausgeführt wird.

\subsubsection{Allgemeine Onkologische Grundsätze}

Die operative Therapie sollte folgende Grundsätze beinhalten:

Die Entfernung des regionären Lymphabflussgebiets mit Absetzung der A. mesenterica inferior zumindest distal des Abgangs der A. colica sinistra. Die abgangsnahe Unterbindung der A. mesenterica inferior hat keine prognostische Bedeutung, sie wird aber häufig aus operationstechnischen Gründen zur ausreichenden Mobilisation des linken Hemikolons zur Rekonstruktion durchgeführt [718]. Allerdings zeigen anatomische Studien, dass in vielen Fällen auch ohne zentrale Absetzung eine tiefe Anastomose möglich ist [719]. Der Wert einer Dissektion der Lymphknoten am Stamm $\operatorname{der} A$. mesenterica inferior proximal des Abgangs der A. colica sinistra ist nicht gesichert (Evidenzstärke 2b) [720-723].

- $\quad$ Die komplette Entfernung des Mesorektums beim Karzinom des mittleren und unteren Rektumdrittels und die partielle Mesorektumexzision beim Karzinom des oberen Rektumdrittels durch scharfe Dissektion entlang anatomischer Strukturen zwischen Fascia pelvis visceralis und parietalis (totale mesorektale Exzision - TME) [724, 725].

- Die Einhaltung eines angemessenen Sicherheitsabstands (siehe unten).

- In der Regel die En-Bloc-Resektion von tumoradhärenten Organen (mutiviszerale Resektion) zur Vermeidung einer örtlichen Tumorzelldissemination [726].

- Die Schonung der autonomen Beckennerven (Nn. hypogastrici, Plexus hypogastrici inferiores et superior) [727, 728]. 


\begin{tabular}{|l|l|}
$\begin{array}{l}\text { 7.26. } \\
\text { Empfehlungsgrad }\end{array}$ & $\begin{array}{l}\text { Evidenzbasierte Empfehlung } \\
\text { Die systematische Dissektion der lateralen Lymphknoten entlang der Art iliaca interna } \\
\text { und ihrer Äste soll ohne begründeten Metastasenverdacht nicht durchgeführt } \\
\text { werden. Sie erhöht die perioperative Morbidität, ohne dass onkologische Vorteile } \\
\text { belegt sind. }\end{array}$ \\
\hline $\begin{array}{l}\text { Level of Evidence } \\
\mathbf{1} \mathbf{0}\end{array}$ & De Novo: [729-732] \\
\hline & Starker Konsens \\
\hline
\end{tabular}

\subsubsection{Vorgehen bei Tumoren des oberen Rektumdrittels}

7.27.

Empfehlungsgrad

B

Level of Evidence

$3 b$

\section{Evidenzbasierte Empfehlung}

Bei Tumoren des oberen Rektumdrittels erfolgt die Durchtrennung des Rektums mit partieller Mesorektumexzision $5 \mathrm{~cm}$ distal des makroskopischen Tumorrands, gemessen in vivo. Das Mesorektum sollte horizontal ohne proximalwärtige Ausdünnung durchtrennt werden (kein Coning).

De Novo: [724, 733-737]

Starker Konsens

\section{Hintergrund}

Die Begründung dieses Vorgehens [736, 737] liegt darin, dass bei T3- und T4-Tumoren in seltenen Fällen Satellitenknoten oder Lymphknotenmetastasen in bis zu $4 \mathrm{~cm}$ distal des makroskopischen Tumorrands, gemessen am histologischen Schnitt nach Fixation des nicht ausgespannten Präparats, vorkommen können.

\subsubsection{Vorgehen bei Tumoren des mittleren und unteren Rektumdrittels}

\begin{tabular}{|l|l|}
$\begin{array}{l}\text { 7.28. } \\
\text { Evidenzbasierte Empfehlung }\end{array}$ & 2008 \\
\hline $\begin{array}{l}\text { Empfehlungsgrad } \\
\text { A }\end{array}$ & $\begin{array}{l}\text { Bei Tumoren des mittleren und unteren Rektumdrittels erfolgt die totale } \\
\text { Mesorektumexzision (TME) bis zum Beckenboden unter Schonung des Plexus } \\
\text { hypogastricus superior, der Nn. hypogastrici und der Plexus hypogastrici inferiores. }\end{array}$ \\
\hline $\begin{array}{l}\text { Level of Evidence } \\
\mathbf{1}\end{array}$ & Quellen: $[727,738,739]$ \\
\hline
\end{tabular}




\begin{tabular}{|l|l|}
$\begin{array}{l}\text { 7.29. } \\
\text { Evidenzbasierte Empfehlung }\end{array}$ & 2008 \\
\hline $\begin{array}{l}\text { Empfehlungsgrad } \\
\mathbf{B}\end{array}$ & $\begin{array}{l}\text { Bei Low-Grade-Tumoren guter oder mäßiger Differenzierung des unteren } \\
\text { Rektumdrittels ist ein Sicherheitsabstand von 1-2cm in situ ausreichend. Bei High- } \\
\text { Grade-Tumoren (G3/4) ist ein größerer Sicherheitsabstand anzustreben. }\end{array}$ \\
\hline $\begin{array}{l}\text { Level of Evidence } \\
\mathbf{2} \mathbf{b}\end{array}$ & \begin{tabular}{l} 
Quellen: $[725,740-743]$ \\
\hline
\end{tabular} \\
\hline
\end{tabular}

\subsection{0.}

Empfehlungsgrad

B

Level of Evidence

$2 b$

\section{Evidenzbasierte Empfehlung}

Nach neoadjuvanter Radiochemotherapie kann zur Abwendung einer ansonsten notwendigen Exstirpation auch ein aboraler Abstand von $0,5 \mathrm{~cm}$ akzeptiert werden. Die Tumorfreiheit des aboralen Resektionsrandes sollte durch intraoperativen Schnellschnitt gesichert werden.

de Novo: [744, 745]

Konsens

\section{Hintergrund}

Bei Karzinomen des unteren Drittels kann als Alternative zu der ansonsten erforderlichen Rektumexstirpation die intersphinktere Rektumresektion (auch als abdomino-peranale Rektumresektion bezeichnet) durchgeführt werden, wenn - unter Wahrung der oben genannten Sicherheitsabstände - die puborektale Schlinge nicht infiltriert ist. Diese Operation setzt besondere Erfahrung voraus.

\subsubsection{Rekonstruktion nach total mesorektaler Exzision}

Nach totaler mesorektaler Resektion mit nachfolgender spinkternaher Anastomose ist potenziell mit u.U. erheblichen funktionellen Störungen zu rechnen. Diese sind abhängig von der Wahl des Rekonstruktionsverfahrens. Als Möglichkeiten stehen zur Verfügung:

- $\quad$ gerade colo-anale Anastomose

- $\quad$ der Colon-J-Pouch

- die transverse Coloplastie

- $\quad$ die Seit-zu-End-Anastomose 


\begin{tabular}{|l|l|}
$\begin{array}{l}\text { 7.31. } \\
\text { Empfehlungsgrad }\end{array}$ & $\begin{array}{l}\text { Bei der Rekonstruktion nach tiefer anteriorer Resektion soll wegen der besseren } \\
\text { funktionellen Ergebnisse in der Regel (wenn anatomisch machbar) keine gerade colo- } \\
\text { anale Anastomose angelegt werden. }\end{array}$ \\
\hline $\begin{array}{l}\text { Level of Evidence } \\
\mathbf{1}\end{array}$ & de Novo: [746] \\
\hline & Starker Konsens \\
\hline
\end{tabular}

\begin{tabular}{|l|l|}
$\begin{array}{l}\text { 7.32. } \\
\text { Level of Evidence }\end{array}$ & $\begin{array}{l}\text { Eon den verschiedenen Rekonstruktions-Formen sind die Vorteile im funktionellen } \\
\mathbf{1} \mathbf{A}\end{array}$ \\
\hline & Ergebnis des Colon-J-Pouches am besten belegt. \\
\hline & de Novo: $[746,747]$ \\
\hline & mehrheitliche Zustimmung \\
\hline
\end{tabular}

\begin{tabular}{|l|l|}
\hline $\begin{array}{l}\text { 7.33. } \\
\text { Level of Evidence }\end{array}$ & $\begin{array}{l}\text { Unter funktionellen Gesichtspunkten ist die transverse Koloplastie dem Colon-J- } \\
\text { Pouch unterlegen. }\end{array}$ \\
\hline $\mathbf{1 0}$ & de Novo: $[747,748]$ \\
\hline & mehrheitliche Zustimmung \\
\hline
\end{tabular}

\begin{tabular}{|l|l|}
$\begin{array}{l}\text { 7.34. } \\
\text { Level of Evidence }\end{array}$ & Möglicherweise ist die Seit-zu-End-Anastomose dem Colon-J-Pouch ebenbürtig. \\
\hline $\mathbf{2}$ & \\
\hline & de Novo: [749] \\
\hline & Starker Konsens \\
\hline
\end{tabular}

\section{Hintergrund}

Die Nachteile einer geraden colo-analen Anastomose sind hinreichend belegt und wirken sich vor allem während der ersten beiden postoperativen Jahre in erhöhter Stuhlfrequenz sowie schlechterer Kontinenz und Lebensqualität aus [746]. Bei Anlage eines J-Pouches sollte zur Vermeidung von Entleerungsproblemen die Schenkellänge nicht über $6 \mathrm{~cm}$ liegen [750]. Die einzige größere prospektiv randomisierte Studie zur Frage des 
differentiellen Einsatzes verschiedener Rekonstruktionen [747] zeigte, dass bei $74 \%$ der Patienten die Anlage eines J-Pouch technisch machbar ist. Verglichen mit einer transversen Coloplastie war in dieser Studie der J-Pouch hinsichtlich Stuhlfrequenz und Inkontinenzscore besser. Eine Metaanalyse unter Einschluss der Fazio-Studie relativierte zwar die Aussage zur Stuhlfrequenz, berücksichtigte aber weder die Langzeitergebnisse der Fazio-Studie, noch die Daten zur Inkontinenz [748]. Zur endgültigen Beurteilung des Stellenwerts der Seit-zu-End-Anastomose fehlen noch Studien mit ausreichender Fallzahl [749].

\subsubsection{Entscheidung zum Sphinkter-Erhalt}

\begin{tabular}{l|l|}
\hline 7.35. & Konsensbasierte Empfehlung \\
\hline EK & $\begin{array}{l}\text { Durch die neoadjuvante Radiochemotherapie und entsprechende Remission kann u. U. } \\
\text { trotz primär nicht für möglich gehaltenen Sphinktererhaltes eine Schließmuskel } \\
\text { erhaltende Rektumresektion möglich werden. Deshalb sollte diesbezüglich frühestens } \\
6 \text { Wochen nach Abschluss der Radiochemotherapie eine Reevaluation stattfinden. }\end{array}$
\end{tabular}

Starker Konsens

\section{Hintergrund}

Eine Reduktion der Tumormasse vor allem bei tiefsitzenden Tumoren ist mit entscheidend für den Erhalt des Sphinkterapparates. Hier zeichnet sich ein Vorteil für die neoadjuvante Radiochemotherapie ab, deren Effekte aber oft verzögert erst nach mehreren Wochen fassbar werden. Dies bedeutet, dass die Entscheidung für das zu wählende Operationsverfahren erst zum Zeitpunkt der Operation nach der erfolgten neoadjuvanten Radiochemotherapie getroffen werden darf [751]. Bildgebende Verfahren sind in der Beurteilung des Response wenig hilfreich [752].

\subsubsection{Vorgehen bei komplettem Response nach neoadjuvanter Therapie}

\begin{tabular}{|c|c|}
\hline 7.36. & Konsensbasierte Empfehlung \\
\hline & $\begin{array}{l}\text { In den seltenen Fällen, in denen nach neoadjuvanter Radiochemotherapie klinisch, } \\
\text { endoskopisch und durch bildgebende Verfahren (Endosonographie und MRT, } \\
\text { alternativ evtl. auch CT) kein Tumor mehr nachweisbar ist, kann auf jegliche Operation } \\
\text { verzichtet werden. Voraussetzung ist die gründliche Aufklärung über die noch } \\
\text { unzureichende Validierung dieses Vorgehens und die Bereitschaft des Patienten, sich } \\
\text { einer sehr engmaschigen mindestens } 5 \text {-jährigen Nachsorge zu unterziehen. }\end{array}$ \\
\hline
\end{tabular}

Konsens

\section{Hintergrund}

In einer Studie aus Brasilien mit 265 Patienten mit Rektumkarzinom war bei 26,8\% nach neoadjuvanter Therapie kein Tumor mehr nachweisbar. Diese Patienten wurden nicht operiert und erhielten eine Nachsorge [753]. Nach einem medianen Follow-up von 57,3 Monaten wurde bei zwei Patienten (2,8\%) ein endoluminales Rezidiv und bei drei Patienten (4,8\%) systemische Metastasen nachgewiesen. Die Autoren postulieren, dass bei Patienten mit komplettem Ansprechen nach neoadjuvanter Radiochemotherapie eine alleinige Nachsorge ohne Operation ausreichend zu sein scheint. Es ist jedoch zu 
bedenken, dass es sich um keine randomisierte Studie handelt und nicht abschließend geklärt ist, ob die Ergebnisse auch außerhalb von Brasilien erreicht werden können.

\subsubsection{Rektum-Exstirpation}

\begin{tabular}{|l|l|}
$\begin{array}{l}\text { 7.37. } \\
\text { Empfehlungsgrad }\end{array}$ & $\begin{array}{l}\text { Bei tiefliegenden Tumoren mit Infiltration des Analkanals/ der Sphinkteren, die nicht } \\
\text { sphinktererhaltend operiert werden können, sollte die abdomino-perineale } \\
\text { Exstirpation im Sinne einer „zylindrischen“ Resektion unter Mitresektion des Levator } \\
\text { ani erfolgen. }\end{array}$ \\
\hline $\begin{array}{l}\text { Level of Evidence } \\
\mathbf{3} \mathbf{b}\end{array}$ & \begin{tabular}{l} 
de Novo: $[709,754,755]$ \\
\hline
\end{tabular} \\
\hline
\end{tabular}

\section{Hintergrund}

Bei der Rektum-Exstirpation ist klassisch die Rate an Präparaten mit unzureichenden lateralen Sicherheitsabständen erhöht [754]. Die onkologischen Ergebnisse sind gegenüber sphinkter-erhaltenden Eingriffen bei vergleichbaren Tumorstadien schlechter [755]. Die extralevatorische „zylindrische“ Resektion führt zu besseren Sicherheitsabständen und muss auch ohne Beweis durch randomisierte Studien als das überlegene Verfahren angesehen werden [709].

\subsection{8. \\ Empfehlungsgrad \\ O \\ Level of Evidence de Novo: [756] \\ $3 b$ \\ Konsens}

Evidenzbasierte Empfehlung

2013

Nach neoadjuvanter Radio-(Chemo-)-Therapie und/oder bei großen perinealen Defekten können die Wundheilungsstörungen perineal durch primäre plastische Deckung mit einem myokutanen Lappen reduziert werden.

\section{Hintergrund}

Die Rate perinealer Wundheilungsstörungen ist insbesondere nach neoadjuvanter Bestrahlung und bei größeren Defekten hoch. Die Anwendung verschiedenen plastischen Rekonstruktionsverfahren bleibt aber eine Einzelfallentscheidung, bei der die individuelle anatomische Verfügbarkeit, die in Kauf zu nehmende Sekundär-morbidität des Hebedefekts, der operative Aufwand und die verfügbare chirurgische Expertise einzubeziehen sind [756]. 


\subsubsection{Stoma-Anlage}

7.39.

Konsensbasierte Empfehlung/ Statement

EK

Bei der radikalen Operation des Rektumkarzinoms mit TME und tiefer Anastomose soll ein temporäres Deviations-Stoma vorgeschaltet werden.

Starker Konsens

7.40 .

Konsensbasiertes Statement

2013

EK

Als Deviationsstoma sind Kolostoma und lleostoma gleichwertig.

Starker Konsens

\section{Hintergrund}

Durch eine protektive Stomaanalage kann die Morbidität, insbesondere hinsichtlich klinisch relevanter Anastomoseninsuffizienen und dringlicher Relaparotomien gesenkt werden [757]. Auch ist langfristig bei primärem Verzicht auf ein Deviationsstoma die Rate permanenter Stomata keineswegs geringer [758]. Zur Art des protektiven Stomas gibt es Argumente für beide Varianten, auch wenn jüngere Metaanalysen eher das Ileostoma favorisieren [759, 760].

7.41

\section{Konsensbasierte Empfehlung}

2013

EK

Die Stomaanlage soll möglichst frühzeitig vor der Operation mit dem Patienten besprochen und geplant werden.

Starker Konsens

7.42 .

Konsensbasierte Empfehlung

2013

EK

Die Stomaposition soll präoperativ angezeichnet werden.

Konsens

7.43 .

Konsensbasierte Empfehlung

2013

EK

Die Ileostomie sollte prominent angelegt werden $(>1 \mathrm{~cm})$. Die Kolostomie sollte leicht erhaben angelegt werden.

Starker Konsens 


\section{Hintergrund}

Die präoperativen Informationen zur Stomaanlage sollten vom behandelnden Arzt und einer entsprechend ausgebildeten Pflegekraft (Stomatherapeut/in) gegeben werden. Ein Gespräch mit einem Betroffenen aus einer Selbsthilfegruppe sollte - sofern verfügbar angeboten werden. Die Markierung des Stomas kann durch eine geschulte Pflegekraft / Stomatherapeut/in erfolgen, die Verantwortung für die korrekte Stoma-markierung und damit auch korrekte Anlage des Stomas liegt aber beim Arzt. Für eine optimale Lage ist es erforderlich, dass die Stomaposition im Liegen, Sitzen und Stehen angezeichnet wird.

Die postoperative Stomatherapie sollte sicherstellen, dass der Patient bzw. - sofern er selbst dazu nicht in der Lage ist - dessen Angehörige oder betreuende Personen, selbstständig die Stomaversorgung durchführen können (Grundplattenwechsel, Stomabeutel leeren und wechseln), die Versorgung mit Stomaartikeln gewährleistet ist und im Bedarfsfall der Zugang zu einem(r) Stomatherapeuten/in gewährleistet ist. Die Irrigation sollte Kolostomieträgern angeboten werden. Durch die präoperative Stomamarkierung und Einsatz von Stomatherapeuten konnte in einzelnen Studien eine Senkung der postoperativen Stoma-Komplikationsrate gezeigt werden [761, 762]. Des weiteren konnte in einer prospektiven Studie gezeigt werden, dass die präoperative Stomaberatung die postoperative Versorgung vereinfacht [763].

\subsubsection{Lokale Operationsverfahren des Rektumkarzinoms}

\begin{tabular}{|l|l|}
\hline 7.44. & Evidenzbasiertes Statement \\
\hline $\begin{array}{l}\text { Level of Evidence } \\
\mathbf{1 ~ b}\end{array}$ & $\begin{array}{l}\text { Eine lokale chirurgische Tumorexzision beim Rektumkarzinom (Vollwandexzision) ist } \\
\text { als alleinige therapeutische Maßnahme unter kurativer Zielsetzung onkologisch } \\
\text { ausreichend bei pT1-Karzinomen mit einem Durchmesser bis zu } 3 \mathrm{~cm}, \text { guter oder } \\
\text { mäßiger Differenzierung, ohne Lymphgefäßinvasion (Low-Risk-Histologie), sofern die } \\
\text { Entfernung komplett erfolgt ist (R0). }\end{array}$ \\
\hline & Quellen: [764-767] \\
\hline & Starker Konsens
\end{tabular}

\section{Hintergrund}

Auch in Low-risk Fällen ist im Vergleich zur radikalen Operation mit einem höheren Lokalrezidiv-Risiko zu rechnen bei gleichzeitig geringerer Morbidität und Letalität und besserem funktionellen Ergebnis, so dass im Einzelfall diese Risiken gegeneinander abzuwägen sind [768, 769]. Es spricht vieles dafür, dass für die lokale Exzison die Technik der transanalen endoskopischen Mikrochirurgie der offenen transanalen Exzision mittels Spreizer überlegen ist [770, 771].

Hinsichtlich des Stellenwertes der sm-Klassifikation für die Einschätzung in „low-risk“ oder "high-risk“ konnte kein Konsens gefunden werden. Tl-Karzinome mit tiefer submucosaler Infiltration (sm3, nach manchen Serien sogar sm2) werden von anderen Autoren und Leitlinien als Hochrisiko-Konstellation eingeschätzt, die einer radikalen Operation zugeführt werden sollte [772-775]. 


\begin{tabular}{|l|l|l|}
$\begin{array}{l}\text { 7.45. } \\
\text { Empfehlungsgrad }\end{array}$ & $\begin{array}{l}\text { Bei T1-High-Risk-Karzinomen (G3/4 u./o. Lymphgefäßinvasion) } \\
\text { B }\end{array}$ & $\begin{array}{l}\text { Karzinomen liegt das Auftreten von Lymphknotenmetastasen bei 10-20\%, sodass die } \\
\text { alleinige lokale Exzision nicht empfohlen werden kann. } \\
\text { (siehe auch Abschnitt 6.4). }\end{array}$ \\
\hline $\begin{array}{l}\text { Level of Evidence } \\
\mathbf{3} \mathbf{b}\end{array}$ & \begin{tabular}{l} 
Quellen: $[772,776]$ \\
\hline
\end{tabular} \\
\hline
\end{tabular}

\section{Hintergrund}

Ist die „high-risk“ Konstellation prätherapeutisch bekannt, sollte primär radikal operiert werden. Stellt sich erst nach transanaler Vollwandresektion eine Hoch-RisikoKonstellation heraus, so ist die sekundäre radikale Nachoperation innerhalb eines Monats nicht mit einer Verschlechterung der Prognose im Vergleich zum primär radikalen Vorgehen assoziiert [772, 776]. Lehnt der Patient in dieser Situation eine radikale Nachoperation ab, so kann eine adjuvante Radiochemotherapie erwogen werden.

\subsection{Laparoskopische Chirurgie}

7.46.

Empfehlungsgrad

A

Level of Evidence

1 a
Evidenzbasierte Empfehlung

2013

Die laparoskopische Resektion des Kolon- und Rektumkarzinoms kann bei entsprechender Expertise des Operateurs und geeigneter Selektion mit gleichen onkologischen Ergebnissen im Vergleich zur offenen OP-Technik durchgeführt werden. ${ }^{7}$

de Novo: [777-781]

Starker Konsens

7.47.

Konsensbasierte Empfehlung

2013

EK

Die Qualität des Präparates soll durch den Pathologen dokumentiert werden.

Starker Konsens

\section{Hintergrund}

Auch wenn laparoskopische kolorektale Resektionen längere Operationszeiten bedingen, sprechen zahlreiche randomisierte Studien dafür, dass im Kurzzeitverlauf die

${ }^{7}$ Das „kann“ im Empfehlungstext steht nicht im Widerspruch zum Empfehlungsgrad A, da sich dieser auf die Gleichwertigkeit der Verfahren bezieht. 
perioperative chirurgische Morbidität niedriger ist als nach konventioneller Operation bei unveränderter Gesamt-Morbidität und Letalität [777]. Im Langzeitverlauf konnten weder für die Rate an Narbenhernien und an adhäsions-bedingten Reoperationen noch für regionäre und systemische Tumorrezidive Unterschiede gefunden werden [778, 779]. Die systematischen Reviews und Metaanalysen zeigten equivalente onkologische Langzeitergebnisse vor allem beim Kolonkarzinom, während die Datenlage zum Rektumkarzinom noch zu wünschen übrig ließ. Inzwischen wurden aber reife Langzeitergebnissen der britischen CLASICC-Studie publiziert, die trotz initial problematischer Surrogat-Parameter in der Untergruppe der Rektumkarzinome [780] die onkologische Sicherheit der laparoskopischen Chirurgie sowohl für das Kolon- als auch das Rektumkarzinom belegen konnten [781]. Für die ohne Zweifel notwendige spezielle laparoskopische Expertise sind bisher keine stringenten Kriterien validiert.

7.48 . Konsensbasierte Empfehlung

Neuere Operationsverfahren (z.B. Robotik, NOTES) können wegen unzureichender Daten außerhalb von Studien nicht empfohlen werden.

Starker Konsens

\section{Hintergrund}

Das DaVinci-Telemanipulationssystem - der einzige in der klinischen Routine etablierte „Roboter“- ist in mehreren Serien zur laparoskopischen total mesorektalen Exzision verwendet worden [782, 783]. Da weder kurzfristige Vorteile noch die längerfristige onkologische Gleichwertigkeit bewiesen sind, ist diese Methode bisher als experimentell anzusehen. Gleiches gilt in noch stärkerem Maße für transgastrale, transvaginale oder transanale Zugänge und Präparate-Bergungen. 


\subsection{Sondersituationen}

\subsubsection{Chirurgische Therapie der Peritonealkarzinose}

\begin{tabular}{|c|c|}
\hline 7.49 . & Evidenzbasierte Empfehlung \\
\hline $\begin{array}{l}\text { Empfehlungsgrad } \\
0\end{array}$ & $\begin{array}{l}\text { Bei Patienten mit einer isolierten und limitierten Peritonealkarzinose kann eine } \\
\text { zytoreduktive Chirurgie gefolgt von einer hyperthermen intraperitonealen } \\
\text { Chemotherapie (HIPEC) durchgeführt werden, wenn folgenden Voraussetzungen } \\
\text { erfüllt sind: } \\
\text { - } \mathrm{PCl} \text { (peritoneal cancer index) }<20 \\
\text { - keine extraabdominellen Metastasen } \\
\text { - Möglichkeit der makroskopisch kompletten Entfernung oder Destruktion } \\
\quad \text { jeglicher Tumormanifestation } \\
\text { - Therapie in einem spezialisierten Zentrum } \\
\text { Die Durchführung im Rahmen von Studien sollte bevorzugt werden. }\end{array}$ \\
\hline $\begin{array}{l}\text { Level of Evidence } \\
2 \mathbf{a}\end{array}$ & de Novo: [784-787] \\
\hline & starker Konsens \\
\hline
\end{tabular}

\section{Hintergrund}

Es existiert hierzu bisher nur eine einzige abgeschlossene randomisierte Studie zum Vergleich zwischen systemischer Chemotherapie und zytoreduktiver Chirurgie mit HIPEC [785]. Diese Studie konnte auch im Langzeitverlauf belegen, dass die chirurgische Therapie der Peritonealkarzinose zu sigifikant besserem krankheits-spezifischem Überleben führt [786]. Ließ sich makroskopische Tumorfreiheit erzielen, lag das 5Jahres-Überleben bei $45 \%$. Einschränkend muss angeführt werden, dass der Kontrollarm lediglich eine 5-FU-basierte systemische Therapie erhielt. Trotz zahlreicher nichtrandomisierter Studien mit ermutigenden Ergebnissen [787] ist damit nicht eindeutig definiert, welche Patienten über eine moderne systemische Polychemo-therapie hinaus von einer chirurgischen Therapie mit HIPEC profitieren.

\subsubsection{Multiviszerale Resektion}

Bei Adhärenz eines Tumors an Nachbarorganen ist makroskopisch nicht sicher zu klären, ob es sich um eine Infiltration des Karzinoms in das Nachbarorgan oder nur um eine peritumoröse Entzündungsreaktion handelt. In solchen Fällen sollten Biopsien und Schnellschnittuntersuchungen strikt vermieden werden, da hierbei stets die Gefahr einer örtlichen Tumorzelldissemination besteht, was mit einer signifikanten Verringerung der Überlebenschancen einhergeht [696]. Daher sollte eine En bloc-Resektion der befallenen Organe durchgeführt werden (multiviszerale Resektion). Im Falle des Rektumkarzinoms können totale Beckenexenterationen notwendig werden.

\subsubsection{Mehrfachkarzinome des Kolorektums}

In diesen Fällen sollte keine regelhafte Kolektomie erfolgen, sondern das Vorgehen unter Berücksichtigung der Erfordernisse der einzelnen Karzinome erfolgen, d.h. auch u. U. die Anlage mehrerer Anastomosen. 


\subsubsection{Notfalloperationen}

Bei lleus, Tumorperforation oder Darmperforation bei stenosierendem Tumor ist das Vorgehen abhängig von der vorliegenden Situation. Nach Möglichkeit ist eine onkologische radikale Resektion entsprechend dem elektiven Vorgehen anzustreben. In geeignet erscheinenden Fällen kann bei einem lleus die Einlage eines endoluminalen Stents diskutiert werden [788]. Ein lleus in Verbindung mit einem Rektumkarzinom geht in der Regel mit weit fortgeschrittenen Karzinomen einher, so dass fast immer eine neoadjuvante Radio-/Chemotherapie anzustreben ist. Aus diesem Grund wird bei dieser Situation häufig primär ein Transversostoma rechts angelegt. Eine tumorbedingte Blutung ist sehr selten relevant für einen Therapieentscheid.

\subsubsection{Resektion von Lebermetastasen}

\begin{tabular}{|c|c|}
\hline 7.50. & Konsensbasiertes Statement \\
\hline & $\begin{array}{l}\text { Die simultane Resektion von Lebermetastasen beeinflusst wahrscheinlich das } \\
\text { Langzeitüberleben im Vergleich zu einem zweizeitigen Vorgehen bei geeigneter } \\
\text { Selektion der Patienten nicht. }\end{array}$ \\
\hline & Starker Konsens \\
\hline
\end{tabular}

7.51 .

Die simultane Lebermetastasenresektion kann bei entsprechender Komorbidität oder höherem Lebensalter (>70 Jahre) zu einer höheren postoperativen Letalität führen.

Konsens

7.52.

Bei multiplen synchronen Lebermetastasen sollte ein zweizeitiges und multimodales Vorgehen gewählt werden.

Konsens

\section{Hintergrund}

Die Entscheidung zur Simultanresektion von Lebermetastasen in Verbindung mit Resektion des Primärtumors hat mehrere Gesichtspunkte zu bedenken:

- $\quad$ Bei rechtsseitigen Kolonresektionen ist der Zugang zur Leber aufgrund der Lagerung des Patienten und der Schnittführung (abgesehen von sehr adipösen Patienten, einer großen Fettleber) in der Regel ohne Schwierigkeiten möglich. Bei linksseitigen Kolon- und Rektumoperationen und der hierfür üblichweise gewählten Lagerung mit Erhöhung des Beckens gestaltet sich der Zugang zur Leber sehr viel schwieriger.

- Periphere Einzelmetastasen können auch bei der Lagerung für linksseitige Resektionen ohne wesentliche Erschwernisse durchgeführt werden, vor allen 
Dingen formale rechtsseitige Resektionen (z.B. Hemihepatektomie re.) gestalten sich nur bei rechtsseitigen Resektionen aufgrund des damit verbundenen Zuganges akzeptabel.

- $\quad$ Gerade kleine, einzelne Metastasen sind häufig von einer weitergehenden metachronen Metastasierung gefolgt, so dass es auch gerechtfertigt ist, trotz gegebener technischer Resektabilität die weitere Entwicklung bzgl. einer möglichen weitergehenden Lebermetastasierung abzuwarten und eventuell eine systemische Chemotherapie zwischenzuschalten.

Als weiteres wird von einzelnen Chirurgen das Konzept der primären Leberresektion ohne jegliche Resektion des Primärtumors oder vorgeschaltete weitere Maßnahmen wie neoadjuvante Chemotherapie bzw. neoadjuvante Radiochemotherapie beim Rektumkarzinom vertreten.

\subsubsection{Ausgedehnte Fernmetastasierung und asymptomatischer Primärtumor}

\subsection{3. \\ Empfehlungsgrad

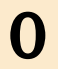 \\ Level of Evidence \\ 4 \\ de Novo: $[789,790]$ \\ starker Konsens}

Evidenzbasierte Empfehlung

2013

Bei Patienten im Stadium IV mit ausgedehnter Lebermetastasierung (Metastasenleber") und asymptomatischem Primärtumor (keine Stenosesymptomatik, keine transfusionspflichtigen Blutungen) kann ohne Resektion des Primärtumors primär eine Chemotherapie durchgeführt werden.

\section{Hintergrund}

Bei Patienten mit ausgedehnter irresektabler Fernmetastasierung sind in der Regel die Fernmetastasen prognosebestimmend. Besteht eine durch den Primärtumor bedingte lleussymptomatik oder eine transfusionspflichtige Blutung ist eine zeitnahe Resektion des Primärtumors bis auf wenige Ausnahmen unerlässlich. Bei einem asympto-matischen Primärtumor ist jedoch unklar, ob eine Resektion für den Patienten einen Vorteil hat. So wird durch die Operation der Beginn einer Chemotherapie hinaus-gezögert. In einer Meta-Analyse von 8 retrospektiven deskriptiven Studien (Zeitraum 1985-2005) fand sich in 7 der Studien ein verlängertes Überleben für Patienten mit Resektion des Primärtumors [789]. Die Studien waren jedoch nicht randomisiert d.h. die Gründe für eine Allokation von Patienten in die primäre OP- oder primäre Chemotherapiegruppe bleiben unklar. Auch erscheint ein medianes Überleben von 6 Monaten in einigen Chemotherapiegruppen deutlich geringer, als mit den heute zur Verfügung stehenden Chemotherapieprotokollen erreichbar. Die Gefahr, dass bei primärer Chemotherapie im Verlauf eine Operation aufgrund eines lleus oder Perforation erforderlich wird, ist nach der derzeitigen Datenlage gering (etwa 7\%) [790]. Die Einleitung einer Chemotherapie mit Belassen eines asymptomatischen Primärtumors bei ausgedehnter Fernmetastasierung erscheint daher gerechtfertigt. Der Stellenwert einer Resektion des Primärtumors bei ausgedehnter Fernmetastasierung wird derzeit im Rahmen einer randomisierten Studie (Synchronous-Studie, Studien-nummer ISRCTN30964555) untersucht. 


\subsubsection{Karzinome auf dem Boden eines hereditären kolorektalen Karzinoms ohne Polyposis (HNPCC)}

\begin{tabular}{|l|l|}
\hline 7.54. & Konsensbasierte Empfehlung \\
\hline EK & $\begin{array}{l}\text { Eine prophylaktische Kolektomie bzw. Proktokolektomie bei HNPCC-Mutations- } \\
\text { trägern soll nicht durchgeführt werden. } \\
\text { Eine subtotale Kolektomie bei Karzinom sollte nicht generell durchgeführt, aber } \\
\text { individuell mit dem Patienten besprochen werden. }\end{array}$ \\
\hline Starker Konsens
\end{tabular}

\subsection{5.}

Empfehlungsgrad

A

Level of Evidence

\section{Evidenzbasierte Empfehlung}

Die koloskopische Überwachung des Patienten nach onkologischer Resektion muss zusätzlich zur üblichen Nachsorge auch postoperativ nach dem gleichen Muster wie vor der Operation fortgesetzt werden. (siehe auch Kapitel 5.2.2.1)

Quellen: [275, 276, 290-293]

Starker Konsens

\section{Hintergrund}

Da durch regelmäßige Vorsorgeuntersuchungen Karzinome bei fast allen Patienten im Stadium UICC I/II oder sogar als prämaligne Adenome entdeckt werden [275, 276] und die Penetranz unvollständig ist, wird von einer prophylaktische Kolektomie bzw. Proktokolektomie abgeraten.

Bei Nachweis eines Karzinoms werden die Patienten nach tumorchirurgischen Gesichtspunkten im Sinne einer onkologischen Resektion operiert. Das Risiko eines kolorektalen Karzinoms im verbliebenen Dickdarm und das Risiko von extrakolischen Neoplasien bleibt jedoch erhöht, so dass diese Patienten einer intensiven postoperativen Nachsorge zugeführt werden müssen. In dieser sollte die Tumornachsorge für sporadische KRK mit dem HNPCC-spezifischen Früherkennungsprogramm für KRK und extrakolische Tumoren kombiniert werden. Ob eine erweiterte prophylaktische Resektion zur Prophylaxe metachroner KRK einer engmaschigen Überwachung überlegen ist, ist derzeit unklar. Die bisherigen Daten aus retrospektiven Fallserien sind unzureichend, und aufgrund nationaler Unterschiede im Bezug auf das ScreeningIntervall nicht auf Deutschland übertragbar (für weitere Informationen siehe Kapitel 5.2.2.1) 


\subsubsection{Karzinome auf dem Boden einer familiären adenomatösen Polyposis (FAP)}

\begin{tabular}{|l|l|}
\hline $\begin{array}{l}\text { 7.56. } \\
\text { Empfehlungsgrad }\end{array}$ & $\begin{array}{l}\text { Evidenzbasierte Empfehlung } \\
\text { B Regeloperation bei FAP-Patienten ist die restaurative Proktokolektomie mit } \\
\text { Dündarm-Pouch und Lymphknotendissektion entsprechend der Lokalisation des } \\
\text { Karzinoms mit den sich hieraus ergebenen Behandlungserfordernissen (z. B. } \\
\text { radikuläre Gefäßdurchtrennung, totale mesorektale Exzision). In Abhängigkeit von } \\
\text { einer eventuellen Sphincterinsuffizienz oder nicht kurablen Tumorerkrankungen } \\
\text { kann auch eine Prokto-Kolektomie oder eine limitierte Resektion durchgeführt } \\
\text { werden. } \\
\text { Bei einer attenuierten FAP mit diskretem Rektumbefall ist eine lleorektostomie zu } \\
\text { empfehlen. (siehe auch 5.2.2.2) }\end{array}$ \\
\hline $\begin{array}{l}\text { Level of Evidence } \\
\mathbf{b}\end{array}$ & \begin{tabular}{l} 
Quellen: [308] \\
\hline
\end{tabular} \\
\hline
\end{tabular}

\subsubsection{Karzinome auf dem Boden einer Colitis ulcerosa}

7.57.
Level of Evidence
$3 \mathbf{b}$

Evidenzbasiertes Statement

Die Regeloperation stellt die restaurative Prokto-Kolektomie mit Dünndarm-Pouch dar, falls aus onkologischen und funktionellen Gründen sinnvoll.

starker Konsens

\section{Hintergrund}

Für weitere Empfehlungen und Informationen z.B. zu Durchführungsbedingungen bei Überwachungskoloskopien, sei auf die S3 Leitlinie Diagnostik und Therapie der Colitis ulcerosa [399] verwiesen. 


\subsection{Postoperative pathohistologische Diagnostik}

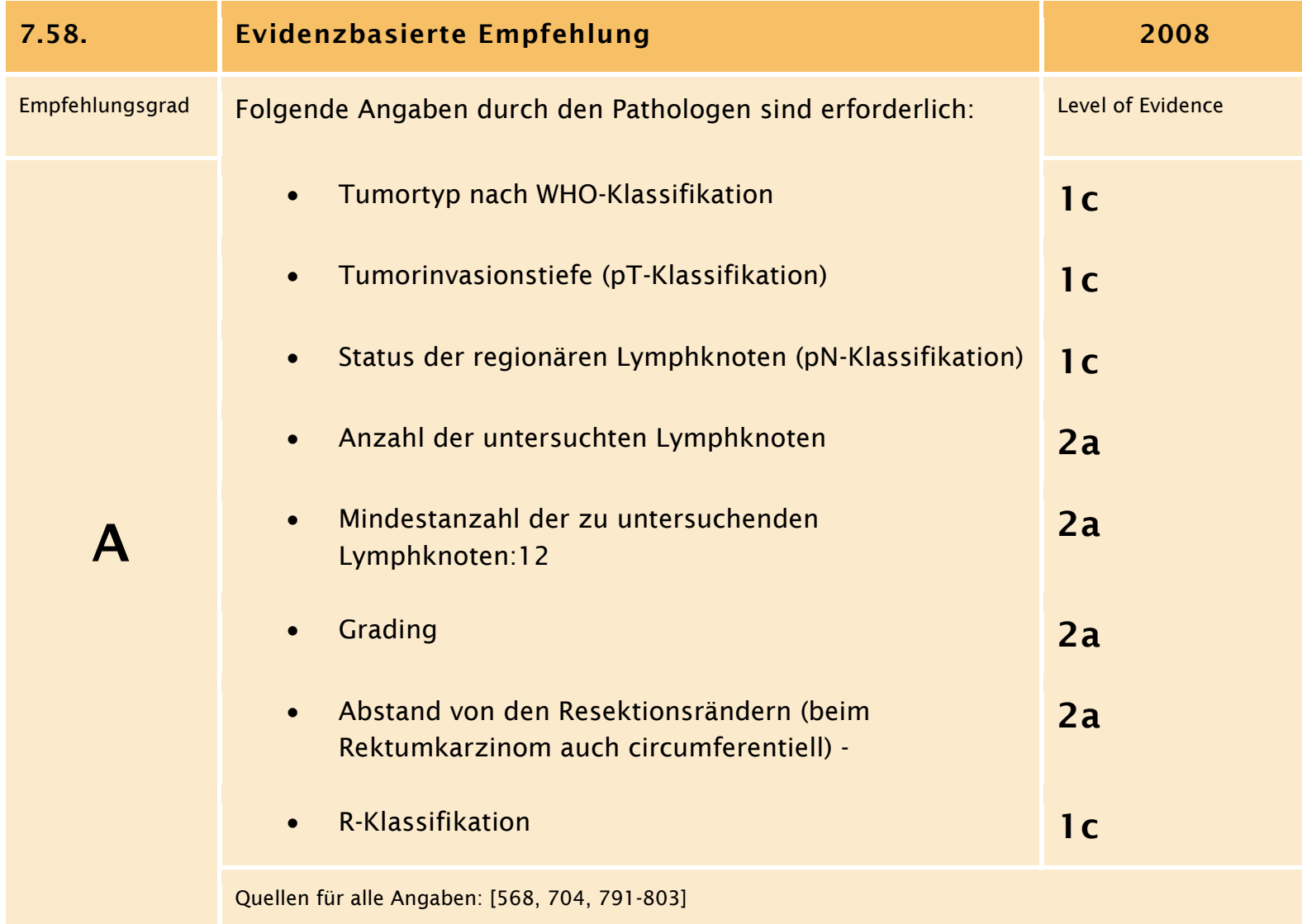

\section{Hintergrund}

Zunehmend wird vor allen Dingen nach neoadjuvanter Radiochemotherapie das Ausmaß der eingetretenen pathohistolgisch fassbaren Remission in Anlehnung an Dworak klassifiziert [803].

\begin{tabular}{l|lc}
\hline 7.59. & Konsensbasierte Empfehlung & 2008 \\
\hline EK & $\begin{array}{l}\text { Die Untersuchung auf Mikrosatelliteninstabilität erfolgt fakultativ bei V.a. Vorliegen } \\
\text { eines HNPCC. }\end{array}$
\end{tabular}

\subsubsection{Karzinom-Graduierung in Abhängigkeit von MSI-H}

\begin{tabular}{|c|c|}
\hline 60. & Konsensbasierte Empfehlung \\
\hline K & $\begin{array}{l}\text { Schlecht differenzierte Adenokarzinome einschließlich muzinöser Adenokarzinome } \\
\text { und undifferenzierte Karzinome sollen immunhistochemisch auf die Expression von } \\
\text { hMLH1 und hMSH2 getestet und bei einem Ausfall von hMLH1 oder hMSH2 als } \\
\text { niedrigaradige (low arade) Karzinome araduiert werden. }\end{array}$ \\
\hline
\end{tabular}

Konsens 


\section{Hintergrund}

Kolorektale Karzinome mit hochgradiger Mikrosatelliteninstabilität (MSI-H) haben eine signifikant günstigere Prognose und niedrigere Fernmetastasierungsrate als kolorektale Karzinome mit Mikrosatellitenstabilität (MSS) oder niedriggradiger Mikrosatelliteninstabilität (MSI-L) [804-807]. Auch wurde gezeigt, dass wenig differenzierte (G3) Adenokarzinome, aber auch muzinöse Adenokarzinome, die bislang als G3 graduiert wurden, bei Vorliegen von MSI-H eine signifikant günstigere Prognose haben als bei Vorliegen von MSS oder MSI-L $[808,809]$. Daher sollen wenig differenzierte Adenokarzinome einschließlich der muzinösen Adenokarzinome und undifferenzierte Karzinome im Dickdarm nicht als hochgradig (high grade) sondern als niedriggradig (low grade) eingestuft werden, wenn Hinweise für MSI-H bestehen.

Der immunhistochemische Nachweis eines Expressionsausfalls für hMLH1 bzw. hMSH2 hat eine vergleichbare prognostische Relevanz wie MSI-H [810] und kann aufgrund seiner Sensitivität und Spezifität als einfacher und kostengünstiger Ersatz für eine molekulare MSI-Testung zur MSI-assoziierten Prognosebewertung eingesetzt werden [811-814]. Die Immunhistochemie für hMLH1 und hMSH2 erfasst die sporadischen MSI-H-Karzinome und einen Teil der MSI-H-Karzinome bei Lynch-Syndrom. Der Zusammen-hang eines kolorektalen Karzinoms mit dem Lynch-Syndrom kann hierdurch nicht zuverlässig bewertet werden und erfordert zusätzliche Analysen.

\subsubsection{Anzahl zu entfernender LK}

7.61 . Konsensbasiertes Statement

EK

12 und mehr Lymphknoten sollen entfernt und untersucht werden.

Starker Konsens

7.62 .

EK

Das Verhältnis von untersuchten zu befallenen Lymphknoten sollte angegeben werden.

Starker Konsens

7.63 .

Konsensbasiertes Statement

2013

EK

Je größer die Zahl der entfernten und untersuchten Lymphknoten, desto besser ist die Prognose der Patienten mit KRK im UICC-Stadium II und III.

Die Zahl der entfernten und untersuchten Lymphknoten kann als Surrogatmarker für Behandlungsqualität gelten.

Die Größe des Lymphknotens korreliert nicht mit der Wahrscheinlichkeit einer Metastase.

Starker Konsens 


\section{Hintergrund}

Die Zahl der entfernten bzw. untersuchten Lymphknoten hängt nicht nur von der Tumorbiologie, sondern auch vom Chirurgen und Pathologen ab. Die 7. Auflage der UICC-TNM-Klassifikation 2010 gibt vor, dass für die Kategorie „pNO“ eine regionäre Lymphadenektomie und histologische Untersuchung von üblicherweise zwölf und mehr Lymphknoten erfolgen sollte.

Obwohl die Studienqualität zur Zahl der Lymphknoten gering ist, gilt jedoch, dass Patienten mit einer größeren Zahl entfernter und untersuchter Lymphknoten eine verbesserte Prognose im UICC-Stadium II und III aufweisen. Der Zusammenhang ist in Kohortenstudien immer wieder gezeigt worden, so an 3411 Patienten im Stadium II und III des sog. Intergroup-Trials [704]. Die sog. INTACC-Studie an 3491 Patienten konnte ebenfalls zeigen, dass die Prognose mit der Zahl der entfernten/untersuchten Lymphknoten korreliert [815]. Dabei ist nicht nur die Zahl der Lymphknotenmetastasen relevant, sondern generell die der Lymphknoten. Beide Studien wiesen auch bei nodalnegativen Tumoren einen prognostischen Effekt nach, der zur Zahl der entfernten/untersuchten Lymphknoten korrelierte [816].

Dieser Effekt wurde in zahlreichen Kohorten nachgewiesen [817-820].

Die Zahl der Lymphknoten kann als Surrogatmarker für die Behandlungs- und Diagnosequalität sowohl für die Chirurgie als auch Pathologie gelten.

Die „optimale Zahl“ der zu entfernenden/untersuchenden Lymphknoten für ein korrektes Staging wird in der Literatur kontrovers diskutiert; sicher ist allerdings, dass zwölf Lymphknoten nicht ausreichend sind. Die alleinige histopathologische Aufarbeitung der größten Lymphknoten am Präparat ist nicht ausreichend, da die Größe des Lymphknotens nicht mit der Wahrscheinlichkeit einer Metastase korreliert.

In der Literatur wird angeregt, das Verhältnis von untersuchten zu befallenen Lymphknoten anzugeben [821-823].

Bei Patienten, die eine präoperative Radiochemotherapie aufgrund eines lokal fortgeschrittenen Rektumkarzinoms erhalten haben, ist die Zahl der Lymphknoten geringer. Eine Analyse von 615 Patienten, die aufgrund eines primären Adeno-karzinoms des Rektums operiert wurden, dass $33 \%$ weniger Lymphknoten gefunden wurden, wenn präoperativ eine neoadjuvante Therapie durchgeführt wurde [824].

\subsubsection{Bedeutung des Tumorabstands zur Resektionsfläche des Mesokolons beim Kolonkarzinom}

\begin{tabular}{|c|c|}
\hline 7.64. & Konsensbasierte Empfehlung \\
\hline EK & $\begin{array}{l}\text { Analog der Qualitätsbewertung von Resektaten beim Rektumkarzinom sollte auch } \\
\text { beim Kolonkarzinom die Qualität des Resektates folgendermaßen kategorisiert } \\
\text { werden: } \\
\text { - Grad } 1 \text { (gut): Mesokolische Schicht erhalten } \\
\text { - Grad } 2 \text { (moderat): Oberflächeneinrisse } \\
\text { - Grad } 3 \text { (schlecht): Einrisse reichen bis auf die Muscularis propria oder Tumor } \\
\text { Diese Bewertungen sind vom Pathologen vorzunehmen. }\end{array}$ \\
\hline
\end{tabular}


Konsens

\section{Hintergrund}

Bezüglich des zirkumferentiellen Sicherheitsabstandes des Primärtumors beim Kolonkarzinom liegen keine vergleichbaren Daten wie zum Rektumkarzinom vor. Zudem besteht bei Kolonkarzinomen sehr viel häufiger die Möglichkeit, trotz ausgedehnter Tiefeninfiltration des Primärtumors die Resektion angrenzender Strukturen oder Organe zu einem ausreichenden zirkumferentiellen Sicherheitsabstand zu kommen. In Ausnahmefällen ist dies nicht möglich (z.B. beim Sigmakarzinom, welches den Iliacalgefäßen aufsitzt).

Gegebenenfalls sollte deshalb analog zum Rektumkarzinom der zirkumferentielle Sicherheitsabstand dokumentiert werden.

Zwischenzeitlich liegen jedoch Publikationen analog zum Rektumkarzinom über die Qualität der Bewertung von Kolonkarzinomresektaten vor. Ebenso wie beim Rektumkarzinom ist inzwischen belegt, dass Einrisse bis auf die Muscularis propria oder auf den Tumor zu einer schlechteren Überlebensrate führen (15\% geringere 5-JahresÜberlebenswahrscheinlichkeit bei Muscularis propria vs. Mesokolischer Schicht), wobei der Unterschied im Falle von Lymphknotenmetastasen (UICC Stadium III) stärker ausgeprägt ist (27\% höhere Überlebenswahrscheinlichkeit nach 5 Jahren Follow-up), als bei fehlenden Lymphknotenmetastasen [825, 826].

\subsubsection{Bedeutung des Abstands vom zirkumferentiellen Resektionsrand (CRM-Klassifikation) beim Kolon- und Rektumkarzinom}

\begin{tabular}{l|l|}
\hline 7.65. & Konsensbasierte Empfehlung \\
\hline EK & $\begin{array}{l}\text { Der zirkumferentielle Sicherheitsabstand ist negativ, wenn er 1 mm oder mehr beträgt } \\
\text { (R0 „wide“). Ein positiver zirkumferentieller Sicherheitsabstand liegt vor, wenn der } \\
\text { zirkumferentielle Sicherheitsabstand weniger als } 1 \mathrm{~mm} \text { beträgt (R0 "close") oder } \\
\text { Tumorgewebe direkt an ihn heranreicht (R1). Der gemessene Abstand soll in Zahlen } \\
\text { dokumentiert werden. }\end{array}$ \\
& Starker Konsens
\end{tabular}

\section{Hintergrund}

Nach den offiziellen Definitionen der AJCC wird der residuale Tumor als R1 definiert, wenn ein Karzinom tatsächlich histologisch bis an den Resektionsrand (RR) heranreicht. 


\title{
R0 (kurative Resektion)
}

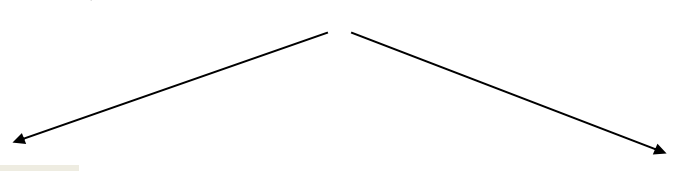

\section{R0 wide}

\section{R0 narrow}

\author{
Abstand $>0,1 \mathrm{~cm}$ \\ Abstand $\leq 0,1 \mathrm{~cm}$ \\ CRM negativ \\ CRM positiv
}

\section{Abstand des Tumors zum RR angeben}

Berücksichtigt werden kontinuierliche Primärtumorausläufer, Satelliten, Lymphgefäß-/Veneninvasion, Perineuralscheideninvasion,

Lymphknotenmetastasen

Vor allem Arbeiten aus den Niederlanden und Großbritannien haben jedoch gezeigt, dass auch bei einem zirkumferentiellen Sicherheitsabstand von weniger als $1 \mathrm{~mm}$ das Lokalrezidivrisiko beim Rektumkarzinom deutlich ansteigt (3-Jahres Lokalrezidivraten: $6 \%$ bei CRM- und $17 \%$ bei CRM+ ; 3-Jahres tumorfreies Überleben $79 \%$ bei CRM- und $50 \%$ bei CRM+ [685, 827].

Ob bereits ein Sicherheitsabstand von weniger als $2 \mathrm{~mm}$ zu einer verschlechternden Prognose führt, wird kontrovers diskutiert [828, 829]. 


\subsubsection{Bedeutung der Angabe der Qualität des TME- Präparats}

7.66.

Konsensbasierte Empfehlung

2013

EK

Da die Qualität eines Operationsresektates unter Berücksichtigung der oben genannten Kategorien Rückschlüsse auf die Prognose bezüglich der Entwicklung eines Lokalrezidives zulässt, soll diese obligat im patho-histologischen Befundbericht wie folgend beschrieben werden:

Die Qualität des Präparates wird beurteilt an der Integrität der mesorektalen Faszie im Falle der Resektion mit 3 Kategorien:

- Grad1 (gut): Mesorektale Faszie erhalten

- $\quad \operatorname{Grad} 2$ (moderat): Intramesorektale Einrisse

- Grad 3 (schlecht): Erreichen der Muscularis propria oder Tumor

Im Falle einer Rektumexstirpation sind bei einer vollständigen Resektion der Levatormuskulatur Präparateinrisse und ein tumorpositiver zirkumferentieller Sicherheitsabstand seltener. [830]

Im patho-histologischen Befundbericht ist deshalb die Beschreibung bezüglich der Radikalität im Bereich der Levatormuskulatur obligat. Hierzu sollen folgende Kategorien Verwendung finden:

- Grad 1 (gut): Levatormuskulatur mitreseziert, keine Eröffnung des Darmes oder des Tumors

- Grad 2 (moderat): Muscularis propria erhalten, keine Eröffnung des Darmes oder des Tumors

- Grad 3 (schlecht): Teile der Muscularis propria fehlen oder Eröffnung des Darmes oder des Tumors

Diese Bewertungen sind vom Pathologen vorzunehmen.

Konsens

\section{Hintergrund}

Die Qualität eines Rektumresektates hat signifikanten Einfluss auf die Lokalrezidivrate.

Bei intaktem Mesorektum betrug das 5-Jahres tumorfreie Überleben $65 \%$ im Vergleich zu 47\% bei Defekten des Mesorektums $(P<0.05)$ [831].

Nach 3 Jahren Follow-up betrug die Lokalrezidivrate bei intakter mesorektaler Faszie 4\% (3-6\%), 7\% (5-11\%) bei intramesorektalen Einrissen, und 13\% (8-21\%) bei Erreichen der Muscularis Propria-Ebene [827].

Bei der Beurteilung eines Präparates nach einer Rektumexstirpation wird zwischen zylindrischer Exzision und Standardexzision unterschieden. Nach einer zylindrischen Exzision ist der zirkumferentielle Resektionsrand signifikant seltener befallen und Perforationen des Präparates sind ebenfalls signifikant seltener. [825, 830]. Daten zur Auswirkung auf Lokalrezidivraten und Überlebensraten liegen bislang nicht vor.

Die Beurteilung der Qualität des chirurgischen Präparates sollte anhand der obenstehenden genannten Kriterien durch den Pathologen und nicht den Chirurgen erfolgen. 


\subsection{Psychoonkologische Aspekte}

\begin{tabular}{|l|l|}
\hline $\begin{array}{l}\text { 7.67. } \\
\text { Evidenzbasierte Empfehlung/ EK }\end{array}$ & 2013 \\
\hline $\mathbf{B}$ & $\begin{array}{l}\text { Psychoonkologische Behandlungsmaßnahmen sollten in das Gesamtkonzept der } \\
\text { onkologischen Therapie integriert werden. }\end{array}$ \\
\hline $\begin{array}{l}\text { Level of Evidence } \\
\mathbf{1} \text { b }\end{array}$ & Quellen: [833-860] \\
\hline
\end{tabular}

\begin{tabular}{|l|l|l|}
\hline 7.68. & Konsensbasierte Empfehlung/ $\mathbf{E K}^{9}$ & 2013 \\
\hline EK & $\begin{array}{l}\text { Alle Patienten sollten von ärztlicher Seite frühzeitig über Möglichkeiten psycho- } \\
\text { onkologischer Hilfestellungen informiert werden. }\end{array}$
\end{tabular}

\section{Hintergrund}

Über den gesamten Verlauf einer Krebserkrankung treten behandlungsbedürftige psychische Belastungen und Störungen mit einer Häufigkeit von 20 - 35\% (Krebspatienten aller Tumorlokalisationen und Krankheitsstadien) auf. Es überwiegen Anpassungsstörungen ( $F$ 43.12), akute Belastungsreaktionen ( $F$ 43.0) gefolgt von depressiven Störungen (Major Depression 8-20\%, Dysthymie 5-15\%) [835-838]. Für die Gruppe der Patienten mit kolorektalen Karzinomen liegen vergleichbare Zahlen vor [839, 840]. Fortgeschrittenes Krankheitsstadium, ausgeprägte funktionelle Beein-trächtigung und hoher somatischer Beschwerdedruck sind mit höherem Risiko psychischer Störungen verbunden [841]. Die zusätzliche Anlage eines Kolostoma bedeutet in der Regel eine eingreifende Veränderung für betroffene Patienten. Die Akzeptanz fällt umso schwerer, je beeinträchtigender die Funktionseinschränkungen sind und je massiver die körperliche Entstellung empfunden wird. Das Selbstwertgefühl der Patienten kann durch ein Stoma deutlich vermindert werden, sodass körperliche, sportliche und soziale Aktivitäten wie auch die Aufnahme der beruflichen Tätigkeit als schwierig und belastend erlebt werden und zu psychischen Beeinträchtigungen führen können. Gerade die äußerlich sichtbare Körperveränderung durch ein Stoma erschwert die Anpassung und führt zu Selbstwert- und Anpassungsstörungen bis hin zu einer Depression [840, 842]. Bei vielen Betroffenen werden Scham- und auch Ekelgefühle sowie Schmutz- und Geruchsängste zu einer großen psychischen Belastung, die das Bedürfnis nach Intimität gänzlich in den Hintergrund treten lassen. Insofern empfinden Stomaträger häufig ihr Sexualleben durch das Stoma negativ beeinflusst [843].

Ein beträchtlicher Prozentsatz psychischer Störungen bei Tumorpatienten wird nicht zutreffend diagnostiziert und bleibt unzureichend behandelt [837, 844, 845] mit

\footnotetext{
8 Entnommen der Interdisziplinären S3-Leitlinie für die Diagnostik, Therapie und Nachsorge des Mammakarzinoms 832. Kreienberg, R., et al. Interdisziplinäre S3-Leitlinie für die Diagnostik, Therapie und Nachsorge des Mammakarzinoms. Informationszentrum für Standards in der Onkologie (ISTO), Deutsche Krebsgesellschaft e.V., 2008. 1. Aktualisierung

9 Entnommen der Interdisziplinären S3-Leitlinie für die Diagnostik, Therapie und Nachsorge des Mammakarzinoms 832. Ibid.
} 
nachteiligen Auswirkungen auf körperliches Befinden, Funktionsstatus, Beschwerden (Schmerzen, Übelkeit, Fatigue) und die Lebensqualität der Patienten. Deshalb sollte das psychische Befinden der Patienten regelmäßig im Krankheitsverlauf, d.h. in allen Krisenphasen und zu Zeiten mit erwartbar hoher Belastung ermittelt werden. Neuere Studien sprechen für die Wirksamkeit von Vorsorge- / Früherkennungs-basierten psychosozialen Interventionen bei Tumorpatienten [836, 846, 847]. Vorsorge- / Früherkennungs-Verfahren beinhalten die Beantwortung einiger einfacher gezielter Fragen durch den Patienten, entweder im persönlichen Kontakt oder mit Hilfe eines Fragebogens. Verschiedene Screening-Verfahren stehen zur Identifizierung behandlungsbedürftiger Patienten mit hoher psychischer Belastung bzw. Komorbidität zur Verfügung. Eine Übersicht mit Darstellung verschiedener Screeningverfahren findet sich bei [848], online erhältlich unter www.pso-ag.de .

Professionelle psychische Unterstützung/Mitbehandlung soll für alle Patienten und deren Angehörige verfügbar sein. Dies kann in Form eines psychoonkologischen, eines psychosomatisch oder psychiatrischen Konsil-/Liaison-Dienstes, durch psychoonkologische Fachkräfte in Organ- und onkologischen Zentren oder durch Einbe-ziehung niedergelassener ärztlicher oder psychologischer Psychotherapeuten mit psychoonkologischer Qualifizierung erfolgen [849-851]. Sie hat in enger Kooperation mit und Rückmeldung an die behandelnden Ärzte und Pflegekräfte zu erfolgen.

Alle Patienten mit kolorektalem Karzinom sollen von ihren medizinischen Behandlern (Ärzten/Pflegekräften) über die Verfügbarkeit professioneller psychischer Unterstützung bzw. Mitbehandlung informiert werden.

Die Wirksamkeit unterschiedlicher psychoedukativer und psychotherapeutischer Interventionen bei Tumorpatienten hinsichtlich Symptomreduktion (Depression, Angst, Schmerzen, Fatigue), Krankheitsverarbeitung und Verbesserung der Lebensqualität ist gesichert [834, 845, 852-860].

Weiterführend wird auf die S3-Leitlinie Psychoonkologie, hingewiesen, AWMF Registernummer: 032/0510L: http://www.leitlinienprogrammonkologie.de/leitlinien/psychoonkologie/ 
8. Adjuvante und neoadjuvante Therapie

8.1. Adjuvante Therapie des Kolonkarzinoms

8.1.1. Indikation zur adjuvanten Behandlung bei Kolonkarzinomen

Voraussetzung für eine adjuvante Therapie ist die R0-Resektion des Primärtumors. Grundlage für die Indikation zur adjuvanten Therapie nach qualitätsgesicherter Tumorresektion [861] ist die pathohistologische Stadienbestimmung, insbesondere die Bestimmung des pN-Status. Zur Festlegung von pNO sollen 12 oder mehr regionäre Lymphknoten untersucht werden (UICC 2002). Immunzytologische Befunde von isolierten Tumorzellen in Knochenmarkbiopsien oder Lymphknoten sowie zytologische Tumorzellbefunde in Peritonealspülungen sind keine Indikation zur adjuvanten Therapie außerhalb von Studien.

Für Patienten mit einem kurativ resezierten Kolonkarzinom im Stadium I ist eine adjuvante Therapie nicht indiziert. Patienten des UICC Stadiums II und III sollten möglichst in kontrollierte Studien eingebracht werden, um Aufschluss über die Indikationsstellung und die optimale adjuvante Therapie zu erhalten. Der Verlauf von Patienten, die außerhalb klinischer Studien behandelt werden, sind im Rahmen der Qualitätssicherung das Auftreten von Rezidiven, die Überlebensrate und Nebenwirkungen zu dokumentieren. Die Durchführung der adjuvanten Chemotherapie erfordert einschlägige Erfahrung und insbesondere die Kenntnis der entsprechenden Dosisreduktionsschemata, die bei auftretender Toxizität eingehalten werden müssen.

\section{Kontraindikationen der adjuvanten Chemotherapie bei Kolonkarzinomen}

- $\quad$ Allgemeinzustand schlechter als 2 (ECOG)

- Unkontrollierte Infektion

- $\quad$ Leberzirrhose Child B und C

- $\quad$ Schwere koronare Herzkrankheit, Herzinsuffizienz (NYHA III und IV)

- $\quad$ Präterminale und terminale Niereninsuffizienz

- $\quad$ Eingeschränkte Knochenmarkfunktion

- $\quad$ Andere, die Lebenserwartung relevant beeinflussende Komorbiditäten

- Unvermögen, an regelmäßigen Kontrolluntersuchungen teilzunehmen

\subsubsection{Altersbeschränkung für die Durchführung einer adjuvanten Chemotherapie}

\begin{tabular}{|l|l|l|} 
8.1. & Evidenzbasierte Empfehlung & 2017
\end{tabular}

EK Alleine aus Altersgründen sollte eine adjuvante Chemotherapie nicht unterlassen werden. Bei Patienten über 75 Jahre gibt es jedoch keine ausreichende Evidenz für die Durchführung einer adjuvanten Chemotherapie.

Starker Konsens 


\section{Hintergrund}

Analysen einer kanadischen Datenbank, welche Patienten $(n=2.801)$ in Ontario aus dem Zeitraum 2002-2008 untersuchten, zeigen, dass Patienten welche 70-79 Jahre alt waren zu $68 \%$ und Patienten welche $>80$ Jahre alt waren immerhin noch zu $24 \%$ im Stadium III eine adjuvante Therapie erhielten [862]. In dieser retrospektiven Analyse profitierten alle Altersgruppen von einer adjuvanten Chemotherapie. Allerdings war die Gabe einer adjuvanten Therapie mit dem Charlson Comorbiditäts Index assoziiert, so dass nur „medical“ fitte ältere Patienten eine adjuvante Therapie erhielten.

Daten aus prospektiven randomisierten Studien zum Einfluss einer adjuvanten Chemotherapie bei älteren Patienten sind durch ihre kleinen Fallzahlen in ihrer Aussagekraft limitiert. Grund hierfür ist, dass in den meisten Studien eine Altersbeschränkung als Einschlusskriterium vorlag. So konnten in die MOSAIC Studie keine Patienten $>75$ Jahre eingeschlossen werden [863]. Die NSAPB C-07 Studie hatte zwar keine Altersbegrenzung, allerdings waren nur 396 der ursprünglich 2.409 Patienten älter als 69 Jahre. Daher muss bei der Frage nach dem Einfluss des Alters auf die Gabe einer adjuvaten Chemotherapie auf gepoolte Analysen klinischer Studien zurückgegriffen werden. Gepoolte Analysen der NSABP-C08, XELOXA, X-ACT und AVANT Studien ergaben, dass in allen Altersgruppen eine Therapie mit FOLFOX/XELOX diskutiert werden kann [864]. Die Hazard Ratio für eine adjuvante Therapie mit Oxaliplatin erreichte bei Patienten >69 Jahre 0,78 für das Gesamtüberleben, wohingegen die jüngeren Patienten mit einer HR von 0,62 einen deutlich höheren Benefit von der Oxaliplatin-haltigen Therapie hatten. Allerdings wiesen ältere Patienten eine höhere Rate an unerwünschten Nebenwirkungen auf. Vergleichbare Daten konnten in der NO16968 Studie eruiert werden. In einer explorativen Analyse war der Nutzen von XELOX gegenüber dem Vergleichsarm 5-FU/FA (Bolus-Regime) auch bei Patienten $>69$ Jahre $(n=$ 409 von 1888 ) erhalten [865]. So war die Hazard Ratio für 7-Jahres OS bei $>69$ Jährigen

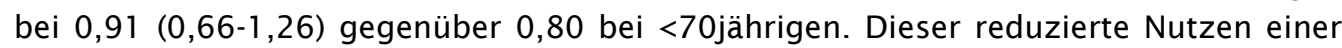
adjuvanten Therapie bei Älteren war auch in einer Analyse der ACCENT Datenbank zu sehen. Während die Gabe von Capecitabine ihre Effektivität auch bei Älteren zeigte, war die Kombination aus Fluropyrimidin mit Oxaliplatin bei Älteren nicht effektiv [866]. Das Lebensalter eines Patienten hat somit keinen alleinigen prädiktiven Wert [867]. Bei älteren Patienten ist der Benefit einer adjuvanten Therapie allerdings kleiner bei gleichzeitig höherer Toxizität.

\begin{tabular}{|c|c|}
\hline 8.2. & Evidenzbasierte Empfehlung \\
\hline $\begin{array}{l}\text { Empfehlungsgrad } \\
\text { B }\end{array}$ & Die adjuvante Chemotherapie sollte baldmöglichst postoperativ eingeleitet werden. \\
\hline $\begin{array}{l}\text { Level of Evidence } \\
3 \mathbf{b}\end{array}$ & Quellen: [868-870] \\
\hline & Starker Konsens \\
\hline
\end{tabular}




\begin{tabular}{|l|l|}
\hline 8.3. & Evidenzbasiertes Statement \\
\hline $\begin{array}{l}\text { Level of Evidence } \\
\text { 1 b }\end{array}$ & $\begin{array}{l}\text { In den randomisierten Studien wurde die adjuvante Chemotherapie innerhalb von } 8 \\
\text { Wochen eingeleitet. }\end{array}$
\end{tabular}

\section{Hintergrund}

Randomisierte Studien zu dieser Fragestellung existieren nicht. In einer retrospektiven Analyse von Kohortenstudien [868]. wurde eine inverse Korrelation zwischen dem Zeitpunkt des Beginns einer adjuvanten Chemotherapie und dem Überleben berechnet. Dies wurde auch in einer anderen retrospektiven Analyse von Kohortenstudien [869] und in einer retrospektiven Registeranalyse bestätigt [870].

Eine kleine retrospektive Studie $(n=186)$ weist darauf hin, dass der Beginn einer adjuvanten Therapie später als 60 Tage nach der Operation zu einem verschlechterten Gesamtüberleben führen kann [871]. Diese wichtige Fragestellung wird auch an 1.053 Pat. mit einen Stadium III Kolonkarzinom untersucht (OP 2000-2005) [872]. Bei 648 Patienten (61\%) wurde die adjuvante Chemotherapie innerhalb von 16 Wochen nach Operation begonnen. Patienten, die adjuvante Chemotherapie später als 12 Wochen nach der Operation erhielten, wiesen ein schlechtes sozioökonomisches Niveau und mehr Komorbiditäten auf. Patienten, die die adjuvante Chemotherapie 12-16 Wochen nach der Operation erhielten, hatten eine 1,4-fach erhöhte Mortalität verglichen mit solchen Patienten, die die Behandlung innerhalb von 8 Wochen nach Operation erhielten. Patienten, die die adjuvante Chemotherapie nicht innerhalb von 16 Wochen erhielten, hatten eine mehr als zweifach erhöhte Mortalitätsrate verglichen mit solchen Patienten, die die Therapie innerhalb von 8 Wochen erhielten. Die karzinomspezifische Mortalität erhöhte sich in diesem Patientenkollektiv um $76 \%$.

In einer weiteren retrospektiven Analyse (1997-2012) wird untersucht, ob der Zeitpunkt des Beginns einer adjuvanten Therapie (<oder > 8 Wo.) oder eine erforderliche Reoperation Einfluss auf die Prognose haben [873]. Eine erforderliche Nachoperation war ein wesentlicher Faktor für die Verschiebung einer adjuvanten Therapie (OR 2,3). Zwischen den Patienten, die keine adjuvante Therapieverzögerung, aber eine Nachoperation hatten, und den Pat., die weder eine adjuvante Therapieverzögerung noch eine Nachoperation hatten, bestand kein Unterschied im Überleben. Patienten, die eine adjuvante Therapieverzögerung ohne Nachoperation hatten, besaßen jedoch eine signifikant schlechtere Prognose als solche Pat., die weder eine adjuvante Therapieverzögerung noch eine Nachoperation hatten (Kolon: HR 1,16; Rektum: HR 1,17). Auch Patienten, die sowohl eine adjuvante Therapieverzögerung als auch eine Nachoperation hatten, hatten ein schlechteres Gesamtüberleben als solche Pat., die beides nicht hatten. 


\subsubsection{UICC-Stadium III}

8.4 .

Empfehlungsgrad

A

Level of Evidence

1 a

\section{Evidenzbasierte Empfehlung}

Bei Patienten mit einem RO resezierten Kolonkarzinom im Stadium III soll eine adjuvante Chemotherapie durchgeführt werden.

Quelle: [874-877]

Starker Konsens

\section{Hintergrund}

In zahlreichen randomisierten Studien konnte ein signifikanter Überlebensvorteil für Patienten mit Kolonkarzinom im Stadium III durch eine adjuvante Chemotherapie nachgewiesen werden [878, 879]. Meta-Analysen und gepoolte Analysen (Gill et al.) bei 3.303 Patienten mit einem Stadium II und III Kolonkarzinom konnten eindeutig zeigen, dass für Patienten mit einer Lymphknoten positiven Erkrankung (Stadium III) die Durchführung einer adjuvanten Chemotherapie zu einer signifikanten Prognoseverbesserung im Vergleich zur alleinigen Operationen führt [874-877].

\subsubsection{UICC-Stadium II}

8.5.
Empfehlungsgrad
$\mathbf{0}$
Level of Evidence
1 b

\section{Evidenzbasierte Empfehlung}

Bei Patienten mit einem kurativ resezierten Kolonkarzinom im Stadium II kann eine adjuvante Chemotherapie durchgeführt werden.

Quellen: [880-884]

Starker Konsens

\section{Hintergrund}

Der Nutzen einer adjuvanten Therapie im UICC Stadium II ohne Risikofaktoren liegt absolut zwischen 2-5\% im 5-Jahresüberleben. Studien und gepoolte Analysen von Studien bei Patienten mit einem Kolonkarzinom im Stadium II fand sich kein signifikanter Überlebensvorteil durch eine postoperative adjuvante Chemotherapie [880-883]. Die gepoolte Analyse von 7 randomisierten Studien, die eine adjuvante Chemotherapie mit einer alleinigen Operation verglichen, zeigte in der univariaten Analyse nur eine signifikante Verbesserung für das 5 Jahres krankheitsfreie Überleben (DFS) (72 versus 76\%; $p=0.049$ ), aber nicht für das 5 Jahres Gesamtüberleben ( 80 versus $81 \% ; p=0.1127$ ) im Stadium II, wobei sich die Einzelstudien deutlich in den Therapiemodi unterschieden und kleine Patientenzahlen einschlossen [875]. In einem Kollektiv von 43.032 Medicare Empfängern (> 66 Jahre; Stadium III 18.185 Pat., $57 \%$ erhielten eine adjuv. Chemotherapie) besaßen im Stadium II 6.234 Pat. kein (19\% mit adjuv. Chemotherapie) und 18.613 Pat. mindestens ein prognostisch ungünstiges Kriterium (21 \% mit adjuv. 
Chemotherapie). Die 5-Jahresüberlebensraten in diesen drei Gruppen betrugen $44 \%$, $69 \%$ und $57 \%$. Während in beiden Stadium II Gruppen die Chemotherapie keinen Einfluss auf das Überleben hatte, verbesserte die Chemotherapie im Stadium III das Überleben signifikant (5-JÜLR: 48,9 \% vs. 35,2\%). [885] .

Die britische QUASAR-Studie ist die für diese Fragestellung größte publizierte Einzelstudie [884]. Hier war in einem Beobachtungszeitraum von (im Median) 5,5 Jahren das relative Risiko für einen Tod jedweder Ursache in der Therapiegruppe signifikant

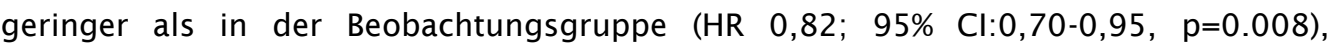
entsprechend einem absoluten Überlebensvorteil von ca. 3,0 \% (95\% Cl:1,0-6,0). Allerdings hat diese Studie methodische Schwächen hinsichtlich ihres heterogenen Studienkollektivs (71\% Kolonkarzinome, 91\% Dukes' B Stadien) und der heterogenen 5FU-haltigen Therapieprotokolle (mit oder ohne Levamisol, Folinsäuredosierung unterschiedlich). Das Relative Risiko für die isolierte Subgruppe Kolonkarzinom im Stadium II war nicht signifikant verringert, allerdings war der Effekt in allen Subgruppen konsistent gleich groß, so dass ein Überlebensvorteil in allen Prognosegruppen angenommen werden kann. Hinsichtlich der Bedeutung dieser Studie für die so definierte "Hochrisikosituation" (s.u.) kann keine Empfehlung abgeleitet werden, da lediglich für ca. $20 \%$ der Patienten Daten für die T-Kategorie und/oder eine vaskuläre Invasion vorliegen und von diesen $20 \%$ die wenigsten Patienten tatsächlich eine T4 oder V1- Situation aufwiesen.

Für den Einsatz von Oxaliplatin im Stadium II liegen keine überzeugenden Daten vor: In einer Untergruppenanalyse wurde über den Effekt einer adjuvanten postoperativen Chemotherapie (FOLFOX4 versus LV5FU2) im Stadium II berichtet [886]. Für das Stadium II konnte weder eine signifikante Verbesserung des krankheitsfreien Überlebens noch ein Überlebensvorteil für die zusätzlich mit Oxaliplatin behandelten Patienten mit einem Stadium II Tumor belegt werden. Aus den bisher vorliegenden randomisierten kontrollierten Studien lässt sich somit ein obligater Einsatz einer adjuvanten Chemotherapie im Stadium II nicht ableiten [887-889]. Aufgrund der positiven Ergebnisse der bislang größten Untersuchung, der QUASAR Studie, kann jedoch - bei allen methodischen Mängeln - der Nutzen einer adjuvanten Therapie im Stadium II ohne Risikofaktoren nicht gänzlich ausgeschlossen werden. Deshalb sollte eine Therapie in diesem Stadium zumindest in Betracht gezogen werden [884], in jedem Fall sollten die Vorteile und Risiken einer solchen Therapie mit dem Patienten besprochen werden. 


\subsubsection{UICC-Stadium II mit Risikofaktoren}

\begin{tabular}{|l|l|}
$\mathbf{8 . 6 .}$ & Evidenzbasierte Empfehlung \\
\hline $\begin{array}{l}\text { Empfehlungsgrad } \\
\mathbf{B}\end{array}$ & $\begin{array}{l}\text { Im Stadium II sollte in ausgewählten Risikosituationen (T4, Tumorperforation/- } \\
\text { einriss, Operation unter Notfallbedingungen, Anzahl untersuchter Lymphknoten zu } \\
\text { gering) eine adjuvante Chemotherapie erwogen werden. }\end{array}$ \\
\hline $\begin{array}{l}\text { Level of Evidence } \\
\mathbf{3} \mathbf{b}\end{array}$ & \begin{tabular}{l} 
Quellen: $[704,879,890]$ \\
\hline
\end{tabular} \\
\hline & Starker Konsens \\
\hline
\end{tabular}

\section{Hintergrund}

Die oben genannten Faktoren sind als prognostisch ungünstig identifiziert worden. Es erscheint daher denkbar, dass Patienten mit diesen Risikofaktoren auch im Stadium II von einer adjuvanten Chemotherapie profitieren könnten. Es existieren jedoch keine prospektiven Daten zum Zusammenhang der aufgeführten Risikocharakteristika und dem Nutzen einer adjuvanten Chemotherapie. Daher sollen vor Einleitung einer adjuvanten Chemotherapie auch in dieser Untergruppe mit dem Patienten in einem intensiven Gespräch die Vor- bzw. Nachteile einer adjuvanten Chemotherapie vor dem Hintergrund der aktuellen Datenlage erörtert und die Patientenpräferenzen angesprochen werden.

In Studien gingen gewisse Risikosituationen wie ein T4-Tumor, eine Tumorperforation und/oder Operation unter Notfallbedingungen sowie eine zu geringe Anzahl untersuchter Lymphknoten mit einer schlechteren Prognose einher [891, 892]. Eine retrospektive Untersuchung an 1306 Patienten mit einem Stadium II Tumor zeigte in einer multivariaten Analyse, dass die Kategorie T4 mit einem schlechten krankheitsfreien Überleben assoziiert war (HR 1,75) [893]. In der Studie von Moertel $(n=318)$ besitzt die T4-Kategorie im Stadium II keine zusätzliche prognostische Bedeutung [887], wohl aber in der Studie von Burdy $(n=108)$ [894], in der Erlanger Analyse $(n=305)$ [891] und in der veröffentlichten Metaanalyse von Gill [875].

Nach einer Notfalloperation resultierte mit 29,8 vs $52,4 \%$ eine signifikant niedrigere 5 Jahres-Überlebensrate $(\mathrm{p}<0.001)$. Dieser Unterschied zeigte sich sowohl im Stadium I/II als auch im Stadium III [895]. Das karzinomspezifische Überleben nach 5 Jahren reduzierte sich von 74,6\% auf 60,9\% bei Nachweis einer Anämie, auf 51,6\% bei Nachweis einer Stenose und auf $46,5 \%$ bei Perforation $(p<0.001)$ [896]. Auch die Anzahl der untersuchten Lymphknoten galt in mehreren Studien als ein unabhängiger prognostischer Faktor [704, 890]. So betrug bei 222 Patienten mit einem KRK im Stadium II die 5-Jahresüberlebensrate 49\% für Patienten, bei denen 6 oder weniger als 6 Lymphknoten untersucht worden waren, verglichen mit 68\% für Patienten mit 7 oder mehr untersuchten Lymphknoten [890]. Le Voyer (INT-0089, n=3.411) untersuchte Patienten im Stadium Dukes B2 oder Dukes C mit adjuvanter Therapie mit 5-FU, Folinsäure (FS) und/oder Levamisol. Sowohl bei N0, N1 als auch N2 hatte die Anzahl der entfernten Lymphknoten eine prognostische Bedeutung. Patienten mit N0-Tumoren hatten das beste Gesamtüberleben, wenn mehr als 20 Lymphknoten analysiert wurden [704]. In einer Untersuchung von 3592 Fällen mit kolorektalem Karzinom konnte eine englische Arbeitsgruppe [897] jeweils einen signifikanten Überlebensvorteil für Patienten mit einer größeren Zahl identifizierter Lymphknoten errechnen (0-4 
Lymphknoten, 5-10 Lymphknoten, >10 Lymphknoten). Dieser Effekt zeigt sich auch in jedem Tumorstadium. In der multivariaten Analyse erwies sich die Zahl untersuchter Lymphknoten als unabhängiger prognostischer Faktor. Eine Analyse der SEER-Datenbank [898] korreliert die Zahl der untersuchten bzw. entfernten Lymphknoten mit dem Langzeitüberleben. In der multivariaten Analyse zeigte sich eine Reduktion der Krebsmortalität um 20,6\% wenn mehr als 15 Lymphknoten untersucht wurden, verglichen mit Patienten bei denen nur 1-7 Lymphknoten untersucht wurden. Dieses Ergebnis war unabhängig vom Tumorstadium und sonstigen Patienten- oder Tumorcharakteristika. Auch wenn die Studienlage hinsichtlich der genauen Anzahl der zu untersuchenden Lymphknoten nicht einheitlich ist, sollten nach Meinung von Experten 12 Lymphknoten als Mindestmaß gefordert werden, auch wenn diese Zahl nicht immer flächendeckend erreicht werden kann. In diesem Zusammenhang sei auf das Kapitel „Chirurgie“ verwiesen.

In einer Studie, in denen Patienten mit Stadium II Tumoren und Hochrisikocharakteristika eine kleine Untergruppe darstellten, konnte kein Benefit einer adjuvanten Chemotherapie gegenüber der alleinigen operativen Therapie gezeigt werden [879]. Im Gegensatz dazu stellten in der MOSAIC-Studie Patienten mit Stadium II Tumoren mit einem T4 Tumor, einer Tumorperforation, einem Ileus, einem schlecht differenzierten Tumor, einer Blutgefäßinvasion und/oder weniger als 10 untersuchten Lymphknoten eine Hochrisikopopulation dar, bei der die postoperative adjuvante FOLFOX4 Chemotherapie im Vergleich zu einer 5FU/FS Chemotherapie zu einer nicht-signifikaten Verbesserung des $10 \mathrm{~J}$. krankheitsfreien Überlebens um 3,7\% Prozent-punkte führte. Es konnte auch keine signifikante Verbesserung des $10 \mathrm{~J}$. Gesamtüberlebens erreicht werden $(75,4$ vs $71,7 \%)$. In der FOLFOX4 Gruppe verstarben 48 von 212 Patienten versus 53 von 222 Patienten in der 5-FU/FS Gruppe [886]*.

\begin{tabular}{|l|l|}
$\begin{array}{l}\text { 8.7. } \\
\text { Empfehlungsgrad }\end{array}$ & $\begin{array}{l}\text { Evidenzbasierte Empfehlung } \\
\text { B }\end{array}$ \\
$\begin{array}{l}\text { Bei Patienten im Stadium II soll vor der Indikationsstellung für eine adjuvante } \\
\text { Chemotherapie der Mikrosatellitenstatus bestimmt werden. Weitere Parameter (z. B. } \\
\text { CEA-Spiegel, Differenzierungsgrad, 18q Verlust, isolierte Tumorzellen in Lymph- } \\
\text { knoten oder im Knochenmark, DNA-Ploidie und TS/p53 Expression, Lymph- und } \\
\text { Blutgefäßinvasion, molekulargenetische Analysen) sollen nicht zur Indikations- } \\
\text { stellung für eine adjuvante Chemotherapie benutzt werden. }\end{array}$ \\
\hline $\begin{array}{l}\text { Level of Evidence } \\
\mathbf{3 b}\end{array}$ & \begin{tabular}{l} 
Quellen: [899-902] \\
\hline
\end{tabular} \\
\hline
\end{tabular}




\begin{tabular}{|l|l|l|}
\hline $\begin{array}{l}\text { 8.8. } \\
\text { Empfehlungsgrad } \\
\mathbf{B}\end{array}$ & $\begin{array}{l}\text { Bei nachgewiesener Mikrosatelliteninstabilität } \\
\text { Chemotherapie im Stadium II nicht erfolgen. }\end{array}$ & 2017 \\
\hline $\begin{array}{l}\text { Level of Evidence } \\
\mathbf{2} \mathbf{a}\end{array}$ & & \\
\hline
\end{tabular}

\section{Hintergrund}

Für eine Reihe der in Empfehlung 8.7. genannten Prognoseparameter konnte in einigen, aber nicht in allen Studien eine prognostische Bedeutung für das kolorektale Karzinom gezeigt werden. Es liegen jedoch keine prospektiven Studien zum Nutzen einer adjuvanten Chemotherapie bei Vorhandensein einzelner oder mehrerer dieser Faktoren vor. Der Differenzierungsgrad wird in manchen Arbeiten sowohl im Stadium III [795, 903] als auch im Stadium II und III [875] als ein unabhängiger prognostischer Faktor gewertet. Hingegen zeigt eine Analyse von Hermanek, dass der Differen-zierungsgrad nur in einer Untergruppe des Stadium III (jedes T N2 M0) eine zusätzliche prognostische Bedeutung besitzt [904].

Der 18q-Alleelverlust besitzt in mehreren Studien auch im Stadium II unabhängige prognostische Bedeutung [905-909]. Eine Studie bei Patienten mit einem kolorektalem Karzinom im Stadium II $(\mathrm{n}=70)$ kommt jedoch zu dem Schluss, dass der 18q-Verlust keine prognostische Bedeutung besitzt [910]. Eine aktuell publizierte Metaanalyse zeigte eine klare negative prognostische Bedeutung der DNA Aneuploidie. Patienten mit einem aneuploiden kolorektalen Karzinom hatten fünf Jahre nach Operation eine deutlich höhere Sterblichkeitsrate als solche mit diploiden Tumoren. Dies galt für alle untersuchten Subgruppen, v. a. auch für das Stadium II. Allerdings waren die Studien retrospektiv angelegt [911].

Mikrosatelliteninstabilität (MSI) kann in 10-15\% der sporadischen kolorektalen Karzinome nachgewiesen werden. Die Ergebnisse einer Untersuchung von 718 Patienten aus Italien zeigen, dass Patienten mit einem Mismatch Repair Protein (MMRP)-negativen Tumor eine bessere Langzeitprognose aufweisen als Patienten mit einem MMRPpositiven Karzinom. Dieser positive prognostische Effekt war sowohl im Stadium II als auch im Stadium III vorhanden (757). In einer ergänzenden Studie der PETACC-3-Studie (5-FU vs. FOLFIRI) wird analysiert, welchen Einfluss der Mikrosatellitenstatus auf die Prognose von Stadium-II/III-Tumoren hat ( $\mathrm{n}=1.254$ Pat.). Die Tumore wurden anhand von 10 Markern eingeteilt in MSI-hoch (MSI-H) (3 oder mehr Marker instabil) oder MS-stabil (MSS). Im Stadium II hatten Pat. mit MSI-H-Tumoren ein signifikant besseres RFS und OS als solche mit MSS-Tumoren (HR 0,26 und 0,16). Im Stadium III war nur ein geringfügig verbessertes RFS zu verzeichnen (HR 0,67), aber kein signifikanter Einfluss auf das OS [912].

Zur Frage des Einflusses des MS-Status auf die Effekte einer adjuvanten Chemotherapie liegen auch nur retrospektive Analysen von prospektiven randomisierten Studien oder Registerauswertungen vor. Bei Patienten mit MMR-Protein-positiven Tumoren bewirkt die adjuvante Chemotherapie in der italienischen Studie eine Prognoseverbesserung [913]. In einer Arbeit von Sinicrope waren Mikrostelliteninstabilität und DNA Diploidie ebenfalls 
mit einer besseren Prognose assoziiert [914]. Bei 570 Patienten im Stadium II (55\%) und Stadium III (45\%) aus der gemeinsamen IMPACT-Analyse führte die adjuvante Chemotherapie zu einer Verbesserung des Überlebens, bei hoher Mikrosatelliteninstabilität führte die adjuvante Chemotherapie eher zu einer Verschlechterung des Überlebens [899]. Eine Untersuchung von 876 Patienten mit einem Stadium III Tumor konnte zeigen, dass in der Gruppe ohne adjuvante Chemotherapie der Mikrosatellitenstatus keine prognostische Bedeutung besaß (5 Jahresüberlebensrate: 43 gegen 36\%), während in der chemotherapeutisch behandelten Gruppe die Patienten mit MSI positiven Tumoren ein signifikant besseres Überleben aufwiesen [915]. Eine ergänzende Analyse von 5 prospektiven Studien ergab, dass MSI Tumoren nach alleiniger Operation ein signifikant verbessertes DFS und einen Trend zu einem verbesserten OS aufweisen. Ein DFS-Benefit durch eine 5-FU basierte Therapie wurde in der Gruppe der MSI-Tumoren im Gegensatz zu der Gruppe mit MSS- Tumoren jedoch nicht beobachtet. Bei Patienten mit Stadium II MSI Tumoren führte die adjuvante 5-FU Therapie sogar zu einem reduzierten OS [900]. Auch in einer spanischen Studie (Stadium II/III) und in einer koreanischen Studie (Stadium II) profitierten Patienten mit MSI Tumoren nicht von einer adjuvanten 5-FU Therapie [901] [902]. Im Gegensatz dazu führte die adjuvante Chemotherapie in einer retrospektiven australischen Studie zu einer Prognoseverbesserung auch bei MSI Tumoren [916] Auch in der PETACC-3-Studie wurde die Prognose von Pat. mit MSI-H-Kolonkarzinomen nicht durch den Therapiearm beeinflusst, d.h. in der Gruppe der 5-FU-therapierten Patienten behielten MSI-H-Tumore ihren prognostischen Vorteil im Vergleich zu MSI-L/S-Tumoren. [912].

Die Daten der MOSAIC Studie $(\mathrm{n}=2.246)$ ( FU/LV vs. FOLFOX) wurden ergänzend analysiert bezüglich der Effekte des Mismatch Repair Status (MMR) und der BRAF Mutation nach einem medianen Follow-up von 9,5 Jahren. Das $10 \mathrm{~J}$. OS betrug 79,5\% in der FU/LV Gruppe vs. 78,4\% in der FOLFOX4 Gruppe im Stadium II (HR 1,00) und 59,0\% vs. 67,1\% im Stadium III (HR 0,80; p=0,016). Defektes MMR (dMMR) wurde bei 9,4\% der Studienpopulation nachgewiesen. Unter FOLFOX4 konnte kein signifikant verbessertes Überleben in der dMMR-Gruppe (Stadium II/III) nachgewiesen werden (HR 0,41; 95\%Cl:0,16-1,07) [886].

Neuere Studien haben ergänzende Parameter untersucht:

- Immunhistochemische Analyse des CDX2-Genprodukts, einem Regulator der intestinalen Entwicklung. CDX2-negative Tumoren (6,9 \% der KRK) wiesen in dieser retrospektiven Studie eine signifikant schlechtere Prognose auf. Auch im Stadium II zeigte sich eine deutliche Verbesserung des 5-J.-DFS bei CDX2positiven (80-87 \%) vs. -negativen Tumoren (49-51 \%). In der CDX2-negativen Gruppe (aus den Studien NCBI-GEO, NCI-CDP, NSABP C-07 und Stanford TMAD rekrutiert) erhöhte eine adjuvante Chemotherapie im Stadium II gegenüber der alleinigen Operation das DFS von 56 auf 91 \%, im Stadium III von 37 auf 74 \% ([917])

- Zirkulierende Tumor-DNA (ctDNA). In der Gruppe, die postoperativ keine adjuvante Chemotherapie erhalten hatten, wurde ctDNA postoperativ bei 14/178 Pat. (7,9 \%) entdeckt, 11 (79\%) davon entwickelten ein Rezidiv nach einem medianen Follow-up von 27 Monaten. Ein Rezidiv trat nur in 16/164 Pat. $(9,8 \%)$ mit negativer ctDNA auf ([918]).

Lokale Entzündung (gemessen am Schnitt als intratumorale chronische Zelldichte (CIC), Lymphozyten, Plasmazellen und Makrophagen) und systemischeEntzündung (gemessen im Blut als Neutrophilen-zuLymphozyten Verhältnis (NLR)) auf die Prognose bestimmt. Ein erhöhter CIC- 
Wert verbesserte das 5-J.-OS signifikant (niedrig: 69,7 \%; hoch: 83,7\%), während ein hoher NLR-Wert das Überleben verschlechtert (niedrig: 82,5 \%; hoch: 60,5\%) ([919]).

\subsubsection{Chemotherapieprotokolle}

\subsubsection{Stadium III}

Oxaliplatin in Kombination mit 5-FU/Folinsäure (FS)

\begin{tabular}{|l|l|}
$\begin{array}{l}\text { 8.9. } \\
\text { Empfehlungsgrad }\end{array}$ & $\begin{array}{l}\text { Für die adjuvante Chemotherapie des Kolonkarzinoms im Stadium III soll eine } \\
\text { Oxaliplatin-haltige Therapie eingesetzt werden. }\end{array}$ \\
\hline $\begin{array}{l}\text { Level of Evidence } \\
\mathbf{1} \mathbf{D}\end{array}$ & \begin{tabular}{l} 
Quellen: $[865,886,920,921]$ \\
\hline
\end{tabular} \\
\hline
\end{tabular}

\section{Therapieschemata}

FOLFOX4: Folinsäure (FS) $\left(200 \mathrm{mg} / \mathrm{m}^{2}\right.$ als 2 Stunden Infusion, Tag 1 und 2) plus 5-FU $\left(400 \mathrm{mg} / \mathrm{m}^{2}\right.$ als Bolus, danach $600 \mathrm{mg} / \mathrm{m}^{2}$ als 22 Stunden Infusion; Tag 1 und 2) in Kombination mit Oxaliplatin $\left(85 \mathrm{mg} / \mathrm{m}^{2}\right.$ als 2 Stunden Infusion; Tag 1), Wiederholung Tag 15.1 Zyklus umfasst 2 Wochen, insgesamt 12 Zyklen.

modifiziertes FOLFOX6 Schema: Oxaliplatin $\left(85 \mathrm{mg} / \mathrm{m}^{2}\right.$ als 2 Stunden Infusion; Tag 1), Folinsäure $\left(400 \mathrm{mg} / \mathrm{m}^{2}\right.$ als 2 Stunden Infusion, Tag 1) + 5-FU (400 mg/m² i.v. Bolus, Tag 1; danach $2400 \mathrm{mg} / \mathrm{m}^{2}$ kontinuierlich i.v. über 46 Stunden). 1 Zyklus umfaßt 2 Wochen, insgesamt 12 Zyklen.

XELOX: Oxaliplatin $130 \mathrm{mg} / \mathrm{m}^{2}$ an Tag 1; Capecitabine $2 \times 1.000 \mathrm{mg} / \mathrm{m}^{2} / \mathrm{Tag}$, Tag 1-14, Wiederholung Tag 22. 1 Zyklus umfasst 3 Wochen, insgesamt 8 Zyklen.

\section{Hintergrund}

Durch die Kombination aus 5-FU und Folinsäure konnte in mehreren randomisierten Studien eine signifikanten Senkung der Rezidivrate sowie eine Steigerung des Gesamtüberlebens gezeigt werden [876, 878, 879].

Die MOSAIC-Studie (2.246 Patienten) verglich eine adjuvante Chemotherapie bestehend aus 5-FU/FS (LV5FU2) mit dem FOLFOX4 Schema (LV5FU2 + Oxaliplatin $85 \mathrm{mg} / \mathrm{m}^{2}$ ) alle 2 Wochen über 12 Zyklen. In dem Gesamtkollektiv führte die FOLFOX4 Chemotherapie zu einer signifikanten Verbesserung des krankheitsfreien Überlebens gegenüber der LV5FU2-Chemotherapie (73,3 versus 67,4\%, $\mathrm{p}=0.003$ ) (10 J Überleben) [886, 920]*. Betrachtet man nur das Stadium III, ergibt sich für die FOLFOX4 Chemotherapie ein Unterschied im krankheitsfreien Überleben um 7,5 Prozentpunkte (HR 0,78; 95\% Cl: 0,65-0,93; $\mathrm{p}=0.005)$ ). Auch das Gesamtüberleben wird im Stadium III durch die FOLFOX4-Chemotherapie signifikant um 4,4 Prozentpunkte verbessert $(p=0.029)$ (250 Todesfälle in der FOLFOX-Gruppe $(n=672)$ vs. 293 Todesfälle in der 5-FU/FS Gruppe 
$(\mathrm{n}=675) .4$ Jahre nach der Therapie lag die Rate der peripher-sensorischen Neuropathie bei 12\% (Grad I), 2,8\% (Grad II) und 0,7\% (Grad III) [886, 920, 922, 923]*.

In die NSABP-Studie C-07 wurden 2407 Patienten im Stadium II (28,6\%) oder im Stadium III eingeschlossen und erhielten entweder das Roswell-Park-Schema mit wöchentlicher 5FU/FS Bolusgabe (3 Zyklen à jeweils 8 Wochen) oder das gleiche 5-FU/FS-Schema mit Oxaliplatin $85 \mathrm{mg} / \mathrm{m}^{2}$ in Woche 1,3 und $5 \mathrm{im}$ achtwöchentlichen Rhythmus (FLOXSchema). In der FLOX-Gruppe wurden $20 \%$ weniger Rezidive beobachtet $(p<0.04)$. Das krankheitsfreie Überleben nach 4 Jahren betrug 73,2\% für die FLOX-Gruppe und 67,0\% für die 5-FU/FS-Behandelten [921]. Bei der Auswahl ist das unterschiedliche Nebenwirkungsprofil der einzelnen Protokolle zu berücksichtigen. Aufgrund der höheren kumulativen Oxaliplatindosis in der MOSAIC-Studie liegt die Rate der beobachteten Grad 3-4 Neuropathien in der NSABP-Studie niedriger (12,4 vs 8,4\%). In dem Bolus FLOX Protokoll wurden jedoch um das Dreifache! häufiger als in dem infusionalen FOLFOX4 Protokoll Grad 3 und 4 Diarrhöen beobachtet (38 vs 10,8\%!). 5 Patienten $(0,4 \%)$ in der NSABP Studie starben innerhalb der ersten 60 Tage nach Beginn der Chemotherapie aufgrund einer chemotherapieinduzierten Enteropathie [921]. Die Toxizität des FLOX Protokolls ist im Vergleich mit dem FOLFOX4 Protokoll bei vergleichbarer Wirksamkeit nicht tolerabel. Daher sollte das FLOX Protokoll in der adjuvanten Situation keine Anwendung finden. International wird derzeit dem modifizierten FOLFOX6 Schema, das nach einem 5-FU Bolus an Tag 1 eine 46 stündige Dauerinfusion von 5-FU vorsieht, der Vorzug gegeben. Dies erspart dem Patienten den 5-FU Bolus und den Pumpenwechsel an Tag 2 der FOLFOX4 Therapie.

Daten der NO16968 Studie (FU/LV vs. XELOX) zeigen nach einem Follow-up von fast 7 Jahren, dass die Addition von Oxaliplatin das $7 \mathrm{~J}$. OS von 67 auf $73 \%$ verbessert. Somit bestätigt diese Langzeitanalyse, dass auch die Kombination der oralen Prodrug Capecitabin in Kombination mit Oxaliplatin einen Stellenwert in der adjuvanten Chemotherapie des Stadium III Kolonkarzinoms hat [865]. In einer Metaanalyse mit 8734 Patienten (NSABPC-08, XELOXA, X-ACT, und AVANT Studien) unterschieden sich das DFS und das OS nicht in Abhängigkeit von dem verabreichten Fluoropyrimidin (5FU/Leucovorin vs. Capecitabin). In einer multivariaten Analyse bestätigten sich die Ergebnisse der MOSAIC-Studie, dass die Addition von Oxaliplatin zu einem Überlebensvorteil führt [924].

\begin{tabular}{|c|c|}
\hline 8.10. & Evidenzbasierte Empfehlung \\
\hline $\begin{array}{l}\text { Empfehlungsgrad } \\
\text { B }\end{array}$ & Bei Patienten über 70 Jahre sollte eine Oxaliplatin-haltige Therapie nicht erfolgen. \\
\hline \multirow{2}{*}{$\begin{array}{l}2 \mathrm{~b} \\
\end{array}$} & Quellen: $[863,925-928]$ \\
\hline & Konsens \\
\hline
\end{tabular}

\section{Hintergrund}

Obwohl 40\% aller KRK-Patienten über 75 Jahre alt sind, sind in den adjuvanten Therapiestudien nur zwischen 1-5\% der randomisierten Pat. $\geq 75$ Jahre. Ob die adjuvante Chemotherapie einen positiven Effekt bei Pat. $\geq 75$ Jahre hat, wurde anhand von 5489 Pat. aus 4 Datenregistern mit einem UICC Stadium III KRK analysiert. Der Einsatz der 
adjuvanten Chemotherapie in diesem Alterskollektiv führte zu einem verbesserten postoperativen Überleben. Die Kombination aus 5-FU mit Oxaliplatin führte nur zu einem verbesserten Überlebenstrend ( $\mathrm{HR} 0,84 ; \mathrm{Cl} 0,69-1,04 ; \mathrm{p}=\mathrm{NS})$, entsprechend einer absoluten Verbesserung des Überlebens von 5 Prozentpunkten nach 3 Jahren. Eine ergänzende Analyse der Datenregister zeigt, dass unter Oxaliplatin bei älteren Pat. mehr Nebenwirkungen beobachtet werden. [925].

Retrospektive Analysen der MOSAIC Studie [863] untersuchten die Wertigkeit der Erweiterung der adjuvanten Therapie um Oxaliplatin auf die Endpunkte DFS (krankheitsfreies Überleben), TTR (Zeit bis zum Rezidiv) und OS (Gesamtüberleben) bei älteren Patienten. Einschränkend muss gesagt werden, dass in der MOSAIC Studie Patienten $>75$ Jahre nicht eingeschlossen werden durften, so dass die Subgruppe der >69jährigen in der MOSAIC Studie nur 315 Patienten, von insgesamt 2.246 Patienten umfasste. In dieser Subgruppenanalyse zeigte sich kein Vorteil für die Gabe von Oxaliplatin. Die HR für das OS lag mit $1.10(95 \% \mathrm{Cl}$ 0.73-1.56) sogar auf der Seite für die alleinige 5-FU/FS Gabe. Für das DFS und TTR lag die HR mit 0.93 (95\% Cl 0.64-1.35) bzw. 0.72 (95\% Cl 0.47-1.11) allerdings wurde das Signifikanzniveau nicht erreicht, was durch die kleine Fallzahl bedingt sein kann.

Eine ähnliche Analyse der amerikanischen NSABP C-07 Studie [926] zeigte bei Patienten >69 Jahre in Bezug auf das DSF keinen Vorteil für die FLOX (5-FU Bolusregime plus Oxaliplatin) Therapie gegenüber der alleinigen FU/FS Gabe (HR 1,03; 95\%Cl 0,77-1,36; $\mathrm{p}=0,87)$ ). Dies galt auch für das Gesamtüberleben (HR 1,1 8; 95\%Cl 0,86-1,62; $\mathrm{p}=0.30$ ). Dabei war die Oxaliplatin-Alters Interaktion auf das OS mit einem p-Wert von 0,0391 signifikant. 396 der ursprünglich 2409 Patienten waren älter als 69 Jahre. Diese Daten werden von kleineren retrospektiven Analysen unterstützt. Eine Arbeitsgruppe aus Dänemark konnte retrospektiv 191 Patienten $>69$ Jahre identifizieren, welche in einem Zentrum adjuvant mit einem Fluoropyrimidin (FP) oder mit FP plus Oxaliplatin behandelt wurden [927]. Auch hier war die Gabe von Oxaliplatin mit einem kürzeren DFS (HR 0,58, $p=0,016)$ und kürzerem OS (HR 0,$49 ; p=0,003)$ bei höherer Toxizität $(H R=3,69 ; p=0,001)$ vergesellschaftet. Eine kanadische Auswertung konnte zeigen, dass bei 90 Patienten, welche $>65$ Jahre alt waren, der Vorteil der adjuvanten Gabe von Oxaliplatin mit dem Alter abnimmt [928].

Die NO16968 Studie (FU/FS vs. XELOX) [865] konnte in der Subgrupenanalyse keinen negativen Effekt des Alters auf das DFS oder OS nachweisen. Inwieweit dies mit Capecitabine als Kombinationspartner zusammenhängt, ist allerdings unklar.

Eine gepoolte Analyse welche die Frage nach Oxaliplatin bei älteren Patienten untersuchte [864] konnte nach Adjustierung für Komorbiditäten keinen negative Effekt des Alters auf die Gabe von Oxaliplatin feststellen. Bei der Analyse von 904 Patienten aus den NSABP C-08, XELOXA, X-ACT, und AVANT Studie, welche entweder eine Fluoropyrimidin (FP) Monotherapie oder FP plus Oxaliplatin erhielten, war der Interaktionstest für Alter und Oxaliplatin in Hinblick auf das OS und das DFS negativ.

Zusammenfassend sollte in der adjuvanten Therapie die Gabe von Oxaliplatin nur in gut begründeten Ausnahmesituationen z.B. bei Patienten ohne Komorbiditäten erfolgen. 


\section{Monotherapie mit Fluoropyrimidinen}

\begin{tabular}{|c|c|}
\hline 8.11. & Evidenzbasierte Empfehlung \\
\hline $\begin{array}{l}\text { Empfehlungsgrad } \\
\text { A }\end{array}$ & $\begin{array}{l}\text { Bei Kontraindikationen gegen Oxaliplatin-haltige Regime soll eine Monotherapie mit } \\
\text { Fluoropyrimidinen durchgeführt werden. Dabei werden orale Fluoropyrimidine den } \\
\text { infusionalen Schemata vorgezogen. Bolusregime sollen wegen der höheren Toxizität } \\
\text { nicht mehr verwendet werden. }\end{array}$ \\
\hline \multirow{2}{*}{ Level of Evidence } & Quellen: [924, 929-934] \\
\hline & Starker Konsens \\
\hline
\end{tabular}

Orale 5-FU Prodrug:

Capecitabin $2 \times 1250 \mathrm{mg} / \mathrm{m}^{2}$ Körperoberfläche p.o. Tag 1-14, alle 3 Wochen.

1 Zyklus umfasst 3 Wochen, insgesamt 8 Zyklen

\section{Hintergrund}

1987 Patienten mit einen Stadium III Kolonkarzinom wurden entweder in das MayoClinic-Schema (983 Patienten) randomisiert oder erhielten Capecitabin als Mono-therapie (1004 Patienten) jeweils über eine Zeit von 24 Wochen (X-ACT Studie). Das primäre Studienziel mit dem Nachweis, dass Capecitabin im krankheitsfreien Überleben mindestens gleichwertig zum Mayo-Schema ist, wurde erreicht. Die Analyse zeigte einen Trend zu Gunsten eines überlegenen krankheitsfreien Überlebens mit Capecitabin (HR 0,87; 95\% Cl: 075-1,00; $\mathrm{p}=0.05)$. Auch das Gesamtüberleben unterschied sich nicht signifikant, mit einem Trend zur Überlegenheit von Capecitabin (81,3\% vs 75,6\%; $p=0.05$ ) [929]. In einer Metaanalyse mit 8734 Patienten (NSABPC-08, XELOXA, X-ACT, und AVANT Studien) unterschieden sich das DFS und das OS nicht in Abhängigkeit von dem verabreichten Fluoropyrimidin (5-FU/Leucovorin vs. Capecitabin) [924].

Auch wenn in einer randomisierten Studie UFT + Folinsäure versus 5-FU/FS [935] kein Unterschied im Gesamtüberleben und im krankheitsfreien Überleben bestand und in einer japanischen Metaanalyse von drei Studien sogar ein signifikanter Vorteil für Gesamtüberleben und DFS erreicht werden konnte [936], wird UFT aktuell nicht empfohlen, da es keine Zulassung zur adjuvanten Chemotherapie des Kolonkarzinoms in Deutschland besitzt.

\section{Infusionales 5-FU/Folinsäure:}

- LV5FU2

z.B. Folinsäure (FS) $\left(200 \mathrm{mg} / \mathrm{m}^{2}\right.$ als 2 Stunden Infusion, Tag 1 und 2) plus 5 FU $\left(400 \mathrm{mg} / \mathrm{m}^{2}\right.$ als Bolus, danach $600 \mathrm{mg} / \mathrm{m}^{2}$ als 22 Stunden Infusion; Tag 1 und 2)

1 Zyklus umfasst 2 Wochen, insgesamt 12 Zyklen

- 5-FU/Folinsäure-Schema

z.B. Folinsäure (FS) $\left(500 \mathrm{mg} / \mathrm{m}^{2}\right.$ als 1-2 Stunden Infusion) plus 5-FU (2600 
$\mathrm{mg} / \mathrm{m}^{2}$ als 24 Stunden Infusion) $1 \times$ pro Woche über 6 Wochen (Tag 1, 8, 15, 22, 29, 36). Erneuter Beginn der Therapie in Woche 9 (Tag 50).

- $\quad$ Insgesamt 2 Zyklen.

- $\quad$ venöse 5-FU Dauerinfusion (PVI)

z.B. 5-FU als Dauerinfusion über insgesamt 12 Wochen $\left(300 \mathrm{mg} / \mathrm{m}^{2} / \mathrm{Tag}\right)$

\section{Hintergrund}

Im Vergleich zu den Bolusschemata zeigen mehrere Therapiestudien mit unterschiedlicher infusionaler Applikationsform keinen Unterschied zu der Bolusgabe von 5FU/FS bezüglich des krankheitsfreien Überlebens und des Gesamtüberlebens. Das deutlich bessere Toxizitätsprofil spricht jedoch eindeutig für die infusionale Applikation $[930,931]^{*}[932,933]$. Ein Vergleich von 12 Wochen Therapie mit der «protracted venous infusion » (PVI) von 5 - $\mathrm{FU}\left(300 \mathrm{mg} / \mathrm{m}^{2}\right.$ pro Tag) gegen ein sechsmonatiges MAYOSchema zeigte keinen signifikanten Unterschied im rezidiv-freien Überleben (RFS) und im Gesamtüberleben bei geringerer Toxizität von PVI 5-FU [934]. Der Beginn der adjuvanten Chemotherapie innerhalb eines Zeitraums von 8 Wochen nach Operation zeigte einen signifikanten Überlebensvorteil [937]. Die optimale Dauer der Chemotherapie beträgt 6 Monate [932, 938, 939].

\begin{tabular}{|c|c|}
\hline 8.12. & Evidenzbasierte Empfehlung \\
\hline Empfehlungsgrad & $\begin{array}{l}\text { Monoklonale Antikörper oder Irinotecan sollen in der adjuvanten Therapie des } \\
\text { Kolonkarzinoms nicht eingesetzt werden. }\end{array}$ \\
\hline \multirow{2}{*}{ Level of Evidence } & Quellen: [940-942] \\
\hline & Konsens \\
\hline
\end{tabular}

\section{Hintergrund}

Eine adjuvante Therapie mit einem irinotecanhaltigen Protokoll ist auf Basis der vorliegenden Daten aus Phase III Studien nicht zu empfehlen [940], [941], [942].

Mehrere Studien haben den Stellenwert der monoklonalen Antikörper in der adjuvanten Situation untersucht. In der N0147 Studie wurden Pat. ( $N=1863)$ mit KRAS Wildtyp Tumoren entweder mit mFOLFOX6 oder mit mFOLFOX6 + Cetuximab adjuvant behandelt (12 Zyklen alle 2 Wo.). Nach einem Follow-up von 28 Monaten konnte kein positiver Effekt durch die zusätzliche Cetuximab Therapie belegt werden: weder das DFS ( 74,6 vs. $71,5 \%$ ) noch das Gesamtüberleben (87,3 vs. $85,6 \%$ ) unterschieden sich. In der Cetuximab Gruppe wurde eine signifikant erhöhte Toxizität beobachtet. In der Subgruppe der über 70jährigen führte die Addition von Cetuximab zu einem deutlich verschlechterten DFS (86,2 vs. 72,5\%). [943]. Auch in der PETACC-08 Studie führte die Addition von Cetuximab zur FOLFOX4 Chemotherapie nicht zu einer Verbesserung der onkologischen Ergebnisse [944].

In der NASABP-Studie C-08 wurde das modifizierte FOLFOX6-Schema (12 Zyklen alle 2 Wochen) mit FOLFOX6 + Bevacizumab verglichen. Bevacizumab wurde für insgesamt 1 Jahr gegeben. In die Studie wurden Patienten mit einem kurativ resezierten 
Kolonkarzinom im Stadium II oder III (75\%) aufgenommen. Der Anteil der über 70jährigen Patienten lag bei nur 15,1 \%. Nach einem medianen Follow-up von 5 Jahren zeigte sich in dieser Studie keine signifikante Verbesserung des DFS nach zusätzlichem Einsatz des Antikörpers (75,1 vs. 77,9\%), auch nicht bei getrennter Auswertung für das Stadium II und Stadium III. Das Gesamtüberleben in beiden Gruppen war vergleichbar. [945]. In der AVANT-Studie ( $\mathrm{n}=3.451$ ) wurde ein ähnliches Therapiedesign wie in der NSABP-C-08-Studie eingesetzt. Der primäre Endpunkt war das DFS. Als zusätzlicher 3. Therapiearm wurde in dieser Studie XELOX plus Bevacizumab getestet. In den Bevacizumab behandelten Gruppen traten mehr schwerwiegende Nebenwirkungen auf als in der alleinigen FOLFOX-Gruppe (25,5\% vs. $20 \%)$. Innerhalb von 60 Tagen nach Beginn der Therapie verstarben 2 Patienten in der FOLFOX-Gruppe, 4 Patienten in der FOLFOX4-plus-Bevacizumab-Gruppe und 6 Patienten in der XELOX-plus-BevacizumabGruppe. Das DFS wurde durch die zusätzliche Gabe von Bevacizumab nicht verbessert. Im Gesamtüberleben wurde sogar ein negativer Einfluss der Bevacizumab-Therapie beobachtet (HR für Bevacizumab-FOLFOX4 vs. FOLFOX4 1,27 ( $p=0,02)$, BevacizumabXELOX vs. FOLFOX4 1,15 ( $p=0,21)$ ). [946]. In der großen QUASAR 2 Studie (Kerr) wurden zwei Therapiegruppen miteinander verglichen nach kurativer Therapie eines Stadium II mit Hochrisikocharakteristika oder III Kolonkarzinoms (Capecitabine versus Capecitabine plus Bevacizumab). Nach einem medianen Follow-up von 4,92 J. bestand kein Unterschied im 3 J. DFS (75,4 vs. 78,4\%). [947]

Die Intensivierung der postoperativen Chemotherapie mit Hilfe einer Anti-körpertherapie bringt im Vergleich zur alleinigen Kombinationschemotherapie keine Verbesserung des Überlebens. Monoklonale Antikörper haben somit keinen Stellenwert in der adjuvanten Therapie des Kolonkarzinoms.

\subsubsection{Stadium II}

8.13.

Empfehlungsgrad

B

Level of Evidence

$1 \mathrm{~b}$

\section{Evidenzbasierte Empfehlung}

Wenn bei Patienten mit Stadium II Tumoren eine adjuvante Chemotherapie durch geführt wird, sollten Fluoropyrimidine als Monotherapie eingesetzt werden.

Quellen: $[863,886]$

Konsens

\section{Hintergrund}

Es gibt bisher keine Hinweise dafür, dass die Addition von Oxaliplatin zu einer höheren Effektivität der adjuvanten Therapie im Stadium II führt (siehe Kapitel 8.1.4). Zur Frage der Effektivität einer adjuvanten Chemotherapie mit dem FOLFOX4 Schema im Stadium II und bei älteren Pat. (70-75 J., N=315) wurden Subgruppenanalysen der MOSAIC Studie durchgeführt. In die MOSAIC Studie wurden 2.246 Patienten nach kurativer Resektion eines Kolonkarzinoms im Stadium II $(\mathrm{N}=899)$ oder III aufgenommen und postoperativ entweder mit LV5FU2 oder FOLFOX4 für 12 Zyklen adjuvant behandelt. HochrisikoStadium II war definiert als: T4 Tumor, Tumorperforation, lleus, schlechter Differenzierungsgrad, Veneninvasion oder weniger als 10 Lymphknoten untersucht. Für das gesamte Stadium II Kollektiv brachte die zusätzliche Oxaliplatingabe keine Verbesserung des DFS oder des Überlebens. Dies galt auch für die Subgruppe der Hochrisikotumore und die Gruppe der älteren Patienten [863]. Dies wurde auch in der 
Langzeitanalyse der MOSAIC Studie für das Hochrisiko-Stadium II bestätigt: die Addition von Oxaliplatin führt nicht zu einer prognostischen Verbesserung gegenüber einer LV5FU2-Chemotherapie [886].

Für das orale 5-FU Prodrug Capecitabine, liegen keine randomisierten Untersuchungen für Patienten mit Kolonkarzinom im Stadium II vor. Im Stadium II liegt eine höhere Medikamenten-Noncompliance vor als im Stadium III [948] .

\subsubsection{Dauer der adjuvanten Therapie}

\begin{tabular}{|c|c|}
\hline 8.14. & Evidenzbasierte Empfehlung \\
\hline Empfehlungsgrad & $\begin{array}{l}\text { A) In der adjuvanten Situation soll die akkumulierende (Neuro-)Toxizität } \\
\text { engmaschig gegen den therapeutischen Nutzen abgewogen werden. }\end{array}$ \\
\hline B & $\begin{array}{l}\text { B) Bei niedrigem Rezidivrisiko }(\mathrm{T} 1-3 \mathrm{~N} 1) \text { sollte deshalb eine 3-monatige } \\
\text { oxaliplatinhaltige Therapie nach dem CAPOX/XELOX-Schema durchgeführt } \\
\text { werden. }\end{array}$ \\
\hline \multirow{2}{*}{$\begin{array}{l}\text { Level of Evidence } \\
1 \text { b }\end{array}$} & Quellen: [949-952] \\
\hline & $\begin{array}{l}\text { A) Starker Konsens } \\
\text { B) Konsens }\end{array}$ \\
\hline
\end{tabular}

\subsection{5.}

Empfehlungsgrad

B

Level of Evidence

$1 \mathrm{~b}$

\section{Evidenzbasierte Empfehlung}

Bei Patienten mit hohem Risiko (T4 oder N2-Stadium) sollte weiterhin eine Oxaliplatinbasierte Therapie (FOLFOX oder CAPOX/XELOX Schema) über 6 Monate geplant werden.

Quellen: [949-952]

Konsens

\section{Hintergrund}

Zur Dauer der adjuvanten Chemotherapie wurde auf dem ASCO Kongress 2017 die prospektive, planmäßig gepoolte Analyse von 6 randomisierten Phase III Studien (SCOT, TOSCA, Alliance/SWOG 80702, IDEA France, ACHIEVE, HORG; $n=12.834$ Pat., medianes Follow-up 39 Mo.) der IDEA („International Duration Evaluation of Adjuvant Therapy“) vorgestellt [953]. Diese Studie ist jetzt voll publiziert [949]. Diese Studie war darauf ausgelegt, die Nichtunterlegenheit einer 3-monatigen vs. 6-monatigen adjuvanten FOLFOX/CAPOX Chemotherapie zu zeigen. Die Nicht-Unterlegenheit wurde angenommen, wenn das 95 \% Konfidenzintervall $(\mathrm{Cl})$ der Hazard Ratio (HR) unter 1,12 lag. $40 \%$ der Pat. erhielten CAPOX. Es muss betont werden, dass keine Randomisation zwischen einer CAPOX- oder FOLFOX-Therapie stattfand. Der primäre Endpunkt war das 
3-Jahre-krankheitsfreie-Überleben (3.-J.-DFS). Daten zum Gesamtüberleben liegen noch nicht vor.

Eine Grad 3/4-Neurotoxizität trat signifikant seltener nach 3 als nach 6 Monaten auf (FOLFOX: 3 \% vs. $16 \%$; CAPOX: 3 \% vs. 9 \%). Die 3-J.-DFS-Rate betrug 74,6 \% (3 Mo.) vs. 75,5 \% (6 Mo.) im Gesamtkollektiv (HR 1,07; $95 \% \mathrm{Cl}$ 1,00-1,15), so dass der primäre Endpunkt des Nachweises der Nichtunterlegenheit, welcher ein oberes Konfidenzintervall $(95 \% \mathrm{Cl}$ ) von (maximal) 1,12 als „nicht unterlegen“ definiert hatte, verfehlt wurde.

Die 3 Mo. vs. 6 Mo. DFS-HRs lagen bei $1,16(1,06-1,26)$ für FOLFOX und bei $0,95(0,85$ 1,06) für CAPOX. Somit ergibt sich eine Unterlegenheit einer dreimonatigen FOLFOX Therapie, während sich für das CAPOX Schema eine Nicht-Unterlegenheit einer dreimonatigen CAPOX Therapie im Vergleich zu einer sechsmonatigen CAPOX Therapie ergab.

Die 3 Mo. vs. 6 Mo. DFS-HRs lagen bei 1,01 $(0,90-1,12)$ in der T1-3N1 Subgruppe und bei 1,12 $(1,03-1,23)$ für die T4 oder N2 Subgruppen. Die Nicht-Unterlegenheit wurde somit nicht für die Gesamtstudie, aber für die Subgruppe im Stadium T1-3 N1 unter 3 Monate CAPOX Therapie gezeigt. Das 3-J.-DFS (\%) zwischen 3 und 6 Monaten Therapie lag in den verschiedenen Untergruppen bei:

N1: 79,7 vs. $80,8 \%$

N2: 61,6 vs. $61,8 \%$

T1-3: 79,0 vs. $79,3 \%$

T4: 58,1 vs. $61,4 \%$

T1-3N1: 83,1 vs. $83,3 \%$

T4 oder N2: 62,7 vs. $64,4 \%$

Die Daten in der N2 Untergruppe unterscheiden sich somit nicht, so dass hier ggf. auch eine Therapieverkürzung diskutiert werden kann.

Der Hauptkritikpunkt an den IDEA-Daten liegt in der Heterogenität der 6 Einzelstudien und der Tatsache, dass Ergebnisse nur für Subgruppen signifikant waren. In der französischen Unterstudie [950] bestand eine Überlegenheit des 6-Monate-Regimes, allerdings wurden nur $10 \%$ der Patienten mit CAPOX behandelt. Auch in der TOSCAStudie [951] schnitten 6 Monate Chemotherapie besser als 3 Monate ab (Power noch $72 \%$ anstatt $80 \%$ ). In die SCOT-Studie [952] wurden auch Rektumkarzinome und Hochrisiko-Stadium-II-Kolonkarzinome aufgenommen. Wie schwierig die Entscheidungsfindung auch unter den Autoren der IDEA-Studie sein kann, zeigt die ausgiebige Diskussion der Ergebnisse in der Arbeit von Sobrero et al. 2018 [954]. Eine einheitliche Entscheidung zu einer Therapieverkürzung bei einer N2-Kategorie war hier nicht vorhanden.

Auf dem ASCO Kongress 2017 wurden auch die Daten einer großen Registerstudie aus Korea ( $n=61.315$; Stadium II: 20.525; Stadium III: 25170) vorgestellt [955]. Hier erwies sich im Gegensatz zu der IDEA-Studie die adjuvante Chemotherapie $<3$ Mo. beim Kolonkarzinom als signifikant schlechter, es wurde zusätzlich auch noch der härtere Endpunkt OS statt DFS angegeben (HR FOLFOX/CAPOX 2,15; HR FL/CAP 3,72). 


\subsection{Perioperative Therapie beim Rektumkarzinom}

\subsubsection{Neoadjuvante Therapie}

8.2.1.1. Stadium I

\begin{tabular}{|c|c|c|}
\hline 8.16. & Evidenzbasierte Empfehlung & 2017 \\
\hline Empfehlungsgrad & $\begin{array}{l}\text { In UICC-Stadium I (cT1-2N0) soll eine präoperative Therapie } \\
\text { werden. }\end{array}$ & nicht durchgeführt \\
\hline \multirow{2}{*}{$\begin{array}{l}\text { Level of Evidence } \\
2 \text { b }\end{array}$} & Quellen: $[956,957]$ & \\
\hline & Starker Konsens & \\
\hline
\end{tabular}

\section{Hintergrund}

Rektumkarzinome im UICC-Stadium I (T1/2N0) haben nach alleiniger radikaler Operation mit adäquater totaler mesorektaler Exzision (TME) für Tumoren im unteren/mittleren Drittel sowie partieller mesorektaler Exzision (PME) für Tumoren im oberen Rektumdrittel niedrige Lokalrezidiv- und Fernmetastasenraten (3 \% Lokalrezidive bzw. weniger als $10 \%$ Fernmetastasen nach 10 Jahren [956]. In den modernen, randomisierten Studien zur Rolle der neoadjuvanten Radiochemotherapie des lokal fortgeschrittenen Rektumkarzinoms (cT3/4 und/oder $\mathrm{cN}+$ ) war dieses frühe Tumorstadium daher nicht eingeschlossen [958] [959] [960] [961] [962].

Randomisierte Studien zur präoperativen Kurzzeit-Vorbestrahlung mit $5 \times 5$ Gy versus alleiniger Operation hatten das Tumorstadium I gleichwohl eingeschlossen. Langzeitergebnisse der schwedischen Studie zeigten zwar einen signifikanten Vorteil hinsichtlich der lokalen Kontrolle für die zusätzlichen Bestrahlung auch für das UICCStadium I (4.5\% versus $14 \%$ nach 13 Jahren, $\mathrm{p}=0.009$ ), allerdings war in dieser Studie das Konzept der TME noch nicht umgesetzt und die Lokalrezidvraten im alleinigen Operationsarm unakzeptabel hoch [963]. Die jüngere holländische „TME-Studie“ zeigte für das UICC-Stadium I ebenfalls eine numerisch signifikante Reduzierung der Lokalrezidivrate im Arm mit präoperativer Kurzzeit-Radiotherapie ( $<1 \%$ mit versus 3\% nach 10 Jahren ohne präoperative Radiotherapie, $p=0.027$ ) [956], die Lokalrezidivraten in diesem Stadium waren aber in beiden Armen gering. Diese niedrige Lokalrezidivrate im Stadium I wurde auch in der jüngsten Britischen Studie zur Kurzzeit-Radiotherapie (MRC-CR07) bestätigt (1.9\% nach 3 Jahren mit Vorbestrahlung versus $2.8 \%$ bei sofortiger Operation, n.s.) [957].

Für Patienten mit tiefsitzenden T1 N0-high-risk (G3/4, L1, V1, Durchmesser größer $3 \mathrm{~cm}$, sm3) oder T2N0-Tumoren, die eine Exstirpation ablehnen, ist die Radio(chemo-) therapie, gefolgt von lokaler Exzision/transanaler endoskopischer Mikrochirurgie oder einer wait-and-see Strategie bei klinisch kompletter Remission eine Behandlungsoption [964-968]. Dies ist jedoch ein noch nicht gesichertes Vorgehen und bedarf weiterer prospektiver Studien. 


\subsubsection{Stadium II/III}

\begin{tabular}{|l|l|}
$\begin{array}{l}\text { 8.17. } \\
\text { Empfehlungsgrad }\end{array}$ & $\begin{array}{l}\text { In den UICC-Stadien II und III (cT3/4 und/oder cN+) soll bei Tumoren des unteren und } \\
\text { mittleren Rektumdrittels eine neoadjuvante Radiochemotherapie oder Kurzzeit- } \\
\text { Radiotherapie erfolgen. }\end{array}$ \\
\hline $\begin{array}{l}\text { Level of Evidence } \\
\mathbf{1}\end{array}$ & Quellen: $[956,958,959,969-973]$ \\
\hline & \\
\hline & Konsens \\
\hline
\end{tabular}

8.18.

In folgenden Ausnahmefällen kann bei Patienten mit Rektumkarzinom im UICCStadium II/III eine primäre Resektion erfolgen:

CT1/2-Tumore im unteren und mittleren Drittel mit bildgebend fraglichen Lymphknotenbefall

cT3a/b-Tumore im mittleren Drittel mit in der MRT nur limitierter Infiltration ins perirektale Fettgewebe (cT3a: <1 mm, cT3b: 1-5 mm) und ohne bildgebenden Verdacht auf Lymphknotenmetastasen oder extra-muraler Gefäßinvasion (EMVI-) bei adäquater Qualitätssicherung der MRT-Diagnostik und der TME-Chirurgie.

Starker Konsens

\section{Hintergrund}

Ein Tumor gilt als Rektumkarzinom, wenn seine aborale Grenze weniger als $16 \mathrm{~cm}$ von der Anokutanlinie, gemessen mit einem starren Rektoskop, entfernt ist [974]. Die Definition der Rektum-Drittel auf Grundlage der Entfernung des Tumorunterpols zur Anokutanlinie wurde in den Studien zur neoadjuvanten Therapie des Rektumkarzinoms nicht einheitlich gehandhabt. Neben der Definition $\leq 6 \mathrm{~cm}$ für das untere Drittel, 6 bis $\leq$ $12 \mathrm{~cm}$ für das mittlere Drittel, und 12 bis $\leq 16 \mathrm{~cm}$ für das obere Drittel [959] [975] [976] , wurden als Grenzen auch 0-5 cm; > 5-10 cm, und > $10-15 \mathrm{~cm}$ verwandt [956] [957].

Die deutsche Studie zur adjuvanten und neoadjuvanten Radiochemotherapie (50,4 Gy in 28 Fraktionen, 5-Fluorouracil $1000 \mathrm{mg} / \mathrm{m}^{2} / \mathrm{Tag} 1$ - 5 in der 1 . und 5. RT-Woche) des Rektumkarzinoms (bis $16 \mathrm{~cm}$ ab Anokutanline) im UICC-Stadium II und III (CAO/ARO/AIO-94) wies eine signifikante Reduzierung der Lokalrezidivrate im neoadjuvanten Arm auf ( $6 \%$ versus $13 \%$ im postoperativen Arm nach 5 Jahren, $p=0.006$ [958] [959]. Die Rate postoperativer Komplikationen war nach präoperativer Radiochemotherapie im Vergleich zur sofortigen Operation nicht erhöht, die akute und chronische Toxizität im präoperativen Radiochemotherapie-Arm insgesamt signifikant niedriger. Bei tiefsitzenden Tumoren, die der Chirurg vor Randomisation als exstirpationspflichtig eingeschätzt hatte, konnte die Rate sphinktererhaltender Operationsverfahren durch die Vorbehandlung im Vergleich zur sofortigen Operation verdoppelt werden (19\% bei sofortiger Operation, $39 \%$ nach neoadjuvanter Radiochemotherapie, $p=0.004)$. Diese Studie etablierte 2004 die neoadjuvante Radiochemotherapie als einen neuen Standard für das lokal fortgeschrittene Rektum-karzinom 
im UICC-Stadium II und III. Meta-Analysen bestätigten eine verbesserte lokale Kontrolle durch die präoperative Radiotherapie (mit einer biologisch-äquivalenten Dosis, BED, $>30 G y$ ) im Vergleich zur alleinigen Operation oder der postoperativen Bestrahlung [972], [973]. Bei konventionell-fraktionierter präoperativer Radiotherapie (1.8-2 Gy Einzeldosis bis 45-50,4 Gy Gesamtdosis) erwies sich die simultane Kombination mit einer 5Fluorouracil-haltigen Chemotherapie in zwei randomisierten Studien hinsichtlich der lokalen Kontrolle als signifikant überlegen $\quad(H R=0.54,95 \% \mathrm{Cl} 0.41-0.72)$ [960] [961] [962] [977]. Dies wurde in Meta-Analysen bestätigt [969] [970] [971].

Die verbesserte lokale Kontrolle hat sich allerdings in keiner dieser Studien/ Metaanalysen in ein verbessertes krankheitsfreies Überleben oder Gesamtüberleben übertragen. Langzeitergebnisse insbesondere der schwedischen und holländischen Studie zur Kurzzeit-Vorbestrahlung zeigten für einige Items eine signifikant verschlechterte krankheitsbezogene Lebensqualität nach Vorbestrahlung mit $5 \times 5 \mathrm{~Gy}$ im Vergleich zur alleinigen Operation (z. B. Häufigkeit Stuhlgang bei Patienten ohne Stoma gemäß EORTC QLQ-CR-29: mittlerer Punkte-Score nach alleiniger Operation 19,4 versus 26,3 nach $5 \times 5$ Gy + OP, $p=0.006$ [978] und eine Verschlechterung funktioneller Ergebnisse (Sexualfunktion: Erektionsstörungen $30 \%$ nach alleiniger Operation versus 50 \% nach $5 \times 5 \mathrm{~Gy}+$ Operation: - Stuhlinkontinenz mit Gebrauch von Vorlagen: $37 \%$ nach alleiniger Operation vs $56 \%$ nach $5 \times 5$ Gy + Operation nach 14 Jahren Nachbeobachtungszeit [979] [980] [981] - bei allerdings auch veralteter Bestrahlungstechnik und Bestrahlungsvolumina.

Daher sollen Kriterien für einen möglichen Verzicht auf die präoperative Radio-/ Radiochemotherapie im UICC-Stadium II und III formuliert werden:

Als Problem jeder neoadjuvanten Therapie muß das potentielle "Overstaging" und die daraus resultierende „Überbehandlung“ von Patienten gewertet werden, bei denen fälschlicherweise ein lymphknotenpositiver Tumor $(\mathrm{cN}+)$ diagnostiziert wurde. Die Schwierigkeit in der bildgebenden Diagnostik für $\mathrm{cN}+$ ist u.a. durch das Auftreten reaktiv vergrößerter Lymphknoten sowie häufiger Mikrometastasen in normal großen Lymphknoten begründet. Da somit die Sensitivität und Spezifität der Beurteilung des Lymphknotenbefalls für alle Staging-Methoden limitiert ist (MRT/endorektaler Ultraschall/Becken-CT: Sensitivität 77\%, 57\%, 79\%, Spezifität 76\%, 80\%, 76\%, [982] wird bei $\mathrm{cTl} / 2$-Tumoren mit bildgebend fraglichen $\mathrm{cN}+$ (formal also UICC-Stadium III) die primäre Operation als sinnvolle Option erachtet.

Als weiteres Selektionskriterium für eine primäre Operation kann bei den wandüberschreitenden cT3-Tumoren im mittleren Drittel das Ausmaß der Infiltration ins perirektale Fettgewebe herangezogen werden. Bei in der Dünnschicht-MRT bestimmter radialer Tumorinfiltration unter $5 \mathrm{~mm}(\mathrm{cT} 3 \mathrm{a} / \mathrm{b})$ und ausreichendem Abstand zur mesorektalen Faszie ist bei adäquater TME ein dem Stadium I vergleichbares Lokalrezidivrisiko anzunehmen [983], sodass in dieser Konstellation auf eine neoadjuvante Radio(chemo)therapie verzichtet werden kann, wenn keine zusätzlichen Risikofaktoren (z.B. tiefliegender Tumor, eindeutiger Lymphknotenbefall, EMVI) vorliegen und eine nachvollziehbar hohe Qualität der MRT sowie der TME-Chirurgie am Zentrum gegeben ist [984]. 


\begin{tabular}{l|l} 
8.19. & Konsensbasierte Empfehlung \\
\hline EK & $\begin{array}{l}\text { Der im Dünnschicht-MRT gemessene radiale Abstand des Primärtumors (oder } \\
\text { bildgebend befallener Lymphknoten) von der mesorektalen Faszie (mrCRM) soll } \\
\text { außerhalb von Studien nicht als Entscheidungskriterium für eine primäre Operation } \\
\text { herangezogen werden. }\end{array}$ \\
\hline Konsens
\end{tabular}

\section{Hintergrund}

Als Selektionskriterium für eine primäre Operation ohne neoadjuvante Therapie werden von einigen Studiengruppen der in der MRT gemessene radiale Abstand des Tumors (oder tumorbefallener Lymphknoten) zur mesorektalen Fazie (= späterer zirkumferentieller Resektionsrand, CRM), untersucht [985] [976]. Beträgt dieser mehr als $1 \mathrm{~mm}$ (mrCRM-), wird eine pathologisch bestätigte CRM-negative Resektion ( $\mathrm{pCRM}>1 \mathrm{~mm}$ ) bei adäquater TME in über 90-95\% der Fälle auch ohne Vorbehandlung erreicht. Die Mercury Study Group berichtet über einen negativ-prädiktiven Wert der MRT bezüglich des zirkumferentiellen Resektionsrandes bei primärer Operation von 94\% (pCRM- bei 192 von 205 Patienten mit mrCRM-). Die absolute Anzahl von Lokal-rezidiven betrug bei einem medianen Follow-up von 62 Monaten in der Gruppe der 205 ausschließlich operierten Patienten mit negativen mrCRM 7\% (14/205) [985].

Sowohl der durch die Dünnschicht-MRT bestimmte mrCRM also auch der histopathologisch bestimmte pCRM haben unzweifelhaft hohe prognostische Bedeutung für die lokale Kontrolle sowie das krankheitsfreie Überleben und Gesamtüberleben [985] [685]. Allerdings belegen Subgruppenanalysen großer, randomisierter Studien, dass die präoperative Radiotherapie gerade bei Erreichen einer pCRM-negativen Resektion zu einer weiteren signifikanten Verbesserung der Lokalrezidivrate führte [956] [827]. Eine multivariate Untersuchung im Rahmen der britischen MRC-CR07-Studie zu Risikofaktoren für ein lokoregionäres Rezidiv identifizierte den Lymphknotenstatus, die Vorbestrahlung mit 5x5 Gy, die Lagebeziehung des Tumors zum anterioren Quadranten sowie die chirurgische TME-Qualität als unabhängige Prognosefaktoren, während der pCRM nur in der univariaten Analyse als signifikanter Faktor bestätigt wurde [827]. Eine ausschließlich auf das Selektionskriterium mrCRM- bezogene Indikationsstellung zur neoadjuvanten Radio-/Radiochemotherapie versus primärer Operation bedarf daher weiterer qualitätsgesicherter, prospektiver Studien.

Die OCUM-Studiengruppe untersucht eine risikobasierte Indikationsstellung zur präoperativen Radiochemotherapie, die im Rahmen dieser nicht-randomisierten, prospektiven Beobachtungsstudie für Tumore $>/=6 \mathrm{~cm}$ ab Anokutanlinie unabhängig von der T- und N-Kategorie nur bei mrCRM+ Konstellation durchgeführt wird, während Patienten im unteren Drittel $(<6 \mathrm{~cm})$ ab einer T3-Kategorie neoadjuvant behandelt werden. Von 642 bislang eingeschlossenen Patienten wurden 389 (61\%) primär operiert, die Rate an pCRM- betrug für diese Patienten 98\% [976]. Allerdings waren in der primär operierten Gruppe insgesamt 192 Patienten (49\%) mit frühen CT1-2-Tumoren und bei 96 Patienten (25\%) lag der Tumor im oberen Rektumdrittel. Langzeitergebnisse zur lokalen Kontrolle und Überlebensdaten liegen noch nicht vor. 


\begin{tabular}{|l|l|}
$\begin{array}{l}\text { 8.20. } \\
\text { Empfehlungsgrad }\end{array}$ & Evidenzbasierte Empfehlung \\
\hline $\mathbf{A}$ & $\begin{array}{l}\text { Rektumkarzinome im oberen Drittel ohne Risikokonstellation für ein Lokal-rezidiv } \\
\text { sollen primär operiert und adjuvant analog zu Kolonkarzinomen behandelt werden. }\end{array}$ \\
\hline $\begin{array}{l}\text { Level of Evidence } \\
\mathbf{1}\end{array}$ & Quellen: $[963,986]$ \\
\hline & \\
\hline
\end{tabular}

\begin{tabular}{|l|l|}
\hline 8.21. & Konsensbasierte Empfehlung \\
\hline EK & $\begin{array}{l}\text { Bei einer Risikokonstellation im oberen Rektumdrittel (z.B. T4, mrCRM+, bildgebend } \\
\text { eindeutiger und ausgedehnter Lymphknotenbefall) kann eine präoperative Radio-/ } \\
\text { Radiochemotherapie erfolgen. }\end{array}$ \\
\hline & Konsens \\
\hline
\end{tabular}

\section{Hintergrund}

Folgende Argumente sprechen dafür, das obere Rektumdrittel (variabel definiert als $>10-15 \mathrm{~cm}$, bzw. $>12-16 \mathrm{~cm}$ ab Anokutanlinie, gemessen mit einem starren Rektoskop) wie ein Kolonkarzinom zu behandeln: Die Daten der amerikanischen Adjuvanzstudien, die ursprünglich die postoperative Radiochemotherapie bei der Behandlung des lokal fortgeschrittenen Rektumkarzinoms etabliert hatten, bezogen sich ausschließlich auf Rektumtumoren mit einem Abstand des distalen Tumorpols von der Anokutanlinie bis $12 \mathrm{~cm}$ [987] [988]. In den moderneren Studien zur neoadjuvanten Radiotherapie/ Radiochemotherapie waren zwar Tumoren im oberen Drittel inkludiert, in der schwedischen und holländischen Studie zur Kurzzeit-Vorbestrahlung versus alleiniger Operation konnte bei Tumoren im oberen Rektumdrittel (hier definiert als > 10-15 cm ab Anokutanlinie) keine signifikante Verbesserung der Lokalrezidivrate durch die zusätzliche Radiotherapie nachgewiesen werden ( $8 \%$ versus $12 \%$ bei alleiniger Operation nach 10 Jahren, $\mathrm{p}=0.3$, in der schwedischen Studie; $3.7 \%$ versus $6.2 \%$ nach 5 Jahren, p=0.12, in der holländischen Studie) [963] [986]. In der britischen MRC CR07Studie betrug allerdings die 3-Jahres-Lokalrezidivrate bei Tumoren $>10-15 \mathrm{~cm}$ ab Anokutanlinie nach Vorbestrahlung $1.2 \%$ versus $6.2 \%$ nach primärer Operation $(\mathrm{HR}=$ 0.19; 95\% Cl 0.07-0.47) [957]. Insgesamt nimmt die Rate an Lokalrezidiven mit zunehmenden Abstand zur Anokutanlinie in allen Studien ab, sodass es gerechtfertigt ist, auf eine generelle Vorbestrahlung für Tumore im oberen Rektumdrittel zu verzichten bzw. diese nur selektiv bei Vorliegen von Faktoren für ein erhöhtes Lokalrezidivrisiko oder eine R1-Resektion (z.B. cT4, mrCRM+, cN2) zu indizieren. 


\begin{tabular}{|l|l|}
$\begin{array}{l}\mathbf{8 . 2 2} . \\
\text { Empfehlungsgrad }\end{array}$ & $\begin{array}{l}\text { Evidenzbasierte Empfehlung } \\
\text { Defolgt von sofortiger Operation oder als konventionell fraktionierte Radio- }\end{array}$ \\
\hline $\mathbf{0}$ & $\begin{array}{l}\text { gefomotherapie (1.8-2.0 Gy bis 45-50.4 Gy) mit einem Intervall von 6-8 Wochen bis } \\
\text { zur Operation erfolgen. }\end{array}$ \\
\hline $\begin{array}{l}\text { Level of Evidence } \\
\mathbf{1}\end{array}$ & \begin{tabular}{l} 
Quellen: [989][994[990-993] \\
\hline
\end{tabular} \\
\hline
\end{tabular}

\begin{tabular}{|l|l|l}
\hline 8.23. & Konsensbasierte Empfehlung & 2017 \\
\hline EK & $\begin{array}{l}\text { Bei T4-Tumoren, Nähe des Tumors zur mesorektalen Fazie }(<1-2 \text { mm }) \text { oder } \\
\text { tiefliegenden Tumoren mit intendiertem Sphinktererhalt sollte eine präoperative } \\
\text { Radiochemotherapie durchgeführt werden. }\end{array}$ \\
\hline Starker Konsens
\end{tabular}

\subsection{4.}

Empfehlungsgrad

0

Level of Evidence

lb

\section{Evidenzbasierte Empfehlung}

Für Patienten bei denen ein Downsizing angestrebt ist, kann auch die Kurzzeitbestrahlung mit längerem Intervall bis zu 12 Wochen zur Operation (mit und ohne neoadjuvante Chemotherapie) durchgeführt werden.

Quellen: [994-996]

Konsens

\section{Hintergrund}

Die präoperative Kurzzeitbestrahlung mit $5 \times 5$ Gy an 5 aufeinander folgenden Tagen, unmittelbar gefolgt von der Operation, wurde in zwei randomisierten Studien mit der präoperativen, konventionell fraktionierten, 5-Fluorouracil-basierten Radiochemotherapie (45 bis 50.4 Gy in 25-28 Fraktionen), gefolgt von der Operation nach 4-8 Wochen, verglichen. Eine polnische Studie zeigte nach neoadjuvanter Radiochemotherapie ein signifikant besseres Downsizing und Downstaging (pCR-Rate 16\% versus $1 \%, \mathrm{p}<0.001$ ) sowie eine signifikant niedrigere Rate an CRM+ Resektionen (4\% versus $16 \%$ nach $5 \times 5 \mathrm{~Gy}, \mathrm{p}=0.02$ ) bei allerdings erhöhter Akuttoxizität im Vergleich zur Kurzzeitbestrahlung (18\% Grad 3-4 Toxizität versus 3\% nach $5 \times 5$ Gy, p<0.001). Die Rate an sphinktererhaltenden Operationsverfahren (primärer Endpunkt: $58 \%$ versus $61 \%$ nach $5 \times 5$ Gy), die lokale (16\% Lokalrezidive nach 4 Jahren versus $11 \%$ nach $5 \times 5$ Gy) und systemische Tumorkontrolle (34,6\% Metastasen nach 4 Jahren versus 31,4\% nach 5 x 5 Gy) - sowie die Spättoxizität (Grad 3-4 7\% versus 10\% nach 5 x 5 Gy) waren in beiden Armen nicht signifikant unterschiedlich [990] [991] [992]. Die australische Trans- 
Tasman-Studie bestätigte diese Ergebnisse. Auch hier war der primäre Endpunkt, die Rate lokoregionärer Rezidive, in beiden Armen nicht signikant unterschiedlich (3-Jahres Ergebnisse: $7.5 \%$ nach $5 \times 5$ Gy versus $4.4 \%$ nach Radiochemotherapie, $p=0.24$ ) [989]. Die postoperativen Komplikationen waren in beiden Armen vergleichbar (53.2\% nach $5 \times 5$ Gy versus $50.4 \%$ nach Radiochemotherapie), die Akuttoxizität während der Radiochemotherapie höher (z.B. Grad 3-4 Diarrhoe $1.3 \%$ versus $14.2 \%, p<0.001$ ) [997]. Innerhalb der ersten 12 Monate konnten auf Grundlage der EORTC Fragebögen QLQ-C30 und OLC-C38 keine signifikanten Unterschiede in der krankheitsbezogenen Lebensqualität zwischen beiden Therapiearmen erhoben werden [993].

Prinzipiell können also beide Fraktionierungsschemata für die präoperative Radiotherapie verwendet werden. In Konstellationen, die eine Tumorschrumpfung vor Operationen anstreben lassen (z.B. T4-Tumore, Nähe des Tumors zur mesorektalen Fazie, tiefliegende Tumore mit intendiertem Sphinktererhalt) sollte der konventionell fraktionierten Radiochemotherapie mit Intervall bis zur Operation der Vorzug vor der Kurzzeitbestrahlung mit unmittelbar darauf erfolgender Operation gegeben werden.

Neuere Studien belegen allerdings, dass mit verlängertem Intervall zwischen KurzzeitRadiotherapie und Operation ebenfalls eine deutliche Tumorregression eintritt. In der randomisierten Stockholm III-Studie erfolgte die Operation entweder unmittelbar nach der Applikation von 5x5 Gy oder nach 4-8 Wochen: die Rate an ypT0-Tumoren betrug bei sofortiger Operation 2.1\% und nach verlängertem Intervall 11.8\% [994] [995]. Eine polnische Phase III-Studie verglich die konventionell-fraktionierte 5-Fluorouracil-basierte Radiochemotherapie (teilweise unter Inklusion von Oxaliplatin) mit einer KurzzeitRadiotherapie gefolgt von 3 Zyklen einer neoadjuvanten Chemotherapie mit FOLFOX-4 und Operation in Woche 12 bei Patienten mit klinisch fixierten T3- oder T4-Tumoren. Der primäre Endpunkt, die R0-Resektionsrate, war in beiden Armen nicht signifikant unterschiedlich $(71 \%$ versus $77 \%, p=0.07)$, ebenso die lokale Kontrolle und das krankheitsfreie Überleben. Die Akuttoxizität über alle Grade (1-4) war im Arm mit Kurzzeit-RT und neoadjuvanter Chemotherapie signifikant niedriger ( $75 \%$ versus $83 \%$, $\mathrm{p}=0.006$ ), höhergradige Grad 3-4 Toxizität (Diarrhoe, Neutropenie) trat in Summe in beiden Armen allerdings gleich häufig auf (23\% versus 21\% [996]. Eine weitere randomisierte Phase III-Studie (RAPIDO) testete bei MRT-definierten Hoch-risikopatienten (T4, mrCRM+, N2, EMVI+) die Kurzzeit-Radiotherapie mit 5x5 Gy, gefolgt von 6 Zyklen CAPOX und Operation in Woche 22-24 versus einer konventionellen Radiochemotherapie mit Capecitabine und Operation in Woche 14-16 [998]. Die Rekrutierung dieser Studie ist seit Juni 2016 abgeschlossen, Ergebnisse liegen noch nicht vor. 


\begin{tabular}{|l|l|}
$\begin{array}{l}\text { 8.25. } \\
\text { Empfehlungsgrad }\end{array}$ & $\begin{array}{l}\text { Evidenzbasierte Empfehlung } \\
\text { Die neoadjuvante Radiochemotherapie soll orales Capecitabin oder infusionales 5- } \\
\text { Fluorouracil beinhalten. }\end{array}$ \\
\hline $\begin{array}{l}\text { Level of Evidence } \\
\mathbf{1} \mathbf{b}\end{array}$ & Quellen: [970,971,999-1001] \\
\hline & Starker Konsens \\
\hline
\end{tabular}

\section{Hintergrund}

Bei konventionell-fraktionierter präoperativer Radiotherapie (1.8-2 Gy Einzeldosis bis 4550.4 Gy Gesamtdosis) erwies sich die simultane Kombination mit einer 5-Fluorouracilhaltigen Chemotherapie in zwei randomisierten Studien (EORTC 22921, FFCD 9203) hinsichtlich der lokalen Kontrolle, nicht jedoch des Krankeitsfreien Überlebens und des Gesamtüberlebens, als signifikant überlegen im Vergleich zur alleinigen Radiotherapie [960] [961] [962]. Dies wurde in Meta-Analysen bestätigt [970] [971]. Im Rahmen der EORTC 22921- und FFCD 9203-Studie erhielten die Patienten in den präoperativen Kombinationsarmen 5-Fluorouracil als Bolusinfusion in einer Dosierung von 350 $\mathrm{mg} / \mathrm{m}^{2} /$ Tag und Folinsäure in einer Dosierung von $20 \mathrm{mg} / \mathrm{m}^{2} / \mathrm{Tag}$ über jeweils 5 Tage in der 1. und 5. Bestrahlungswoche. In der deutschen CAO/ARO/AIO-94-Studie wurde 5Fluorouracil ohne Modulation mit Folinsäure in der 1. und 5. Bestrahlungswoche in einer Dosierung von $1000 \mathrm{mg} / \mathrm{m}^{2} / \mathrm{Tag}$ als 120-stündige Dauerinfusion appliziert [958].

Zwei folgende prospektiv-randomisierte Studien untersuchten den Ersatz von infusionalem 5-Fluorouracil durch orales Capecitabin [999] [1000] [1001] (siehe Evidenztabelle im Leitlinienreport). Die deutsche Phase III-Studie begann 2002 als adjuvante Studie und wurde nach Publikation der CAO/ARO/AIO-94-Studie im Jahre 2004 um ein neoadjuvantes Stratum erweitert. Im adjuvanten Stratum wurden nach Resektion zunächst zwei Zyklen Capecitabin in einer Dosierung von 2500 mg/m²/Tag über 14 Tage (Wiederholung Tag 22), gefolgt von einer Radiochemotherapie (1.8 Gy bis 50.4 Gy) mit Capecitabin $1650 \mathrm{mg} / \mathrm{m}^{2} / \mathrm{Tag}$ während der gesamten Radiotherapie und weitere drei Zyklen Capecitabin verabreicht. Patienten im 5-Fluorouracil-Arm erhielten nach Operation zwei Zyklen Bolus 5-FU (500 mg/m²/Tag 1-5, Wiederholung Tag 29), gefolgt von der Radiotherapie mit infusionalem 5 -FU in einer Dosierung von $225 \mathrm{mg} / \mathrm{m}^{2} / \mathrm{Tag}$ während der gesamten Radiotherapie und zwei weitere Zyklen Bolus 5-FU. Im neoadjuvanten Stratum wurde die Radiochemotherapie mit Capecitabin, gefolgt von der Operation und fünf adjuvanten Zyklen Capecitabin in denselben Dosen wie in dem adjuvanten Stratum durchgeführt. Die neoadjuvante Kohorte im infusionalem 5-FU-Arm erhielt eine präoperative Radiochemotherapie analog der CAO/ARO/AIO-94-Studie (1000 $\mathrm{mg} / \mathrm{m}^{2} /$ Tag als 120 -stündige Dauerinfusion in der 1. und 5. Woche). In Anschluss an der Operation wurden 4 Zyklen Bolus-5-FU (500 mg/m²/Tag 1-5, Wiederholung Tag 29) verabreicht. Der primäre Endpunkt der Studie war das Gesamtüberleben (NichtUnterlegenheitsheitsgrenze 12.5\%). Bei 392 auswertbaren Patienten und einer medianen Nachbeobachtungszeit von 52 Monaten betrug das 5-Jahres-Überleben im CapecitabinArm 76\% und im 5-Fluorouracil-Arm 67\% ( $p=0.0004$ für Nicht-Unterlegenheit). Im neoadjuvanten Stratum ( $n=161$ Patienten) fanden sich in der Capecitabin-Gruppe Hinweise auf eine bessere Antitumorwirkung: Pathologische Komplettremissionen (pCR) fanden sich häufiger im Capecitabin-Arm (14\% versus 5\%, $\mathrm{p}=0.09$ ) und mehr Patienten wiesen bei Resektion nodal-negative Tumore auf ( $71 \%$ versus $57 \%, p=0.08)$ [999]. Die 
Resultate der amerikanischen NSABP R04-Studie, die in einem $2 \times 2$-faktoriellen Design neben der Frage der neoadjuvante Radiochemotherapie mit infusionalem 5-Fluorouracil (225 mg/m²/Tag während der RT) versus Capecitabin (1650 mg/m²/Tag während der RT) auch den Einsatz von Oxaliplatin untersuchte (siehe unten), bestätigte die Deutsche Studie, soweit es chirurgische Resultate, pCR-Raten und die Verträglichkeit der Therapie betrifft [1000]. Auch die Rate an lokoregionären Rezidiven nach 3 Jahren (4.0\% vs. 3.9\%) sowie das Krankheitsfreie Überleben (66.4\% vs. $67.7 \%)$ und Gesamtüberleben $(79.9 \%$ vs. 80.8\%) nach 5 Jahren waren in den Armen mit infusionalem 5-FU und Capecitabine nahezu identisch [1001].

Die neoadjuvante 5-Fluorouracil- oder Capecitabin-basierte Radiochemotherapie unter Einschluss von Oxaliplatin zeigte in einer Vielzahl von Phase II-Studien pCR-Raten bis zu 30\% (Übersicht: [1002]). Der Stellenwert dieser Kombinationsbehandlungen wurde daraufhin in insgesamt sieben randomisierten Phase III-Studien geprüft [1000] [1001] [1003] [1004] [1005] [1006] [1007] [975] [1008] [1009] (Tabelle 1). Diese Studien unterschieden sich wesentlich in der Applikationsweise und Dosierung von 5Fluorouracil/Capecitabin und Oxaliplatin während der simultanen Radiochemotherapie. Fünf der Studien untersuchten den Stellenwert von Oxaliplatin lediglich während der neoadjuvanten Radiochemotherapie, bei zwei schloss die Randomisierung auch die adjuvante Chemotherapie mit oder ohne Oxaliplatin ein [975] [1007]. Die Studien unterschieden sich ebenfalls hinsichtlich des gewählten primären Endpunktes (pCR-Rate [1005], lokale Kontrolle [1001], krankheitsfreies Überleben [975] [924] [1009] ], Gesamtüberleben [1004] [1008] sowie der darauf beruhenden Fallzahl-Kalkulationen und in ihren Nachbeobachtungszeiten. In der deutschen CAO/ARO/AIO-04- [975] sowie der chinesischen FOWARC-Studie [1009] konnte eine signifikante Verbesserung der pCRRate als sekundärer Endpunkt nach neoadjuvanter Radiochemotherapie mit Inklusion von Oxaliplatin festgestellt werden, während in den fünf weiteren Einzelstudien kein signifikanter Unterschied der pCR-Raten bestand. Eine Metaanalyse mit Inklusion der ACCORD 12, STAR-01, NSAPB R-04, und CAO/ARO/AIO-04-Studie beschrieb eine signifikant erhöhte $\mathrm{pCR}$-Rate (HR 1.20;95\% Cl, 1.01-1.42; $\mathrm{p}=0.04)$, allerdings auch eine signifikant erhöhte Akuttoxizität Grad 3-4 (HR 2.29; 95\% Cl, 1.31-4.00, p=0.004) nach neoadjuvanter Radiochemotherapie mit Oxaliplatin [1010].

In der deutschen CAO/ARO/AIO-04 Studie konnte ein signifikant verbessertes krankheitsfreies Überleben (als primärer Endpunkt) nachgewiesen werden; eine Metaanalyse unter Einschluss von 4 Studien mit Angaben zum krankheitsfreien Überleben beschrieb einen marginal signifikanten Effekt auf diesen Endpunkt (HR 0.89; $95 \% \mathrm{Cl}, 0.78-1.00, \mathrm{p}=0.05$ ) [1011]. In fünf der sieben Einzelstudien konnte allerdings keine signifikante Verbesserung des krankheitsfreien Überlebens durch Inklusion von Oxaliplatin gezeigt werden, Langzeitergebnisse zu FOWARC-Studie stehen diesbezüglich noch aus. Insgesamt ist somit ein Vorteil des Einsatzes von Oxaliplatin nicht gesichert. 


\begin{tabular}{|l|l|}
\hline $\begin{array}{l}\text { 8.26. } \\
\text { Empfehlungsgrad } \\
\mathbf{B}\end{array}$ & Die Operation sollte 6-8 Wochen nach neoadjuvanter Radiochemotherapie erfolgen. \\
\hline $\begin{array}{l}\text { Level of Evidence } \\
\mathbf{3} \mathbf{A}\end{array}$ & Quellen: [1012-1015] \\
\hline & Konsens \\
\hline
\end{tabular}

\subsection{7 .}

Empfehlungsgrad

B

Level of Evidence

3b

\section{Evidenzbasierte Empfehlung}

Nach Kurzzeit-Bestrahlung ( 5 × 5 Gy) sollte die Operation entweder innerhalb von 10 Tagen nach Beginn der Radiotherapie oder nach 4-8 Wochen erfolgen.

Quellen: [995, 1016]

Starker Konsens

\section{Hintergrund}

In den prospektiven Studien zur Etablierung der präoperativen Radiochemotherapie erfolgte die Operation in Abständen von 3-10 Wochen (Median: 5.4 Wochen; EORTC 22921, FFCD 9203 [960] [962] bzw. 6 Wochen (CAO/ARO/AIO-94 und CAO/ARO/AIO-04 [958] [975] nach Abschluss der neoadjuvanten Therapie. Die kürzlich publizierte GRECCAR-6-Studie randomisierte 265 Patienten nach neoadjuvanter Radiochemotherapie (45 - $50 \mathrm{~Gy}$, infusionales 5-FU oder Capecitabin) in zwei Arme mit Operation nach 7 Wochen versus 11 Wochen [1012] (siehe Evidenztabelle im Leitlinienreport). Der primäre Endpunkt dieser Studie, die pCR-Rate, war nicht signifikant unterschiedlich (15\% nach 7 Wochen vs. $17.4 \%$ nach 11 Wochen, $\mathrm{p}=0.6$ ), allerdings traten nach Operation in Woche 11 mehr postoperative Komplikationen (32\% vs. 44.5\%, p=0.04) auf und die TMEQualität war schlechter (komplette TME $90 \%$ vs $78.7 \%, p=0.02$ ). Langzeitergebnisse zu onkologischen Endpunkten liegen für diese Phase III-Studie noch nicht vor. Zwei MetaAnalysen und ein systematischer Review auf Grundlage nicht-randomisierter Daten mit Sekundäranalysen von prospektiven Studien oder Beobachtungsstudien geben Hinweise, dass ein Intervall länger als die üblichen 6-8 Wochen nach Radiochemotherapie mit einer erhöhten pCR-Rate bei nicht erhöhter Komplikationsrate einherzugehen scheint [1013] [1014] [1015] (siehe Evidenztabelle im Leitlinienreport), Unterschiede in der Lokalrezidivrate, dem Krankheitsfreien Überleben oder Gesamtüberleben wurden nicht beobachtet. Eine höhergradige Evidenz zur Verlängerung des Intervalls zwischen Abschluss der Radiochemotherapie und der Operation über die standardmäßigen 6-8 Wochen hinaus liegt somit nicht vor.

In den prospektiven Studien zur Etablierung der Kurzzeit-Radiotherapie mit $5 \times 5$ Gy sollte die Operation innerhalb einer Woche nach Abschluss der Radiotherapie erfolgen. Eine Sekundäranalyse der holländischen TME-Studie zeigte, dass insbesondere ältere Patienten (=/> 75 Jahre) ein schlechteres Überleben hatten, wenn sie mehr als 3 Tage 
(d.h. OP am Tag 4-7) nach Abschluss der Radiotherapie operiert worden waren [1016]; als Gesamtbehandlungszeit (Beginn RT bis OP) wird von dieser Gruppe daher ein Intervall von maximal 10 Tagen empfohlen.

In der randomisierten Stockholm III Studie erfolgte die Operation entweder unmittelbar nach der Applikation von 5x5 Gy oder nach 4-8 Wochen: die Rate an ypT0-Tumoren betrug bei sofortiger Operation 2.1\% und nach verlängertem Intervall $11.8 \%$, die Rate an pCRM+ Resektionen und an abdominoperinealen Exstirpationen unterschieden sich jedoch nicht [995]. Die postoperativen Komplikationen waren nach $5 \times 5$ Gy und längerem Intervall niedriger als bei sofortiger Operation, die Langzeitergebnisse zu onkologischen Endpunkten (Lokalrezidive, Fernmetastasen) zeigten keine Unterschiede [1017] (siehe Evidenztabelle im Leitlinienreport). Eine weitere randomisierte, monozentrische Studie aus Polen mit insgesamt 154 Patienten ergab ebenfalls ein signifikant verbessertes Tumor-Downstaging nach verlängertem Interval (4-5 Wochen nach $5 \times 5 \mathrm{~Gy}$ ) bei allerdings identischer R0-Resektionsrate, Rate an schließmuskelerhaltenden Operationen und nicht signifikant unterschiedlichem Gesamtüberleben (primärer Endpunkt) [1018]. Ein systematische Review von Bujko et al. erlaubt aufgrund der Heterogenität der eingeschlossenen Studien keine klare Aussage [1019]. Insgesamt ist bei alleiniger präoperativer Radiotherapie mit 5×5 Gy und Operation eine Gesamtbehandlungszeit von 10 Tagen anzustreben, ein verlängertes Intervall zur Operation von 4-8 Wochen kann aber nach Publikation der Stockholm III-Studie ebenfalls angeboten werden und eignet sich insbesondere bei Patienten mit notwendigem Downsizing, die sich nicht für eine Chemotherapie eignen oder diese ablehnen, als Alternative zur konventionellfraktionierten Radiochemotherapie. Zur Addition einer neoadjuvanten Chemotherapie nach $5 \times 5$ Gy und verlängertem Intervall bis zur Operation siehe Empfehlung 8.24. mit Hintergrundtext.

Für Patienten, die nach neoadjuvanter Therapie und vor geplanter Operation auf Grundlage der rektal-digitalen Untersuchung, der Rektoskopie und der Dünnschicht-MRT des Beckens eine klinisch komplette Remission aufweisen, wird zunehmend eine „waitand-see" Strategie unter Verzicht auf die radikale Operation diskutiert. Dieses Behandlungskonzept wird derzeit in prospektiven Studien untersucht und stellt noch kein gesichertes Vorgehen dar. Lehnt die Patientin/der Patient die Operation (zumeist im Sinne einer Exstirpation) trotz Aufklärung zu dieser ungesicherten Datenlage ab, ist eine engmaschige Nachsorge unter Einsatz der rektal-digitalen Untersuchung, der Rektoskopie und der Dünnschicht-MRT im 3-monatigen Intervall während der ersten beiden Jahren zu empfehlen, um ein lokales Wiederauftreten des Tumors (,re-growth“) möglichst frühzeitig erkennen und behandeln zu können.

Kommentar zur Durchführung eines MRT nach Abschluss der neoadjuvanten Therapie: Bei unzureichender Datenlage wurde diese Frage innerhalb der Arbeitsgruppe nicht diskutiert. Es soll eine individuelle Entscheidung vor Ort getroffen werden.

\begin{tabular}{|l|l|}
\hline 8.28. & Konsensbasierte Empfehlung \\
\hline EK & $\begin{array}{l}\text { Die neoadjuvante Chemotherapie vor oder nach Radiochemotherapie (oder als } \\
\text { alleinige neoadjuvante Therapie ohne Radio-/Radiochemotherapie) soll außerhalb } \\
\text { von Studien nicht durchgeführt werden. }\end{array}$ \\
\hline Starker Konsens
\end{tabular}


8.29 .

Die Kurzzeit-RT mit 5 × 5 Gy gefolgt von neoadjuvanter Chemotherapie und Operation im Intervall kann bei synchroner Metastasierung erfolgen.

Konsens

\section{Hintergrund}

Bislang haben nur wenige Studien eine neoadjuvante Chemotherapie vor oder nach Radiochemotherapie, oder als alleinige Therapie ohne RT/RCT, gestestet. Eine kleine, randomisierte Phase II-Studie aus Spanien $(n=108)$ verglich 4 Zyklen einer InduktionsChemotherapie mit Capecitabin/Oxaliplatin, gefolgt von einer Radiochemotherapie mit Capecitabin/Oxaliplatin und Operation, mit der „klassischen“ Sequenz: Radiochemotherapie, Operation, adjuvante Chemotherapie bei ansonsten identischen Substanzen und Dosierungen (Evidenztablelle 3) [1020, 1021]. Der primäre Endpunkt, die pCR-Rate sowie onkologische Langzeitendpunkte waren nicht unterschiedlich (wofür diese randomisierte Phase-II-Studie aber auch nicht ausreichend gepowert war), jedoch war die Verträglichkeit und Durchführbarkeit der Induktions-Chemotherapie im Vergleich zur adjuvanten Chemotherapie signifikant verbessert. Phase-III-Studien zur dieser Fragestellung liegen nicht vor.

Eine prospektive Kohortenstudie des "Timing of Rectal Cancer Response to Chemoradiation Consortium" in den USA untersuchte nach neaodjuvanter Radiochemotherapie mit infusionalem 5-Fluorouracil die Applikation von null, zwei, vier, und sechs Zyklen einer Chemotherapie mit FOLFOX, gefolgt von Operation 6, 11, 15, und 19 Wochen nach Abschluss der Radiochemotherapie (siehe Evidenztabelle im Leitlinienreport). Die pCR-Rate nahm in den Kohorten sukzessiv zu (18\%, 25\%, 30\%, 38\%), ohne dass erhöhte chirurgische Komplikationen auftraten [1022]. Zwei randomisierte Phase II-Studien rekrutieren derzeit (Stand 04/2017) Patienten mit der Fragestellung, welche Sequenz (neoadjuvante Chemotherapie vor oder nach Radiochemotherapie) hinsichtlich Wirksamkeit, Durchführbarkeit und Toxizität überlegen ist [1023] und CAO/ARO/AIO-12]. Ergebnisse werden 2018 erwartet.

Die alleinige neoadjuvante Chemotherapie ohne Radio-/Radiochemotherapie zeigte in Phase II-Studien [1024] [1025] sowie in der randomisierten FORWARC-Studie [1009] R0Resektions-, Downstaging- und pCR-Raten. Eine randomisierte Phase III-Studie (PROSPECT) in den USA untersucht eine alleinige neoadjuvante Chemotherapie (6 Zyklen FOLFOX) versus der Standard-Radiochemotherapie mit 5-FU/Capecitabin bei selektionierten Patienten (cT1/2N1; cT3N0/N1 mit mrCRM-, Schließmuskelerhalt möglich). Daten dazu werden 2018/2019 erwartet.

Zur neoadjuvanten Chemotherapie nach $5 \times 5$ Gy bei Rektumprimärtumor ohne synchrone Fernmetasasierung siehe Hintergrundtext zu Empfehlung 8.17. Im Falle eines Rektumprimärtumors mit synchroner, (potentiell) operabler Metastasierung kann die Radiotherapie mit 5×5 Gy, frühzeitigt gefolgt von einer wirksamen Systemtherapie und Operation von Primärtumor/Metastasen im Verlauf eine sinnvolle Option sein [1026] . 


\subsubsection{Adjuvante Therapie}

8.2.2.1. Adjuvante Therapie des Rektumkarzinoms bei primärer Operation (ohne Vorbehandlung)

\begin{tabular}{|c|c|}
\hline 8.30. & Evidenzbasierte Empfehlung \\
\hline $\begin{array}{l}\text { Empfehlungsgrad } \\
\text { A }\end{array}$ & $\begin{array}{l}\text { Im UICC-Stadium I ( } \mathrm{pT} 1 / 2 \mathrm{~N} 0) \text { soll nach R0-Resektion eine adjuvante Therapie nicht } \\
\text { erfolgen. }\end{array}$ \\
\hline Level of Evidence & Quellen: [956] \\
\hline & Starker Konsens \\
\hline
\end{tabular}

\section{Hintergrund}

In allen randomisierten Studien zur adjuvanten Therapie des Rektumkarzinoms waren Patienten mit Stadium UICC I nach R0-Resektion wegen niedriger Lokalrezidiv- und Fernmetastasenraten (3\% bzw. weniger als 10\% nach 10 Jahren [956] ausgeschlossen.

\subsection{1 .}

EK

\section{Konsensbasierte Empfehlung}

Bei histopathologisch bestätigten Risikofaktoren für ein lokoregionäres Rezidiv (u.a. R1-Resektion, intraoperativer Tumoreinriss, pCRM+, unzu-reichender TME-Qualität, pT4, pT3c/d, pN2, extranodale Tumorherde im Mesorektum, pT3 im unteren Rektumdrittel) sollte eine adjuvante Radio-chemotherapie durchgeführt werden.

Konsens

\section{Hintergrund}

In Phase III-Studien aus den siebziger und achtziger Jahren des 20. Jahrhunderts zur adjuvanten, multimodalen Therapie des Rektumkarzinoms im UICC-Stadium II und III (pT3-4 und/oder pN+, bis $12 \mathrm{~cm}$ ab Anokutanlinie) konnte durch Hinzunahme einer 5Fluorouracil-basierten simultanen und adjuvanten Chemotherapie zu einer postoperativen Bestrahlung sowohl die Lokalrezidivrate gesenkt (absolute Differenz: 1015\%) als auch das Gesamtüberleben im Vergleich zur alleinigen Operation oder zur unimodalen Adjuvanztherapie (absolute Differenz: 5-15\%) verbessert werden [Übersicht: [1027]. Die chirurgischen Prinzipien der totalen mesorektalen Exzision waren in diesen veralteten Studien aber nicht umgesetzt und die Lokalrezidivraten nach alleiniger Operation hoch. Eine gepoolten Subgruppen-Analyse von fünf dieser frühen nordamerikanischen Studien legte außerdem nahe, dass insbesondere Patienten mit pT1-2N1- sowie pT3N0-Tumoren nach R0-Resektion von der zusätzlichen Bestrahlung nicht profitieren [1028].

Zum Stellenwert der adjuvanten Radiochemotherapie bei Patienten im UICC-Stadium II und III liegen seit Etablierung der neoadjuvanten Radio-/Radiochemotherapie keine modernen Studien mit qualitätsgesicherter TME-Chirurgie vor. Da die postoperative Radiochemotherapie weniger effektiv sowie deutlich nebenwirkungsreicher ist als die präoperative (siehe Hintergrundtext zu 8.18. [958], ist das Nutzen-/Risikoverhältnis der 
postoperativen Radiochemotherapie bei strikter Anwendung der Prinzipien der TME nicht ausreichend geklärt [1029]. Eine generelle Empfehlung zur postoperativen Radiochemotherapie für alle Patienten mit Tumoren im UICC-Stadium II und III kann somit nicht erfolgen. Diese sollte Patienten mit histopathologisch-bestätigten Risikofaktoren für ein erhöhtes Lokalrezidivrisiko (u.a. R1-Resektion, intraoperativer Tumoreinriss, pCRM+, unzureichende TME-Qualität, pT4, pT3c/d, pN2, extranodale Tumorherde im Mesorektum, pT3 im unteren Rektumdrittel) vorbehalten bleiben.

\begin{tabular}{|l|l|l|}
\hline 8.32. & Konsensbasierte Empfehlung & 2017 \\
\hline EK & $\begin{array}{l}\text { Wird nach primärer RO-Resektion im Stadium II/III keine adjuvante } \\
\text { Radiochemotherapie durchgeführt, sollte eine adjuvante Chemotherapie analog zu } \\
\text { den Indikationskriterien und Schemata beim Kolonkarzinom erfolgen. }\end{array}$ \\
& Starker Konsens
\end{tabular}

\section{Hintergrund}

Ein systematischer Review zur postoperativen Chemotherapie versus Beobachtung nach kurativer Resektion eines Rektumkarzinoms (mit Einschluss von 21 randomisierten Studien zwischen 1975-2011 und insgesamt 9785 Patienten) bestätigte eine signifikante Reduzierung der Todesrate $(\mathrm{HR}=0.83,95 \% \mathrm{Cl}: 0.76-0.91)$ und der Rezidivrate $(\mathrm{HR}=0.75$; 95\% Cl:0.68-0.83) nach 5-Fluorouracil-basierter adjuvanter Chemotherapie [1030]. Die hierbei eingeschlossenen Studien sind jedoch sehr inhomogen, inkludieren auch solche mit präoperativer und postoperativer Radiotherapie, und erlauben keine differentiellen Aussagen nach UICC-Stadien.

Die randomisierte QUASAR-Studie zur adjuvanten Chemotherapie (5-FU + Folinsäure oder Levamisol) versus Beobachtung für Patienten mit kolorektalem Karzinom und unsicherer Indikation für eine Chemotherapie (zumeist Stadium II) zeigte nach einer medianen Nachbeobachtungszeit von 5,5 Jahren für die Subgruppe der Patienten mit Rektumkarzinom ( $n=948)$ eine signifikant reduzierte Rezidivrate $(\mathrm{HR}=0.68,95 \% \mathrm{Cl}$ : 0.52 0.88) und ein verbessertes Gesamtüberleben ( $\mathrm{HR}=0.77,95 \% \mathrm{Cl}$ : 0.54-1.00) [884], allerdings hatte 203 dieser Patienten auch eine präoperative und 264 Patienten eine zusätzliche postoperativer Radiotherapie erhalten. QoL-Untersuchungen in Bezug auf typische chemotherapieassoziierte Nebenwirkungen (Diarrhoe, Übelkeit, Fatique, Appetitlosigkeit, Mundtrockenheit) zeigten für alle Kategorien eine signifikante Verschlechterung im Therapie- versus Obervationsarm, waren aber auf die Zeit der Chemotherapie limitiert. Zwei randomisierte japanische Studien zeigten einen signifikanten Vorteil einer postoperativen Chemotherapie (mit Uracil-Tegafur für 1 Jahr) versus Beobachtung hinsichtlich des krankheitsfreien und Gesamtüberlebens für Patienten mit Rektumkarzinom im UICC-Stadium III nach TME und selektiver, extendierter lateraler Lymphknotenresektion [1031] [1032] .

Die Evidenz für eine adjuvante Chemotherapie ist für Patienten mit Rektumkarzinom (ohne Vorbehandlung und ohne Indikation zur postoperativen Radiochemotherapie) somit ungleich geringer als für das Kolonkarzinom. Obgleich Phase III-Studien unter Einsatz moderner adjuvanter Kombinations-Chemotherapien als alleinige adjuvante Therapie nach primärer Resektion eines Rektumkarzinoms ohne Vorbehandlung nicht vorliegen, wurde als Expertenkonsens formuliert, in einer solchen Konstellation analog der Indikationskriterien und Schemata des Kolonkarzinoms zu verfahren. 


\subsubsection{Adjuvante Therapie des Rektumkarzinoms nach präoperativer Radio-} /Radiochemotherapie

\begin{tabular}{|l|l|}
\hline $\begin{array}{l}\text { 8.33. } \\
\text { Empfehlungsgrad }\end{array}$ & $\begin{array}{l}\text { Eine Empfehlung für oder gegen eine adjuvante Chemotherapie nach erfolgter } \\
\text { neoadjuvanter Radiochemotherapie kann auf Grundlage der vorhandenen Datenlage } \\
\text { beim Rektumkarzinom nicht geben werden. }\end{array}$ \\
\hline $\begin{array}{l}\text { Level of Evidence } \\
\mathbf{5}\end{array}$ & \begin{tabular}{l} 
Quellen: $[960,961,1033-1035]$ \\
\hline
\end{tabular} \\
\hline
\end{tabular}

\section{Hintergrund}

Zur Frage der adjuvanten Chemotherapie versus Beobachtung nach erfolgter präoperativer Radiotherapie bzw. 5-Fluorouracil-basierter Radiochemotherapie wurden vier randomisierte Phase-III Studien durchgeführt (siehe Evidenztabelle im Leitlinienreport). In keiner dieser Studien konnte eine signifikante Verbesserung des krankheitsfreien Überlebens oder Gesamtüberlebens durch die adjuvante 5-Fluorouracilbasierte Chemotherapie nachgewiesen werden [960, 961, 1033-1035].

Die vier Phase III-Studien unterscheiden sich deutlich in der Wahl und Applikationsweise der neoadjuvanten Radio-/Radiochemotherapie und adjuvanten 5-Fluorouracil-basierten Therapie und verwenden teils veraltete und suboptimale 5-FU-Bolus Regime. Nur die CHRONICLE-Studie inkludierte Oxaliplatin in die adjuvante Chemotherapie, wurde wegen schlechter Rekrutierung aber vorzeitig abgebrochen [1034]. Auffallend ist in allen Studien insbesondere die schlechte Compliance bezüglich der adjuvanten Chemotherapie und die hohe Rate an Patienten (27-28\%), die nach Operation keine adjuvante Behandlung erhalten konnten. Dies gilt insbesondere für Studien, bei denen die Randomisierung vor neoadjuvanter Behandlung und Operation erfolgte [961] [1033]. Zwei der vier Studien mussten wegen schlechter Rekrutierung vorzeitig geschlossen werden und sind somit für die primären Endpunkte „underpowered“ [1034, 1035].

Eine auf diesen vier randomisierten Studien beruhende Meta-Analyse auf Grundlage individueller Patientendaten fand für die Subgruppe der Patienten mit ypTNM II oder III Tumoren nach R0-Resektion $(n=1196)$ ebenfalls keine signifikanten Unterschiede im Krankheitsfreien Überleben (HR 0.91; 95\% Cl 0.77-1.07, p=0.23) oder Gesamtüberleben (HR 0.97, 95\% Cl 0.81-1.17, p=0.775) für Patienten mit oder ohne adjuvanter Chemotherapie. Subgruppen-Analysen lassen allenfalls für Patienten mit Rektumtumoren im oberen Drittel (hier: 10-15 cm ab Anokutanlinie) einen Benefit der adjuvanten Chemotherapie vermuten [1036]. Patienten mit ypTNM-Stadium 0-I nach neoadjuvanter Therapie waren aus dieser Meta-Analyse allerdings ausgeschlossen, da sie in zwei der vier Einzelstudien nicht inkludiert waren. Problematisch ist des Weiteren, dass Patienten mit alleiniger neoadjuvanter, konventionell-fraktionierter Radiotherapie nicht ausgeschlossen wurden, obwohl diese Therapie keinen neoadjuvanten Standard darstellt und die Hazard Ratios für das Krankheitsfreies Überleben- und Gesamtüberleben in dieser Patientengruppe besonders zuungunsten der adjuvanten Chemottherapie ausfallen, die Ergebnisse der gepoolten Analyse also verschlechtern.

Weitere Meta-Analysen zur Rolle der adjuvanten Chemotherapie nach neoadjuvanter Radio-/Radiochemotherapie mit Inklusion überwiegend nicht-randomisierter, 
retrospektiver Serien ließen einen Benefit der adjuvanten Chemotherapie für die Subgruppe von Patienten mit Downstaging [1037], bzw. mit ypN0 (weniger für Patienten mit ypTONO oder ypN+ vermuten [1038]. Die Evidenz dieser retrospektiven Daten und Meta-Analysen ist allerdings als niedrig zu bewerten und das Risiko für Verzerrungen hoch. Die 10-Jahres-Daten der randomisierten EORTC 22921 bestätigten deren initiale Beobachtung jedenfalls nicht, dass insbesondere Patienten mit Downstaging (ypT0-2) nach neoadjuvanter Therapie von der adjuvanten Chemotherapie profitieren [961].

Eine randomisierte Phase II-Studie aus Südkorea (ADORE) fand ein signifikant verbessertes Krankheitsfreies Überleben für Patienten im ypTNM-Stadium II/III nach neoadjuvanter 5-Fluorouracil-basierter Radiochemotherapie für die adjuvante Chemotherapie mit FOLFOX versus 5-FU/Folinsäure-Bolus-Regime [1039]. Eine Subgruppenanalyse zeigte, dass dies insbesondere für das ypTNM-Stadium III galt. Das mediane Alter der Studienpatienten lag bei (nur) 54 Jahren, die Compliance der adjuvanten Chemotherapie war exzeptionell hoch und es gab keinen alleinigen Beobachtungsarm. Zwei randomisierte Phase-III Studien (CAO/ARO/AIO-04, PETACC-6) inkludierten Oxaliplatin sowohl in die neoadjuvanten Radiochemotherapie als auch die adjuvante Chemotherapie versus 5-Fluorouracil-Monotherapien. Die CAO/ARO/AIO-04Studie konnte eine signifikante Verbesserung des DFS im Oxaliplatin-Arm nachweisen, allerdings bleibt unklar, ob dies der neoadjuvanten, adjuvanten Inklusion oder beiden zuzuschreiben ist [975]. Die PETACC-6-Studie zeigte keinen Benefit für die Inklusion von Oxaliplatin [1007].

Auf Grundlage der beschriebenen Phase III-Daten und Meta-Analysen kann somit keine eindeutige Empfehlung für oder gegen eine adjuvante Chemotherapie nach erfolgter präoperativer Radio-/Radiochemotherapie gemacht werden und auch keine Subgruppe identifiziert werden, die davon präferentiell profitiert. Dies trifft auch auf die präoperative Kurzzeit-Radiotherapie mit unmittelbar gefolgter Operation zu, denn in den Phase III-Studien zur Etablierung der Vorbestrahlung mit $5 \times 5$ Gy versus alleiniger Operation erfolgte generell keine adjuvante Chemotherapie, und die einzige Phase-IIIStudie zur adjuvanten Chemotherapie nach 5x5 Gy (SKRIPT) war „underpowered“ und negativ. Methodisch sind diese Studien allerdings aufgrund der teils suboptimalen 5-FUBolus-Gabe, der reduzierten Compliance sowie der teils vorzeitigen Studienabbrüche limitiert.

Als Argument für eine adjuvante Chemotherapie nach neoadjuvanter Radiochemotherapie kann gelten, dass die Großzahl der Studien zur multimodalen Behandlung des Rektumkarzinoms die adjuvante Chemotherapie als obligaten Bestandteil inkludierten und somit gut etablierte Behandlungsmodalitäten darstellen [958] [999] [975]. Entscheiden sich Patient und Arzt nach ausführlicher Erörterung des Für und Wider für eine adjuvante Chemotherapie, legen die Daten nahe, auf Bolus-5-FU in der Adjuvanz zugunsten von Capecitabin oder infusionalem 5-FU zu verzichten (vergleiche dazu Stellungnahme der Arbeitsgruppe Kolon-/Rektumkarzinom der AIO; http://www.aioportal.de). Eine zusätzliche Oxaliplatingabe post- oder perioperativ sollte anhand des individuellen Falls im Tumorboard und gemeinsam mit den Patienten besprochen und festgelegt werden. 


\section{Therapeutisches Vorgehen bei Metastasierung und in der palliativen Situation}

Der folgende Teil der S3-Leitlinie enthält 2017 aktualisierte Empfehlungen zur Tumortherapie beim metastasierten Kolorektalkarzinom (mKRK), die vor allem Erkenntnisse aus Studien der Jahre 2003-2016 Jahre widerspiegeln. Auf die primär resektablen Metastasen wird ebenso eingegangen wie auf die besondere Situation einer sekundären Resektabilität in einem primär palliativen Therapiekonzept. Der Verfügbarkeit chemotherapeutischer und biologischer Substanzen wird in einer kommentierten Auflistung möglicher Kombinationen in Abhängigkeit vom Therapieziel sowie tumor- und patientenbezogener Kriterien Rechnung getragen. Die Einteilung der Patienten in Subgruppen soll die Entscheidungsfindung erleichtern. Die Entscheidung über das therapeutische Vorgehen bei Nachweis einer Metastasierung beginnt mit der Beurteilung des Allgemeinzustandes (siehe Abbildung 4 Therapiealgorithmus in der Behandlung des mKRK

Patienten in gutem Allgemeinzustand können einer intensiven Behandlung, d.h. einer Operation oder Chemotherapie zugeführt werden. Bei resektablen Tumormanifestationen und günstiger Risikokonstellation soll primär die Metastasenresektion angestrebt werden (siehe 9.7.1). Diejenigen Patienten, für die primär keine chirurgische Interventionsmöglichkeit besteht, sollten eine möglichst effektive systemische Chemotherapie erhalten. Als primäres Therapieziel wird die maximale Tumorreduktion angestrebt. Die Wahl des Chemotherapieregimes hängt entscheidend von dem molekularpathologischen Profil des Tumors ab. Bei Patienten mit RAS-Wildtyp Tumoren kommt als weitere Entscheidungsgrundlage die Lokalisation des Primärtumors hinzu.

Die therapeutische Strategie in der Behandlung der metastasierten Erkrankung sollte im Rahmen einer Tumorkonferenz interdisziplinär festgelegt werden. Die Patienten sind ihren individuellen Erfordernissen entsprechend ausführlich über die Therapie-optionen aufzuklären und in die Entscheidung miteinzubeziehen. Neben der Tumortherapie, die im Folgenden dargestellt wird, sind die Sicherstellung einer adäquaten Schmerztherapie und Ernährung, eine bedarfsgerechte psychosoziale und psychoonkologische Versorgung sowie supportive Therapiemaßnahmen integrale Bestandteile des palliativen Therapiekonzepts (siehe themenspezifische Leitlinien unter http://www.leitlinienprogramm-onkologie.de/leitlinien/ und http://www.awmfleitlinien.de).

Palliativmedizin ist definiert als ein Ansatz zur Verbesserung der Lebensqualität von Patienten und ihren Familien, die mit Problemen konfrontiert sind, welche mit einer lebensbedrohlichen Erkrankung einhergehen. Dies geschieht durch Vorbeugen und Lindern von Leiden durch frühzeitige Erkennung, sorgfältige Einschätzung und Behandlung von Schmerzen sowie anderen Problemen körperlicher, psychosozialer und spiritueller Art.

Bezüglich palliativmedizinischer Aspekte unabhängig der zugrunde liegenden Diagnose wird auf die S3-Leitlinie Palliativmedizin des Leitlinienprogramms Onkologie verwiesen (http://www.leitlinienprogramm-onkologie.de/leitlinien/palliativmedizin/). Weitere Quellen, Stand 04/2017: S3-Leitlinie Klinische Ernährung in der Onkologie, AWMFRegisternummer: 073/006; S3-Leitlinie Supportivtherapie, AWMF-Registernummer: 032/054OL: $\quad$ http://www.leitlinienprogramm-onkologie.de/leitlinien/supportivetherapie/). 
In Hinblick auf die Ziele der Tumortherapie kommt der krankheits- und therapiebezogenen Lebensqualität ein zunehmend größerer Stellenwert zu. Als sehr gut messbarer Parameter wird sie immer häufiger als ein sekundärer Endpunkt klinischer Studien erhoben. Dem Wunsch der Patienten nach Informationen über alle relevanten verfügbaren Maßnahmen (tumorspezifische, supportive, psychosoziale, psychoonkologische Therapieoptionen) und Hilfsangebote (z. B. Krebsberatungsstellen, Selbsthilfegruppen) ist nachzukommen. Auch komplementäre / unkonventionelle Behandlungsmethoden sollten offen mit den Patienten besprochen werden, auch um ungünstige Wechselwirkungen mit anderen Therapeutika zu vermeiden.

\section{Abbildung 4 Therapiealgorithmus in der Behandlung des mKRK}

\section{Figur 1: Therapiealgorithmus Erstlinientherapie des mKRK}

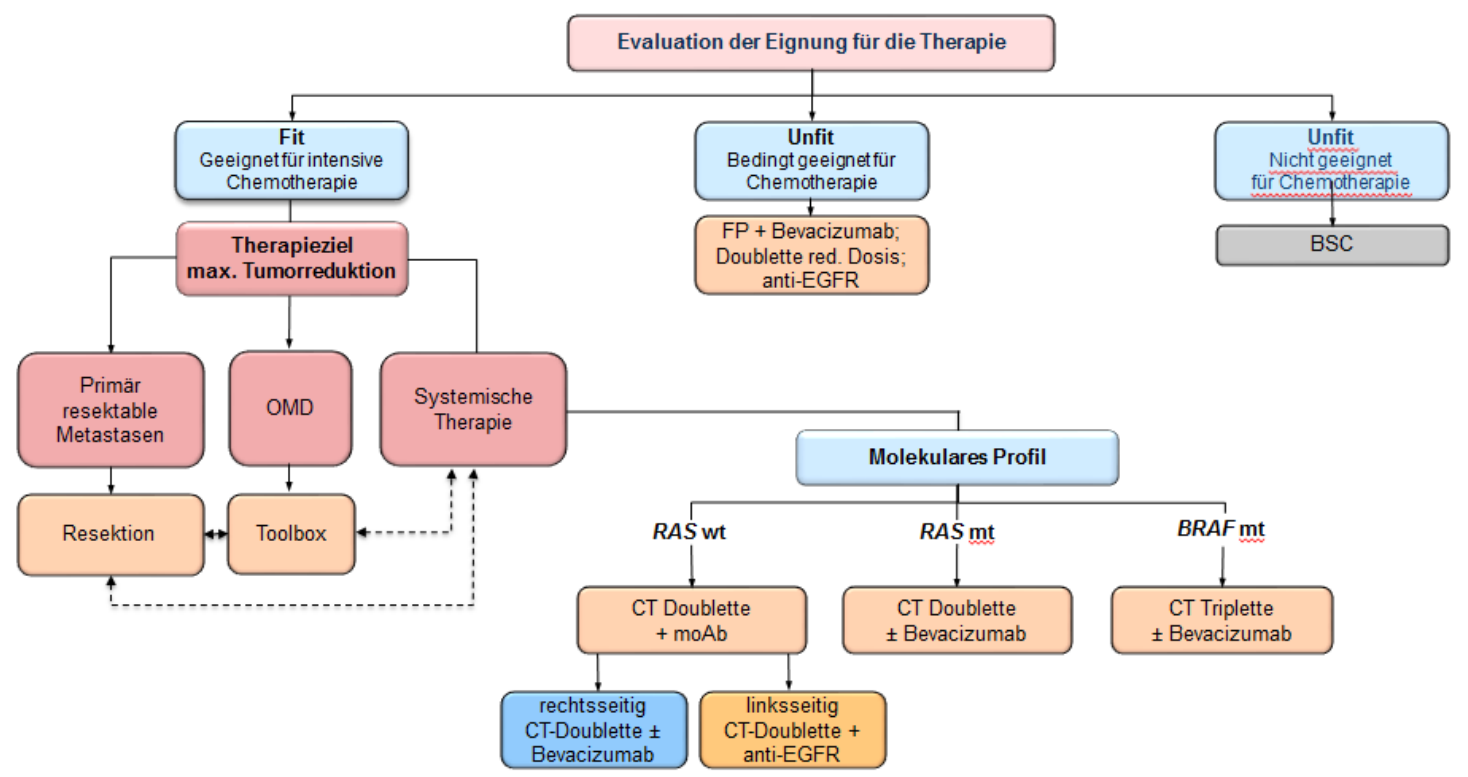




\subsection{Behandlungsstrategie}

\begin{tabular}{|l|l|}
\hline 9.1. & Konsensbasierte Empfehlung \\
\hline EK & $\begin{array}{l}\text { Grundsätzlich sollen die Patienten im Laufe ihrer Erkrankung Zugang zu allen } \\
\text { Therapiemodalitäten, vorzugsweise in zertifizierten Zentren, haben. }\end{array}$ \\
\hline Konsens &
\end{tabular}

9.2 .

\section{EK}

Besteht die Indikation zu einer medikamentösen Tumortherapie, so soll diese zum Zeitpunkt des Nachweises der Metastasen unabhängig von metastasenbezogenen Symptomen eingeleitet werden. Bei der Indikationsstellung sind mögliche Kontraindikationen zu berücksichtigen. Alter per se stellt keine Kontraindikation dar.

Konsens

\section{Hintergrund}

Die therapeutische Strategie wird auf dem Boden patientenspezifischer und tumorspezifischer Eigenschaften festgelegt. Setzt man den Behandlungswillen (partizipative Entscheidungsfindung) des Patienten voraus, so steht hinsichtlich der patientenbezogenen Parameter (wie Allgemeinzustand, Komorbiditäten, Lebenserwartung) die Eignung für eine intensive bzw. weniger intensive Therapie im Vordergrund. Unter den tumorbezogenen Charakteristika kommt der pathologischen und molekularen Zuordnung die größte Bedeutung zu.

Definition von Subgruppen nach klinischen Situationen/Therapiezielen:

9.3.

\section{EK}

\section{Konsensbasierte Empfehlung}

Um die Wahl der optimalen Erstlinientherapie zu ermöglichen, kann ein Entscheidungsalgorithmus angeboten werden, der die Patienten definierten Behandlungsgruppen zuordnet. Es können drei Entscheidungsebenen voneinander unterschieden werden:

- Allgemeinzustand (Tolerabilität einer intensiven Therapie)

- Krankheitsausdehnung inklusive Lokalisation (therapeutische Optionen beinhalten Fragen nach Resektabilität oder lokoregionäre Intervention)

- Molekularbiologie des Tumors (Definition der optimalen gezielten Therapie)

Starker Konsens

\section{Hintergrund}

Aufgrund der sich unter der Behandlung verändernden Molekularbiologie des Tumors und der damit verbundenen Resistenzentwicklung sind in späteren Therapielinien verabreichte Behandlungen deutlich weniger wirksam und können daher die in der Erstlinientherapie möglichen Effekte mutmaßlich nicht aufwiegen. Darüber hinaus kommt es im Therapieverlauf zu einer deutlichen Abnahme der behandelbaren 
Patienten, sodass in der Zweitlinientherapie etwa $70 \%$ und in der Drittlinientherapie meist nicht mehr als $50 \%$ der initial behandelbaren Patienten eine Therapie erhalten können [1040, 1041]. Unter der Erstlinientherapie können mit modernen Kombinationstherapien Remissionsraten von 50-70\% erreicht werden, in der Zweitlinientherapie liegen diese nur noch bei 10-30\%, dagegen in der Drittlinientherapie zumeist unter $10 \%$.

\subsubsection{Zuordnung entsprechend des Allgemeinzustandes}

Die erste Entscheidungsebene stellt den Patienten in den Vordergrund. Sie befasst sich bei gegebener Motivation von Seiten des Patienten mit der Frage der Therapiefähigkeit. Im Vordergrund stehen der Allgemeinzustand bzw. die Eignung für eine möglichst effektive (intensive) Therapie. Diese Bewertung ist vor dem Hintergrund der in Studien untersuchten Populationen mit einem medianen Alter von 60-65 Jahren und überwiegend gutem Performance Status (ECOG 0-1) zu verstehen. Die Wahl der für den einzelnen Patienten optimalen Behandlung erfolgt also zunächst auf der Grundlage der folgenden Zuordnungen:

1. Für intensive Therapie nicht geeignet

2. Für intensive Therapie geeignet

Weitere Details sind in den Kapiteln 9.6 und 9.7 festgelegt.

\subsubsection{Zuordnung hinsichtlich der Krankheitsausdehnung}

Die zweite Ebene der Entscheidungsfindung betrifft die Ausdehnung der metasta-sierten Erkrankung. Hier steht die Frage nach Iokalen, lokoregionären oder primär systemischen Behandlungsansätzen im Vordergrund. Für die Wahl der optimalen Behandlungsstrategie sind daher folgende Zuordnungen hilfreich:

1. Bestehen einer kurativen Behandlungsoption

a. Resektable Erkrankung

b. Potenziell resektable Erkrankung

2. Eher palliative Therapieausrichtung

a. Oligometastasierung

b. Wahrscheinlich nicht/nie resektable, disseminierte Erkrankung

Die Kriterien für die Zuordnung sind unter 9.7.2 aufgeführt.

\subsubsection{Zuordnung hinsichtlich der Molekularbiologie des Tumors}

Die ausreichende Kenntnis der Molekularbiologie des Tumors ist die Grundlage für eine adäquate Therapieentscheidung hinsichtlich der Frage nach der optimalen zielrichteten Behandlung.

Weitere Details sind in Kapitel 9.8 festgelegt.

\subsubsection{Wahl der späteren Therapielinien}

Die Wahl der optimalen Zweitlinientherapie bzw. späterer Therapielinien richtet sich nach der Molekularpathologie des Tumors, nach der Tumorlokalisation und nach der Wahl, dem Ansprechen und der Toxizität der vorangegangenen Therapie. 


\subsection{Initiale molekularbiologische Diagnostik vor Therapieeinleitung}

Das vorrangige Ziel besteht zunächst in der molekularpathologischen Charakteri-sierung der Erkrankung. Diese dient der prognostischen Einschätzung sowie der Gewinnung prädiktiver Informationen hinsichtlich der Therapieauswahl. Die molekularpathologischen Untersuchungen kolorektaler Karzinome umfassen essentiell eine Analyse des Mutationszustands der RAS-Gene KRAS und NRAS (hotspot-Regionen der Exone 2, 3 und 4), des BRAF-Gens (hotspot region in Exon 15) sowie des Status der Mikrosatelliteninstabilität (MSS, Mikrosatelliten-stabil; MSI-H, hochgradig Mikrosatelliten-instabil).

Bei hohem Behandlungsdruck (rasch progrediente oder symptomatische Erkrankung) kann die Chemotherapie begonnen und um die beste adäquate zielgerichtete Therapie ergänzt werden, sobald das Ergebnis der molekularpathologischen Testung vorliegt.

\subsection{1. (ALL) RAS- und BRAF-Diagnostik vor Beginn der Erstlinientherapie}

\begin{tabular}{|l|l|}
\hline $\begin{array}{l}\text { 9.4. } \\
\text { Empfehlungsgrad }\end{array}$ & Evidenzba \\
\hline $\mathbf{A}$ & $\begin{array}{l}\text { Die Bestimm } \\
\text { Einleitung de }\end{array}$ \\
\hline $\begin{array}{l}\text { Level of Evidence } \\
\mathbf{1} \mathbf{a}\end{array}$ & Quelle: [1042] \\
\hline & Starker Konsens \\
\hline
\end{tabular}

\section{Hintergrund}

Durch die Bestimmung von RAS- und BRAF-Mutationen noch vor Einleitung der Erstlinientherapie kann gewährleistet werden, dass die Patienten einer möglichst effektiven Behandlung zugeführt werden. Die molekularpathologische Evaluation ist daher eine notwendige Voraussetzung für die Behandlung innerhalb des vorgegebenen Therapiealgorithmus.

\section{RAS Mutation}

Etwa 50\% der mKRK Tumore zeigen eine KRAS- oder NRAS Mutation [1043], [1044], [1042] [1045]. Bis zu $90 \%$ der aktivierenden Mutationen im KRAS Gen werden in den Codons 12 und 13 festgestellt. Etwa $70 \%$ der KRAS Exon 2 Mutationen zeigen sich im Codon 12, weitere $30 \%$ im Codon 13 [1046].

Eine retrospective Analyse der PRIME Studie zeigte, dass $17 \%$ der Tumoren, die initial als KRAS Exon 2 Wildtyp eingestuft worden waren, weitere RAS Mutationen in den KRAS Codons 61, 117 und 146 oder in den NRAS Codons 12, 13 und 61 aufwiesen. Diese anderen RAS Mutationen waren, wie auch die KRAS Exon 2 Mutationen mit einer Resistenz gegen Panitumumab verbunden [1043]. Vergleichbare Daten wurden auch aus der OPUS Studie gewonnen. Beide Studien weisen darauf hin, dass die Zugabe eines antiEGFR Antikörpers (Panitumumab oder Cetuximab) zu einer FOLFOX Chemotherapie bei 
Vorliegen einer RAS Mutation, mit einem ungünstigeren Therapie-ergebnis (PFS und OS) verbunden ist als die Chemotherapie allein [1043] [1047].

Eine post hoc Analyse der CRYSTAL Studie identifizierte in der Population der KRAS Exon 2 wild type Tumoren 15\% andere RAS Mutationen. Auch diese Evaluation zeigte, dass Cetuximab bei RAS mutierten Tumoren nicht wirksam war, allerdings wurde hier kein negativer Einfluss auf Effektivitäsparameter beobachtet [1044].

Die negative prädiktive Bedeutung der RAS Mutation wurde für die Erstlinientherapie in einer Meta-Analyse von 9 Studien bestätigt [1042]. Im Vergleich zur alleinigen Chemotherapie wurde durch Zugabe eines anti-EGFR Antikörpers keine Verbesserung von PFS (HR 1,12, $p=0,20$ ) oder OS (HR 1,08, $p=0,14)$ beobachtet. Vergleichbare Daten wurden für das Gesamtüberleben auch in der Zweitlinientherapie (OS: HR 0,93, p=0,482) gefunden [1048]. Bei intensiv vorbehandelten Patienten und Vorliegen einer KRAS Mutation konnte durch Cetuximab im Vergleich zu „best supportive care“ (BSC) kein Überlebensvorteil erreicht werden (HR 1,01, $p=0,97$ ), während eine hochgsignifikante OS-Verlängerung bei Patienten mit KRAS-Wildtyp Tumoren beobachtet wurde (HR 0,55, $\mathrm{p}<0,001)$ [1 049].

Zusammenfassend kann die RAS-Mutation in Hinblick auf die Wirksamkeit einer antiEGFR Therapie als negativer prädiktiver Marker betrachtet werden. Entsprechend ist vor Beginn einer anti-EGFR Therapie der Ausschluss einer RAS Mutation erforderlich.

\subsection{Bestimmung des RAS Mutationsstatus}

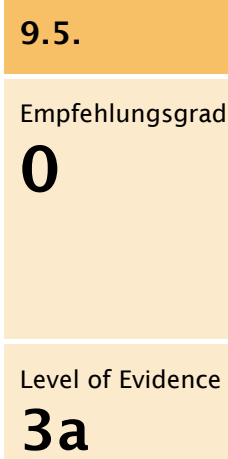

Evidenzbasierte Empfehlung

Die Bestimmungen des RAS-Mutationsstatus können entweder am Primärtumorgewebe oder an Metastasen erfolgen. Falls die Bestimmung des RASMutationsstatus aus dem Gewebe nicht möglich ist, kann in Betracht gezogen werden, den RAS-Mutationsstatus aus der im Blut zirkulierenden Tumor-DNA zu ermitteln.

Quellen: [1050-1052]

Konsens

\section{Hintergrund}

Grundsätzlich kann man von einer hohen Konkordanz des RAS Mutationsstatus im Primärtumor und im metastatischen Gewebe ausgehen. Dies gilt insbesondere für Lebermetastasen (Diskordanzrate etwa 5 - 15\%), jedoch in deutlich geringerem Maße für Lymphknotenmetastasen (Diskordanzrate 25\%) [1050], [1051], [1052].

Die Bestimmung des RAS- und BRAF-Mutationsstatus kann im Rahmen einer Stufendiagnostik erfolgen. Die BRAF Analytik erfolgt dann erst nach Ausschluss einer RAS-Mutation, da sich in aller Regel RAS- und BRAF-Mutationen im Tumor ausschließen. Als alternative Methodik kommt u.a. die Panel-Diagnostik in Betracht, die in der Lage ist, mehrere Mutationen in einem Schritt zu evaluieren.

Die Lokalisation des Primärtumors hat eine prognostische Bedeutung und scheint darüber hinaus - in Ergänzung zum RAS- und BRAF-Mutationsstatus - eine prädiktive 
Rolle zu spielen [1053], [1054] [1055], [1056]. Somit sollte diese Information, besonders bei RAS-Wildtyp-Patienten, vor Beginn der Therapie einer metastasierten KRK Erkrankung vorliegen. Retrospektive Analysen mehrerer großer Studien zur Erstlinientherapie von Patienten mit RAS-Wildtyp-Tumoren legen nahe, dass sich bei Patienten mit linksseitigem Primärtumor durch Hinzunahme einer anti-EGFR Therapie zu einer Kombinationschemotherapie eine erhebliche Verbesserung des Gesamt-überlebens gegenüber dieser alleine oder in Kombination mit Bevacizumab erzielen lässt, während dies bei rechtsseitigen Tumoren nicht der Fall ist. Weitere Details werden in Kapitel 9.8.2 dargelegt.

\subsubsection{BRAF-Mutation}

Aktivierende Mutationen im BRAF-Gen werden bei etwa 8-12\% der Patienten mit mKRK beschrieben [1057], [1058]. Gleichzeitiger Mutationen von RAS- und BRAF Genen sind sehr selten $(0.001 \%)$ und werden daher als sich gegenseitig ausschließend betrachtet [1059]. Weit überwiegend handelt es sich um BRAF V600 Mutationen, die mit einer sehr schlechten Prognose assoziiert sind [1057].

BRAF V600 Mutationen treten häufiger zusammen mit MSI- als mit MSS-Status auf [1060]. Bei gleichzeitigen Auftreten von BRAFV600 Mutationen und MSI handelt es sich um sporadisch auftretende Defekte der Mismatch-Reparatur (dMMR). Dagegen werden BRAF V600 Mutationen nicht bei Vorliegen einer Keimbahnmutation der MMR (Lynch-Syndrom) beobachtet [1061].

Beim Vergleich der BRAF V600 Mutation mit den deutlich selteneren BRAF Mutationen in Kodons 594 und 596 fällt auf, dass die BRAF V600 Mutationen häufiger in rechtsseitigen und muzinösen Primärtumoren mit peritonealer Metastasierung gefunden werden. Dagegen waren die BRAF 594- und 596-Mutationen häufiger mit rektalen und nichtmuzinösen Tumoren ohne peritoneale Metastasierung assoziiert. Die untersuchten BRAF 594- und 596-Tumoren waren alle Mikrosatelliten-stabil und waren darüber hinaus mit erheblich längeren Überlebenszeiten (Median 62,0 vs 12,6 Monate; HR 0,36, p=0,002) verbunden [1062].

Die klinische Relevanz der BRAF Mutation hinsichtlich der optimalen Wahl einer molekularbiologisch gezielten Therapie ist nicht vollständig geklärt. (siehe auch 9.8.4). Gleichwohl wird die Bestimmung der BRAF V600-Mutation bereits bei Erst-diagnose der metastasierten Erkrankung empfohlen. Liegt eine BRAF V600 Mutation vor, so kann aufgrund der schlechten Prognose bereits frühzeitig eine intensivierte Chemotherapie (z.B. mit FOLFOXIRI + Bevacizumab) eingeleitet werden. Andererseits sollten gerade bei diesen Patienten auch innovative Behandlungsansätze im Rahmen klinischer Studien frühzeitig in Betracht gezogen werden. 


\subsection{Pharmakogenetische Diagnostik vor Beginn der Erstlinientherapie}

Mehrere genetische Tests sind verfügbar, die eine Prädiktion der Toxizität der in der Behandlung des mKRK eingesetzten Medikamente ermöglichen. Gleichwohl ist die Evidenz, welche den Nutzen dieser Testung belegt, immer noch limitiert [1063]. So stehen prospektive Validierungsstudien derzeit noch aus.

\subsubsection{UDP Glucuronosyltransferase}

\begin{tabular}{|l|l|}
\hline $\mathbf{9 . 6 .}$ & Evidenzbasierte Empfehlung \\
\hline $\begin{array}{l}\text { Empfehlungsgrad } \\
\mathbf{0}\end{array}$ & $\begin{array}{l}\text { Die regelhafte Bestimmung von UGTIA1 vor Beginn einer palliativen CTX mit } \\
\text { Irinotecan wird nicht empfohlen. Sie kann aber durchgeführt werden, V. a. bei Morbus } \\
\text { Meulengracht oder anderen Bilirubin-Konjugationsstörungen. }\end{array}$ \\
\hline $\begin{array}{l}\text { Level of Evidence } \\
\mathbf{3}\end{array}$ & \begin{tabular}{l} 
Quellen: [1064] \\
\hline
\end{tabular} \\
\hline
\end{tabular}

\section{Hintergrund}

Die UDP Glucuronosyltransferase ist für die Glucuronidierung von SN-38, dem aktiven Metaboliten von Irinotecan, zu dem inaktiven Metaboliten SN-38G verantwortlich. Die benigne unkonjugierte Hyperbilirubinämie (Morbus Meulengracht) wird vorwiegend durch eine Variante im UGTIAl-Gen hervorgerufen. Bisher wurden mehr als 60 Polymorphismen des UGT1A21 Gens identifiziert. UGT1A1*1 ist das Wildtyp Allel, während *28,*93,*60, and *6 zu den häufigsten polymorphen Varianten zählen. Die bei Kaukasieren am häufigsten anzutreffende Variante ist das UGT1A1*28 Allel, welches eine Minderung der Genexpression von bis zu 70\% induziert und eine deutliche Steigerung der Irinotecan-Toxizität zur Folge hat.

Die Häufigkeit des *28 Allels beträgt $39 \%$ bei Europäern, $16 \%$ bei Asiaten und $43 \%$ bei Patienten aus Afrika. Etwa 10-20\% Kaukasier und Amerikaner mit afrikanischem Ursprung sind homozygot für *28, während dies für weniger als $5 \%$ der Asiaten zutrifft [1065].

Bei einer Irinotecan-Therapie werden bei verminderter Metabolisierung von SN-38 erhöhte Nebenwirkungsraten, insbesondere Neutropenie und Diarrhoe, beobachtet.

Die gegenwärtig verfügbaren Daten sind nicht ausreichend für die grundsätzliche Empfehlung einer prätherapeutischen UGT1A1-Genotypisierung ([1064]). In einer MetaAnalyse von 12 klinischen Studien wurde der UGT1A1*28 Polymorphismus nicht als zuverlässiger Prädiktor der Behandlungseffektivität (Response, PFS) identifiziert. Bei Nachweis des UGT1A1*28 Allels wurde in zwei Modellen zwar kein statistisch signifikantes Ergebnis, jedoch ein Trend zu einem höheren Mortalitätsrisiko festgestellt [1064].

Bei spontan erhöhtem Serum-Bilirubin (insbesondere bei niedrigem konjugiertem Bilirubin) kann jedoch ein M. Meulengracht vorliegen und damit ein gesteigertes Risiko Irinotecan-assoziierter Nebenwirkungen bestehen. Laut Fachinformation für Irinotecan sollten Patienten mit bekannter Homozygotie für UGT1A1*28 die normalerweise (unter Berücksichtigung des Bilirubinwertes) indizierte Anfangsdosis erhalten, sie sind jedoch 
auf hämatologische Toxizitäten zu überwachen. Bei Patienten mit unter der vorherigen Therapie aufgetretener hämatologischer Toxizität ist eine herabgesetzte IrinotecanAnfangsdosis in Betracht zu ziehen.

\subsubsection{Dihydropyrimidin Dehydrogenase}

\begin{tabular}{|c|c|}
\hline 9.7 . & Evidenzbasierte Empfehlung \\
\hline $\begin{array}{l}\text { Empfehlungsgrad } \\
0\end{array}$ & $\begin{array}{l}\text { Die Bestimmung des DPD-Mangels ist eine diagnostische Option vor Beginn einer } \\
\text { Fluoropyrimidin-Therapie. Die regelhafte Evaluation des DPYD*2A -Polymorphismus } \\
\text { kann durchgeführt werden. }\end{array}$ \\
\hline $\begin{array}{l}\text { Level of Evidence } \\
\mathbf{2 b}\end{array}$ & Quellen: $[1066,1067]$ \\
\hline & Konsens \\
\hline
\end{tabular}

\section{Hintergrund}

Die Dihydropyrimidin-Dehydrogenase (DPD) ist das Schlüsselenzym für den 5-FU Metabolismus. DPD inaktiviert etwa 80-90\% des verabreichten 5-FU zu 5,6Dihydrofluorouracil. Der Genpolymorphismus des kodierenden DPYD Gens ist die am besten beschriebene Ursache eines DPD Mangels. Etwa 3-5\% der Kaukasier weisen einen partiellen und 0.2\% einen kompletten DPD-Mangel auf [1066]. Nicht funktionelle Allele sind u.a. die Varianten DPYD*2A und DPYD*13, DPYD*9A sowie die SNP-Variante rs67376798.

Der DPYD*2A Polymorphismus ist mit einer Häufigkeit von 1-2\% in den westlichen Nationen die klinisch relevanteste Variante. Bei heterozygoten Trägern wird eine Dosisreduktion von 5-FU empfohlen, bei den sehr viel selteneren homozygoten Trägern ist die Gabe von 5-FU aufgrund von potentiell lebensbedrohlicher Toxizität (Neutropenie) kontraindiziert [1067].

In der Populationsanalyse $(n=2038)$ von Deenen et al. [1066] erwies sich die Testung des DPYD*2A Polymorphismus als kostensparend. Fokussiert man die Testung allein auf DPYD*2A, dann können allerdings nur 25\% aller DPD-defizienten Patienten identifiziert werden. Es verbleibt also ein signifikantes Restrisiko. Letztlich muss auch die Übertragbarkeit der Studienergebnisse in das deutsche Gesundheitswesen in Betracht gezogen werden.

\subsection{Diagnostik ohne unmittelbare Relevanz für die Erstlinientherapie}

Die Bestimmung der Mikrosatelliteninstabilität sowie der HER2 Amplifikation/ Überexpression hat in der Erstlinientherapie keine unmittelbare Relevanz. Sie kann aber in späteren Therapielinien Bedeutung für die Behandlungsführung erlangen. Die Messung dieser Parameter kann daher empfohlen werden.

\subsubsection{Testung der Mikrosatelliteninstabilität (MSI)}

Beim mKRK ist die MSI-Testung einerseits für die Indikationsstellung einer humangenetischen Beratung hilfreich. Andererseits kann sie in Hinblick auf die Behandlung 
mit Immun-Checkpointinhibitoren bei der Festlegung einer linienübergreifenden Therapiestrategie sinnvoll sein.

Zum Zeitpunkt der Leitlinienerstellung hat der MSI-Status noch keine Bedeutung für die Wahl der Erstlinientherapie. Zu vorbehandelten Patienten liegen allerdings Daten vor, die bei Patienten mit Mikrosatelliteninstabilität (MSI-H) auf die hohe Effektivität einer Behandlung mit dem Checkpoint-Inhibitor Pembrolizumab hinweisen. Bei 82\% (9/11) der untersuchten Patienten lag jedoch eine hereditäre Komponente (Lynch-Syndrom) vor, so dass die Übertragbarkeit auf Patienten mit sporadisch auftretendem MMR-Defekt noch belegt werden muss [1068].

\section{Hintergrund}

Bei Hinweis auf familiären Darmkrebs sollte die Mikrosatellitenanalyse (mittels der Untersuchung der immunhistochemischen Expression der DNA-Mismatch-ReparaturProteine MLH1, MSH2, MSH6 und PMS2 oder der Untersuchung auf MikrosatellitenInstabilität) und gegebenenfalls gekoppelt mit einer Methylierungsanalyse des MLH1 Promoter/ Exon 1 Bereichs oder, wenn diese nicht vorhanden ist, mit Hilfe einer BRAF Diagnostik (hereditär vs. sporadisch) zwingend erfolgen.

Die Trennschärfe einer BRAF-Mutationsanalyse zur Abgrenzung spontaner- von hereditären kolorektalen Karzinomen liegt bei etwa 50\% [1069], die einer Methylierungsanalyse ist vollständig ( 100\%) [1070].

\subsubsection{HER2-neu Amplifikation/Überexpression}

Der Nachweis einer HER2-neu Amplifikation/Überexpression hat zum gegenwärtigen Zeitpunkt keinen gesicherten Stellenwert in der Wahl der Erstlinientherapie [1071]. Allerdings liegen erste Daten vor, welche darauf hinweisen, dass eine HER2-neu Amplifikation mit einer Resistenz gegen anti-EGFR Substanzen verbunden sein kann [1072], [1073]. Darüber hinaus zeigt die HERACLES Studie, dass Patienten mit RAS-wt mKRK, die gegen eine Standardtherapie (einschließlich Cetuximab oder Panitumumab) refraktär waren und eine HER2 Amlifikation/Überexpression aufwiesen, von einer Behandlung mit Trastuzumab plus Lapatinib profitierten [1074] (siehe 9.8.6).

\subsection{Diagnostik während der Therapie des mKRK}

Zur Diagnostik während der Therapie konnten angesichts der fehlenden oder kontroversen Datenlage keine klaren Empfehlungen gemacht werden.

\subsubsection{Therapiemonitoring unter Therapie}

Bei Patienten mit RAS- /BRAF-Wildtyp mKRK, kann unter einer Folgetherapie eine erneute molekulargenetische Testung des RAS- und BRAF-Mutationsstatus (Re-Biopsie) erfolgen, wenn zuvor keine anti-EGFR-Therapie erfolgt ist. Falls die Bestimmung des RAS- oder BRAF-Mutationsstatus aus dem Gewebe nicht möglich ist, kann in Betracht gezogen werden, den Mutationsstatus aus der im Blut zirkulierenden Tumor-DNA zu ermitteln [1075]. Diese Analysen ermöglichen im Einzelfall den Nachweis einer anti-EGFR Resistenz, die sich auf dem Boden einer Expansion RAS-mutierter Klone entwickelt [1076], [1077], [1078]. Sie dienen allein der Verlaufsbeurteilung und sollen keinesfalls die zum Beginn der Erstlinientherapie empfohlenen Testungen des Tumorgewebes ersetzen. 


\subsubsection{Analysen zur Indikationsstellung gezielter Therapien nach Versagen der Erstlinientherapie}

Der Nachweis eines Mikrosatelliten-instabilen Karzinoms (MSI) kann die Indikationsstellung für eine Behandlung mit Checkpoint-Inhibitoren unterstützen (siehe 9.4.1 Testung der Mikrosatelliteninstabilität (MSI)). Derzeit liegt hierfür keine Zulassung vor.

Gleichfalls kann bei RAS-Wildtyp im späteren Verlauf der Behandlung und nach Durchlaufen einer anti-EGFR Therapie bei HER2-neu Amplifikation/Überexpression erwogen werden, die Indikation für eine Kombinationsbehandlung mit Trastuzumab/ Lapatinib zu stellen (siehe 9.4.2 HER2-neu Amplifikation/Überexpression). Derzeit liegt hierfür keine Zulassung vor.

\subsection{Behandlung von Patienten, die nicht für eine intensive Therapie in Frage kommen}

Entsprechend der unter Punkt 9.1.1 durchgeführten Gruppenzuordnung können grundsätzlich zwei Behandlungsgruppen unterschieden werden:

(I) Patienten, die für eine intensive Therapie nicht geeignet sind.

(II) Patienten, die für eine intensive Therapie geeignet sind.

Bei Patienten, die für eine intensive Behandlung nicht geeignet sind, geht es um solche, die aufgrund ihres Allgemeinzustandes nicht für eine primäre Operation oder eine intensive Kombinationstherapie qualifizieren oder die eine intensivierte Behandlung aufgrund der damit verbundenen Nebenwirkungen ablehnen. In der Beurteilung der Eignung für eine Therapie steht explizit nicht das numerische Alter der Patienten im Vordergrund. Entscheidend sind die biologischen Rahmenbedingungen des Patienten und des Tumors.

\subsubsection{Primär resektable Erkrankung bei reduziertem Allgemeinzustand}

\begin{tabular}{|l|l|c|}
\hline 9.8. & Konsensbasierte Empfehlung & 2017 \\
\hline EK & $\begin{array}{l}\text { Bei primär resektabler Metastasierung soll die Operationsfähigkeit geprüft werden. } \\
\text { Ist diese primär nicht gegeben, so sollte die Operabilität / Resektabilität in } \\
\text { regelmäßigen, nachfolgenden Untersuchungen (z.B. alle 8 Wochen) geklärt werden. }\end{array}$ \\
\hline & Konsens
\end{tabular}

\section{Hintergrund}

Bei Patienten, die für eine intensive Therapie primär nicht in Betracht kommen, sollte die Option nebenwirkungsarmer „Bridging“-Therapien, z.B. mit einem Fluorpyrimidin, geprüft werden. Bevacizumab kann dann hinzugegeben werden, wenn die Operation nicht rasch angestrebt wird. Bei RAS-Wildtyp kommt ggf. auch eine anti-EGFRMonotherapie in Betracht.

Grundsätzlich besteht das Ziel darin, die Therapiefähigkeit wieder herzustellen und einen Zustand zu erreichen, in dem auch intensivere Kombinationschemotherapien vertragen werden. Wichtig ist, dass das Behandlungskonzept in Abhängigkeit von Allgemeinzustand und Motivation des Patienten sowie von der Verträglichkeit und Effektivität regelmäßig neu abgestimmt wird. 


\subsubsection{Primär nicht resektable Erkrankung bei reduziertem Allgemeinzustand}

\begin{tabular}{|l|l|}
\hline 9.9. & Konsensbasierte Empfehlung \\
\hline EK & $\begin{array}{l}\text { Bei reduziertem Allgemeinzustand, der eine intensive Chemotherapie nicht erlaubt, } \\
\text { steht eine primär palliative, symptomatische Behandlung im Vordergrund. } \\
\text { Es kann eine initiale Behandlung mit einem Fluoropyrimidin + Bevacizumab oder eine } \\
\text { dosisreduzierten Chemotherapiedoublette (+/- Bevacizumab) durchgeführt werden. } \\
\text { Bei Vorliegen von RAS-WT Tumoren im linksseitigen Kolon (ab linke Flexur) oder im } \\
\text { Rektum kann darüber hinaus eine anti-EGFR-Monotherapie erfolgen. }\end{array}$ \\
\hline & starker Konsens
\end{tabular}

\subsubsection{Schlechter Allgemeinzustand aufgrund der Tumorerkrankung}

\begin{tabular}{|l|l|c|}
\hline 9.10. & Konsensbasierte Empfehlung & 2017 \\
\hline EK & $\begin{array}{l}\text { Wenn der schlechte Allgemeinzustand vorrangig durch die Tumorerkrankung } \\
\text { bedingt ist, kann auch bei schlechtem Performance Status (ECOC >1) nach } \\
\text { Abschätzung aller Risiken eine intensivierte Tumortherapie primär zum Einsatz } \\
\text { kommen. }\end{array}$ \\
\hline & \\
Konsens
\end{tabular}

\subsection{Behandlung von Patienten, die für eine intensive Therapie geeignet sind}

9.11 .

EK

\section{Konsensbasierte Empfehlung}

Grundsätzlich sollen Patienten Zugang zu der effektivsten und noch tolerablen Therapie haben. Wenn eine kurative Zielsetzung besteht und keine Einschränkungen hinsichtlich der (potentiellen) Therapieauswahl vorliegen, sollten zur Festlegung des optimalen multimodalen Vorgehens prinzipiell folgende Parameter in die Entscheidungsfindung miteinbezogen werden:

a) chirurgische Kriterien (Operabilität, Resektabilität inklusive lokal ablativer Verfahren),

b) prognostische Kriterien.

Starker Konsens

\section{Hintergrund}

Bei primär resektabler Erkrankung besteht die grundsätzliche Frage, in wie weit durch eine primäre Resektion (oder Intervention) ein längeres krankheitsfreies Intervall oder im besten Fall eine Heilung erreicht werden können. Bei ungünstigen Prognosefaktoren kann im Einzelfall eine neoadjuvante Chemotherapie die bessere Behandlungsoption darstellen. 
In diesem Zusammenhang sollte zwischen Patienten mit synchroner und metachroner Metastasierung unterschieden werden [1079], [1080], [1081]. Die synchrone wird im Vergleich zur metachronen Metastasierung als prognostisch ungünstiger betrachtet. Darüber hinaus liegt bei synchroner Metastastasierung keine Information zur Krankheitsdynamik vor. Der Nutzen einer primären Resektion ist daher in dieser Patientengruppe unsicherer als bei Patienten mit metachroner Metastasierung. Weitere prognostische Faktoren, die bei der Entscheidungsfindung hinzugezogen werden können, sind u.a. die Zahl der metastatischen Läsionen, das Vorliegen einer extrahepatischen Metastasierung oder der FONG-Score [1082].

\subsubsection{Technisch primär resektable Erkrankung}

\subsubsection{Primär resektable Erkrankung bei onkologisch günstiger Prognose}

\begin{tabular}{|l|l|}
\hline 9.12. & Konsensbasierte Empfehlung \\
\hline EK & $\begin{array}{l}\text { Bei resektablen Tumormanifestationen und prognostisch günstigen Kriterien soll die } \\
\text { primäre Metastasenresektion durchgeführt werden. }\end{array}$ \\
\hline & Konsens
\end{tabular}

\subsubsection{Beurteilung der Resektabilität}

\begin{tabular}{|l|l|}
\hline 9.13. & Konsensbasierte Empfehlung \\
\hline EK & $\begin{array}{l}\text { Die Beurteilung soll durch ein Tumorboard unter Beteiligung eines in der } \\
\text { Metastasenchirurgie erfahrenen Chirurgen erfolgen. }\end{array}$ \\
\hline & Konsens
\end{tabular}

\section{Hintergrund}

Die Resektabilität von Metastasen sollte von einem erfahrenen Organchirurgen (Leber/ Lunge/Peritoneum) entschieden werden. Das Gesamttherapiekonzept und die Einordnung der möglichen Resektion in das Therapiekonzept müssen von einem multidisziplinären Tumorboard entschieden werden. Bisher gibt es keine sichere Definition der Kriterien, die einen in der Metastasenchirurgie erfahrenen Chirurgen charakterisieren.

Hinsichtlich der chirurgischen Resektabilität der Metastasen ist nicht allein die Größe oder Zahl der Metastasen entscheidend, sondern die Einschätzung in der Zusammenschau aller klinischer Faktoren (Allgemeinzustand, Lokalisation der Metastasen, Größe der Restleber, erkrankungsfreies Intervall, ggf. Risiko-Scores etc). Risiko-Scores wie der von Fong et al. können in der Entscheidungsfindung hilfreich sein, sind allein aber nicht ausreichend [1082].

Bei Resektion von Lebermetastasen kann ein 5-Jahresüberleben im Bereich von 25-35\% erreicht werden. Zur Resektion von Lungenmetastasen liegen deutlich weniger Daten vor. Gleichwohl sollte ein chirurgisches Vorgehen in Betracht gezogen werden, wenn eine R0-Resektion erreicht werden kann [1050].

Option der Einholung einer Zweitmeinung 
Es wird sehr deutlich darauf hingewiesen, dass von der Einholung einer Zweitmeinung insbesondere auch im Bereich der Metastasenchirurgie Gebrauch gemacht werden sollte. Nach Möglichkeit sollten Zweitmeinungen durch ausgewiesene Zentren unter Miteinbeziehung multidisziplinärer Tumorkonferenzen durchgeführt werden.

\subsubsection{Primär resektable Erkrankung bei prognostisch ungünstigen Kriterien}

\begin{tabular}{|l|l|}
\hline 9.14. & Konsensbasierte Empfehlung \\
\hline EK & $\begin{array}{l}\text { Bei primär resektabler Erkrankung und prognostisch ungünstigen Kriterien (z. B. } \\
\text { kurzes krankheitsfreies Intervall oder synchrone Metastasierung) kann primär eine } \\
\text { systemische Therapie erfolgen. }\end{array}$ \\
\hline Konsens
\end{tabular}

\section{Hintergrund}

Die präoperative Therapie kann dann eingesetzt werden, wenn die Dynamik der Tumorerkrankung gerade bei synchroner Metastasierung unklar ist und eine präoperative Beobachtungsphase unter Chemotherapie hilfreich ist, um die Geschwindigkeit und das Muster der Metastasierung einzuordnen.

\subsubsection{Optimaler Zeitpunkt der Resektion}

\begin{tabular}{l|l|}
\hline 9.15. & Konsensbasierte Empfehlung \\
\hline EK & $\begin{array}{l}\text { Kann durch die systemische Therapie eine Stabilisierung der Erkrankung erreicht } \\
\text { werden, so soll die Resektion möglichst zeitnah (d. h. nach 2-3 Monaten) angestrebt } \\
\text { werden. }\end{array}$ \\
\hline Konsens
\end{tabular}

\section{Hintergrund}

Bei metachroner Metastasierung kann die Dynamik der Erkrankung auf dem Boden der Dauer des krankheitsfreien Intervalls (DFS) abgeschätzt werden. Bei langem DFS kann von einer günstigeren Prognose ausgegangen werden [1083]. Im Gegensatz dazu wird die synchrone Metastasierung als prognostisch eher ungünstig betrachtet. Eine synchrone Metastasierung liegt dann vor, wenn zum Zeitpunkt der Erstdiagnose des KRK bereits eine metastatische Ausbreitung der Erkrankung besteht. Bei kurzem DFS $(<6$ Monate) oder synchroner Metastasierung kann eine primäre Chemotherapie empfohlen werden. Die Evaluation der Krankheitsdynamik und des Ansprechens auf die Therapie helfen bei der prognostischen Einordnung der Erkrankung [1084]. 


\subsubsection{Vorgehen bei sehr kleinen Metastasen}

\begin{tabular}{|l|l|}
\hline 9.16. & Konsensbasierte Empfehlung \\
\hline EK & $\begin{array}{l}\text { Kleine Metastasen }(\leq 1 \mathrm{~cm}) \text { können primär entfernt werden, da diese unter einer } \\
\text { initialen Chemotherapie möglicherweise verschwinden würden und dann durch den } \\
\text { Chirurgen intraoperativ nicht mehr aufgefunden werden. }\end{array}$ \\
\hline Konsens
\end{tabular}

\subsubsection{Neoadjuvante Therapie resektabler Lebermetastasen}

\begin{tabular}{|l|l|}
\hline $\begin{array}{l}\text { 9.17. } \\
\text { Empfehlungsgrad }\end{array}$ & $\begin{array}{l}\text { Evidenzbasierte Empfehlung } \\
\text { B neoadjuvante Therapie von primär resektablen Lebermetastasen sollte nicht } \\
\text { durchgeführt werden. }\end{array}$ \\
\hline $\begin{array}{l}\text { Level of Evidence } \\
\mathbf{2} \mathbf{D}\end{array}$ & Quelle: [1085] \\
\hline & \\
\hline
\end{tabular}

\section{Hintergrund}

Bei technischer Resektabilität und günstigen Prognosekriterien ist der klinische Nutzen einer neoadjuvanten/präoperativen Therapie nicht sicher belegt. Dieser wurde in einem systematischen Review [1085], mit überwiegend retrospektiven kontrollierten Beobachtungsstudien, einer randomisierten Studie [1086], [1087] sowie mehreren nicht kontrollierten Analysen untersucht. Insgesamt ist kein Einfluss auf das Gesamtüberleben (OS) nachweisbar. Fehlt der Nutzen, so wird die Indikationsstellung obsolet, auch wenn ein Schaden durch die präoperative Therapie nicht konkretisiert werden kann.

In der Bewertung der präoperativen Therapie kommt der EORTC-40983-Studie eine zentrale Bedeutung zu. In diese Studie wurden Patienten mit überwiegend günstigen Risikofaktoren eingeschlossen (1-4 resektable Lebermetastasen, 52\% 1 Leber-metastase, 26\% 2 Lebermetastasen, 65\% metachrone Metastasierung). Bei primär resektabler Lebermetastasierung wurden eine perioperative Chemotherapie (6 Zyklen FOLFOX4 jeweils vor und nach der Operation) mit der alleinigen Operation [1086] verglichen. Primärer Endpunkt war das progressionsfreie Überleben. Das Ziel, durch eine perioperative Chemotherapie eine PFS-Verlängerung mit einer Hazard Ratio von $\leq 0.71$ zu erreichen, wurde bei den randomisierten Patienten knapp verfehlt (HR 0.79; $95 \% \mathrm{Cl}$, 0.62-1.02; $p=0.058$ ). Darüber hinaus zeigte die Auswertung des 5-Jahresüberlebens keinen Überlebensvorteil. Im Arm der perioperativen Chemotherapie lag die 5Jahresüberlebensrate bei $51 \%(95 \% \mathrm{Cl} 45-58)$ im Vergleich zu 48\% (95\% Cl 40-55) in der Gruppe der primär operierten Patienten [1087]. Einschränkend muss hier darauf hingewiesen werden, dass das statistische Design (Power) der Studie nicht dazu ausgelegt war, um einen Überlebensvorteil zu belegen.

Die Schlussfolgerungen aus der EPOC-Studie gelten für eine prognostisch günstigere Patientenpopulation. Entsprechend sollte bei günstiger Prognose (z.B. langes krankheitsfreies Intervall bei metachroner Metastasierung) und technisch einfacher Resektabilität (geringe Anzahl und günstige Lokalisation der Metastasen), eine primäre 
Metastasenresektion angestrebt werden (siehe auch 9.7.1.1.). Bei Vorliegen prognostisch ungünstiger Kriterien kann dagegen primär eine systemische Therapie erfolgen (siehe 9.7.1.3.).

\subsubsection{Leberresektion nach Chemotherapie}

\begin{tabular}{|l|l|}
$\begin{array}{l}\text { 9.18. } \\
\text { Evidenzbasiertes Statement }\end{array}$ & $\mathbf{2 0 1 7}$ \\
\hline $\begin{array}{l}\text { Empfehlungsgrad } \\
\text { Ob bei einer Leberresektion nach Chemotherapie auch die Segmente mit nicht mehr } \\
\text { nachweisbaren Metastasen reseziert werden müssen, kann derzeit mangels } \\
\text { ausreichender Evidenz nicht konkret beantwortet werden. }\end{array}$ \\
\hline $\begin{array}{l}\text { Level of Evidence } \\
\text { Quellen: [1088-1096] }\end{array}$ \\
\hline
\end{tabular}

\section{Hintergrund}

Im Vordergrund steht die Frage, ob bei einer Leberresektion nach Chemotherapie auch die Segmente mit nicht mehr nachweisbaren Metastasen reseziert werden müssen. $\mathrm{Zu}$ diesem Thema liegen ausschließlich retrospektive Fallserien vor [1088], [1089], [1090], [1091], [1 092], [1 093], [1 094], [1095], [1096]. Der Anteil der im Chemotherapieverlauf bildgebend nicht mehr nachweisbaren Lebermetastasen variiert zwischen 6 und 24\%.

Intraoperativ konnten makroskopisch oder durch intraoperativen Ultraschall zwischen $27 \%$ und $67 \%$ der in der Bildgebung nicht mehr detektierbaren Metastasen nachgewiesen werden. Der Anteil an Metastasen mit vitalen Tumorzellen betrug bei resezierten Patienten $0 \%$ bis $80 \%$. Ein Teil der Patienten erhielt eine intraarterielle Chemotherapie. Zu bedenken ist auch die Qualität der Bildgebung. Hinsichtlich der Metastasendetektion in der Leber bieten hier die MRT-Untersuchung mit leberspezifischem Kontrastmittel sowie die kontrastmittelverstärkte Sonographie die höchste Sensitivität [1 097, 1098].

Ob Areale mit bildgebend nicht mehr nachweisbaren Metastasen eine Metastasenresektion zwingend erfordern, ist nicht eindeutig geklärt. Auf jeden Fall ist eine engmaschige Kontrolle erforderlich. In den Fallserien erhielten die Patienten nach Metastasenresektion in der Mehrzahl eine additive Chemotherapie (keine Aussage zur optimalen Therapiedauer). 


\subsubsection{Adjuvante / additive Therapie nach Resektion von Lebermetastasen}

\begin{tabular}{|l|l|}
\hline $\mathbf{9 . 1 9 .}$ & Evidenzbasierte Empfehlung \\
\hline $\begin{array}{l}\text { Empfehlungsgrad } \\
\mathbf{B}\end{array}$ & $\begin{array}{l}\text { Eine adjuvante/additive Chemotherapie nach Resektion von Metastasen sollte nicht } \\
\text { erfolgen. }\end{array}$ \\
\hline $\begin{array}{l}\text { Level of Evidence } \\
\mathbf{2} \mathbf{A}\end{array}$ & Quellen: [1099-1101] \\
\hline & Konsens \\
\hline
\end{tabular}

\section{Hintergrund}

Der Nutzen einer adjuvanten/additiven Chemotherapie nach R0-Resektion von Metastasen ist nicht sicher belegt. Trotz R0-Resektion von Lebermetastasen bleiben nur ca. 30\% der Patienten langfristig rezidivfrei. Zur Fragestellung des möglichen Nutzens einer adjuvanten Therapie liegen eine gepoolte Analyse von 2 prospektiven randomisierten Studien sowie mehrere retrospektive Analysen vor [1099], [1100], [ $\left.\begin{array}{llll}1 & 1 & 0 & 1\end{array}\right]$, [ 11102$]$, [ 11103$]$, [ 11104$]$, [ 11105$]$, [ 11106$]$, [ 11107$]$, [1 087]. In der gepoolten Analyse wurde eine Verbesserung des 5J-DFS (36,7\% vs. 27,7\%) gefunden, die aber knapp das Signifikanzniveau verfehlte $(p=0.058)$ ([ $\left.\left.\begin{array}{lll}1 & 1 & 01\end{array}\right]\right)$. Gleichfalls wurde auch keine signifikante Verbesserung des 5 J-OS $(52,8$ vs. 39,6$)$ erreicht $(p=0.095)$. Darüber hinaus ist das 5 -FUBolus-Regime, das bei den beiden Studien eingesetzt wurde, heute nicht mehr als Standard anzusehen. Der Nutzen einer nach aktuellen Standards durchgeführten adjuvanten/additiven Chemotherapie ist nicht belegt.

In den retrospektiven Analysen wurde ein Einfluss auf das DFS und teilweise OS beschrieben. Allerdings besteht bei fehlender Randomisierung ein beträchtliches Risiko für eine Fehlbeurteilung, insbesondere da Unterschiede in der Zusammensetzung der verglichenen Kohorten (z. B. Patientenzahl, Alter, extrahepatische Metastasierung) bestanden. In einigen Studien wurde kein Einfluss auf das OS gefunden. Einzelne Studien legen einen Nutzen bei höherem Rezidivrisiko (z.B. anhand des Memorial Sloan-Kettering Cancer Center Clinical Risk Score (MSKCC-CRS)) nahe. Insgesamt muss die verfügbare Datenbasis als unbefriedigend bezeichnet werden. Eine weitere Meta-Analyse beinhaltet sowohl perioperative als auch additive Therapie, in einer Netzwerk-Meta-Analyse wurden v.a. verschiedene CTX-Regime miteinander verglichen, so dass keine konklusive Aussage zu Chemotherapie vs. keiner Chemotherapie nach R0-Resektion von Lebermetastasen gemacht werden kann [1099], [1 100].

\subsubsection{Oligometastasierung}

Unter Oligometastasierung wird eine limitierte Ausbreitung sowie eine potentiell resektable oder lokal interventionell angehbare Metastasierung verstanden, in der Regel unter Beschränkung der Ausbreitung auf z.B. 1-5 Metastasen und wenige Organsysteme (1-3 Organe). Aufgrund der deutlich schlechteren Prognose werden Metastasen in Lymphknoten, Gehirn oder Knochen in diese Zuordnung nicht mitein-bezogen.

Hinsichtlich der Definition und Behandlung der oligometastasierten Erkrankung konnte in Ermangelung belastbarer Daten keine Einigung erreicht werden.

\section{Hintergrund}


Eine verbindliche Definition der Oligometastasierung liegt derzeit nicht vor. In der Begrifflichkeit wird neben dem Ausmaß der Metastasierung auch berücksichtigt, dass insbesondere lokal ablative oder lokoregionäre Maßnahmen zur Tumorbehandlung eingesetzt werden können. Unter günstigen Voraussetzungen kann bei Oligometastasierung auch ein kurativer Behandlungsansatz in Betracht gezogen werden. Dieser Definition liegen die folgenden Annahmen zu Grunde:

a) Die spezifische Tumorbiologie lässt einen Verlauf möglich erscheinen, bei dem die Oligometastasierung für zumindest ein relevantes Intervall eine limitierte Metastasierung widerspiegelt.

b) Chirurgische, lokal ablative oder lokoregionäre Maßnahmen zur Tumorbehandlung können in Ergänzung zur Systemtherapie eingesetzt werden.

c) Die Kontrolle der Metastasenlokalisation ist prognostisch relevant und der Einsatz der lokal ablativen oder resezierenden Verfahren kann eine Unterbrechung der Systemtherapie ermöglichen.

Bei lokal behandelbarer Metastasierung soll, wenn möglich und vertretbar, primär die chirurgische Resektion angestrebt werden. Lokal ablative Verfahren sind: Thermoablation (RFA, MWA), Strahlentherapie (z. B. SBRT, Brachytherapie) oder Elektroporation (siehe Kapitel 9.13 Lokal ablative Verfahren).

Zu den lokoregionären Verfahren gehören die intraarterielle Chemotherapie der Leber (HAI) oder die selektive intraarterielle Radiotherapie (SIRT) sowie auch die Zyto-reduktion mit hyperthermer Chemotherapie (HIPEC) (siehe auch 9.14.1-9.14.3). Letztlich ist natürlich auch die Kombination chirurgischer und ablativer Verfahren möglich. Die Indikationsstellung zu lokal ablativen Verfahren sollte in multidisziplinären Tumorkonferenzen erfolgen (siehe 9.14 Lokoregionär wirksame Verfahren).

\subsubsection{Primär nicht resektable Metastasierung}

\begin{tabular}{|l|l|}
\hline 9.20. & Konsenbasierte Empfehlung \\
\hline EK & $\begin{array}{l}\text { Bei primärer Irresektabilität soll zunächst eine systemische Tumortherapie erfolgen. } \\
\text { Entsprechend der Tumor- und Patientencharakteristika soll die wirksamste Therapie } \\
\text { an den Anfang der Behandlung gestellt werden. }\end{array}$ \\
\hline Konsens
\end{tabular}

\section{Hintergrund}

Als primäres Therapieziel wird die maximale Tumorreduktion angestrebt. Diese Strategie wird übereinstimmend für Patienten mit rasch progredienter oder symptomatischer, aber auch bei asymptomatischer Metastasierung eingesetzt. Das beste Gesamtüberleben wird durch ein multimodales, ggf. sequenzielles, Therapiekonzept erreicht. Es soll daher primär die situativ effektivste verfügbare systemische Kombinationstherapie, unter Berücksichtigung der Patientenpräferenz und der tumorerkrankungsunabhängigen Faktoren (wie Komorbidität) angewandt werden (intensivierte Therapie). Die Möglichkeit einer sekundären Resektion bzw. die Durchführbarkeit lokal ablativer Maßnahmen soll mittels regelhaft eingesetzter Folgeuntersuchungen durch multi-disziplinäre Tumorkonferenzen überprüft werden (z.B. im Abstand von 2-3 Monaten). 
Entsprechend diesen Vorgaben kann bei primärer Indikation zu einer systemischen Therapie der Primärtumor zunächst belassen werden. Ausnahmen können ein symptomatisch stenosierendes Tumorwachstum und/oder eine Hb-relevante Blutung sein.

\subsection{Wahl der systemischen Therapie in Abhängigkeit von der molekularpathologischen Subgruppe und der Tumorlokalisation}

Das Grundprinzip in der optimalen Behandlung des mKRK besteht darin, in Abhängigkeit von patientenabhängigen Faktoren (wie Motivation und Toxizitätsprofil) und den tumorerkrankungsunabhängigen Faktoren (wie dem Allgemeinzustand des Patienten, Komorbidität, etc.), die effektivste Primärtherapie auszuwählen. Ent-sprechend der aktuell verfügbaren Erkenntnisse ist dies auf dem Boden der Lokalisation und der Molekularpathologie des Primärtumors möglich.

\subsubsection{RAS Wildtyp}

\subsection{1 .}

Empfehlungsgrad

A

Level of Evidence

\section{Evidenzbasierte Empfehlung}

Patienten, die in einer erweiterten RAS-Analytik (KRAS und NRAS, Exone 2-4) einen RAS-Wildtyp (RAS-wt) zeigen und eine linksseitige Lokalisation des Primärtumors (Kolonkarzinom) aufweisen, sollen in der Erstlinientherapie der metastasierten Erkrankung präferentiell mit einer Chemotherapie-Doublette plus anti-EGFRTherapie behandelt werden.

Quellen: [1 108]

Konsens

\section{Hintergrund}

Ein direkter Vergleich von anti-EGFR Antikörpern (Cetuximab und Panitumumab) mit dem anti-VEGF Antikörper Bevacizumab erfolgte in drei randomisierten Studien (FIRE-3, PEAK, CALGB 80405), die in der Erstlinienbehandlung des mKRK durchgeführt wurden [1045], [ 11109$]$, [ $\left.\begin{array}{lll}1 & 1 & 1\end{array}\right]$. Die FIRE-3-Studie (Phase III) verglich FOLFIRI plus Cetuximab mit FOLFIRI plus Bevacizumab und evaluierte die ORR als primären Endpunkt. Die Evaluation der RAS Wildtyp (wt) Population zeigte keinen signifikanten Unterschied in ORR und PFS. Dagegen wurde ein deutlicher Überlebensvorteil im Cetuximab-Arm beobachtet (HR 0.70, $\mathrm{p}=0.0059$ ) [1045].

Die PEAK-Studie wurde als randomierte Phase II Studie durchgeführt und verglich FOLFOX plus Panitumumab mit FOLFOX plus Bevacizumab. Dabei wurde das PFS als primäreres Zielkriterium untersucht. In der Subgruppe der Patienten mit RAS-wt Tumoren wurde eine Überlegenheit des Panitumumab-Arms in Hinblick auf PFS (HR 0.65, $p=0.029$ ) und OS (41.3 vs 28.9 Monate) gefunden. Bei niedriger Eventrate $(<50 \%)$ wurde hinsichtlich des OS keine statistische Signifikanz erreicht (HR 0.63, 0.058) [1 109].

Die CALGB/SWOG-80405-Studie (Phase III) führte einen randomisierten Vergleich von (jeglicher) Chemotherapie plus Cetuximab versus Chemotherapie plus Bevacizumab durch. Die Wahl des Chemotherapieregimes, FOLFOX (76\%) oder FOLFIRI (24\%) war den 
teilnehmenden Zentren überlassen. Diese Studie berichtete eine signifikant höhere ORR im Cetuximab-Arm (69\% vs 54\%, p<0.01). Dagegen wurde in Hinblick auf PFS und OS kein signifikanter Unterschied zwischen den Behandlungsarmen festgestellt [ 11110$]$.

Eine Meta-Analyse basierend auf den publizierten Ergebnissen der drei Vergleichsstudien unterstützte die Überlegenheit der anti-EGFR Behandlung in Bezug auf ORR (odds ratio 1.46, $p=0.004$ ) und Gesamtüberleben (HR 0.77, $p=0.016)$. Das PFS (HR 0.92, $p=0.5$ ) zwischen den Behandlungsarmen war vergleichbar [1 108].

Als nebenwirkungsreichere therapeutische Alternative kommt bei diesen Patienten auch eine Behandlung mit der Chemotherapie-Triplette mit FOLFOXIRI (ggf. + Bevacizumab) in Betracht. In der TRIBE-Studie wurde bei unselektierten Patienten FOLFIRI plus Bevacizumab mit FOLFOXIRI plus Bevacizumab verglichen. Die Triplette zeigte im Vergleich zur Doublette eine signifikante Überlegenheit in Hinblick auf die ORR (65\% vs 54\%; OR 1,59, $\mathrm{p}=0.013)$, das PFS ( 12.3 vs 9.7 Monate; HR 0.77, $\mathrm{p}=0.006)$ als auch das OS (29.8 vs 25.8 Monate; HR $0.80, p=0.03$ ) [1111]. Erwartungsgemäß war die Behandlung mit FOLFOXIRI plus Bevacizumab im Vergleich zur Gabe von FOLFIRI plus Bevacizumab von einer signifikanten Steigerung der Grad 3-4 Nebenwirkungen in Hinblick auf Neutropenie, Diarrhoe und periphere Neuropathie begleitet [1 1112$]$.

Während Phase II Studien auf die hohe Effektivität einer Triplette plus anti-EGFR-Therapie hinweisen, so liegen dazu jedoch keine Ergebnisse aus Phase III Studien vor.

\subsubsection{Relevanz der Tumorlokalisation in der Behandlung von RAS Wildtyp Tumoren}

Retrospektive Untersuchungen klinischer Studien weisen darauf hin, dass die Lokalisation des Primärtumors nicht nur eine prognostische Relevanz hat, sondern auch eine wichtige Determinante der therapeutischen Effektivität ist und daher in Therapieentscheidungen miteinbezogen werden sollte [1113], [1114], [1056]. Präklinische Analysen unterstützen die unterschiedlichen Muster der Genmutation und Genexpression bei rechts- und linksseitigen Tumoren [1 1115$]$. [1 1116$]$. Als Trennlinie wird in der Mehrzahl der Untersuchungen die splenische Flexur betrachtet [1055], [1053]. Prinzipiell würde man die Trennlinie zwischen rechts- und linksseitigen Tumoren zwischen den proximalen zwei Dritteln und dem distalen Drittel des Colon transversum ziehen. Angesichts der retrospektiven Evaluationen wurde jedoch in der Mehrzahl der Untersuchungen aus pragmatischen Gründen die splenische Flexur als Trennlinie betrachtet [1055], [1053]. Entsprechend werden Coecum, Colon ascendens und Colon transversum dem rechtsseitigen Colon zugerechnet, während Tumoren des Colon descendens, Sigma und Rektum als linksseitig betrachtet werden. Rechtsseitige Tumoren sind weniger häufig als linksseitige (30\% versus $70 \%$ ). Der Anteil an weiblichen und älteren Patientienten ist höher. Sie zeichnen sich aus durch eine höhere Mutationslast und eine höhere Immunogenität. Molekularbiologisch findet sich bei rechtsseitigen Tumoren eine höhere Rate an CIMP (CPG-island methylation phenotype), BRAF-Mutationen und Mikrosatelliteninstabilität [ $\left[\begin{array}{llll}1 & 1 & 3\end{array}\right]$.

Die derzeit verfügbaren Daten weisen darauf hin, dass linksseitige Tumoren in hohem Maße von einer Behandlung mit anti-EGFR Substanzen profitieren. In der Erstlinientherapie wurden insbesondere Chemotherapie-Doubletten untersucht, die in Kombination mit anti-EGFR Substanzen bei linksseitigen Tumoren hinsichtlich ORR, PFS und OS deutlich effektiver waren als vergleichbare Kombinationen mit Bevacizumab oder ohne monoklonalen Antikörper [1053], [1054]. 
Hingegen sind rechtsseitige Tumoren durch eine ungünstigere Prognose mit schlechterem Ansprechen auf Standardtherapien und Anti-EGFR-Antikörper charakterisiert. In zwei Studien (FIRE-3, CALGB 80405) war die Kombination einer Chemotherapie-Doublette mit dem anti-EGFR Antikörper Cetuximab weniger effektiv als die Kombination der gleichen Chemotherapie mit Bevacizumab [1053], [1055]. Bei geringer Fallzahl wurde in der Subgruppenanalyse das Signifikanzniveau nicht erreicht.

Im Unterschied zu EGFR-Antikörpern liegen bisher keine Hinweise für eine lokalisationsabhängige Wirksamkeit von Bevacizumab vor. Bei rechtsseitigen Tumoren wurden in der N016966- und der AVF2107g Studie durch Zugabe von Bevacizumab längere Überlebenszeiten erreicht als mit Chemotherapie allein. Die lokalisationsbezogenen Interaktionstests waren negativ [1056]. Auf dem Boden der gegenwärtigen Datenlage wird bei rechtsseitigen Primärtumoren in der Erstlinientherapie der metastasierten Erkrankung eine Chemotherapie-Doublette oder Triplette (+/- Bev) empfohlen.

\subsubsection{RAS Mutation: Triplette / Doublette}

\subsection{2 .}

Empfehlungsgrad

B

Level of Evidence

\section{Evidenzbasierte Empfehlung}

Bei Vorliegen einer RAS Mutation sollte primär eine Chemotherapie-Doublette zum Einsatz kommen. Ob eine Triplette besser als eine Doublette ist, oder ob Bevacizumab zum Einsatz kommen sollte, ist nicht belegt.

Quellen: [ 111111$]$

Konsens

\section{Hintergrund}

Anti-EGFR Antikörper zeigen keine Effektivität bei Vorliegen einer RAS Mutation und sollen daher nicht eingesetzt werden [1043], [1044], [1047]. Prospektive Untersuchungen, welche die Effektivität von anti-VEGF Substanzen in der Erstlinientherapie von Tumoren mit RAS-Mutation untersuchen, liegen nicht vor. Eine retrospektive Subgruppenanalyse der FIRE-3 Studie zeigt bei Vorliegen einer KRASMutation vergleichbare Überlebenszeiten wenn Patienten FOLFIRI plus Bevacizumab oder FOLFIRI plus Cetuximab erhielten [1 1117 ]. Dagegen weist eine retrospektive Analyse der TML Studie angesichts negativer Interaktionstests darauf hin, dass die Effektivität einer Behandlung mit Bevacizumab in der Zweitlinientherapie unabhängig vom KRAS Status war [1 11118$]$.

In einer Subgruppenanalyse der TRIBE-Studie wurde bei Patienten mit RAS mutierten Tumoren die Effektivität der Chemotherapie-Triplette (FOLFOXIRI plus Bevacizumab) mit der Doublette (FOLFIRI plus Bevacizumab) verglichen [1111]. Dabei wurden mit der Triplette sowohl im Gesamtüberleben (HR 0,88; 95\% Cl 0,65-1,18), als auch im PFS (HR 0,78; 95\% Cl, 0,60-1,02) oder der Responserate (OR 1,55; 95\% Cl, 0,91-2,62) eine höhere Effektivität berichtet. In keinem der Effektivitätsparameter wurde jedoch das Signifikanzniveau erreicht. Entsprechend kann derzeit bei Vorliegen einer RAS-Mutation keine sichere Empfehlung zum Einsatz einer Chemotherapie-Triplette gemacht werden. 


\subsubsection{BRAF Mutation}

\subsection{3.}

Empfehlungsgrad

B

Level of Evidence

4

\section{Evidenzbasierte Empfehlung}

Liegt eine BRAF-Mutation vor, sollte primär eine möglichst effektive Chemotherapie z. B. mit einer Triplette oder der Einschluss in eine klinische Studie erfolgen.

Quellen: $[1062,1119]$

Konsens

\section{Hintergrund}

Eine BRAF V600 Mutation wird bei 8-12\% der mKRK Patienten beobachtet. Häufiger sind Frauen betroffen, das Erkankungsalter ist meist höher. Bei etwa zwei Dritteln der Betroffenen ist der Tumor im rechten Kolon lokalisiert; histologisch werden vermehrt muzinöse Subtypen beobachtet.

Klinisch fällt eine höhere Rate an Lymphknotenmetastasierung und Peritonealkarzinose auf. Molekularpathologisch stehen Mikrosatelliteninstabilität und ein „Methylator Phänotyp“ im Vordergrund [1120], [1057]. Die Prognose der Patienten mit BRAF-V600Mutation ist außerordentlich schlecht, so dass in zahlreichen Studien mediane PFS-Zeiten von weniger als 6 Monaten und mediane Überlebenszeiten von weniger als einem Jahr berichtet werden [1059].

Derzeit wird bei Vorliegen einer BRAF-V600-Mutation eine Chemotherapie-Triplette, mit dem FOLFOXIRI-Regime, empfohlen. Diese Empfehlung gründet sich allerdings auf eine Subgruppenanalyse von nur 28 Patienten mit BRAF-Mutation, die im Rahmen der TRIBEStudie behandelt wurden. Diese erzielten unter einer Behandlung mit FOLFOXIRI plus Bevacizumab $(n=16)$ im Vergleich zu FOLFIRI plus Bevacizumab $(n=12)$ deutlich günstigere Outcomedaten: Ein deutlich längeres OS (19,0 vs. 10,7 Monate; HR 0,54), ein längeres PFS (7,5 vs 5,5 Monate; HR 0,57) und eine höhere Remissionsrate (56 \% vs 42 \%; OR 1,87) [1062]. Einerseits können die Ergebnisse dieser Analyse aufgrund der geringen Fallzahl nur als hypothesengenerierend betrachtet werden, andererseits liegen weitere Untersuchungen derselben Arbeitsgruppe vor, welche die Effektivität von FOLFOXIRI plus Bevacizumab bei Vorliegen einer BRAF-Mutation unterstützen [ $\left.\begin{array}{llll}1 & 1 & 1 & 9\end{array}\right]$.

Ob anti-EGFR-Substanzen bei Vorliegen einer BRAF-Mutation effektiv sind, ist Gegenstand einer kontroversen Debatte. Zwei Meta-Analysen kommen hier zu unterschiedlichen Bewertungen. In der Analyse von Pietrantonio et al. wird duch Gabe von anti-EGFR-Antikörpern keine signifikante Verlängerung von PFS (HR 0,88, $p=0,33$ ) oder OS (HR 0,91, $p=0,63$ ) gefunden [1059]. Dagegen argumentieren Rowland und Mitarbeiter, dass die Evidenz nicht ausreicht, um definitiv auszuschließen, dass antiEGFR-Antikörper bei BRAF-Mutation einen anderen Behandlungseffekt haben als bei BRAF Wildtyp [1 1121 ].

Letztlich sind die vorliegenden Analysen durch kleine Fallzahlen charakterisiert, die weder für sich genommen noch in der gemeinsamen meta-analytischen Betrachtung definitive Schlussfolgerungen erlauben. 
Die Frage nach dem Stellenwert einer Bevacizumab-basierten im Vergleich zu einer Cetuximab-basierten Therapie wurde in einer Subgruppenanalyse der FIRE-3-Studie adressiert. Bei 48 evaluierbaren Patienten mit RAS-wt/BRAF-mut mKRK, war das OS in beiden Therapiearmen kurz und vergleichbar (Median 12.3 vs 13.7 Monate) unab-hängig davon, ob Cetuximab oder Bevacizumab zusammen mit FOLFIRI gegeben worden war [1 122]. Diese Analyse führte zu der Hypothese, dass gleichermaßen, weder eine antiEGFR- noch eine anti-VEGF Strategie, in der Lage sind, das therapeutische Ergebnis zu verbessern.

Aufgrund der schlechten Prognose BRAF-mutierter Tumoren können in der Zweitlinientherapie individuelle (derzeit nicht zugelassene) Therapieansätze, z. B. mit einem BRAF-Inhibitor, MEK-Inhibitor und Anti-EGFR-Antikörper oder wenn möglich die Behandlung im Rahmen einer klinischen Studie in Betracht gezogen werden [1123].

\subsubsection{MSI}

Immun-Checkpoint Inhibitoren haben in ersten klinischen Untersuchungen bei vorbehandelten mKRK Patienten mit Mikrosatelliteninstabilität (MSI) Aktivität gezeigt. Angesichts der derzeit noch limitierten Datenlage wird bei Nachweis einer MSI zu-nächst eine Erstlinienbehandlung entsprechend dem RAS-Mutationsstatus empfohlen. In späteren Therapielinien sollte die Möglichkeit einer Behandlung mit CheckpointInhibitoren evaluiert werden.

\section{Hintergrund}

Mutationen in den Mismatch Reparaturgenen (MLH1, MSH2, MSH6 und PMS2) führen zu einer fehlerhaften DNS-Replikation, die sich anhand variabler Längen der Mikrosatelliten-DNS als Mikrosatelliteninstabilität (MSI) manifestiert. Die defekte Mismatch Reparatur (MMRd) ist im Vergleich zur profizienten Mismatch Reparatur (MMRp) für eine um das 10-100-fache gesteigerte Mutationsrate ([1069]) verantwortlich. Diese bedingt eine gesteigerte Immunogenität und letztlich die deutlich vermehrten lymphozytäre Infiltrate der MSI-Tumoren ([ 11124$])$. Im Sinne eines „immune escape“ Mechanismus wird gerade bei MSI-Tumoren eine Hochregulation von Immun-Checkpoints, wie dem „programmed death“ (PD-1) Pathway nachgewiesen.

Beim kolorektalen Karzinom entsteht die MMRd sowohl im Rahmen von Keimbahnmutationen in einem der vier Mismatch Reparaturgene (hereditäres nonpolyposis kolorektales Karzinom (HNPCC oder Lynch-Syndrom), als auch durch somatische Mutationen oder durch epigenetisches Silencing [1068].

Erste klinische Daten bestätigen die Hypothese, dass MSI-Tumoren - im Gegensatz zu MSS-Tumoren - gut auf eine PD-1 Blockade ansprechen. Im Rahmen einer Phase II Studie wurden 32 Patienten untersucht, die mindestens 2 vorangegangene Chemotherapieregime erhalten hatten [1068]. Davon wurden 11 Patienten als MMRd und 21 als MMRp klassifiziert. Unter einer Behandlung mit dem PD-1 Inhibitor Pembrolizumab zeigten MMRd Patienten im Vergleich zu MMRp-Patienten eine deutlich höhere Rate an ORR ( $40 \%$ vs $0 \%$ ) und SD ( $50 \%$ vs $11 \%$ ) sowie eine hochsignifikante Verlängerung von PFS und OS (Mediane nicht erreicht).

Zum Zeitpunkt der Leitlinienerstellung sind immunologische Checkpointinhibitoren derzeit nicht für die Behandlung des mKRK zugelassen. 


\subsubsection{HER-2 Amplifikation}

Liegt bei metastasiertem KRK eine HER-2-Amplifikation vor, so wird derzeit zunächst eine Behandlung gemäß des RAS- und BRAF-Mutationsstatus empfohlen. Bei behandlungsrefraktären Tumoren kann dann eine molekularbiologisch auf den HER-2 Status gezielte Behandlung erwogen werden. In einer multizentrischen „Proof of Concept“Studie (HERACLES) wurde bei therapierefraktärer Erkrankung (KRAS Wildtyp) die Effektivität einer Kombination aus Trastuzumab und Lapatinib nachgewiesen. Die Kombination von Trastuzumab und Lapatinib ist zum Zeitpunkt der Leitlinienerstellung für die Behandlung des kolorektalen Karzinoms nicht zugelassen.

\section{Hintergrund}

Präklinische Untersuchungen zeigten, dass eine Amplifikation des HER-2 Onkogens mit einer Resistenz gegen anti-EGFR Substanzen wie Cetuximab assoziiert sein kann [1073]. Darüber hinaus wurde anhand von Xenograft-Modellen gezeigt, dass bei HER-2 Amplifikation oder Überexpression eine kombinierte Behandlung mit Trastuzumab und Lapatinib effektiv ist, während kein Ansprechen auf die Einzelsubstanzen verzeichnet wurde.

Die HERACLES Studie wurde als offene, multizentrische "proof-of-concept" Phase-2 Studie durchgeführt [1074]. Eingeschlossen wurden mKRK-Patienten, die auf die StandardChemotherapie (einschließlich Cetuximab oder Panitumumab) refraktär waren. Bei 914 Patienten mit KRAS Exon 2 Wildtyp wurden 48 Patienten (5\%) mit HER2-positiven Tumoren identifiziert. Die Tumoren wurden als HER2-positiv klassifiziert, wenn entweder ein IHC-Score 3+ oder IHC-Score 2+ mit positivem FISH-Test vorlag. Bei 27 in die Studie eingeschlossenen Patienten lag die ORR bei 30\% (95\% Cl 14-50), die SD-Rate bei $44 \%$ (95\% Cl 25-63),, das mediane PFS bei 21 Wochen (95\% Cl 16-32) und das mediane OS bei 46 (95\% Cl 33-68) Wochen.

\subsection{Durchführung der Erstlinienchemotherapie}

Die Gesamtschau aller Daten der aktuell verfügbaren Studien zur Erstlinientherapie des metastasierten kolorektalen Karzinoms weist darauf hin, dass effektivere und damit häufig auch intensivere Behandlungsregime mit einem Überlebensvorteil verbunden sind (Tabelle 10 - 
Tabelle 16).

Entsprechend sollte allen Patienten eine möglichst effektive Erstlinientherapie angeboten werden. Die Strategie, alle für die Therapie in Frage kommenden Medikamente im Verlauf der Behandlung sequenziell anzubieten, wurde zwar durch ältere Studien ohne Einsatz monoklonaler Antikörper (FOCUS, CAIRO) unterstützt, nachdem diese Studien aber in der "Vor-Antikörper-Ära" durchgeführt wurden, liegen aufgrund der limitierten therapeutischen Optionen auch die Überlebenszeiten deutlich unter 20 Monaten. Entsprechend sind die genannten Studien für die aktuelle Therapieführung nur noch von begrenzter Relevanz. 


\subsubsection{Erstlinienchemotherapie bei gutem Allgemeinzustand}

9.24 .

Empfehlungsgrad

A

Level of Evidence

$1 \mathrm{a}$
Evidenzbasierte Empfehlung

In der Erstlinienchemotherapie sollen bei gutem Allgemeinzustand und hoher Motivation in erster Linie Fluoropyrimidin-basierte Kombinationsregime mit infusionaler Verabreichung von 5-Fluorouracil, wie FOLFIRI, FOLFOX oder FOLFOXIRI oder mit dem oralen Fluoropyrimidin Capecitabin (vorwiegend mit Oxaliplatin, CAPOX) eingesetzt werden.

Quellen: [1125], [1126]

Konsens

\section{Hintergrund}

Voraussetzung für eine intensive Kombinationschemotherapie ist ein guter Allgemeinzustand des Patienten. Nachdem prinzipiell auch die Option einer weniger effektiven, aber deutlich nebenwirkungsärmeren Fluoropyrimidin-Monotherapie plus Bevacizumab besteht, ist es wichtig, die Patienten in die Diskussion miteinzubeziehen und die Motivationslage in Hinblick auf eine potenzielle Überlebensverlängerung zu definieren [1125], [1 126].

\subsection{5 . Konsensbasierte Empfehlung \\ EK Gesamtüberleben erreicht werden kann. \\ Konsens \\ 9.9.2. Erstlinienchemotherapie bei herabgesetztem Allgemeinzustand ab ECOG 2}

\section{7}

Die Kombination mit einer zielrichtet wirksamen Substanz (anti-EGFR oder anti-VEGF) sollte sich in erster Linie nach den vorrangigen Therapiezielen, den molekularbiologischen Charakteristika des Tumors und der Tumorlokalisation richten (siehe 9.8.2). Im Vordergrund der Therapieentscheidungen steht die Frage nach der Behandlung, mit der bei akzeptabler Verträglichkeit das längste

9.26 . Konsensbasierte Empfehlung

Bei herabgesetztem Allgemeinzustand können in Hinblick auf die Chemotherapie Fluoropyrimidin-Monotherapien (5-Fluorouracil/Folinsäure oder Capecitabin) gewöhnlich in Kombination mit Bevacizumab eingesetzt werden.

Konsens

\section{Hintergrund}

Die AVEX-Studie evaluierte die Effektivität einer Kombination aus Capecitabin plus Bevacizumab bei älteren Patienten ( $\geq 70$ Jahre) und verglich diese im Rahmen eines Phase III Designs mit einer Capecitabin Monotherapie [1126]. Überwiegend wurden Patienten 
mit einem Performance Status ECOG 0 (46\%) und ECOG 1 (45\%) eingeschlossen. Die Häufigkeit von Patienten mit ECOG $\geq 2$ betrug unter 10\%. Als primärer Endpunkt wurde das PFS untersucht. Unter der Behandlung mit Capecitabin plus Bevacizumab wurde eine signifikante Verlängerung des PFS (9,1 vs 5,1 Monate; HR 0,53, p<0,0001; primärer Studienendpunkt) sowie eine Steigerung der ORR (19\% vs 10\%; $p=0,04)$ erreicht. Das Gesamtüberleben (sekundärer Endpunkt) betrug 16,8 Monate im Kontrollarm und 20,7 Monate in der mit Capecitabin und Bevacizumab behandelten Gruppe ( $\mathrm{HR} 0,79,95 \% \mathrm{CI}$ 0,57-1,09; $p=0,18)$, dieser Unterschied war nicht signifikant. Die Behandlung kann grundsätzlich als gut verträglich eingestuft werden. Die Häufigkeit schwerwiegender Nebenwirkungen (SAEs) lag unter der Capecitabin-Monotherapie bei 31\%, unter der Kombination von Capecitabin und Bevacizumab bei 30\%. Analysen der Lebensqualität wurden nicht durchgeführt [ $\left[\begin{array}{ll}1 & 126\end{array}\right]$.

Diese Daten werden durch eine randomisierte Phase II Studie unterstützt, die bei Patienten durchgeführt wurde, die für eine Erstlinientherapie mit Irinotecan nicht in Betracht kamen [1 125]. Auch in dieser Studie lag die Häufigkeit der Patienten mit ECOG 2 unterhalb von 10\%. In dieser Population wurde Bevacizumab plus 5-FU/LV mit einer alleinigen 5-FU/LV-Behandlung verglichen. Die Zugabe von Bevacizumab zu 5-FU/LV induzierte eine signifikante Steigerung des PFS (9.2 vs 5.5 Monate; HR 0.50, p=0.0002) sowie eine nicht signifikante Steigerung des OS (16.6 vs 12.9 Monate; HR 0.79, $p=0.16$ ) und der ORR (26.0\% vs 15.2\%, p=0.055). Die Evaluation des FACT-C Scores zeigte keinen negativen Effekt von Bevacizumab auf die Lebensqualität (QOL). Die mediane Zeit bis zur Verschlechterung der Lebensqualität lag im Bevacizumab-Arm bei 3,2 Monaten und im Placebo-Arm bei 2,3 Monaten (HR 0,66; $p=0,016$ ).

Zusammengefasst weisen diese Studienergebnisse darauf hin, dass die Erstlinienchemotherapie mit einem Fluoropyrimidin und Bevcizumab bei älteren Patienten und solchen, die für eine initiale Irinotecan-basierte Therapie nicht geeignet waren, effektiv ist und damit für diese Patientenpopulation eine sinnvolle Behandlungsoption darstellt.

\subsubsection{Einsatz von FOLFOXIRI in der Erstlinientherapie}

\begin{tabular}{|c|c|}
\hline 9.27. & Evidenzbasierte Empfehlung \\
\hline $\begin{array}{l}\text { Empfehlungsgrad } \\
\text { B }\end{array}$ & $\begin{array}{l}\text { FOLFOXIRI gehört zu den effektivsten Chemotherapie-Regimen, sollte aber aufgrund } \\
\text { seiner erhöhten Nebenwirkungsrate nur bei Patienten mit gutem Allgemeinzustand } \\
\text { (ECOG Performance Status 0-1) zum Einsatz kommen. }\end{array}$ \\
\hline $\begin{array}{l}\text { Level of } \\
\text { Evidence }\end{array}$ & Quellen: $[1111,1112,1127-1130]$ \\
\hline & Konsens \\
\hline
\end{tabular}

\section{Hintergrund}

Es liegen eine „Meta“-Analyse von 2 prospektiven randomisierten Studien sowie zwei weitere prospektive randomisierte Studien vor [1127], [1128], [1129], [11112], [11111], [1130]. In der Meta-Anlayse wurde FOLFOXIRI mit FOLFIRI verglichen, in den beiden anderen Studien FOLFOXIRI + Bevacizumab mit FOLFIRI + Bevacizumab. Die Daten zeigen übereinstimmend eine höhere Response-Rate, ein verlängertes PFS und OS, sowie in den Studien, die dies untersuchten, eine höhere sekundäre R0-Resektionsrate von 
Lebermetastasen (Tabelle 10). Die Grad 3-4 Nebenwirkungen waren unter der Behandlung mit FOLFOXIRI plus Bevacizumab signifikant höher als in der Gruppe der mit FOLFIRI plus Bevacizumab behandelten Patienten. Im Vordergrund standen dabei Neutropenie (50\% vs. 20,5\%, p<0,001), Diarrhoe (18,8 vs. 10,6, p=0,01) und pheriphere Neuropathie $(5,2$ vs. $0 \%, p<0,001)$.

Tabelle 10: Randomisierte Studien zur Erstlinientherapie mit FOLFOXIRI bei unselektierten Patienten

\begin{tabular}{|c|c|c|c|c|c|c|c|c|}
\hline Studie & Regime & $\begin{array}{c}\mathbf{N} \\
\text { Patien } \\
\text {-ten }\end{array}$ & $\begin{array}{c}\text { ORR } \\
(\%)\end{array}$ & $\begin{array}{c}\text { OR } \\
\text { (P- } \\
\text { Wert) }\end{array}$ & $\begin{array}{l}\text { PFS } \\
\text { (Mo) }\end{array}$ & $\begin{array}{l}\text { HR PFS } \\
\text { (P-Wert) }\end{array}$ & $\begin{array}{c}\text { OS } \\
(\mathrm{Mo})\end{array}$ & $\begin{array}{l}\text { HR OS } \\
\text { (P-Wert) }\end{array}$ \\
\hline $\begin{array}{l}\text { Falcone } \\
{\left[\begin{array}{llll}1 & 1 & 28\end{array}\right]}\end{array}$ & $\begin{array}{l}\text { FOLFOXIRI } \\
\text { FOLFIRI }\end{array}$ & $\begin{array}{l}122 \\
122\end{array}$ & $\begin{array}{l}66^{*} \\
41^{*}\end{array}$ & $\begin{array}{l}\text { na } \\
(0.0002)\end{array}$ & $\begin{array}{l}9.8 \\
6.9\end{array}$ & $\begin{array}{l}0.63 \\
(0.0006)\end{array}$ & $\begin{array}{l}22.6 \\
16.7\end{array}$ & $\begin{array}{l}0.70 \\
(0.032)\end{array}$ \\
\hline $\begin{array}{l}\text { Souglakos } \\
{\left[\begin{array}{llll}1 & 1 & 27\end{array}\right]}\end{array}$ & $\begin{array}{l}\text { FOLFOXIRI } \\
\text { FOLFIRI }\end{array}$ & $\begin{array}{l}137 \\
146\end{array}$ & $\begin{array}{l}43 \\
33.6\end{array}$ & $\begin{array}{l}\text { na } \\
(0.168)\end{array}$ & $\begin{array}{l}8.4^{\#} \\
6.9^{\#}\end{array}$ & $\begin{array}{l}\text { na } \\
(0.17)\end{array}$ & $\begin{array}{l}21.5 \\
19.5\end{array}$ & $(0.337)$ \\
\hline $\begin{array}{l}\text { Loupakis } \\
{\left[\begin{array}{llll}1 & 1 & 1 & 2\end{array}\right]} \\
\text { Cremolini } \\
{\left[\begin{array}{llll}1 & 1 & 1 & 1\end{array}\right]}\end{array}$ & $\begin{array}{l}\text { FOLFOXIRI + } \\
\text { Bev } \\
\text { FOLFIRI + } \\
\text { Bev }\end{array}$ & $\begin{array}{l}252 \\
256\end{array}$ & $\begin{array}{l}65.1 \\
53.1\end{array}$ & $\begin{array}{l}1.64 \\
(0.006)\end{array}$ & $\begin{array}{l}12.1 \\
9.7\end{array}$ & $\begin{array}{l}0.75 \\
(0.003)\end{array}$ & $\begin{array}{l}29.8^{\S} \\
25.8^{\S}\end{array}$ & $\begin{array}{l}0.80^{\S} \\
(0.03)^{\S}\end{array}$ \\
\hline
\end{tabular}

\subsubsection{Kombination von Chemotherapie mit anti-EGFR Substanzen}

\begin{tabular}{|l|l|}
$\begin{array}{l}\text { 9.28. } \\
\text { Empfehlungsgrad }\end{array}$ & $\begin{array}{l}\text { Eie Zugabe von anti-EGFR AK (Cetuximab oder Panitumumab) zu einer Chemo- } \\
\text { therapie führt zu einer signifikanten Effektivitätssteigerung in Hinblick auf ORR, PFS } \\
\text { und OS. Anti-EGFR-AK sollen erst bei Nachweis eines all-RAS-Wildtyps im Tumor } \\
\text { gegeben werden. }\end{array}$ \\
\hline $\begin{array}{l}\text { Level of Evidence } \\
\mathbf{1}\end{array}$ & $\begin{array}{l}\text { Meta-Analyse: siehe Leitlinienreport } \\
\text { Primärstudien: [1041][1045][1040][1 129][1056][1130][1131] }\end{array}$ \\
\hline & Konsens \\
\hline
\end{tabular}

\section{Hintergrund}

Gegen den EGFR gerichtete monoklonale Antikörper wie Cetuximab oder Panitumumab sind nur bei Patienten mit RAS-Wildtyp Tumoren effektiv (Tabelle 11). 
Tabelle 11: Randomisierte Studien zur Erstlinientherapie mit anti-EGFR Substanzen bei RAS-wt Patienten

\begin{tabular}{|c|c|c|c|c|c|c|c|c|}
\hline Studie & Regime & $\begin{array}{l}\mathbf{N} \\
\text { Patien } \\
\text {-ten }\end{array}$ & $\begin{array}{l}\text { ORR } \\
(\%)\end{array}$ & $\begin{array}{l}\text { OR } \\
\text { (P- } \\
\text { Wert) }\end{array}$ & $\begin{array}{l}\text { PFS } \\
\text { (Mo) }\end{array}$ & $\begin{array}{l}\text { HR PFS } \\
\text { (P-Wert) }\end{array}$ & $\begin{array}{l}\text { OS } \\
\text { (Mo) }\end{array}$ & $\begin{array}{l}\text { HR OS } \\
\text { (P-Wert) }\end{array}$ \\
\hline $\begin{array}{l}\text { CRYSTAL } \\
\text { [1 044] }\end{array}$ & $\begin{array}{l}\text { FOLFIRI + Cet } \\
\text { FOLFIRI }\end{array}$ & $\begin{array}{l}178 \\
189\end{array}$ & $\begin{array}{l}66.3 \\
38.6\end{array}$ & $\begin{array}{l}3.11 \\
(<0.001)\end{array}$ & $\begin{array}{l}11.4 \\
8.4\end{array}$ & $\begin{array}{l}0.56 \\
(<0.001)\end{array}$ & $\begin{array}{l}28.4 \\
20.2\end{array}$ & $\begin{array}{l}0.69 \\
(0.0024)\end{array}$ \\
\hline $\begin{array}{l}\text { OPUS } \\
{[1047]}\end{array}$ & $\begin{array}{l}\text { FOLFOX + Cet } \\
\text { FOLFOX }\end{array}$ & $\begin{array}{l}38 \\
49\end{array}$ & $\begin{array}{l}58 \\
29\end{array}$ & $\begin{array}{l}3.33 \\
(0.0084)\end{array}$ & $\begin{array}{l}12 \\
5.8\end{array}$ & $\begin{array}{l}0.53 \\
(0.0615)\end{array}$ & $\begin{array}{l}198 \\
17.8\end{array}$ & $\begin{array}{l}0.94 \\
(0.80)\end{array}$ \\
\hline $\begin{array}{l}\text { PRIME } \\
\text { [1 043] }\end{array}$ & $\begin{array}{l}\text { FOLFOX + Pani } \\
\text { FOLFOX }\end{array}$ & $\begin{array}{l}259 \\
253\end{array}$ & $\begin{array}{l}60 \\
47\end{array}$ & $\begin{array}{l}\text { NR } \\
(0.003)\end{array}$ & $\begin{array}{l}10.1 \\
7.9\end{array}$ & $\begin{array}{l}0.72 \\
(0.004)\end{array}$ & $\begin{array}{l}26.0 \\
20.2\end{array}$ & $\begin{array}{l}0.78 \\
(0.04)\end{array}$ \\
\hline $\begin{array}{l}\text { COIN\# } \\
{\left[\begin{array}{llll}1 & 1 & 3 & 1\end{array}\right]}\end{array}$ & $\begin{array}{l}\text { FU/LV or Cape }+ \\
\text { Ox } \\
\text { FU/LV or Cape* } \\
+ \text { Ox + Cet }\end{array}$ & $\begin{array}{l}367 \\
362\end{array}$ & $\begin{array}{l}57 \\
64\end{array}$ & $(0.049)$ & $\begin{array}{l}8.6 \\
8.6\end{array}$ & $\begin{array}{l}0.96 \\
(0.60)\end{array}$ & $\begin{array}{l}17.9 \\
17.0\end{array}$ & $\begin{array}{l}1.04 \\
(0.67)\end{array}$ \\
\hline $\begin{array}{l}\text { NORDIC\# } \\
{[1058]}\end{array}$ & $\begin{array}{l}\text { FLOX } \\
\text { FLOX + Cet }\end{array}$ & $\begin{array}{l}97 \\
97\end{array}$ & $\begin{array}{l}47 \\
46\end{array}$ & $\begin{array}{l}0.96 \\
(0.89)\end{array}$ & $\begin{array}{l}8.7 \\
7.9\end{array}$ & $\begin{array}{l}1.07 \\
(0.66)\end{array}$ & $\begin{array}{l}22.0 \\
20.1\end{array}$ & $\begin{array}{l}1.14 \\
(0.48)\end{array}$ \\
\hline $\begin{array}{l}\text { FIRE-3 } \\
{\left[\begin{array}{lll}1 & 1 & 32\end{array}\right]}\end{array}$ & $\begin{array}{l}\text { FOLFIRI + Cet } \\
\text { FOLFIRI + Bev }\end{array}$ & $\begin{array}{l}199 \\
201\end{array}$ & $\begin{array}{l}65.3 \\
58.7\end{array}$ & $\begin{array}{l}1.33 \\
(0.18)\end{array}$ & $\begin{array}{l}10.3 \\
10.2\end{array}$ & $\begin{array}{l}0.97 \\
(0.77)\end{array}$ & $\begin{array}{l}33.1 \\
25.0\end{array}$ & $\begin{array}{l}0.697 \\
(0.0059)\end{array}$ \\
\hline $\begin{array}{l}\text { GALGB } \\
80405 \\
{[1133]}\end{array}$ & $\begin{array}{l}\text { FOLFOX/ } \\
\text { FOLFIRI + Cet } \\
\text { FOLFOX/ } \\
\text { FOLFIRI + Bev }\end{array}$ & $\begin{array}{l}270 \\
256\end{array}$ & $\begin{array}{l}68.6 \\
53.8\end{array}$ & $\begin{array}{l}1.75 \\
(<0.01)\end{array}$ & $\begin{array}{l}11.4 \\
11.3\end{array}$ & $\begin{array}{l}1.1 \\
(0.31)\end{array}$ & $\begin{array}{l}32.0 \\
31.2\end{array}$ & $\begin{array}{l}0.9 \\
(0.40)\end{array}$ \\
\hline $\begin{array}{l}\text { PEAK } \\
{\left[\begin{array}{lll}1 & 1 & 09\end{array}\right]}\end{array}$ & $\begin{array}{l}\text { FOLFOX + Pani } \\
\text { FOLFOX + Bev }\end{array}$ & $\begin{array}{l}88 \\
82\end{array}$ & $\begin{array}{l}63.6 \\
60.5\end{array}$ & NR & $\begin{array}{l}13.0 \\
9.5\end{array}$ & $\begin{array}{l}0.65 \\
(0.029)\end{array}$ & $\begin{array}{l}41.3 \\
28.9\end{array}$ & $\begin{array}{l}0.63 \\
0.058\end{array}$ \\
\hline
\end{tabular}

Die Zugabe von Cetuximab zu einer FOLFIRI-Chemotherapie führte bei RAS-wt Patienten in einer randomisierten Phase-III Studie (CRYSTAL) zu einer Verlängerung des medianen Überlebens von 20,2 auf 28,4 Monate (HR 0,69, p=0,0024) [1044]. Vergleichbare Daten wurden in der PRIME-Studie beschrieben. In dieser randomisierten Phase III Studie wurde durch Zugabe von Panitumumab zu dem FOLFOX-Regime eine OS-Verängerung von 20,2 auf 26,0 Monate erzielt (HR 0,78, p=0,04).

In einer meta-analytischen Evaluation (siehe Leitlinienreport) der verfügbaren Studien führt die Hinzunahme von anti-EGFR-AK zu Kombinationschemotherapien bei Patienten mit KRAS-Wildtyp Tumoren zu einer signifikanten Verlängerung von PFS (HR 0.83 , $\mathrm{p}=0.0001)$ und OS (HR 0.89, $\mathrm{p}=0.02$ ).

Angesichts der Studienergebnisse wurde rückblickend postuliert, dass anti-EGFRSubstanzen in Kombination mit Bolus-5-FU Regimen oder oralen Fluoropyrimdinen keine ausreichende Wirksamkeit entfalten. Nimmt man diese Studien (COIN und NORDIC) nun 
aus der meta-analytischen Betrachtung heraus, so sind die therapeutischen Effekte durch die Hinzunahme der anti-EGFR-AK für PFS (HR 0.72, p<0.00001) und OS (HR 0.79, $\mathrm{p}=0.0003$ ) deutlich stärker.

Der Einsatz von Panitumumab in Kombination mit FOLFOX führte in der PRIME-Studie im Vergleich zur alleinigen Behandlung mit FOLFOX zu einer deutlichen Steigerung von Grad 3-4 Nebenwirkungen, wie Hauttoxizität ( $36 \%$ vs $2 \%$ ), Diarrhoe (18\% vs $9 \%$ ), Fatigue ( $9 \%$ vs $3 \%$ ) oder Hypomagnesiämie (6\% vs $<1 \%$ ). Die Rate der Grad 3 Infusions-reaktionen nach Gabe des humanen IgG2 Antikörpers Panitumumab lag bei 0,3\% [1134]. Ähnliche Nebenwirkungen wurden auch in der CRYSTAL Studie beschrieben. Die Kombination von FOLFIRI plus Cetuximab führte im Vergleich zur alleinigen Gabe von FOLFIRI zu einer Steigerung von Grad 3-4 Nebenwirkungen wie akneiformem Exanthem (16,2\% vs 0\%) oder Diarrhoe (15,7\% vs 10,5\%). Die Rate der Grad 3-4 Infusionsreaktionen nach Gabe des chimären IgG1 Antikörpers Cetuximab lag bei 2,5\% [940].

Zum Einsatz von anti-EGFR Substanzen in Abhängigkeit von der Lokalisation des Primärtumors siehe 9.8.2.

Tabelle 12: Zusammenfassung der Meta-Analysen zu anti-EGFR Substanzen - OS

\begin{tabular}{|c|c|c|c|c|}
\hline OS (KRAS wt) & Studien & HR & $(95 \% \mathrm{CI})$ & $\mathbf{p}$ \\
\hline $\mathrm{CT}+/$ - anti EGFR alle & 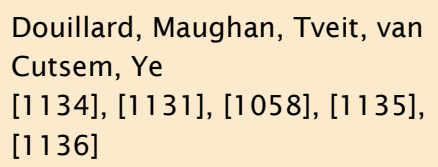 & 0.89 & $0.80-0.98$ & 0.02 \\
\hline $\begin{array}{l}\mathrm{CT}+/ \text { - anti-EGFR } \\
\text { (keine Bolus- oder oralen Regime)* }\end{array}$ & $\begin{array}{l}\text { Douillard, van Cutsem, Ye } \\
\text { [1 1 134], [ } 1135],\left[\begin{array}{lll}1 & 1 & 136\end{array}\right]\end{array}$ & 0.79 & $0.69-0.90$ & 0.0003 \\
\hline
\end{tabular}

Tabelle 13 Zusammenfassung der Meta-Analysen zu anti-EGFR Substanzen - PFS

\begin{tabular}{|c|c|c|c|c|}
\hline PFS (KRAS wt) & Studien & HR & $(95 \% \mathrm{CI})$ & $\mathbf{p}$ \\
\hline $\mathrm{CT}+/$ - anti EGFR alle & 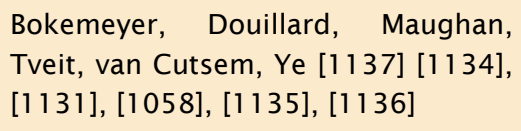 & 0.83 & $0.76-0.91$ & 0.0001 \\
\hline $\begin{array}{l}C T+/ \text { - anti-EGFR } \\
\text { (keine Bolus- oder oralen Regime)* }\end{array}$ & $\begin{array}{l}\text { Bokemeyer, Douillard, van Cutsem, } \\
\left.\text { Ye [ } \begin{array}{lll}1 & 137\end{array}\right],\left[\begin{array}{lll}1 & 1 & 34\end{array}\right],\left[\begin{array}{lll}1 & 135\end{array}\right],\left[\begin{array}{lll}1 & 1 & 36\end{array}\right]\end{array}$ & 0.72 & $0.63-0.82$ & $<0.00001$ \\
\hline
\end{tabular}




\subsubsection{Kombination mit anti-VEGF Substanzen}

9.29 .

Level of Evidence

la
Evidenzbasiertes Statement

2017

Die Zugabe von Bevacizumab zu einer infusionalen Kombinationschemotherapie führt in der meta-analytischen Aufarbeitung der Studiendaten zu einer signifikanten Effektivitätssteigerung in Hinblick auf PFS, aber nicht auf ORR und OS. Dagegen führt die Zugabe von Bevacizumab zu einer Monochemotherapie mit einem Fluoropyrimidin zu einer signifikanten Effektivitätssteigerung in Hinblick auf ORR, PFS und OS.

Meta-Analyse: Siehe Leitlinienreport

Primärstudien: $[1125,1126,1138-1144]$

Konsens

\section{Hintergrund}

Die Effektivität einer gegen VEGF gerichteten Behandlung mit Bevacizumab wurde in zahlreichen Studien zur Erstlinientherapie des mKRK untersucht (Tabelle 14). Dabei handelte es sich um molekularpathologisch unselektierte Patientenpopulationen. In der meta-analytischen Evaluation aller verfügbaren Studien führt die Hinzunahme von Bevacizumab zu einer Fluoropyrimidin-basierten Chemotherapie (Monotherapie oder Kombinationstherapie) zu einer signifikanten Verlängerung von PFS (HR 0.71, $p<0.00001$ ) und OS (HR 0.85, p=0.0008).

Beschänkt man die Analyse auf eine Fluoropyrimidin-Monotherapie (5-FU Bolus oder infusionales 5-FU, Capecitabin) so führt die Hinzugabe von Bevacizumab zu einer hoch signifikanten Verlängerung von PFS (HR 0.57, p<0.00001) und OS (HR 0.83, p=0.03).

Nimmt man die nicht mehr gebräuchlichen Bolus-5-FU Regime (IFL) aus der Analyse und fokussiert die Evaluation auf infusionale Kombinationschemotherapien, so führt die Zugabe von Bevacizumab zwar zu einer signifikanten Verlängerung des PFS (HR 0.79, $\mathrm{p}<0.0001$ ), nicht aber des Gesamtüberlebens (HR 0.92, $\mathrm{p}=0.18$ ). 
Tabelle 14: Randomisierte Studien zur Erstlinientherapie mit Bevacizumab bei unselektierten Patienten

\begin{tabular}{|c|c|c|c|c|c|c|c|c|}
\hline Studie & Regime & $\begin{array}{c}\text { N } \\
\text { Pat. }\end{array}$ & $\begin{array}{c}\text { ORR } \\
(\%)\end{array}$ & $\begin{array}{c}\text { OR } \\
\text { (P-Wert) }\end{array}$ & $\begin{array}{l}\text { PFS } \\
\text { (Mo) }\end{array}$ & $\begin{array}{l}\text { HR PFS } \\
\text { (P) }\end{array}$ & $\begin{array}{c}\text { OS } \\
(\mathrm{Mo})\end{array}$ & $\begin{array}{l}\text { HR OS } \\
\text { (P-Wert) }\end{array}$ \\
\hline $\begin{array}{l}\text { Hurwitz } \\
\text { (Phase III) } \\
{\left[\begin{array}{lll}1 & 138\end{array}\right]}\end{array}$ & $\begin{array}{l}\mathrm{IFL}+\mathrm{Bev} \\
\mathrm{IFL}\end{array}$ & $\begin{array}{l}402 \\
411\end{array}$ & $\begin{array}{l}44.8 \\
34.8\end{array}$ & $(0.004)$ & $\begin{array}{l}10.6 \\
6.2\end{array}$ & $\begin{array}{l}0.54 \\
(<0.001)\end{array}$ & $\begin{array}{l}20.3 \\
15.6\end{array}$ & $\begin{array}{l}0.66 \\
(<0.001)\end{array}$ \\
\hline $\begin{array}{l}\text { Stathopoulos } \\
\text { (Phase III) } \\
{\left[\begin{array}{lll}1 & 139\end{array}\right.}\end{array}$ & $\begin{array}{l}\mathrm{IFL}+\mathrm{Bev} \\
\mathrm{Bev}\end{array}$ & $\begin{array}{l}114 \\
108\end{array}$ & $\begin{array}{l}36.8 \\
35.2\end{array}$ & (n.s.) & NR & NR & $\begin{array}{l}22 \\
25\end{array}$ & $\begin{array}{l}1.05 \\
(0.139)\end{array}$ \\
\hline $\begin{array}{l}\text { ARTIST } \\
\text { (Phase III) } \\
{\left[\begin{array}{lll}1 & 140]\end{array}\right.}\end{array}$ & $\begin{array}{l}\mathrm{mlFL}+\mathrm{Bev} \\
\mathrm{mIFL}\end{array}$ & $\begin{array}{l}142 \\
72\end{array}$ & $\begin{array}{l}35.3 \\
17.2\end{array}$ & $(0.013)$ & $\begin{array}{l}8.3 \\
4.2\end{array}$ & $\begin{array}{l}0.44 \\
(<0.001)\end{array}$ & $\begin{array}{l}18.7 \\
13.4\end{array}$ & $\begin{array}{l}0.62 \\
(0.014)\end{array}$ \\
\hline $\begin{array}{l}\text { N016966 } \\
\text { (Phase III) } \\
{\left[\begin{array}{llll}1141]\end{array}\right.}\end{array}$ & $\begin{array}{l}\text { FOLFOX/XELO } \\
\text { X }+ \text { BeV } \\
\text { FOLFOX/XELO } \\
\text { X }\end{array}$ & $\begin{array}{l}699 \\
701\end{array}$ & $\begin{array}{l}47 \\
49\end{array}$ & $\begin{array}{l}0.90 \\
(0.31)\end{array}$ & $\begin{array}{l}9.4 \\
8.0\end{array}$ & $\begin{array}{l}0.83 \\
(0.0023)\end{array}$ & $\begin{array}{l}21.3 \\
19.9\end{array}$ & $\begin{array}{l}0.89 \\
(0.077)\end{array}$ \\
\hline $\begin{array}{l}\text { ITACa } \\
\text { (Phase III) } \\
{\left[\begin{array}{lll}1 & 142]\end{array}\right.}\end{array}$ & $\begin{array}{l}\text { FOLFOX4/ } \\
\text { FOLFIRI + Bev } \\
\text { FOLFOX4/ } \\
\text { FOLFIRI }\end{array}$ & $\begin{array}{l}176 \\
194\end{array}$ & $\begin{array}{l}50.6 \\
50\end{array}$ & $(0.865)$ & $\begin{array}{l}9.6 \\
8.4\end{array}$ & $\begin{array}{l}0.86 \\
(0.182)\end{array}$ & $\begin{array}{l}20.8 \\
21.3\end{array}$ & $\begin{array}{l}1.13 \\
(0.317)\end{array}$ \\
\hline $\begin{array}{l}\text { Kabbinavar } \\
\text { (Phase II) } 2003 \\
{\left[\begin{array}{lll}1143]\end{array}\right.}\end{array}$ & $\begin{array}{l}5-\mathrm{FU} / \mathrm{LV} \\
5-\mathrm{FU} / \mathrm{LV}+\mathrm{BeV} \\
5 \mathrm{mg} / \mathrm{kg} \\
5-\mathrm{FU} / \mathrm{LV}+\mathrm{Bev} \\
10 \mathrm{mg} / \mathrm{kg}\end{array}$ & $\begin{array}{l}36 \\
35 \\
33\end{array}$ & $\begin{array}{l}17 \\
40 \\
24\end{array}$ & & $\begin{array}{l}5.2 \\
9.0 \\
7.2\end{array}$ & & $\begin{array}{l}13.8 \\
21.5 \\
16.1\end{array}$ & \\
\hline $\begin{array}{l}\text { Kabbinavar } \\
\text { (Phase II) } 2005 \\
{\left[\begin{array}{lll}1 & 125]\end{array}\right.}\end{array}$ & $\begin{array}{l}\mathrm{FU} / \mathrm{LV}+\mathrm{BeV} \\
\mathrm{FU} / \mathrm{LV}\end{array}$ & $\begin{array}{l}104 \\
105\end{array}$ & $\begin{array}{l}26.0 \\
15.2\end{array}$ & $(0.055)$ & $\begin{array}{l}9.2 \\
5,5\end{array}$ & $\begin{array}{l}0.50 \\
(0.0002)\end{array}$ & $\begin{array}{l}16.6 \\
12.9\end{array}$ & $\begin{array}{l}0.79 \\
(0.16)\end{array}$ \\
\hline $\begin{array}{l}\text { AVEX } \\
\text { (Phase III) } \\
{\left[\begin{array}{lll}1 & 126]\end{array}\right.}\end{array}$ & $\begin{array}{l}\text { Cape + Bev } \\
\text { Cape }\end{array}$ & $\begin{array}{l}140 \\
140\end{array}$ & $\begin{array}{l}19 \\
10\end{array}$ & $(0.04)$ & $\begin{array}{l}9.1 \\
5.1\end{array}$ & $\begin{array}{l}0.53 \\
(<0.0001)\end{array}$ & $\begin{array}{l}20.7 \\
16.8\end{array}$ & $\begin{array}{l}0.79 \\
(0.18)\end{array}$ \\
\hline $\begin{array}{l}\text { MAX } \\
\text { (Phase III) } \\
{\left[\begin{array}{lll}1 & 144]\end{array}\right.}\end{array}$ & $\begin{array}{l}\text { Cape }+ \text { Bev } \\
\text { Cape }\end{array}$ & $\begin{array}{l}157 \\
156\end{array}$ & $\begin{array}{l}38.1 \\
30.3\end{array}$ & $\begin{array}{l}\text { NR } \\
(0.16)\end{array}$ & $\begin{array}{l}8.5 \\
5.7\end{array}$ & $\begin{array}{l}0.62 \\
(<0.001)\end{array}$ & NR & $\begin{array}{l}0.88 \\
(0.314)\end{array}$ \\
\hline
\end{tabular}

Zusammenfassend kann festgehalten werden, dass in der meta-analytischen Aufarbeitung der verfügbaren Studien eine Verlängerung des Gesamtüberlebens dann erreicht wurde, wenn Bevacizumab zu einer Fluoropyrimidin-Monotherapie hinzugefügt wurde, während dies bei Zugabe zu einer infusionalen Kombinationschemotherapie nicht gelang. 
In der Zulassungsstudie führte die Zugabe von Bevacizumab zu dem IFL-Regime im Vergleich zur alleinigen Behandlung mit IFL zu einer Steigerung von Grad 3-4 Nebenwirkungen wie Diarrhoe $(32,4 \%$ vs $24,7 \%)$, Hypertonie (11\% vs $2,3 \%$ ) oder thrombotischen Ereignissen (19,4\% vs 16,2\%). Zusätzlich wurde bei insgesamt geringer Häufigkeit eine Steigerung von Grad 3-4 Blutungsereignissen (3,1\% vs 2,5\%) sowie gastrointestinalen Perforationen ( $1,5 \%$ vs. $0 \%$ ) beobachtet [1 138]).

Bei Zugabe von Bevacizumab zu einem Oxaliplatin-basierten Behandlungsregime (FOLFOX oder XELOX) wurden in der NO16966-Studie folgende Bevacizumab-spezifische Nebenwirkungen beschrieben: venöse thromboembolische Ereignisse ( $8 \%$ vs 5\%), arterielle thomboembolische Ereignisse (2\% vs 1\%), Blutungsereignisse (2\% vs 1\%), Hypertonie (4\% vs 1\%) [1 141].

Die Zugabe von Bevacizumab zu einer Behandlung mit Capecitabin war im Vergleich zur Capecitabin-Monotherapie mit vergleichsweise geringen Nebenwirkungen vergesellschaftet. Folgende Grad 3-4 Nebenwirkungen wurden in der AVEX-Studie beschrieben: Hand-Fuß-Syndrom (16\% vs 7\%), Diarrhoe (7\% vs 6\%), venöse thromboembolische Ereignisse ( $6 \%$ vs $4 \%$ ), Blutungsereignisse ( $0 \%$ vs $0 \%$ ), Hypertonie ( $0 \%$ vs $1 \%)$ [1 1126$]$.

Tabelle 15: Zusammenfassung der Meta-Analysen zu Bevacizumab - OS

\begin{tabular}{|c|c|c|c|c|}
\hline OS & Studien & HR & $(95 \% \mathrm{CI})$ & $\mathbf{p}$ \\
\hline $\begin{array}{l}\mathrm{CT}+/ \text { - Bevacizumab } \\
\text { (alle verfügbaren Studien) }\end{array}$ & 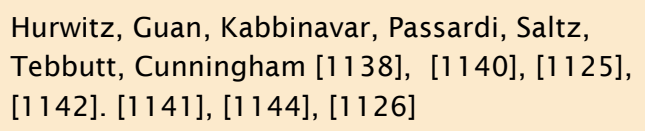 & 0.85 & $0.78-0.94$ & 0.0008 \\
\hline $\begin{array}{l}\mathrm{CT}+/- \text { Bevacizumab } \\
\text { (nur aktuell verwendete } \\
\text { Standardregime)* }\end{array}$ & 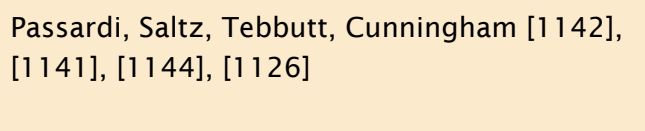 & 0.92 & $0.83-1.03$ & 0.13 \\
\hline $\begin{array}{l}\text { Fluoropyrimidin +/- } \\
\text { Bevacizumab }\end{array}$ & $\begin{array}{l}\text { Cunningham, Kabbinavar,Tebbutt [ }\left[\begin{array}{lll}1 & 126\end{array}\right] \\
{\left[\begin{array}{lll}1 & 1 & 25\end{array}\right],\left[\begin{array}{lll}1 & 1 & 44\end{array}\right]}\end{array}$ & 0.83 & $0.70-0.98$ & 0.03 \\
\hline $\begin{array}{l}\text { Infusionale Kombi-CT +/- } \\
\text { Bevacizumab }\end{array}$ & 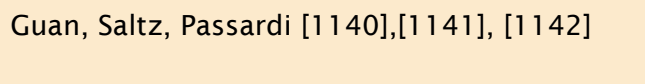 & 0.92 & $0.81-1.04$ & 0.18 \\
\hline
\end{tabular}


Tabelle 16: Zusammenfassung der Meta-Analysen zu Bevacizumab - PFS

\begin{tabular}{|c|c|c|c|c|}
\hline PFS & Studien & HR & $(95 \% \mathrm{CI})$ & $\mathbf{p}$ \\
\hline $\mathrm{CT}+/-$ Bevacizumab alle & 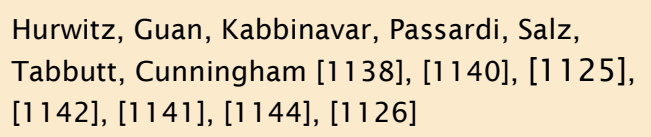 & 0.71 & $0.65-0.77$ & $<0.00001$ \\
\hline $\begin{array}{l}\mathrm{CT}+\text { /- Bevacizumab } \\
\text { (nur aktuell verwendete } \\
\text { Standardregime)* }\end{array}$ & 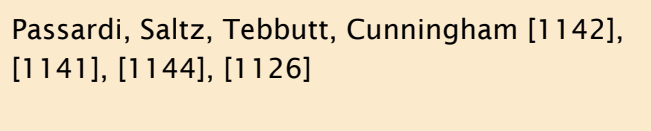 & 0.75 & $0.68-0.82$ & $<0.00001$ \\
\hline $\begin{array}{l}\text { Fluoropyrimidin +/- } \\
\text { Bevacizumab }\end{array}$ & $\begin{array}{l}\text { Cunningham, Kabbinavar, Tebbutt [ }\left[\begin{array}{ll}1 & 126\end{array}\right] \\
{\left[\begin{array}{lll}1 & 1 & 25\end{array}\right],\left[\begin{array}{lll}1 & 144\end{array}\right]}\end{array}$ & 0.57 & $0.48-0.66$ & $<0.00001$ \\
\hline $\begin{array}{l}\text { Infusionale Kombi-CT +/- } \\
\text { Bevacizumab }\end{array}$ & $\begin{array}{l}\text { Guan, Saltz, Passardi } \\
\text { [1 } 1140],\left[\begin{array}{lll}1 & 1 & 4\end{array}\right],\left[\begin{array}{lll}1 & 1 & 42\end{array}\right]\end{array}$ & 0.79 & $0.71-0.88$ & $<0.0001$ \\
\hline
\end{tabular}

\subsubsection{Kombination von anti-EGFR- und anti-VEGF Substanzen}

\begin{tabular}{|c|c|}
\hline 9.30. & Evidenzbasierte Empfehlung \\
\hline $\begin{array}{l}\text { Empfehlungsgrad } \\
\text { A }\end{array}$ & $\begin{array}{l}\text { Die Kombinationstherapie mit einem anti-EGFR-AK plus VEGF-Signalweg-Inhibitor } \\
\text { soll nicht eingesetzt werden. }\end{array}$ \\
\hline $\begin{array}{l}\text { Level of } \\
\text { Evidence }\end{array}$ & Quellen: [1 145-1147] \\
\hline & Starker Konsens \\
\hline
\end{tabular}

\section{Hintergrund}

Drei prospektive Studien zeigen übereinstimmend, dass eine Polychemotherapie in Kombination mit anti-EGFR-AK und mit Bevacizumab gegenüber einer Polychemotherapie in Kombination mit Bevacizumab mit einem verminderten PFS und mit erhöhter Toxizität einhergeht. In allen drei Studien wurde eine oxaliplatinhaltige Therapie eingesetzt, in einer Studie zusätzlich eine Irinotecan-basierte Therapie [1145], [1146], [1 1147$]$. 


\subsubsection{Dauer der Induktionstherapie}

9.31 . Konsensbasierte Empfehlung

\section{EK}

Eine Oxaliplatin-basierte Induktionstherapie (FOLFOX, CAPOX, FOLFOXIRI) sollte nach Möglichkeit über einen Zeitraum von 4 - 6 Monaten durchgeführt werden, bevor auf eine Oxaliplatin-freie Behandlung deeskaliert wird.

Limitierend für den Einsatz von Oxaliplatin sind nicht nur allergische Reaktionen, sondern auch die Entwicklung einer peripheren Polyneuropathie, deren Inzidenz und Schwere mit der kumulativen Dosis von Oxaliplatin zunimmt.

Konsens

\section{Hintergrund}

Aufgrund der durch Oxaliplatin induzierten Polyneuropathie kann FOLFOX oder CAPOX im Regelfall nicht länger als 4-6 Monate verabreicht werden. In der MACRO- sowie auch der CAIRO3-Studie wurden vor Beginn der Erhaltungstherapie 6 Zyklen CAPOX (4,5 Monate) gegeben [1148], [1149]. In der AIO KRK-0207 Studie folgte die Erhaltungstherapie einer über 6 Monate durchgeführten Induktionstherapie [1150]. Gleichfalls wurde auch in der TRIBE-Studie die Dauer der Induktionstherapie auf maximal 12 Zyklen der 2-wöchentlich gegebenen Chemotherapie beschränkt, worauf-hin eine Erhaltungstherapie mit 5-FU plus Bevacizumab einsetzte und bis zur Progression fortgeführt wurde [1056].

Die optimale Dauer einer Induktionstherapie mit FOLFIRI ist nicht klar. Diese Behandlung kann daher bis zum Erreichen eines optimalen Ansprechens fortgesetzt werden. In jedem Fall wird man aber auch hier eine initiale Therapiedauer von mindestens 4-6 Monaten anstreben.

Bei Patienten, die eine initiale Monochemotherapie mit einem Fluoropyrimidin erhalten, sollte diese Behandlung bis zur Progression fortgesetzt werden.

\subsubsection{Erhaltungstherapie und Therapiepause}

\begin{tabular}{|l|l|}
\hline $\begin{array}{l}\text { 9.32. } \\
\text { Empfehlungsgrad } \\
\mathbf{0}\end{array}$ & $\begin{array}{l}\text { Nach einer Induktionschemotherapie kann die Behandlung pausiert oder in eine } \\
\text { Erhaltungstherapie deeskaliert werden. }\end{array}$ \\
\hline $\begin{array}{l}\text { Level of } \\
\text { Evidence }\end{array}$ & Quellen: [1150-1155] \\
\hline $\mathbf{1}$ & \\
\hline
\end{tabular}

\section{Hintergrund}

Nach 4-6 Monaten einer Oxaliplatin-basierten Induktionstherapie sollte überprüft werden, ob zur Minderung der Toxizität und Steigerung der Lebensqualität eine Erhaltungstherapie durchgeführt werden kann oder ob eine Therapiepause möglich ist. 


\subsubsection{Erhaltungstherapie vs Fortführung der Induktionstherapie}

Die Frage, ob eine Erhaltungstherapie im Vergleich zur Fortführung der Induktionstherapie einen Einfluss auf das Gesamtüberleben hat, wurde in drei Meta-Analysen und einem systematischen Review beleuchtet [1151], [1152], [1153], [1154]. Diese zeigen übereinstimmend, dass eine Erhaltungstherapie im Vergleich zu einer kontinuierlichen Therapie ohne signfikante Änderung des OS durchgeführt werden kann. Im Vergleich einer kontinuierlichen Chemotherapie mit einer Unterbrechung der Therapie war der Unterschied im OS gering (HR um 0.10 vermindert), aber teilweise signifikant. Die Nebenwirkungsrate scheint durch eine intermittierende Therapie bzw. Therapieunterbrechung zum Teil geringer zu sein. Es zeichnet sich ein Trend zu einer besseren Lebensqualität im Unterbrechungsarm ab. Dieser Parameter wurde jedoch nur in wenigen Studien erfasst und dies mit unterschiedlichen Scores.

\subsubsection{Erhaltungstherapie vs Therapiepause}

Basierend auf den Daten der AIO KRK-0207-Studie sowie der CAIRO3-Studie ermöglicht die Erhaltungstherapie im Vergleich zur Therapiepause eine prolongierte progressionsfreie Zeit, hat aber keinen Einfluss auf das Gesamtüberleben ([1150], [1155]).

Nach Induktionstherapie mit einer Doublette /Triplette plus Bevacizumab wird eine Maintenance-Therapie mit einem Fluoropyrimidin plus Bevacizumab als bevorzugte Behandlungsoption betrachtet. Eine alleinige Erhaltungstherapie mit Bevacizumab wird dagegen nicht empfohlen [1150]. Die Fortführung der Behandlung durch eine Erhaltungstherapie mit einem Fluoropyrimidin plus Bevacizumab führte im Vergleich zur Bevacizumab-Monotherapie oder zur Therapiepause nicht zu einer Absenkung des globalen Lebensqualitätsscores (GHS/QoL) [1156].

Wählt man nach einer Induktionschemotherapie eine Therapiepause, so sollte diese als „kontrollierte Pause" durchgeführt werden und nach entsprechender Staging-Diagnostik zu einem geplanten Wiedervorstellungstermin führen.

\subsection{Zweitlinientherapie}

\subsubsection{Durchführung der Zweitlinien-Chemotherapie}

\begin{tabular}{|l|l|}
$\begin{array}{l}\text { 9.33. } \\
\begin{array}{l}\text { Empfehlungsgrad } \\
\text { B }\end{array}\end{array}$ & $\begin{array}{l}\text { Die Effektivität der Zweitlinientherapie ist gewöhnlich deutlich geringer als die der } \\
\text { Erstlinientherapie. Die Wahl einer Zweitlinientherapie sollte sich im Rahmen des } \\
\text { sequenziellen Einsatzes aktiver Substanzen in erster Linie nach der Effektivität und } \\
\text { den Nebenwirkungen der Vortherapie richten. }\end{array}$ \\
\hline $\begin{array}{l}\text { Level of } \\
\text { Evidence } \\
\mathbf{1}\end{array}$ & \begin{tabular}{l} 
Quellen: [1157-1159] \\
\hline
\end{tabular} \\
\hline
\end{tabular}

\section{Hintergrund}

Metastasierte KRK-Patienten sollen im Verlauf der Behandlung nach Möglichkeit Zugang zu allen verfügbaren Medikamenten erhalten. Der Stellenwert einer effektiven 
Zweitlinien-therapie für das Gesamtüberleben wurde in mehreren Phase III Studien untersucht. So erbrachte eine Zweitlinientherapie mit Irinotecan nach Versagen einer Fluorouracil Monotherapie im Vergleich zu BSC (OS 9,2 vs 6,5 Monate) [1159] oder infusionalem 5-FU/FS [1158] (OS 10,8 vs 8,5 Monate) einen signifikanten Vorteil im Gesamtüberleben.

Beim Vergleich von Irinotecan mit infusionalem 5-FU war das schmerzfreie Überleben unter der Behandlung mit Irinotecan länger (10,3 vs 8,5 Monate), gleichzeitig waren die Verträglichkeit sowie auch die Lebensqualität in beiden Behandlungsarmen vergleichbar [1158]. Im direkten Vergleich mit "best supportive care" induzierte Irinotecan eine signifikante Verlängerung der Zeit ohne Verschlechterung des Performance Status. Bei der Analyse der Lebensqualität schnitt die Behandlung mit Irinotecan in allen Belangen (außer Diarroe) besser ab [1159].

Eine Kombinationstherapie mit Oxaliplatin und Fluororuracil nach Versagen eines irinotecanhaltigen Protokolls in der Erstlinientherapie war einer 5-FU/FS bzw. Oxaliplatin Monotherapie sowohl bezüglich der erzielten Ansprechraten als auch bezüglich der Zeit bis zur Progression überlegen [1157]. Die Kombinationstherapie von 5-FU/FS mit Oxaliplatin bzw. Irinotecan, die als Erstlinientherapie jeweils Ansprechraten (CR + PR) von 40-55\% zeigen, erreichen in der Zweitlinientherapie Ansprechraten von 4\% (FOLFIRI) bis 15\% (FOLFOX) und ein progressionsfreies Überleben von ungefähr 2,5-4.2 Monaten. Das mediane Überleben der Patienten betrug für beide Therapiesequenzen (FOLFOX gefolgt von FOLFIRI bzw. FOLFIRI gefolgt von FOLFOX) jeweils etwa 20 Monate [1160]. Entsprechend der eingesetzten Medikamente waren die Toxiziätsspektren erwartungsgemäß unterschiedlich. Analysen zur Lebensqualität liegen aus dieser Studie nicht vor.

\subsubsection{Zweitlinientherapie mit anti-VEGF- bzw. anti-VEGFR- Substanzen}

Mehrere randomisierte Studien belegen den Nutzen von Bevacizumab (E3200, TML, BEBYP), Aflibercept (VELOUR) und Ramucirumab (RAISE) in der Zweitlinientherapie. Die Therapieeffekte sind in hohem Maße konsistent. Die evaluierbaren Studien zeigen einheitlich, dass eine signifikante Verlängerung von PFS und OS erreicht werden kann, wenn die anti-VEGF Substanzen Bevacizumab oder Aflibercept bzw. der anti-VEGFRAntikörper Ramucirumab zu einer Zweitlinienchemotherapie hinzugegeben werden. Einschränkend muss darauf hingewiesen werden, dass die absoluten Überlebenszeitgewinne im Vergleich der medianen OS-Zeiten durchwegs moderat sind und überwiegend in einem Bereich von 1-2 Monaten liegen.

Die antiangiogene Therapie ist mit den für sie charakteristischen Nebenwirkungen verbunden. So wurden z.B. bei Zugabe von Aflibercept zu FOLFIRI im Vergleich zur alleinigen Behandlung mit FOLFIRI folgende Grad 3-4 Nebenwirkungen beobachtet: Hypertonie $(19,3 \%$ vs $1,5 \%)$, Blutungen $(2,9 \% \vee 1,7 \%)$, arterielle thromboembolische Ereignisse $(1,8 \% \vee 0,5 \%)$, und venöse thromboembolische Ereignisse $(7,9 \% \vee 6,3 \%)$. Darüber hinaus wurde z.T. aber auch eine Verstärkung von chemotherapieassoziierten Toxizitäten wie Diarrhoe oder Stomatitis beobachtet [1161].

In ähnlicher Weise wurde auch bei der Kombination des VEGFR-Inhibitors Ramucirumab mit FOLFIRI im Vergleich zur FOLFIRI-Chemotherapie eine Steigerung der Nebenwirkungen festgestellt. Diese betraf insbesondere Grad 3-4 Nebenwirkungen wie Neutropenie (38\% vs 23\%), Hypertonie (11\% vs 3\%), Blutungen (1,9\% vs 1,5\%) oder gastrointestinale Perforationen (1,5\% vs 0,6\%) [1 162]. 
Tabelle 17: Randomisierte Studien zur Zweitlinientherapie mit anti-VEGF Substanzen

\begin{tabular}{|c|c|c|c|c|c|c|c|c|c|}
\hline Studie & $\begin{array}{l}\text { Vor- } \\
\text { behandlung }\end{array}$ & Regime & $\begin{array}{l}\mathbf{N} \\
\text { Pat. }\end{array}$ & $\begin{array}{l}\text { ORR } \\
(\%)\end{array}$ & $\begin{array}{l}\text { OR } \\
\text { (P- } \\
\text { Wert) }\end{array}$ & $\begin{array}{l}\text { PFS } \\
\text { (Mo) }\end{array}$ & $\begin{array}{l}\text { HR PFS } \\
\text { (P- } \\
\text { Wert) }\end{array}$ & $\begin{array}{l}\text { OS } \\
\text { (Mo) }\end{array}$ & $\begin{array}{l}\text { HR OS (P- } \\
\text { Wert) }\end{array}$ \\
\hline $\begin{array}{l}\text { E3200 } \\
\text { (Phase III) } \\
{\left[\begin{array}{lll}1 & 163]\end{array}\right.}\end{array}$ & $\begin{array}{l}\text { Fluoropyrimidne } \\
\text { and irinotecan } \\
(0 \% \mathrm{Bev})\end{array}$ & $\begin{array}{l}\text { FOFOX4 + } \\
\text { Bev } \\
\text { FOLFOX4 }\end{array}$ & $\begin{array}{l}286 \\
291\end{array}$ & $\begin{array}{l}22,7 \\
8.6\end{array}$ & $(<0.0001)$ & $\begin{array}{l}7.3 \\
4.7\end{array}$ & $\begin{array}{l}0.61 \\
(<0.0001)\end{array}$ & $\begin{array}{l}12.9 \\
10.8\end{array}$ & $\begin{array}{l}0.75 \\
(0.0011)\end{array}$ \\
\hline $\begin{array}{l}\text { TML } \\
\text { (Phase III) } \\
{\left[\begin{array}{lll}1 & 164]\end{array}\right.}\end{array}$ & $\begin{array}{l}\text { Chemotherapy } \\
\text { (100\% Bev) }\end{array}$ & $\begin{array}{l}\text { Chemo- } \\
\text { therapy + } \\
\text { Bev } \\
\text { Chemo- } \\
\text { therapy }\end{array}$ & $\begin{array}{l}409 \\
411\end{array}$ & 5 & (n.s) & 5.7 & $\begin{array}{l}0.68 \\
(<0.0001)\end{array}$ & $\begin{array}{l}11.2 \\
9.8\end{array}$ & $\begin{array}{l}0.81 \\
(0.0062)\end{array}$ \\
\hline $\begin{array}{l}\text { BEBYP } \\
\text { (Phase III) } \\
{\left[\begin{array}{ll}1165]\end{array}\right.}\end{array}$ & $\begin{array}{l}\text { Chemotherapy } \\
\text { (100\% Bev) }\end{array}$ & $\begin{array}{l}\text { Chemo- } \\
\text { therapy }+ \\
\text { Bev } \\
\text { Chemo- } \\
\text { therapy }\end{array}$ & 92 & $\begin{array}{l}21 \\
17\end{array}$ & $(0.573)$ & 5.0 & $\begin{array}{l}0.70 \\
(0.010)\end{array}$ & $\begin{array}{l}15.5 \\
14.1\end{array}$ & $\begin{array}{l}0.77 \\
(0.043)\end{array}$ \\
\hline $\begin{array}{l}\text { Chinese } \\
\text { (Phase II) } \\
{\left[\begin{array}{lll}1 & 166]\end{array}\right.}\end{array}$ & $\begin{array}{l}\text { Oxaliplatin- } \\
\text { based (0\% Bev) }\end{array}$ & $\begin{array}{l}\text { FOLFIRI + } \\
\text { BEV } \\
\text { FOLFIRI }\end{array}$ & $\begin{array}{l}65 \\
77\end{array}$ & $\begin{array}{l}47.7 \\
28.5\end{array}$ & $(<0.001)$ & $\begin{array}{l}8.5 \\
5.1\end{array}$ & NR & $\begin{array}{l}15.2 \\
11.3\end{array}$ & NR \\
\hline 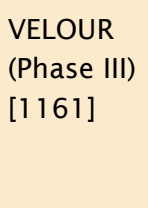 & $\begin{array}{l}\text { Oxaliplatin- } \\
\text { based }(30.4 \% \\
\text { Bev) }\end{array}$ & $\begin{array}{l}\text { FOLFIR + } \\
\text { Afliberce } \\
\text { pt } \\
\text { FOLFIRI + } \\
\text { Placebo }\end{array}$ & $\begin{array}{l}612 \\
614\end{array}$ & $\begin{array}{l}19.8 \\
11.1\end{array}$ & $(0.0001)$ & $\begin{array}{l}6.9 \\
4.7\end{array}$ & $\begin{array}{l}0.76 \\
(<0.0001)\end{array}$ & $\begin{array}{l}13.5 \\
12.1\end{array}$ & $\begin{array}{l}0.82 \\
(0.0032)\end{array}$ \\
\hline $\begin{array}{l}\text { RAISE } \\
\text { (Phase III) } \\
{\left[\begin{array}{lll}1 & 162]\end{array}\right.}\end{array}$ & $\begin{array}{l}\text { Fluoropyrimidine } \\
\text { and oxaliplatin } \\
(100 \% \mathrm{Bev})\end{array}$ & $\begin{array}{l}\text { FOLFIRI + } \\
\text { Ramu- } \\
\text { cirumab } \\
\text { FOLFIRI + } \\
\text { Placebo }\end{array}$ & $\begin{array}{l}536 \\
536\end{array}$ & $\begin{array}{l}13.4 \\
12.5\end{array}$ & $(0.63)$ & 4.5 & $\begin{array}{l}0.79 \\
(0.0005)\end{array}$ & $\begin{array}{l}13.3 \\
11.7\end{array}$ & $\begin{array}{l}0.84 \\
(0.0219)\end{array}$ \\
\hline
\end{tabular}

\subsubsection{Zweitlinientherapie mit anti-EGFR Substanzen}

Randomisierte Studien belegen den Nutzen von Panitumumab und Cetuximab in der Zweitlinientherapie. Diese Medikamente können daher entsprechend ihrer Zulassung nach Durchlaufen der Erstlinientherapie eingesetzt werden.

Zwei randomisierte Studien (EPIC und 181-Studie) belegen die Effektivität der anti-EGFR Substanzen Cetuximab und Panitumumab in der. Beide Studien zeigen eine signifikante Steigerung von ORR und PFS, wenn anti-EGFR Substanzen in der Zweitlinientherapie zu einer FOLFIRI-Chemotherapie hinzugefügt werden. Allerdings wurde in keiner der Studien ein signifikanter Überlebensgewinn erreicht (Tabelle 18).

Eine typische Nebenwirkung der anti-EGFR Therapie ist das akneiforme Exanthem, welches z.B. in der EPIC-Studie in einer Gesamthäufigkeit von 81,2\% und einer Grad 3-4 
Inzidenz von $8,2 \%$ auftrat. Auch bei Gabe von anti-EGFR-Substanzen wird eine Steigerung der chemotherapieassoziierten Toxizität z. B. der Diarrhoe (28,4 \% vs 15,7 \%) beobachtet [1167]. In der EPIC-Studie war die Gabe von Cetuximab mit einer signifikanten Steigerung des "global health" Scores verbunden. Kritisch muss allerdings angemerkt werden, dass die üblichen Instrumente zur Analyse der Lebensqualität, wie der EORTC QLQ-C30 Fragebogen, keinen exanthemrelvanten Score beinhalten und daher nicht geeignet sind, diesbezügliche Bewertungen zu untersuchen [1167].

Tabelle 18: Randomisierte Studien zur Zweitlinientherapie mit anti-EGFR Substanzen

\begin{tabular}{|c|c|c|c|c|c|c|c|c|c|}
\hline Studie & $\begin{array}{l}\text { Vor- } \\
\text { behandlung }\end{array}$ & Regime & $\begin{array}{l}\text { N } \\
\text { Pat. }\end{array}$ & ORR & $\begin{array}{l}\text { OR } \\
\text { (P-Wert) }\end{array}$ & PFS & $\begin{array}{l}\text { HR PFS } \\
\text { (P) }\end{array}$ & OS & $\begin{array}{l}\text { HR OS } \\
\text { (P) }\end{array}$ \\
\hline $\begin{array}{l}\text { EPIC } \\
{\left[\begin{array}{lll}1 & 167\end{array}\right]}\end{array}$ & $\begin{array}{l}\text { Fluoropyrimidin + } \\
\text { Oxaliplatin }\end{array}$ & $\begin{array}{l}\text { FOLFIRI + } \\
\text { Cet } \\
\text { FOLFIRI }\end{array}$ & $\begin{array}{l}648 \\
650\end{array}$ & $\begin{array}{l}16.4 \\
4.2\end{array}$ & $\begin{array}{l}\text { NR } \\
(<0.0001)\end{array}$ & $\begin{array}{l}4.0 \\
2.6\end{array}$ & $\begin{array}{l}0.692 \\
(<0.0001)\end{array}$ & $\begin{array}{l}10.7 \\
10.0\end{array}$ & $\begin{array}{l}0.975 \\
(0.71)\end{array}$ \\
\hline $\begin{array}{l}191^{* * *} \\
{[1168]}\end{array}$ & $\begin{array}{l}\text { Fluoropyrimidin- } \\
\text { basierte Therapie } \\
\text { (66\% Oxaliplatin } \\
19 \% \mathrm{Bev})\end{array}$ & $\begin{array}{l}\text { FOLFIRI + } \\
\text { Pani } \\
\text { FOLFIRI }\end{array}$ & $\begin{array}{l}303 \\
294\end{array}$ & $\begin{array}{l}35 \\
10\end{array}$ & $(<0.001)$ & $\begin{array}{l}5.9 \\
3.9\end{array}$ & $\begin{array}{l}0.73 \\
(0.004)\end{array}$ & $\begin{array}{l}14.5 \\
12.5\end{array}$ & $\begin{array}{l}0.85 \\
(0.12)\end{array}$ \\
\hline
\end{tabular}




\subsection{Therapiesequenz}

\begin{tabular}{|c|c|}
\hline 9.34 . & Evidenzbasierte Empfehlung \\
\hline $\begin{array}{l}\text { Empfehlungsgrad } \\
\text { B }\end{array}$ & $\begin{array}{l}\text { Bei Patienten mit RAS Wildtyptumoren ist die Lokalisation des Primärtumors eine } \\
\text { wichtige Determinante in der Beurteilung der optimalen Therapiesequenz (siehe } \\
\text { 9.8.2.). Bei Patienten mit linksseitigem mKRK und RAS Wildtyp sollte in der } \\
\text { Erstlinientherapie ein anti-EGFR-AK in Kombination mit einer Chemotherapie zum } \\
\text { Einsatz kommen. In dieser Konstellation kommt eine anti-VEGF Therapie erst ab der } \\
\text { Zweitlinie in Betracht. }\end{array}$ \\
\hline \multirow[t]{2}{*}{$\begin{array}{l}\text { Level of Evidence } \\
\mathbf{2 b}\end{array}$} & Quellen: [1 130][1131] \\
\hline & Konsens \\
\hline
\end{tabular}

\begin{tabular}{|l|l|l|}
\hline $\mathbf{9 . 3 5 .}$ & Evidenzbasierte Empfehlung & \multicolumn{1}{|c|}{2017} \\
\hline \begin{tabular}{l|l} 
Empfehlungsgrad \\
$\mathbf{B}$
\end{tabular} & $\begin{array}{l}\text { Bei Patienten mit rechtseitigem mKRK und RAS Wildtyp sollten in der } \\
\text { Erstlinientherapie keine anti-EGFR-AK in Kombination mit einer Chemotherapie zum } \\
\text { Einsatz kommen. }\end{array}$ \\
\hline $\begin{array}{l}\text { Level of Evidence } \\
\mathbf{2} \mathbf{b}\end{array}$ & \begin{tabular}{l} 
Quellen: [1130][1131] \\
\hline
\end{tabular} & \\
\hline & Starker Konsens \\
\hline
\end{tabular}

\section{Hintergrund}

Abgesehen von der Lokalisation des Primärtumors (siehe 9.8.2) gibt es derzeit keine konkret belastbare Evidenz, die eine optimale Sequenz molekularbiologisch gezielter Substanzen zwingend belegt. Die bisher verfügbaren Daten kommen überwiegend (I) von theoretischen molekularbiologischen Abhandlungen / Erwägungen, (II) von mehr oder minder ungeplanten Erfassungen von Zweitlinientherapien nach Erstlinienrandomisation (FIRE-3, CALGB, PEAK) oder (III) von ebenso ungeplanten retrospektiven Erfassungen der Erstliniensituation in randomisierten Zweitlinientherapien (TML, VELOUR; PRIME, PEAK).

\subsubsection{Fortführung der anti-VEGF Therapie in der Zweitlinienbehandlung}

Die klinischen Daten der TML-Studie belegen, dass bei unselektierten mKRK Patienten die Fortführung einer anti-VEGF-Therapie mit Bevacizumab nach Progression unter einer Bevacizumab-basierten Erstlinientherapie eine effektive Behandlungsstrategie darstellt. Patienten, die in der Zweitlinientherapie Bevacizumab plus Chemotherapie erhielten, zeigten im Vergleich zur alleinigen Chemotherapie ein längeres medianes Gesamtüberleben 11,2 Monate $(95 \% \mathrm{Cl}, 10,4-12,2)$ für Bevacizumab plus Chemotherapie und 9,8 Monate $(95 \% \mathrm{Cl}, 8,9-10,7)$ für Chemotherapie allein (HR 0.81, $\mathrm{p}=0.0062)$ [1 164]. 
Die häufigsten, in der TML-Studie berichteten, Grad 3-5 Nebenwirkungen waren Neutropenie (16\% vs 13\%), Diarrhoe (10\% vs $8 \%$ ) und Asthenie (6\% vs $4 \%$ ). Unter der Behandlung mit Bevacizumab plus Chemotherapie traten im Vergleich zur alleinigen Behandlung mit Chemotherapie folgende Grad 3-5 Ereignisse häufiger auf: Blutungen/Hämorrhagie ( $2 \%$ vs $<1 \%$ ), gastrointestinale Perforationen $(2 \%$ vs $<1 \%$ ) und venöse thromboembolische Ereignisse (5\% vs 3\%).

\subsubsection{Fortführung der anti-EGFR Therapie in der Zweitlinienbehandlung}

Die CAPRI-GOIM-Studie untersuchte KRAS-Wildtyp mKRK Patienten, die nach einer Erstlinientherapie mit FOLFIRI plus Cetuximab im randomisierten Vergleich entweder FOLFOX plus Cetuximab oder nur FOLFOX erhielten. Die Fortführung der Behandlung mit Cetuximab über die Progression hinaus (experimenteller Arm) führte in der Gesamtgruppe der untersuchten Patienten zu einer nicht signifikanten Verlängerung des PFS (6,4 vs 4,5 Monate, $p=0,19)$. Dagegen wurde bei Patienten mit KRAS, NRAS, BRAF and PIK3CA Wildtyp Tumoren im experimentellen Arm eine signifikante Verlängerung des 2nd-line PFS (HR, 0,56, p=0,025) beschrieben, für das Gesamtüberleben wurde bei kleiner Fallzahl $(n=66)$ das Signifikanzniveuau nicht erreicht $(H R, 0,57, p=0,056)$ [1 169].

\subsubsection{Sequenzieller Einsatz von anti-EGFR und anti-VEGF-Therapie}

Retrospektive klinische Untersuchungen weisen darauf hin, dass eine anti-EGFR Therapie dann weniger wirksam ist, wenn ihr eine anti-VEGF-Therapie voranging ([1170]). Präklinische Daten stützen diese Hypothese [1 1171 ] [ 11172$])$.

In der FIRE-3 Studie konnte nach initialer anti-EGFR Therapie eine deutlich längere antiVEGF Therapie in der Zweitlinienbehandlung gezeigt werden als dies in der umgekehrten Sequenz der Fall war [1040]. Während die Kombination von Panitumumab mit einer Kombinationschemotherapie in der Erstlinientherapie (PEAK-Studie) deutlich effektiver war als die Bevacizumab-basierte Vergleichstherapie [1109], so konnte dieser Effekt in der Zweitlinientherapie (SPIRITT-Studie) nach Bevacizumab-Vorbehandlung nicht reproduziert werden [1173]. Vergleichbare Daten wurden auch in der Prodige 18 UNICANCER GI Studie erhoben, die KRAS-wt mKRK Patienten nach Progression unter einer Bevacizumab-baiserten Chemotherapie untersuchte. Die Fortführung von Bevacizumab in Kombination mit einer "crossover" Chemotherapie war mit einem (nicht statistisch signifikant) längeren medianen PFS und OS verbunden als die Behandlung mit Cetuximab plus Chemotherapie [1174]. Bisher sind die Ergebnisse dieser Studie allerdings nur in Abstractform verfügbar.

Während die verfügbaren Daten die Sequenz einer anti-VEGF- gefolgt von einer antiEGFR-Therapie als eher ungünstig erscheinen lassen, so steht eine letztendliche Bewertung, die auch die Tumorlokalisation mit einbezieht, noch aus.

Die Daten der 181-Studie (FOLFIRI +/- Panitumumab) sprechen dafür, dass die anti-EGFR Therapie in der Zweitlinienbehandlung bei linksseitigen Primärtumoren effektiver ist als bei rechtsseitigen [1175]. Dies drückt sich bei linksseitigen RAS-Wildtyp Tumoren in günstigeren Effektivitätsparametern hinsichtlich ORR (50\% vs $13 \%$ ), PFS (8,0 vs 4,8 Monate) und OS (20,1 vs 10,3 Monate) aus.

\subsection{Chemotherapie in späteren Therapielinien}

Die therapeutische Aktivität von anti-EGFR Antikörpern, sowie von Trifluridin/Tipiracil und Regorafenib wurde in randomisierten Placebo-kontrollierten Studien untersucht, die bei intensiv vorbehandelten Patienten nach Durchlaufen der Standardtherapien durchgeführt wurden. 


\subsubsection{Effektivität von anti-EGFR Antikörpern}

Zwei große randomisierten Studien verglichen eine Behandlung mit "best supportive care" (BSC) plus anti-EGFR AK versus BSC allein bei Patienten mit chemotherapierefraktärem mKRK [1 176], [1 177]. Darüber hinaus zeigten Cetuximab und Panitumumab im direkten head-to-head Vergleich vergleichbare Effektivitäts- und Toxizitätsdaten [1 178]. Bei hinsichtlich des RAS-Status unselektierten Patienten führte die Zugabe von Panitumumab zu BSC zu einer signifikanten Verbesserung der Ansprechrate ( $10 \%$ vs $0 \%$ ) und des PFS (HR 0,54, $p<0,0001$ ). Allerdings wurde keine Verlängerung des Überlebens erreicht (HR 1,00), was durch den hohen Anteil von "cross-over" Patienten (76\%) erklärt wurde [1176].

Eine weitere Studie evaluierte die Zugabe von Cetuximab zu BSC [1177]. In einer retrospektiven Analyse von Patienten mit KRAS Wildtyp Tumoren führte die Zugabe von Cetuximab zu einer ORR von $12,8 \%$ vs $0 \%$, sowie einer signifikanten Verlängerung von PFS ( 3,7 vs 1,9 Monate; HR 0,40, p<0.001) und OS (9,5 vs 4,8 Monate; HR 0,55, p<0.001). Dagegen wurde in der Subgruppe der Patienten mit KRAS-mutierten Tumoren kein Nutzen beobachtet [1049]. Die Lebensqualität wurde in der Cetuximab-Gruppe bei geringerer Verschlechterung der "physical function-" und "global health status" Scores (both $\mathrm{P}<0.05$ ) signifikant länger aufrechterhalten [1 177]. Beim Vergleich von Cetuximab mit BSC allein wurden folgende Grad 3-4 Nebenwirkungen beobachtet: Infusionsreaktionen ( $4,5 \%$ vs $0 \%)$, Rash ( $11,8 \%$ vs $0,4 \%)$, Hypomagnesiämie $(5,2 \%$ vs $0 \%)$ [1 1177$])$.

\subsubsection{Effektivität von Trifluridin/Tipiracil}

\begin{tabular}{|l|l|}
\hline $\mathbf{9 . 3 6 .}$ & Evidenzbasierte Empfehlung \\
\hline $\begin{array}{l}\text { Empfehlungsgrad } \\
\mathbf{B}\end{array}$ & $\begin{array}{l}\text { Trifluridin/Tipiracil sollte bei Patienten, welche alle verfügbaren Chemotherapien/ } \\
\text { Antikörper durchlaufen haben oder für diese nicht geeignet sind, eingesetzt werden. }\end{array}$ \\
\hline $\begin{array}{l}\text { Level of Evidence } \\
\mathbf{1} \mathbf{D}\end{array}$ & \begin{tabular}{l} 
Quellen: $[1179,1180]$ \\
\hline
\end{tabular} \\
\hline
\end{tabular}

\section{Hintergrund}

Trifluridin/Tipiracil (TAS102) wurde in zwei randomisierten Studien (Phase II und Phase III) bei vorbehandelten KRK Patienten untersucht und gegen Pacebo verglichen [1180], [1179]. Einschlusskriterium war die Vorbehandlung mit mindestens zwei vorangegangenen Chemotherapieregimen, mit monoklonalen Antikörpern. Beide Studien zeigten eine Verlängerung des Gesamtüberlebens (OS 1.8 bzw. 2.4 Monate). In der Phase III Studie lag die Hazard Ratio für die Verbesserung des Gesamtüberlebens bei 0.68 $(95 \% \mathrm{Cl}$ 0.58-0.81, p<0.001). Die Therapie war auch wirksam bei Patienten, die vorher FU-Therapie erhalten hatten. Die Nebenwirkungsrate wurde als moderat eingestuft. Im Vordergrund stehen hämatologische Grad 3-4 Nebenwirkungen wie: Neutropenie (38\% vs $0 \%$ ), febrile Neutropenie ( $4 \%$ vs $0 \%$ ), Anämie ( $18 \%$ vs $3 \%$ ) und Thrombozytopenie ( $5 \%$ vs. $<1 \%)$. 


\subsubsection{Regorafenib}

\begin{tabular}{|l|l|}
\hline $\mathbf{9 . 3 7 .}$ & Evidenzbasierte Empfehlung \\
\hline $\begin{array}{l}\text { Empfehlungsgrad } \\
\mathbf{0}\end{array}$ & $\begin{array}{l}\text { Regorafenib kann bei mit allen verfügbaren Chemotherapien/Antikörpern vorbehan- } \\
\text { delten Patienten eingesetzt werden. }\end{array}$ \\
\hline $\begin{array}{l}\text { Level of Evidence } \\
\mathbf{1} \mathbf{0}\end{array}$ & Quellen: $[1181,1182]$ \\
\hline & Konsens \\
\hline
\end{tabular}

\section{Hintergrund}

Regorafenib ist ein Multikinaseinhibitor, der im Rahmen der CORRECT-Studie bei vorbehandelten mKRK Patienten gegen Placebo getestet wurde [1182]. Die Studie erreichte ihren primären Endpunkt und zeigte einen signifikanten Überlebensvorteil für Regorafenib (OS: 6,4 vs 5,0 Monate; HR, 0,77; 95\% Cl 0,64-0,94). Diese Ergebnisse wurden durch die CONCUR Studie, die ausschließlich bei asiatischen Patienten durchgeführt wurde, bestätigt [ 11181 ]. Der Einsatz von Regorafenib wurde aufgrund der behandlungsassoziierten Toxizität kritisch bewertet. Zu den wichtigsten Grad 3-4 Nebenwirkungen gehören Hand-Fuß-Syndrom (17\% vs <1\%), Fatigue (10\% vs 5\%) und Diarrhoe (7\% vs 1\%) [1 182].

\subsubsection{Re-Induktion / Re-,,challenge“}

Die Reinduktion von antineoplastischen Substanzen, deren Effektivität in frühen Therapielinien nachgewiesen wurde, ist zwar eine in der Onkologie bewährte Therapiestrategie, Nachweise für die klinische Effektivität dieses Vorgehens sind jedoch limitiert.

Santini und Mitarbeiter untersuchten im Rahmen einer kleinen Studie $(n=39)$ die Effektivität einer „Re-challenge“ mit Cetuximab. Patienten, die auf eine Cetuximabbasierte Erstlinientherapie mit einer CR, PR oder SD > 6 Monate angesprochen hatten und unter einer Cetuximab-freien „window therapy“ progredient waren konnte mit einer erneuten Cetuximab-basierten Therapie eine ORR von 54\% und ein PFS von 6.6 Monaten erreicht werden [1 183]. Aufgrund der sehr kleinen Fallzahl der Studie können diese Daten derzeit nur als hypothesengenerierend betrachtet werden.

\subsubsection{Weitere Medikamente}

Weitere Medikamente, die nach Durchlaufen der Standardtherapien im Rahmen der Zulassung in Betracht kommen, sind derzeit nicht verfügbar. Mitomycin sollte aufgrund seiner vergleichsweisen geringen Wirksamkeit in diesem Setting nicht eingesetzt werden. 


\subsection{Lokal ablative Verfahren}

\begin{tabular}{|c|c|}
\hline 9.38. & Evidenzbasierte Empfehlung \\
\hline Empfehlungsgrad & $\begin{array}{l}\text { Lokal ablative Verfahren können eingesetzt werden, wenn nicht resektable } \\
\text { Metastasen vorliegen oder der Allgemeinzustand des Patienten eine Resektion nicht } \\
\text { zulässt, insbesondere nach vorangegangener Lebermetastasenresektion. }\end{array}$ \\
\hline \multirow{2}{*}{$\begin{array}{l}\text { Level of Evidence } \\
36\end{array}$} & Quellen: [1 $1184-11187]$ \\
\hline & Starker Konsens \\
\hline
\end{tabular}

\section{Hintergrund}

Primär werden lokal ablative Verfahren dann eingesetzt, wenn eine chirurgische Resektion technisch oder aufgrund patientenbezogener Faktoren nicht möglich ist. Lokal ablative Verfahren können aber auch in Kombination mit einer chirurgischen Resektion durchgeführt werden [1184-1186]. Grundsätzlich muss in diesem Zusammenhang festgehalten werden, dass die Evidenz zu diesem Thema limitiert ist und ausreichend große prospektive, randomisierte Studien fehlen.

Bei primär resektablen Lebermetastasen, die nach Größe und Lokalisation auf für lokal ablative Verfahren geeignet sind, sollen diese alternativ angeboten und im interdisziplinären Tumorboard mit einem in der Leberchirurgie erfahrenen Chirurgen sowie mit einem erfahrenen interventionellen Radiologen diskutiert werden. Die Indikationsstellung zu lokal ablativen Verfahren sollte in multidisziplinären Tumorkonferenzen erfolgen. Das beste Gesamtüberleben wird durch ein multimodales ggf. sequenzielles Therapiekonzept erreicht. Die Möglichkeit einer sekundären Resektabilität bzw. die Durchführbarkeit lokal ablativer Maßnahmen soll mittels regelhaft eingesetzter Folgeuntersuchungen durch regelmäßige, multidisziplinäre Tumorkonferenzen überprüft werden.

Im Rahmen der verfügbaren thermischen Verfahren werden die Radiofrequenzablation (RFA), die Mikrowellenablation oder hochkonformale, hypofraktionierte Bestrahlungen, wie die stereotaktische Bestrahlung oder [HDR]-Brachytherapie in Abwesenheit randomisierter Vergleichsstudien in ihrer Wirkung als vergleichbar betrachtet. Je nach Verfahren variiert aber die Indikationsstellung in Abhängigkeit von Lokalisation oder Gefäßnähe. Der potentielle Nutzen einer RFA (plus chirurgische) Behandlung zusätzlich zur Chemotherapie deutet sich in der CLOCC Studie trotz kleiner Fallzahl an [1187]. In dieser Studie wurde bei nicht resektabler Lebermetastasierung eine RFA (intraoperativ, laparoskopisch oder perkutan) zusätzlich zu einer Chemotherapie (6 Monate) mit einer alleinigen Chemotherapie verglichen. Diese Studie zeigte im RFA/Chemotherapie-Arm sowohl eine signifikante Verlängerung des PFS (9,9 vs 16,8 Monate; HR 0,57, p=0,005), sondern auch des Gesamtüberlebens (45,6 vs 40,5 Monate; HR 0,58, p=0,01).

Angesichts weiterhin fehlender prospektiv kontrollierter Studien kann eine LITT zur Behandlung von Lebermetastasen bei KRK außerhalb klinischer Studien nicht empfohlen werden. 


\subsubsection{Lokal ablative Verfahren zur Behandlung von Lebermetastasen}

\subsubsection{Thermoablation}

Die Thermoablation (RFA, MWA) ist wie die chirurgische Resektion kolorektaler Metastasen ein lokales Therapieverfahren. Der Vergleich mit der chirurgischen Resektion anhand der vorliegenden Datenlage ist aufgrund der unterschiedlichen Patientenselektion in seiner Aussagekraft stark limitiert. Lokal ablative Verfahren können eingesetzt werden, wenn nicht resektable Metastasen vorliegen, wenn der Allgemeinzustand des Patienten eine Resektion nicht zulässt, nach vorangegangener Leberresektion oder in Kombination mit der Resektion ([1184-1186]). Gillams und Mitarbeiter konnten nach perkutaner RFA irresektabler hepatischer kolorektaler Metastasen bei 309 systemisch vortherapierten Patienten in der Subgruppe von Patienten mit $<5$ Metastasen mit jeweils $<5 \mathrm{~cm}$ Durchmesser ein mittleres Überleben von 28 Monaten nachweisen ([ 1188$]$ ). In der Subgruppe von Patienten mit $>5$ Metastasen und mit Metastasen $>5 \mathrm{~cm}$ lag das mediane Überleben demgegenüber bei 14 Monaten. Neben der Anzahl und dem Durchmesser der hepatischen Metastasen hatte das Vorliegen extrahepatischer Metastasen einen signifikanten Einfluss auf das mediane Überleben (28 Monate bei ausschließlich hepatischen Metastasen vs. 14 Monate bei extrahepatischer Metasta-sierung). Zusammenfassend ist das Langzeitüberleben von Patienten bis zu 10 Jahre nach Thermoablation Oligolebermetastasen mittlerweile gut dokumentiert ([1 189], [1 190]).

Darüberhinaus liegen Kohortensstudien und retrospektive Analysen vor, die im Vergleich von Resektion und Thermoablation bei Metastasen bis 3-4 cm Durchmesser keinen Unterschied bzgl. des Überlebens erbrachten ([1191], [1192]), so dass bei entsprechender klinischer Konstellation die Thermoablation als Alternativverfahren zur Resektion angeboten werden kann. Eine aktuelle Metanalyse von einer internationalen Expertengruppe ([1193]) berichtet über vergleichbare Ergebnisse zwischen RFA und Chirurgie für kolorektale Metastasen bis $3 \mathrm{~cm}$ und in selektierten Fällen sogar bis zu einer Tumorgröße von $5 \mathrm{~cm}$, mit einem mittleren 5 Jahre Überleben von $31 \%$ trotz negativer Selektion bei meist nicht operablen Patienten. Eine retrospective Analyse von 2 prospektiv randomisierten EORTC-Studien zur lokalen Rezidivsrate nach Ablation + Chemotherapie (6\%) oder nach Resektion +/- Chemotherapie (5,5\%) von kolorektalen Lebermetastasen zeigte zwischen Resektion und Thermoablation ein vergleichbares Ergebnis für Metastasen bis zu 4 cm ([1 194]). Allenfalls sollte ein Sicherheitsabstand zwischen Metastase und induzierter Koagulation von $5 \mathrm{~mm}$ angestrebt werden ([1 195]).

In den letzten Jahren werden auch zunehmend Daten größerer Patientenkollektive und/oder mit längerem Nachsorgezeitraum verfügbar. Diese Studien unterstreichen u.a. die Vorteile der Ablation mit der Möglichkeit einer Wiederholung der Intervention. In einer Langzeit-Studie mit 99 Patienten mit kolorektalen Lebermetastasen gab es bei einer Nachbeobachtungszeit von über 10 Jahren keinen statistisch signifikanten Unterschied im Überleben zwischen der Gruppe ohne Rezidiv und der mit abladierbarem Rezidiv ([1196]). Eine Empfehlung kann dabei derzeit nur für die Radiofrequenz- und Mikrowellenablation (MWA) ausgesprochen werden. Erste Ergebnisse zeigen eine niedrigere lokale Rezidivrate bei der Behandlung von kolorektalen Lebermetastasen nahe zu großen Gefäßen mit der MWA in Vergleich zur RFA ([1 1 97]). Hervorzuheben sind dabei die Langzeitergebnisse einer prospektiv randomisierten Vergleichstudie zwischen Resektion kombiniert mit Thermoablation und Chemotherapie versus Chemotherapie alleine, bei denen sich nach 8 Jahren ein signifikanter Überlebensvorteil durch die Kombinationstherapie Resektion+RFA+Chemotherapie versus Chemotherapie allein 
zeigt $(\mathrm{HR}=0,58,95 \% \mathrm{Cl}: 0,38-0.88, \mathrm{p}=0,01) .3-$, 5- und 8-Jahren Überleben waren, $57 \%$, $43 \%$ und $36 \%$ für die Kombination SR+RFA+Chemotherapie versus 55\%, 30\% und 9\% für die Chemotherapie allein. Medianes Überleben war 45,6 Mo. (95\% Cl: 30.3 - 67.8) für die Kombinations-therapie vs. 40,5 Mo. (95\% Cl: 27.5 - 47.7) für die Chemotherapie Gruppe ([1 198]). Angesichts weiterhin fehlender prospektiv kontrollierter Studien kann eine LITT oder eine Elektroporation zur Behandlung von Lebermetastasen bei KRK außerhalb klinischer Studien nicht empfohlen werden.

\subsubsection{Weitere lokal wirksame interventionelle Verfahren}

Weitere lokale Verfahren für die Behandlung von Lebermetastasen mit noch geringeren (SBRT, Brachytherapie, Kryotherapie) oder fehlenden Evidenzen (Irreversible Elektroporation) sind die stereotaktische Bestrahlung (SBRT), die Brachytherapie, die Kryotherapie und die irreversible Elektroporation (IRE).

\subsection{SBRT}

Die SBRT ist ein Verfahren, das modernste Techniken der Bestrahlungapplikation und planung benötigt, die sog. „intensity modulated radiotherapy“ ([1199]). In der Literatur liegt das 2-Jahres-Überleben zwischen 32\% und 83\% ([1200], [1201]). Die aktuelle Literatur berichtet über ca. 250 Patienten mit kolorektalen Lebermetastasen. Studien mit 5-Jahres-Überleben mit größeren Patientenkohorten oder randomisierte Studien SBRT versus Resektion oder Thermoablation liegen für kolorektalen Lebermetastasen nicht vor.

\subsection{Brachytherapie}

Bei der Brachytherapie (interstitielle high-dose-rate (HDR)-Brachytherapie) wird im Nachladeverfahren eine quasi punktförmige 192Iridium-Strahlenquelle ferngesteuert durch einen vorher mittels CT oder MR-Führung eingebrachten Applikator innerhalb des Tumorgewebes platziert ([1 189]). In einer Studie mit 73 eingeschlossenen, extensiv systemisch und chirurgisch vorbehandelten Patienten mit insgesamt 199 kolorektalen Lebermetastasen konnte durch die HDR-Brachytherapie ein medianes Überleben von bis zu 23,4 Monaten erzielt werden. Durch einzeitige Applikation von $\geq 20$ Gray (Gy) können Lokalrezidive effektiv vermieden werden ([1202]). Langzeitergebnisse liegen für die irreversible Elektroporation, SBRT und Brachytherapie noch nicht vor.

\subsubsection{Behandlung von Lungenmetastasen}

Die Resektabilität von Lungenmetastasen und die lokale Behandlung mit den Möglichkeiten der Ablation (RFA, MWA) oder der Strahlentherapie (SBRT) sollen von einem multidisziplinären Tumorboard, in dem ein erfahrener Organchirurg (Thoraxchirurgie), ein erfahrener interventioneller Onkologe und ein Radioonkologe vertreten sind, entschieden werden. Gemäß Literatur von De Baere et al oder Vogl TJ et al. eignen sich Lungenmetastasen bis zu $3 \mathrm{~cm}$ mit einer Zahl von bis zu 3 Metastasen pro Lunge für die thermischen Ablationsverfahren. Die lokale Kontrolle lässt sich dabei in Abhängigkeit von der Technik in Werten zwischen 69,2\% und 88,3\% erzielen ([1203]; [1204]). Randomisierte Studien liegen nicht vor. Alternativ kann die stereotaktische BodyStrahlentherapie (SBRT) zum Einsatz kommen. Dabei sind jedoch technische Voraussetzungen zu berücksichtigen, wie die Intensity Modulated Radiotherapy (IMRT) oder auch eine Weiterentwicklung der IMRT mit der volumetric-modulated-arc-therapy (VMAT). Für die SBRT von Lungenmetastasen liegen keine randomisierten Studien vor bei noch gering publizierten Fallzahlen mit Risiken der radiogen-induzierten Strahlenpneumonitis und Einschränkung des funktions-tüchtigen Lungenvolumens. 


\subsection{Lokoregionär wirksame Verfahren}

Zu den lokoregionären Verfahren gehören die die selektive intraarterielle Radiotherapie (SIRT) oder die intraarterielle Chemotherapie der Leber (HAI, TACE).

\subsubsection{Selektive interne Radioembolisation (SIRT)}

\begin{tabular}{|l|l|}
\hline $\mathbf{9 . 3 9 .}$ & Evidenzbasierte Empfehlung \\
\hline $\begin{array}{l}\text { Empfehlungsgrad } \\
\mathbf{0}\end{array}$ & $\begin{array}{l}\text { Eine SIRT kann zur Behandlung von disseminierten Lebermetastasen bei KRK bei } \\
\text { solchen Patienten eingesetzt werden, für die keine andere gleichwertige Therapie- } \\
\text { option in Frage kommt. }\end{array}$ \\
\hline $\begin{array}{l}\text { Level of Evidence } \\
\mathbf{2} \mathbf{b}\end{array}$ & \begin{tabular}{l} 
Quellen: [1205] \\
\hline
\end{tabular} \\
\hline
\end{tabular}

\section{Hintergrund}

In vergleichsweise kleinen randomisierten Studien war die Kombination der SIRT (selektive intraarterielle Radioembolisation mit Yttrium-90 Harz-Mikrosphären) mit 5-FU effektiver als die Chemotherapie allein. Bei mehrfach vorbehandelten, „chemorefraktären" Patienten ( $n=44)$ induzierte die Hinzunahme der SIRT zu einer 5-FUMonotherapie im Vergleich zur alleinigen Behandlung mit 5-FU eine Verbesserung von Ansprechrate (10\% vs 0\%) und TTP (4.5 vs 2.1 Monate, $\mathrm{P}<0.03$ ) [1205].

In der Erstlinientherapie konnte bei 21 Patienten im randomisierten Vergleich von SIRT plus 5-FU versus 5-FU eine signifikante Steigerung von TTP (18.6 versus 3.6 Monate, $\mathrm{p}<0.0005)$ und OS (29.4 vs 12.8 Monate, $\mathrm{p}=0.025)$ erreicht werden ([1206]). Basierend auf den Daten einer Phase I Studie wurde die Kombination von SIRT mit einer FOLFOX Chemotherapie in 3 randomisierten Multicenterstudien (SIRFLOX, FOXFIRE und FOXFIRE global) untersucht [1207].

In der SIRFLOX Studie wurde das PFS (primärer Endpunkt) durch Zugabe von SIRT zu der FOLFOX-Chemotherapie in der Erstlinientherapie nicht verbessert [1208]. Die Analyse des Gesamtüberlebens erfolgt als primärer Endpunkt in der gemeinsamen Evaluation aller drei Studien unter dem Dach der FOXFIRE global Studie. Auf dem ASCO Kongress 2017 wurden diese Ergebnisse der FOXFIRE-global Studie erstmals präsentiert. In der Intent-to-treat population $(n=1103)$ wurde durch die Zugabe von SIRT zu einer Oxaliplatin/5-FU-basierten Erstlinienchemotherapie weder ein Vorteil im PFS noch im Gesamtüberleben gezeigt. Allerdings wurden durch die Behandlung mit SIRT zusätzliche Nebenwirkungen induziert [1209]. Auffallend ist in dieser Studien-population der hohe Anteil an synchroner hepatischer (87\%) und extrahepatischer Metastasierung (35\%), von $50-55$ \% Primärtumoren in situ, und von 36 \% ECOG 1 Patienten, was den geringen Effekt einer isoliert Leber-assozierten Therapie wie der SIRT auf das Gesamtüberleben mindestens zum Teil erklären dürfte. Die Zugabe von SIRT zu einer 5-FU/Oxaliplatin (+ Bevacizumab) -basierten Chemotherapie führte in der ITT-Population der FOXFIRE global Studie zu einer signifikanten Verbesserung der Responserate (Odds Ratio 1.52, $p=0.001$ ). Obwohl gleichfalls ein signifikanter Vorteil der SIRT-Therapie in Hinblick auf das hepatische Ansprechen beobachtet wurde, war die Resektionsrate in beiden Behandlungsarmen annähernd gleich (16\% vs 17\%, $p=0.669)$. Die sekundäre 
Resektabilität wurde darüber hinaus in der SIRFLOX-Studie durch ein Panel von hepatobiliären Chirurgen evaluiert. In dieser verblindeten Analyse der Bildgebung zum Zeitpunkt der "best response" wurde im SIRT-Arm eine signifikant höhere potentielle Resektabilität gefunden als im Kontrollarm (Garlipp et al. ASCO 2017) [1210].

\subsubsection{Hepatische intraarterielle Chemotherapie}

\subsubsection{Hepatisch intraarterielle Chemotherapie (HAI)}

$\mathrm{Zu}$ den lokoregionär wirksamen Verfahren gehört die hepatische intraarterielle Chemotherapie (HAl). Dieses Verfahren wurde erfolgreich an dafür spezialisierten Zentren eingesetzt [1211], [1212]. Wie eine aktuelle europäische Multicenter Phase II Studie (OPTILIV Studie; [1213]) zeigt, läßt sich in der 2. Linientherapie durch eine dosisintensivierte, intraarterielle Chemotherapie (HAI, Irinotecan, Oxaliplatin, 5-FU) Resektabiltät herstellen, mit einer 4-Jahres Überlebensrate von 37,4 \% unter Berücksichtigung von R0 und R1 Resektionen. Eine Einigung hinsichtlich einer Empfehlung zur Durchführung der HAl bei leberdominanter Metastasierung in dafür spezialisierten Zentren konnte nicht erreicht werden.

\subsubsection{Einsatz von Irinotecan-beladenen Microbeads}

Wenn auch die Datenlage überschaubar ist, so zeigte sich in einer prospektiv randomisierten Studie in der palliativen Situation bei Patienten mit Progress nach 2. und 3.Linien Chemotherapie ein verbessertes Überleben mit besserer Lebensqualität nach intraarterieller Applikation Irinotecan-beladener Partikeln ([1214]). Eine weitere prospektive randomisierte Studie berichtet über bessere Ansprechraten mit besserem progressionsfreien Überleben durch die Kombination von FOLFOX +/-Bevacizumab mit Irinotecan-beladenen Partikeln im Vergleich zur FOLFOX Gabe +/-Bevacizumab ([ $\left.\left.1 \begin{array}{llll}1 & 2 & 1 & 5\end{array}\right]\right)$.

\subsubsection{Hypertherme intraperitoneale Chemotherapie (HIPEC)}

\begin{tabular}{|c|c|}
\hline 9.40. & Evidenzbasierte Empfehlung \\
\hline $\begin{array}{l}\text { Empfehlungsgrad } \\
0\end{array}$ & 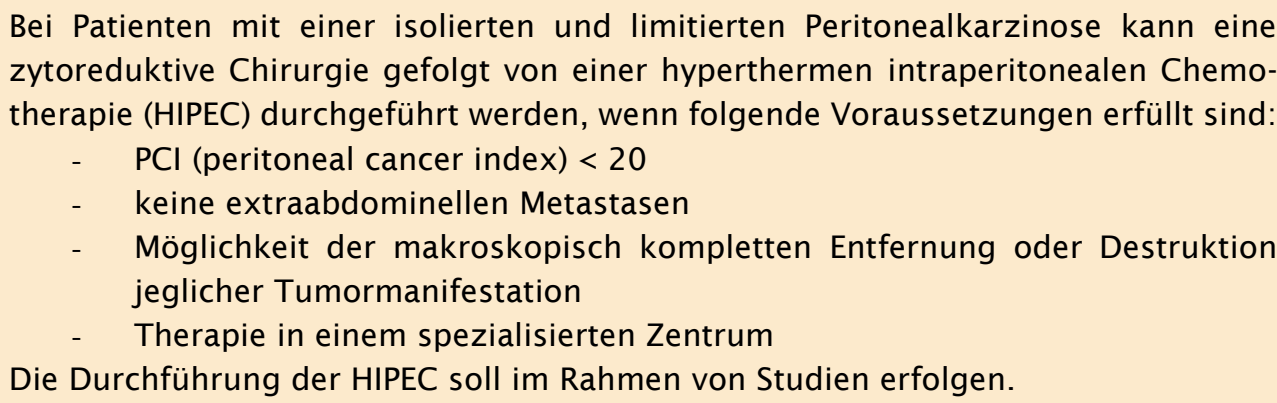 \\
\hline \multirow{2}{*}{$\begin{array}{l}\text { Level of Evidence } \\
4\end{array}$} & Quellen: $[785,786,1216-1218]$ \\
\hline & Konsens \\
\hline
\end{tabular}

\section{Hintergrund}

Die Durchführung einer HIPEC-Therapie ist aufgrund der damit verbundenen Morbidität an ausgewiesene „high-volume“ Zentren gebunden [1216]. Die Effektivität der 
zytoreduktiven Chirurgie ist insbesondere vom Ausmaß der Peritonealkarzinose abhängig. Entsprechend kommen für dieses Verfahren nur Patienten mit einer limitierten peritonealen Metastasierung in Betracht. Als Entscheidungshilfe dient der $\mathrm{PCl}$ (peritoneal cancer index) [1217]. Rezidive sind nach zytoreduktiver Therapie und intraperitonealer Chemotherapie häufig und gelten als ungünstige prognostische Faktoren [1218].

Die konkrete Wirksamkeit der HIPEC-Therapie wurde bisher nur in einer kleineren randomisierten Studie aus dem Jahr 2003 untersucht [785], [786]. Diese Studie schloss 105 Patienten ein und verglich eine Behandlung mit 5-FU/Leucovorin +/- chirurgische Resektion mit chirurgischer Zytoreduktion plus hyperthermer Chemotherapie. Das mediane Überleben war im experimentellen Behandlungsarm deutlich länger (22,3 vs. 12,6 Monate, $p=0,032$ ) als im Standardarm, allerdings war die Morbidität hoch und die Mortalität lag bei $8 \%$ ([785]). Zusätzlich muss einschränkend darauf hingewiesen werden, dass in dieser Studie weder Oxaliplatin noch Irinotecan, noch molekular-biologisch gezielte Substanzen (anti-EGFR oder anti-VEGF) zum Einsatz kamen.

Die Relevanz der HIPEC in der derzeitigen Therapielandschaft bleibt angesichts der limitierten, in prospektiven, kontrollierten Studien erhobenen Evidenz weitgehend unklar. Gleichfalls muss auch der Stellenwert der verschiedenen Komponenten der HIPEC Therapie (Zytoreduktion, Hyperthermie, intraperitoneale Chemotherapie, Wahl der chemotherapeutischen Agenzien) klar definiert werden. Entsprechend kann die Durchführung der HIPEC außerhalb von Studien nicht generell unterstützt werden.

\subsection{Therapiebegleitendes interprofessionelles Management von Symptomen, Nebenwirkungen und Toxizitäten}

\begin{tabular}{|l|l|}
\hline 9.41. & Konsensbasierte Empfehlung \\
\hline EK & $\begin{array}{l}\text { Unter Chemotherapie bei Metastasierung und in der palliativen Situation soll bei allen } \\
\text { Patienten ein regelmäßiges Assessment krankheits- und therapieinduzierter } \\
\text { Nebenwirkungen sowie eine gezielte Symptombehandlung durchgeführt werden. } \\
\text { Primäres Ziel ist eine Verlängerung des progressionsfreien Überlebens und des } \\
\text { Gesamtüberlebens bei geringer Toxizität und guter Lebensqualität. }\end{array}$ \\
& $\begin{array}{l}\text { Eine Anleitung der Patienten zu einem effektiven Selbst-Management der Symptome } \\
\text { soll regelmäßig durchgeführt werden. }\end{array}$ \\
& Konsens
\end{tabular}

\section{Hintergrund}

Bis zu 30\% aller mKRK-Patienten leiden an krankheits- und therapiebedingten Toxizitäten CTC Grad III-IV, insbesondere Diarrhoe, Übelkeit und Erbrechen, Mucositis/ Stomatitis, Obstipation und Neuropathien ([1219], [1220]). Zur Vermeidung von non-intentionalen Deeskalationen und Therapieabbrüchen soll das Auftreten bzw. die Aggravierung therapie- und krankheitsbedingter Symptome und Belastungen regelmäßig erfasst werden. Dazu liegen validierte Assessment-Instrumente wie der National Cancer Institute Common Toxicity Criteria Scale (NCI-CTC 4.0) vor. 
Insbesondere bei Patienten über 65 Jahre besteht ohne ein systematisches Monitoring ein hohes Risiko für endgültige Therapieabbrüche von bis zu $30 \%$ wegen anhaltend hoher Nebenwirkungen, verbunden mit einem reduzierten Gesamtüberleben [1221].

Studien belegen die Verbesserung der gesundheitsbezogenen Lebensqualität [1222], die Reduktion der Syptombelastung [1223] und die Vermeidung von Therapie-abbrüchen [1224] durch ein regelmäßiges Assessment von auftretenden Toxizitäten, Nebenwirkungen und unerwünschten Ereignissen.

Für deren Management wird auf die Leitlinie „Supportive Therapie bei onkologischen PatientInnen“, AWMF Registernummer: 032/054OL (http://www.leitlinienprogrammonkologie.de/leitlinien/supportive-therapie/) verwiesen. Die Symptom-Therapie soll zudem unter Einbeziehung der individuellen Patientenmerkmale (Alter, Geschlecht, Komorbidität, Symptombelastung) sowie der informierten Patientenentscheidung ausgewählt werden [1050].

Darüber hinaus liegt Evidenz für die Wirksamkeit eines effektiven Selbst-Assessment, Selbst-Management und der Fähigkeit einer angemessenen Kommunikation zur Reduktion und Vermeidung auftretender Symptome und Belastungen vor [1223], [1225]. Patienten soll daher regelmäßig eine Anleitung und Schulung zum Selbst-Management angeboten werden. 


\section{Nachsorge}

Nach Diagnose und Therapie eines kolorektalen Karzinoms ist unabhängig vom Tumorstadium eine adäquate ärztliche Betreuung sinnvoll. Nach kurativer Therapie besteht für 5 Jahre ein erhöhtes Risiko für ein lokales oder lokoregionäres Rezidiv (324\%), Fernmetastasen (25\%) oder einen metachronen Zweittumor (1,5-10\%) [1226-1235]. Das Risiko ist höher bei genetischer Veranlagung [1230] und bei fortge-schrittenen Tumorstadien [1236, 1237]. Hieraus leitet sich grundsätzlich die Begründung für eine Nachsorge dieser Patienten ab: Ein Rezidiv sollte so früh entdeckt werden, dass ein operativer Zweiteingriff in kurativer Absicht möglich ist. Objektive Ziele sind neben der Heilung oder Lebensverlängerung bei Rezidiv, der Nachweis von Zweittumoren sowie der Nachweis und die Beseitigung nicht Rezidiv bedingter Folgen der Tumorkrankheit oder der operativen Therapie. Subjektive Ziele der Nachsorge sind auf die Verbesserung der Lebensqualität des Patienten ausgerichtet [1238].

Ein zusätzliches Ziel stellt die Qualitätskontrolle der durchgeführten diagnostischen und therapeutischen Maßnahmen dar. Die Effektivität der Nachsorge scheint jedoch marginal mit im Mittel $1 \%$ verbessertem Überleben im Gesamtkollektiv der nach-gesorgten Patienten [1239]. Daten aus 267 Artikeln zu diesem Thema wurden in einer Metaanalyse ausgewertet [1240]. Um einem Patienten mit kolorektalem Karzinom ein Langzeitüberleben zu ermöglichen, wurden 360 positive Nachsorgeteste und 11 Zweitoperationen benötigt. Die übrigen 359 Nachsorgemaßnahmen und 10 Operationen waren entweder ohne therapeutischen Gewinn oder mit negativen Auswirkungen verbunden [1240].

In einem Cochrane Review von 2008 mit 8 eingeschlossenen Studien konnte gezeigt werden, dass eine intensive Nachsorge im Vergleich mit keiner oder einer minimalen Nachsorge mit einer geringeren Mortalität einhergeht (OR 0.73 ( $95 \% \mathrm{Cl} 0.59$ to 0.91$)$ ).

\subsection{Nachsorge bei Patienten im UICC-Stadium I}

\begin{tabular}{|l|l|c|}
\hline 10.1. & Konsensbasierte Empfehlung & 2013 \\
\hline EK & $\begin{array}{l}\text { Eine regelmäßige Nachsorge bei Patienten mit kolorektalem Karzinom und frühem } \\
\text { Tumorstadium (UICC I) ist nach RO-Resektion in Anbetracht der geringen Rezidivrate } \\
\text { und der günstigen Prognose nicht zu empfehlen. Eine rein koloskopische Nachsorge } \\
\text { soll entsprechend Kapitel 10.3.9.1 erfolgen. }\end{array}$
\end{tabular}

\section{Hintergrund}

Patienten im UICC Stadium I haben eine gute Prognose nach kurativer Resektion. Dabei erleiden häufiger Patienten mit pT2 Tumoren (UICC Ib) (13\%) als mit pT1-Tumoren (UICC la) (4\%) ein Rezidiv [1241]. Insgesamt ist das Langzeitüberleben im Stadium UICC I entsprechend dieser prospektiven Kohortenstudie mit $86 \%$ sehr gut und erlaubt einen weitgehenden Verzicht auf eine programmierte Nachsorge. Zu einem ähnlichen Ergebnis kam eine retrospektive Studie an 541 Patienten mit einem kolorektalen Karzinom im UICC Stadium 1, wobei pTINOMO (UICC la) Tumoren 2,9\% Rezidive, und pT2 Karzinome (UICC Ib) 5.6\% Rezidive aufwiesen [1242]. Im Stadium Dukes A wurden Rezidive nur bei Patienten mit Rektumkarzinom $(11 \%, n=6 / 55)$, nicht dagegen beim Kolonkarzinom beobachtet [1243]. 
Abweichend kann im Einzelfall nach leitliniengerechter Therapie bei Annahme eines hohen lokalen Rezidivrisikos aufgrund des endoskopischen oder intraoperativen Befundes (z. B. nach intraoperativer Tumoreröffnung) oder eines pathologischen Befundes (z. B. erhöhtes Risiko für Fernmetastasen bei Invasion perikolischer Venen [1244, 1245], angiolymphatischer Invasion [1246, 1247], G3/G4 Tumoren oder pT2Tumoren) eine engmaschigere Nachsorge angezeigt sein. Hierbei erwies sich die alleinige Bestimmung des CEA als ausreichend [1242].

Da Patienten auch im Stadium I ein erhöhtes Risiko für die Entwicklung metachroner Zweittumoren besitzen, ist eine koloskopische Nachsorge entsprechend Kapitel 0 sinnvoll.

\subsection{Nachsorge bei Patienten im UICC-Stadium II und III}

\begin{tabular}{|l|l|}
$\begin{array}{l}\text { 10.2. } \\
\text { Empfehlungsgrad } \\
\mathbf{A}\end{array}$ & $\begin{array}{l}\text { Nach R0-Resektion von kolorektalen Karzinomen des UICC-Stadiums II und III sind } \\
\text { regelmäßige Nachsorgeuntersuchungen (siehe Empfehlung 10.1.) indiziert. }\end{array}$ \\
\hline $\begin{array}{l}\text { Level of Evidence } \\
\mathbf{1}\end{array}$ & Quellen: [1239, 1240, 1248-1257] \\
\hline & Starker Konsens \\
\hline
\end{tabular}

\begin{tabular}{|l|l|l|}
\hline 10.3. & Konsensbasierte Empfehlung & 2008 \\
\hline EK & $\begin{array}{l}\text { Diese sollten jedoch nur durchgeführt werden, wenn bei einem Rezidiv } \\
\text { therapeutische Konsequenzen zu erwarten sind. }\end{array}$ \\
\hline Starker Konsens
\end{tabular}

\section{Hintergrund}

Bei fortgeschrittenem kolorektalen Karzinomen (UICC Stadium II und UICC Stadium III) ist das Rezidivrisiko wesentlich höher [1226-1237]. Zum Nutzen von Nachsorgemaßnahmen bei diesem Tumor gibt es 6 randomisierte kontrollierte Studien [12521257], von denen nur 2 einen positiven Effekt auf das 5-Jahresüberleben einer intensivierten Nachsorge im Vergleich zu einer "Standardnachsorge" nachweisen konnten [1255, 1257]. Fast alle Studien schlossen auch Patienten im UICC Stadium I ein.

Verschiedene Metaanalysen von 5 randomisierten und kontrollierten Studien (1 positive, 4 negative) [1239, 1240, 1248-1251] ergaben einen geringen Überlebens-vorteil für die Durchführung von mehr Maßnahmen in der Nachsorge gegenüber weniger Testen. Eine Bildgebung zur Beurteilung der Leber erwies sich ebenfalls in der Nachsorge als signifikant besser. Die Signifikanz ging jedoch verloren, wenn beide Ergebnisse nicht als Odds Ratio, sondern als Risikodifferenz berechnet wurden [1250]. Eine aktive Nachsorge führte nur zu einem marginalen Überlebensvorteil nach 5 Jahren von 0,5 bis 2\% [1239].

Eine kürzliche Cochrane Publikation [1258] schloss 3 weitere Studien in diese Metaanalyse ein $[1257,1259,1260]$. Es fand sich ein Überlebensvorteil für intensiver 
nachgesorgte Patienten nach 5 Jahren (OR 0,73; 95\% Cl 0,59-0,91), wobei aber die absolute Zahl an entdeckten Rezidiven in beiden Gruppen gleich war [30].

Eine retrospektive Studie untersuchte die Auswirkungen einer regelmäßigen Nachsorge entsprechend den ASCO-Leitlinien $(>70 \%$ der Nachsorgetermine wurden wahrgenommen) mit geringer (<70\%) und keiner Nachsorge [1261]. Bei regelmäßiger Nachsorge war im Vergleich zu den anderen Gruppen das 5 und 10-Jahres-Überleben statistisch signifikant besser. Dies gilt auch für die Prognose dieser Patienten bei einem Rezidiv. Auch ein psychologischer Nutzen ist für die betroffenen Patienten aus Nachsorgemaßnahmen abzuleiten [1262].

Leider wird den Leitlinien nicht immer gefolgt. So zeigte sich, dass bei Menschen jenseits des 65.Lebensjahres nur in 73,6\% der Fälle die empfohlenen Koloskopien und lediglich bei $46,7 \%$ eine Bestimmung des CEA erfolgte, während nicht empfohlene Maßnahmen wie CT und PET-CT bei $48 \%$ bzw. 7\% durchgeführt wurden [1263].

Aussagen zu Art und Häufigkeit der Nachsorgeuntersuchungen können nicht sicher gemacht werden, da hierzu gute Studien fehlen [1264-1266]. Eine Nachsorge adaptiert an die UICC Stadien oder die Auswirkungen eines kompletten Verzichtes auf eine Nachsorge wurden bisher in keiner prospektiven Studie geprüft.

Wegen der insgesamt unbefriedigenden Datenlage legte sich die Expertenkonferenz trotz eines Härtegrades 1 a bei Vorliegen von mehreren Metaanalysen lediglich auf den Empfehlungsgrad B für das programmierte Vorgehen in der Nachsorge des kolorektalen Karzinoms des UICC Stadiums II und III fest.

In 
Tabelle 19 ist das empfohlene Nachsorgschema bei Patienten mit einem kolorektalem Karzinom UICC II oder III dargestellt. 
Tabelle 19: Programmierte Untersuchungen im Rahmen der Nachsorge bei kolorektalem Karzinom UICC II oder III

\begin{tabular}{|c|c|c|c|c|c|c|c|c|c|c|c|c|}
\hline \multirow{2}{*}{\multicolumn{2}{|c|}{ Untersuchung }} & \multicolumn{11}{|c|}{ Monate } \\
\hline & & 3 & 6 & 9 & 12 & 15 & 18 & 21 & 24 & 36 & 48 & 60 \\
\hline \multicolumn{2}{|c|}{ Anamnese, körp.Untersuch., CEA } & & $X$ & & $X$ & & $X$ & & $X$ & $X$ & $X$ & $X$ \\
\hline \multicolumn{2}{|c|}{ Koloskopie } & & $X^{*}$ & & $X * *$ & & & & & & & $X^{* * *}$ \\
\hline \multicolumn{2}{|c|}{ Abdomensonografie $* * *$} & & $\mathrm{X}$ & & $\mathrm{X}$ & & $X$ & & $\mathrm{X}$ & $\mathrm{X}$ & $\mathrm{X}$ & $\mathrm{X}$ \\
\hline \multicolumn{2}{|c|}{ Sigmoidoskopie (Rektoskopie) $* * * *$} & & $X$ & & $X$ & & $X$ & & $X$ & & & \\
\hline \multicolumn{2}{|c|}{ Spiralcomputertomografie $x$} & $\mathrm{X}$ & & & & & & & & & & \\
\hline \multicolumn{2}{|c|}{ Röntgen Thorax ***** } & & & & $X$ & & & & $\mathrm{X}$ & $X$ & $X$ & $\mathrm{X}$ \\
\hline \multicolumn{13}{|c|}{ * $\quad$ wenn keine vollständige Koloskopie präoperativ erfolgt ist } \\
\hline$* * *$ & bei unauffälligem Befund (kein Ader & $\mathrm{m}, \mathrm{k}$ & Karz & nom & lächst & Kolosk & ie nac & $5 \mathrm{Jahr}$ & & & & \\
\hline \multirow{4}{*}{$\begin{array}{l}* * * * * \\
* * * * * * \\
x\end{array}$} & $\begin{array}{l}\text { eine Metaanalyse ergab einen Vorte } \\
\text { Nachsorge. Aus diesem Grund ents } \\
\text { anzuwenden. }\end{array}$ & $\begin{array}{l}\text { für e } \\
\text { ied s }\end{array}$ & bildg & spenc & $\begin{array}{l}5 \text { Verfa } \\
\text { nkomn }\end{array}$ & $\begin{array}{l}\text { en zu } \\
\text { sion, }\end{array}$ & $\begin{array}{l}\text { Nachv } \\
\text { is einf }\end{array}$ & $\begin{array}{l}\text { is von } \\
\text { hste } u\end{array}$ & berme & $\begin{array}{l}\text { staser } \\
\text { ünsti }\end{array}$ & der & Iren \\
\hline & nur beim Rektumkarzinom ohne ne & djuv & te od & adj & lante $\mathrm{R}$ & dioche & othera & & & & & \\
\hline & nur beim Rektumkarzinom kann jäh & ch e & Rön & en & oraxur & ersuch & ig dur & gefüh & werde & & & \\
\hline & $\begin{array}{l}\text { nur beim Rektumkarzinom } 3 \text { Monat } \\
\text { Strahlen-/Chemotherapie) als Ausga }\end{array}$ & $\begin{array}{l}\text { hach } \\
\text { gsbe }\end{array}$ & $\begin{array}{l}\text { sschlt } \\
\text { id. }\end{array}$ & $s \mathrm{~d}$ & tumor & ezifisc & en The & apie $(\mathrm{O}$ & ration & & vante & \\
\hline
\end{tabular}




\subsection{Wertigkeit diagnostischer Methoden in der Nachsorge}

Zu den Inhalten der Nachsorge werden folgende Empfehlungen gegeben:

\subsubsection{Anamneseerhebung}

\begin{tabular}{|l|l|}
\hline 10.4. & Konsensbasierte Empfehlung \\
\hline EK & $\begin{array}{l}\text { Eine symptomorientierte Anamneseerhebung und körperliche Untersuchung sind } \\
\text { grundsätzlicher Bestandteil der Nachsorge. }\end{array}$ \\
\hline & Starker Konsens
\end{tabular}

\section{Hintergrund}

Anamnese und körperliche Untersuchung tragen wenig zur frühzeitigen Entdeckung eines Rezidivs bei kolorektalem Karzinom bei. Dennoch sollten diese ärztlichen Basismaßnahmen vor jeder weiteren Untersuchung erfolgen [1265, 1267]. Hierfür sprachen sich alle Teilnehmer der Konsensuskonferenz aus.

\subsubsection{CEA Bestimmung}

\begin{tabular}{|l|l|}
\hline $\mathbf{1 0 . 5 .}$ & Evidenzbasierte Emp \\
\hline $\begin{array}{l}\text { Empfehlungsgrad } \\
\mathbf{B}\end{array}$ & $\begin{array}{l}\text { Die Bestimmung des k } \\
\text { mindestens 2 Jahre e } \\
\text { Diagnostik, berechtigt a } \\
\text { Verdacht auf ein metast }\end{array}$ \\
\hline $\begin{array}{l}\text { Level of Evidence } \\
\mathbf{4}\end{array}$ & Quellen: [1255, 1268, 1269] \\
\hline & Starker Konsens \\
\hline
\end{tabular}

\section{Hintergrund}

CEA erwies sich in der Nachsorge bei der frühzeitigen Entdeckung von Leber-metastasen besser als Koloskopie, Computertomografie und Sonografie [1255, 1268, 1269]. Eine Metaanalyse von 7 nicht randomisierten Studien zeigte einen Überlebensvorteil von 9\% für Patienten, bei denen im Nachsorgeprogramm CEA enthalten war [1248]. Andere Studien führten zu keinem oder nur einem minimalen Nutzen [1253, 1270]. CEA wird in der Nachsorge in einer Literaturübersicht nicht empfohlen [1266]. Amerikanische (ASCO) und europäische (EGTM, European Group on Tumour Markers) Leitlinien zur Nachsorge enthalten jedoch das CEA [1265, 1267, 1269], wobei die Bestimmung alle 2-3 Monate in den ersten 2 Jahren empfohlen wird.

Die adjuvante Therapie mit 5-Fluoruracil kann zu falsch erhöhten Werten führen, so dass ein ausreichender Abstand zu dieser Behandlung eingehalten werden sollte [1271]. 30\% aller kolorektalen Tumoren setzen CEA nicht frei [1271, 1272], während 44\% der Patienten mit normalen präoperativen Werten postoperativ einen Anstieg zeigen [1273]. Bei der weiteren Abklärung des erhöhten CEA-Wertes sind bildgebende Verfahren und 
gegebenenfalls 18-Fluorodeoxyglukose-Positronenemissionstomografie [1274, 1275] einzusetzen.

Wegen der insgesamt widersprüchlichen Daten für einen Einsatz von CEA in der Nachsorge kolorektaler Karzinome, wich die Expertenkonferenz von den ASCO- und EGTM-Empfehlungen ab und legte halbjährliche statt vierteljährliche Bestimmungsintervalle in den ersten 2 Jahren und danach jährlich über drei Jahre fest.

\subsubsection{Weitere Laborwerte}

\begin{tabular}{|l|l|}
\hline $\begin{array}{l}\text { 10.6. } \\
\text { Empfehlungsgrad }\end{array}$ & $\begin{array}{l}\text { Evidenzbasierte Empfehlung } \\
\text { Bweckmäßig. }\end{array}$ \\
\hline $\begin{array}{l}\text { Level of Evidence } \\
\mathbf{3} \mathbf{b}\end{array}$ & Quellen: $[1264,1267]$ \\
\hline & \\
\hline
\end{tabular}

\section{Hintergrund}

In zahlreichen Studien zur Nachsorge war die Bestimmung von Leberenzymen im Serum Bestandteil des Nachsorgeprogramms. Eine Studie zeigte jedoch, dass CEA und andere bildgebende Verfahren früher pathologisch ausfallen als die Leber-funktionsproben [1264]. Aus diesem Grunde wird von einer routinemäßigen Bestimmung dieser Serumparameter abgeraten. Ähnliches gilt für die Bestimmung eines Blutbildes [1267].

\subsubsection{FOBT}

\begin{tabular}{|l|l|}
\hline $\begin{array}{l}\text { 10.7. } \\
\text { Empfehlungsgrad }\end{array}$ & Die Untersuchung auf okkultes Blut im Stuhl ist für die Nachsorge nicht geeignet. \\
\hline $\mathbf{A}$ & \\
\hline $\begin{array}{l}\text { Level of Evidence } \\
\mathbf{3}\end{array}$ & Quellen: [1272, 1276] \\
\hline & Starker Konsens \\
\hline
\end{tabular}

\section{Hintergrund}

Die Untersuchung auf okkultes Blut im Stuhl ist für die Nachsorge nicht geeignet. Nur 12\% der lokalen Tumorrezidive führten zu einer oberflächlichen Verletzung der Schleimhaut [1272]. Reihenuntersuchungen bei 1.217 Patienten nach durchgemachtem kolorektalen Karzinom zeigten eine sehr niedrige Sensitivität und Spezifität dieses Testes für einen Rezidivtumor oder Polypen [1276]. 


\subsubsection{Sonografie}

10.8 .

Empfehlungsgrad

A

Level of Evidence

5

\section{Evidenzbasierte Empfehlung}

Die Sonografie ist zur Erkennung von Lebermetastasen technisch geeignet. Ihr Routineeinsatz ist aufgrund der Datenlage nicht gesichert. Die Expertenkommission bewertet die Sonografie als einfachstes und kostengünstigstes Verfahren und schlägt daher ihre Nutzung zur Diagnostik auf Lebermetastasen vor.

Quellen: [1250, 1252, 1266]

Starker Konsens

\section{Hintergrund}

Die Sensitivität der Sonografie zum Nachweis von Lebermetastasen schwankt in einem weitem Bereich zwischen 53\% und 82\% [1266]. Sie ist der Computertomografie in dieser Hinsicht in den meisten Studien unterlegen. In einer kontrollierten randomisierten Studie [1252] erwies sich der Einsatz von Sonografie und Computer-tomografie ohne Einfluss auf Überleben und Resektionsrate der nachgesorgten Patienten. Lediglich in einer Metaanalyse mehrerer randomisierter Studien stellte sich der Einsatz eines bildgebenden Verfahrens zur Beurteilung der Leber als statistisch signifikanter Überlebensvorteil dar [1250]. Erfolgte die Berechnung dieses Ergebnisses nicht als Odds Ratio, sondern als Risikodifferenz, so war dieser Vorteil nicht mehr nachweisbar [1250]. Da die Sonografie schneller und kostengünstiger als andere bildgebende Verfahren ist, wurde von den Teilnehmern der Konsensuskonferenz der abdominelle Ultraschall zur Entdeckung von Lebermetastasen in der Nachsorge empfohlen.

\subsubsection{Endosonografie}

\begin{tabular}{|l|l|}
$\begin{array}{l}\text { 10.9. } \\
\text { Empfehlungsgrad } \\
\mathbf{B}\end{array}$ & $\begin{array}{l}\text { Die Endosonografie (EUS) ist zur Erkennung von Lokalrezidiven beim } \\
\text { Rektumkarzinom insbesondere in Kombination mit der EUS-gesteuerten Biopsie } \\
\text { geeignet. Zum routinemäßigen primären Einsatz in der Nachsorge kann derzeit keine } \\
\text { Empfehlung gegeben werden. }\end{array}$ \\
\hline $\begin{array}{l}\text { Level of Evidence } \\
\mathbf{3} \mathbf{0}\end{array}$ & \begin{tabular}{l} 
Quellen: [1277] \\
\hline
\end{tabular} \\
\hline
\end{tabular}

\section{Hintergrund}

Endosonografische Nachsorgeuntersuchungen beim Rektumkarzinom erwiesen sich in einer Studie zur Erkennung von lokoregionären Rezidiven nach sphinkter-erhaltender Rektumresektion als hilfreich, wenn dieses Verfahren mit einer EUS-gesteuerten Biopsie kombiniert wurde [1277]. 68 perirektalen Läsionen in der EUS standen 36 tatsächliche Lokalrezidive bei 312 Patienten gegenüber. 12 Rezidive konnten mittels Proktoskopie erkannt werden. Bei 22 der endosonographisch gesehenen Läsionen war die Histologie 
positiv. Bei 41 Läsionen war sie negativ und bei 5 wurde kein verwertbares Ergebnis erhalten. Bei 18 der 68 Patienten beeinflusste die Endosonografie das weitere Vorgehen [1277]. Zur primären Diagnostik wird die EUS in der Nachsorge wegen der mit der Biopsie verbundenen Invasivität nicht empfohlen. Sie ist jedoch bei der weiteren Abklärung von mit anderen Methoden entdeckten lokoregionären Rezidiven des Rektumkarzinoms nützlich.

\subsubsection{Röntgen-Thorax Untersuchung}

\begin{tabular}{|l|l|}
\hline $\begin{array}{l}\mathbf{1 0 . 1 0 .} \\
\begin{array}{l}\text { Empfehlungsgrad } \\
\mathbf{0}\end{array}\end{array}$ & $\begin{array}{l}\text { Ein Röntgen-Thorax kann bei Patienten mit Rektumkarzinom im Stadium II und III bis } \\
\text { zum fünften Jahr jährlich durchgeführt werden. }\end{array}$ \\
\hline $\begin{array}{l}\text { Level of Evidence } \\
\mathbf{3} \mathbf{0}\end{array}$ & \begin{tabular}{l} 
de Novo: [1278] \\
\hline
\end{tabular} \\
\hline
\end{tabular}

\section{Hintergrund}

In einer Kohortenstudie aus Frankreich traten bei 5,8\% der Patienten innerhalb von 5 Jahren nach kurativer Resektion eines kolorektalen Karzinoms im Verlauf Lungenmetastasen auf [1278]. Die Rate an Lungenmetastasen nach 1 Jahr betrug 0,9\%, nach 3 Jahren 4,2\%. Das Risiko von Lungenmetastasen bei Patienten mit Rektumkarzinom war deutlich höher als bei Patienten mit Kolonkarzinom (OR 2,6 95Cl 1,65-4,75). Das 3Jahresüberleben bei Nachweis von metachronen Lungenmetastasen betrug 13,8\%. Nach kurativer Resektion dieser Metastasen betrug das relative 3-Jahresüberleben $59 \%$.

In einer systematischen Literatursuche zum Stellenwert der Durchführung des RöntgenThorax in der Nachsorge wurden 18 Studien zu diesem Thema identifiziert. Hierbei zeigte sich, dass in der Nachsorge durch einen Röntgen-Thorax bei 0,8 bis 7,0\% aller Patienten Lungenmetastasen bzw. zwischen 3,4 und 29,4\% aller Rezidive durch diese Bildgebung entdeckt wurden. Die Rate an kurativen Resektionen der detektierten Metastasen in diesen Studien betrug 0-100\%. Nähere Aussagen zu Einfluss von Tumorlokalisation und -stadium und idealer Abstand der Röntgenuntersuchungen sind aufgrund der Angaben in den Studien, der geringen Fallzahlen bzw. der Heterogenität der Studien nicht möglich.

Insgesamt scheint der Nutzen der Lungenbildgebung aufgrund der Daten von Mitry bei Patienten mit Rektumkarzinomen höher zu sein, so dass der Einsatz in der Nachsorge von Kolonkarzinompatienten aufgrund des unklaren Nutzens weiterhin nicht empfohlen werden kann. 


\subsubsection{Computertomografie}

\begin{tabular}{|l|l|}
$\begin{array}{l}\text { 10.11. } \\
\text { Empfehlungsgrad }\end{array}$ & $\begin{array}{l}\text { Evidenzbasierte Empfehlung } \\
\text { Die Computertomografie ist zur Erkennung von Lebermetastasen, von Lokalrezidiven } \\
\text { im kleinen Becken sowie von Lungenmetastasen technisch geeignet. Die Datenlage } \\
\text { spricht gegen einen routinemäßigen Einsatz der Computertomografie in der } \\
\text { Nachsorge. }\end{array}$ \\
\hline $\begin{array}{l}\text { Level of Evidence } \\
\mathbf{1}\end{array}$ & \begin{tabular}{l} 
Evidenz aus Aktualisierungsrecherche: $[1255,1256,1258,1279-1281]$ \\
\hline
\end{tabular} \\
\hline
\end{tabular}

\section{Hintergrund}

In einer randomisierten kontrollierten Studie zeigte der Einsatz der Computertomografie (CT) in der Nachsorge keinen Einfluss auf das Überleben der Patienten [1256]. Zwar wurden die Leberherde hierdurch etwas früher entdeckt (12 von 20 waren asymptomatisch), aber das CT steigerte nicht die Zahl kurativer Leberresektionen. CEA zeigte in einigen Studien ein Tumorrezidiv früher an als regelmäßige CT-Untersuchungen $[1255,1256,1281]$.

Eine Aktualisierung der ASCO Leitlinien im Jahr 2005 führte zur Empfehlung von jährlichen CT-Abdomen Untersuchungen über 3 Jahre [1279]. Die Kommission begründete seine Entscheidung für den Einsatz des Abdomen-CTs in der Nachsorge mit der Arbeit von Chau et al. [1280]. Bei 530 Patienten wurden retrospektiv 154 Tumorrezidive ausgewertet. Die Studie war ursprünglich als Therapiestudie und nicht zur Prüfung der Rolle des CT in der Nachsorge angelegt. 65 Rezidive wurden durch Symptome, 45 durch wiederholte CEA-Bestimmungen und 49 durch ein CT 12 und 24 Monate nach Beginn der adjuvanten Chemotherapie entdeckt. 14 dieser Patienten hatten auch ein erhöhtes CEA und wurden in beiden Gruppen aufgeführt. Häufigere Resektionen erfolgten in der CT-Gruppe $(n=13,26,5 \%)$ und in der CEA-Gruppe $(n=8,17,8 \%)$. In der symptomatischen Gruppe wurden 2 Patienten operiert (3,1\%). Diese Unterschiede waren signifikant. Die Wertigkeit der Sonographie im Vergleich zum CT wurde jedoch nicht untersucht. Auch neuere Metaanalysen lassen keinen Rückschluß auf die Art der einzusetzenden Verfahren zu [1258], so dass derzeit keine Empfehlung zu regelmäßigen CT-Untersuchungen bei asymptomatischen Patienten in der Nachsorge gemacht werden kann. 


\subsubsection{Endoskopische Verfahren}

\subsubsection{Koloskopie}

\begin{tabular}{|c|c|}
\hline 10.12. & Evidenzbasierte Empfehlung \\
\hline $\begin{array}{l}\text { Empfehlungsgrad } \\
\text { B }\end{array}$ & $\begin{array}{l}\text { Die Koloskopie ist geeignet, Lokalrezidive oder Zweittumoren zu erkennen. Alle } \\
\text { Patienten sollten prä- oder innerhalb von } 6 \text { Monaten postoperativ eine komplette } \\
\text { Koloskopie erhalten. } \\
\text { Eine Koloskopie sollte nach } 1 \text { Jahr und anschließend bei unauffälligem Befund alle } 5 \\
\text { Jahre erfolgen, um metachrone Karzinome oder Polypen zu erkennen. Ist die } \\
\text { komplette Koloskopie postoperativ im Zeitraum von } 6 \text { Monaten erfolgt, sollte die } \\
\text { nächste Koloskopie nach } 5 \text { Jahren durchgeführt werden. } \\
\text { Findet sich bei der Koloskopie nach } 6 \text { Monaten oder } 12 \text { Monaten eine Neoplasie, } \\
\text { sollte entsprechend den Empfehlungen } 6.15 \text {. bis } 6.21 \text {. verfahren werden. }\end{array}$ \\
\hline \multirow[t]{2}{*}{$\begin{array}{l}\text { Level of Evidence } \\
\mathbf{2 b}\end{array}$} & de Novo: [1251, 1282-1285] \\
\hline & \\
\hline
\end{tabular}

\subsubsection{Sigmoidoskopie}

\begin{tabular}{|l|l|}
$\begin{array}{l}\text { 10.13. } \\
\text { Empfehlungsgrad } \\
\mathbf{B}\end{array}$ & $\begin{array}{l}\text { Evidenzbasierte Empfehlung } \\
\text { Bereich zu erkennen. Zusätzliche Sigmoidoskopien sind nur beim Rektumkarzinom } \\
\text { vom UICC-Stadium II und III bei Patienten durchzuführen, die keine neoadjuvante oder } \\
\text { adjuvante Radiochemotherapie erhielten. }\end{array}$ \\
\hline $\begin{array}{l}\text { Level of Evidence } \\
\mathbf{4}\end{array}$ & \\
\hline & Starker Konsens \\
\hline
\end{tabular}




\subsubsection{Rektoskopie}

\begin{tabular}{|c|c|}
\hline 10.14. & Evidenzbasierte Empfehlung \\
\hline $\begin{array}{l}\text { Empfehlungsgrad } \\
\text { B }\end{array}$ & $\begin{array}{l}\text { Die starre Rektoskopie ist geeignet, beim Rektumkarzinom Lokalrezidive und } \\
\text { Anastomosenveränderungen zu erkennen. Sie kann als alternatives Verfahren zur } \\
\text { Sigmoidoskopie angewandt werden. }\end{array}$ \\
\hline \multicolumn{2}{|l|}{$\begin{array}{l}\text { Level of Evidence } \\
4\end{array}$} \\
\hline & Starker Konsens \\
\hline
\end{tabular}

\section{Hintergrund}

Ziel einer endoskopischen Nachsorge ist es, metachrone Neoplasien und Anastomosenrezidive in einem kurativem Zustand zu entdecken. Hierbei sollte bedacht werden, dass in einer Metaanalyse verschiedener randomisierter kontrollierter Studien gezeigt wurde, dass die koloskopische Nachsorge weniger effektiv als die Suche nach extramuralen Rezidiven ist [1251]. Ferner geht die Koloskopie mit Kosten und einer - wenn auch geringen - Komplikationsrate einher.

In den im Rahmen der Literatursuche identifizierten Artikeln (für Einzelheiten siehe Evidenzbericht) variierte die Rate an metachronen Karzinomen zwischen 0 und 6,4\% und die der Anastomosenrezidive zwischen 0 und 12\% innerhalb des Follow-ups (24 bis 94 Monate). In den Studien, in denen die entsprechenden Angaben vorhanden waren, betrug die errechnete jährliche Inzidenz metachroner Karzinome 0-2,3\%. Berücksichtigt man zusätzlich endoluminale Anastomosenrezidive betrug die jährliche Inzidenz von Karzinomen 0 bis 2,6\%. Die Rate fortgeschrittener Adenome, die im Rahmen der Nachsorge detektiert wurde, wurde nur in wenigen Studien angegeben und variierte zwischen 3,7 und 13\% innerhalb des Follow-ups.

Die Rate an kurativen Resektionen der metachronen Karzinome und / oder Anastomosenrezidive betrug 27 bis $100 \%$.

Die erforderliche Häufigkeit von Koloskopien in der Nachsorge wurde in einer Studie untersucht, die eine intensive koloskopische Nachsorge (im ersten Jahr alle 3 Monate, im 2. und 3. Jahr alle 6 Monate und danach jährlich) mit einem weniger intensiven Protokoll (nach 6, 30 und 60 Monaten) verglich [1282]. Rezidive wurden bei 8,1\% der intensiv nachgesorgten und bei $11,4 \%$ der weniger häufig koloskopierten Patienten gefunden. Während sich das Gesamtüberleben nicht unterschied, zeigte sich eine statistische Signifikanz für mehr asymptomatische Rezidive und eine bessere Prognose der erneut operierten Patienten. Die Häufigkeit von metachronen Tumoren wird in dieser Studie nicht angegeben. Die hohe Frequenz an Koloskopien wirkte sich nicht auf das Gesamtüberleben aus, zumal die meisten Rezidive extraluminal entstehen, so dass ein weniger belastendes Protokoll für die Patienten ausreichend effektiv ist.

In einer Studie wiesen 20 von 1.002 Patienten (3,1 \%) ein Zweitkarzinom auf, wovon 9 innerhalb von 18 Monaten entdeckt wurden [1283]. Eine fortgeschrittene Neoplasie (definiert als Adenom $>1 \mathrm{~cm}$, villöse Histologie, HGD oder Karzinom) fand sich häufiger $(15,5 \%)$ bei einer Koloskopie nach 36-60 Monaten im Vergleich zu einer frühzeitigeren Untersuchung innerhalb von 18 Monaten (6,9\%). Eine frühzeitigere Koloskopie war zwar nicht mit einem besseren Überleben in der Nachsorge assoziiert, die Autoren kommen 
aber zu dem Schluss, dass eine erste Koloskopie 12 Monate nach der Operation gemacht werden sollte, um möglicherweise bei der Indexuntersuchung übersehene metachrone Karzinome rechtzeitig zu entdecken. Aufgrund dieser Daten erfolgt jetzt die Empfehlung die erste Nachsorgekoloskopie 12 Monate nach dem Zeitpunkt der Operation durchzuführen.

Auch Rex et al. betonen, dass das primäre Ziel der Koloskopie in der Nachsorge weniger die Entdeckung eines seltenen Anastomosenrezidivsals das Auffinden metachroner Neoplasien ist [1284]. 2-7\% der Patienten wiesen mindestens einen metachronen Tumor auf. Inwieweit es sich um echte metachrone Läsionen handelte oder um übersehene synchrone Karzinome bei der ersten Koloskopie, kann anhand der Daten nicht entschieden werden. Dennoch empfehlen auch die Autoren dieser Leitlinie, eine erste Koloskopie 12 Monate nach Tumorresektion durchzuführen. Sie begründen diese Empfehlung mit einer Publikation aus dem Jahr 1993 eines Tumorregisters in Nebraska, das eine jährliche Inzidenz von metachronen Tumoren von 0,35\% ergab [1227]. Da ein lebenslanges Risiko für metachrone Tumoren besteht, ist eine dauerhafte koloskopische Nachsorge analog der Vorsorge auf ein Kolonkarzinom indiziert [1285].

\subsubsection{Kolonkontrasteinlauf, virtuelle Kolonografie und PET}

\begin{tabular}{|l|l|}
$\begin{array}{l}\mathbf{1 0 . 1 5 .} \\
\begin{array}{l}\text { Empfehlungsgrad } \\
\mathbf{B}\end{array}\end{array}$ & $\begin{array}{l}\text { Kolonkontrasteinlauf, virtuelle Kolonographie und PET, PET-CT und PET-MRT haben } \\
\text { in der programmierten Nachsorge keinen Stellenwert. }\end{array}$ \\
\hline $\begin{array}{l}\text { Level of Evidence } \\
\mathbf{4}\end{array}$ & \begin{tabular}{l} 
de Novo: $[194,1286,1287]$ \\
\hline
\end{tabular} \\
\hline
\end{tabular}

\section{Hintergrund}

Zum Einsatz der PET in der Nachsorge existieren nur wenige Daten. Die überwiegende Mehrzahl der Studien schließt nur Patienten mit V.a. Rezidiv ein und überprüft bei diesen die Wertigkeit der PET. Dieses Vorgehen war nicht Ziel der Literatursuche, sondern es ging um den Einsatz bei asymptomatischen Patienten ohne V.a. Rezidiv.

In einer randomisierten Studie [1286] mit sehr engmaschigen Nachsorgeuntersuchungen wurden durch eine PET nach 9 und 15 Monaten Rezidive früher erkannt und konnten häufiger R0-reseziert werden.

In einer prospektiv randomisierten Studie [194] sollte die Rolle der PET-CT in der Nachsorge des kolorektalen Karzinoms geklärt werden. Nach 9 und 15 Monaten erfolgte zusätzlich zu einer Nachsorge bestehend aus Abdomensonographie, Röntgen- Thorax, Tumormarkerbestimmung und Abdomen-CT im Vergleich zur Kontrollgruppe eine PETCT. Die Rezidivrate war in beiden Gruppen nicht unterschiedlich. Rezidive wurden in der PET-Gruppe im Mittel 3,2 Monate früher entdeckt. Zwar wurden häufiger Rezidivoperationen in der PET-CT Gruppe induziert (15 vs 2), eine Aussage zu einem besseren Überleben konnte aber nicht gemacht werden, da die Studie vorzeitig mit Erscheinen einer neuen PET-CT Generation abgebrochen wurde. Bedenklich ist auch, dass in drei Fällen falsch positive Befunde durch die PET-CT erhoben wurden, was zu unnötigen diagnostischen Maßnahmen oder operativen Eingriffen führte. 
In einer prospektive Fallserie von 31 Patienten [1287] wurde eine PET nach 2 Jahren durchgeführt. Die Patienten hatten zuvor regelmäßig CT/MRT erhalten und galten als rezidivfrei. Bei 6 Patienten war die PET positiv, bei 5 Patienten konnte ein Rezidiv im Verlauf gesichert werden. Es bleibt unklar, wie das Outcome dieser Pat. war. Insgesamt wurde die Datenlage durch die Teilnehmer als nicht ausreichend eingeschätzt, um die PET/PET-CT/PET-MRT-Untersuchung in die Routinenachsorge aufzunehmen.

Kolonkontrasteinlauf und die virtuelle Kolonographie wurden in der Nachsorge bisher nicht evaluiert und können die endoskopischen Verfahren in der Nachsorge nicht ersetzen.

\subsection{Zeitlicher Ablauf der Nachsorge}

\subsection{6.}

EK

\section{Konsensbasierte Empfehlung}

Der Beginn der Nachsorge errechnet sich ab dem Zeitpunkt der Operation.

Starker Konsens

\section{Hintergrund}

In den ersten beiden Jahren nach der Operation eines kolorektalen Karzinoms treten $80 \%$ der Rezidive auf, wobei nach 5 Jahren praktisch keine neuen Rezidive mehr festgestellt werden [1288]. Dies gilt auch für das Rektumkarzinom, obwohl bei dieser Tumorlokalisation auch später noch lokoregionäre Rezidive beobachtet wurden [1233]. Dies rechtfertigt jedoch nicht, die Nachsorge über das 5. postoperative Jahr hinaus auszudehnen.

In den meisten Studien wurden die Nachsorgeintervalle im 1. und 2. postoperativen Jahr mit 3 Monaten kürzer gehalten als in den darauffolgenden Jahren [1252, 1253, 1255, 1256]. 3-monatliche Intervalle erwiesen sich in einer Studie bei ansonsten gleichen Untersuchungsmethoden besser als Kontrollen in 6-monatlichen Abständen [1255]. Allerdings erhielten die Patienten in der 3-monatlich nachgesorgten Gruppe jährlich noch zusätzlich ein CT, das sich in einer anderen Studie in der Nachsorge jedoch als nutzlos erwies [1256]. Die Konsensuskonferenz entschied sich bei Fehlen eindeutiger Daten für Untersuchungsintervalle von 6 Monaten in den ersten 2 Jahren. Über den Zeitpunkt nach 5 Jahren sollten lediglich Koloskopien zum Ausschluss eines Zweitkarzinoms erfolgen.

\subsection{Altersbegrenzung der Nachsorge}

In kontrollierten Studien zur Nachsorge wurden Patienten bis 87 Jahre eingeschlossen [1252-1257]. Aus diesen Studien kann keine Altersbegrenzung abgeleitet werden. Sinnvollerweise sollten jedoch Operabilität, biologisches Alter, Begleiterkrankungen und der Wille, sich gegebenenfalls erneut operieren zu lassen, die Art und Dauer der Nachsorge bestimmen. 


\subsection{Sonderfälle}

Bzgl. der Nachsorge nach lokaler Abtragung von low-risk T1 Karzinomen wird auf die Empfehlung 6.15.verwiesen.

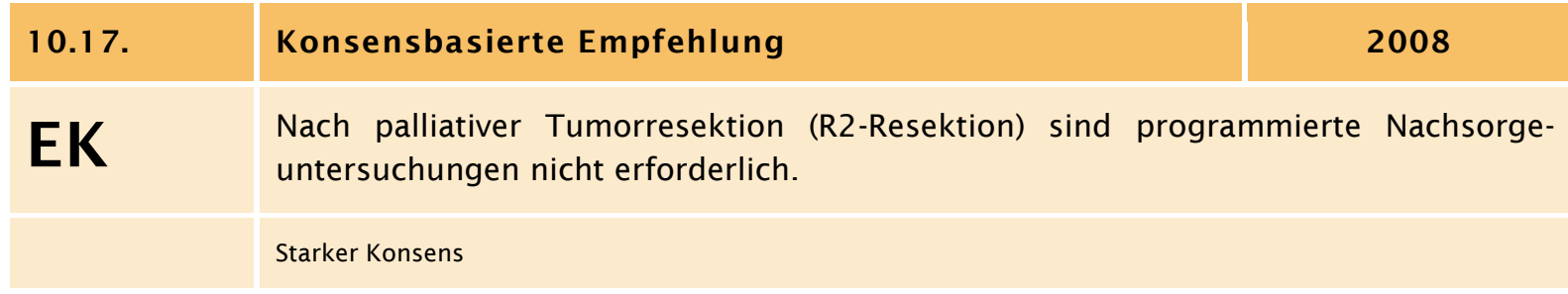

\begin{tabular}{|l|l|l|}
\hline 10.18. & Konsensbasierte Empfehlung & 2013 \\
\hline EK & $\begin{array}{l}\text { Patienten im Stadium IV nach kurativer Metastasentherapie sollten einer } \\
\text { programmierten Nachsorge unterworfen werden. }\end{array}$ \\
\hline & Starker Konsens
\end{tabular}

10.19.

Empfehlungsgrad

A

Level of Evidence

$2 \mathrm{a}$

\section{Evidenzbasierte Empfehlung}

Bei Patienten mit einem Karzinom bei HNPCC sind nach Hemikolektomie koloskopische Untersuchungen und nach subtotaler Kolektomie rektoskopische Untersuchungen in jährlichen Intervallen angezeigt (siehe auch Empfehlung 5.23.).

Starker Konsens 
10.20 .

Empfehlungsgrad

A

Level of Evidence

$2 \mathrm{a}$

\section{Evidenzbasierte Empfehlung}

Bei Patienten mit einem Kolonkarzinom bei familiärer Adenomatosis coli (FAP) sollten nach Anlage eines lleum-Pouches jährlich eine Pouchoskopie durchgeführt werden (siehe auch Empfehlung 5.35.).

Starker Konsens

10.21

Empfehlungsgrad

A

Level of Evidence

$2 \mathrm{a}$

\section{Evidenzbasierte Empfehlung}

Nach Ileorektostomie ist die Rektoskopie in halbjährlichen Abständen erforderlich (siehe auch Empfehlung 5.35.).

Starker Konsens

\subsection{Rehabilitation nach Resektion eines kolorektalen Karzinoms}

10.22 .

\section{EK}

Nach Abschluss der Primärtherapie sollte eine AHB allen rehabilitationsfähigen Patienten angeboten werden. Wenn die AHB unmittelbar postoperativ erfolgt, muss sichergestellt werden, dass eine indizierte adjuvante Chemotherapie zeitgerecht eingeleitet wird. Alternativ kann die AHB nach Abschluss der adjuvanten Chemotherapie erfolgen.

Starker Konsens

\section{Hintergrund}

Auftrag der Rehabilitation ist die möglichst weitgehende Beseitigung tumor- oder therapiebedingter Folgen sowie die Hilfestellung bei der Akzeptanz verbleibender Behinderungen mit dem Ziel einer selbstbestimmten Teilhabe am beruflichen, privaten und sozialen Leben. Zum Stellenwert rehabilitativer Maßnahmen bei Patienten mit kolorektalem Karzinom liegt keine auswertbare Literatur vor. Für die rehabilitative Maßnahme sind ausgewiesene Reha-Zentren bzw. Kliniken mit gastrointestinaler und onkologischer Expertise zu bevorzugen, die den Standards des Qualitätssicherungsverfahrens der DRV und Anforderungen von Darmzentren entsprechen. 
Der Rehabilitationsbedarf nach Behandlung von kolorektalen Tumoren ist äußerst variabel und im Wesentlichen abhängig von Art und Ausmaß des operativen Vorgehens sowie der Therapiefolgen (Kontinenzprobleme, sexuelle Funktionsstörungen, Stoma usw.).

Eine psychosoziale Beratung und gegebenenfalls Betreuung ist wünschenswert bei Problemen der psychischen Verarbeitung des Tumorleidens, bei Therapiefolgen, bei sozialen Anpassungsstörungen sowie bei der beruflichen Wiedereingliederung [1262, 1289, 1290].

Kontakte mit erfahrenen Gleichbetroffenen können gerade bei der psychischen Verarbeitung oder der Anpassung an eine veränderte Lebenssituation die wesentliche Hilfe für einen Neubetroffenen darstellen. Gleichbetroffene können durch ihr eigenes Beispiel sowie ihre Erfahrungen im Alltagsleben mit Krankheit und Behinderung glaubwürdig vermitteln, dass eine hohe Lebensqualität auch dann möglich ist. Deswegen sollten Kontaktaufnahmen oder Vermittlung zu Selbsthilfeorganisationen erfolgen.

Grundsätzlich sollten die Patienten in Abstimmung mit dem familiären Umfeld erst nach Abschluss der Primärtherapie in die Rehabilitation gehen. Falls die Rehabilitationsmaßnahme vor einer indizierten adjuvanten Chemotherapie erfolgen soll, muss die Durchführung der Chemotherapie während des Rehabilitations-verfahrens gewährleistet sein. Dazu werden neben dem Operationsbericht einschließlich der pathologischen Begutachtung (Tumorformel) auch die Entscheidung des Tumorboards benötigt und sollten daher der Rehabilitationseinrichtung zur Verfügung stehen.

\subsection{Tertiärprävention}

\subsubsection{Versorgungskontinuität und Fortführung der gesundheitsförderlichen Aktivitäten nach Akuttherapie und Rehabilitation}

Auch nach den Phasen der Akuttherapie und einer adjuvanten Chemotherapie sollten alle an der Behandlung und Nachversorgung beteiligten Berufsgruppen, wie z.B. niedergelassenen Ärzte, Pflegekräfte oder Physiotherapeuten die Patienten weiter beraten und begleiten.

\subsubsection{Verbesserung der Versorgungskontinuität}

Ein wichtiges Ziel sollte die Verbesserung der Versorgungskoordination sein, um die vorzeitige Beendigung oder den verzögerten Beginn des nachstationären Therapieverlaufs oder eine regionale Unterversorgung mit Versorgungslücken zu vermeiden. Das Übergangsmanagement von Patienten zwischen primärer Therapie, Rehabilitation und weiterer Unterstützung im Hinblick auf krankheits- oder therapiebezogene Nebenwirkungen und Beschwerden ist nach wie vor defizitär [1291, 1292]. Insbesondere nach der Entlassung aus der Klinik ist die ambulante Weiterbetreuung der Patienten durch niedergelassene Ärzte und andere Versorger (Psychoonkologie, Physiotherapie, Ergotherapie u. a.) wenig koordiniert. Häufig stehen die Patienten einer Situation von untereinander konkurrierenden und unverbundenen Gesundheits-anbietern gegenüber. „Access“ und „Support“ sind besonders eingeschränkt in strukturschwachen ländlichen Regionen mit niedrigem Versorgungsstand [1293, 1294]. In Studien wurde der Vorteil eines Fall-bezogenen Managements (Case-Management) durch begleitende professionelle Steuerung des Behandlungs- und Nachversorgungsverlaufs für den Patienten belegt [1295]. Wie aktuelle systematische Übersichten belegen, kann die 
Versorgungskontinuität insbesondere durch pflegerische Interventionen optimiert werden [1296, 1297]. Durch multiprofessionelle Steuerung des Behandlungs- und Nachsorgeverlaufs kann eine verbesserte Symptomkontrolle, eine erhöhte Leitlinientreue, eine verbesserte Patienten-zufriedenheit und höhere gesundheitsbezogene Lebensqualität erzielt werden [1295-1298].

Auch bis zum Beginn der adjuvanten Therapie sollten Patienten durch ein multiprofessionelles "Case Management" unterstützt werden, um Versorgungskontinuität zu gewährleisten. Als signifikante Risikofaktoren für Versorgungsbrüche im Übergang von primär chirurgischer zu adjuvanter (Radio)Chemotherapie wurde ein hohes Alter, das Vorliegen von Komorbiditäten, ein niedriger sozioökonomischer Status, die Versorgungsstufe der erstversorgenden Einrichtung und der Zugang oder die Wohnortentfernung zu subspezialisierten Fachärzten identifiziert [1291].

\subsubsection{Fortführung der gesundheitsförderlichen Aktivitäten}

Die Patienten sollten verstärkt dazu angehalten werden, ihre für Gesundheit und Lebensqualität förderlichen Lifestyle-Aktivitäten weiter zu praktizieren. Darüber hinaus belegen Studien, dass Patienten davon profitieren, wenn sie das Management ihrer Beschwerden und Nebenwirkungen (Übelkeit, Erbrechen, Schmerz, Fatigue, Depression, Stoma-Versorgung u. a.) selbst in die Hand nehmen können [1299, 1300]. Dies kann durch einfache Maßnahmen wie telefonisches „Follow up“, schriftliches Informationsmaterial, das Führen eines Patiententagebuchs mit integrierter Ergebniskontrolle („Patient Reported Outcomes“, PRO) unterstützt werden [1295, 1297, 1298, 1301]. Bei der Umsetzung dieser Maßnahmen kommt den Krebs-Beratungsstellen sowie den Selbsthilfegruppen eine wichtige Rolle zu.

\subsubsection{Tertiärprävention: Physikalische Aktivität, Ernährung sowie komplementäre und alternative Therapie}

10.8.2.1. Physische Aktivität

\begin{tabular}{|l|l|l|}
$\begin{array}{l}\text { 10.23. } \\
\text { Empfehlungsgrad } \\
\mathbf{B}\end{array}$ & $\begin{array}{l}\text { Kohortenstudien weisen auf einem Zusammenhang von körperlicher Aktivität und } \\
\text { erniedrigter Rezidivrate und verbessertem Überleben hin. Patienten sollten zu } \\
\text { körperlicher Aktivität angehalten werden. }\end{array}$ \\
\hline $\begin{array}{l}\text { Level of Evidence } \\
\mathbf{2} \mathbf{D}\end{array}$ & \begin{tabular}{l} 
Evidenz aus Aktualisierungsrecherche: [1302-1304] \\
\hline
\end{tabular} \\
\hline & Konsens \\
\hline
\end{tabular}

\section{Hintergrund}

Drei Kohortenstudien [1302-1304] zeigen, dass durch körperliche Aktivität nach kurativer Therapie eines kolorektalen Karzinoms sowohl die Rezidivrate als auch die Mortalität abgesenkt werden. Die Aktivität bei unbehandeltem Karzinom beeinflusst diese Parameter nicht. Der positive Effekt körperlicher Aktivität findet sich auch bei übergewichtigen Patienten. Eine alleinige Gewichtsveränderung im Anschluss an die adjuvante Therapie hat dagegen keinen Einfluss auf die Prognose. 
Eine statistisch signifikante Risikoreduktion für die krankheitsspezifische Mortalität tritt ab einer wöchentlichen körperlichen Belastung von 3 Stunden auf.

\subsubsection{Ernährung}

Für die Tertiärprävention gibt es keine Studien, aus denen sich konkrete Ernährungsempfehlungen für Patienten nach kurativer Behandlung eines kolorektalen Karzinoms ableiten lassen.

Für einen gesunden Lebensstil gelten die Empfehlungen der Deutschen Gesellschaft für Ernährung. Ein erhöhter BMI ist nicht mit der Rezidivrate oder dem Überleben korreliert [1302]. Eine Gewichtsabnahme bei übergewichtigen Patienten vermindert die Rezidivrate nicht [1302]. Allerdings sollte bei Vorliegen eines erheblichen Übergewichtes im Sinne der allgemeinen Prävention eine Gewichtsreduktion angestrebt werden.

Es gibt keinen Hinweis dafür, dass die Einnahme von Nahrungsergänzungsmitteln (Vitaminen und Spurenelementen) einen positiven Einfluss auf die Rezidivrate hat [1305]. Vitamine und Spurenelemente sollten nur bei nachgewiesenem Mangel substituiert werden.

\subsubsection{Komplementäre und alternative Medizin}

Komplementäre Verfahren beruhen auf unterschiedlichen Methoden und Substanzen, die zum Teil aus der Naturheilkunde stammen oder auf andere Weise Gedanken eines ganzheitlichen Therapiekonzepts verfolgen.

Sie ersetzen keine aktive antitumorale oder supportive Therapie, sondern stellen ergänzende Methoden dar, die es dem Patienten ermöglichen, selbstständig tätig zu werden.

Komplementäre Therapien können Neben- und Wechselwirkungen haben. Deshalb ist es sinnvoll, dass eine Beratung zu komplementärmedizinischen Behandlungen durch onkologisch erfahrene Ärzte durchgeführt wird.

Eine der am häufigsten genutzten komplementären Therapien ist die Misteltherapie. Ein Review und das Cochrane-Review [75,76] fassen zusammen, dass die meisten der bisher veröffentlichten Studien von unzureichender Qualität sind. Die wenigen methodisch gut durchgeführten Studien zeigen keine positiven Effekte auf das Überleben bei verschiedenen Tumorarten. Für das kolorektale Karzinom gibt es zwei retrospektive Untersuchungen, die einen Überlebensvorteil zeigen [1306, 1307]. Beide weisen jedoch deutliche methodische Mängel auf. Sowohl das Cochrane-Review, wie das systematische Review, finden nur schwache Hinweise auf eine Verbesserung der Lebensqualität durch Misteltherapie.

Zum Einfluss der Misteltherapie auf die Lebensqualität ist zuletzt 2010 ein Review aus 26 randomisiert kontrollierten Studien und 10 nicht randomisierten kontrollierten Studien publiziert worden [1308]. Es wurden Verbesserungen v.a. im Hinblick auf Coping, Fatigue, Schlaf, Erschöpfung, Übelkeit, Appetit, Depression und Angst gezeigt. Die von den Autoren als methodisch gut in dem Review charakterisierten Studien weisen allerdings ebenfalls erhebliche Mängel auf.

Für eine Reihe von hauptsächlich pflanzlichen Stoffen liegen präklinisch-experimentelle Daten vor, die auf eine antitumorale Wirkung hindeuten [1309, 1310]. Diese rechtfertigen einen klinischen Einsatz außerhalb von Studien derzeit nicht. Bisher wurden 2 kleine Studien zu Grünteeextrakt nach Kolonpolyp bzw. Kolonkarzinom [1310] 
publiziert. In beiden Studien war in der Therapiegruppe die Rate an Adenom- bzw. Karzinomrezidiven signifikant vermindert.

Es gibt eine Reihe von Publikationen zum Einsatz von Heilpilzen bei kurativ behandelten Patienten mit kolorektalen Karzinom [1311, 1312]. Diese Studien stammen aus China und Japan und sind in den Originalsprachen publiziert. Ob die dort durchgehend gezeigten positiven Effekte auf die Überlebensraten wissenschaftlich fundiert sind, ist unklar. Aufgrund seltener aber bedrohlicher Nebenwirkungen ist der Einsatz von Präparaten aus Heilpilzen nicht empfehlenswert.

In pflanzlichen Präparaten aus Asien im Kontext der traditionellen chinesischen Medizin und des Ayurveda wurden wiederholt Beimengungen aus Schwermetallen, Pestiziden und medizinischen Substanzen wie Corticoide und Coumarine gefunden. Der Einsatz ist bei fehlendem Nutzennachweis nicht empfehlenswert.

Ein therapeutischer Nutzen der Homöopathie als supportive Therapie ist beim kolorektalen Karzinom nicht erwiesen. [1297, 1313]. Eine Cochrane-Analyse [1313] schloss eine sehr heterogene Gruppe von Studien ein. Die beiden positiv bewerteten Studien beinhalteten keine Homöopathie im eigentlichen Sinn, sodass insgesamt keine Studie den positiven Effekt der Homöopathie bei Tumorpatienten belegen konnte [1313].

\subsubsection{Alternative Heilmethoden}

Es gibt eine Reihe von „alternativ“ zur wissenschaftlich empfohlenen Therapie angebotenen Therapien, für deren Einsatz es keine Rationale gibt. Hierzu gehören u.a.: Ukrain, Vitamin B 17 (Aprikosenkerne, Bittermandel), „insulinpotenzierte Therapie“, kohlenhydratarme Diät, „Vitamine nach Dr. Rath“, „Neue Germanische Medizin ${ }^{\circledR}$ “, Eigenblutzytokine, Zapper, „Redifferenzierungstherapie“. Es ist wichtig Patienten vor diesen Angeboten durch klare Stellungnahmen zu schützen. 


\section{Qualitätsindikatoren}

Qualitätsindikatoren (QI) sind Messgrößen, deren Erhebung der Beurteilung der Qualität der zugrunde liegenden Strukturen, Prozesse bzw. Ergebnisse dient [1314]. Qualitätsindikatoren sind ein wichtiges Instrument des Qualitätsmanagements. Ziel ihres Einsatzes ist die stetige Verbesserung der Versorgung indem die Ergebnisse der Versorgung dargestellt, kritisch reflektiert und wenn nötig verbessert werden. Die vorliegende Auswahl von Qualitätsindikatoren wurde gemäß der Methodik des Leitlinienprogramms Onkologie erstellt [1315]. Für den Ableitungsprozess konstituierte sich eine Arbeitsgruppe „AG Qualitätsindikatoren“. Diese erstellte das finale Set der Qualitätsindikatoren auf Grundlage der bereits bestehenden Qualitätsindikatoren der Leitlinie 2013, der neuen starken Empfehlungen (Empfehlungsstärke A, „soll“) der aktualisierten Leitlinie, den Ergebnissen der bestehenden Qualitätsindikatoren aus den zertifizierten Darmkrebszentren der Deutschen Krebsgesellschaft ${ }^{10}$ und den Ergebnissen der Recherche nach bestehenden nationalen und internationalen QIs. Die genaue Vorgehensweise und die Zusammensetzung der AG ist ist im Leitlinienreport dargelegt. Nach einer Präsenzsitzung und einer Telefonkonferenz dieser AG wurden 4 neue Indikatoren angenommen (QI 1-4). Diese kommen zu den 7 bereits bestehenden Indikatoren (QI 5 - 11) hinzu, so dass das finale Set aus 11 Qualitätsindikatoren besteht.

Tabelle 20: Qualitätsindikatoren 2017

\section{Qualitätsindikator \\ (Der Zähler ist stets eine \\ Teilmenge des Nenners)}

\section{Referenz Empfehlung}

Evidenzgrundlage/ weitere Informationen

\section{QI 1: Erfassung Familienanamnese (neu 2017)}

\section{Zähler:}

Anzahl Pat. mit ausgefülltem

Patientenfragebogen

Nenner:

Alle Patienten mit

Erstdiagnose KRK

\section{Keine}

Begründung dieses QI:

Die Analyse der international bereits verwendeten QI (hier v.a. ASCO) hat gezeigt, dass international QI für die Erfassung der Familienanamnese beschrieben sind. Die Leitliniengruppe hält den Bereich für relevant, so dass sie ohne begleitende starke Empfehlung in der Leitlinie einen QI definiert.

\section{Qualitätsziel:}

Möglichst häufig Ausfüllen des Patientenfragebogens für die Erfassung der Familienanamnese

Anmerkung: Patientenfragebogen:

https://www.krebsgesellschaft.de/zertdokumente.html?file=files/dkg/deutsche-

krebsgesellschaft/content/pdf/Zertifizierung/Erhebungs-

\%20und\%20Kennzahlenboegen/PatientenFragebogen\%20familiaerer\%20Darmkrebs\%20\%2803032017\%2 9.pdf

${ }^{10}$ Verfügbar unter Deutsche Krebsgesellschaft. Jahresbericht der zertifizierten Darmkrebszentren. Verfügbar: https://www.krebsgesellschaft.de/jahresberichte.html?file=files/dkg/deutschekrebsgesellschaft/content/pdf/Zertifizierung/Jahresberichte\%20mit\%20DOI\%20und\%20ISBN/dz_jahresb ericht-2017-A1_170616.pdf 


\begin{tabular}{l|l} 
Qualitätsindikator & Referenz Empfehlung \\
(Der Zähler ist stets eine & \\
Teilmenge des Nenners) &
\end{tabular}

\section{QI 2: Vollständige Befundberichte nach Tumorresektion bei KRK (neu 2017)}

\section{Zähler:}

Anzahl Patienten mit Befundbericht mit Angabe von:

- Tumortyp nach WHOKlassifikation

- Tumorinvasionstiefe (pT-Klassifikation)

- $\quad$ Status der regionären Lymphknoten ( $\mathrm{pN}$ Klassifikation)

- Anzahl der untersuchten Lymphknoten

- Grading

- Abstand von den Resektionsrändern (beim Rektumkarzinom auch circumferentiell)

- R-Klassifikation

Nenner:

Alle Pat. mit KRK und operativer Resektion
7.58.

Folgende Angaben durch den Pathologen sind erforderlich:

- Tumortyp nach WHOKlassifikation (Evidenzl. 1c)

- Tumorinvasionstiefe (pTKlassifikation) (Evidenzl. 1c)

- Status der regionären Lymphknoten ( $\mathrm{pN}$ Klassifikation) (Evidenzl. 1c)

- Anzahl der untersuchten Lymphknoten (Evidenzl. 2a)

- $\quad$ Grading (Evidenzl. 2a)

- Abstand von den Resektionsrändern (beim Rektumkarzinom auch circumferentiell) (Evidenzl. 2a)

- R-Klassifikation (Evidenzl. 1c)
EG A

\section{Qualitätsziel:}

Möglichst häufig vollständige

Befundberichte nach

Tumorresektion bei KRK

\section{QI 3: Mutationsbestimmung bei mKRK (neu 2017)}

Zähler:

Anzahl Pat. mit Bestimmung RAS- (= KRAS u NRAS-

Mutationen) sowie BRAF-

Mutation zu Beginn der

Erstlinientherapie

\section{Nenner:}

Alle Patienten mit mKRK und Erstlinientherapie

\section{4 .}

Die Bestimmung von (ALL) RAS- und BRAF-Mutationen soll möglichst noch vor Einleitung der Erstlinientherapie erfolgen.

\section{EG A, LoE 1}

\section{Qualitätsziel:}

Möglichst häufig

Mutationsbestimmung vor

Erstlinientherapie bei mKRK.

\section{Anmerkung:}

Festlegung „zu Beginn“ = Datum Mutationsbestimmung max. +15d von Datum Beginn Erstlinientherapie

\section{QI 4: Kombinationschemotherapie bei mKRK (neu 2017)}

\section{Zähler:}

Anzahl Pat. mit Kombinationschemotherapie
9.24 .

In der Erstlinienchemotherapie sollen bei gutem Allgemeinzustand und hoher Motivation in erster Linie Fluoropyrimidin-basierte

\section{EG A, LoE 1a}

Qualitätsziel:

Möglichst häufig

Kombinationschemotherapie bei 


\begin{tabular}{l|l|}
$\begin{array}{l}\text { Qualitätsindikator } \\
\text { (Der Zähler ist stets eine } \\
\text { Teilmenge des Nenners) }\end{array}$ & Referenz Empfehlung \\
\hline $\begin{array}{l}\text { Nenner: } \\
\text { Alle Patienten mit mKRK, } \\
\text { ECOG 0-1 und systemischer } \\
\text { Erstlinientherapie }\end{array}$ & $\begin{array}{l}\text { Kombinationsregime mit } \\
\text { infusionaler Verabreichung von 5- } \\
\text { Fluorouracil, wie FOLFIRI, FOLFOX } \\
\text { oder FOLFOXIRI oder mit dem } \\
\text { oralen Fluoropyrimidin Capecitabin } \\
\text { (vorwiegend mit Oxaliplatin, } \\
\text { CAPOX) eingesetzt werden. }\end{array}$ \\
\hline
\end{tabular}

Evidenzgrundlage/ weitere Informationen

Erstlinientherapie von Patienten mit mKRK, ECOG 0-1

\section{QI 5: Angabe Abstand mesorektale Faszie (seit 2013, vormals: KRK 1)}

\section{Zähler:}

Alle Patienten mit Angabe des Abstands zur mesorektalen Faszie im Befundbericht

\section{Nenner:}

Alle Patienten mit Rektumkarzinom und MRT oder Dünnschicht-CT des Beckens
7.17.

Die Befundbeschreibung soll eine Aussage über den Abstand zur mesorektalen Faszie beinhalten.

\section{EK}

(Expertenkonsens)

\section{Qualitätsziel:}

Möglichst häufig Angabe des Abstandes der mesorektalen Faszie, wenn ein MRT/CT bei Rektumkarzinom durchgeführt wurde.

\section{QI 6: Qualität TME (seit 2013, vormals: KRK 3)}

\section{Zähler:}

Anzahl aller Patienten mit guter oder moderater Qualität (Grad 1:

Mesorektale Faszie erhalten oder Grad 2:

Intramesorektale Einrisse) der TME

\section{Nenner:}

Alle Patienten mit radikal operiertem Rektumkarzinom

\subsection{6.}

Da die Qualität eines Operationsresektates unter Berücksichtigung der oben genannten Kategorien Rückschlüsse auf die Prognose bezüglich der Entwicklung eines Lokalrezidives zuläßt, soll diese obligat im pathohistologischen Befundbericht wie folgt beschrieben werden

Die Qualität des Präparates wird beurteilt an der Integrität der mesorektalen Faszie im Falle der Resektion mit den 3 Kategorien

- Mesorektale Faszie erhalten

- Intramesorektale Einrisse

- Erreichen der Muscularis propria oder Tumor.

Im Falle einer Rektumexstirpation sind bei einer vollständigen Resektion der Levatormuskulatur Präparateinrisse und ein tumorpositiver zirkumferentieller Sicherheitsabstand seltener.
EK

(Expertenkonsens)

\section{Qualitätsziel:}

Möglichst häufig gute bzw moderate Qualität der TME bei

Rektumkarzinom 


\begin{tabular}{|c|c|}
\hline $\begin{array}{l}\text { Qualitätsindikator } \\
\text { (Der Zähler ist stets eine } \\
\text { Teilmenge des Nenners) }\end{array}$ & Referenz Empfehlung \\
\hline & $\begin{array}{l}\text { Im patho-histologischen Befund- } \\
\text { bericht ist deshalb die Beschrei- } \\
\text { bung bezüglich der Radikalität im } \\
\text { Bereich der Levatormuskulatur } \\
\text { obligat. Hierzu sollen folgende } \\
\text { Kategorien Verwendung finden: } \\
\text { Teile der Muscularis propria fehlen } \\
\text { oder Eröffnung des Darmes oder } \\
\text { des Tumors } \\
\text { Muscularis propria erhalten, keine } \\
\text { Eröffnung des Darmes oder des } \\
\text { Tumors } \\
\text { Levatormuskulatur mitreseziert, } \\
\text { keine Eröffnung des Darmes oder } \\
\text { des Tumors } \\
\text { Diese Bewertungen sind vom } \\
\text { Pathologen vorzunehmen. }\end{array}$ \\
\hline
\end{tabular}

QI 7: Vorstellung Tumorkonferenz (seit 2013, vormals: KRK 5)

Zähler:

Anzahl der Patienten, die prätherapeutisch in einer interdisziplinären

Tumorkonferenz vorgestellt wurden

\section{Nenner:}

Alle Patienten mit Rektumkarzinom und alle Pat. mit Kolonkarzinom Stad IV
7.1.

Alle Patienten mit KRK sollen nach Abschluss der Primärtherapie (z.B. Operation, Chemotherapie) in einer interdisziplinären Tumorkonferenz vorgestellt werden.

Bereits prätherapeutisch sollen

Patienten in folgenden

Konstellationen vorgestellt werden Nenner:

- mit Rektumkarzinom

- mit Kolonkarzinom, im Stadium IV

- mit metachronen Fermetastasen

- mit Lokalrezidiven

- vor jeder lokal ablativen

Maßnahme RFA/LITT/SIRT

\section{Evidenzgrundlage/ weitere} Informationen

\section{EK}

(Expertenkonsens)

\section{Qualitätsziel:}

Möglichst häufig Vorstellung der Pat. mit RektumCa und der Pat. mit KolonCa Stad. IV in der prätherapeutischen Tumorkonferenz

\section{QI 8: Adjuvante Chemotherapie (seit 2013, vormals: KRK 6)}

\section{Zähler:}

Anzahl der Patienten, die eine adjuvante Chemotherapie erhalten haben.

\section{Nenner:}

Alle Patienten mit

Kolonkarzinom im UICC

Stadium III, bei denen eine

R0-Resektion des

Primärtumors erfolgte.

\section{4}

Bei Patienten mit einem R0 resezierten Kolonkarzinom im Stadium III ist eine adjuvante Chemotherapie indiziert.
Empfehlung LoE 1a, starker Konsens

\section{Qualitätsziel:}

Adäquate Durchführung der adjuvanten Chemotherapie nach R0-Resektion KolonCa Stad. III 


\section{Qualitätsindikator \\ (Der Zähler ist stets eine \\ Teilmenge des Nenners)}

Referenz Empfehlung

Referenz Empfehlung
Evidenzgrundlage/ weitere Informationen

\section{QI 9: Anastomoseninsuffizienz Rektumkarzinom (seit 2013, vormals: KRK 8)}

\section{Zähler:}

Anzahl der Patienten mit

Anastomoseninsuffizienz

Grad B (mit Antibiotikagabe

o. interventioneller Drainage

o.transanaler

Lavage/Drainage) oder C ((Re-)-Laparotomie) nach elektiven Eingriffen

\section{Nenner:}

Alle Patienten mit

Rektumkarzinom, bei denen

in einer elektiven primären

Tumorresektion eine

Anastomose angelegt wurde.

\section{Keine}

Begründung dieses QI:

\section{Anmerkung:}

Die Leitlinienkommission hat beschlossen, dass nicht nur Strukturqualitätsziele, sondern auch Ergebnisqualitätsziele berücksichtigt werden sollen. Daraus ergibt sich die Aufnahme dieses Indikators auch ohne konsentierte starke Empfehlung in der Leitlinie.

\section{QI 10: Anastomoseninsuffizienz Kolonkarzinom (seit 2013, vormals: KRK 9)}

\section{Zähler:}

Re-Interventionsbedürftige

Anastomoseninsuffizienzen

Kolon nach elektiven

Eingriffen

\section{Nenner:}

Alle Patienten mit

Kolonkarzinom, bei denen in einer elektiven

Tumorresektion eine

Anastomose angelegt wurde.

\section{Keine}

Begründung dieses QI:

\section{Anmerkung:}

Die Leitlinienkommission hat beschlossen, dass nicht nur Strukturqualitätsziele, sondern auch Ergebnisqualitätsziele berücksichtigt werden sollen. Daraus ergibt sich die Aufnahme dieses Indikators auch ohne konsentierte starke Empfehlung in der Leitlinie.
Keine Evidenzgrundlage, die einer starken Empfehlung zugeordnet ist, da dieser Indikator nicht aus einer solchen abgeleitet wurde.

\section{Qualitätsziel:}

Möglichst selten Anastomoseninsuffizienzen Grad B oder C nach Anastomosenanlage bei operiertem Rektumkarzinom.

\section{QI 11: Anzeichnung Stomaposition (seit 2013, vormals: KRK 10)}

Zähler:

Anzahl der Pat. mit präoperativer Anzeichnung der Stomaposition

\section{Nenner:}

Alle Pat. mit Rektumkarzinom, bei denen eine Operation mit Stomaanlage durchgeführt wurde

\subsection{2 .}

Die Stomaposition soll präoperativ angezeichnet werden.
Keine Evidenzgrundlage, die einer starken Empfehlung zugeordnet ist, da dieser Indikator nicht aus einer solchen abgeleitet wurde.

\section{Qualitätsziel:}

Möglichst selten re-interventionsbedürftige Anastomoseninsuffizienzen $G$ nach

Anastomosenanlage bei operiertem Kolonkarzinom

EK

(Expertenkonsens)

\section{Qualitätsziel:}

Möglichste häufig präoperative Anzeichnung der Stomaposition 


\section{Anhang}

\subsection{UICC-Klassifikation des Kolorektalen Karzinoms}

Die UICC-Stadieneinteilung wurde von der „Union Internationale Contre le Cancer“ (UICC) eingeführt. Sie basiert auf statistischen Untersuchungen und gibt Auskunft über die Ausbreitung der Tumorerkrankung. Sie ist Grundlage bei Prognose- und Therapieplanerstellung.

Tabelle 21: UICC-Klassifikation des Kolorektalen Karzinoms

\begin{tabular}{|c|c|c|c|}
\hline UICC 2010 & $\mathrm{~T}$ & $\mathbf{N}$ & M \\
\hline Stadium 0 & Tis & NO & MO \\
\hline Stadium I & T1/ T2 & NO & MO \\
\hline $\begin{array}{l}\text { Stadium II } \\
\text { IIA } \\
\text { IIB } \\
\text { IIC }\end{array}$ & $\begin{array}{l}\text { T3/ T4 } \\
\text { T3 } \\
\text { T4a } \\
\text { T4b }\end{array}$ & $\begin{array}{l}\text { NO } \\
\text { NO } \\
\text { NO } \\
\text { NO }\end{array}$ & $\begin{array}{l}\text { M0 } \\
\text { M0 } \\
\text { M0 } \\
\text { M0 }\end{array}$ \\
\hline $\begin{array}{l}\text { Stadium III } \\
\qquad \text { IIIA } \\
\text { IIIB } \\
\text { IIIC }\end{array}$ & $\begin{array}{l}\text { jedes T } \\
\text { T1/ T2 } \\
\text { T1 } \\
\text { T3/ T4 } \\
\text { T2/ T3 } \\
\text { T1/ T2 } \\
\text { T4a } \\
\text { T3/ T4a } \\
\text { T4b }\end{array}$ & $\begin{array}{l}\mathrm{N} 1 / \mathrm{N} 2 \\
\mathrm{~N} 1 \\
\mathrm{~N} 2 \mathrm{a} \\
\mathrm{N} 1 \\
\mathrm{~N} 2 \mathrm{a} \\
\mathrm{N} 2 \mathrm{~b} \\
\mathrm{~N} 2 \mathrm{a} \\
\mathrm{N} 2 \mathrm{~b} \\
\mathrm{~N} 1 / \mathrm{N} 2\end{array}$ & $\begin{array}{l}\text { M0 } \\
\text { M0 } \\
\text { M0 } \\
\text { M0 } \\
\text { M0 } \\
\text { M0 } \\
\text { M0 } \\
\text { M0 } \\
\text { M0 }\end{array}$ \\
\hline $\begin{array}{r}\text { Stadium IV } \\
\qquad \begin{array}{l}\text { IVA } \\
\text { IVB }\end{array}\end{array}$ & $\begin{array}{l}\text { jedes } T \\
\text { jedes } T \\
\text { jedes } T\end{array}$ & $\begin{array}{l}\text { jedes } N \\
\text { jedes } N \\
\text { jedes } N\end{array}$ & $\begin{array}{l}\text { M1 } \\
\text { M1a } \\
\text { M1b }\end{array}$ \\
\hline
\end{tabular}

\subsection{Amsterdam Kriterien}

\section{Amsterdam Kriterien (AC)}

$A C 1$ = nur KRK, AC2 = auch extrakolische Manifestationen [237, 1316]

1.

2.

3.

4.

5.
Mindestens drei Familienmitglieder mit HNPCC-assoz. Karzinomen (Kolon/Rektum, Endometrium, Dünndarm, Urothel (Ureter/Nierenbecken))

Mindestens zwei aufeinanderfolgende Generationen betroffen

Ein Familienmitglied erstgradig verwandt mit den beiden anderen

Ein Erkrankter zum Zeitpunkt der Diagnose jünger als 50 Jahre

Ausschluss einer familiären adenomatösen Polyposis 


\subsection{Revidierte Bethesda-Kriterien}

Tumoren von Patienten, die eines der folgenden Kriterien erfüllen, sollten auf eine Mikrosatelliteninstabilität untersucht werden:

\section{Revidierte Bethesda-Kriterien [249]}

Patient mit KRK vor dem 50. Lj.

Patient mit syn- oder metachronen kolorektalen oder anderen HNPCC-assoziierten Tumoren (Kolon, Rektum, Endometrium, Magen, Ovar, Pankreas, Ureter, Nierenbecken, biliäres System, Gehirn (v.a. Glioblastom), Haut (Talgdrüsenadenome und -karzinome, Keratoakanthome, Dünndarm)) unabhängig vom Alter bei Diagnose.

Patient mit KRK vor dem 60. Lj. mit typischer Histologie eines MSI-H- Tumors (Tumor-infiltrierende Lymphozyten, Crohn's like Lesions, muzinöse oder siegelringzellige Differenzierung, medulläres Karzinom).

Patient mit KRK, der einen Verwandten 1. Grades mit einem KRK oder einem HNPCC-assoziierten Tumor vor dem 50. Lebensjahr hat.

Patient mit KRK (unabhängig vom Alter), der mindestens zwei Verwandte 1. oder 2. Grades hat, bei denen ein KRK oder ein HNPCC-assoziierter Tumor (unabhängig vom Alter) diagnostiziert wurde. 


\section{4. Änderungen der Empfehlungen durch Aktualisierung 2017 und 2019 (Versionen 2.0 und 2.1)}

Version $2.0(2017)$

Kapitel 8.1.6.3: Dauer der adjuvanten Therapie
Version 2.1 (2019)

In der adjuvanten Situation soll die akkumulierende (Neuro-)Toxizität engmaschig gegen den therapeutischen Nutzen abgewogen werden.

Bei niedrigem Rezidivrisiko ( $\mathrm{T} 1-3 \mathrm{NI}$ ) sollte deshalb eine 3-monatige oxaliplatinhaltige Therapie nach dem CAPOX/XELOX Schema durchgeführt werden.

Bei Patienten mit hohem Risiko (T4 oder N2Stadium) sollte weiterhin eine Oxaliplatin-basierte Therapie (FOLFOX oder CAPOX/XELOX Schema) über 6 Monate geplant werden.

Version 1 (2013)

Version $2.0(2017)$

Kapitel 6.1. Stellenwert der Endoskopie in der Diagnostik von Polypen und kolorektalen Karzinomen

Bei inkompletter Koloskopie aufgrund eines stenosierenden Tumors kann präoperativ zusätzlich eine CT- oder MR-Kolonographie erfolgen. Postoperativ soll eine komplette Koloskopie erfolgen.

(2008: Empfehlung 6.2.)

Bei inkompletter Koloskopie infolge anderer Ursachen (z. B. Adhäsionen) sollte eine CT- oder MR-Kolonographie erfolgen.

(2008: Empfehlung 6.3.)

Bei positivem FOBT-Test, bei Tumorverdacht oder sigmoidoskopischem Nachweis eines neoplastischen Polypen soll eine vollständige Koloskopie durchgeführt werden.

(2008: Empfehlung 6.4.)

Eine Chromoendoskopie kann bei Patienten mit einer chronisch entzündlichen Darmerkrankung
Bei inkompletter Koloskopie aufgrund eines stenosierenden Tumors kann präoperativ zusätzlich eine CT-Kolonographie erfolgen.

Postoperativ soll eine komplette Koloskopie erfolgen.

(2017: Empfehlung 6.2.)

Bei inkompletter Koloskopie aufgrund anderer Ursachen (z. B. Adhäsionen) sollte eine CTKolonographie erfolgen.

(2017: Empfehlung 6.3.)

Bei positivem FOBT/FIT-Test, bei Tumorverdacht oder sigmoidoskopischem Nach-weis eines neoplastischen Polypen soll eine vollständige Koloskopie durchgeführt werden.

(2017: Empfehlung 6.4.)

Eine Chromoendoskopie kann bei Patienten mit einer chronisch entzündlichen Darmerkrankung 


\section{Version 1 (2013)}

und HNPCC zur besseren Erkennung von neoplastischen Läsionen eingesetzt werden. Sie kann darüber hinaus zur besseren Abgrenzung flacher und eingesenkter Läsionen vor endoskopischer Therapie verwendet werden.

(2008: Empfehlung 6.5.)

Die Durchführung der Zoomendoskopie zur Klassifikation des „pit-pattern“ Musters ist derzeit kein Standardverfahren.

(2008: Empfehlung 6.6.)

\section{Kapitel 6.2. Polypektomiedurchführung}

Um eine repräsentative histologische Aussage zu erhalten und zur definitiven Therapie, sollen Polypen > $>\mathrm{mm}$ vollständig durch Schlingenektomie entfernt werden. Polypen $\leq 5$ $\mathrm{mm}$ sollten generell mit der Zange komplett entfernt werden.

Grundsätzlich sollen diagnostische Koloskopien nur dann durchgeführt werden, wenn in gleicher Sitzung die Möglichkeit zur Schlingenektomie besteht.

(2008: Statement 6.8.)

\section{Kapitel 6.3. Histologische Untersuchungen}

Die histologische Untersuchung jedes Polypen ist obligat. Die histologische Befundung der Polypen erfolgt entsprechend der WHO-Kriterien [568] mit einer Aussage zur Vollständigkeit der Abtragung. Konventionelle Adenome werden klassifiziert nach histologischem Wachstumstyp (tubulär, tubulovillös und villös) und dem Grad der intraepithelialen Neoplasie (niedrig- und hochgradige intraepitheliale Neoplasie); serratierte Läsionen werden unterteilt in hyperplastische Polypen, sessile serratierte Adenome, gemischte Polypen (mit Angabe des IEN-Grades) und traditionelle serratierte Adenome (mit Angabe des IEN-Grades) [569, 570]. (2008: Empfehlung 6.9.)

\section{Version 2.0 (2017)}

und HNPCC zur besseren Erkennung von neoplastischen Läsionen eingesetzt werden. (2017: Empfehlung 6.5.)

\section{gestrichen}

Um eine repräsentative histologische Aussage zu erhalten und zur definitiven Therapie, sollen Polypen > $5 \mathrm{~mm}$ vollständig durch Schlingenektomie entfernt werden.

Grundsätzlich sollen diagnostische Koloskopien nur dann durchgeführt werden, wenn in gleicher Sitzung die Möglichkeit zur Schlingenektomie besteht.

(2017: Empfehlung 6.7.)

Die histologische Untersuchung jedes Polypen ist obligat. Die histologische Befundung der Polypen soll entsprechend der WHO-Kriterien [568] mit einer Aussage zur Vollständigkeit der Abtragung erfolgen. Konventionelle Adenome werden klassifiziert nach histologischem Wachstumstyp (tubulär, tubulovillös und villös) und dem Grad der intraepithelialen Neoplasie (niedrig- und hochgradige intraepitheliale Neoplasie); serratierte Läsionen werden unterteilt in hyperplastische Polypen, sessile serratierte Adenome, mixed Polypen (mit Angabe des IENGrades) und traditionelle serratierte Adenome (mit Angabe des IEN-Grades) [569, 570].

(2017: Empfehlung 6.8.)

Das Ausmaß des Tumorbuddings kann als zusätzlicher Parameter gewertet werden.

(2017: Empfehlung 6.10.) 


\section{Version 1 (2013)}

\section{Kapitel 6.4. Vorgehen bei pT1-Karzinomen}

Ergibt die histologische Untersuchung eines endoskopisch R0-entfernten Polypen ein pT1Karzinom, soll auf eine onkologische Nachresektion verzichtet werden, wenn es sich um eine Low-risk Situation bei histologisch karzinomfreier Polypenbasis (R0) handelt [589, 1317-1319]. In der High-risk Situation ist die radikale chirurgische Behandlung erforderlich, auch wenn die Läsion komplett entfernt wurde.

(2008: Empfehlung 6.12.)

Bei inkompletter Abtragung eines Low-risk pT1Karzinoms soll eine komplette endoskopische oder lokale chirurgische Entfernung erfolgen [1320]. Wenn eine R0-Situation nicht erreichbar ist oder Zweifel am Vorliegen einer pT1-Situation bestehen, so ist die onkologisch-chirurgische Resektion erforderlich.

(2008: Empfehlung 6.13.)

Die endoskopische lokale Nachsorge soll nach kompletter Entfernung (R0) von Low-risk ( $\mathrm{pT} 1$, low grade (G1, G2, L0)) Karzinomen nach einem halben Jahr und nach zwei Jahren erfolgen.

(2008: Empfehlung 6.14.)

\section{Kapitel 6.5. Polypenmanagement (Nachsorge)}

Nach Abtragung kleiner einzelner nicht neoplastischer Polypen besteht keine Notwendigkeit einer endoskopischen Nachsorge. (2008: Empfehlung 6.15.))

Nach kompletter Abtragung neoplastischer Polypen (Adenome) ist eine Kontrollendoskopie erforderlich. Der Zeitpunkt der Kontrollkoloskopie sollte von Anzahl, Größe und Histologie der entfernten Adenome abhängig gemacht werden. Bei Patienten mit 1 oder 2 Adenomen $<1 \quad \mathrm{~cm}$ ohne höhergradige

\section{Version $2.0(2017)$}

Tumorzellbudding größer als 1 kann ebenfalls als „High-risk“ gewertet werden. (2017: Empfehlung 6.12.)

Ergibt die histologische Untersuchung eines endoskopisch R0-entfernten Polypen ein pT1Karzinom, soll auf eine onkologische Nachresektion verzichtet werden, wenn es sich um eine Low-risk Situation bei histologisch karzinomfreier Polypenbasis (R0) handelt. In der High-risk Situation soll die radikale chirurgische Behandlung erfolgen, auch wenn die Läsion komplett entfernt wurde.

(2017: Empfehlung 6.13.)

Bei inkompletter Abtragung eines Low-risk pT1Karzinoms soll eine komplette endoskopische oder lokale chirurgische Entfernung erfolgen. Wenn eine R0-Situation nicht erreichbar ist oder Zweifel am Vorliegen einer pTl-Situation bestehen, soll die onkologisch-chirurgische Resektion erfolgen.

(2017: Empfehlung 6.14.)

Die endoskopische lokale Nachsorge soll nach kompletter Entfernung (R0) von Low risk (pT1, low grade (G1, G2, L0)) Karzinomen nach einem halben Jahr erfolgen

Eine komplette Koloskopie soll nach 3 Jahren erfolgen.

(2017: Empfehlung 6.15.)

Nach Abtragung kleiner einzelner nicht neoplastischer Polypen sollte keine endoskopische Nachsorge erfolgen. (2017: Empfehlung 6.16.)

Der Zeitpunkt der Kontrollkoloskopie nach kompletter Abtragung neoplastischer Polypen (Adenome) soll von Anzahl, Größe und Histologie der entfernten Adenome abhängig gemacht werden.

(2017: Empfehlung 6.17.) 


\section{Version 1 (2013)}

intraepitheliale Neoplasie ist eine Kontrollkoloskopie nach 5 Jahren ausreichend. (2008: Empfehlung 6.16.)

\section{Version $2.0(2017)$}

Bei Patienten mit 1 oder 2 Adenomen $<1 \mathrm{~cm}$ ohne höhergradige intraepitheliale Neoplasie sollte eine Kontrollkoloskopie nach 5-10 Jahren erfolgen.

(2017: Empfehlung 6.18.)

Werden bei dieser Kontrollkoloskopie wiederum keine oder nur 1 - 2 Adenome < $10 \mathrm{~mm}$ ohne überwiegend villöse Histologie oder HGIEN gefunden, sollte die nächste Kontrollkoloskopie nach 10 Jahren erfolgen.

(2017: Empfehlung 6.19.)

Bei Patienten mit 3-4 Adenomen oder einem Adenom $\geq 1 \mathrm{~cm}$ oder einem Adenom mit überwiegend villöser Histologie oder HGIEN sollte die erste Kontrollkoloskopie nach 3 Jahren erfolgen.

(2017: Empfehlung 6.20.)

Bei Patienten mit Adenom mit hochgradiger intraepithelialer Neoplasie und histologisch bestätigter vollständiger Abtragung sollte eine Kontrollkoloskopie nach 3 Jahren erfolgen.

(2017: Empfehlung 6.21.)

Bei histologisch nicht bestätigter vollständiger Abtragung von Adenomen $>5 \mathrm{~mm}$ sollte auch bei makroskopisch kompletter Abtragung eine Kontrolle nach 6 Monaten erfolgen.

(2017: Empfehlung 6.22.)

Bei Nachweis von $\geqq 5$ Adenomen jeder Größe sollte das Kontrollintervall < 3 Jahre betragen. (2017: Empfehlung 6.23.)

Nach Abtragung großer Adenome in PiecemealTechnik soll eine kurzfristige Kontrolle der Abtragungsstelle nach 2-6 Monaten erfolgen. (2017: Empfehlung 6.24.) 


\section{Version 1 (2013)}

Nach unauffälliger Kontrollendoskopie sind weitere Kontrollen in 5-jährigen Abständen angezeigt. Nach kompletter Abtragung eines traditionellen serratierten Adenoms, eines gemischten Schleimhautpolypen oder eines sessilen serratierten Adenoms sollte aufgrund des potentiell erhöhten Karzinomrisikos unabhängig vom IEN-Grad eine Kontrollkoloskopie nach 3 Jahren erfolgen.

(2008: Empfehlung 6.22.)

\section{Version $2.0(2017)$}

Nach kompletter Abtragung eines traditionellen serratierten Adenoms oder eines sessilen serratierten Adenoms sollte die Nachsorge analog zu klassischen Adenomen erfolgen.

(2017: Empfehlung 6.25.)

gestrichen

\section{Kapitel 8.1. Adjuvante Therapie des Kolonkarzinoms}

Eine Altersbeschränkung für die Durchführung einer adjuvanten Chemotherapie existiert nicht, allgemeine Kontraindikationen (s.o.) sind $\mathrm{zu}$ berücksichtigen.

(2008: Empfehlung 8.1.)
Alleine aus Altersgründen sollte eine adjuvante Chemotherapie nicht unterlassen werden. Bei Patienten über 75 Jahre gibt es jedoch keine ausreichende Evidenz für die Durchführung einer adjuvanten Chemotherapie.

(2017: Empfehlung 8.1.)

Die adjuvante Chemotherapie sollte baldmöglichst postoperativ eingeleitet werden. (2017: Empfehlung 8.2.)

In den randomisierten Studien wurde die adjuvante Chemotherapie innerhalb von 8 Wochen eingeleitet.

(2017: Empfehlung 8.3.)

Bei Patienten mit einem R0 resezierten Kolonkarzinom im Stadium III ist eine adjuvante Chemotherapie indiziert.

(2008: Empfehlung 8.2.)

Bei Patienten mit einem R0 resezierten Kolonkarzinom im Stadium III soll eine adjuvante Chemotherapie durchgeführt werden.

(2017: Empfehlung 8.4.)

Zusätzliche Parameter (z. B. CEA-Spiegel, Differenzierungsgrad, $18 q$ Verlust, isolierte Tumorzellen in Lymphknoten oder im Knochenmark, Mikrosatelliten-Status, DNA-Ploidie und TS/p53 Expression, Lymph- und Blutgefäßinvasion) sollen momentan nicht zur Indikationsstellung für eine adjuvante Chemotherapie benutzt werden.

(2008: Empfehlung 8.5.)
Bei Patienten im Stadium II soll vor der Indikationsstellung für eine adjuvante Chemotherapie der Mikrosatellitenstatus bestimmt werden. Weitere Parameter (z. B. CEASpiegel, Differenzierungsgrad, 18q Verlust, isolierte Tumorzellen in Lymphknoten oder im Knochenmark, DNA-Ploidie und TS/p53 Expression, Lymph- und Blutgefäßinvasion, molekulargenetische Analysen) sollen nicht zur 


\section{Version 1 (2013)}

Sollte bei Patienten mit Stadium II Tumoren eine adjuvante Chemotherapie durchgeführt werden, können Fluoropyrimidine als Monotherapie eingesetzt werden.

(2008: Empfehlung 8.8.)

\section{Version $2.0(2017)$}

Indikationsstellung für eine adjuvante Chemotherapie benutzt werden. (2017: Empfehlung 8.7.)

Bei nachgewiesener Mikrosatelliteninstabilität (MSI-H) sollte eine adjuvante Chemotherapie im Stadium II nicht erfolgen.

(2017: Empfehlung 8.8.)

Bei Patienten über 70 Jahre sollte eine Oxaliplatinhaltige Therapie nicht erfolgen.

(2017: Empfehlung 8.10.)

Monoklonale Antikörper oder Irinotecan sollen in der adjuvanten Therapie des Kolonkarzinoms nicht eingesetzt werden.

(2017: Empfehlung 8.12.)

Wenn bei Patienten mit Stadium II Tumoren eine adjuvante Chemotherapie durchgeführt wird, sollten Fluoropyrimidine als Monotherapie eingesetzt werden.

(2017: Empfehlung 8.13.)

\section{Kapitel 8.2. Perioperative Therapie beim Rektumkarzinom}

Im Stadium I ist eine perioperative Therapie nicht indiziert.

(2008: Empfehlung 8.9.)

Im UICC-Stadium II und III ist die neoadjuvante Radio- oder Radiochemotherapie indiziert. Eine Sondersituation besteht bei cT1/2-Karzinomen mit fraglichem Lymphknotenbefall; hier ist auch die primäre Operation (mit ggf. adjuvanter Radiochemotherapie bei $\mathrm{pN}+$ ) eine mögliche Behandlungsoption.

(2008: Empfehlung 8.10.)
In UICC-Stadium I (cT1-2N0) soll eine präoperative Therapie nicht durchgeführt werden.

(2017: Empfehlung 8.14.)

In den UICC-Stadien II und III (cT3/4 und/oder $\mathrm{cN}+$ ) soll bei Tumoren des unteren und mittleren Rektumdrittels eine neoadjuvante Radiochemotherapie oder Kurzzeit-Radiotherapie erfolgen.

(2017: Empfehlung 8.17.)

In folgenden Ausnahmefällen kann bei Patienten mit Rektumkarzinom im UICC-Stadium II/III eine primäre Resektion erfolgen:

CT1/2-Tumore im unteren und mittleren Drittel mit bildgebend fraglichen Lymphknotenbefall cT3a/b-Tumore im mittleren Drittel mit in der MRT nur limitierter Infiltration ins perirektale Fettgewebe (cT3a: <1 mm, cT3b: 1-5 mm) und ohne bildgebenden Verdacht auf Lymphknotenmetastasen oder extra-muraler Gefäßinvasion (EMVI-) bei adäquater 


\section{Version 1 (2013)}

Der Stellenwert der Strahlentherapie des Rektumkarzinoms im oberen Drittel wird kontrovers diskutiert. Es kann eine adjuvante Therapie wie beim Kolonkarzinom oder eine perioperative Radio(chemo-)therapie wie beim Rektumkarzinom durchgeführt werden.

(2008: Empfehlung 8.11.)

\section{Version $2.0(2017)$}

Qualitätssicherung der MRT-Diagnostik und der TME-Chirurgie.

(2017: Empfehlung 8.16.)

Der im Dünnschicht-MRT gemessene radiale Abstand des Primärtumors (oder bildgebend befallener Lymphknoten) von der mesorektalen Faszie (mrCRM) soll außerhalb von Studien nicht als Entscheidungskriterium für eine primäre Operation herangezogen werden.

(2017: Empfehlung 8.17.)

Rektumkarzinome im oberen Drittel ohne Risikokonstellation für ein Lokalrezidiv sollen primär operiert und adjuvant analog zu Kolonkarzinomen behandelt werden.

(2017: Empfehlung 8.18.)

Bei einer Risikokonstellation im oberen Rektumdrittel (z.B. T4, mrCRM+, bildgebend eindeutiger und ausgedehnter Lymphknotenbefall) kann eine präoperative Radio-/ Radiochemotherapie erfolgen.

(2017: Empfehlung 8.19.)

Die neoadjuvante Radiotherapie kann entweder als Kurzzeitbestrahlung mit $5 \times 5$ Gy gefolgt von sofortiger Operation oder als konventionell fraktionierte Radiochemotherapie (1.8-2.0 Gy bis 45-50.4 Gy) mit einem Intervall von 6-8 Wochen bis zur Operation erfolgen.

(2017: Empfehlung 8.20.)

In Situationen, in denen ein Downsizing angestrebt wird (T4-Tumore, nicht ausreichender Sicherheitsabstand im Dünnschicht-MRT zur mesorektalen Faszie - Abstand $1 \mathrm{~mm}$ oder weniger - oder erwünschter Sphinktererhalt bei Tumoren im unteren Drittel), soll der präoperativen Radiochemotherapie der Vorzug vor einer Kurzzeit-Radiotherapie gegeben werden. Bei cT3-Tumoren oder cN+Tumoren, bei denen kein Downsizing angestrebt wird, kann die präoperative Therapie entweder als Radiochemotherapie oder als Kurzzeitbestrahlung erfolgen. (2008: Empfehlung 8.12.)
Bei T4-Tumoren, Nähe des Tumors zur mesorektalen Fazie $(<1-2 \mathrm{~mm}$ ) oder tiefliegenden Tumoren mit intendiertem Sphinktererhalt sollte eine präoperative Radiochemotherapie durchgeführt werden.

(2017: Empfehlung 8.21.)

Für Patienten bei denen ein Downsizing angestrebt ist, kann auch die Kurzzeitbestrahlung mit längerem Intervall bis zu 12 Wochen zur Operation (mit und ohne neoadjuvante Chemotherapie) durchgeführt werden.

(2017: Empfehlung 8.22.) 


\section{Version 1 (2013)}

Die neoadjuvante Radiochemotherapie soll eine 5Fluorouracil-Monochemotherapie mit oder ohne Folinsäure beinhalten.

(2008: Empfehlung 8.13.)

\section{Version $2.0(2017)$}

Die neoadjuvante Radiochemotherapie soll orales Capecitabin oder infusionales 5-Fluorouracil beinhalten.

(2017: Empfehlung 8.23.)

Die Operation sollte 6-8 Wochen nach neoadjuvanter Radiochemotherapie erfolgen.

(2017: Empfehlung 8.24.)

Nach Kurzzeit-Bestrahlung ( $5 \times 5$ Gy) sollte die Operation entweder innerhalb von 10 Tagen nach Beginn der Radiotherapie oder nach 4-8 Wochen erfolgen.

(2017: Empfehlung 8.25.)

Die neoadjuvante Chemotherapie vor oder nach Radiochemotherapie (oder als alleinige neoadjuvante Therapie ohne Radio/Radiochemotherapie) soll außerhalb von Studien nicht durchgeführt werden.

(2017: Empfehlung 8.26.)

Die Kurzzeit-RT mit $5 \times 5$ Gy gefolgt von neoadjuvanter Chemotherapie und Operation im Intervall kann bei synchroner Metastasierung erfolgen.

(2017: Empfehlung 8.27.)

\section{Kapitel 8.2.2. Adjuvante Therapie}

Im Stadium I ist nach R0-Resektion eine adjuvante Therapie nicht indiziert.

(2008: Empfehlung 8.14.)

Nach R1-Resektion oder intraoperativem Tumoreinriss sollte postoperativ radiochemotherapiert werden, falls keine neoadjuvante Radio(chemo)therapie vorangegangen ist.

(2008: Empfehlung 8.16.)

Bei Patienten im UICC-Stadium II und III, die keine neoadjuvante Radiochemotherapie oder KurzzeitRadiotherapie erhalten haben, soll eine adjuvante Radiochemotherapie erfolgen.

(2008: Empfehlung 8.15.)
Im UICC-Stadium I (pTl/2N0) soll nach RO Resektion eine adjuvante Therapie nicht erfolgen. (2017: Empfehlung 8.28.)

Bei histopathologisch bestätigten Risikofaktoren für ein lokoregionäres Rezidiv (u.a. R1-Resektion, intraoperativer Tumoreinriss, pCRM+, unzureichender TME-Qualität, pT4, pT3c/d, pN2, extranodale Tumorherde im Mesorektum, pT3 im unteren Rektumdrittel) sollte eine adjuvante Radiochemotherapie durchgeführt werden. (2017: Empfehlung 8.29.)

Wird nach primärer R0-Resektion im Stadium II/III keine adjuvante Radiochemotherapie durchgeführt, sollte eine adjuvante Chemotherapie analog der Indikationskriterien und Schemata beim Kolonkarzinom erfolgen.

(2017: Empfehlung 8.30.) 


\section{Version 1 (2013)}

Die adjuvante Therapie sollte 4-6 Wochen nach der Operation beginnen.

(2008: Empfehlung 8.17.)

Die Strahlentherapie kann zeitgleich zum 1. und 2. Chemotherapiezyklus oder zum 3. und 4. Zyklus erfolgen.

(2008: Empfehlung 8.18.)

Die Strahlentherapie soll mit einer 5-FUMonochemotherapie kombiniert werden.

(2008: Empfehlung 8.19.)

Standard für die adjuvante Therapie des Rektumkarzinoms ist die kombinierte Radiochemotherapie. Eine Indikation für eine alleinige (adjuvante) Chemo- oder Radiotherapie beim Rektumkarzinom besteht nicht. Eine Ausnahme stellt nur die Kontraindikation gegen eine der beiden Therapieformen dar.

(2008: Empfehlung 8.20.)

Nach neoadjuvanter Radiochemotherapie ist eine adjuvante Chemotherapie unabhängig vom postoperativen Tumorstadium (also auch bei kompletter Remission oder UICC-Stadium I und II) indiziert.

(2008: Empfehlung 8.21.)

Die adjuvante Chemotherapie sollte entweder als 5-FU Monotherapie oder als Kombination aus 5FU/Folinsäure durchgeführt werden.

(2008: Empfehlung 8.22.)

\section{Version $2.0(2017)$}

Eine Empfehlung für oder gegen eine adjuvante Chemotherapie nach erfolgter neoadjuvanter Radiochemotherapie kann auf Grundlage der vorhandenen Datenlage beim Rektumkarzinom nicht geben werden.

(2017: Empfehlung 8.31.)

gestrichen

gestrichen

gestrichen

gestrichen

gestrichen

\section{gestrichen}

Grundsätzlich sollen die Patienten im Laufe ihrer Erkrankung Zugang zu allen Therapiemodalitäten, vorzugsweise in zertifizierten Zentren, haben.

(2017: Empfehlung 9.1.) 


\section{Version 1 (2013)}

Kapitel 9.2. Initiale molekularbiologische Diagnostik vor Therapieeinleitung (Version 2017)

Die Bestimmung von (ALL) RAS- und BRAFMutationen soll möglichst noch vor Einleitung der Erstlinientherapie erfolgen.

(2017: Empfehlung 9.4.)

Die Bestimmungen des RAS-Mutationsstatus können entweder am Primär-tumorgewebe oder an Metastasen erfolgen. Falls die Bestimmung des RAS-Mutationsstatus aus dem Gewebe nicht möglich ist, kann in Betracht gezogen werden, den RAS-Mutationsstatus aus der im Blut zirkulierenden Tumor-DNA zu ermitteln.

(2017: Empfehlung 9.5.)

\section{Kapitel 9.3. Pharmakogenetische Diagnostik vor Beginn der Erstlinientherapie (Version 2017)}

Die regelhafte Bestimmung von UGT1Al vor Beginn einer palliativen CTX mit Irinotecan wird nicht empfohlen. Sie kann aber durchgeführt werden, V. a. bei Morbus Meulengracht oder anderen Bilirubin-Konjugationsstörungen.

(2017: Empfehlung 9.6.)

Die Bestimmung des DPD-Mangels ist eine diagnostische Option vor Beginn einer 
Version 1 (2013)

Kapitel 9.6. Behandlung von Patienten, die nicht für eine intensive Therapie in Frage kommen (Version 2017)

\section{Version $2.0(2017)$}

Fluoropyrimidin-Therapie. Die regelhafte Evaluation des DPYD*2A -Polymorphismus kann durchgeführt werden.

(2008: Empfehlung 9.7.)

\begin{tabular}{|l|l|}
\hline $\begin{array}{l}\text { Bei primär resektabler Metastasierung soll die } \\
\text { Operationsfähigkeit geprüft werden. Ist diese } \\
\text { primär nicht gegeben, so sollte die Operabilität / } \\
\text { Resektabilität in regelmäßigen, nachfolgenden } \\
\text { Untersuchungen (z.B. alle } 8 \text { Wochen) geklärt } \\
\text { werden. } \\
\text { (2017: Empfehlung 9.8.) }\end{array}$ \\
$\begin{array}{l}\text { Bei reduziertem Allgemeinzustand, der eine } \\
\text { intensive Chemotherapie nicht erlaubt, steht eine } \\
\text { primär palliative, symptomatische Behandlung im } \\
\text { Vordergrund. } \\
\text { Es kann eine initiale Behandlung mit einem } \\
\text { Fluoropyrimidin + Bevacizumab oder eine } \\
\text { dosisreduzierten Chemotherapiedoublette (t) } \\
\text { Bevacizumab) durchgeführt werden. Bei Vorliegen } \\
\text { von RAS-WT Tumoren im linksseitigen Kolon (ab } \\
\text { linke Flexur) oder im Rektum kann darüber hinaus } \\
\text { eine anti-EGFR-Monotherapie erfolgen. } \\
\text { (2017: Empfehlung 9.9.) }\end{array}$ \\
\hline $\begin{array}{l}\text { Wenn der schlechte Allgemeinzustand vorrangig } \\
\text { durch die Tumorerkrankung bedingt ist, kann } \\
\text { auch bei schlechtem Performance Status (ECOG } \\
>1) \text { nach Abschätzung aller Risiken eine } \\
\text { intensivierte Tumortherapie primär zum Einsatz } \\
\text { kommen. } \\
\text { (2017: Empfehlung 9.10.) }\end{array}$ \\
\hline
\end{tabular}

Kapitel 9.7. Behandlung von Patienten, die für eine intensive Therapie geeignet sind (Version 2017)

Grundsätzlich sollen die Patienten im Laufe ihrer Therapie Zugang zu allen verfügbaren Medikamenten haben.

(2008: Empfehlung 9.11.)
Grundsätzlich sollen Patienten Zugang zu der effektivsten und noch tolerablen Therapie haben. Wenn eine kurative Zielsetzung besteht und keine Einschränkungen hinsichtlich der (potentiellen) Therapieauswahl vorliegen, sollten zur Festlegung des optimalen multimodalen Vorgehens prinzipiell folgende Parameter in die Entscheidungsfindung miteinbezogen werden: 


\section{Version 1 (2013)}

Resektable Lungenmetastasen sollen reseziert werden.

(2008: Empfehlung 9.1.)

Auf die Leber beschränkte R0-resektable Metastasen sollen reseziert werden.

(2008: Empfehlung 9.2.)

Die Resektabilität von Metastasen soll durch einen in der Metastasenchirurgie erfahrenen Chirurgen beurteilt werden.

(2008: Empfehlung 9.3.)

\section{Version $2.0(2017)$}

chirurgische Kriterien (Operabilität, Resektabilität inklusive lokal ablativer Verfahren)

prognostische Kriterien

(2017: Empfehlung 9.11.)

Bei resektablen Tumormanifestationen und prognostisch günstigen Kriterien soll die primäre Metastasenresektion durchgeführt werden.

(2017: Empfehlung 9.12.)

Beurteilung soll durch ein Tumorboard unter Beteiligung eines in der Metastasenchirurgie erfahrenen Chirurgen erfolgen.

(2017: Empfehlung 9.13.)

Bei primär resektabler Erkrankung und prognostisch ungünstigen Kriterien (z.B. kurzes krankheitsfreies Intervall oder synchrone Metastasierung) kann primär eine systemische Therapie erfolgen.

(2017: Empfehlung 9.14.)

Kann durch die systemische Therapie eine Stabilisierung der Erkrankung erreicht werden, so soll die Resektion möglichst zeitnah (d.h. nach 2 3 Monaten) angestrebt werden.

(2017: Empfehlung 9.15.)

Kleine Metastasen $(\leq 1 \mathrm{~cm})$ können primär entfernt werden, da diese unter einer initialen Chemotherapie möglicherweise verschwinden würden und dann durch den Chirurgen intraoperativ nicht mehr aufgefunden werden. (2017: Empfehlung 9.16.)

Eine neoadjuvante systemische Therapie Die neoadjuvante Therapie von primär resektablen Lebermetastasen sollte nicht durchgeführt werden.

(2017: Empfehlung 9.17.)

Ob bei einer Leberresektion nach Chemotherapie auch die Segmente mit nicht mehr nachweisbaren Metastasen reseziert werden müssen, kann derzeit mangels ausreichender Evidenz nicht konkret beantwortet werden.

(2017: Empfehlung 9.18.) 


\section{Version 1 (2013)}

Nach R0-Resektion synchroner oder metachroner Lebermetastasen kann eine adjuvante Chemotherapie erwogen werden.

(2008: Empfehlung 9.7.)

Bei primärer Irresektabilität soll eine systemische Chemotherapie erfolgen.

(2008: Empfehlung 9.12.)

Eine medikamentöse Tumortherapie ist grundsätzlich indiziert, da ein Überlebensvorteil nachgewiesen ist.

(2008: Empfehlung 9.8.)

Besteht die Indikation zu einer medikamentösen Tumortherapie, so soll diese zum Zeitpunkt des Nachweises der Metastasen unabhängig von metastasenbezogenen Symptomen eingeleitet werden. Bei der Indikationsstellung sind mögliche Kontraindikationen zu berücksichtigen. Alter per se stellt keine Kontraindikation dar.

(2008: Empfehlung 9.9.)

Bei Indikation zur systemischen Therapie (z. B. inoperable Leber-/Lungenfiliae) kann der Primärtumor belassen werden. Ausnahmen können ein symptomatisch stenosierendes Tumorwachstum und/oder eine Hb-relevante Blutung sein.

(2008: Empfehlung 9.10.

Bei primär irresektablen Lebermetastasen soll eine systemische Therapie begonnen werden. Wichtig ist die regelmäßige Evaluation einer möglichen sekundären Resektabilität nach Remissionsinduktion. Ist das Therapieziel die Remissionsinduktion mit sekundärer Metastasenresektion, dann soll primär die effektivste jeweils verfügbare systemische Kombinationstherapie angewandt werden (intensivierte Therapie).

(2008: Empfehlung 9.13.)

Die Hepatotoxizität o.a. Protokolle z. B. „Blue Liver"/Chemotherapieassoziierte Steatohepatitis

\section{Version $2.0(2017)$}

Eine adjuvante/additive Chemotherapie nach Resektion von Metastasen sollte nicht erfolgen. (2017: Empfehlung 9.19.)

Bei primärer Irresektabilität soll zunächst eine systemische Tumortherapie erfolgen. Entsprechend der Tumor- und Patientencharakteristika soll die wirksamste Therapie an den Anfang der Behandlung gestellt werden.

(2017: Empfehlung 9.20.)

gestrichen

gestrichen

\section{gestrichen}




\section{Version 1 (2013)}

(CASH) sollte dabei in die differentialtherapeutische Entscheidung und OPPlanung miteinbezogen werden.

(2008: Empfehlung 9.14.)

Intraoperativ sollte eine Exploration der Leber anhand der Metastasenlokalisation in der Ausgangs-Bildgebung erfolgen. Es sollte eine chirurgische Therapie aller Ausgangsläsionen angestrebt werden.

(2008: Empfehlung 9.15.)

Eine RFA kann durchgeführt werden, wenn nicht resektable Lebermetastasen vorliegen oder der Allgemeinzustand des Patienten eine Resektion nicht zulässt, insbesondere nach vorangegangener Leberresektion.

(2008: Empfehlung 9.16.)

Eine SIRT zur Behandlung von disseminierten Lebermetastasen bei KRK sollte nur bei Patienten, für die keine andere Therapieoption infrage kommt, und dann nur innerhalb klinischer Studien durchgeführt werden.

(2008: Empfehlung 9.17.)

Eine LITT zur Behandlung von Lebermetastasen bei KRK sollte nur innerhalb klinischer Studien durchgeführt werden.

(2008: Empfehlung 9.18.)
Version $2.0(2017)$

Im Kapitel 9.1 gestrichen, aber im Kapitel 7.5.2.3 beibehalten.

Im Kapitel 9.1 gestrichen, aber im Kapitel 7.5.2.3 beibehalten.

Im Kapitel 9.1 gestrichen, aber im Kapitel 7.5.2.3 beibehalten.

Kapitel 9.8. Wahl der systemischen Therapie in Abhängigkeit von der molekularpathologischen Subgruppe und der Tumorlokalisation (Version 2017)

Patienten, die in einer erweiterten RAS-Analytik (KRAS und NRAS, Exone 2-4) einen RAS-Wildtyp (RAS-wt) zeigen und eine linksseitige Lokalisation des Primärtumors (Kolonkarzinom) aufweisen, sollen in der Erstlinientherapie der metastasierten Erkrankung präferentiell mit einer Chemotherapie-Doublette plus anti-EGFRTherapie behandelt werden.

(2017: Empfehlung 9.21.)

Bei Vorliegen einer RAS Mutation sollte primär eine Chemotherapie-Doublette zum Einsatz kommen. $\mathrm{Ob}$ eine Triplette besser als eine Doublette ist, oder ob Bevacizumab zum Einsatz kommen sollte, ist nicht belegt. 
Version 1 (2013)

\section{Version $2.0(2017)$}

(2017: Empfehlung 9.22.)

Liegt eine BRAF-Mutation vor, sollte primär eine möglichst effektive Chemotherapie z. B. mit einer Triplette oder der Einschluss in eine klinische Studie erfolgen.

(2017: Empfehlung 9.23.)

\section{Kapitel 9.9. Durchführung der Erstlinienchemotherapie (Version 2017)}

Patienten mit tumorbedingten Symptomen, Organkomplikationen oder raschem Progress sollten unter Berücksichtigung des Allgemeinzustandes des Patienten eine möglichst effektive Kombinationstherapie erhalten (intensivierte Therapie).

(2008: Empfehlung 9.19.)

Bei Patienten mit multiplen Metastasen ohne Option für Resektion nach Metastasenrückbildung ohne tumorbezogene Symptome oder Organkomplikationen und/oder schwerer Komorbidität kann eine Monotherapie als Erstlinientherapie eingesetzt werden.

(2008: Empfehlung 9.20.)

Für den Fall, dass eine FluoropyrimidinMonotherapie gegeben wird, sollte eine orale der intravenösen 5-FU-Gabe vorgezogen werden. Bei den zur Auswahl stehenden Infusionsprotokollen sollte dem de Gramont-Schema der Vorzug gegenüber dem AIO-Schema gegeben werden, da das de Gramont-Schema bei wahrscheinlich gleicher Wirksamkeit durch die 14-tägige Applikation eine geringere Belastung für den Patienten darstellt.

(2008: Empfehlung 9.21.)
In der Erstlinienchemotherapie sollen bei gutem Allgemeinzustand und hoher Motivation in erster Linie Fluoropyrimidin-basierte Kombinationsregime mit infusionaler Verabreichung von 5-Fluorouracil, wie FOLFIRI, FOLFOX oder FOLFOXIRI oder mit dem oralen Fluoropyrimidin Capecitabin (vorwiegend mit Oxaliplatin, (APOX) eingesetzt werden. (2017: Empfehlung 9.24.) 


\section{Version 1 (2013)}

\section{Version 2.0 (2017)}

Die Kombination mit einer zielrichtet wirksamen Substanz (anti-EGFR oder anti-VEGF) sollte sich in erster Linie nach den vorrangigen Therapiezielen, den molekularbiologischen Charakteristika des Tumors und der Tumorlokalisation richten. Im Vordergrund der Therapieentscheidungen steht die Frage nach der Behandlung, mit der bei akzeptabler Verträglichkeit das längste Gesamtüberleben erreicht werden kann.

(2017: Empfehlung 9.25.)

Bei herabgesetztem Allgemeinzustand können in Hinblick auf die Chemotherapie FluoropyrimidinMonotherapien (5-Fluorouracil/Folinsäure oder Capecitabin) gewöhnlich in Kombination mit Bevacizumab eingesetzt werden.

(2017: Empfehlung 9.26.)

FOLFOXIRI gehört zu den effektivsten Chemotherapie-Regimen, sollte aber aufgrund seiner erhöhten Nebenwirkungsrate nur bei Patienten mit gutem Allgemeinzustand (ECOC Performance Status 0-1) zum Einsatz kommen. (2017: Empfehlung 9.27.)

Die Zugabe von anti-EGFR AK (Cetuximab oder Panitumumab) zu einer Chemo-therapie führt zu einer signifikanten Effektivitätssteigerung in Hinblick auf ORR, PFS und OS. Anti-EGFR-AK sollen erst bei Nachweis eines all-RAS-Wildtyps im Tumor gegeben werden.

(2017: Empfehlung 9.28.)

Die Zugabe von Bevacizumab zu einer infusionalen Kombinationschemotherapie führt in der meta-analytischen Aufarbeitung der Studiendaten $\mathrm{zu}$ einer signifikanten Effektivitätssteigerung in Hinblick auf PFS, aber nicht auf ORR und OS. Dagegen führt die Zugabe von Bevacizumab zu einer Monochemotherapie mit einem Fluoro-pyrimidin zu einer signifikanten Effektivitätssteigerung in Hinblick auf ORR, PFS und OS.

(2017: Empfehlung 9.29.)

Die Kombinationstherapie mit einem anti-EGFR-AK plus VEGF-Signalweg-Inhibitor soll nicht eingesetzt werden. 


\section{Version 1 (2013)}

Es gibt keine ausreichende Evidenz, die ein Absetzen einer einmal begonnenen medikamentösen Therapie bis zum erneuten Progress rechtfertigen würde. (2008: Empfehlung 9.22.)

Kapitel 9.10. Zweitlinientherapie (Version 2017)

Kapitel 9.11. Therapiesequenz (Version 2017)

\section{Version 2.0 (2017)}

(2017: Empfehlung 9.30.)

Eine Oxaliplatin-basierte Induktionstherapie (FOLFOX, CAPOX, FOLFOXIRI) sollte nach Möglichkeit über einen Zeitraum von 4 - 6 Monaten durchgeführt werden, bevor auf eine Oxaliplatin-freie Behandlung deeskaliert wird.

Limitierend für den Einsatz von Oxaliplatin sind nicht nur allergische Reaktionen, sondern auch die Entwicklung einer peripheren Polyneuropathie, deren Inzidenz und Schwere mit der kumulativen Dosis von Oxaliplatin zunimmt. (2017: Empfehlung 9.31.)

Nach einer Induktionschemotherapie kann die Behandlung pausiert oder in eine Erhaltungstherapie deeskaliert werden.

(2017: Empfehlung 9.32.)

Die Effektivität der Zweitlinientherapie ist gewöhnlich deutlich geringer als die der Erstlinientherapie. Die Wahl einer Zweitlinientherapie sollte sich im Rahmen des sequenziellen Einsatzes aktiver Substanzen in erster Linie nach der Effektivität und den Nebenwirkungen der Vortherapie richten.

(2017: Empfehlung 9.33.)

Bei Patienten mit RAS Wildtyptumoren ist die Lokalisation des Primärtumors eine wichtige Determinante in der Beurteilung der optimalen Therapiesequenz. Bei Patienten mit linksseitigem mKRK und RAS Wildtyp sollte in der Erstlinientherapie ein anti-EGFR-AK in Kombination mit einer Chemotherapie zum Einsatz kommen. In dieser Konstellation kommt eine anti-VEGF Therapie erst ab der Zweitlinie in Betracht.

(2017: Empfehlung 9.34) 


\section{Version 1 (2013)}

\section{Kapitel 9.12. Chemotherapie in späteren Therapielinien (Version 2017)}

Trifluridin/Tipiracil sollte bei Patienten, welche alle verfügbaren Chemotherapien/ Antikörper durchlaufen haben oder für diese nicht geeignet sind, eingesetzt werden.

(2017: Empfehlung 9.36.)

Regorafenib kann bei mit allen verfügbaren Chemotherapien/Antikörpern Patienten eingesetzt werden.

(2017: Empfehlung 9.37.)

Kapite 9.13. Lokal ablative Verfahren (Version 2017)

Lokal ablative Verfahren können eingesetzt werden, wenn nicht resektable Metastasen vorliegen oder der Allgemeinzustand des Patienten eine Resektion nicht zulässt, insbesondere nach vorangegangener Lebermetastasenresektion.

(2017: Empfehlung 9.38.)

Kapitel 9.14. Lokoregionär wirksame Verfahren (Version 2017)

Bei Patienten mit einer isolierten und limitierten Peritonealkarzinose kann eine zytoreduktive Chirurgie gefolgt von einer hyperthermen intraperitonealen Chemotherapie (HIPEC) durchgeführt werden, wenn folgenden Voraussetzungen erfüllt sind:

- $\quad \mathrm{PCl}$ (peritoneal cancer index) $<20$

- keine extraabdominellen Metastasen
Eine SIRT kann zur Behandlung von disseminierten Lebermetastasen bei KRK bei solchen Patienten eingesetzt werden, für die keine andere gleichwertige Therapieoption in Frage kommt.

(2017: Empfehlung 9.39.)

Bei Patienten mit einer isolierten und limitierten Peritonealkarzinose kann eine zytoreduktive Chirurgie gefolgt von einer hyperthermen intraperitonealen Chemotherapie (HIPEC) durchgeführt werden, wenn folgende Voraussetzungen erfüllt sind:

- $\quad \mathrm{PCl}$ (peritoneal cancer index) $<20$

- keine extraabdominellen Metastasen 


\section{Version 1 (2013)}

- Möglichkeit der makroskopisch kompletten Entfernung oder Destruktion jeglicher Tumormanifestation

- Therapie in einem spezialisierten Zentrum

Die Durchführung im Rahmen von Studien sollte bevorzugt werden.

\section{Version 2.0 (2017)}

- Möglichkeit der makroskopisch kompletten Entfernung oder Destruktion jeglicher Tumormanifestation

- Therapie in einem spezialisierten Zentrum

Die Durchführung der HIPEC soll im Rahmen von Studien erfolgen. (2017: Empfehlung 9.40.)

Kapitel 9.15. Therapiebegleitendes interprofessionelles Management von Symptomen, Nebenwirkungen und Toxizitäten

Unter Chemotherapie bei Metastasierung und in der palliativen Situation soll bei allen Patienten ein regelmäßiges Assessment krankheits- und therapieinduzierter Nebenwirkungen sowie eine gezielte Symptombehandlung durchgeführt werden. Primäres Ziel ist eine Verlängerung des progressionsfreien und des Gesamt-überlebens bei geringer Toxizität und guter Lebensqualität.

Eine Anleitung der Patienten zu einem effektiven Selbst-Management der Symptome soll regelmäßig durchgeführt werden.

(2017: Empfehlung 9.41.)

\section{Altes Kapitel 9.1: Primär resektable Lungenmetastasen (Version 2013)}

Eine PET-CT kann bei Patienten mit resektablen Lebermetastasen eines kolorektalen Karzinoms durchgeführt werden.

(2008: Empfehlung 9.4.)

Eine PET-CT sollte nicht innerhalb von 4 Wochen nach Gabe einer systemischen Chemotherapie oder Antikörpertherapie durchgeführt werden, da die Sensitivität deutlich reduziert ist.

(2008: Empfehlung 9.5.)

Aufgrund unzureichender Evidenz soll mit Ausnahme der Fluoropyrimidine oder der Gabe von Irinotecan in Kombination mit Cetuximab nach Versagen einer irinotecanhaltigen Therapie keines der oben beschriebenen Therapeutika nach dokumentiertem Progress unter Therapie weiter appliziert werden. Dies gilt auch für Cetuximab und Bevacizumab.

(2008: Empfehlung 9.23.)
Im Kapitel 9.1 gestrichen, aber im Kapitel 7.4.2.3 beibehalten.

Im Kapitel 9.1 gestrichen, aber im Kapitel 7.4.2.3 beibehalten.

Hintergrundtext siehe Kapitel 9.12.4 Re-Induktion / Re-, ,challenge“ 


\section{Abbildungsverzeichnis}

Abbildung 1: Algorithmus zum Ablauf der genetischen Diagnostik bei Patienten mit V.a. ein hereditäres Tumordispositions-Syndrom am Beispiel des HNPCC-/Lynch-Syndroms. Zum Nachweis der MSI bei V.a. HNPCC-/Lynch-Syndroms wird auf Abbildung 2 verwiesen.

Abbildung 2: Algorithmus zum Ablauf der molekularpathologischen Abklärung eines MismatchReparaturdefektes bei klinischem V.a. HNPCC-/Lynch-Syndrom. Zur sich ggf. anschließenden genetischen Diagnostik wird auf Abbildung 1 verwiesen.

Abbildung 3: Staging beim kolorektalen Karzinom

\section{Tabellenverzeichnis}

Tabelle 1: Beteiligte Fachgesellschaften und Organisationen ................................................. 13

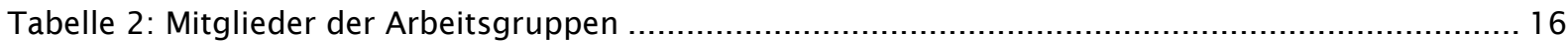

Tabelle 3: Schema der Evidenzgraduierung nach Oxford (Version 2009) .......................................22

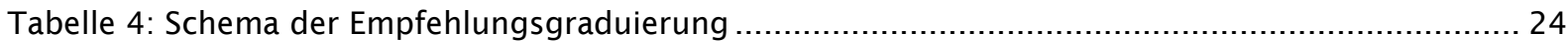

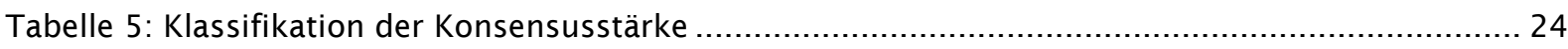

Tabelle 6: Empfohlenes Krebsfrüherkennungsprogramm bei HNPCC ..................................... 81

Tabelle 7: Klassifikation der Ausprägung der Duodenalpolyposis nach der Spigelman-Klassifikation (modifiziert nach [310]) ........................................................................... 82

Tabelle 8:Übersicht leitlinienbasierte prozedurenspezifischen Qualitätsparameter der Koloskopie......84 84

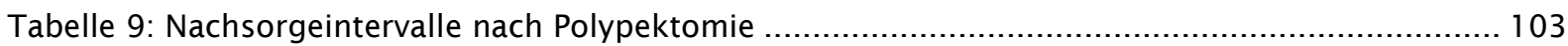
Tabelle 10: Randomisierte Studien zur Erstlinientherapie mit FOLFOXIRI bei unselektierten Patienten208 Tabelle 11: Randomisierte Studien zur Erstlinientherapie mit anti-EGFR Substanzen bei RAS-wt

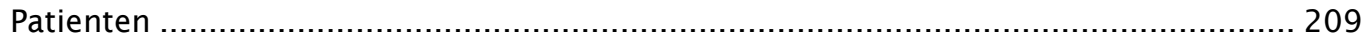

Tabelle 12: Zusammenfassung der Meta-Analysen zu anti-EGFR Substanzen - OS ............................2 210

Tabelle 13 Zusammenfassung der Meta-Analysen zu anti-EGFR Substanzen - PFS ........................ 210

Tabelle 14: Randomisierte Studien zur Erstlinientherapie mit Bevacizumab bei unselektierten

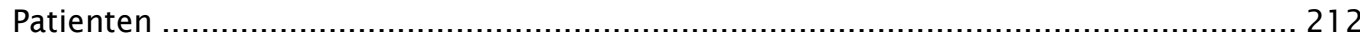

Tabelle 15: Zusammenfassung der Meta-Analysen zu Bevacizumab - OS ......................................213

Tabelle 16: Zusammenfassung der Meta-Analysen zu Bevacizumab - PFS ................................. 214

Tabelle 17: Randomisierte Studien zur Zweitlinientherapie mit anti-VEGF Substanzen ................... 218

Tabelle 18: Randomisierte Studien zur Zweitlinientherapie mit anti-EGFR Substanzen .................... 219

Tabelle 19: Programmierte Untersuchungen im Rahmen der Nachsorge bei kolorektalem Karzinom

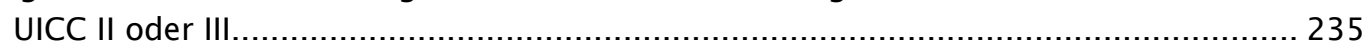

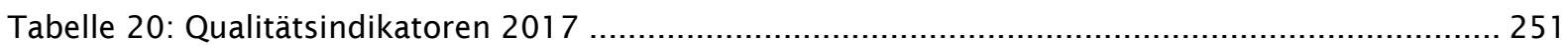

Tabelle 21: UICC-Klassifikation des Kolorektalen Karzinoms ............................................... 256 


\section{Literaturverzeichnis}

1. Arbeitsgemeinschaft der Wissenschaftlichen Medizinischen Fachgesellschaften - Ständige Kommission Leitlinien. AWMF-Regelwerk "Leitlinien". 1. Auflage 2012 [cited 2013 09.12.]; Available from: http://www.awmf.org/leitlinien/awmf-regelwerk/awmf-regelwerk.html.

2. Kirkegaard, H., et al., Association of adherence to lifestyle recommendations and risk of colorectal cancer: a prospective Danish cohort study. BMJ, 2010.341 : p. c5504.

3. Halle, M. and M.H. Schoenberg, Physical activity in the prevention and treatment of colorectal carcinoma. Dtsch Arztebl Int, 2009. 106(44): p. 722-7.

4. Wolin, K.Y., et al., Physical activity and colon cancer prevention: a meta-analysis. Br J Cancer, 2009. 100(4): p. 611-6.

5. Wolin, K.Y., Y. Yan, and G.A. Colditz, Physical activity and risk of colon adenoma: a metaanalysis. Br J Cancer, 2011 . 104(5): p. 882-5.

6. Wolin, K.Y., et al., Leisure-time physical activity patterns and risk of colon cancer in women. Int J Cancer, 2007. 121(12): p. 2776-81.

7. Friedenreich, C., et al., Physical activity and risk of colon and rectal cancers: the European prospective investigation into cancer and nutrition. Cancer Epidemiol Biomarkers Prev, 2006. 15(12): p. 2398-407.

8. Lubin, F., et al., Nutritional and lifestyle habits and water-fiber interaction in colorectal adenoma etiology. Cancer Epidemiol Biomarkers Prev, 1997. 6(2): p. 79-85.

9. Giacosa, A., et al., Energy intake, overweight, physical exercise and colorectal cancer risk. Eur J Cancer Prev, 1999. 8 Suppl 1: p. S53-60.

10. Friedenreich, C.M. and M.R. Orenstein, Physical activity and cancer prevention: etiologic evidence and biological mechanisms. J Nutr, 2002. 132(11 Suppl): p. 3456S-3464S

11. Terry, M.B., et al., Risk factors for advanced colorectal adenomas: a pooled analysis. Cancer Epidemiol Biomarkers Prev, 2002. 11(7): p. 622-9.

12. Lee, I.M., Physical activity and cancer prevention--data from epidemiologic studies. Med Sci Sports Exerc, 2003. 35(11): p. 1823-7.

13. Wei, E.K., et al., Comparison of risk factors for colon and rectal cancer. Int J Cancer, 2004. 108(3): p. 433-42.

14. Martinez, M.E., et al., Physical activity, body mass index, and prostaglandin E2 levels in rectal mucosa. J Natl Cancer Inst, 1999. 91(11): p. 950-3

15. Giovannucci, E., Modifiable risk factors for colon cancer. Gastroenterol Clin North Am, 2002. 31 (4): p. 925-43.

16. Giovannucci, E., Diet, body weight, and colorectal cancer: a summary of the epidemiologic evidence. J Womens Health (Larchmt), 2003. 12(2): p. 173-82.

17. Renehan, A.G., et al., Body-mass index and incidence of cancer: a systematic review and metaanalysis of prospective observational studies. Lancet, 2008. 371(9612): p. 569-78.

18. Pischon, T., et al., Body size and risk of colon and rectal cancer in the European Prospective Investigation Into Cancer and Nutrition (EPIC). J Natl Cancer Inst, 2006. 98(13): p. 920-31.

19. Ning, Y., L. Wang, and E.L. Giovannucci, A quantitative analysis of body mass index and colorectal cancer: findings from 56 observational studies. Obes Rev, 2010. 11(1): p. 19-30.

20. Terry, M.B. and A.I. Neugut, Cigarette smoking and the colorectal adenoma-carcinoma sequence: a hypothesis to explain the paradox. Am J Epidemiol, 1998. 147(10): p. 903-10.

21. Almendingen, K., et al., Smoking and colorectal adenomas: a case-control study. Eur J Cancer Prev, 2000. 9(3): p. 193-203.

22. Chao, A., et al., Cigarette smoking and colorectal cancer mortality in the cancer prevention study II. J Natl Cancer Inst, 2000. 92(23): p. 1888-96.

23. Giovannucci, E., An updated review of the epidemiological evidence that cigarette smoking increases risk of colorectal cancer. Cancer Epidemiol Biomarkers Prev, 2001. 10(7): p. 725-31.

24. Reid, M.E., et al., Smoking exposure as a risk factor for prevalent and recurrent colorectal adenomas. Cancer Epidemiol Biomarkers Prev, 2003. 12(10): p. 1006-11.

25. Botteri, E., et al., Cigarette smoking and adenomatous polyps: a meta-analysis. Gastroenterology, 2008. 134(2): p. 388-95.

26. Botteri, E., et al., Smoking and colorectal cancer: a meta-analysis. JAMA, 2008. 300(23): p. 2765-78.

27. Miller, P.E., et al., Dietary patterns and colorectal adenoma and cancer risk: a review of the epidemiological evidence. Nutr Cancer, 2010. 62(4): p. 413-24.

28. Cottet, V., et al., Dietary patterns and the risk of colorectal adenoma recurrence in a European intervention trial. Eur J Cancer Prev, 2005. 14(1): p. 21-9.

29. Mai, V., et al., Diet quality and subsequent cancer incidence and mortality in a prospective cohort of women. Int J Epidemiol, 2005. 34(1): p. 54-60. 
30. Sansbury, L.B., et al., The effect of strict adherence to a high-fiber, high-fruit and -vegetable, and low-fat eating pattern on adenoma recurrence. Am J Epidemiol, 2009. 170(5): p. 576-84.

31. Wakai, K., et al., Dietary risk factors for colon and rectal cancers: a comparative case-control study. J Epidemiol, 2006. 16(3): p. 125-35.

32. Beresford, S.A., et al., Low-fat dietary pattern and risk of colorectal cancer: the Women's Health Initiative Randomized Controlled Dietary Modification Trial. JAMA, 2006. 295(6): p. 64354.

33. Lanza, E., et al., The polyp prevention trial continued follow-up study: no effect of a low-fat, high-fiber, high-fruit, and-vegetable diet on adenoma recurrence eight years after randomization. Cancer Epidemiol Biomarkers Prev, 2007. 16(9): p. 1745-52.

34. Dahm, C.C., et al., Dietary fiber and colorectal cancer risk: a nested case-control study using food diaries. J Natl Cancer Inst, 2010. 102(9): p. 614-26.

35. Ishikawa, H., et al., Randomized trial of dietary fiber and Lactobacillus casei administration for prevention of colorectal tumors. Int J Cancer, 2005. 116(5): p. 762-7.

36. Jacobs, E.T., et al., Fiber, sex, and colorectal adenoma: results of a pooled analysis. Am J Clin Nutr, 2006. 83(2): p. 343-9.

37. Park, Y., et al., Dietary fiber intake and risk of colorectal cancer: a pooled analysis of prospective cohort studies. JAMA, 2005. 294(22): p. 2849-57.

38. Robertson, D.J., et al., Fat, fiber, meat and the risk of colorectal adenomas. Am J Gastroenterol, 2005. 100(12): p. 2789-95.

39. Austin, G.L., et al., Moderate alcohol consumption protects against colorectal adenomas in smokers. Dig Dis Sci, 2008. 53(1): p. 116-22.

40. Cho, E., et al., Alcohol intake and colorectal cancer: a pooled analysis of 8 cohort studies. Ann Intern Med, 2004. 140(8): p. 603-13.

41. Mizoue, T., et al., Alcohol drinking and colorectal cancer in Japanese: a pooled analysis of results from five cohort studies. Am J Epidemiol, 2008. 167(12): p. 1397-406.

42. Moskal, A., et al., Alcohol intake and colorectal cancer risk: a dose-response meta-analysis of published cohort studies. Int J Cancer, 2007. 120(3): p. 664-71.

43. Chao, A., et al., Meat consumption and risk of colorectal cancer. JAMA, 2005. 293(2): p. 172 82.

44. Larsson, S.C. and A. Wolk, Meat consumption and risk of colorectal cancer: a meta-analysis of prospective studies. Int J Cancer, 2006. 119(11): p. 2657-64.

45. Mathew, A., et al., Meat intake and the recurrence of colorectal adenomas. Eur J Cancer Prev, 2004. 13(3): p. 159-64.

46. Norat, T., et al., Meat, fish, and colorectal cancer risk: the European Prospective Investigation into cancer and nutrition. J Natl Cancer Inst, 2005. 97(12): p. 906-16.

47. Sinha, R., et al., Meat, meat cooking methods and preservation, and risk for colorectal adenoma. Cancer Res, 2005. 65(17): p. 8034-41.

48. Geelen, A., et al., Fish consumption, $n-3$ fatty acids, and colorectal cancer: $a$ meta-analysis of prospective cohort studies. Am J Epidemiol, 2007. 166(10): p. 1116-25.

49. Sugawara, Y., et al., Fish consumption and the risk of colorectal cancer: the Ohsaki Cohort Study. Br J Cancer, 2009. 101(5): p. 849-54.

50. Pot, G.K., et al., Fish consumption and markers of colorectal cancer risk: a multicenter randomized controlled trial. Am J Clin Nutr, 2009. 90(2): p. 354-61.

51. Je, Y., W. Liu, and E. Giovannucci, Coffee consumption and risk of colorectal cancer: a systematic review and meta-analysis of prospective cohort studies. Int J Cancer, 2009. 124(7): p. 1662-8.

52. Sun, C.L., et al., Green tea, black tea and colorectal cancer risk: a meta-analysis of epidemiologic studies. Carcinogenesis, 2006. 27(7): p. 1301-9.

53. Zhang, X., et al., Risk of colon cancer and coffee, tea, and sugar-sweetened soft drink intake: pooled analysis of prospective cohort studies. J Natl Cancer Inst, 2010. 102(11): p. 771-83.

54. Foschi, R., et al., Citrus fruit and cancer risk in a network of case-control studies. Cancer Causes Control, 2010. 21(2): p. 237-42.

55. Millen, A.E., et al., Fruit and vegetable intake and prevalence of colorectal adenoma in a cancer screening trial. Am J Clin Nutr, 2007. 86(6): p. 1754-64.

56. Koushik, A., et al., Fruits, vegetables, and colon cancer risk in a pooled analysis of 14 cohort studies. J Natl Cancer Inst, 2007. 99(19): p. 1471-83.

57. Limburg, P.J., et al., Prospective evaluation of trans-fatty acid intake and colorectal cancer risk in the lowa Women's Health Study. Int J Cancer, 2008. 123(11): p. 2717-9.

58. Alexander, D.D., et al., Meta-analysis of animal fat or animal protein intake and colorectal cancer. Am J Clin Nutr, 2009. 89(5): p. 1402-9.

59. Galeone, C., et al., Fried foods, olive oil and colorectal cancer. Ann Oncol, 2007. 18(1): p. 36-9. 
60. Nakamura, T., et al., Excessive fat restriction might promote the recurrence of colorectal tumors. Nutr Cancer, 2010. 62(2): p. 154-63.

61. Larsson, S.C., et al., Dietary acrylamide intake and risk of colorectal cancer in a prospective cohort of men. Eur J Cancer, 2009. 45(4): p. 513-6.

62. Hogervorst, J.G., et al., Dietary acrylamide intake is not associated with gastrointestinal cancer risk. J Nutr, 2008. 138(11): p. 2229-36.

63. Mucci, L.A. and K.M. Wilson, Acrylamide intake through diet and human cancer risk. J Agric Food Chem, 2008. 56(15): p. 6013-9.

64. Mucci, L.A., H.O. Adami, and A. Wolk, Prospective study of dietary acrylamide and risk of colorectal cancer among women. Int J Cancer, 2006. 118(1): p. 169-73.

65. Bjelakovic, G., et al., Mortality in randomized trials of antioxidant supplements for primary and secondary prevention: systematic review and meta-analysis. JAMA, 2007. 297(8): p. 842-57.

66. Weingarten, M.A., A. Zalmanovici, and J. Yaphe, Dietary calcium supplementation for preventing colorectal cancer and adenomatous polyps. Cochrane Database Syst Rev, 2008(1): p. CD003548.

67. Wactawski-Wende, J., et al., Calcium plus vitamin D supplementation and the risk of colorectal cancer. N Engl J Med, 2006. 354(7): p. 684-96.

68. Sanjoaquin, M.A., et al., Folate intake and colorectal cancer risk: a meta-analytical approach. Int J Cancer, 2005. 113(5): p. 825-8.

69. Wu, K., et al., A randomized trial on folic acid supplementation and risk of recurrent colorectal adenoma. Am J Clin Nutr, 2009. 90(6): p. 1623-31.

70. Jaszewski, R., et al., Folic acid supplementation inhibits recurrence of colorectal adenomas: a randomized chemoprevention trial. World J Gastroenterol, 2008. 14(28): p. 4492-8.

71. Cole, B.F., et al., Folic acid for the prevention of colorectal adenomas: a randomized clinical trial. JAMA, 2007. 297(21): p. 2351-9.

72. Logan, R.F., et al., Aspirin and folic acid for the prevention of recurrent colorectal adenomas. Gastroenterology, 2008. 134(1): p. 29-38.

73. Grau, M.V., et al., Prolonged effect of calcium supplementation on risk of colorectal adenomas in a randomized trial. J Natl Cancer Inst, 2007. 99(2): p. 129-36.

74. Wallace, K., et al., Effect of calcium supplementation on the risk of large bowel polyps. J Natl Cancer Inst, 2004. 96(12): p. 921-5.

75. Hartman, T.J., et al., The association of calcium and vitamin D with risk of colorectal adenomas. J Nutr, 2005. 135(2): p. 252-9.

76. Lin, J., et al., Total magnesium intake and colorectal cancer incidence in women. Cancer Epidemiol Biomarkers Prev, 2006. 15(10): p. 2006-9.

77. Jacobs, E.T., et al., Selenium and colorectal adenoma: results of a pooled analysis. J Natl Cancer Inst, 2004. 96(22): p. 1669-75.

78. Peters, U., et al., High serum selenium and reduced risk of advanced colorectal adenoma in a colorectal cancer early detection program. Cancer Epidemiol Biomarkers Prev, 2006. 15(2): p. 315-20.

79. Vinogradova, Y., et al., Risk of colorectal cancer in patients prescribed statins, nonsteroidal anti-inflammatory drugs, and cyclooxygenase-2 inhibitors: nested case-control study. Gastroenterology, 2007. 133(2): p. 393-402.

80. Bertagnolli, M.M., et al., Celecoxib for the prevention of sporadic colorectal adenomas. N Engl J Med, 2006. 355(9): p. 873-84.

81. Arber, N., et al., Celecoxib for the prevention of colorectal adenomatous polyps. N Engl J Med, 2006. 355(9): p. 885-95.

82. Baron, J.A., et al., A randomized trial of rofecoxib for the chemoprevention of colorectal adenomas. Gastroenterology, 2006. 131(6): p. 1674-82.

83. Bardou, M., A. Barkun, and M. Martel, Effect of statin therapy on colorectal cancer. Gut, 2010. 59(11): p. 1572-85.

84. Routine aspirin or nonsteroidal anti-inflammatory drugs for the primary prevention of colorectal cancer: U.S. Preventive Services Task Force recommendation statement. Ann Intern Med, 2007. 146(5): p. 361-4.

85. Flossmann, E. and P.M. Rothwell, Effect of aspirin on long-term risk of colorectal cancer: consistent evidence from randomised and observational studies. Lancet, 2007. 369(9573): p. 1603-13.

86. Rothwell, P.M., et al., Effect of daily aspirin on long-term risk of death due to cancer: analysis of individual patient data from randomised trials. Lancet, 2011 . 377(9759): p. 31-41.

87. Chan, A.T., et al., Long-term use of aspirin and nonsteroidal anti-inflammatory drugs and risk of colorectal cancer. JAMA, 2005. 294(8): p. 914-23. 
88. Hormone therapy for the prevention of chronic conditions in postmenopausal women: recommendations from the U.S. Preventive Services Task Force. Ann Intern Med, 2005. 142(10): p. 855-60.

89. Farquhar, C., et al., Long term hormone therapy for perimenopausal and postmenopausal women. Cochrane Database Syst Rev, 2009(2): p. CD004143.

90. Alberts, D.S., et al., Phase III trial of ursodeoxycholic acid to prevent colorectal adenoma recurrence. J Natl Cancer Inst, 2005. 97(11): p. 846-53.

91. DGGG, Hormontherapie (HT) in der Peri- und Postmenopause. 2009.

92. Birkner, B.R., [Evidence-based prevention of colorectal carcinoma]. Dtsch Med Wochenschr, 2003. 128(49): p. 2598-603.

93. Winawer, S.J., et al., Colorectal cancer screening: clinical guidelines and rationale. Gastroenterology, 1997. 112(2): p. 594-642.

94. Imperiale, T.F., et al., Results of screening colonoscopy among persons 40 to 49 years of age. $\mathrm{N}$ Engl J Med, 2002. 346(23): p. 1781-5.

95. Whitlock, E.P., et al., Screening for colorectal cancer: a targeted, updated systematic review for the U.S. Preventive Services Task Force. Ann Intern Med, 2008. 149(9): p. 638-58.

96. Stevens, T. and C.A. Burke, Colonoscopy screening in the elderly: when to stop? Am J Gastroenterol, 2003. 98(8): p. 1881-5.

97. Kirchgatterer, A., et al., [Colonoscopy and sigmoidoscopy in patients aged eighty years or older]. Z Gastroenterol, 2002. 40(12): p. 951-6.

98. Pox, C.P., et al., Efficacy of a nationwide screening colonoscopy program for colorectal cancer. Gastroenterology, 2012. 142(7): p. 1460-7.e2.

99. Zhang, B., A. Fattah, and H. Nakama, Characteristics and survival rate of elderly patients with colorectal cancer detected by immunochemical occult blood screening. Hepatogastroenterology, 2000. 47(32): p. 414-8.

100. Winawer, S.J., et al., Prevention of colorectal cancer by colonoscopic polypectomy. The National Polyp Study Workgroup. N Engl J Med, 1993. 329(27): p. 1977-81.

101. Citarda, F., et al., Efficacy in standard clinical practice of colonoscopic polypectomy in reducing colorectal cancer incidence. Gut, 2001. 48(6): p. 812-5.

102. Zauber, A.G., et al., Colonoscopic polypectomy and long-term prevention of colorectal-cancer deaths. N Engl J Med, 2012. 366(8): p. 687-96.

103. Hol, L., et al., Screening for colorectal cancer: randomised trial comparing guaiac-based and immunochemical faecal occult blood testing and flexible sigmoidoscopy. Gut, 2010. 59(1): p. 62-8.

104. Quintero, E., et al., Colonoscopy versus fecal immunochemical testing in colorectal-cancer screening. N Engl J Med, 2012. 366(8): p. 697-706.

105. Pox, C.P., et al., Efficacy of a Nationwide Screening Colonoscopy Program for Colorectal Cancer. Gastroenterology, 2012: p. 1460-7.e2.

106. Huppe, D., et al., [Effectiveness of screening colonoscopy in a community-based study]. Z Gastroenterol, 2008. 46(2): p. 193-200.

107. Kahi, C.J., et al., Effect of screening colonoscopy on colorectal cancer incidence and mortality. Clin Gastroenterol Hepatol, 2009. 7(7): p. 770-5; quiz 711.

108. Imperiale, T.F., et al., Risk of advanced proximal neoplasms in asymptomatic adults according to the distal colorectal findings. N Engl J Med, 2000. 343(3): p. 169-74.

109. Lieberman, D.A., et al., Use of colonoscopy to screen asymptomatic adults for colorectal cancer. Veterans Affairs Cooperative Study Group 380. N Engl J Med, 2000. 343(3): p. 162-8.

110. Atkin, W., et al., Single flexible sigmoidoscopy screening to prevent colorectal cancer: baseline findings of a UK multicentre randomised trial. Lancet, 2002. 359(9314): p. 1291-300.

111. Muller, A.D. and A. Sonnenberg, Protection by endoscopy against death from colorectal cancer. A case-control study among veterans. Arch Intern Med, 1995. 155(16): p. 1741-8.

112. Newcomb, P.A., et al., Screening sigmoidoscopy and colorectal cancer mortality. J Natl Cancer Inst, 1992. 84(20): p. 1572-5.

113. Selby, J.V., et al., A case-control study of screening sigmoidoscopy and mortality from colorectal cancer. N Engl J Med, 1992. 326(10): p. 653-7.

114. Singh, H., et al., Predictors of colorectal cancer after negative colonoscopy: a population-based study. Am J Gastroenterol, 2010. 105(3): p. 663-73; quiz 674.

115. Brenner, H., et al., Protection from right- and left-sided colorectal neoplasms after colonoscopy: population-based study. J Natl Cancer Inst, 2010. 102(2): p. 89-95.

116. Brenner, H., et al., Protection from colorectal cancer after colonoscopy: a population-based, case-control study. Ann Intern Med, 2011. 154(1): p. 22-30.

117. Sieg, A., U. Hachmoeller-Eisenbach, and T. Eisenbach, Prospective evaluation of complications in outpatient Gl endoscopy: a survey among German gastroenterologists. Gastrointest Endosc, 2001. 53: p. 620-7. 
118. Rex, D.K., et al., Colonoscopic miss rates of adenomas determined by back-to-back colonoscopies. Gastroenterology, 1997. 112(1): p. 24-8.

119. Rex, D.K., et al., 5-year incidence of adenomas after negative colonoscopy in asymptomatic average-risk persons [see comment]. Gastroenterology, 1996. 111 (5): p. 1178-81.

120. Brenner, H., et al., Long-term risk of colorectal cancer after negative colonoscopy. J Clin Oncol, $2011.29(28)$ : p. 3761-7.

121. Bekanntmachungen der Bundesärztekammer: Richtlinien zur prädiktiven genetischen Diagnostik. Dt Ärztebl, 2003. 100(19): p. A1297-1305.

122. Bekanntmachungen: Richtlinien des Bundesausschusses der Ärzte und Krankenkassen über die Früherkennung von Krebserkrankungen („Krebsfrüherkennungs-Richtlinien“)*. Dtsch Arztebl Int, 2002. 1(11): p. 518.

123. Atkin, W.S., et al., Once-only flexible sigmoidoscopy screening in prevention of colorectal cancer: a multicentre randomised controlled trial. Lancet, 2010. 375(9726): p. 1624-33.

124. Muller, A.D. and A. Sonnenberg, Prevention of colorectal cancer by flexible endoscopy and polypectomy. A case-control study of 32,702 veterans. Ann Intern Med, 1995. 123(12): p. 90410.

125. Newcomb, P.A., et al., Long-term efficacy of sigmoidoscopy in the reduction of colorectal cancer incidence. J Natl Cancer Inst, 2003. 95(8): p. 622-5.

126. Schoen, R.E., et al., Results of repeat sigmoidoscopy 3 years after a negative examination. Jama, 2003. 290(1): p. 41-8.

127. Burke, C.A., K. Elder, and R. Lopez, Screening for colorectal cancer with flexible sigmoidoscopy: is a 5-yr interval appropriate? A comparison of the detection of neoplasia $3 \mathrm{yr}$ versus $5 \mathrm{yr}$ after a normal examination. Am J Gastroenterol, 2006. 101(6): p. 1329-32.

128. Winawer, S.J., et al., Screening for colorectal cancer with fecal occult blood testing and sigmoidoscopy. J Natl Cancer Inst, 1993. 85(16): p. 1311-8.

129. Lieberman, D.A. and D.G. Weiss, One-time screening for colorectal cancer with combined fecal occult-blood testing and examination of the distal colon. N Engl J Med, 2001. 345(8): p. 555-60.

130. Gondal, G., et al., The Norwegian Colorectal Cancer Prevention (NORCCAP) screening study: baseline findings and implementations for clinical work-up in age groups 50-64 years. Scand J Gastroenterol, 2003. 38(6): p. 635-42.

131. Kato, J., et al., Combination of sigmoidoscopy and a fecal immunochemical test to detect proximal colon neoplasia. Clin Gastroenterol Hepatol, 2009. 7(12): p. 1341-6.

132. Eliakim, R., et al., Evaluation of the PillCam Colon capsule in the detection of colonic pathology: results of the first multicenter, prospective, comparative study. Endoscopy, 2006. 38(10): p. 963-70.

133. Schoofs, N., J. Deviere, and A. Van Gossum, PillCam colon capsule endoscopy compared with colonoscopy for colorectal tumor diagnosis: a prospective pilot study. Endoscopy, 2006. 38(10): p. 971-7.

134. Van Gossum, A., et al., Capsule endoscopy versus colonoscopy for the detection of polyps and cancer. N Engl J Med, 2009. 361(3): p. 264-70.

135. Spada, C., et al., Meta-analysis shows colon capsule endoscopy is effective in detecting colorectal polyps. Clin Gastroenterol Hepatol, 2010. 8(6): p. 516-22.

136. Gay, G., et al., Could the colonic capsule PillCam Colon be clinically useful for selecting patients who deserve a complete colonoscopy?: results of clinical comparison with colonoscopy in the perspective of colorectal cancer screening. Am J Gastroenterol, 2010. 105(5): p. 1076-86.

137. Eliakim, R., et al., Prospective multicenter performance evaluation of the second-generation colon capsule compared with colonoscopy. Endoscopy, 2009. 41(12): p. 1026-31.

138. Spada, C., et al., Second-generation colon capsule endoscopy compared with colonoscopy. Gastrointest Endosc, 2011. 74(3): p. 581-589 e1.

139. Mandel, J.S., et al., Reducing mortality from colorectal cancer by screening for fecal occult blood. Minnesota Colon Cancer Control Study. N Engl J Med, 1993. 328(19): p. 1365-71.

140. Zhu, M.M., et al., Comparison of immunochemical and guaiac-based fecal occult blood test in screening and surveillance for advanced colorectal neoplasms: a meta-analysis. J Dig Dis, 2010. 11(3): p. 148-60.

141. van Rossum, L.G., et al., Colorectal cancer screening comparing no screening, immunochemical and guaiac fecal occult blood tests: a cost-effectiveness analysis. Int J Cancer, 2011 . 128(8): p. 1908-17.

142. Hundt, S., U. Haug, and H. Brenner, Comparative evaluation of immunochemical fecal occult blood tests for colorectal adenoma detection. Ann Intern Med, 2009. 150(3): p. 162-9.

143. Brenner, H., U. Haug, and S. Hundt, Inter-test agreement and quantitative cross-validation of immunochromatographical fecal occult blood tests. Int J Cancer, 2010. 127(7): p. 1643-9.

144. Haug, U., et al., Sensitivity of immunochemical faecal occult blood testing for detecting left- vs right-sided colorectal neoplasia. Br J Cancer, 2011. 104(11): p. 1779-85. 
145. Allison, J.E., et al., Screening for colorectal neoplasms with new fecal occult blood tests: update on performance characteristics. J Natl Cancer Inst, 2007. 99(19): p. 1462-70.

146. Park, D.I., et al., Comparison of guaiac-based and quantitative immunochemical fecal occult blood testing in a population at average risk undergoing colorectal cancer screening. Am J Gastroenterol, 2010. 105(9): p. 2017-25.

147. Launoy, G.D., et al., Evaluation of an immunochemical fecal occult blood test with automated reading in screening for colorectal cancer in a general average-risk population. Int J Cancer, 2005. 11 (3): p. 493-6.

148. van Roon, A.H., et al., Diagnostic yield improves with collection of 2 samples in fecal immunochemical test screening without affecting attendance. Clin Gastroenterol Hepatol, 2011 . 9(4): p. 333-9.

149. Haug, U., S. Hundt, and H. Brenner, Quantitative immunochemical fecal occult blood testing for colorectal adenoma detection: evaluation in the target population of screening and comparison with qualitative tests. Am J Gastroenterol, 2010. 105(3): p. 682-90.

150. Doran, J. and J.D. Hardcastle, Bleeding patterns in colorectal cancer: the effect of aspirin and the implications for faecal occult blood testing. Br J Surg, 1982. 69(12): p. 711-3.

151. Ahlquist, D.A., et al., Patterns of occult bleeding in asymptomatic colorectal cancer. Cancer, 1989. 63(9): p. 1826-30.

152. Macrae, F.A. and D.J. St. John, Relationship between patterns of bleeding and hemoccult sensitivity in patients with colorectal cancers or adenomas. Gastroenterology, 1982. 82: p. 891-8.

153. Greegor, D.H., Occult blood testing for detection of asymptomatic colon cancer. Cancer, 1971. 28: p. 131-4.

154. Hardcastle, J.D., et al., Randomised controlled trial of faecal-occult-blood screening for colorectal cancer. Lancet, 1996. 348(9040): p. 1472-7.

155. Kronborg, O., et al., Randomised study of screening for colorectal cancer with faecal-occultblood test. Lancet, 1996. 348(9040): p. 1467-71.

156. Hewitson, P., et al., Screening for colorectal cancer using the faecal occult blood test, Hemoccult. Cochrane Database Syst Rev, 2007(1): p. CD001216.

157. Levin, B., K. Hess, and C. Johnson, Screening for colorectal cancer. A comparison of 3 fecal occult blood tests. Arch Intern Med, 1997. 157(9): p. 970-6.

158. Gnauck, R., F.A. Macrae, and M. Fleisher, How to perform the fecal occult blood test. CA Cancer J Clin, 1984. 34: p. 134-47.

159. Jaffe, R.M., et al., False-negative stool occult blood tests caused by ingestion of ascorbic acid (vitamin C). Ann Intern Med, 1975. 83(6): p. 824-6.

160. Macrae, F.A., et al., Optimal dietary conditions for hemoccult testing. Gastroenterology, 1982. 82(5 Pt 1): p. 899-903.

161. Rozen, P., J. Knaani, and Z. Samuel, Eliminating the need for dietary restrictions when using a sensitive guaiac fecal occult blood test. Dig Dis Sci, 1999. 44(4): p. 756-60.

162. Pignone, M., et al., Meta-analysis of dietary restriction during fecal occult blood testing. Eff Clin Pract, 2001. 4(4): p. 150-6.

163. van Rossum, L.G., et al., Random comparison of guaiac and immunochemical fecal occult blood tests for colorectal cancer in a screening population. Gastroenterology, 2008. 135(1): p. 82-90.

164. Webendorfer, S., et al., [Precautions for intestinal cancer in the workplace. An initiative for secondary prevention in the BASF joint-stock company]. Dtsch Med Wochenschr, 2004. 129(6): p. 239-43.

165. Mandel, J.S., et al., The effect of fecal occult-blood screening on the incidence of colorectal cancer. N Engl J Med, 2000. 343(22): p. 1603-7.

166. Traverso, G., et al., Detection of APC mutations in fecal DNA from patients with colorectal tumors. N Engl J Med, 2002. 346(5): p. 311-20.

167. Ahlquist, D.A., et al., Colorectal cancer screening by detection of altered human DNA in stool: feasibility of a multitarget assay panel. Gastroenterology, 2000. 119(5): p. 1219-27.

168. Dong, S.M., et al., Detecting colorectal cancer in stool with the use of multiple genetic targets. J Natl Cancer Inst, 2001. 93(11): p. 858-65.

169. Tagore, K.S., et al., Sensitivity and specificity of a stool DNA multitarget assay panel for the detection of advanced colorectal neoplasia. Clin Colorectal Cancer, 2003. 3(1): p. 47-53.

170. Imperiale, T.F., et al., Fecal DNA versus fecal occult blood for colorectal-cancer screening in an average-risk population. N Engl J Med, 2004. 351(26): p. 2704-14.

171. Ahlquist, D.A., et al., Stool DNA and occult blood testing for screen detection of colorectal neoplasia. Ann Intern Med, 2008. 149(7): p. 441-50, W81.

172. Haug, U., S. Hundt, and H. Brenner, Sensitivity and specificity of faecal tumour M2 pyruvate kinase for detection of colorectal adenomas in a large screening study. Br J Cancer, 2008. 99(1): p. 133-5. 
173. Moslein, G., et al., [Analysis of the statistical value of various commercially available stool tests - a comparison of one stool sample in correlation to colonoscopy]. Dtsch Med Wochenschr, 2010. 135(12): p. 557-62.

174. de Haan, M.C., et al., Diagnostic value of CT-colonography as compared to colonoscopy in an asymptomatic screening population: a meta-analysis. Eur Radiol, 2011.21 (8): p. 1747-63.

175. El-Maraghi, R.H. and A.Z. Kielar, CT colonography versus optical colonoscopy for screening asymptomatic patients for colorectal cancer a patient, intervention, comparison, outcome (PICO) analysis. Acad Radiol, 2009. 16(5): p. 564-71.

176. Pox, C.P. and W. Schmiegel, Role of CT colonography in colorectal cancer screening: risks and benefits. Gut, 2010. 59(5): p. 692-700.

177. Frazier, A.L., et al., Cost-effectiveness of screening for colorectal cancer in the general population. Jama, 2000. 284(15): p. 1954-61.

178. Geul, K.W., et al., Prevention of colorectal cancer. Costs and effectiveness of sigmoidoscopy. Scand J Gastroenterol Suppl, 1997. 223: p. 79-87.

179. Inadomi, J.M., Update on the cost-effectiveness of screening for colorectal neoplasia. Curr Opin Gastroenterol, 2003. 29: p. 44-50.

180. Khandker, R.K., et al., A decision model and cost-effectiveness analysis of colorectal cancer screening and surveillance guidelines for average-risk adults. Int J Technol Assess Health Care, 2000. 16(3): p. 799-810.

181. McGrath, J.S., T.P. Ponich, and J.C. Gregor, Screening for colorectal cancer: the cost to find an advanced adenoma. Am J Gastroenterol, 2002. 97(11): p. 2902-7.

182. Norum, J., Prevention of colorectal cancer: a cost-effectiveness approach to a screening model employing sigmoidoscopy. Ann Oncol, 1998. 9(6): p. 613-8.

183. Pignone, M., et al., Cost-effectiveness analyses of colorectal cancer screening: a systematic review for the U.S. Preventive Services Task Force. Ann Intern Med, 2002. 137(2): p. 96-104.

184. Sonnenberg, A. and F. Delco, Cost-effectiveness of a single colonoscopy in screening for colorectal cancer. Arch Intern Med, 2002. 162(2): p. 163-8.

185. Vijan, S., et al., Which colon cancer screening test? A comparison of costs, effectiveness, and compliance. Am J Med, 2001. 111(8): p. 593-601.

186. Zauber, A.G., et al., Evaluating test strategies for colorectal cancer screening: a decision analysis for the U.S. Preventive Services Task Force. Ann Intern Med, 2008. 149(9): p. 659-69.

187. Lansdorp-Vogelaar, I., A.B. Knudsen, and H. Brenner, Cost-effectiveness of colorectal cancer screening. Epidemiol Rev, 2011 . 33(1): p. 88-100.

188. Sung, J.J., et al., Asia Pacific consensus recommendations for colorectal cancer screening. Gut, 2008. 57(8): p. 1166-76.

189. Jasperson, K.W., et al., Hereditary and familial colon cancer. Gastroenterology, 2010. 138(6): p. 2044-58.

190. Andrieu, N., et al., Familial relative risk of colorectal cancer: a population-based study. Eur J Cancer, 2003. 39(13): p. 1904-11.

191. Johns, L.E. and R.S. Houlston, A systematic review and meta-analysis of familial colorectal cancer risk. Am J Gastroenterol, 2001. 96(10): p. 2992-3003.

192. Lichtenstein, P., et al., Environmental and heritable factors in the causation of cancer--analyses of cohorts of twins from Sweden, Denmark, and Finland. N Engl J Med, 2000. 343(2): p. 78-85.

193. Slattery, M.L., et al., Family history and colorectal cancer: predictors of risk. Cancer Causes Control, 2003. 14(9): p. 879-87.

194. Winawer, S., et al., Colorectal cancer screening and surveillance: clinical guidelines and rationale-Update based on new evidence. Gastroenterology, 2003. 124(2): p. 544-60.

195. Bonelli, L., et al., Family history of colorectal cancer as a risk factor for benign and malignant tumours of the large bowel. A case-control study. Int J Cancer, 1988. 41(4): p. 513-7.

196. Fuchs, C.S., et al., A prospective study of family history and the risk of colorectal cancer. $\mathrm{N}$ Engl J Med, 1994. 331(25): p. 1669-74.

197. Guillem, J.G., et al., Clustering of colorectal cancer in families of probands under 40 years of age. Dis Colon Rectum, 1996. 39(9): p. 1004-7.

198. Guillem, J.G., et al., Colonoscopic screening for neoplasms in asymptomatic first-degree relatives of colon cancer patients. A controlled, prospective study. Dis Colon Rectum, 1992. 35(6): p. 523-9.

199. Kune, G.A., S. Kune, and L.F. Watson, The role of heredity in the etiology of large bowel cancer: data from the Melbourne Colorectal Cancer Study. World J Surg, 1989. 13(1): p. 124-9.

200. Rozen, P., et al., Family history of colorectal cancer as a marker of potential malignancy within a screening programm. Cancer, 1987. 60(2): p. 248-54.

201. Sondergaard, J.O., S. Bulow, and E. Lynge, Cancer incidence among parents of patients with colorectal cancer. Int J Cancer, 1991. 47(2): p. 202-6. 
202. St John, D.J., et al., Cancer risk in relatives of patients with common colorectal cancer. Ann Int Med, 1993. 118(10): p. 785-90.

203. Butterworth, A.S., J.P. Higgins, and P. Pharoah, Relative and absolute risk of colorectal cancer for individuals with a family history: a meta-analysis. Eur J Cancer, 2006. 42(2): p. 216-27.

204. Hall, N.R., et al., Hereditary susceptibility to colorectal cancer. Relatives of early onset cases are particularly at risk. Dis Colon Rectum, 1996. 39(7): p. 739-43.

205. Hunt, L.M., et al., Endoscopic screening of relatives of patients with colorectal cancer. Gut, 1998. 42(1): p. 71-5.

206. Winawer, S.J., et al., Risk of colorectal cancer in the families of patients with adenomatous polyps. National Polyp Study Workgroup. N Engl J Med, 1996. 334(2): p. 82-7.

207. Ahsan, H., et al., Family history of colorectal adenomatous polyps and increased risk for colorectal cancer. Ann Intern Med, 1998. 128(11): p. 900-5.

208. Nakama, H., et al., Family history of colorectal adenomatous polyps as a risk factor for colorectal cancer. Eur J Cancer, 2000. 36(16): p. 2111-4.

209. Almendingen, K., B. Hofstad, and M.H. Vatn, Does a family history of cancer increase the risk of occurrence, growth, and recurrence of colorectal adenomas? Gut, 2003. 52(5): p. 747-51.

210. Winawer, S.J., et al., Risk and surveillance of individuals with colorectal polyps. WHO Collaborating Centre for the Prevention of Colorectal Cancer. Bull World Health Organ, 1990. 68(6): p. 789-95.

211. Atkin, W.S., B.C. Morson, and J. Cuzick, Long-term risk of colorectal cancer after excision of rectosigmoid adenomas. N Engl J Med, 1992. 326(10): p. 658-62.

212. Otchy, D.P., et al., Metachronous colon cancer in persons who have had a large adenomatous polyp. Am J Gastroenterol, 1996. 91(3): p. 448-54.

213. van Stolck, R.U., et al., Adenoma characteristics at first colonoscopy as predictors of adenoma recurrence and characteristics at follow-up. The Polyp Prevention Study Group.

Gastroenterology, 1998. 115(1): p. 13-8.

214. Noshirwani, K.C., et al., Adenoma size and number are predictive of adenoma recurrence: implications for surveillance colonoscopy. Gastrointest Endosc, 2000. 51: p. 433-7.

215. Aldridge, A.J. and J.N. Simson, Histological assessment of colorectal adenomas by size. Are polyps less than $10 \mathrm{~mm}$ in size clinically important? Eur J Surg, 2001. 167(10): p. 777-81.

216. Avidan, B., et al., New occurrence and recurrence of neoplasms within 5 years of a screening colonoscopy. Am J Gastroenterol, 2002. 97(6): p. 1524-9.

217. Bertario, L., et al., Predictors of metachronous colorectal neoplasms in sporadic adenoma patients. Int J Cancer, 2003. 105(1): p. 82-7.

218. Chen, C.D., et al., A case-cohort study for the disease natural history of adenoma-carcinoma and de novo carcinoma and surveillance of colon and rectum after polypectomy: implication for efficacy of colonoscopy. Br J Cancer, 2003. 88(12): p. 1866-73.

219. Bensen, S., et al., The colonoscopic miss rate and true one-year recurrence of colorectal neoplastic polyps. Polyp Prevention Study Group. Am J Gastroenterol, 1999. 94(1): p. 194-9.

220. Katalinic, A., H. Raspe, and A. Waldmann, [Positive family history of colorectal cancer--use of a questionnaire]. Z Gastroenterol, 2009. 47(11): p. 1125-31.

221. Baron, J.A., et al., Neoplastic and antineoplastic effects of beta-carotene on colorectal adenoma recurrence: results of a randomized trial. J Natl Cancer Inst, 2003. 95(10): p. 71 7-22.

222. Karnes, W.E., Epidemiology and etiology of sporadic colorectal adenocarcinoma. Current opinion in Gastroenterology, 1994. 10: p. 19-26.

223. Sandler, R.S., et al., A randomized trial of aspirin to prevent colorectal adenomas in patients with previous colorectal cancer. N Engl J Med, 2003. 348(10): p. 883-90.

224. Rothwell, P.M., et al., Long-term effect of aspirin on colorectal cancer incidence and mortality: 20-year follow-up of five randomised trials. Lancet, 2010. 376(9754): p. 1741-50.

225. Burt, R.W., Screening of patients with a positive family history of colorectal cancer. Gastrointest Endosc Clin N Am, 1997. 7(1): p. 65-79.

226. Gilbert, J.M., et al., Feasibility study of colonoscopy as the primary screening investigation in relatives of patients with colorectal cancer. Ann R Coll Surg Engl, 2001. 83(6): p. 415-9.

227. Syrigos, K.N., et al., Colonoscopy in asymptomatic individuals with a family history of colorectal cancer. Ann Surg Oncol, 2002. 9(5): p. 439-43.

228. Bradshaw, N., et al., Colonoscopy surveillance of individuals at risk of familial colorectal cancer. Gut, 2003. 52(12): p. 1748-51.

229. Brenner, H., M. Hoffmeister, and U. Haug, Family history and age at initiation of colorectal cancer screening. Am J Gastroenterol, 2008. 103(9): p. 2326-31.

230. Waldmann, A., H. Raspe, and A. Katalinic, [Colon cancer risk in persons at familial or hereditary risk aged < 55 years]. Z Gastroenterol, 2009. 47(10): p. 1052-8.

231. Levin, B., et al., Screening and surveillance for the early detection of colorectal cancer and adenomatous polyps, 2008: a joint guideline from the American Cancer Society, the US Multi- 
Society Task Force on Colorectal Cancer, and the American College of Radiology. Gastroenterology, 2008. 134(5): p. 1570-95.

232. Engel, C., et al., Efficacy of annual colonoscopic surveillance in individuals with hereditary nonpolyposis colorectal cancer. Clin Gastroenterol Hepatol, 2010. 8(2): p. 174-82.

233. Dove-Edwin, I., et al., Prevention of colorectal cancer by colonoscopic surveillance in individuals with a family history of colorectal cancer: 16 year, prospective, follow-up study. BMJ, 2005. 331 (7524): p. 1047.

234. Duncan, R.E., et al., The challenge of developmentally appropriate care: predictive genetic testing in young people for familial adenomatous polyposis. Fam Cancer, 2010. 9(1): p. 27-35.

235. Douma, K.F., et al., Psychosocial issues in genetic testing for familial adenomatous polyposis: a review of the literature. Psychooncology, 2008. 17(8): p. 737-45.

236. Douma, K.F., et al., Psychological distress and use of psychosocial support in familial adenomatous polyposis. Psychooncology, 2010. 19(3): p. 289-98.

237. Vasen, H.F., et al., The International Collaborative Group on Hereditary Non-Polyposis Colorectal Cancer (ICG-HNPCC). Dis Colon Rectum, 1991. 34(5): p. 424-5.

238. Rodriguez-Bigas, M.A., et al., A National Cancer Institute Workshop on Hereditary Nonpolyposis Colorectal Cancer Syndrome: meeting highlights and Bethesda guidelines. J Natl Cancer Inst, 1997. 89(23): p. 1758-62.

239. Schulmann, K., et al., Small bowel cancer risk in Lynch syndrome. Gut, 2008. 57(1 1): p. 162930.

240. ten Kate, G.L., et al., Is surveillance of the small bowel indicated for Lynch syndrome families? Gut, 2007. 56(9): p. $1198-201$.

241. Schulmann, K., et al., HNPCC-associated small bowel cancer: clinical and molecular characteristics. Gastroenterology, 2005. 128(3): p. 590-9.

242. van der Post, R.S., et al., Risk of urothelial bladder cancer in Lynch syndrome is increased, in particular among MSH2 mutation carriers. J Med Genet, 2010. 47(7): p. 464-70.

243. Kastrinos, F., et al., Risk of pancreatic cancer in families with Lynch syndrome. JAMA, 2009. 302(16): p. 1790-5.

244. Win, A.K., et al., Colorectal and other cancer risks for carriers and noncarriers from families with a DNA mismatch repair gene mutation: a prospective cohort study. J Clin Oncol, 2012. 30(9): p. 958-64.

245. Vasen, H.F., et al., MSH2 mutation carriers are at higher risk of cancer than MLH1 mutation carriers: a study of hereditary nonpolyposis colorectal cancer families. J Clin Oncol, 2001. 19(20): p. 4074-80.

246. Aarnio, M., et al., Life-time risk of different cancers in hereditary non-polyposis colorectal cancer (HNPCC) syndrome. Int J Cancer, 1995. 64(6): p. 430-3.

247. Vasen, H.F., et al., The risk of brain tumours in hereditary non-polyposis colorectal cancer (HNPCC). Int J Cancer, 1996. 65(4): p. 422-5.

248. Kruse, R., et al., Muir-Torre phenotype has a frequency of DNA mismatch-repair-gene mutations similar to that in hereditary nonpolyposis colorectal cancer families defined by the Amsterdam criteria. Am J Hum Genet, 1998. 63(1): p. 63-70.

249. Umar, A., et al., Revised Bethesda Guidelines for hereditary nonpolyposis colorectal cancer (Lynch syndrome) and microsatellite instability. J Natl Cancer Inst, 2004. 96(4): p. 261-8.

250. Shia, J., Immunohistochemistry versus microsatellite instability testing for screening colorectal cancer patients at risk for hereditary nonpolyposis colorectal cancer syndrome. Part I. The utility of immunohistochemistry. J Mol Diagn, 2008. 10(4): p. 293-300.

251. Deng, G., et al., BRAF mutation is frequently present in sporadic colorectal cancer with methylated $h M L H 1$, but not in hereditary nonpolyposis colorectal cancer. Clin Cancer Res, 2004. 10(1 Pt 1): p. 191-5.

252. Domingo, E., et al., BRAF screening as a low-cost effective strategy for simplifying HNPCC genetic testing. J Med Genet, 2004. 41(9): p. 664-8.

253. Loughrey, M.B., et al., Incorporation of somatic BRAF mutation testing into an algorithm for the investigation of hereditary non-polyposis colorectal cancer. Fam Cancer, 2007. 6(3): p. 301-10.

254. McGivern, A., et al., Promoter hypermethylation frequency and BRAF mutations distinguish hereditary non-polyposis colon cancer from sporadic MSI-H colon cancer. Fam Cancer, 2004. 3(2): p. $101-7$.

255. Levine, A.J., et al., Cancer risks for the relatives of colorectal cancer cases with a methylated MLH1 promoter region: data from the Colorectal Cancer Family Registry. Cancer Prev Res (Phila), 2012. 5(2): p. 328-35.

256. Foulkes, W.D., A tale of four syndromes: familial adenomatous polyposis, Gardner syndrome, attenuated APC and Turcot syndrome. QJM, 1995. 88(12): p. 853-63.

257. Lynch, H.T., et al., Attenuated familial adenomatous polyposis (AFAP). A phenotypically and genotypically distinctive variant of FAP. Cancer, 1995. 76(12): p. 2427-33. 
258. Lynch, H.T. and T.C. Smyrk, Classification of familial adenomatous polyposis: a diagnostic nightmare. Am J Hum Genet, 1998. 62(6): p. 1288-9.

259. Soravia, C., et al., Genotype-phenotype correlations in attenuated adenomatous polyposis coli. Am J Hum Genet, 1998. 62: p. 1290-1301.

260. Hernegger, G.S., H.G. Moore, and J.G. Guillem, Attenuated familial adenomatous polyposis: an evolving and poorly understood entity. Dis Colon Rectum, 2002. 45(1): p. 127-34; discussion 134-6.

261. Knudsen, A.L., M.L. Bisgaard, and S. Bulow, Attenuated familial adenomatous polyposis (AFAP). A review of the literature. Fam Cancer, 2003. 2(1): p. 43-55.

262. Al-Tassan, N., et al., Inherited variants of MYH associated with somatic G:C-->T:A mutations in colorectal tumors. Nat Genet, 2002. 30(2): p. 227-32.

263. Cao, Y., et al., Challenge in the differentiation between attenuated familial adenomatous polyposis and hereditary nonpolyposis colorectal cancer: case report with review of the literature. Am J Gastroenterol, 2002. 97(7): p. 1822-7.

264. Aretz, S., The differential diagnosis and surveillance of hereditary gastrointestinal polyposis syndromes. Dtsch Arztebl Int, 2010. 107(10): p. 163-73.

265. Aretz, S., M. Genuardi, and F.J. Hes, Clinical utility gene card for: MUTYH-associated polyposis (MAP), autosomal recessive colorectal adenomatous polyposis. 2012, Eur J Hum Genet.

266. Sampson, J.R. and N. Jones, MUTYH-associated polyposis. Best Pract Res Clin Gastroenterol, 2009. 23(2): p. 209-18.

267. Morreau, H., R. Riddel, and S. Aretz, MUTYH-associated polyposis., in WHO Classification of Tumours of the Digestive System, F.T. Bosman, et al., Editors. 2010, IARC/ WHO Press: Lyon. p. 156-159.

268. Aretz, S., et al., MUTYH-associated polyposis: 70 of 71 patients with biallelic mutations present with an attenuated or atypical phenotype. Int J Cancer, 2006. 119(4): p. 807-14.

269. Lubbe, S.J., et al., Clinical implications of the colorectal cancer risk associated with MUTYH mutation. J Clin Oncol, 2009. 27(24): p. 3975-80.

270. Cleary, S.P., et al., Germline MutY human homologue mutations and colorectal cancer: a multisite case-control study. Gastroenterology, 2009. 136(4): p. 1251-60.

271. Boparai, K.S., et al., Hyperplastic polyps and sessile serrated adenomas as a phenotypic expression of MYH-associated polyposis. Gastroenterology, 2008. 135(6): p. 2014-8.

272. Vogt, S., et al., Expanded extracolonic tumor spectrum in MUTYH-associated polyposis. Gastroenterology, 2009. 137(6): p. 1976-85 e1-10.

273. Papadopoulos, N. and A. Lindblom, Molecular basis of HNPCC: mutations of MMR genes. Hum Mut, 1997. 10: p. 89-99.

274. Kuiper, R.P., et al., Recurrence and variability of germline EPCAM deletions in Lynch syndrome. Hum Mutat, $2011.32(4)$ : p. 407-14.

275. Jarvinen, H.J., et al., Controlled 15-year trial on screening for colorectal cancer in families with hereditary nonpolyposis colorectal cancer. Gastroenterology, 2000. 118(5): p. 829-34.

276. de Vos tot Nederveen Cappel, W.H., et al., Surveillance for hereditary nonpolyposis colorectal cancer: a long-term study on 114 families. Dis Colon Rectum, 2002. 45(12): p. 1588-94.

277. Edelstein, D.L., et al., Rapid development of colorectal neoplasia in patients with Lynch syndrome. Clin Gastroenterol Hepatol, 2011 . 9(4): p. 340-3.

278. Renkonen-Sinisalo, L., et al., Surveillance improves survival of colorectal cancer in patients with hereditary nonpolyposis colorectal cancer. Cancer Detect Prev, 2000. 24(2): p. 137-42.

279. Huneburg, R., et al., Chromocolonoscopy detects more adenomas than white light colonoscopy or narrow band imaging colonoscopy in hereditary nonpolyposis colorectal cancer screening. Endoscopy, 2009. 41(4): p. 316-22.

280. Stoffel, E.M., et al., Missed adenomas during colonoscopic surveillance in individuals with Lynch Syndrome (hereditary nonpolyposis colorectal cancer). Cancer Prev Res (Phila), 2008. 1(6): p. 470-5.

281. Hurlstone, D.P., et al., High magnification chromoscopic colonoscopy or high frequency $20 \mathrm{MHz}$ mini probe endoscopic ultrasound staging for early colorectal neoplasia: a comparative prospective analysis. Gut, 2005. 54(11): p. 1585-9.

282. Aarnio, M., et al., Cancer risk in mutation carriers of DNA-mismatch-repair genes. Int J Cancer, 1999. 81(2): p. 214-8.

283. Dove-Edwin, l., et al., The outcome of endometrial carcinoma surveillance by ultrasound scan in women at risk of hereditary nonpolyposis colorectal carcinoma and familial colorectal carcinoma. Cancer, 2002. 94(6): p. 1708-12.

284. Rijcken, F.E., et al., Gynecologic screening in hereditary nonpolyposis colorectal cancer. Gynecol Oncol, 2003. 91(1): p. 74-80.

285. Renkonen-Sinisalo, L., et al., Surveillance for endometrial cancer in hereditary nonpolyposis colorectal cancer syndrome. Int J Cancer, 2007. 120(4): p. 821-4. 
286. Vasen, H.F., et al., Guidelines for the clinical management of Lynch syndrome (hereditary nonpolyposis cancer). J Med Genet, 2007. 44(6): p. 353-62.

287. Goecke, T., et al., Genotype-phenotype comparison of German MLH1 and MSH2 mutation carriers clinically affected with Lynch syndrome: a report by the German HNPCC Consortium. J Clin Oncol, 2006. 24(26): p. 4285-92.

288. Burn, J., et al., Effect of aspirin or resistant starch on colorectal neoplasia in the Lynch syndrome. N Engl J Med, 2008. 359(24): p. 2567-78.

289. Burn, J., et al., Long-term effect of aspirin on cancer risk in carriers of hereditary colorectal cancer: an analysis from the CAPP2 randomised controlled trial. Lancet, 2011 . 378(9809): $p$. 2081-7.

290. Natarajan, N., et al., Comparison of extended colectomy and limited resection in patients with Lynch syndrome. Dis Colon Rectum, 2010. 53(1): p. 77-82.

291. Kalady, M.F., et al., Risk of colorectal adenoma and carcinoma after colectomy for colorectal cancer in patients meeting Amsterdam criteria. Ann Surg, 2010. 252(3): p. 507-11; discussion 511-3.

292. Parry, S., et al., Metachronous colorectal cancer risk for mismatch repair gene mutation carriers: the advantage of more extensive colon surgery. Gut, 2011 . 60(7): p. 950-7.

293. Stupart, D.A., et al., Surgery for colonic cancer in HNPCC: total vs segmental colectomy. Colorectal Dis, 2011 . 13(12): p. 1395-9.

294. Schmeler, K.M., et al., Prophylactic surgery to reduce the risk of gynecologic cancers in the Lynch syndrome. N Engl J Med, 2006. 354(3): p. 261-9.

295. Church, J.M., et al., Teenagers with familial adenomatous polyposis: what is their risk for colorectal cancer? Dis Colon Rectum, 2002. 45(7): p. 887-9.

296. King, J.E., et al., Care of patients and their families with familial adenomatous polyposis. Mayo Clin Proc, 2000. 75(1): p. 57-67.

297. Bertario, L., et al., Causes of death and postsurgical survival in familial adenomatous polyposis: results from the Italian Registry. Italian Registry of Familial Polyposis Writing Committee. Semin Surg Oncol, 1994. 10(3): p. 225-34.

298. Galle, T.S., K. Juel, and S. Bulow, Causes of death in familial adenomatous polyposis. Scand J Gastroenterol, 1999. 34(8): p. 808-12.

299. Heiskanen, I., T. Luostarinen, and H.J. Jarvinen, Impact of screening examinations on survival in familial adenomatous polyposis. Scand J Gastroenterol, 2000. 35(12): p. 1284-7.

300. Vasen, H.F., et al., Decision analysis in the surgical treatment of patients with familial adenomatous polyposis: a Dutch-Scandinavian collaborative study including 659 patients. Gut, 2001. 49(2): p. 231-5.

301. Bulow, S., Results of national registration of familial adenomatous polyposis. Gut, 2003. 52(5): p. 742-6.

302. Ficari, F., et al., APC gene mutations and colorectal adenomatosis in familial adenomatous polyposis. Br J Cancer, 2000. 82(2): p. 348-53.

303. Bulow, S., Clinical features in familial polyposis coli. Results of the Danish Polyposis Register. Dis Colon Rectum, 1986. 29(2): p. 102-7.

304. De Cosse, J.J., et al., Rectal cancer risk in patients treated for familial adenomatous polyposis. The Leeds Castle Polyposis Group. Br J Surg, 1992. 79(12): p. 1372-5.

305. Nyam, D.C., et al., Ileal pouch-anal canal anastomosis for familial adenomatous polyposis: early and late results. Ann Surg, 1997. 226(4): p. 514-9; discussion 519-21.

306. Parc, Y.R., et al., Familial adenomatous polyposis: results after ileal pouch-anal anastomosis in teenagers. Dis Colon Rectum, 2000. 43(7): p. 893-8; discussion 898-902.

307. Van Duijvendijk, P., et al., Quality of life after total colectomy with ileorectal anastomosis or proctocolectomy and ileal pouch-anal anastomosis for familial adenomatous polyposis. $\mathrm{Br} \mathrm{J}$ Surg, 2000. 87(5): p. 590-6.

308. Church, J., et al., Risk of rectal cancer in patients after colectomy and ileorectal anastomosis for familial adenomatous polyposis: a function of available surgical options. Dis Colon Rectum, 2003. 46(9): p. $1175-81$.

309. Will, O.C., et al., Familial adenomatous polyposis and the small bowel: a loco-regional review and current management strategies. Pathol Res Pract, 2008. 204(7): p. 449-58.

310. Spigelman, A.D., et al., Upper gastrointestinal cancer in patients with familial adenomatous polyposis. Lancet, 1989. 2(8666): p. 783-5.

311. Burke, C.A., et al., The natural history of untreated duodenal and ampullary adenomas in patients with familial adenomatous polyposis followed in an endoscopic surveillance program. Gastrointest Endosc, 1999. 49(3 Pt 1): p. 358-64.

312. Matsumoto, T., et al., Natural history of ampullary adenoma in familial adenomatous polyposis: reconfirmation of benign nature during extended surveillance. Am J Gastroenterol, 2000. 95(6): p. 1557-62. 
313. Moozar, K.L., et al., Slow progression of periampullary neoplasia in familial adenomatous polyposis. J Gastrointest Surg, 2002. 6(6): p. 831-7; discussion 837.

314. Saurin, J.C., et al., The influence of mutation site and age on the severity of duodenal polyposis in patients with familial adenomatous polyposis. Gastrointest Endosc, 2002. 55(3): p. 342-7.

315. Saurin, J.C., et al., Surveillance of duodenal adenomas in familial adenomatous polyposis reveals high cumulative risk of advanced disease. J Clin Oncol, 2004. 22(3): p. 493-8.

316. Vasen, H.F., et al., Decision analysis in the management of duodenal adenomatosis in familial adenomatous polyposis. Gut, 1997. 40: p. 716-9.

317. Bulow, S., et al., Duodenal adenomatosis in familial adenomatous polyposis. Gut, 2004. 53(3): p. 381-6.

318. Kadmon, M., A. Tandara, and C. Herfarth, Duodenal adenomatosis in familial adenomatous polyposis coli. A review of the literature and results from the Heidelberg Polyposis Register. Int J Colorectal Dis, 2001. 16(2): p. 63-75.

319. Norton, I.D., et al., Safety and outcome of endoscopic snare excision of the major duodenal papilla. Gastrointest Endosc, 2002. 56(2): p. 239-43.

320. Kalady, M.F., et al., Pancreas-preserving duodenectomy in the management of duodenal familial adenomatous polyposis. J Gastrointest Surg, 2002. 6(1): p. 82-7.

321. Parc, Y., J.Y. Mabrut, and C. Shields, Surgical management of the duodenal manifestations of familial adenomatous polyposis. Br J Surg, 2011 . 98(4): p. 480-4.

322. de Vos tot Nederveen Cappel, W.H., et al., Worldwide survey among polyposis registries of surgical management of severe duodenal adenomatosis in familial adenomatous polyposis. $\mathrm{Br} \mathrm{J}$ Surg, 2003. 90(6): p. 705-10.

323. Heiskanen, I., I. Kellokumpu, and H. Jarvinen, Management of duodenal adenomas in 98 patients with familial adenomatous polyposis. Endoscopy, 1999. 31(6): p. 412-6.

324. Norton, I.D., et al., Endoscopic surveillance and ablative therapy for periampullary adenomas. Am J Gastroenterol, 2001. 96(1): p. 101-6.

325. Bleau, B.L. and C.J. Gostout, Endoscopic treatment of ampullary adenomas in familial adenomatous polyposis. J Clin Gastroenterol, 1996. 22(3): p. 237-41.

326. Soravia, C., et al., Desmoid disease in patients with familial adenomatous polyposis. Dis Colon Rectum, 2000. 43(3): p. 363-9.

327. Bertario, L., et al., Multiple approach to the exploration of genotype-phenotype correlations in familial adenomatous polyposis. J Clin Oncol, 2003. 21 (9): p. 1698-707.

328. Friedl, W., et al., Can APC mutation analysis contribute to therapeutic decisions in familial adenomatous polyposis? Experience from 680 FAP families. Gut, 2001. 48(4): p. 515-21.

329. Janinis, J., et al., The pharmacological treatment of aggressive fibromatosis: a systematic review. Ann Oncol, 2003. 14(2): p. 181-90.

330. Tonelli, F., et al., Treatment of desmoids and mesenteric fibromatosis in familial adenomatous polyposis with raloxifene. Tumori, 2003. 89(4): p. 391-6.

331. Gega, M., et al., Successful chemotherapeutic modality of doxorubicin plus dacarbazine for the treatment of desmoid tumors in association with familial adenomatous polyposis. J Clin Oncol, 2006. 24(1): p. 102-5.

332. Skapek, S.X., et al., Vinblastine and methotrexate for desmoid fibromatosis in children: results of a Pediatric Oncology Group Phase II Trial. J Clin Oncol, 2007. 25(5): p. 501-6.

333. Latchford, A.R., et al., A 10-year review of surgery for desmoid disease associated with familial adenomatous polyposis. Br J Surg, 2006. 93(10): p. 1258-64.

334. Möslein, G., Current ideas in desmoid tumours: invited commentary. Fam Cancer, 2006. 5: p. 287-288.

335. Clark, S.K. and R.K. Phillips, Desmoids in familial adenomatous polyposis. Br J Surg, 1996. 83(11): p. 1494-504.

336. Nieuwenhuis, M.H., et al., Evaluation of management of desmoid tumours associated with familial adenomatous polyposis in Dutch patients. Br J Cancer, 2011 . 104(1): p. 37-42.

337. Huang, K., et al., Prognostic factors for extra-abdominal and abdominal wall desmoids: a 20year experience at a single institution. J Surg Oncol, 2009. 100(7): p. 563-9.

338. Salas, S., et al., Prognostic factors influencing progression-free survival determined from a series of sporadic desmoid tumors: a wait-and-see policy according to tumor presentation. $J$ Clin Oncol, 2011 . 29(26): p. 3553-8.

339. Bulow, C. and S. Bulow, Is screening for thyroid carcinoma indicated in familial adenomatous polyposis? The Leeds Castle Polyposis Group. Int J Colorectal Dis, 1997. 12(4): p. 240-2.

340. Jarrar, A.M., et al., Screening for thyroid cancer in patients with familial adenomatous polyposis. Ann Surg, 2011. 253(3): p. 515-21.

341. Martayan, A., et al., Gene variants associated to malignant thyroid disease in familial adenomatous polyposis: a novel APC germline mutation. J Endocrinol Invest, 2010. 33(9): p. 603-6. 
342. Herraiz, M., et al., Prevalence of thyroid cancer in familial adenomatous polyposis syndrome and the role of screening ultrasound examinations. Clin Gastroenterol Hepatol, 2007. 5(3): $p$. 367-73.

343. Bell, B. and E.L. Mazzaferri, Familial adenomatous polyposis (Gardner's syndrome) and thyroid carcinoma. A case report and review of the literature. Dig Dis Sci, 1993. 38(1): p. 185-90.

344. Perrier, N.D., et al., Thyroid cancer in patients with familial adenomatous polyposis. World J Surg, 1998. 22(7): p. 738-42; discussion 743.

345. Hughes, L.J. and V.V. Michels, Risk of hepatoblastoma in familial adenomatous polyposis. Am J Med Genet, 1992. 43(6): p. 1023-5.

346. Thomas, D., et al., Familial hepatoblastoma and APC gene mutations: renewed call for molecular research. Eur J Cancer, 2003. 39(15): p. 2200-4.

347. Aretz, S., et al., Should children at risk for familial adenomatous polyposis be screened for hepatoblastoma and children with apparently sporadic hepatoblastoma be screened for APC germline mutations? Pediatr Blood Cancer, 2006. 47(6): p. 811-8.

348. Vasen, H.F., et al., Guidelines for the clinical management of familial adenomatous polyposis (FAP). Gut, 2008. 57(5): p. 704-13.

349. Waddell, W.R. and R.W. Loughry, Sulindac for polyposis of the colon. J Surg Oncol, 1983. 24(1): p. 83-7.

350. Labayle, D., et al., Sulindac causes regression of rectal polyps in familial adenomatous polyposis. Gastroenterology, 1991. 101(3): p. 635-9.

351. Giardiello, F.M., et al., Treatment of colonic and rectal adenomas with sulindac in familial adenomatous polyposis. N Engl J Med, 1993. 328(18): p. 1313-6.

352. Cruz-Correa, M., et al., Long-term treatment with sulindac in familial adenomatous polyposis: a prospective cohort study. Gastroenterology, 2002. 122(3): p. 641-5.

353. Winde, G., et al., Complete reversion and prevention of rectal adenomas in colectomized patients with familial adenomatous polyposis by rectal low-dose sulindac maintenance treatment. Advantages of low-dose nonsteroidal anti-inflammatory drug regimen in reversing adenomas exceeding 33 months. Dis Colon Rectum, 1995. 38(8): p. 813-30.

354. Giardiello, F.M., et al., Primary chemoprevention of familial adenomatous polyposis with sulindac. N Engl J Med, 2002. 346(14): p. 1054-9.

355. Steinbach, G., et al., The effect of celecoxib, a cyclooxygenase-2 inhibitor, in familial adenomatous polyposis. N Engl J Med, 2000. 342(26): p. 1946-52.

356. Phillips, R.K., et al., A randomised, double blind, placebo controlled study of celecoxib, a selective cyclooxygenase 2 inhibitor, on duodenal polyposis in familial adenomatous polyposis. Gut, 2002. 50(6): p. 857-60.

357. Solomon, S.D., et al., Cardiovascular risk of celecoxib in 6 randomized placebo-controlled trials: the cross trial safety analysis. Circulation, 2008. 117(16): p. 2104-13.

358. Matsuo, S., et al., Attenuated familial adenomatous polyposis associated with advanced rectal cancer in a 16-year-old boy: report of a case. Surg Today, 2001. 31(11): p. 1020-3.

359. Leggett, B.A., et al., Severe upper gastrointestinal polyposis associated with sparse colonic polyposis in a familial adenomatous polyposis family with an APC mutation at codon 1520. Gut, 1997. 41(4): p. 518-21.

360. Zwick, A., et al., Gastric adenocarcinoma and dysplasia in fundic gland polyps of a patient with attenuated adenomatous polyposis coli. Gastroenterology, 1997. 113(2): p. 659-63.

361. Hofgartner, W.T., et al., Gastric adenocarcinoma associated with fundic gland polyps in a patient with attenuated familial adenomatous polyposis. Am J Gastroenterol, 1999. 94(8): p. 2275-81.

362. Jones, N., et al., Increased colorectal cancer incidence in obligate carriers of heterozygous mutations in MUTYH. Gastroenterology, 2009. 137(2): p. 489-94, 494 e 1 ; quiz 725-6.

363. Farrington, S.M., et al., Germline susceptibility to colorectal cancer due to base-excision repair gene defects. Am J Hum Genet, 2005. 77(1): p. 112-9.

364. Nielsen, M., et al., Analysis of MUTYH genotypes and colorectal phenotypes in patients With MUTYH-associated polyposis. Gastroenterology, 2009. 136(2): p. 471-6.

365. Nascimbeni, R., et al., Rectum-sparing surgery may be appropriate for biallelic MutYHassociated polyposis. Dis Colon Rectum, 2010. 53(12): p. 1670-5.

366. Nielsen, M., et al., Duodenal carcinoma in MUTYH-associated polyposis. J Clin Pathol, 2006. 59(11): p. 1212-5.

367. Zbuk, K.M. and C. Eng, Hamartomatous polyposis syndromes. Nat Clin Pract Gastroenterol Hepatol, 2007. 4(9): p. 492-502.

368. Aretz, S., et al., High proportion of large genomic STK1 1 deletions in Peutz-Jeghers syndrome. Hum Mutat, 2005. 26(6): p. 513-9.

369. Hinds, R., et al., Complications of childhood Peutz-Jeghers syndrome: implications for pediatric screening. J Pediatr Gastroenterol Nutr, 2004. 39(2): p. 219-20. 
370. Reid, J.D., Intestinal carcinoma in the Peutz-Jeghers syndrome. Jama, 1974. 229(7): p. 833-4.

371. Burdick, D. and J.T. Prior, Peutz-Jeghers syndrome. A clinicopathologic study of a large family with a 27-year follow-up. Cancer, 1982. 50(10): p. 2139-46.

372. Giardiello, F.M., et al., Increased risk of cancer in the Peutz-Jeghers syndrome. N Engl J Med, 1987. 316(24): p. 1511-4.

373. Foley, T.R., T.J. McGarrity, and A.B. Abt, Peutz-Jeghers syndrome: a clinicopathologic survey of the "Harrisburg family" with a 49-year follow-up. Gastroenterology, 1988. 95(6): p. 1535-40.

374. Spigelman, A.D., V. Murday, and R.K. Phillips, Cancer and the Peutz-Jeghers syndrome. Gut, 1989. 30(1 1): p. 1588-90.

375. Hizawa, K., et al., Neoplastic transformation arising in Peutz-Jeghers polyposis. Dis Colon Rectum, 1993. 36: p. 953-57.

376. Boardman, L.A., et al., Increased risk for cancer in patients with the Peutz-Jeghers syndrome. Ann Intern Med, 1998. 128(11): p. 896-9.

377. Mehenni, H., et al., Cancer risks in LKB1 germline mutation carriers. Gut, 2006. 55(7): p. 98490.

378. Giardiello, F.M., et al., Very high risk of cancer in familial Peutz-Jeghers syndrome. Gastroenterology, 2000. 119(6): p. 1447-53.

379. Hearle, N., et al., Frequency and spectrum of cancers in the Peutz-Jeghers syndrome. Clin Cancer Res, 2006. 12(10): p. 3209-15.

380. Sweet, K., et al., Molecular classification of patients with unexplained hamartomatous and hyperplastic polyposis. JAMA, 2005. 294(19): p. 2465-73.

381. Aretz, S., et al., High proportion of large genomic deletions and a genotype phenotype update in 80 unrelated families with juvenile polyposis syndrome. J Med Genet, 2007. 44(1 1): p. 7029.

382. Gallione, C.J., et al., A combined syndrome of juvenile polyposis and hereditary haemorrhagic telangiectasia associated with mutations in MADH4 (SMAD4). Lancet, 2004. 363(9412): p. 8529.

383. Watanabe, A., et al., Familial juvenile polyposis of the stomach. Gastroenterology, 1979. 77(1): p. 148-51.

384. Jass, J.R., et al., Juvenile polyposis--a precancerous condition. Histopathology, 1988. 13(6): p. 619-30.

385. Sassatelli, R., et al., Generalized juvenile polyposis with mixed pattern and gastric cancer. Gastroenterology, 1993. 104(3): p. 910-5.

386. Coburn, M.C., et al., Malignant potential in intestinal juvenile polyposis syndromes. Ann Surg Oncol, 1995. 2(5): p. 386-91.

387. Desai, D.C., et al., Juvenile polyposis. Br J Surg, 1995. 82(1): p. 14-7.

388. Delnatte, C., et al., Contiguous gene deletion within chromosome arm $10 \mathrm{q}$ is associated with juvenile polyposis of infancy, reflecting cooperation between the BMPR IA and PTEN tumorsuppressor genes. Am J Hum Genet, 2006. 78(6): p. 1066-74.

389. Hobert, J.A. and C. Eng, PTEN hamartoma tumor syndrome: an overview. Genet Med, 2009. 11 (10): p. 687-94.

390. Tan, M.H., et al., Lifetime cancer risks in individuals with germline PTEN mutations. Clin Cancer Res, 2012. 18(2): p. 400-7.

391. Orloff, M.S. and C. Eng, Genetic and phenotypic heterogeneity in the PTEN hamartoma tumour syndrome. Oncogene, 2008. 27(41): p. 5387-97.

392. Marsh, D.J., et al., PTEN mutation spectrum and genotype-phenotype correlations in BannayanRiley-Ruvalcaba syndrome suggest a single entity with Cowden syndrome. Hum Mol Genet, 1999. 8(8): p. 1461-72.

393. Jeevaratnam, P., et al., Familial giant hyperplastic polyposis predisposing to colorectal cancer: a new hereditary bowel cancer syndrome. J Pathol, 1996. 179(1): p. 20-5.

394. Leggett, B.A., et al., Hyperplastic polyposis: association with colorectal cancer. Am J Surg Pathol, 2001. 25(2): p. 177-84.

395. Renaut, A.J., P.R. Douglas, and G.L. Newstead, Hyperplastic polyposis of the colon and rectum. Colorectal Dis, 2002. 4(3): p. 213-215.

396. Jaeger, E.E., et al., An ancestral Ashkenazi haplotype at the HMPS/CRAC1 locus on 15q13-q14 is associated with hereditary mixed polyposis syndrome. Am J Hum Genet, 2003. 72(5): p. 1261-7.

397. Rozen, P., Z. Samuel, and E. Brazowski, A prospective study of the clinical, genetic, screening, and pathologic features of a family with hereditary mixed polyposis syndrome. Am J Gastroenterol, 2003. 98(10): p. 2317-20.

398. Beggs, A.D., et al., Peutz-Jeghers syndrome: a systematic review and recommendations for management. Gut, 2010. 59(7): p. 975-86. 
399. Dignass, A., et al., [Updated German guideline on diagnosis and treatment of ulcerative colitis, 2011]. Z Gastroenterol, 2011 . 49(9): p. 1276-341.

400. Collins, P.D., et al., Strategies for detecting colon cancer and/or dysplasia in patients with inflammatory bowel disease. Cochrane Database Syst Rev, 2006(2): p. CD000279.

401. Rutter, M.D., et al., Thirty-year analysis of a colonoscopic surveillance program for neoplasia in ulcerative colitis. Gastroenterology, 2006. 130(4): p. 1030-8.

402. Lutgens, M.W., et al., High frequency of early colorectal cancer in inflammatory bowel disease. Gut, 2008. 57(9): p. 1246-51.

403. Lim, C.H., et al., Ten year follow up of ulcerative colitis patients with and without low grade dysplasia. Gut, 2003. 52(8): p. 1127-32.

404. Connell, W.R., et al., Factors affecting the outcome of endoscopic surveillance for cancer in ulcerative colitis. Gastroenterology, 1994. 107(4): p. 934-44.

405. Claessen, M.M., et al., More right-sided IBD-associated colorectal cancer in patients with primary sclerosing cholangitis. Inflamm Bowel Dis, 2009. 15(9): p. 1331-6.

406. Soetikno, R.M., et al., Increased risk of colorectal neoplasia in patients with primary sclerosing cholangitis and ulcerative colitis: a meta-analysis. Gastrointest Endosc, 2002. 56(1): p. 48-54.

407. Scarpa, M., et al., Systematic review of dysplasia after restorative proctocolectomy for ulcerative colitis. Br J Surg, 2007. 94(5): p. 534-45.

408. Hoffmann, J.C., et al., [Clinical practice guideline on diagnosis and treatment of Crohn's disease]. Z Gastroenterol, 2008. 46(9): p. 1094-146.

409. Javeri, K., T.R. Williams, and J.W. Bonnett, An overview of the method, application, and various findings of computed tomographic colonography in patients after incomplete colonoscopy. Curr Probl Diagn Radiol, 2010. 39(6): p. 262-74.

410. Copel, L., et al., CT colonography in 546 patients with incomplete colonoscopy. Radiology, 2007. 244(2): p. 471-8.

411. Neri, E., et al., Colorectal cancer: role of CT colonography in preoperative evaluation after incomplete colonoscopy. Radiology, 2002. 223(3): p. 615-9.

412. Morrin, M.M., et al., Role of virtual computed tomographic colonography in patients with colorectal cancers and obstructing colorectal lesions. Dis Colon Rectum, 2000. 43(3): p. 30311.

413. Pickhardt, P.J., et al., Colorectal cancer: CT colonography and colonoscopy for detection-systematic review and meta-analysis. Radiology, 201 1. 259(2): p. 393-405.

414. Corley, D.A., et al., Adenoma detection rate and risk of colorectal cancer and death. $\mathrm{N}$ Engl J Med, 2014. 370(14): p. 1298-306.

415. Kaminski, M.F., et al., Quality indicators for colonoscopy and the risk of interval cancer. N Engl J Med, 2010. 362(19): p. 1795-803.

416. Denzer, U., et al., [S2k guideline: quality requirements for gastrointestinal endoscopy, AWMF registry no. 021-022]. Z Gastroenterol, 2015. 53(12): p. E1-e227.

417. Rembacken, B., et al., Quality in screening colonoscopy: position statement of the European Society of Gastrointestinal Endoscopy (ESGE). Endoscopy, 2012. 44(10): p. 957-68.

418. Valori, R., et al., European guidelines for quality assurance in colorectal cancer screening and diagnosis. First Edition--Quality assurance in endoscopy in colorectal cancer screening and diagnosis. Endoscopy, 2012. 44 Suppl 3: p. Se88-105.

419. Rees, C.J., et al., UK key performance indicators and quality assurance standards for colonoscopy. Gut, 2016. 65(12): p. 1923-1929.

420. Rex, D.K., et al., Quality indicators for colonoscopy. Am J Gastroenterol, 2006. 101 (4): p. 87385.

421. Aranda-Hernandez, J., J. Hwang, and G. Kandel, Seeing better--Evidence based recommendations on optimizing colonoscopy adenoma detection rate. World J Gastroenterol, 2016. 22(5): p. 1767-78.

422. Barclay, R.L., et al., Colonoscopic withdrawal times and adenoma detection during screening colonoscopy. N Engl J Med, 2006. 355(24): p. 2533-41.

423. Barclay, R.L., J.J. Vicari, and R.L. Greenlaw, Effect of a time-dependent colonoscopic withdrawal protocol on adenoma detection during screening colonoscopy. Clin Gastroenterol Hepatol, 2008. 6(10): p. 1091-8.

424. Butterly, L., et al., Serrated and adenomatous polyp detection increases with longer withdrawal time: results from the New Hampshire Colonoscopy Registry. Am J Gastroenterol, 2014. 109(3): p. 417-26.

425. Sawhney, M.S., et al., Effect of institution-wide policy of colonoscopy withdrawal time $>$ or $=7$ minutes on polyp detection. Gastroenterology, 2008. 135(6): p. 1892-8.

426. Adler, A., et al., Factors determining the quality of screening colonoscopy: a prospective study on adenoma detection rates, from 12,134 examinations (Berlin colonoscopy project 3, BECOP3). Gut, 2013. 62(2): p. 236-41. 
427. Gellad, Z.F., et al., Colonoscopy withdrawal time and risk of neoplasia at 5 years: results from VA Cooperative Studies Program 380. Am J Gastroenterol, 2010. 105(8): p. 1746-52.

428. Moritz, V., et al., Withdrawal time as a quality indicator for colonoscopy - a nationwide analysis. Endoscopy, 2012. 44(5): p. 476-81.

429. Robertson, D.J., et al., Colorectal cancer in patients under close colonoscopic surveillance. Gastroenterology, 2005. 129(1): p. 34-41.

430. Pabby, A., et al., Analysis of colorectal cancer occurrence during surveillance colonoscopy in the dietary Polyp Prevention Trial. Gastrointest Endosc, 2005. 61(3): p. 385-91.

431. Sawhney, M.S., et al., Microsatellite instability in interval colon cancers. Gastroenterology, 2006. 131(6): p. 1700-5.

432. Hosokawa, O., et al., Invasive colorectal cancer detected up to 3 years after a colonoscopy negative for cancer. Endoscopy, 2003. 35(6): p. 506-10.

433. Bressler, B., et al., Colonoscopic miss rates for right-sided colon cancer: a population-based analysis. Gastroenterology, 2004. 127(2): p. 452-6.

434. Leaper, M., et al., Reasons for failure to diagnose colorectal carcinoma at colonoscopy. Endoscopy, 2004. 36(6): p. 499-503.

435. Murthy, S.K., Ducharme R., Rostom A., Dube C., James P.D., Benchimol E., Post-Colonoscopy Colorectal Cancer Rates Have Decreased Over Time in Average-Risk Patients Aged 50 to 74: A Population-Based Study from Ontario, Canada. Gastroenterology, 2016. 150(4): p. S. 120.

436. Plumb, A.A., et al., Sensitivity and specificity of CT colonography for the detection of colonic neoplasia after positive faecal occult blood testing: systematic review and meta-analysis. Eur Radiol, 2014. 24(5): p. 1049-58.

437. Lin, O.S., et al., Preference for colonoscopy versus computerized tomographic colonography: a systematic review and meta-analysis of observational studies. J Gen Intern Med, 2012. 27(10): p. 1349-60.

438. Zijta, F.M., S. Bipat, and J. Stoker, Magnetic resonance (MR) colonography in the detection of colorectal lesions: a systematic review of prospective studies. Eur Radiol, 2010. 20(5): p. 1031 46.

439. Hartmann, D., et al., Incomplete conventional colonoscopy: magnetic resonance colonography in the evaluation of the proximal colon. Endoscopy, 2005. 37(9): p. 816-20.

440. Ajaj, W., et al., MR colonography in patients with incomplete conventional colonoscopy. Radiology, 2005. 234(2): p. 452-9.

441. Spada, C., et al., Clinical indications for computed tomographic colonography: European Society of Gastrointestinal Endoscopy (ESGE) and European Society of Gastrointestinal and Abdominal Radiology (ESGAR) Guideline. Endoscopy, 2014. 46(10): p. 897-915.

442. Spada, C., et al., Colon capsule versus CT colonography in patients with incomplete colonoscopy: a prospective, comparative trial. Gut, 2015. 64(2): p. 272-81.

443. Spada, C., et al., Accuracy of First- and Second-Generation Colon Capsules in Endoscopic Detection of Colorectal Polyps: A Systematic Review and Meta-analysis. Clin Gastroenterol Hepatol, 2016. 14(11): p. 1533-1543.e8.

444. Rex, D.K., et al., Accuracy of capsule colonoscopy in detecting colorectal polyps in a screening population. Gastroenterology, 2015. 148(5): p. 948-957 e2.

445. Reumkens, A., et al., Post-Colonoscopy Complications: A Systematic Review, Time Trends, and Meta-Analysis of Population-Based Studies. Am J Gastroenterol, 2016. 111 (8): p. 1092-101.

446. Boroff, E.S., et al., Polyp and adenoma detection rates in the proximal and distal colon. Am J Gastroenterol, 2013. 108(6): p. 993-9.

447. Dodou, D. and J.C. de Winter, The relationship between distal and proximal colonic neoplasia: a meta-analysis. J Gen Intern Med, 2012. 27(3): p. 361-70.

448. Lin, O.S., et al., Risk of proximal colon neoplasia with distal hyperplastic polyps: a metaanalysis. Arch Intern Med, 2005. 165(4): p. 382-90.

449. Arora, A. and P. Singh, Colonoscopy in patients 80 years of age and older is safe, with high success rate and diagnostic yield. Gastrointest Endosc, 2004. 60(3): p. 408-13.

450. Karajeh, M.A., D.S. Sanders, and D.P. Hurlstone, Colonoscopy in elderly people is a safe procedure with a high diagnostic yield: a prospective comparative study of 2000 patients. Endoscopy, 2006. 38(3): p. 226-30.

451. Jafri, S.M., K. Monkemuller, and F.J. Lukens, Endoscopy in the elderly: a review of the efficacy and safety of colonoscopy, esophagogastroduodenoscopy, and endoscopic retrograde cholangiopancreatography. J Clin Gastroenterol, 2010. 44(3): p. 161-6.

452. Travis, A.C., D. Pievsky, and J.R. Saltzman, Endoscopy in the elderly. Am J Gastroenterol, 2012. 107(10): p. 1495-501; quiz 1494, 1502.

453. Khan, S., et al., Colonoscopy in the octogenarian population: diagnostic and survival outcomes from a large series of patients. Surgeon, 2011 . 9(4): p. 195-9. 
454. Lim, Y.J. and S.J. Hong, What is the best strategy for successful bowel preparation under special conditions? World J Gastroenterol, 2014. 20(11): p. 2741-5.

455. Lin, O.S., Performing colonoscopy in elderly and very elderly patients: Risks, costs and benefits. World J Gastrointest Endosc, 2014. 6(6): p. 220-6.

456. Lin, O.S., et al., Screening colonoscopy in very elderly patients: prevalence of neoplasia and estimated impact on life expectancy. JAMA, 2006. 295(20): p. 2357-65.

457. Yano, H., et al., Oxygen desaturation during sedation for colonoscopy in elderly patients. Hepatogastroenterology, 1998. 45(24): p. 2138-41.

458. Schreuders, E.H., et al., Colorectal cancer screening: a global overview of existing programmes. Gut, 2015. 64(10): p. 1637-49.

459. Kiesslich, R., et al., Methylene blue-aided chromoendoscopy for the detection of intraepithelial neoplasia and colon cancer in ulcerative colitis. Gastroenterology, 2003. 124(4): p. 880-8.

460. Freire, P., et al., Surveillance in ulcerative colitis: is chromoendoscopy-guided endomicroscopy always better than conventional colonoscopy? A randomized trial. Inflamm Bowel Dis, 2014. 20(11): p. 2038-45.

461. Mooiweer, E., et al., Chromoendoscopy for Surveillance in Inflammatory Bowel Disease Does Not Increase Neoplasia Detection Compared With Conventional Colonoscopy With Random Biopsies: Results From a Large Retrospective Study. Am J Gastroenterol, $2015.110(7)$ : p. $1014-$ 21.

462. Wu, L., et al., The diagnostic accuracy of chromoendoscopy for dysplasia in ulcerative colitis: meta-analysis of six randomized controlled trials. Colorectal Dis, 2012. 14(4): p. 416-20.

463. Iannone, A., et al., Chromoendoscopy for Surveillance in Ulcerative Colitis and Crohn's Disease: A Systematic Review of Randomized Trials. Clin Gastroenterol Hepatol, 2017. 15(1 1): p. 16841697 e 11.

464. Rahmi, G., et al., Impact of chromoscopy on adenoma detection in patients with Lynch syndrome: a prospective, multicenter, blinded, tandem colonoscopy study. Am J Gastroenterol, 2015. 110(2): p. 288-98.

465. Lecomte, T., et al., Chromoendoscopic colonoscopy for detecting preneoplastic lesions in hereditary nonpolyposis colorectal cancer syndrome. Clin Gastroenterol Hepatol, 2005. 3(9): p. 897-902.

466. Dinesen, L., T.J. Chua, and A.J. Kaffes, Meta-analysis of narrow-band imaging versus conventional colonoscopy for adenoma detection. Gastrointest Endosc, 2012. 75(3): p. 604-11.

467. Omata, F., et al., Image-enhanced, chromo, and cap-assisted colonoscopy for improving adenoma/neoplasia detection rate: a systematic review and meta-analysis. Scand J Gastroenterol, 2014. 49(2): p. 222-37.

468. Sabbagh, L.C., et al., Narrow-band imaging does not improve detection of colorectal polyps when compared to conventional colonoscopy: a randomized controlled trial and meta-analysis of published studies. BMC Gastroenterol, 2011.11: p. 100.

469. Pasha, S.F., et al., Comparison of the yield and miss rate of narrow band imaging and white light endoscopy in patients undergoing screening or surveillance colonoscopy: a meta-analysis. Am J Gastroenterol, 2012. 107(3): p. 363-70; quiz 371.

470. Jin, X.F., et al., Meta-analysis for evaluating the accuracy of endoscopy with narrow band imaging in detecting colorectal adenomas. J Gastroenterol Hepatol, 2012. 27(5): p. 882-7.

471. Nagorni, A., G. Bjelakovic, and B. Petrovic, Narrow band imaging versus conventional white light colonoscopy for the detection of colorectal polyps. Cochrane Database Syst Rev, 2012. 1 : p. CD008361.

472. Chung, S.J., et al., Comparison of detection and miss rates of narrow band imaging, flexible spectral imaging chromoendoscopy and white light at screening colonoscopy: a randomised controlled back-to-back study. Gut, 2014. 63(5): p. 785-91.

473. Hoffman, A., et al., High definition plus colonoscopy combined with i-scan tone enhancement vs. high definition colonoscopy for colorectal neoplasia: A randomized trial. Dig Liver Dis, 2014. 46(11): p. 991-6.

474. Hoffman, A., et al., High definition colonoscopy combined with i-Scan is superior in the detection of colorectal neoplasias compared with standard video colonoscopy: a prospective randomized controlled trial. Endoscopy, 2010. 42(10): p. 827-33.

475. Hong, S.N., et al., Prospective, randomized, back-to-back trial evaluating the usefulness of $i$ SCAN in screening colonoscopy. Gastrointest Endosc, 2012. 75(5): p. 1011-1021 e2.

476. Kudo, S., et al., Colonoscopic diagnosis and management of nonpolypoid early colorectal cancer. World J Surg, 2000. 24(9): p. 1081-90.

477. Kiesslich, R., et al., Chromoendoscopy with indigocarmine improves the detection of adenomatous and nonadenomatous lesions in the colon. Endoscopy, 2001. 33(12): p. 1001-6.

478. Kudo, S., et al., Pit pattern in colorectal neoplasia: endoscopic magnifying view. Endoscopy, 2001. 33(4): p. 367-73. 
479. Brooker, J.C., et al., Total colonic dye-spray increases the detection of diminutive adenomas during routine colonoscopy: a randomized controlled trial. Gastrointest Endosc, 2002. 56(3): p. 333-8.

480. Eisen, G.M., et al., High-resolution chromoendoscopy for classifying colonic polyps: a multicenter study. Gastrointest Endosc, 2002. 55(6): p. 687-94.

481. Tsuda, S., et al., Flat and depressed colorectal tumours in a southern Swedish population: a prospective chromoendoscopic and histopathological study. Gut, 2002. 51(4): p. 550-5.

482. He, Q., et al., Cap-assisted colonoscopy versus conventional colonoscopy: systematic review and meta-analysis. Int J Colorectal Dis, 2013. 28(2): p. 279-81.

483. Morgan, J.L., et al., Transparent cap colonoscopy versus standard colonoscopy: a systematic review and meta-analysis. Tech Coloproctol, 2013. 17(4): p. 353-60.

484. Ng, S.C., et al., The efficacy of cap-assisted colonoscopy in polyp detection and cecal intubation: a meta-analysis of randomized controlled trials. Am J Gastroenterol, 2012. 107(8): p. 1165-73.

485. Westwood, D.A., N. Alexakis, and S.J. Connor, Transparent cap-assisted colonoscopy versus standard adult colonoscopy: a systematic review and meta-analysis. Dis Colon Rectum, 2012. 55(2): p. 218-25.

486. Biecker, E., et al., Novel endocuff-assisted colonoscopy significantly increases the polyp detection rate: a randomized controlled trial. J Clin Gastroenterol, 2015. 49(5): p. 413-8.

487. Floer, M., et al., Higher adenoma detection rates with endocuff-assisted colonoscopy - a randomized controlled multicenter trial. PLoS One, 2014. 9(12): p. e114267.

488. van Doorn, S.C., et al., Adenoma detection with Endocuff colonoscopy versus conventional colonoscopy: a multicentre randomised controlled trial. Gut, $2017.66(3)$ : p. 438-445.

489. Ngu WS, B.R., Tsiamoulos Z, Bassett P, Hoare Z, Rutter M,Totton N, Lee TJ, Ramadas A, Silcock J, Painter J, Neilson LJ, Saunders BP, Rees CJ, Improved adenoma detection with endocuff vision - a multicentre randomised controlled trial. UEGW late breaking abstract, 2016.

490. Halpern, Z., et al., Comparison of adenoma detection and miss rates between a novel balloon colonoscope and standard colonoscopy: a randomized tandem study. Endoscopy, 2015. 47(4): p. 301.

491. The Paris endoscopic classification of superficial neoplastic lesions: esophagus, stomach, and colon: November 30 to December 1, 2002. Gastrointest Endosc, 2003. 58(6 Suppl): p. S3-43.

492. Abu Dayyeh, B.K., et al., ASGE Technology Committee systematic review and meta-analysis assessing the ASGE PIVI thresholds for adopting real-time endoscopic assessment of the histology of diminutive colorectal polyps. Gastrointest Endosc, 2015. 81(3): p. 502.e1-502.e16.

493. Hewett, D.G., et al., Validation of a simple classification system for endoscopic diagnosis of small colorectal polyps using narrow-band imaging. Gastroenterology, 2012. 143(3): p. 599607 e 1 .

494. Ignjatovic, A., et al., Narrow band imaging for detection of dysplasia in colitis: a randomized controlled trial. Am J Gastroenterol, 2012. 107(6): p. 885-90.

495. Hassan, C., et al., Colonic polyps: are we ready to resect and discard? Gastrointest Endosc Clin N Am, 2013. 23(3): p. 663-78.

496. Hassan, C., P.J. Pickhardt, and D.K. Rex, A resect and discard strategy would improve costeffectiveness of colorectal cancer screening. Clin Gastroenterol Hepatol, 2010. 8(10): p. 865-9, 869 e $1-3$.

497. Kessler, W.R., et al., A quantitative assessment of the risks and cost savings of forgoing histologic examination of diminutive polyps. Endoscopy, 2011 . 43(8): p. 683-91.

498. Kiesslich, R., et al., Confocal laser endoscopy for diagnosing intraepithelial neoplasias and colorectal cancer in vivo. Gastroenterology, 2004. 127(3): p. 706-13.

499. Gondal, G., et al., Biopsy of colorectal polyps is not adequate for grading of neoplasia. Endoscopy, 2005. 37(12): p. 1193-7.

500. Nivatvongs, S., Complications in colonoscopic polypectomy. An experience with 1,555 polypectomies. Dis Colon Rectum, 1986. 29(12): p. 825-30.

501. Reiertsen, O., et al., Complications of fiberoptic gastrointestinal endoscopy--five years' experience in a central hospital. Endoscopy, 1987. 19(1): p. 1-6.

502. Waye, J.D., B.S. Lewis, and S. Yessayan, Colonoscopy: a prospective report of complications. J Clin Gastroenterol, 1992. 15(4): p. 347-51.

503. Waye, J.D., O. Kahn, and M.E. Auerbach, Complications of colonoscopy and flexible sigmoidoscopy. Gastrointest Endosc Clin N Am, 1996. 6(2): p. 343-77.

504. Hsieh, Y.H., et al., Is submucosal epinephrine injection necessary before polypectomy? A prospective, comparative study. Hepatogastroenterology, 2001. 48(41): p. 1379-82.

505. Heitman, S.J., D.J. Tate, and M.J. Bourke, Optimizing Resection of Large Colorectal Polyps. Curr Treat Options Gastroenterol, 2017. 
506. Imai, K., et al., Should laterally spreading tumors granular type be resected en bloc in endoscopic resections? Surg Endosc, 2014. 28(7): p. 2167-73.

507. Oka, S., et al., Therapeutic strategy for colorectal laterally spreading tumor. Dig Endosc, 2009. 21 Suppl 1: p. S43-6.

508. Saito, Y., et al., Endoscopic treatment for laterally spreading tumors in the colon. Endoscopy, 2001. 33(8): p. 682-6.

509. Saito, Y., et al., Endoscopic submucosal dissection (ESD) for colorectal tumors. Dig Endosc, 2009. 21 Suppl 1: p. S7-12.

510. Uraoka, T., et al., Endoscopic indications for endoscopic mucosal resection of laterally spreading tumours in the colorectum. Gut, 2006. 55(11): p. 1592-7.

511. Moss, A., et al., Endoscopic mucosal resection outcomes and prediction of submucosal cancer from advanced colonic mucosal neoplasia. Gastroenterology, 2011 . 140(7): p. 1909-18.

512. Rotondano, G., et al., The Cooperative Italian FLIN Study Group: prevalence and clinicopathological features of colorectal laterally spreading tumors. Endoscopy, 2011 . 43(10): p. 856-61.

513. Ishiguro, A., et al., Correlation of lifting versus non-lifting and microscopic depth of invasion in early colorectal cancer. Gastrointest Endosc, 1999. 50(3): p. 329-33.

514. Uno, Y. and A. Munakata, The non-lifting sign of invasive colon cancer. Gastrointest Endosc, 1994. 40(4): p. 485-9.

515. Kobayashi, N., et al., Determining the treatment strategy for colorectal neoplastic lesions: endoscopic assessment or the non-lifting sign for diagnosing invasion depth? Endoscopy, 2007. 39(8): p. $701-5$.

516. Voloyiannis, T., et al., Management of the difficult colon polyp referred for resection: resect or rescope? Dis Colon Rectum, 2008. 51(3): p. 292-5.

517. Aziz Aadam, A., et al., Physician assessment and management of complex colon polyps: a multicenter video-based survey study. Am J Gastroenterol, 2014. 109(9): p. 1312-24.

518. Lipof, T., et al., Preoperative colonoscopy decreases the need for laparoscopic management of colonic polyps. Dis Colon Rectum, 2005. 48(5): p. 1076-80.

519. Church, J.M., Avoiding surgery in patients with colorectal polyps. Dis Colon Rectum, 2003. 46(11): p. 1513-6.

520. Buchner, A.M., C. Guarner-Argente, and G.G. Ginsberg, Outcomes of EMR of defiant colorectal lesions directed to an endoscopy referral center. Gastrointest Endosc, 2012. 76(2): p. 255-63.

521. Friedland, S., et al., Outcomes of repeat colonoscopy in patients with polyps referred for surgery without biopsy-proven cancer. Gastrointest Endosc, 2014. 79(1): p. 101-7.

522. Efthymiou, M., et al., Biopsy forceps is inadequate for the resection of diminutive polyps. Endoscopy, 2011 . 43(4): p. 312-6.

523. Deenadayalu, V.P. and D.K. Rex, Colon polyp retrieval after cold snaring. Gastrointest Endosc, 2005. 62(2): p. 253-6.

524. Repici, A., et al., Safety of cold polypectomy for <10 $\mathrm{mm}$ polyps at colonoscopy: a prospective multicenter study. Endoscopy, 2012. 44(1): p. 27-31.

525. Tappero, G., et al., Cold snare excision of small colorectal polyps. Gastrointest Endosc, 1992. 38(3): p. 310-3.

526. Kim, J.S., et al., Cold snare polypectomy versus cold forceps polypectomy for diminutive and small colorectal polyps: a randomized controlled trial. Gastrointest Endosc, 2015. 81(3): p. 741-7.

527. Lee, C.K., J.J. Shim, and J.Y. Jang, Cold snare polypectomy vs. Cold forceps polypectomy using double-biopsy technique for removal of diminutive colorectal polyps: a prospective randomized study. Am J Gastroenterol, 2013. 108(10): p. 1593-600.

528. Moss, A., et al., Long-term adenoma recurrence following wide-field endoscopic mucosal resection (WF-EMR) for advanced colonic mucosal neoplasia is infrequent: results and risk factors in 1000 cases from the Australian Colonic EMR (ACE) study. Gut, 2015. 64(1): p. 57-65.

529. Knabe, M., et al., Standardized long-term follow-up after endoscopic resection of large, nonpedunculated colorectal lesions: a prospective two-center study. Am J Gastroenterol, 2014. 109(2): p. 183-9.

530. Seidel, J., et al., Complication and local recurrence rate after endoscopic resection of large high-risk colorectal adenomas of $>/=3 \mathrm{~cm}$ in size. Int J Colorectal Dis, 2016. 31(3): p. 603-11.

531. Cipolletta, L., et al., Endoscopic resection for superficial colorectal neoplasia in Italy: a prospective multicentre study. Dig Liver Dis, 2014. 46(2): p. 146-51.

532. Heresbach, D., et al., A national survey of endoscopic mucosal resection for superficial gastrointestinal neoplasia. Endoscopy, 2010. 42(10): p. 806-13.

533. Woodward, T.A., et al., Predictors of complete endoscopic mucosal resection of flat and depressed gastrointestinal neoplasia of the colon. Am J Gastroenterol, 2012. 107(5): p. 650-4. 
534. Repici, A., et al., Efficacy and safety of endoscopic submucosal dissection for colorectal neoplasia: a systematic review. Endoscopy, 2012. 44(2): p. 137-50.

535. Kim, Y.J., et al., Comparison of clinical outcomes among different endoscopic resection methods for treating colorectal neoplasia. Dig Dis Sci, 2013. 58(6): p. 1727-36.

536. Kobayashi, N., et al., Matched case-control study comparing endoscopic submucosal dissection and endoscopic mucosal resection for colorectal tumors. J Gastroenterol Hepatol, 2012. 27(4): p. 728-33.

537. Saito, Y., et al., Clinical outcome of endoscopic submucosal dissection versus endoscopic mucosal resection of large colorectal tumors as determined by curative resection. Surg Endosc, 2010. 24(2): p. 343-52.

538. Tajika, M., et al., Comparison of endoscopic submucosal dissection and endoscopic mucosal resection for large colorectal tumors. Eur J Gastroenterol Hepatol, 2011 . 23(1 1): p. 1042-9.

539. Terasaki, M., et al., Clinical outcomes of endoscopic submucosal dissection and endoscopic mucosal resection for laterally spreading tumors larger than $20 \mathrm{~mm}$. J Gastroenterol Hepatol, 2012. 27(4): p. 734-40.

540. Fujiya, M., et al., Efficacy and adverse events of EMR and endoscopic submucosal dissection for the treatment of colon neoplasms: a meta-analysis of studies comparing EMR and endoscopic submucosal dissection. Gastrointest Endosc, 2015. 81(3): p. 583-95.

541. Wang, J., et al., Endoscopic submucosal dissection vs endoscopic mucosal resection for colorectal tumors: a meta-analysis. World J Gastroenterol, 2014. 20(25): p. 8282-7.

542. Sauer, M., et al., Endoscopic submucosal dissection for flat or sessile colorectal neoplasia $>20$ $\mathrm{mm}$ : A European single-center series of 182 cases. Endosc Int Open, 2016. 4(8): p. E895-900.

543. Probst, A., et al., Endoscopic submucosal dissection in large sessile lesions of the rectosigmoid: learning curve in a European center. Endoscopy, 2012. 44(7): p. 660-7.

544. Rahmi, G., et al., Endoscopic submucosal dissection for superficial rectal tumors: prospective evaluation in France. Endoscopy, 2014. 46(8): p. 670-6.

545. Repici, A., et al., High efficacy of endoscopic submucosal dissection for rectal laterally spreading tumors larger than $3 \mathrm{~cm}$. Gastrointest Endosc, 2013. 77(1): p. 96-101.

546. Probst, A., et al., Endoscopic submucosal dissection for early rectal neoplasia: experience from a European center. Endoscopy, 2017. 49(3): p. 222-232.

547. Schmidt, A.D., M. Gubier, C. Caca, K. Bauerfeind, P., Endoskopische Vollwandresektion im unteren GI-Trakt mit dem FTRD-System: eine retrospektive Studie. Zeitschrift für Gastroenterologie, 2014. 52: p. 52 - 56.

548. Fisher, D.A., et al., Complications of colonoscopy. Gastrointest Endosc, 2011 . 74(4): p. 745-52.

549. Niv, Y., et al., Screening colonoscopy for colorectal cancer in asymptomatic people: a metaanalysis. Dig Dis Sci, 2008. 53(12): p. 3049-54.

550. Adler, A., et al., Data quality of the German screening colonoscopy registry. Endoscopy, 2013. 45(10): p. 813-8.

551. Bokemeyer, B., et al., Screening colonoscopy for colorectal cancer prevention: results from a German online registry on 269000 cases. Eur J Gastroenterol Hepatol, 2009. 21(6): p. 650-5.

552. Crispin, A., et al., Process quality and incidence of acute complications in a series of more than 230,000 outpatient colonoscopies. Endoscopy, 2009. 41(12): p. 1018-25.

553. Eckardt, V.F., et al., Complications and adverse effects of colonoscopy with selective sedation. Gastrointest Endosc, 1999. 49(5): p. 560-5.

554. Hagel, A.F., et al., Colonoscopy-associated perforation: a 7-year survey of in-hospital frequency, treatment and outcome in a German university hospital. Colorectal Dis, 2012. 14(9): p. $1121-5$.

555. Sieg, A., U. Hachmoeller-Eisenbach, and T. Eisenbach, Prospective evaluation of complications in outpatient Gl endoscopy: a survey among German gastroenterologists. Gastrointest Endosc, 2001. 53(6): p. 620-7.

556. Stock, C., et al., Adverse events requiring hospitalization within 30 days after outpatient screening and nonscreening colonoscopies. Gastrointest Endosc, 2013. 77(3): p. 419-29.

557. Heldwein, W., et al., The Munich Polypectomy Study (MUPS): prospective analysis of complications and risk factors in 4000 colonic snare polypectomies. Endoscopy, 2005. 37(11): p. $1116-22$.

558. Bowles, C.J., et al., A prospective study of colonoscopy practice in the UK today: are we adequately prepared for national colorectal cancer screening tomorrow? Gut, 2004. 53(2): p. 277-83.

559. Kang, H.Y., et al., Incidence and management of colonoscopic perforations in Korea. Digestion, 2008. 78(4): p. 218-23.

560. Ko, C.W., et al., Serious complications within 30 days of screening and surveillance colonoscopy are uncommon. Clin Gastroenterol Hepatol, 2010. 8(2): p. 166-73. 
561. Nelson, D.B., et al., Procedural success and complications of large-scale screening colonoscopy. Gastrointest Endosc, 2002. 55(3): p. 307-14.

562. Niv, Y., et al., Complications in colonoscopy: analysis of 7-year physician-reported adverse events. Eur J Gastroenterol Hepatol, 2011 . 23(6): p. 492-8.

563. Rabeneck, L., et al., Bleeding and perforation after outpatient colonoscopy and their risk factors in usual clinical practice. Gastroenterology, 2008. 135(6): p. 1899-1906, 1906 el.

564. Singh, H., et al., Colonoscopy and its complications across a Canadian regional health authority. Gastrointest Endosc, 2009. 69(3 Pt 2): p. 665-71.

565. Warren, J.L., et al., Adverse events after outpatient colonoscopy in the Medicare population. Ann Intern Med, 2009. 150(12): p. 849-57, W152.

566. Zubarik, R., et al., Prospective analysis of complications 30 days after outpatient colonoscopy. Gastrointest Endosc, 1999. 50(3): p. 322-8.

567. Zubarik, R., et al., Procedure-related abdominal discomfort in patients undergoing colorectal cancer screening: a comparison of colonoscopy and flexible sigmoidoscopy. Am J Gastroenterol, 2002. 97(12): p. 3056-61.

568. Hamilton, S.R. and L.A. Aaltonen, Pathology and Genetics of Tumours of the Digestive System. World Health Organization Classifecation of Tumours, ed. . 2000, Lyon: IARC Press.

569. Jass, J.R., Classification of colorectal cancer based on correlation of clinical, morphological and molecular features. Histopathology, 2007. 50(1): p. 113-30.

570. Makinen, M.J., Colorectal serrated adenocarcinoma. Histopathology, 2007. 50(1): p. 131-50.

571. Chetty, R., Traditional serrated adenoma (TSA): morphological questions, queries and quandaries. J Clin Pathol, 2016. 69(1): p. 6-11.

572. Gibson, J.A. and R.D. Odze, Pathology of premalignant colorectal neoplasia. Dig Endosc, 2016. 28(3): p. 312-23.

573. Bosman F.T., C.F., Hruban R.H., Theise N.D. , WHO Classification of Tumours of the Digestive System. 2010.4 ed. World Health Organisation, 2010.

574. Gao, Q., et al., Serrated polyps and the risk of synchronous colorectal advanced neoplasia: a systematic review and meta-analysis. Am J Gastroenterol, 2015. 110(4): p. 501-9; quiz 510.

575. Holme, O., et al., Long-term risk of colorectal cancer in individuals with serrated polyps. Gut, 2015. 64(6): p. 929-36.

576. Erichsen, R., et al., Increased Risk of Colorectal Cancer Development Among Patients With Serrated Polyps. Gastroenterology, 2016. 150(4): p. 895-902 e5.

577. Hassan, C., et al., New paradigms for colonoscopic management of diminutive colorectal polyps: predict, resect, and discard or do not resect? Clin Endosc, 2013. 46(2): p. 130-7.

578. Baretton, G.B., A. Tannapfel, and W. Schmitt, [Standardized and structured histopathological evaluation of colorectal polyps: a practical checklist against the background of the new WHO classification]. Pathologe, 2011 . 32(4): p. 289-96.

579. Wada, H., et al., Systematic review and meta-analysis of histopathological predictive factors for lymph node metastasis in T1 colorectal cancer. J Gastroenterol, 2015. 50(7): p. 727-34.

580. Kawachi, H., et al., A three-tier classification system based on the depth of submucosal invasion and budding/sprouting can improve the treatment strategy for T1 colorectal cancer: a retrospective multicenter study. Mod Pathol, 2015. 28(6): p. 872-9.

581. Mou, S., et al., Pathologic predictive factors for lymph node metastasis in submucosal invasive (T1) colorectal cancer: a systematic review and meta-analysis. Surg Endosc, 2013. 27(8): p. 2692-703.

582. Beaton, C., et al., Systematic review and meta-analysis of histopathological factors influencing the risk of lymph node metastasis in early colorectal cancer. Colorectal Dis, 2013. 15(7): p. 788-97.

583. Bosch, S.L., et al., Predicting lymph node metastasis in pT1 colorectal cancer: a systematic review of risk factors providing rationale for therapy decisions. Endoscopy, 2013. 45(10): p. 827-34.

584. Choi, J.Y., et al., Meta-analysis of predictive clinicopathologic factors for lymph node metastasis in patients with early colorectal carcinoma. J Korean Med Sci, 2015. 30(4): p. 398406.

585. Ueno, H., et al., Risk factors for an adverse outcome in early invasive colorectal carcinoma. Gastroenterology, 2004. 127(2): p. 385-94.

586. Hassan, C., et al., Histologic risk factors and clinical outcome in colorectal malignant polyp: a pooled-data analysis. Dis Colon Rectum, 2005. 48(8): p. 1588-96.

587. Blackstock, W., et al., ACR Appropriateness Criteria: local excision in early-stage rectal cancer. Curr Probl Cancer, 2010. 34(3): p. 193-200.

588. Russo, S., et al., ACR Appropriateness Criteria(R) Local Excision in Early Stage Rectal Cancer. Am J Clin Oncol, 2015. 38(5): p. 520-5. 
589. Kikuchi, R., et al., Management of early invasive colorectal cancer. Risk of recurrence and clinical guidelines. Dis Colon Rectum, 1995. 38(12): p. 1286-95.

590. Di Gregorio, C., et al., Clinical outcome of low- and high-risk malignant colorectal polyps: results of a population-based study and meta-analysis of the available literature. Intern Emerg Med, 2014. 9(2): p. 151-60.

591. Fischer, J., et al., Management of malignant colorectal polyps in New Zealand. ANZ J Surg, 2017. 87(5): p. 350-355.

592. Wu, X.R., J. Liang, and J.M. Church, Management of sessile malignant polyps: is colonoscopic polypectomy enough? Surg Endosc, 2015. 29(10): p. 2947-52.

593. Hassan, C., et al., Endoscopic follow-up after colorectal cancer resection: an Italian multicentre study. Dig Liver Dis, 2006. 38(1): p. 45-50.

594. Seitz, U., et al., Is endoscopic polypectomy an adequate therapy for malignant colorectal adenomas? Presentation of 114 patients and review of the literature. Dis Colon Rectum, 2004. 47(11): p. 1789-96; discussion 1796-7.

595. Meining, A., et al., Risk factors for unfavorable outcomes after endoscopic removal of submucosal invasive colorectal tumors. Clin Gastroenterol Hepatol, 2011. 9(7): p. 590-4.

596. Nascimbeni, R., et al., Risk of lymph node metastasis in T1 carcinoma of the colon and rectum. Dis Colon Rectum, 2002. 45(2): p. 200-6.

597. Ramirez, M., et al., Management of the malignant polyp. Clin Colon Rectal Surg, 2008. 21 (4): p. 286-90.

598. Matsuda, T., et al., Risk of lymph node metastasis in patients with pedunculated type early invasive colorectal cancer: a retrospective multicenter study. Cancer Sci, 2011 . 102(9): p. 1693-7.

599. Kitajima, K., et al., Correlations between lymph node metastasis and depth of submucosal invasion in submucosal invasive colorectal carcinoma: a Japanese collaborative study. J Gastroenterol, 2004. 39(6): p. 534-43.

600. Suh, J.H., et al., Predictors for lymph node metastasis in T1 colorectal cancer. Endoscopy, 2012. 44(6): p. 590-5.

601. Okabe, S., et al., Lymph node metastasis in T1 adenocarcinoma of the colon and rectum. J Gastrointest Surg, 2004. 8(8): p. 1032-9; discussion 1039-40.

602. Nakadoi, K., et al., Management of T1 colorectal carcinoma with special reference to criteria for curative endoscopic resection. J Gastroenterol Hepatol, 2012. 27(6): p. 1057-62.

603. Ikematsu, H., et al., Long-term outcomes after resection for submucosal invasive colorectal cancers. Gastroenterology, 2013. 144(3): p. 551-9; quiz el 4.

604. Cooper, G.S., et al., Management of malignant colonic polyps: a population-based analysis of colonoscopic polypectomy versus surgery. Cancer, 2012. 118(3): p. 651-9.

605. Provenzale, D., et al., Risk for colon adenomas in patients with rectosigmoid hyperplastic polyps. Ann Intern Med, 1990. 113: p. 760-3.

606. Rex, D.K., et al., Distal colonic hyperplastic polyps do not predict proximal adenomas in asymptomatic average-risk subjects. Gastroenterology, 1992. 102: p. 317-9.

607. Sciallero, S., et al., Distal hyperplastic polyps do not predict proximal adenomas: results from a multicentric study of colorectal adenomas. Gastrointest Endosc, 1997. 46(2): p. 124-30.

608. Koide, N., et al., A case of hyperplastic polyposis of the colon with adenocarcinomas in hyperplastic polyps after long-term follow-up. Endoscopy, 2002. 34(6): p. 499-502.

609. Martinez, M.E., et al., A pooled analysis of advanced colorectal neoplasia diagnoses after colonoscopic polypectomy. Gastroenterology, 2009. 136(3): p. 832-41.

610. Cottet, V., et al., Long-term risk of colorectal cancer after adenoma removal: a populationbased cohort study. Gut, 2012.61(8): p. 1180-6.

611 . Loberg, M., et al., Long-term colorectal-cancer mortality after adenoma removal. N Engl J Med, 2014. 371(9): p. 799-807.

612. Winawer, S.J., et al., Randomized comparison of surveillance intervals after colonoscopic removal of newly diagnosed adenomatous polyps. The National Polyp Study Workgroup. N Engl J Med, 1993. 328(13): p. 901-6.

613. Higaki, S., et al., Long-term follow-up of large flat colorectal tumors resected endoscopically. Endoscopy, 2003. 35(10): p. 845-9.

614. Regula, J., et al., Argon plasma coagulation after piecemeal polypectomy of sessile colorectal adenomas: long-term follow-up study. Endoscopy, 2003. 35(3): p. 212-8.

615. Seitz, U., et al., Long-term results of endoscopic removal of large colorectal adenomas. Endoscopy, 2003. 35(8): p. S41-4.

616. Hurlstone, D.P., et al., A prospective analysis of extended endoscopic mucosal resection for large rectal villous adenomas: an alternative technique to transanal endoscopic microsurgery. Colorectal Dis, 2005. 7(4): p. 339-44. 
617. Boix, J., et al., Endoscopic removal of large sessile colorectal adenomas: is it safe and effective? Dig Dis Sci, 2007. 52(3): p. 840-4.

618. Pereyra, L., et al., Risk of Metachronous Advanced Neoplastic Lesions in Patients with Sporadic Sessile Serrated Adenomas Undergoing Colonoscopic Surveillance. Am J Gastroenterol, 2016. $111(6)$ : p. 871-8.

619. Macaron, C., et al., Risk of Metachronous Polyps in Individuals With Serrated Polyps. Dis Colon Rectum, 2015. 58(8): p. 762-8.

620. Lieberman, D.A., et al., Utilization of colonoscopy in the United States: results from a national consortium. Gastrointest Endosc, 2005. 62(6): p. 875-83.

621. Pickhardt, P.J., et al., Computed tomographic virtual colonoscopy to screen for colorectal neoplasia in asymptomatic adults. N Engl J Med, 2003. 349(23): p. 2191-200.

622. Vemulapalli, K.C. and D.K. Rex, Risk of advanced lesions at first follow-up colonoscopy in highrisk groups as defined by the United Kingdom post-polypectomy surveillance guideline: data from a single U.S. center. Gastrointest Endosc, 2014. 80(2): p. 299-306.

623. Park, H.W., et al., Probability of high-risk colorectal neoplasm recurrence based on the results of two previous colonoscopies. Dig Dis Sci, 2015. 60(1): p. 226-33.

624. Suh, K.H., et al., Risk of adenomas with high-risk characteristics based on two previous colonoscopy. J Gastroenterol Hepatol, 2014. 29(12): p. 1985-90.

625. Laiyemo, A.O., et al., Utilization and yield of surveillance colonoscopy in the continued followup study of the polyp prevention trial. Clin Gastroenterol Hepatol, 2009. 7(5): p. 562-7; quiz 497.

626. Robertson, D.J., et al., Estimated Risk of Advanced and Multiple Adenomas Based On the Results of Two Prior Colonoscopies. Ann Intern Med, 2009. 151(2): p. 103-109.

627. Robertson, D.J., et al., Colorectal cancers soon after colonoscopy: a pooled multicohort analysis. Gut, 2014. 63(6): p. 949-56.

628. Ferlitsch, M., et al., Colorectal polypectomy and endoscopic mucosal resection (EMR): European Society of Gastrointestinal Endoscopy (ESGE) Clinical Guideline. Endoscopy, 2017. 49(3): p. 270297.

629. Baron, J.A., et al., A randomized trial of aspirin to prevent colorectal adenomas. N Engl J Med, 2003. 348(10): p. 891-9.

630. Benamouzig, R., et al., Daily soluble aspirin and prevention of colorectal adenoma recurrence: one-year results of the APACC trial. Gastroenterology, 2003. 125(2): p. 328-36.

631. Weingarten, M.A., A. Zalmanovici, and J. Yaphe, Dietary calcium supplementation for preventing colorectal cancer and adenomatous polyps. Cochrane Database Syst Rev, 2005(3): p. CD003548.

632. Imperiale, T.F., Aspirin and the prevention of colorectal cancer. N Engl J Med, 2003. 348(10): p. 879-80.

633. Bresalier, R.S., et al., Cardiovascular events associated with rofecoxib in a colorectal adenoma chemoprevention trial. N Engl J Med, 2005. 352(11): p. 1092-102.

634. Solomon, S.D., et al., Cardiovascular risk associated with celecoxib in a clinical trial for colorectal adenoma prevention. N Engl J Med, 2005. 352(11): p. 1071-80.

635. Psaty, B.M. and J.D. Potter, Risks and benefits of celecoxib to prevent recurrent adenomas. $\mathrm{N}$ Engl J Med, 2006. 355(9): p. 950-2.

636. Fielding, L.P., et al., Clinicopathological staging for colorectal cancer: an International Documentation System (IDS) and an International Comprehensive Anatomical Terminology (ICAT). J Gastroenterol Hepatol, 1991. 6(4): p. 325-44.

637. Soreide, O., et al., International standardization and documentation of the treatment of rectal cancer., in Rectal cancer surgery. Optimisation - standardization - documentation., O. Soreide and J. Norstein, Editors. 1997, Springer: Berlin Heidelberg New York. p. 405-45.

638. UICC, TNM Classification of Malignant Tumours. 6th Edition ed, ed. L.H. Sobin and C. Wittekind. 2002, New York: John Wiley \& Sons.

639. NIH consensus conference. Adjuvant therapy for patients with colon and rectal cancer. Jama, 1990. 264(1 1): p. 1444-50.

640. Nelson, H., et al., Guidelines 2000 for colon and rectal cancer surgery. J Natl Cancer Inst, 2001. 93(8): p. 583-96.

641. Pilipshen, S.J., et al., Patterns of pelvic recurrence following definitive resections of rectal cancer. Cancer, 1984. 53(6): p. 1354-62.

642. Burton, S., et al., MRI directed multidisciplinary team preoperative treatment strategy: the way to eliminate positive circumferential margins? Br J Cancer, 2006. 94(3): p. 351-7.

643. Segelman, J., et al., Differences in multidisciplinary team assessment and treatment between patients with stage IV colon and rectal cancer. Colorectal Dis, 2009. 11(7): p. 768-74.

644. MacDermid, E., et al., Improving patient survival with the colorectal cancer multi-disciplinary team. Colorectal Dis, 2009. 11(3): p. 291-5. 
645. Barillari, P., et al., Effect of preoperative colonoscopy on the incidence of synchronous and metachronous neoplasms. Acta Chir Scand, 1990. 156(2): p. 163-6.

646. Faivre, J., [Endoscopic monitoring after excision of colorectal cancer]. J Chir (Paris), 1997. 134(7-8): p. 283-5.

647. Chen, H.S. and S.M. Sheen-Chen, Synchronous and "early" metachronous colorectal adenocarcinoma: analysis of prognosis and current trends. Dis Colon Rectum, 2000. 43(8): p. 1093-9.

648. Floriani, I., et al., Performance of imaging modalities in diagnosis of liver metastases from colorectal cancer: a systematic review and meta-analysis. J Magn Reson Imaging, 2010. 31(1): p. 19-31.

649. Rafaelsen, S.R. and A. Jakobsen, Contrast-enhanced ultrasound vs multidetector-computed tomography for detecting liver metastases in colorectal cancer: a prospective, blinded, patientby-patient analysis. Colorectal Dis, 2011 . 13(4): p. 420-5.

650. Quaia, E., et al., Comparison of contrast-enhanced ultrasonography versus baseline ultrasound and contrast-enhanced computed tomography in metastatic disease of the liver: diagnostic performance and confidence. Eur Radiol, 2006. 16(7): p. 1599-609.

651. Niekel, M.C., S. Bipat, and J. Stoker, Diagnostic imaging of colorectal liver metastases with CT, $M R$ imaging, FDG PET, and/or FDG PET/CT: a meta-analysis of prospective studies including patients who have not previously undergone treatment. Radiology, 2010. 257(3): p. 674-84.

652. Mainenti, P.P., et al., Detection of colo-rectal liver metastases: prospective comparison of contrast enhanced US, multidetector CT, PET/CT, and 1.5 Tesla MR with extracellular and reticulo-endothelial cell specific contrast agents. Abdom Imaging, 2010. 35(5): p. 511-21.

653. McAndrew, M.R. and A.K. Saba, Efficacy of routine preoperative computed tomography scans in colon cancer. Am Surg, 1999. 65(3): p. 205-8.

654. Barton, J.B., et al., The utility of routine preoperative computed tomography scanning in the management of veterans with colon cancer. Am J Surg, 2002. 183(5): p. 499-503.

655. Dighe, S., et al., Diagnostic precision of CT in local staging of colon cancers: a meta-analysis. Clin Radiol, 2010. 65(9): p. 708-19.

656. Abdel-Nabi, H., et al., Staging of primary colorectal carcinomas with fluorine- 18 fluorodeoxyglucose whole-body PET: correlation with histopathologic and CT findings. Radiology, 1998. 206(3): p. 755-60.

657. Akiyoshi, T., et al., Comparison of preoperative whole-body positron emission tomography with MDCT in patients with primary colorectal cancer. Colorectal Dis, 2009. 11 (5): p. 464-9.

658. Furukawa, H., et al., Positron emission tomography scanning is not superior to whole body multidetector helical computed tomography in the preoperative staging of colorectal cancer. Gut, 2006. 55(7): p. 1007-11.

659. Kam, M.H., et al., Comparison of magnetic resonance imaging-fluorodeoxy-glucose positron emission tomography fusion with pathological staging in rectal cancer. Br J Surg, 2010. 97(2): p. 266-8.

660. Kinner, S., et al., Whole-body PET/CT-colonography: a possible new concept for colorectal cancer staging. Abdom Imaging, 2007. 32(5): p. 606-12.

661. Llamas-Elvira, J.M., et al., Fluorine-18 fluorodeoxyglucose PET in the preoperative staging of colorectal cancer. Eur J Nucl Med Mol Imaging, 2007. 34(6): p. 859-67.

662. Ono, K., et al., Comparison of diffusion-weighted MRI and 2-[fluorine-18]-fluoro-2-deoxy-Dglucose positron emission tomography (FDG-PET) for detecting primary colorectal cancer and regional lymph node metastases. J Magn Reson Imaging, 2009. 29(2): p. 336-40.

663. Tateishi, U., et al., Non-enhanced CT versus contrast-enhanced CT in integrated PET/CT studies for nodal staging of rectal cancer. Eur J Nucl Med Mol Imaging, 2007. 34(10): p. 1627-34.

664. Veit-Haibach, P., et al., Diagnostic accuracy of colorectal cancer staging with whole-body PET/CT colonography. JAMA, 2006. 296(21): p. 2590-600.

665. Bassi, M.C., et al., FDG-PET/CT imaging for staging and target volume delineation in preoperative conformal radiotherapy of rectal cancer. Int J Radiat Oncol Biol Phys, 2008. 70(5): p. 1423-6.

666. Davey, K., et al., The impact of 18-fluorodeoxyglucose positron emission tomography-computed tomography on the staging and management of primary rectal cancer. Dis Colon Rectum, 2008. 51(7): p. 997-1003.

667. Gearhart, S.L., et al., Improved staging with pretreatment positron emission tomography/computed tomography in low rectal cancer. Ann Surg Oncol, 2006. 13(3): p. 397 404.

668. Kantorova, I., et al., Routine (18)F-FDG PET preoperative staging of colorectal cancer: comparison with conventional staging and its impact on treatment decision making. J Nucl Med, 2003. 44(11): p. 1784-8. 
669. Nagata, K., et al., PET/CT colonography for the preoperative evaluation of the colon proximal to the obstructive colorectal cancer. Dis Colon Rectum, 2008. 51(6): p. 882-90.

670. Park, I.J., et al., Efficacy of PET/CT in the accurate evaluation of primary colorectal carcinoma. Eur J Surg Oncol, 2006. 32(9): p. 941-7.

671. Paskeviciute, B., et al., Impact of (18)F-FDG-PET/CT on staging and irradiation of patients with locally advanced rectal cancer. Strahlenther Onkol, 2009. 185(4): p. 260-5.

672. Ruers, T.J., et al., Improved selection of patients for hepatic surgery of colorectal liver metastases with (18)F-FDG PET: a randomized study. J Nucl Med, 2009. 50(7): p. 1036-41.

673. Moulton, C.A., An Ontario Clinical Oncology Group (OCOG) randomized controlled trial (RCT) assessing FDG PET/CT in resectable liver colorectal adenocarcinoma metastases (CAM). J Clin Oncol, 2011 (suppl): p. abstr 3520.

674. Glazer, E.S., et al., Effectiveness of positron emission tomography for predicting chemotherapy response in colorectal cancer liver metastases. Arch Surg, 2010. 145(4): p. 340-5; discussion 345.

675. Lubezky, N., et al., The role and limitations of 18-fluoro-2-deoxy-D-glucose positron emission tomography (FDG-PET) scan and computerized tomography (CT) in restaging patients with hepatic colorectal metastases following neoadjuvant chemotherapy: comparison with operative and pathological findings. J Gastrointest Surg, 2007. 11(4): p. 472-8.

676. Spatz, J., et al., Neoadjuvant chemotherapy affects staging of colorectal liver metastasis--a comparison of PET, CT and intraoperative ultrasound. Int J Colorectal Dis, 201 1. 26(2): p. 16571.

677. IQWIG, Positronenemissionstomographie (PET und PET/CT) bei rezidivierendem kolorektalen Karzinom - Vorbericht (vorläufige Nutzenbewertung). 2011.

678. Patel, S., et al., Positron emission tomography/computed tomographic scans compared to computed tomographic scans for detecting colorectal liver metastases: a systematic review. Ann Surg, $2011.253(4)$ : p. 666-71.

679. Taylor, R.A., et al., Evaluation with positron emission tomography before hepatic resection for metastatic colorectal cancer improves survival in patients with a high clinical risk score. 2007 Gastrointestinal Cancers Symposium, Abstract No: 240, 2007.

680. Adie, S., et al., Resection of liver metastases from colorectal cancer: does preoperative chemotherapy affect the accuracy of PET in preoperative planning? ANZ J Surg, 2009. 79(5): $p$. 358-61.

681. Levy, M., et al., Tumor markers in staging and prognosis of colorectal carcinoma. Neoplasma, 2008. 55(2): p. 138-42.

682. Lewis, M.R., et al., Metastatic colorectal adenocarcinoma involving the ovary with elevated serum CA125: a potential diagnostic pitfall. Gynecol Oncol, 2007. 105(2): p. 395-8.

683. Chen, C.C., et al., Is it reasonable to add preoperative serum level of CEA and CA19-9 to staging for colorectal cancer? J Surg Res, 2005. 124(2): p. 169-74.

684. Merkel, S., et al., The prognostic inhomogeneity in pT3 rectal carcinomas. Int J Colorectal Dis, 2001. 16(5): p. 298-304.

685. Nagtegaal, I.D. and P. Quirke, What is the role for the circumferential margin in the modern treatment of rectal cancer? J Clin Oncol, 2008. 26(2): p. 303-12.

686. Bipat, S., et al., Rectal cancer: local staging and assessment of lymph node involvement with endoluminal US, CT, and MR imaging--a meta-analysis. Radiology, 2004. 232(3): p. 773-83.

687. Puli, S.R., et al., Accuracy of endoscopic ultrasound to diagnose nodal invasion by rectal cancers: a meta-analysis and systematic review. Ann Surg Oncol, 2009. 16(5): p. 1255-65.

688. Ahmetoglu, A., et al., MDCT with multiplanar reconstruction in the preoperative local staging of rectal tumor. Abdom Imaging, 2011. 36(1): p. 31-7.

689. Kim, C.K., et al., Preoperative staging of rectal cancer: accuracy of 3-Tesla magnetic resonance imaging. Eur Radiol, 2006. 16(5): p. 972-80.

690. Kim, Y.W., et al., A prospective comparison study for predicting circumferential resection margin between preoperative MRI and whole mount sections in mid-rectal cancer: significance of different scan planes. Eur J Surg Oncol, 2008. 34(6): p. 648-54.

691. Kim, Y.W., et al., Factors related to preoperative assessment of the circumferential resection margin and the extent of mesorectal invasion by magnetic resonance imaging in rectal cancer: a prospective comparison study. World J Surg, 2009. 33(9): p. 1952-60.

692. Matsuoka, H., et al., MRI diagnosis of mesorectal lymph node metastasis in patients with rectal carcinoma. what is the optimal criterion? Anticancer Res, 2004. 24(6): p. 4097-101.

693. Wolberink, S.V., et al., Multislice CT as a primary screening tool for the prediction of an involved mesorectal fascia and distant metastases in primary rectal cancer: a multicenter study. Dis Colon Rectum, 2009. 52(5): p. 928-34. 
694. Purkayastha, S., et al., Magnetic resonance colonography vs computed tomography colonography for the diagnosis of colorectal cancer: an indirect comparison. Colorectal Dis, 2007. 9(2): p. 100-11.

695. Torzilli, G., et al., Contrast-enhanced intraoperative ultrasonography during hepatectomies for colorectal cancer liver metastases. J Gastrointest Surg, 2005. 9(8): p. 1148-53; discussion $1153-4$.

696. Zirngibl, H., B. Husemann, and P. Hermanek, Intraoperative spillage of tumor cells in surgery for rectal cancer. Dis Colon Rectum, 1990. 33(7): p. 610-4.

697. Cahill, R.A., J. Leroy, and J. Marescaux, Could lymphatic mapping and sentinel node biopsy provide oncological providence for local resectional techniques for colon cancer? A review of the literature. BMC Surg, 2008. 8: p. 17.

698. Wiese, D., et al., Ultrastaging of sentinel lymph nodes (SLNs) vs. non-SLNs in colorectal cancer-do we need both? Am J Surg, 2010. 199(3): p. 354-8; discussion 358.

699. Goligher, J.C., Incidence and pathology of carcinoma of the colon and rectum, in Surgery of the anus, rectum and colon. 1984, Balliére Tindall: London. p. S. 445.

700. Toyota, S., H. Ohta, and S. Anazawa, Rationale for extent of lymph node dissection for right colon cancer. Dis Colon Rectum, 1995. 38(7): p. 705-11.

701. Tan, K.Y., et al., Distribution of the first metastatic lymph node in colon cancer and its clinical significance. Colorectal Dis, 2010. 12(1): p. 44-7.

702. Lan, Y.T., et al., Significance of lymph node retrieval from the terminal ileum for patients with cecal and ascending colonic cancers. Ann Surg Oncol, 2011 . 18(1): p. 146-52.

703. Chang, G.J., et al., Lymph node evaluation and survival after curative resection of colon cancer: systematic review. J Natl Cancer Inst, 2007. 99(6): p. 433-41.

704. Le Voyer, T.E., et al., Colon cancer survival is associated with increasing number of lymph nodes analyzed: a secondary survey of intergroup trial INT-0089. J Clin Oncol, 2003. 21 (15): p. 2912-9.

705. Van Damme, J.-P., Vascular anatomy in abdominal surgery. 1990, Stuttgart: Thieme.

706. Kanemitsu, Y., et al., Survival benefit of high ligation of the inferior mesenteric artery in sigmoid colon or rectal cancer surgery. Br J Surg, 2006. 93(5): p. 609-15.

707. Chin, C.C., et al., Carcinoma obstruction of the proximal colon cancer and long-term prognosis-obstruction is a predictor of worse outcome in TNM stage II tumor. Int J Colorectal Dis, 2010. 25(7): p. 817-22.

708. Hohenberger, W., et al., Standardized surgery for colonic cancer: complete mesocolic excision and central ligation--technical notes and outcome. Colorectal Dis, 2009. 11(4): p. 354-64; discussion 364-5.

709. West, N.P., et al., Multicentre experience with extralevator abdominoperineal excision for low rectal cancer. Br J Surg, 2010. 97(4): p. 588-99.

710. Bertelsen, C.A., et al., Can the quality of colonic surgery be improved by standardization of surgical technique with complete mesocolic excision? Colorectal Dis, 2011. 13(10): p. 11231129.

711. (NICE), N.I.f.C.E., Radiofrequency ablation for the treatment of colorectal metastases in the liver. . 2011 : London.

712. Kim, K.H., et al., Comparative analysis of radiofrequency ablation and surgical resection for colorectal liver metastases. J Korean Surg Soc, 2011. 81(1): p. 25-34.

713. Mulier, S., et al., Radiofrequency ablation versus resection for resectable colorectal liver metastases: time for a randomized trial? Ann Surg Oncol, 2008. 15(1): p. 144-57.

714. (NICE), N.I.f.C.E., Selective internal radiation therapy for colorectal metastases in the liver. . 2011: London.

715. Townsend, A., T. Price, and C. Karapetis, Selective internal radiation therapy for liver metastases from colorectal cancer. Cochrane Database Syst Rev, 2009(4): p. CD007045.

716. Christophi, C., et al., Long-term survival of patients with unresectable colorectal liver metastases treated by percutaneous interstitial laser thermotherapy. World J Surg, 2004. 28(10): p. 987-94.

717. Wietzke-Braun, P., et al., Quality of life and outcome of ultrasound-guided laser interstitial thermo-therapy for non-resectable liver metastases of colorectal cancer. Eur J Gastroenterol Hepatol, 2004. 16(4): p. 389-95.

718. Abcarian, H. and R.K. Pearl, Simple technique for high ligation of the inferior mesenteric artery and vein. Dis Colon Rectum, 1991. 34(12): p. 1138.

719. Buunen, M., et al., Level of arterial ligation in total mesorectal excision (TME): an anatomical study. Int J Colorectal Dis, 2009. 24(11): p. 1317-20.

720. Pezim, M.E. and R.J. Nicholls, Survival after high or low ligation of the inferior mesenteric artery during curative surgery for rectal cancer. Ann Surg, 1984. 200(6): p. 729-33. 
721. Surtees, P., J.K. Ritchie, and R.K. Phillips, High versus low ligation of the inferior mesenteric artery in rectal cancer. Br J Surg, 1990. 77(6): p. 618-21.

722. Slanetz, C.A., Jr. and R. Grimson, Effect of high and intermediate ligation on survival and recurrence rates following curative resection of colorectal cancer. Dis Colon Rectum, 1997. 40(10): p. 1205-18; discussion 1218-9.

723. Lange, M.M., et al., Level of arterial ligation in rectal cancer surgery: low tie preferred over high tie. A review. Dis Colon Rectum, 2008. 51(7): p. 1139-45.

724. Heald, R.J., E.M. Husband, and R.D. Ryall, The mesorectum in rectal cancer surgery--the clue to pelvic recurrence? Br J Surg, 1982. 69(10): p. 613-6.

725. Bokey, E.L., et al., Local recurrence after curative excision of the rectum for cancer without adjuvant therapy: role of total anatomical dissection. Br J Surg, 1999. 86(9): p. 1164-70.

726. Hermanek, P.j., Multiviszerale Resektion beim kolorektalen Karzinom. Erfahrungen der SGKRKStudie. Langenbecks Arch Surg, 1992. Kongressband: p. 95-100.

727. Havenga, K., et al., Male and female sexual and urinary function after total mesorectal excision with autonomic nerve preservation for carcinoma of the rectum. J Am Coll Surg, 1996. 182(6): p. 495-502.

728. Enker, W.E., et al., Abdominoperineal resection via total mesorectal excision and autonomic nerve preservation for low rectal cancer. World J Surg, 1997. 21(7): p. 715-20.

729. Georgiou, P., et al., Extended lymphadenectomy versus conventional surgery for rectal cancer: a meta-analysis. Lancet Oncol, 2009. 10(11): p. 1053-62.

730. Gohl, J., W. Hohenberger, and S. Merkel, Lymph node dissection in rectal carcinoma: TME and what else? Onkologie, 2009. 32(1-2): p. 57-61.

731. Mortenson, M.M., et al., Total mesorectal excision and pelvic node dissection for rectal cancer: an appraisal. Surg Oncol Clin N Am, 2007. 16(1): p. 177-97.

732. Koch, M., et al., Is the lateral lymph node compartment relevant? Recent Results Cancer Res, 2005. 165: p. 40-5.

733. Scott, N., et al., Total mesorectal excision and local recurrence: a study of tumour spread in the mesorectum distal to rectal cancer. Br J Surg, 1995. 82(8): p. 1031-3.

734. Reynolds, J.V., et al., Pathological evidence in support of total mesorectal excision in the management of rectal cancer. Br J Surg, 1996. 83(8): p. 1112-5.

735. Hida, J., et al., Lymph node metastases detected in the mesorectum distal to carcinoma of the rectum by the clearing method: justification of total mesorectal excision. J Am Coll Surg, 1997. 184(6): p. 584-8.

736. Zaheer, S., et al., Surgical treatment of adenocarcinoma of the rectum. Ann Surg, 1998. 227(6): p. 800-11.

737. Leong, A.F., Selective total mesorectal excision for rectal cancer. Dis Colon Rectum, 2000. 43(9): p. 1237-40.

738. Heald, R.J. and N.D. Karanjia, Results of radical surgery for rectal cancer. World J Surg, 1992. 16(5): p. 848-57.

739. Enker, W.E., Total mesorectal excision--the new golden standard of surgery for rectal cancer. Ann Med, 1997. 29(2): p. 127-33.

740. Lazorthes, F., et al., Distal intramural spread of carcinoma of the rectum correlated with lymph nodal involvement. Surg Gynecol Obstet, 1990. 170(1): p. 45-8.

741. Kwok, S.P., et al., Prospective analysis of the distal margin of clearance in anterior resection for rectal carcinoma. Br J Surg, 1996. 83(7): p. 969-72.

742. Andreola, S., et al., Distal intramural spread in adenocarcinoma of the lower third of the rectum treated with total rectal resection and coloanal anastomosis. Dis Colon Rectum, 1997. 40(1): p. 25-9.

743. Goldstein, N.S., A. Soman, and J. Sacksner, Disparate surgical margin lengths of colorectal resection specimens between in vivo and in vitro measurements. The effects of surgical resection and formalin fixation on organ shrinkage. Am J Clin Pathol, 1999. 111 (3): p. 349-51.

744. Kiran, R.P., L. Lian, and I.C. Lavery, Does a subcentimeter distal resection margin adversely influence oncologic outcomes in patients with rectal cancer undergoing restorative proctectomy? Dis Colon Rectum, 2011. 54(2): p. 157-63.

745. Rutkowski, A., et al., Distal bowel surgical margin shorter than $1 \mathrm{~cm}$ after preoperative radiation for rectal cancer: is it safe? Ann Surg Oncol, 2008. 15(11): p. 3124-31.

746. Brown, C.J., D.S. Fenech, and R.S. McLeod, Reconstructive techniques after rectal resection for rectal cancer. Cochrane Database Syst Rev, 2008(2): p. CD006040.

747. Fazio, V.W., et al., A randomized multicenter trial to compare long-term functional outcome, quality of life, and complications of surgical procedures for low rectal cancers. Ann Surg, 2007. 246(3): p. 481-8; discussion 488-90.

748. Liao, C., et al., Meta-analysis of the colon J-pouch vs transverse coloplasty pouch after anterior resection for rectal cancer. Colorectal Dis, 2010. 12(7): p. 624-31. 
749. Siddiqui, M.R., et al., A meta-analysis comparing side to end with colonic J-pouch formation after anterior resection for rectal cancer. Tech Coloproctol, 2010. 14(2): p. 113-23.

750. Hida, J., et al., Functional outcome after low anterior resection with low anastomosis for rectal cancer using the colonic J-pouch. Prospective randomized study for determination of optimum pouch size. Dis Colon Rectum, 1996. 39(9): p. 986-91.

751. Hohenberger, W., et al., [Neoadjuvant radiochemotherapy for rectal cancer]. Chirurg, 2009. 80(4): p. 294-302.

752. Artioukh, D.Y., Controversial aspects of rectal cancer surgery following preoperative chemoradiation. Colorectal Dis, 2010. 12 Suppl 2: p. 25-9.

753. Habr-Gama, A., et al., Operative versus nonoperative treatment for stage 0 distal rectal cancer following chemoradiation therapy: long-term results. Ann Surg, 2004. 240(4): p. 71 1-7; discussion 717-8.

754. Tekkis, P.P., et al., Comparison of circumferential margin involvement between restorative and nonrestorative resections for rectal cancer. Colorectal Dis, 2005. 7(4): p. 369-74.

755. Nagtegaal, I.D., et al., Low rectal cancer: a call for a change of approach in abdominoperineal resection. J Clin Oncol, 2005. 23(36): p. 9257-64.

756. Nisar, P.J. and H.J. Scott, Myocutaneous flap reconstruction of the pelvis after abdominoperineal excision. Colorectal Dis, 2009. 11(8): p. 806-16.

757. Montedori, A., et al., Covering ileo- or colostomy in anterior resection for rectal carcinoma. Cochrane Database Syst Rev, 2010(5): p. CD006878.

758. Lindgren, R., et al., What is the risk for a permanent stoma after low anterior resection of the rectum for cancer? A six-year follow-up of a multicenter trial. Dis Colon Rectum, 2011. 54(1): p. $41-7$.

759. Klink, C.D., et al., [Protective ileostoma versus protective transverse stoma. What evidence is available?]. Chirurg, 2010. 81(11): p. 974-7.

760. Rondelli, F., et al., Loop ileostomy versus loop colostomy for fecal diversion after colorectal or coloanal anastomosis: a meta-analysis. Int J Colorectal Dis, 2009. 24(5): p. 479-88.

761. Bass, E.M., et al., Does preoperative stoma marking and education by the enterostomal therapist affect outcome? Dis Colon Rectum, 1997. 40(4): p. 440-2.

762. Millan, M., et al., Preoperative stoma siting and education by stomatherapists of colorectal cancer patients: a descriptive study in twelve Spanish colorectal surgical units. Colorectal Dis, 2010. 12(7 Online): p. e88-92.

763. Chaudhri, S., et al., Preoperative intensive, community-based vs. traditional stoma education: a randomized, controlled trial. Dis Colon Rectum, 2005. 48(3): p. 504-9.

764. Hermanek, P. and G.P. Marzoli, Lokale Therapie des Rektumkarzinoms. Verfahren in kurativer Intention. 1994, Berlin Heidelberg Ney York Tokyo: Springer.

765. Winde, G., et al., Surgical cure for early rectal carcinomas (T1). Transanal endoscopic microsurgery vs. anterior resection. Dis Colon Rectum, 1996. 39(9): p. 969-76.

766. Sengupta, S. and J.J. Tjandra, Local excision of rectal cancer: what is the evidence? Dis Colon Rectum, 2001. 44(9): p. 1345-61.

767. Matzel, K.E., S. Merkel, and W. Hohenberger, Lokale Therapieprinzipien beim Rektumkarzinom. Chirurg, 2003. 74(10): p. 897-904.

768. Ptok, H., et al., Oncological outcome of local vs radical resection of low-risk pT1 rectal cancer. Arch Surg, 2007. 142(7): p. 649-55; discussion 656.

769. You, Y.N., et al., Is the increasing rate of local excision for stage I rectal cancer in the United States justified?: a nationwide cohort study from the National Cancer Database. Ann Surg, 2007. 245(5): p. 726-33.

770. Moore, J.S., et al., Transanal endoscopic microsurgery is more effective than traditional transanal excision for resection of rectal masses. Dis Colon Rectum, 2008. 51 (7): p. 1026-30; discussion 1030-1.

771. Sgourakis, G., et al., Transanal endoscopic microsurgery for T1 and T2 rectal cancers: a metaanalysis and meta-regression analysis of outcomes. Am Surg, 2011. 77(6): p. 761-72.

772. Bach, S.P., et al., A predictive model for local recurrence after transanal endoscopic microsurgery for rectal cancer. Br J Surg, 2009. 96(3): p. 280-90.

773. Glimelius, B., L. Pahlman, and A. Cervantes, Rectal cancer: ESMO Clinical Practice Guidelines for diagnosis, treatment and follow-up. Ann Oncol, 2010. 21 Suppl 5: p. v82-6.

774. Suppiah, A., et al., Transanal endoscopic microsurgery in early rectal cancer: time for a trial? Colorectal Dis, 2008. 10(4): p. 314-27; discussion 327-9.

775. Valentini, V., et al., Multidisciplinary Rectal Cancer Management: 2nd European Rectal Cancer Consensus Conference (EURECA-CC2). Radiother Oncol, 2009. 92(2): p. 148-63.

776. Hahnloser, D., et al., Immediate radical resection after local excision of rectal cancer: an oncologic compromise? Dis Colon Rectum, 2005. 48(3): p. 429-37. 
777. Schwenk, W., et al., Short term benefits for laparoscopic colorectal resection. Cochrane Database Syst Rev, 2005(3): p. CD003145.

778. Liang, Y., et al., Laparoscopic versus open colorectal resection for cancer: a meta-analysis of results of randomized controlled trials on recurrence. Eur J Surg Oncol, 2008. 34(11): p. 1217 24.

779. Kuhry, E., et al., Long-term results of laparoscopic colorectal cancer resection. Cochrane Database Syst Rev, 2008(2): p. CD003432.

780. Guillou, P.J., et al., Short-term endpoints of conventional versus laparoscopic-assisted surgery in patients with colorectal cancer (MRC CLASICC trial): multicentre, randomised controlled trial. Lancet, 2005. 365(9472): p. 1718-26.

781. Jayne, D.G., et al., Five-year follow-up of the Medical Research Council CLASICC trial of laparoscopically assisted versus open surgery for colorectal cancer. Br J Surg, 2010. 97(11): p. 1638-45.

782. Kwak, J.M., et al., Robotic vs laparoscopic resection of rectal cancer: short-term outcomes of a case-control study. Dis Colon Rectum, 2011. 54(2): p. 151-6.

783. Pigazzi, A., et al., Multicentric study on robotic tumor-specific mesorectal excision for the treatment of rectal cancer. Ann Surg Oncol, 2010. 17(6): p. 1614-20.

784. Jacquet, P. and P.H. Sugarbaker, Clinical research methodologies in diagnosis and staging of patients with peritoneal carcinomatosis. Cancer Treat Res, 1996. 82: p. 359-74.

785. Verwaal, V.J., et al., Randomized trial of cytoreduction and hyperthermic intraperitoneal chemotherapy versus systemic chemotherapy and palliative surgery in patients with peritoneal carcinomatosis of colorectal cancer. J Clin Oncol, 2003. 21 (20): p. 3737-43.

786. Verwaal, V.J., et al., 8-year follow-up of randomized trial: cytoreduction and hyperthermic intraperitoneal chemotherapy versus systemic chemotherapy in patients with peritoneal carcinomatosis of colorectal cancer. Ann Surg Oncol, 2008. 15(9): p. 2426-32.

787. Cao, C., et al., A systematic review and meta-analysis of cytoreductive surgery with perioperative intraperitoneal chemotherapy for peritoneal carcinomatosis of colorectal origin. Ann Surg Oncol, 2009. 16(8): p. 2152-65.

788. Khot, U.P., et al., Systematic review of the efficacy and safety of colorectal stents. Br J Surg, 2002. 89(9): p. 1096-102.

789. Stillwell, A.P., P.G. Buettner, and Y.H. Ho, Meta-analysis of survival of patients with stage IV colorectal cancer managed with surgical resection versus chemotherapy alone. World J Surg, 2010. 34(4): p. 797-807.

790. Poultsides, G.A., et al., Outcome of primary tumor in patients with synchronous stage IV colorectal cancer receiving combination chemotherapy without surgery as initial treatment. J Clin Oncol, 2009. 27(20): p. 3379-84.

791. Hermanek, P., I. Guggenmoos-Holzmann, and F.P. Gall, Prognostic factors in rectal carcinoma. A contribution to the further development of tumor classification. Dis Colon Rectum, 1989. 32(7): p. 593-9.

792. Hermanek, P. and W. Hohenberger, The importance of volume in colorectal cancer surgery. Eur J Surg Oncol, 1996. 22(3): p. 213-5.

793. Compton, C.C., Pathology report in colon cancer: what is prognostically important? Dig Dis, 1999. 17(2): p. 67-79.

794. Wong, J.H., et al., Number of nodes examined and staging accuracy in colorectal carcinoma. J Clin Oncol, 1999. 17(9): p. 2896-900.

795. Compton, C., et al., American Joint Committee on Cancer Prognostic Factors Consensus Conference: Colorectal Working Group. Cancer, 2000. 88(7): p. 1739-57.

796. Compton, C.C., Updated protocol for the examination of specimens from patients with carcinomas of the colon and rectum, excluding carcinoid tumors, lymphomas, sarcomas, and tumors of the vermiform appendix: a basis for checklists. Cancer Committee. Arch Pathol Lab Med, 2000. 124(7): p. 1016-25.

797. Hermanek, P., Methodik der histopathologischen Untersuchung von Resektionen kolorektaler Karzinome. Chir Gastroenterol, 2000. 16: p. 255-59.

798. Hermanek, P., [Lymph nodes and malignant tumors]. Zentralbl Chir, 2000. 125(10): p. 790-5.

799. Junginger, T., P. Hermanek, and M. Klimpfinger, Klassifikation maligner Tumoren des Gastrointestinaltraktes I. 2002, Berlin Heidelberg New York: Springer Verlag.

800. Wittekind, C., et al., TNM residual tumor classification revisited. Cancer, 2002. 94(9): p. 2511 6.

801. Leibl, S., O. Tsybrovskyy, and H. Denk, How many lymph nodes are necessary to stage early and advanced adenocarcinoma of the sigmoid colon and upper rectum? Virchows Arch, 2003. 443(2): p. 133-8.

802. Sobin, L.H., TNM, sixth edition: new developments in general concepts and rules. Semin Surg Oncol, 2003. 21(1): p. 19-22. 
803. Dworak, O., L. Keilholz, and A. Hoffmann, Pathological features of rectal cancer after preoperative radiochemotherapy. Int J Colorectal Dis, 1997. 12(1): p. 19-23.

804. Wright, C.M., et al., Prognostic significance of extensive microsatellite instability in sporadic clinicopathological stage C colorectal cancer. Br J Surg, 2000. 87(9): p. 1197-202.

805. Popat, S., R. Hubner, and R.S. Houlston, Systematic review of microsatellite instability and colorectal cancer prognosis. J Clin Oncol, 2005. 23(3): p. 609-18.

806. Benatti, P., et al., Microsatellite instability and colorectal cancer prognosis. Clin Cancer Res, 2005. 11 (23): p. 8332-40.

807. Malesci, A., et al., Reduced likelihood of metastases in patients with microsatellite-unstable colorectal cancer. Clin Cancer Res, 2007. 13(13): p. 3831-9.

808. Leopoldo, S., et al., Two subtypes of mucinous adenocarcinoma of the colorectum: clinicopathological and genetic features. Ann Surg Oncol, 2008. 15(5): p. 1429-39.

809. Lanza, G., et al., Medullary-type poorly differentiated adenocarcinoma of the large bowel: a distinct clinicopathologic entity characterized by microsatellite instability and improved survival. J Clin Oncol, 1999. 17(8): p. 2429-38.

810. Parc, Y., et al., Prognostic significance of microsatellite instability determined by immunohistochemical staining of MSH2 and MLH1 in sporadic T3NOMO colon cancer. Gut, 2004. 53(3): p. 371-5.

811. Gafa, R., et al., Sporadic colorectal adenocarcinomas with high-frequency microsatellite instability. Cancer, 2000. 89(10): p. 2025-37.

812. Lanza, G., et al., Immunohistochemical pattern of MLH1/MSH2 expression is related to clinical and pathological features in colorectal adenocarcinomas with microsatellite instability. Mod Pathol, 2002. 15(7): p. 741-9.

813. Lindor, N.M., et al., Immunohistochemistry versus microsatellite instability testing in phenotyping colorectal tumors. J Clin Oncol, 2002. 20(4): p. 1043-8.

814. Overbeek, L.I., et al., Interpretation of immunohistochemistry for mismatch repair proteins is only reliable in a specialized setting. Am J Surg Pathol, 2008. 32(8): p. 1246-51.

815. Prandi, M., et al., Prognostic evaluation of stage B colon cancer patients is improved by an adequate lymphadenectomy: results of a secondary analysis of a large scale adjuvant trial. Ann Surg, 2002. 235(4): p. 458-63.

816. Ogino, S., et al., Negative lymph node count is associated with survival of colorectal cancer patients, independent of tumoral molecular alterations and lymphocytic reaction. Am J Gastroenterol, 2010. 105(2): p. 420-33.

817. Swanson, R.S., et al., The prognosis of T3NO colon cancer is dependent on the number of lymph nodes examined. Ann Surg Oncol, 2003. 10(1): p. 65-71.

818. Carloss, H., et al., The impact of number of lymph nodes removed on five-year survival in stage II colon and rectal cancer. J Ky Med Assoc, 2004. 102(8): p. 345-7.

819. Fazio, L., et al., Association between colonic screening, subject characteristics, and stage of colorectal cancer. Am J Gastroenterol, 2005. 100(11): p. 2531-9.

820. Wong, S.L., et al., Hospital lymph node examination rates and survival after resection for colon cancer. JAMA, 2007. 298(18): p. 2149-54.

821. Qiu, H.B., et al., Ratio of metastatic to resected lymph nodes enhances to predict survival in patients with stage III colorectal cancer. Ann Surg Oncol, 2011 . 18(6): p. 1568-74.

822. Wang, J., et al., Should total number of lymph nodes be used as a quality of care measure for stage III colon cancer? Ann Surg, 2009. 249(4): p. 559-63.

823. Chen, S.L., et al., Lymph node ratio as a quality and prognostic indicator in stage III colon cancer. Ann Surg, 2011. 253(1): p. 82-7.

824. Ha, Y.H., et al., Influence of preoperative chemoradiotherapy on the number of lymph nodes retrieved in rectal cancer. Ann Surg, 2010. 252(2): p. 336-40.

825. West, N.P., et al., Complete mesocolic excision with central vascular ligation produces an oncologically superior specimen compared with standard surgery for carcinoma of the colon. J Clin Oncol, 2010. 28(2): p. 272-8.

826. West, N.P., et al., Pathology grading of colon cancer surgical resection and its association with survival: a retrospective observational study. Lancet Oncol, 2008. 9(9): p. 857-65.

827. Quirke, P., et al., Effect of the plane of surgery achieved on local recurrence in patients with operable rectal cancer: a prospective study using data from the MRC CRO7 and NCIC-CTC CO16 randomised clinical trial. Lancet, 2009. 373(9666): p. 821-8.

828. Tilney, H.S., et al., The influence of circumferential resection margins on long-term outcomes following rectal cancer surgery. Dis Colon Rectum, 2009. 52(10): p. 1723-9.

829. Bernstein, T.E., et al., Circumferential resection margin as a prognostic factor in rectal cancer. Br J Surg, 2009. 96(11): p. 1348-57.

830. West, N.P., et al., Evidence of the oncologic superiority of cylindrical abdominoperineal excision for low rectal cancer. J Clin Oncol, 2008. 26(21): p. 3517-22. 
831. Leite, J.S., et al., Clinical significance of macroscopic completeness of mesorectal resection in rectal cancer. Colorectal Dis, 2011. 13(4): p. 381-6.

832. Kreienberg, R., et al. Interdisziplinäre S3-Leitlinie für die Diagnostik, Therapie und Nachsorge des Mammakarzinoms. Informationszentrum für Standards in der Onkologie (ISTO), Deutsche Krebsgesellschaft e.V., 2008. 1. Aktualisierung

833. Edwards, A.G., S. Hailey, and M. Maxwell, Psychological interventions for women with metastatic breast cancer. Cochrane Database Syst Rev, 2004(2): p. CD004253.

834. Sheard, T. and P. Maguire, The effect of psychological interventions on anxiety and depression in cancer patients: results of two meta-analyses. Br J Cancer, 1999. 80(11): p. 1770-80.

835. Keller, M., et al., Recognition of distress and psychiatric morbidity in cancer patients: a multimethod approach. Ann Oncol, 2004. 15(8): p. 1243-9.

836. Sharpe, M., et al., Major depression in outpatients attending a regional cancer centre: screening and unmet treatment needs. Br J Cancer, 2004. 90(2): p. 314-20.

837. Singer, S., J. Das-Munshi, and E. Brahler, Prevalence of mental health conditions in cancer patients in acute care--a meta-analysis. Ann Oncol, 2010. 21(5): p. 925-30.

838. Zabora, J., et al., The prevalence of psychological distress by cancer site. Psychooncology, 2001. 10(1): p. 19-28.

839. Jansen, L., et al., Health-related quality of life during the 10 years after diagnosis of colorectal cancer: a population-based study. J Clin Oncol, 2011 . 29(24): p. 3263-9.

840. Lynch, B.M., et al., Describing and predicting psychological distress after colorectal cancer. Cancer, 2008. 112(6): p. 1363-70.

841. Miovic, M. and S. Block, Psychiatric disorders in advanced cancer. Cancer, 2007. 110(8): p. 1665-76.

842. Ramsey, S.D., et al., Quality of life in survivors of colorectal carcinoma. Cancer, 2000. 88(6): p. 1294-303.

843. Zettl, S. and J. Hartlapp, eds. Sexualstörungen durch Krankheit und Therapie. ed. Springer. 1997: Heidelberg. S. 59.

844. Fallowfield, L., et al., Psychiatric morbidity and its recognition by doctors in patients with cancer. Br J Cancer, 2001. 84(8): p. 1011-5.

845. Sollner, W., et al., How successful are oncologists in identifying patient distress, perceived social support, and need for psychosocial counselling? Br J Cancer, 2001 . 84(2): p. 179-85.

846. Ell, K., et al., Randomized controlled trial of collaborative care management of depression among low-income patients with cancer. J Clin Oncol, 2008. 26(27): p. 4488-96.

847. Strong, V., et al., Management of depression for people with cancer (SMaRT oncology 1): a randomised trial. Lancet, 2008. 372(9632): p. 40-8.

848. Herschbach, P. and J. Weis, eds. Screeningverfahren in der Psychoonkologie. 2008, Deutsche Krebsgesellschaft: Berlin.

849. NHMRC Clinical practice guidelines for the psychosocial care of adults with cancer. 2003. 1237.

850. Turner, J., et al., Clinical practice guidelines for the psychosocial care of adults with cancer. Psychooncology, 2005. 14(3): p. 159-73.

851. Weis, J., et al., Psychoonkologie: Konzepte und Aufgaben einer jungen Fachdisziplin. . Onkologe, 2007. 13(2): p. 185-194.

852. Edwards, A.G., N. Hulbert-Williams, and R.D. Neal, Psychological interventions for women with metastatic breast cancer. Cochrane Database Syst Rev, 2008(3): p. CD004253.

853. Faller, H., ., et al., Effects of psychooncological interventions on emotional distress and quality of life in adult cancer patients: systematic review and meta-analysis. Nat J Cancer in press.

854. Goedendorp, M.M., et al., Psychosocial interventions for reducing fatigue during cancer treatment in adults. Cochrane Database Syst Rev, 2009(1): p. CD006953.

855. Jacobsen, P.B. and H.S. Jim, Psychosocial interventions for anxiety and depression in adult cancer patients: achievements and challenges. CA Cancer J Clin, 2008. 58(4): p. 214-30.

856. Luebbert, K., B. Dahme, and M. Hasenbring, The effectiveness of relaxation training in reducing treatment-related symptoms and improving emotional adjustment in acute non-surgical cancer treatment: a meta-analytical review. Psychooncology, 2001. 10(6): p. 490-502.

857. Osborn, R.L., A.C. Demoncada, and M. Feuerstein, Psychosocial interventions for depression, anxiety, and quality of life in cancer survivors: meta-analyses. Int J Psychiatry Med, 2006. 36(1): p. 13-34.

858. Rehse, B. and R. Pukrop, Effects of psychosocial interventions on quality of life in adult cancer patients: meta analysis of 37 published controlled outcome studies. Patient Educ Couns, 2003. 50(2): p. 179-86.

859. Uitterhoeve, R.J., et al., Psychosocial interventions for patients with advanced cancer - $a$ systematic review of the literature. Br J Cancer, 2004. 91(6): p. 1050-62. 
860. Williams, S. and J. Dale, The effectiveness of treatment for depression/depressive symptoms in adults with cancer: a systematic review. Br J Cancer, 2006. 94(3): p. 372-90.

861. Merkel, S., et al., Prognosis of patients with colonic carcinoma before, during and after implementation of complete mesocolic excision. Br J Surg, 2016. 103(9): p. 1220-9.

862. Booth, C.M., et al., Use and Effectiveness of Adjuvant Chemotherapy for Stage III Colon Cancer: A Population-Based Study. J Natl Compr Canc Netw, 2016. 14(1): p. 47-56.

863. Tournigand, C., et al., Adjuvant therapy with fluorouracil and oxaliplatin in stage II and elderly patients (between ages 70 and 75 years) with colon cancer: subgroup analyses of the Multicenter International Study of Oxaliplatin, Fluorouracil, and Leucovorin in the Adjuvant Treatment of Colon Cancer trial. J Clin Oncol, 2012. 30(27): p. 3353-60.

864. Haller, D.G., et al., Impact of age and medical comorbidity on adjuvant treatment outcomes for stage III colon cancer: a pooled analysis of individual patient data from four randomized, controlled trials. Ann Oncol, 2015. 26(4): p. 715-24.

865. Schmoll, H.J., et al., Capecitabine Plus Oxaliplatin Compared With Fluorouracil/Folinic Acid As Adjuvant Therapy for Stage III Colon Cancer: Final Results of the NO16968 Randomized Controlled Phase III Trial. J Clin Oncol, 2015. 33(32): p. 3733-40.

866. McCleary, N.J., et al., Impact of age on the efficacy of newer adjuvant therapies in patients with stage II/III colon cancer: findings from the ACCENT database. J Clin Oncol, 2013. 31(20): p. 2600-6.

867. Fata, F., et al., Efficacy and toxicity of adjuvant chemotherapy in elderly patients with colon carcinoma: a 10-year experience of the Geisinger Medical Center. Cancer, 2002. 94(7): p. 1931-8.

868. Biagi, J.J., et al., Association between time to initiation of adjuvant chemotherapy and survival in colorectal cancer: a systematic review and meta-analysis. JAMA, 2011 . 305(22): p. 2335-42.

869. Des Guetz, G., et al., Does delaying adjuvant chemotherapy after curative surgery for colorectal cancer impair survival? A meta-analysis. Eur J Cancer, 2010. 46(6): p. 1049-55.

870. Bos, A.C., et al., Timing of adjuvant chemotherapy and its relation to survival among patients with stage III colon cancer. Eur J Cancer, 2015. 51(17): p. 2553-61.

871. Bayraktar, U.D., et al., Does delay of adjuvant chemotherapy impact survival in patients with resected stage II and III colon adenocarcinoma? Cancer, 2011 . 117(11): p. 2364-70.

872. Lima, I.S., et al., Association between receipt and timing of adjuvant chemotherapy and survival for patients with stage III colon cancer in Alberta, Canada. Cancer, 2011. 117(16): p. 3833-40.

873. Nachiappan, S., et al., Initiation of adjuvant chemotherapy within 8 weeks of elective colorectal resection improves overall survival regardless of reoperation. Colorectal Dis, 2016. 18(11): $p$. 1041-1049.

874. Dube, S., F. Heyen, and M. Jenicek, Adjuvant chemotherapy in colorectal carcinoma: results of a meta-analysis. Dis Colon Rectum, 1997. 40(1): p. 35-41.

875. Gill, S., et al., Pooled analysis of fluorouracil-based adjuvant therapy for stage II and III colon cancer: who benefits and by how much? J Clin Oncol, 2004. 22(10): p. 1797-806.

876. Efficacy of adjuvant fluorouracil and folinic acid in colon cancer. International Multicentre Pooled Analysis of Colon Cancer Trials (IMPACT) investigators. Lancet, 1995. 345(8955): p. 939-44.

877. Sargent, D.J., et al., A pooled analysis of adjuvant chemotherapy for resected colon cancer in elderly patients. N Engl J Med, 2001. 345(15): p. 1091-7.

878. Francini, G., et al., Folinic acid and 5-fluorouracil as adjuvant chemotherapy in colon cancer. Gastroenterology, 1994. 106(4): p. 899-906.

879. O'Connell, M.J., et al., Controlled trial of fluorouracil and low-dose leucovorin given for 6 months as postoperative adjuvant therapy for colon cancer. J Clin Oncol, 1997. 15(1): p. 24650.

880. Figueredo, A., et al., Adjuvant therapy for stage II colon cancer after complete resection. Provincial Gastrointestinal Disease Site Group. Cancer Prev Control, 1997. 1(5): p. 379-92.

881. Efficacy of adjuvant fluorouracil and folinic acid in B2 colon cancer. International Multicentre Pooled Analysis of B2 Colon Cancer Trials (IMPACT B2) Investigators. J Clin Oncol, 1999. 17(5): p. 1356-63.

882. Mamounas, E., et al., Comparative efficacy of adjuvant chemotherapy in patients with Dukes' B versus Dukes' C colon cancer: results from four National Surgical Adjuvant Breast and Bowel Project adjuvant studies (C-01, C-02, C-03, and C-04). J Clin Oncol, 1999. 17(5): p. 1349-55.

883. Benson, A.B., 3rd, et al., American Society of Clinical Oncology recommendations on adjuvant chemotherapy for stage II colon cancer. J Clin Oncol, 2004. 22(16): p. 3408-19.

884. Quasar Collaborative, G., et al., Adjuvant chemotherapy versus observation in patients with colorectal cancer: a randomised study. Lancet, 2007. 370(9604): p. 2020-9. 
885. O'Connor, E.S., et al., Adjuvant chemotherapy for stage II colon cancer with poor prognostic features. J Clin Oncol, 2011. 29(25): p. 3381-8.

886. Andre, T., et al., Adjuvant Fluorouracil, Leucovorin, and Oxaliplatin in Stage II to III Colon Cancer: Updated 10-Year Survival and Outcomes According to BRAF Mutation and Mismatch Repair Status of the MOSAIC Study. J Clin Oncol, 201 5. 33(35): p. 4176-87.

887. Moertel, C.G., et al., Intergroup study of fluorouracil plus levamisole as adjuvant therapy for stage II/Dukes' B2 colon cancer. J Clin Oncol, 1995. 13(12): p. 2936-43.

888. Taal, B.G., H. Van Tinteren, and F.A. Zoetmulder, Adjuvant 5FU plus levamisole in colonic or rectal cancer: improved survival in stage II and III. Br J Cancer, 2001 . 85(10): p. 1437-43.

889. Schrag, D., et al., Adjuvant chemotherapy use for Medicare beneficiaries with stage II colon cancer. J Clin Oncol, 2002. 20(19): p. 3999-4005.

890. Caplin, S., et al., For patients with Dukes' B (TNM Stage II) colorectal carcinoma, examination of six or fewer lymph nodes is related to poor prognosis. Cancer, 1998. 83(4): p. 666-72.

891. Merkel, S., et al., High-risk groups of patients with Stage II colon carcinoma. Cancer, 2001. 92(6): p. 1435-43.

892. Petersen, V.C., et al., Identification of objective pathological prognostic determinants and models of prognosis in Dukes' B colon cancer. Gut, 2002. 51(1): p. 65-9.

893. Morris, M., et al., Population-based study of prognostic factors in stage II colonic cancer. $\mathrm{Br} \mathrm{J}$ Surg, 2006. 93(7): p. 866-71.

894. Burdy, G., et al., Identifying patients with T3-T4 node-negative colon cancer at high risk of recurrence. Dis Colon Rectum, 2001. 44(11): p. 1682-8.

895. Jestin, P., et al., Emergency surgery for colonic cancer in a defined population. Br J Surg, 2005. 92(1): p. 94-100.

896. McArdle, C.S., D.C. McMillan, and D.J. Hole, The impact of blood loss, obstruction and perforation on survival in patients undergoing curative resection for colon cancer. Br J Surg, 2006. 93(4): p. 483-8.

897. George, S., et al., Will Rogers revisited: prospective observational study of survival of 3592 patients with colorectal cancer according to number of nodes examined by pathologists. $\mathrm{Br} \mathrm{J}$ Cancer, 2006. 95(7): p. 841-7.

898. Chen, S.L. and A.J. Bilchik, More extensive nodal dissection improves survival for stages I to III of colon cancer: a population-based study. Ann Surg, 2006. 244(4): p. 602-10.

899. Ribic, C.M., et al., Tumor microsatellite-instability status as a predictor of benefit from fluorouracil-based adjuvant chemotherapy for colon cancer. N Engl J Med, 2003. 349(3): p. 247-57.

900. Sargent, D.J., et al., Defective mismatch repair as a predictive marker for lack of efficacy of fluorouracil-based adjuvant therapy in colon cancer. J Clin Oncol, 2010. 28(20): p. 3219-26.

901. Jover, R., et al., Mismatch repair status in the prediction of benefit from adjuvant fluorouracil chemotherapy in colorectal cancer. Gut, 2006. 55(6): p. 848-55.

902. Kim, J.E., et al., Defective Mismatch Repair Status was not Associated with DFS and OS in Stage II Colon Cancer Treated with Adjuvant Chemotherapy. Ann Surg Oncol, 2015. 22 Suppl 3: p. 630-7.

903. Moertel, C.G., et al., Levamisole and fluorouracil for adjuvant therapy of resected colon carcinoma. N Engl J Med, 1990. 322(6): p. 352-8.

904. Hermanek, P., R.V. Hutter, and L.H. Sobin, Prognostic grouping: the next step in tumor classification. J Cancer Res Clin Oncol, 1990. 116(5): p. 513-6.

905. Martinez-Lopez, E., et al., Allelic loss on chromosome 189 as a prognostic marker in stage II colorectal cancer. Gastroenterology, 1998. 114(6): p. 1180-7.

906. Ogunbiyi, O.A., et al., Confirmation that chromosome 189 allelic loss in colon cancer is a prognostic indicator. J Clin Oncol, 1998. 16(2): p. 427-33.

907. Jernvall, P., et al., Loss of heterozygosity at 18921 is indicative of recurrence and therefore poor prognosis in a subset of colorectal cancers. Br J Cancer, 1999. 79(5-6): p. 903-8.

908. Font, A., et al., Prognostic value of K-ras mutations and allelic imbalance on chromosome 189 in patients with resected colorectal cancer. Dis Colon Rectum, 2001. 44(4): p. 549-57.

909. Diep, C.B., et al., Genetic tumor markers with prognostic impact in Dukes' stages B and C colorectal cancer patients. J Clin Oncol, 2003. 21(5): p. 820-9.

910. Carethers, J.M., et al., Prognostic significance of allelic lost at chromosome 18921 for stage II colorectal cancer. Gastroenterology, 1998. 114(6): p. 1188-95.

911. Araujo, S.E., et al., DNA ploidy status and prognosis in colorectal cancer: a meta-analysis of published data. Dis Colon Rectum, 2007. 50(11): p. 1800-10.

912. Klingbiel, D., et al., Prognosis of stage II and III colon cancer treated with adjuvant 5fluorouracil or FOLFIRI in relation to microsatellite status: results of the PETACC-3 trial. Ann Oncol, $2015.26(1)$ : p. 126-32. 
913. Lanza, G., et al., Immunohistochemical test for $\mathrm{MLH1}$ and MSH2 expression predicts clinical outcome in stage II and III colorectal cancer patients. J Clin Oncol, 2006. 24(15): p. 2359-67.

914. Sinicrope, F.A., et al., Prognostic impact of microsatellite instability and DNA ploidy in human colon carcinoma patients. Gastroenterology, 2006. 131(3): p. 729-37.

915. Elsaleh, H. and B. lacopetta, Microsatellite instability is a predictive marker for survival benefit from adjuvant chemotherapy in a population-based series of stage III colorectal carcinoma. Clin Colorectal Cancer, 2001. 1(2): p. 104-9.

916. Thomas, M.L., et al., Clinicopathological predictors of benefit from adjuvant chemotherapy for stage C colorectal cancer: Microsatellite unstable cases benefit. Asia Pac J Clin Oncol, 2015. $11(4)$ : p. 343-51.

917. Dalerba, P., et al., CDX2 as a Prognostic Biomarker in Stage II and Stage III Colon Cancer. N Engl J Med, 2016. 374(3): p. 211-22.

918. Tie, J., et al., Circulating tumor DNA analysis detects minimal residual disease and predicts recurrence in patients with stage II colon cancer. Sci Transl Med, 2016. 8(346): p. 346ra92.

919. Turner, N., et al., Analysis of local chronic inflammatory cell infiltrate combined with systemic inflammation improves prognostication in stage II colon cancer independent of standard clinicopathologic criteria. Int J Cancer, 2016. 138(3): p. 671-8.

920. Andre, T., et al., Oxaliplatin, fluorouracil, and leucovorin as adjuvant treatment for colon cancer. N Engl J Med, 2004. 350(23): p. 2343-51.

921. Kuebler, J.P., et al., Oxaliplatin combined with weekly bolus fluorouracil and leucovorin as surgical adjuvant chemotherapy for stage II and III colon cancer: results from NSABP C-07. J Clin Oncol, 2007. 25(16): p. 2198-204.

922. Kidwell, K.M., et al., Long-term neurotoxicity effects of oxaliplatin added to fluorouracil and leucovorin as adjuvant therapy for colon cancer: results from National Surgical Adjuvant Breast and Bowel Project trials C-07 and LTS-01. Cancer, 2012. 118(22): p. 5614-22.

923. de Gramont, A., et al., Oxaliplatin/5FU/LV in adjuvant colon cancer: Updated efficacy results of the MOSAIC trial, including survival, with a median follow-up of six years. Journal of Clinical Oncology, 2007 ASCO Annual Meeting Proceedings (Post-Meeting Edition) Vol 25, No 18 S (June 20 Supplement), 2007: 4007, 2007.

924. Schmoll, H.J., et al., Effect of adjuvant capecitabine or fluorouracil, with or without oxaliplatin, on survival outcomes in stage III colon cancer and the effect of oxaliplatin on post-relapse survival: a pooled analysis of individual patient data from four randomised controlled trials. Lancet Oncol, 2014. 15(13): p. 1481-92.

925. Sanoff, H.K., et al., Effect of adjuvant chemotherapy on survival of patients with stage III colon cancer diagnosed after age 75 years. J Clin Oncol, 2012. 30(21): p. 2624-34.

926. Yothers, G., et al., Oxaliplatin as adjuvant therapy for colon cancer: updated results of NSABP C-07 trial, including survival and subset analyses. J Clin Oncol, 2011 . 29(28): p. 3768-74.

927. Lund, C.M., et al., Efficacy and toxicity of adjuvant chemotherapy in elderly patients with colorectal cancer: the ACCORE study. ESMO Open, 2016. 1(5): p. e000087.

928. Kim, C.A., et al., Efficacy and safety of single agent or combination adjuvant chemotherapy in elderly patients with colon cancer: a Canadian cancer institute experience. Clin Colorectal Cancer, 2014. 13(3): p. 199-206.

929. Twelves, C., et al., Capecitabine as adjuvant treatment for stage III colon cancer. N Engl J Med, 2005. 352(26): p. 2696-704.

930. Andre, T., et al., Updated results at 6 year of the GERCOR C96. 1 phase III study comparing LV5FU2 to monthly 5FU-leucovorin (mFufol) as adjuvant treatment for Dukes B2 and C colon cancer patients. Journal of Clinical Oncology, 2005 ASCO Annual Meeting Proceedings. Vol 23, No 16S (June 1 Supplement), 2005: 3522, 2005.

931. Carrato, A., et al., Folinic acid modulated bolus 5-FU or infusional 5-FU for adjuvant treatment of patients of UICC stage III colon cancer: Preliminary analysis of the PETACC-2-study. Journal of Clinical Oncology, 2006 ASCO Annual Meeting Proceedings (Post-Meeting Edition). Vol 24, No 18 S (June 20 Supplement), 2006: 3563, 2006.

932. Andre, T., et al., Semimonthly versus monthly regimen of fluorouracil and leucovorin administered for 24 or 36 weeks as adjuvant therapy in stage II and III colon cancer: results of a randomized trial. J Clin Oncol, 2003. 21(15): p. 2896-903.

933. Arkenau, H.T., K. Rettig, and R. Porschen, Adjuvant chemotherapy in curative resected colon carcinoma: 5-fluorouracil/leucovorin versus high-dose 5-fluorouracil 24-h infusion/leucovorin versus high-dose 5-fluorouracil 24-h infusion. Int J Colorectal Dis, 2005. 20(3): p. 258-61.

934. Chau, I., et al., A randomised comparison between 6 months of bolus fluorouracil/leucovorin and 12 weeks of protracted venous infusion fluorouracil as adjuvant treatment in colorectal cancer. Ann Oncol, 2005. 16(4): p. 549-57.

935. Lembersky, B.C., et al., Oral uracil and tegafur plus leucovorin compared with intravenous fluorouracil and leucovorin in stage II and III carcinoma of the colon: results from National 
Surgical Adjuvant Breast and Bowel Project Protocol C-06. J Clin Oncol, 2006. 24(13): p. 2059 64.

936. Sakamoto, J., et al., Efficacy of oral adjuvant therapy after resection of colorectal cancer: 5 year results from three randomized trials. J Clin Oncol, 2004. 22(3): p. 484-92.

937. Poplin, E.A., et al., Phase III Southwest Oncology Group 9415/Intergroup 0153 randomized trial of fluorouracil, leucovorin, and levamisole versus fluorouracil continuous infusion and levamisole for adjuvant treatment of stage III and high-risk stage II colon cancer. J Clin Oncol, 2005. 23(9): p. 1819-25.

938. O'Connell, M.J., et al., Prospectively randomized trial of postoperative adjuvant chemotherapy in patients with high-risk colon cancer. J Clin Oncol, 1998. 16(1): p. 295-300.

939. Haller, D.G., et al., Phase III study of fluorouracil, leucovorin, and levamisole in high-risk stage II and III colon cancer: final report of Intergroup 0089. J Clin Oncol, 2005. 23(34): p. 8671-8.

940. Van Cutsem, E., et al., Randomized phase III trial comparing biweekly infusional fluorouracil/leucovorin alone or with irinotecan in the adjuvant treatment of stage III colon cancer: PETACC-3. J Clin Oncol, 2009. 27(19): p. 3117-25.

941. Ychou, M., et al., A phase III randomised trial of LV5FU2 + irinotecan versus LV5FU2 alone in adjuvant high-risk colon cancer (FNCLCC Accord02/FFCD9802). Ann Oncol, 2009. 20(4): p. 674-80.

942. Saltz, L.B., et al., Irinotecan fluorouracil plus leucovorin is not superior to fluorouracil plus leucovorin alone as adjuvant treatment for stage III colon cancer: results of CALGB 89803. J Clin Oncol, 2007. 25(23): p. 3456-61.

943. Alberts, S.R., et al., Effect of oxaliplatin, fluorouracil, and leucovorin with or without cetuximab on survival among patients with resected stage III colon cancer: a randomized trial. JAMA, 2012. 307(13): p. 1383-93.

944. Taieb, J., et al., Oxaliplatin, fluorouracil, and leucovorin with or without cetuximab in patients with resected stage III colon cancer (PETACC-8): an open-label, randomised phase 3 trial. Lancet Oncol, 2014. 15(8): p. 862-73.

945. Allegra, C.J., et al., Bevacizumab in stage II-III colon cancer: 5-year update of the National Surgical Adjuvant Breast and Bowel Project C-08 trial. J Clin Oncol, 2013. 31(3): p. 359-64.

946. de Gramont, A., et al., Bevacizumab plus oxaliplatin-based chemotherapy as adjuvant treatment for colon cancer (AVANT): a phase 3 randomised controlled trial. Lancet Oncol, 2012. 13(12): p. 1225-33.

947. Kerr, R.S., et al., Adjuvant capecitabine plus bevacizumab versus capecitabine alone in patients with colorectal cancer (QUASAR 2): an open-label, randomised phase 3 trial. Lancet Oncol, 2016. 17(11): p. 1543-1557.

948. Amlani, A., et al., Compliance with adjuvant capecitabine in patients with stage II and III colon cancer: comparison of administrative versus medical record data. Cancer Med, 2016. 5(8): $\mathrm{p}$. $1776-82$.

949. Grothey, A., et al., Duration of Adjuvant Chemotherapy for Stage III Colon Cancer. N Engl J Med, 2018. 378(13): p. 1177-1188.

950. Andre, T., et al., Three Versus 6 Months of Oxaliplatin-Based Adjuvant Chemotherapy for Patients With Stage III Colon Cancer: Disease-Free Survival Results From a Randomized, OpenLabel, International Duration Evaluation of Adjuvant (IDEA) France, Phase III Trial. J Clin Oncol, 2018. 36(15): p. 1469-1477.

951. Sobrero, A., et al., FOLFOX or CAPOX in Stage II to III Colon Cancer: Efficacy Results of the Italian Three or Six Colon Adjuvant Trial. J Clin Oncol, 2018. 36(15): p. 1478-1485.

952. Iveson, T.J., et al., 3 versus 6 months of adjuvant oxaliplatin-fluoropyrimidine combination therapy for colorectal cancer (SCOT): an international, randomised, phase 3, non-inferiority trial. Lancet Oncol, 2018. 19(4): p. 562-578.

953. Shi, Q., Alberto F. Sobrero, Anthony Frank Shields, Takayuki Yoshino, James Paul, Julien Taieb, ... loannis Sougklakos, Rachel Kerr, Roberto Labianca, Jeffrey A. Meyerhardt, Franck Bonnetain, Toshiaki Watanabe, loannis Boukovinas, Lindsay A. Renfro, Axel Grothey, Donna Niedzwiecki, Valter Torri, Thierry Andre, Daniel J. Sargent, Timothy Iveson, Prospective pooled analysis of six phase III trials investigating duration of adjuvant (adjuv) oxaliplatin-based therapy (3 vs 6 months) for patients (pts) with stage III colon cancer (CC): The IDEA (International Duration Evaluation of Adjuvant chemotherapy) collaboration. . J Clin Oncol, 2017. 35: p. Suppl.

954. Sobrero, A., et al., The hard road to data interpretation: 3 or 6 months of adjuvant chemotherapy for patients with stage III colon cancer? Ann Oncol, 2018. 29(5): p. 1099-1107.

955. Hwang, I.G., et al., Association between timing and duration of adjuvant chemotherapy and survival for colorectal cancer in korea, 2011-2014: A nationwide study based on the database of quality assessment and the health insurance. Journal of Clinical Oncology, 2017. 35(15_suppl): p. 3605-3605. 
956. van Gijn, W., et al., Preoperative radiotherapy combined with total mesorectal excision for resectable rectal cancer: 12-year follow-up of the multicentre, randomised controlled TME trial. Lancet Oncol, 2011 . 12(6): p. 575-82.

957. Sebag-Montefiore, D., et al., Preoperative radiotherapy versus selective postoperative chemoradiotherapy in patients with rectal cancer (MRC CR07 and NCIC-CTC C016): a multicentre, randomised trial. Lancet, 2009. 373(9666): p. 811-20.

958. Sauer, R., et al., Preoperative versus postoperative chemoradiotherapy for rectal cancer. N Engl J Med, 2004. 351(17): p. 1731-40.

959. Sauer, R., et al., Preoperative versus postoperative chemoradiotherapy for locally advanced rectal cancer: results of the German CAO/ARO/AIO-94 randomized phase III trial after a median follow-up of 11 years. J Clin Oncol, 2012. 30(16): p. 1926-33.

960. Bosset, J.F., et al., Chemotherapy with preoperative radiotherapy in rectal cancer. $\mathrm{N}$ Engl J Med, 2006. 355(11): p. $1114-23$.

961. Bosset, J.F., et al., Fluorouracil-based adjuvant chemotherapy after preoperative chemoradiotherapy in rectal cancer: long-term results of the EORTC 22921 randomised study. Lancet Oncol, 2014. 15(2): p. 184-90.

962. Gerard, J.P., et al., Preoperative radiotherapy with or without concurrent fluorouracil and leucovorin in T3-4 rectal cancers: results of FFCD 9203. J Clin Oncol, 2006. 24(28): p. 4620-5.

963. Folkesson, J., et al., Swedish Rectal Cancer Trial: long lasting benefits from radiotherapy on survival and local recurrence rate. J Clin Oncol, 2005. 23(24): p. 5644-50.

964. Marijnen, C.A., Organ preservation in rectal cancer: have all questions been answered? Lancet Oncol, 2015. 16(1): p. e13-22.

965. Lezoche, E., et al., Randomized clinical trial of endoluminal locoregional resection versus laparoscopic total mesorectal excision for $T 2$ rectal cancer after neoadjuvant therapy. Br J Surg, 2012. 99(9): p. 1211-8.

966. Bujko, K., et al., Preoperative radiotherapy and local excision of rectal cancer with immediate radical re-operation for poor responders: a prospective multicentre study. Radiother Oncol, 2013. 106(2): p. 198-205.

967. Verseveld, M., et al., Chemoradiation therapy for rectal cancer in the distal rectum followed by organ-sparing transanal endoscopic microsurgery (CARTS study). Br J Surg, 2015. 102(7): p. 853-60.

968. Garcia-Aguilar, J., et al., Organ preservation for clinical T2NO distal rectal cancer using neoadjuvant chemoradiotherapy and local excision (ACOSOG Z6041): results of an open-label, single-arm, multi-institutional, phase 2 trial. Lancet Oncol, 2015. 16(15): p. 1537-46.

969. Wong, R.K., et al., Pre-operative radiotherapy and curative surgery for the management of localized rectal carcinoma. Cochrane Database Syst Rev, 2007(2): p. Cd002102.

970. Fiorica, F., et al., Can chemotherapy concomitantly delivered with radiotherapy improve survival of patients with resectable rectal cancer? A meta-analysis of literature data. Cancer Treat Rev, 2010. 36(7): p. 539-49.

971. Ceelen, W.P., Y. Van Nieuwenhove, and K. Fierens, Preoperative chemoradiation versus radiation alone for stage II and III resectable rectal cancer. Cochrane Database Syst Rev, 2009(1): p. Cd006041.

972. Camma, C., et al., Preoperative radiotherapy for resectable rectal cancer: A meta-analysis. Jama, 2000. 284(8): p. 1008-15.

973. Adjuvant radiotherapy for rectal cancer: a systematic overview of 8,507 patients from 22 randomised trials. Lancet, 2001. 358(9290): p. 1291-304.

974. FL, G., et al., AJCC Cancer Staging Atlas. New York. Springer, 2006.

975. Rodel, C., et al., Oxaliplatin added to fluorouracil-based preoperative chemoradiotherapy and postoperative chemotherapy of locally advanced rectal cancer (the German CAO/ARO/AIO-04 study): final results of the multicentre, open-label, randomised, phase 3 trial. Lancet Oncol, 2015. 16(8): p. 979-89.

976. Kreis, M.E., et al., Use of Preoperative Magnetic Resonance Imaging to Select Patients with Rectal Cancer for Neoadjuvant Chemoradiation--Interim Analysis of the German OCUM Trial (NCT01325649). J Gastrointest Surg, 2016. 20(1): p. 25-32; discussion 32-3.

977. Bonnetain, F., et al., What is the clinical benefit of preoperative chemoradiotherapy with 5FU/leucovorin for T3-4 rectal cancer in a pooled analysis of EORTC 22921 and FFCD 9203 trials: surrogacy in question? Eur J Cancer, 2012. 48(12): p. 1781-90.

978. Wiltink, L.M., et al., Health-related quality of life 14 years after preoperative short-term radiotherapy and total mesorectal excision for rectal cancer: report of a multicenter randomised trial. Eur J Cancer, 2014. 50(14): p. 2390-8.

979. Birgisson, H., et al., Adverse effects of preoperative radiation therapy for rectal cancer: longterm follow-up of the Swedish Rectal Cancer Trial. J Clin Oncol, 2005. 23(34): p. 8697-705. 
980. Chen, T.Y., et al., Bowel function 14 years after preoperative short-course radiotherapy and total mesorectal excision for rectal cancer: report of a multicenter randomized trial. Clin Colorectal Cancer, 2015. 14(2): p. 106-14.

981. Wiltink, L.M., et al., A comprehensive longitudinal overview of health-related quality of life and symptoms after treatment for rectal cancer in the TME trial. Acta Oncol, 2016. 55(4): p. 502-8.

982. Li, X.T., et al., Evaluating local lymph node metastasis with magnetic resonance imaging, endoluminal ultrasound and computed tomography in rectal cancer: a meta-analysis. Colorectal Dis, 2015. 17(6): p. 0129-35.

983. Merkel, S., et al., Prognostic subdivision of ypT3 rectal tumours according to extension beyond the muscularis propria. Br J Surg, 2014. 101(5): p. 566-72.

984. Taylor, F.G., et al., Preoperative high-resolution magnetic resonance imaging can identify good prognosis stage I, II, and III rectal cancer best managed by surgery alone: a prospective, multicenter, European study. Ann Surg, 2011 . 253(4): p. 711-9.

985. Taylor, F.G., et al., Preoperative magnetic resonance imaging assessment of circumferential resection margin predicts disease-free survival and local recurrence: 5-year follow-up results of the MERCURY study. J Clin Oncol, 2014. 32(1): p. 34-43.

986. Peeters, K.C., et al., The TME trial after a median follow-up of 6 years: increased local control but no survival benefit in irradiated patients with resectable rectal carcinoma. Ann Surg, 2007. 246(5): p. 693-701.

987. Krook, J.E., et al., Effective surgical adjuvant therapy for high-risk rectal carcinoma. N Engl J Med, 1991. 324(11): p. 709-15.

988. Smalley, S.R., et al., Phase III trial of fluorouracil-based chemotherapy regimens plus radiotherapy in postoperative adjuvant rectal cancer: GI INT 0144. J Clin Oncol, 2006. 24(22): p. 3542-7.

989. Ngan, S.Y., et al., Randomized trial of short-course radiotherapy versus long-course chemoradiation comparing rates of local recurrence in patients with $T 3$ rectal cancer: TransTasman Radiation Oncology Group trial 01.04. J Clin Oncol, 2012. 30(31): p. 3827-33.

990. Bujko, K., et al., Sphincter preservation following preoperative radiotherapy for rectal cancer: report of a randomised trial comparing short-term radiotherapy vs. conventionally fractionated radiochemotherapy. Radiother Oncol, 2004. 72(1): p. 15-24.

991. Bujko, K., et al., Long-term results of a randomized trial comparing preoperative short-course radiotherapy with preoperative conventionally fractionated chemoradiation for rectal cancer. Br J Surg, 2006. 93(10): p. 1215-23.

992. Pietrzak, L., et al., Quality of life, anorectal and sexual functions after preoperative radiotherapy for rectal cancer: report of a randomised trial. Radiother Oncol, 2007. 84(3): p. 217-25.

993. McLachlan, S.A., et al., The impact on health-related quality of life in the first 12 months: A randomised comparison of preoperative short-course radiation versus long-course chemoradiation for T3 rectal cancer (Trans-Tasman Radiation Oncology Group Trial 01.04). Eur J Cancer, 2016. 55: p. 15-26.

994. Pettersson, D., et al., Interim analysis of the Stockholm III trial of preoperative radiotherapy regimens for rectal cancer. Br J Surg, 2010. 97(4): p. 580-7.

995. Pettersson, D., et al., Tumour regression in the randomized Stockholm III Trial of radiotherapy regimens for rectal cancer. Br J Surg, 2015. 102(8): p. 972-8; discussion 978.

996. Bujko, K., et al., Long-course oxaliplatin-based preoperative chemoradiation versus $5 \times 5 \mathrm{~Gy}$ and consolidation chemotherapy for CT4 or fixed CT3 rectal cancer: results of a randomized phase III study. Ann Oncol, 2016. 27(5): p. 834-42.

997. Ansari, N., et al., Acute Adverse Events and Postoperative Complications in a Randomized Trial of Preoperative Short-course Radiotherapy Versus Long-course Chemoradiotherapy for T3 Adenocarcinoma of the Rectum: Trans-Tasman Radiation Oncology Group Trial (TROG 01.04). Ann Surg, 2017. 265(5): p. 882-888.

998. Nilsson, P.J., et al., Short-course radiotherapy followed by neo-adjuvant chemotherapy in locally advanced rectal cancer--the RAPIDO trial. BMC Cancer, 2013. 13: p. 279.

999. Hofheinz, R.D., et al., Chemoradiotherapy with capecitabine versus fluorouracil for locally advanced rectal cancer: a randomised, multicentre, non-inferiority, phase 3 trial. Lancet Oncol, 2012. 13(6): p. 579-88.

1000. O'Connell, M.J., et al., Capecitabine and oxaliplatin in the preoperative multimodality treatment of rectal cancer: surgical end points from National Surgical Adjuvant Breast and Bowel Project trial R-04. J Clin Oncol, 2014. 32(18): p. 1927-34.

1001. Allegra, C.J., et al., Neoadjuvant 5-FU or Capecitabine Plus Radiation With or Without Oxaliplatin in Rectal Cancer Patients: A Phase III Randomized Clinical Trial. J Natl Cancer Inst, 2015. 107(11). 
1002. Rodel, C., R. Hofheinz, and T. Liersch, Rectal cancer: state of the art in 2012. Curr Opin Oncol, 2012. 24(4): p. 441-7.

1003. Aschele, C., et al., Primary tumor response to preoperative chemoradiation with or without oxaliplatin in locally advanced rectal cancer: pathologic results of the STAR-01 randomized phase III trial. J Clin Oncol, 2011 . 29(20): p. 2773-80.

1004. Aschele, C., S. Lonardi, and L. Cionini, Final results of STAR-01: A randomized phase III trial comparing preoperative chemoradiation with or without oxaliplatin in locally advanced rectal cancer. J Clin Oncol, 2016. 34((suppl; abstr 3521)).

1005. Gerard, J.P., et al., Clinical outcome of the ACCORD 12/0405 PRODIGE 2 randomized trial in rectal cancer. J Clin Oncol, 2012. 30(36): p. 4558-65.

1006. Francois, E., et al., ACCORD12/0405-Prodige 2 phase III trial neoadjuvant treatment in rectal cancer: Results after 5 years of follow-up. J Clin Oncol, 2016. 34((supp 4S; abstr 490)).

1007. Schmoll, H., et al., Preoperative chemoradiotherapy and postoperative chemothrapy with capecitabine and oxaliplatin versus capecitabine alone in locally advanced rectal cancer: disease-free survial at interim analysis. Proc Am Soc Clin Oncol, 2014. 32(abstract 3501).

1008. Jiao, D., et al., Fluorouracil-based preoperative chemoradiotherapy with or without oxaliplatin for stage II/III rectal cancer: a 3-year follow-up study. Chin J Cancer Res, 2015. 27(6): p. 58896.

1009. Deng, Y., et al., Modified FOLFOX6 With or Without Radiation Versus Fluorouracil and Leucovorin With Radiation in Neoadjuvant Treatment of Locally Advanced Rectal Cancer: Initial Results of the Chinese FOWARC Multicenter, Open-Label, Randomized Three-Arm Phase III Trial. J Clin Oncol, 2016. 34(27): p. 3300-7.

1010. An, X., et al., Short term results of neoadjuvant chemoradiotherapy with fluoropyrimidine alone or in combination with oxaliplatin in locally advanced rectal cancer: a meta analysis. Eur J Cancer, $2013.49(4)$ : p. 843-51.

1011. Yang, Y.J., et al., Fluorouracil-based neoadjuvant chemoradiotherapy with or without oxaliplatin for treatment of locally advanced rectal cancer: An updated systematic review and meta-analysis. Oncotarget, 2016. 7(29): p. 45513-45524.

1012. Lefevre, J.H., et al., Effect of Interval ( 7 or 11 weeks) Between Neoadjuvant Radiochemotherapy and Surgery on Complete Pathologic Response in Rectal Cancer: A Multicenter, Randomized, Controlled Trial (GRECCAR-6). J Clin Oncol, 2016.

1013. Wang, X.J., et al., Effect of Interval between Neoadjuvant Chemoradiotherapy and Surgery on Oncological Outcome for Rectal Cancer: A Systematic Review and Meta-Analysis. Gastroenterol Res Pract, 2016. 2016: p. 6756859.

1014. Petrelli, F., et al., Increasing the Interval Between Neoadjuvant Chemoradiotherapy and Surgery in Rectal Cancer: A Meta-analysis of Published Studies. Ann Surg, 2016. 263(3): p. 458-64.

1015. Foster, J.D., et al., Timing of surgery after long-course neoadjuvant chemoradiotherapy for rectal cancer: a systematic review of the literature. Dis Colon Rectum, 2013. 56(7): p. 921-30.

1016. van den Broek, C.B., et al., Impact of the interval between short-course radiotherapy and surgery on outcomes of rectal cancer patients. Eur J Cancer, 2013. 49(15): p. 3131-9.

1017. Erlandsson, J., et al., Optimal fractionation of preoperative radiotherapy and timing to surgery for rectal cancer (Stockholm III): a multicentre, randomised, non-blinded, phase 3, noninferiority trial. Lancet Oncol, 2017. 18(3): p. 336-346.

1018. Pach, R., et al., Randomized clinical trial on preoperative radiotherapy 25 Gy in rectal cancer-treatment results at 5-year follow-up. Langenbecks Arch Surg, 2012. 397(5): p. 801-7.

1019. Bujko, K., M. Partycki, and L. Pietrzak, Neoadjuvant radiotherapy ( 5 x 5 Gy): immediate versus delayed surgery. Recent Results Cancer Res, 2014. 203: p. 171-87.

1020. Fernandez-Martos, C., et al., Phase II, randomized study of concomitant chemoradiotherapy followed by surgery and adjuvant capecitabine plus oxaliplatin (CAPOX) compared with induction CAPOX followed by concomitant chemoradiotherapy and surgery in magnetic resonance imaging-defined, locally advanced rectal cancer: Grupo cancer de recto 3 study. J Clin Oncol, 2010. 28(5): p. 859-65.

1021. Fernandez-Martos, C., et al., Chemoradiation, surgery and adjuvant chemotherapy versus induction chemotherapy followed by chemoradiation and surgery: long-term results of the Spanish GCR-3 phase II randomized trialdagger. Ann Oncol, 2015. 26(8): p. 1722-8.

1022. Garcia-Aguilar, J., et al., Effect of adding mFOLFOX6 after neoadjuvant chemoradiation in locally advanced rectal cancer: a multicentre, phase 2 trial. Lancet Oncol, 2015. 16(8): p. 957 66.

1023. Smith, J.J., et al., Organ Preservation in Rectal Adenocarcinoma: a phase II randomized controlled trial evaluating 3-year disease-free survival in patients with locally advanced rectal cancer treated with chemoradiation plus induction or consolidation chemotherapy, and total mesorectal excision or nonoperative management. BMC Cancer, 2015. 15: p. 767. 
1024. Schrag, D., et al., Neoadjuvant chemotherapy without routine use of radiation therapy for patients with locally advanced rectal cancer: a pilot trial. J Clin Oncol, 2014. 32(6): p. 513-8.

1025. Fernandez-Martos, C., et al., Preoperative chemotherapy in patients with intermediate-risk rectal adenocarcinoma selected by high-resolution magnetic resonance imaging: the GEMCAD 0801 Phase II Multicenter Trial. Oncologist, 2014. 19(10): p. 1042-3.

1026. van Dijk, T.H., et al., Evaluation of short-course radiotherapy followed by neoadjuvant bevacizumab, capecitabine, and oxaliplatin and subsequent radical surgical treatment in primary stage IV rectal cancer. Ann Oncol, 2013. 24(7): p. 1762-9.

1027. Rodel, C. and R. Sauer, Radiotherapy and concurrent radiochemotherapy for rectal cancer. Surg Oncol, 2004. 13(2-3): p. 93-101.

1028. Gunderson, L.L., et al., Impact of $T$ and $N$ stage and treatment on survival and relapse in adjuvant rectal cancer: a pooled analysis. J Clin Oncol, 2004. 22(10): p. 1785-96.

1029. Valentini, V., et al., EURECCA consensus conference highlights about rectal cancer clinical management: the radiation oncologist's expert review. Radiother Oncol, 2014. 110(1): p. 1958.

1030. Petersen, S.H., et al., Postoperative adjuvant chemotherapy in rectal cancer operated for cure. Cochrane Database Syst Rev, 2012(3): p. Cd004078.

1031. Akasu, T., et al., Adjuvant chemotherapy with uracil-tegafur for pathological stage III rectal cancer after mesorectal excision with selective lateral pelvic lymphadenectomy: a multicenter randomized controlled trial. Jpn J Clin Oncol, 2006. 36(4): p. 237-44.

1032. Hamaguchi, T., et al., Final results of randomized trials by the National Surgical Adjuvant Study of Colorectal Cancer (NSAS-CC). Cancer Chemother Pharmacol, 201 1. 67(3): p. 587-96.

1033. Sainato, A., et al., No benefit of adjuvant Fluorouracil Leucovorin chemotherapy after neoadjuvant chemoradiotherapy in locally advanced cancer of the rectum (LARC): Long term results of a randomized trial (I-CNR-RT). Radiother Oncol, 2014. 113(2): p. 223-9.

1034. Glynne-Jones, R., et al., Chronicle: results of a randomised phase III trial in locally advanced rectal cancer after neoadjuvant chemoradiation randomising postoperative adjuvant capecitabine plus oxaliplatin (XELOX) versus control. Ann Oncol, 2014. 25(7): p. 1356-62.

1035. Breugom, A.J., et al., Adjuvant chemotherapy for rectal cancer patients treated with preoperative (chemo)radiotherapy and total mesorectal excision: a Dutch Colorectal Cancer Group (DCCG) randomized phase III trial. Ann Oncol, 2015. 26(4): p. 696-701.

1036. Breugom, A.J., et al., Adjuvant chemotherapy after preoperative (chemo)radiotherapy and surgery for patients with rectal cancer: a systematic review and meta-analysis of individual patient data. Lancet Oncol, 2015. 16(2): p. 200-7.

1037. Petrelli, F., et al., A systematic review and meta-analysis of adjuvant chemotherapy after neoadjuvant treatment and surgery for rectal cancer. Int J Colorectal Dis, 2015. 30(4): p. 44757.

1038. Maas, M., et al., Adjuvant chemotherapy in rectal cancer: defining subgroups who may benefit after neoadjuvant chemoradiation and resection: a pooled analysis of 3,313 patients. Int J Cancer, 2015. 137(1): p. 212-20.

1039. Hong, Y.S., et al., Oxaliplatin, fluorouracil, and leucovorin versus fluorouracil and leucovorin as adjuvant chemotherapy for locally advanced rectal cancer after preoperative chemoradiotherapy (ADORE): an open-label, multicentre, phase 2, randomised controlled trial. Lancet Oncol, 2014. 15(11): p. 1245-53.

1040. Modest, D.P., et al., Impact of Subsequent Therapies on Outcome of the FIRE-3/AIO KRKO306 Trial: First-Line Therapy With FOLFIRI Plus Cetuximab or Bevacizumab in Patients With KRAS Wild-Type Tumors in Metastatic Colorectal Cancer. J Clin Oncol, 2015. 33(32): p. 3718-26.

1041. Abrams, T.A., et al., Chemotherapy usage patterns in a US-wide cohort of patients with metastatic colorectal cancer. J Natl Cancer Inst, 2014. 106(2): p. djt371.

1042. Sorich, M.J., et al., Extended RAS mutations and anti-EGFR monoclonal antibody survival benefit in metastatic colorectal cancer: a meta-analysis of randomized, controlled trials. Ann Oncol, 2015. 26(1): p. 13-21.

1043. Douillard, J.Y., et al., Panitumumab-FOLFOX4 treatment and RAS mutations in colorectal cancer. N Engl J Med, 2013. 369(11): p. 1023-34.

1044. Van Cutsem, E., et al., Fluorouracil, leucovorin, and irinotecan plus cetuximab treatment and RAS mutations in colorectal cancer. J Clin Oncol, 2015. 33(7): p. 692-700.

1045. Stintzing, S., et al., FOLFIRI plus cetuximab versus FOLFIRI plus bevacizumab for metastatic colorectal cancer (FIRE-3): a post-hoc analysis of tumour dynamics in the final RAS wild-type subgroup of this randomised open-label phase 3 trial. Lancet Oncol, 2016. 17(10): p. 14261434.

1046. Frattini, M., et al., PTEN loss of expression predicts cetuximab efficacy in metastatic colorectal cancer patients. Br J Cancer, 2007. 97(8): p. 1139-45. 
1047. Bokemeyer, C., et al., FOLFOX4 plus cetuximab treatment and RAS mutations in colorectal cancer. Eur J Cancer, 2015. 51(10): p. 1243-52.

1048. Peeters, M., et al., Final results from a randomized phase 3 study of FOLFIRI $\{+/-\}$ panitumumab for second-line treatment of metastatic colorectal cancer. Ann Oncol, 2014. 25(1): p. 107-16.

1049. Karapetis, C.S., et al., K-ras mutations and benefit from cetuximab in advanced colorectal cancer. N Engl J Med, 2008. 359(17): p. 1757-65.

1050. Van Cutsem, E., et al., ESMO consensus guidelines for the management of patients with metastatic colorectal cancer. Ann Oncol, 2016. 27(8): p. 1386-422.

1051. Knijn, N., et al., KRAS mutation analysis: a comparison between primary tumours and matched liver metastases in 305 colorectal cancer patients. Br J Cancer, 201 1. 104(6): p. 1020-6.

1052. Loes, I.M., et al., Impact of KRAS, BRAF, PIK3CA, TP53 status and intraindividual mutation heterogeneity on outcome after liver resection for colorectal cancer metastases. Int J Cancer, 2016. 139(3): p. 647-56.

1053. Holch, J.W., et al., The relevance of primary tumour location in patients with metastatic colorectal cancer: A meta-analysis of first-line clinical trials. Eur J Cancer, 201 7. 70: p. 87-98.

1054. Arnold, D., et al., Prognostic and predictive value of primary tumour side in patients with RAS wild-type metastatic colorectal cancer treated with chemotherapy and EGFR directed antibodies in six randomized trials. Ann Oncol, 2017. 28(8): p. 1713-1729.

1055. Tejpar, S., et al., Prognostic and Predictive Relevance of Primary Tumor Location in Patients With RAS Wild-Type Metastatic Colorectal Cancer: Retrospective Analyses of the CRYSTAL and FIRE-3 Trials. JAMA Oncol, 2016.

1056. Loupakis, F., et al., Primary tumor location as a prognostic factor in metastatic colorectal cancer. J Natl Cancer Inst, 2015. 107(3).

1057. Tran, B., et al., Impact of BRAF mutation and microsatellite instability on the pattern of metastatic spread and prognosis in metastatic colorectal cancer. Cancer, 2011. 117(20): p. 4623-32.

1058. Tveit, K.M., et al., Phase III trial of cetuximab with continuous or intermittent fluorouracil, leucovorin, and oxaliplatin (Nordic FLOX) versus FLOX alone in first-line treatment of metastatic colorectal cancer: the NORDIC-VII study. J Clin Oncol, 2012. 30(15): p. 1755-62.

1059. Pietrantonio, F., et al., Predictive role of BRAF mutations in patients with advanced colorectal cancer receiving cetuximab and panitumumab: a meta-analysis. Eur J Cancer, 2015. 51(5): p. 587-94.

1060. Venderbosch, S., et al., Mismatch repair status and BRAF mutation status in metastatic colorectal cancer patients: a pooled analysis of the CAIRO, CAIRO2, COIN, and FOCUS studies. Clin Cancer Res, 2014. 20(20): p. 5322-30.

1061. Domingo, E., et al., BRAF-V600E is not involved in the colorectal tumorigenesis of HNPCC in patients with functional MLH1 and MSH2 genes. Oncogene, 2005. 24(24): p. 3995-8.

1062. Cremolini, C., et al., BRAF codons 594 and 596 mutations identify a new molecular subtype of metastatic colorectal cancer at favorable prognosis. Ann Oncol, 2015. 26(10): p. 2092-7.

1063. Goldstein, D.A., W.L. Shaib, and C.R. Flowers, Costs and effectiveness of genomic testing in the management of colorectal cancer. Oncology (Williston Park), 2015. 29(3): p. 175-83.

1064. Liu, X., et al., Association of UGT1 Al $* 28$ polymorphisms with irinotecan-induced toxicities in colorectal cancer: a meta-analysis in Caucasians. Pharmacogenomics J, 2014. 14(2): p. 120-9.

1065. Perera, M.A., F. Innocenti, and M.J. Ratain, Pharmacogenetic testing for uridine diphosphate glucuronosyltransferase 1A1 polymorphisms: are we there yet? Pharmacotherapy, 2008. 28(6): p. 755-68.

1066. Deenen, M.J., et al., Upfront Genotyping of DPYD*2A to Individualize Fluoropyrimidine Therapy: A Safety and Cost Analysis. J Clin Oncol, 2016. 34(3): p. 227-34.

1067. Meulendijks, D., et al., Clinical relevance of DPYD variants C.1679T>G, c.1236G>A/HapB3, and c. $1601 \mathrm{G}>\mathrm{A}$ as predictors of severe fluoropyrimidine-associated toxicity: a systematic review and meta-analysis of individual patient data. Lancet Oncol, 2015. 16(16): p. 1639-50.

1068. Le, D.T., et al., PD-1 Blockade in Tumors with Mismatch-Repair Deficiency. N Engl J Med, 2015. 372(26): p. 2509-20.

1069. The Cancer Genome Atlas. Comprehensive molecular characterization of human colon and rectal cancer. Nature, 2012. 487(7407): p. 330-7.

1070. Funkhouser, W.K., Jr., et al., Relevance, pathogenesis, and testing algorithm for mismatch repair-defective colorectal carcinomas: a report of the association for molecular pathology. J Mol Diagn, 2012. 14(2): p. 91-103.

1071. Valtorta, E., et al., Assessment of a HER2 scoring system for colorectal cancer: results from a validation study. Mod Pathol, 2015. 28(11): p. 1481-91. 
1072. Bertotti, A., et al., A molecularly annotated platform of patient-derived xenografts ("xenopatients") identifies HER2 as an effective therapeutic target in cetuximab-resistant colorectal cancer. Cancer Discov, 2011. 1(6): p. 508-23.

1073. Bertotti, A., et al., The genomic landscape of response to EGFR blockade in colorectal cancer. Nature, 2015. 526(7572): p. 263-7.

1074. Sartore-Bianchi, A., et al., Dual-targeted therapy with trastuzumab and lapatinib in treatmentrefractory, KRAS codon 12/13 wild-type, HER2-positive metastatic colorectal cancer (HERACLES): a proof-of-concept, multicentre, open-label, phase 2 trial. Lancet Oncol, 2016. 17(6): p. 738-46.

1075. Schmiegel, W., et al., Blood-based detection of RAS mutations to guide anti-EGFR therapy in colorectal cancer patients: concordance of results from circulating tumor DNA and tissuebased RAS testing. Mol Oncol, 2017. 11(2): p. 208-219.

1076. Diaz, L.A., Jr., et al., The molecular evolution of acquired resistance to targeted EGFR blockade in colorectal cancers. Nature, 2012. 486(7404): p. 537-40.

1077. Misale, S., et al., Emergence of KRAS mutations and acquired resistance to anti-EGFR therapy in colorectal cancer. Nature, 2012. 486(7404): p. 532-6.

1078. Misale, S., et al., Vertical suppression of the EGFR pathway prevents onset of resistance in colorectal cancers. Nat Commun, 2015. 6: p. 8305.

1079. Mekenkamp, L.J., et al., Clinicopathological features and outcome in advanced colorectal cancer patients with synchronous vs metachronous metastases. Br J Cancer, 2010. 103(2): p. 159-64.

1080. van der Pool, A.E., et al., 'Staged' liver resection in synchronous and metachronous colorectal hepatic metastases: differences in clinicopathological features and outcome. Colorectal Dis, 2010. 12(10 Online): p. e229-35.

1081. Slesser, A.A., et al., The tumour biology of synchronous and metachronous colorectal liver metastases: a systematic review. Clin Exp Metastasis, 2013. 30(4): p. 457-70.

1082. Fong Y, F.J., Sun RL, Brennan MF, Blumgart LH, Clinical score for predicting recurrence after hepatic resection for metastatic colorectal cancer: analysis of 1001 consecutive cases. Ann Surg. 1999 Sep;230(3):309-18; discussion 318-21., 1999.

1083. de Gramont, A., et al., Association between disease-free survival and overall survival when survival is prolonged after recurrence in patients receiving cytotoxic adjuvant therapy for colon cancer: simulations based on the 20,800 patient ACCENT data set. J Clin Oncol, 2010. 28(3): p. 460-5.

1084. Van Loon, K. and A.P. Venook, Curable patient with metastatic colorectal cancer: balancing effective therapies and toxicities. J Clin Oncol, 2014. 32(10): p. 991-6.

1085. Nigri, G., Neoadjuvant chemotherapy for resectable colorectal liver metastases: What ist the evidence? Results of a systematic review of comparative studies. The Surgeon, 2014. 13/2015 p. $83-90$.

1086. Nordlinger, B., et al., Perioperative chemotherapy with FOLFOX4 and surgery versus surgery alone for resectable liver metastases from colorectal cancer (EORTC Intergroup trial 40983): a randomised controlled trial. Lancet, 2008. 371(9617): p. 1007-16.

1087. Nordlinger, B., et al., Perioperative FOLFOX4 chemotherapy and surgery versus surgery alone for resectable liver metastases from colorectal cancer (EORTC 40983): long-term results of a randomised, controlled, phase 3 trial. Lancet Oncol, 2013. 14(12): p. 1208-15.

1088. Ono, T., et al., Outcome in disappearing colorectal cancer liver metastases during oxaliplatinbased chemotherapy. Oncol Lett, 2012. 4(5): p. 905-909.

1089. Ferrero, A., et al., Intraoperative detection of disappearing colorectal liver metastases as a predictor of residual disease. J Gastrointest Surg, 2012. 16(4): p. 806-14.

1090. van Vledder, M.G., et al., Disappearing colorectal liver metastases after chemotherapy: should we be concerned? J Gastrointest Surg, 2010. 14(11): p. 1691-700.

1091. Auer, R.C., et al., Predictors of a true complete response among disappearing liver metastases from colorectal cancer after chemotherapy. Cancer, 2010. 116(6): p. 1502-9.

1092. Tanaka, K., et al., Importance of complete pathologic response to prehepatectomy chemotherapy in treating colorectal cancer metastases. Ann Surg, 2009. 250(6): p. 935-42.

1093. Fiorentini, G., et al., Complete response of colorectal liver metastases after intra-arterial chemotherapy. Tumori, 2008. 94(4): p. 489-92.

1094. Elias, D., et al., Outcome of posthepatectomy-missing colorectal liver metastases after complete response to chemotherapy: impact of adjuvant intra-arterial hepatic oxaliplatin. Ann Surg Oncol, 2007. 14(11): p. 3188-94.

1095. Benoist, S., et al., Complete response of colorectal liver metastases after chemotherapy: does it mean cure? J Clin Oncol, 2006. 24(24): p. 3939-45.

1096. Elias, D., et al., Evolution of missing colorectal liver metastases following inductive chemotherapy and hepatectomy. J Surg Oncol, 2004. 86(1): p. 4-9. 
1097. Kim, H.J., et al., Incremental value of liver MR imaging in patients with potentially curable colorectal hepatic metastasis detected at CT: a prospective comparison of diffusion-weighted imaging, gadoxetic acid-enhanced MR imaging, and a combination of both MR techniques. Radiology, 2015. 274(3): p. 712-22.

1098. Westwood, M., et al., Contrast-enhanced ultrasound using SonoVue(R) (sulphur hexafluoride microbubbles) compared with contrast-enhanced computed tomography and contrastenhanced magnetic resonance imaging for the characterisation of focal liver lesions and detection of liver metastases: a systematic review and cost-effectiveness analysis. Health Technol Assess, 2013. 17(16): p. 1-243.

1099. Ciliberto, D., Role of systemic chemothreapy in the management of rsected or resectable colorectal liver metastases: A systematic review and meta-analysis of randomized controlled trials. Oncology Reports, 2012. 27: p. 1849 - 1856.

1100. Zhu, G.-Q., Systematic Review with network Meta-Analysis. Medicine (Baltimore), 2015. 94.

1101. Mitry, E., et al., Adjuvant chemotherapy after potentially curative resection of metastases from colorectal cancer: a pooled analysis of two randomized trials. J Clin Oncol, 2008. 26(30): p. 4906-11.

1102. Kobayashi, H., et al., Impact of adjuvant chemotherapy in patients with curatively resected stage IV colorectal cancer. Medicine (Baltimore), 2015. 94(17): p. e696.

1103. Nakai, T., The clinical risk score predicts the effectiveness of adjuvant chemotherapy for colorectal liver metastasis. World J Surg, 2015. 39: p. 1527 - 1536.

1104. Rahbari, N., Adjuvant thrapy after resection of colorectal liver metastases: the predictive value of the MSKCC clinical risk score in the era of modern chemotherapy. BMC Cancer, 2014. 14(174).

1105. Hirokawa, F., Reconsideration of the indications for adjuvant chemotherapy for liver metastases from colorectal cancer after initial hepatectomy. Ann Surg Oncol, 2014. 21 : p. 139 $-146$.

1106. Brandi, G., et al., Adjuvant systemic chemotherapy after putative curative resection of colorectal liver and lung metastases. Clin Colorectal Cancer, 2013. 12(3): p. 188-94.

1107. Faron, M., Impact of preoperative and postoperative FOLFOX chemotherapies in patients with resectable colorectal liver metastasis. J Gastrointest Cancer, 2014. September.

1108. Heinemann, V., et al., A study-level meta-analysis of efficacy data from head-to-head first-line trials of epidermal growth factor receptor inhibitors versus bevacizumab in patients with RAS wild-type metastatic colorectal cancer. Eur J Cancer, 2016. 67: p. 11-20.

1109. Schwartzberg, L.S., et al., PEAK: a randomized, multicenter phase II study of panitumumab plus modified fluorouracil, leucovorin, and oxaliplatin (mFOLFOX6) or bevacizumab plus mFOLFOX6 in patients with previously untreated, unresectable, wild-type KRAS exon 2 metastatic colorectal cancer. J Clin Oncol, 2014. 32(21): p. 2240-7.

1110. Lenz, H.J., D. Niedzwiecki, and F. Innocenti, CALGB/SWOG 80405: phase III trial of irinotecan/5-FU/leucovorin (FOLFIRI) or oxaliplatin/5-FU/leucovorin (mFOLFOX6) with bevacizumab (BV) or cetuximab (CET) for patients (pts) with expanded RAS analyses untreated metastatic adenocarcinoma of the colon or rectum (mCRC). . Ann Oncol, 2014. 25(Suppl 5): p. 298 - 306.

1111. Cremolini, C., et al., FOLFOXIRI plus bevacizumab versus FOLFIRI plus bevacizumab as first-line treatment of patients with metastatic colorectal cancer: updated overall survival and molecular subgroup analyses of the open-label, phase 3 TRIBE study. Lancet Oncol, 2015. 16(13): p. 1306-15.

1112. Loupakis, F., et al., Initial therapy with FOLFOXIRI and bevacizumab for metastatic colorectal cancer. N Engl J Med, 2014. 371(17): p. 1609-18.

1113. Yamauchi, M., et al., Assessment of colorectal cancer molecular features along bowel subsites challenges the conception of distinct dichotomy of proximal versus distal colorectum. Gut, 2012. 61(6): p. 847-54.

1114. von Einem, J.C., et al., Left-sided primary tumors are associated with favorable prognosis in patients with KRAS codon 12/13 wild-type metastatic colorectal cancer treated with cetuximab plus chemotherapy: an analysis of the AIO KRK-0104 trial. J Cancer Res Clin Oncol, 2014. 140(9): p. 1607-14

1115. Missiaglia, E., et al., Distal and proximal colon cancers differ in terms of molecular, pathological, and clinical features. Ann Oncol, 2014. 25(10): p. 1995-2001.

1116. Guinney, J., et al., The consensus molecular subtypes of colorectal cancer. Nat Med, 2015. 21(11): p. 1350-6.

1117. Stintzing, S., et al., FOLFIRI plus cetuximab versus FOLFIRI plus bevacizumab as first-line treatment for patients with metastatic colorectal cancer-subgroup analysis of patients with KRAS: mutated tumours in the randomised German AlO study KRK-0306. Ann Oncol, 2012. 23(7): p. 1693-9. 
1118. Kubicka, S., et al., Bevacizumab plus chemotherapy continued beyond first progression in patients with metastatic colorectal cancer previously treated with bevacizumab plus chemotherapy: ML18147 study KRAS subgroup findings. Ann Oncol, 2013. 24(9): p. 2342-9.

1119 . Loupakis, F., et al., FOLFOXIRI plus bevacizumab as first-line treatment in BRAF mutant metastatic colorectal cancer. Eur J Cancer, 2014. 50(1): p. 57-63.

1120. Samowitz, W.S., et al., Association of smoking, CPG island methylator phenotype, and V600E BRAF mutations in colon cancer. J Natl Cancer Inst, 2006. 98(23): p. 1731-8.

1121. Rowland, A., et al., Meta-analysis of BRAF mutation as a predictive biomarker of benefit from anti-EGFR monoclonal antibody therapy for RAS wild-type metastatic colorectal cancer. Br J Cancer, 2015. 112(12): p. 1888-94.

1122. Stintzing, S., L. Jung, and D.P. Rossius, Mutations within the EGFR signaling pathway: Influence on efficacy in FIRE-3: A randomized phase III study of FOLFIRI plus cetuximab or bevacizumab as first-line treatment for wild-type (WT) KRAS (exon 2) metastatic colorectal cancer (mCRC) patients. J Clin Oncol 2014. 32: p. suppl 3; abstract 445.

1123. Kopetz, S., et al., Phase II Pilot Study of Vemurafenib in Patients With Metastatic BRAF-Mutated Colorectal Cancer. J Clin Oncol, 2015. 33(34): p. 4032-8.

1124. Young, J., et al., Features of colorectal cancers with high-level microsatellite instability occurring in familial and sporadic settings: parallel pathways of tumorigenesis. Am J Pathol, 2001. 159(6): p. 2107-16.

1125. Kabbinavar, F.F., et al., Addition of bevacizumab to bolus fluorouracil and leucovorin in firstline metastatic colorectal cancer: results of a randomized phase II trial. J Clin Oncol, 2005. 23(16): p. 3697-705.

1126. Cunningham, D., et al., Bevacizumab plus capecitabine versus capecitabine alone in elderly patients with previously untreated metastatic colorectal cancer (AVEX): an open-label, randomised phase 3 trial. Lancet Oncol, 2013. 14(11): p. 1077-85.

1127. Souglakos, J., et al., FOLFOXIRI (folinic acid, 5-fluorouracil, oxaliplatin and irinotecan) vs FOLFIRI (folinic acid, 5-fluorouracil and irinotecan) as first-line treatment in metastatic colorectal cancer (MCC): a multicentre randomised phase III trial from the Hellenic Oncology Research Group (HORG). Br J Cancer, 2006. 94(6): p. 798-805.

1128. Falcone, A., et al., Phase III trial of infusional fluorouracil, leucovorin, oxaliplatin, and irinotecan (FOLFOXIRI) compared with infusional fluorouracil, leucovorin, and irinotecan (FOLFIRI) as first-line treatment for metastatic colorectal cancer: the Gruppo Oncologico Nord Ovest. J Clin Oncol, 2007. 25(13): p. 1670-6.

1129. Montagnani, F., A systematic review of FOLFOXIRI chemotherapy for the firstline treatment of metastatic colorectal cancer: improved efficacy at the cost of increased toxicity. Colorectal Disease, 2011.13 : p. 846 - 854.

1130. Gruenberger, T., et al., Bevacizumab plus mFOLFOX-6 or FOLFOXIRI in patients with initially unresectable liver metastases from colorectal cancer: the OLIVIA multinational randomised phase II trial. Ann Oncol, 2015. 26(4): p. 702-8.

1131. Maughan, T.S., et al., Addition of cetuximab to oxaliplatin-based first-line combination chemotherapy for treatment of advanced colorectal cancer: results of the randomised phase 3 MRC COIN trial. Lancet, 2011. 377(9783): p. 2103-14.

1132. Heinemann, V., et al., FOLFIRI plus cetuximab versus FOLFIRI plus bevacizumab as first-line treatment for patients with metastatic colorectal cancer (FIRE-3): a randomised, open-label, phase 3 trial. Lancet Oncol, 2014. 15(10): p. 1065-75.

1133. Venook AP, N.D., Lenz HJ, et al. , CALGB/SWOG 80405: Phase III trial of irinotecan/5FU/leucovorin (FOLFIRI) or oxaliplatin/5-FU/leucovorin (mFOLFOX6) with bevacizumab (BV) or cetuximab (CET) for patients (pts) with KRAS wild-type (wt) untreated metastatic adenocarcinoma of the colon or rectum (MCRC). . J Clin Oncol 2014. 32:5s.

1134. Douillard, J.Y., et al., Randomized, phase III trial of panitumumab with infusional fluorouracil, leucovorin, and oxaliplatin (FOLFOX4) versus FOLFOX4 alone as first-line treatment in patients with previously untreated metastatic colorectal cancer: the PRIME study. J Clin Oncol, 2010. 28(31): p. 4697-705.

1135. Van Cutsem, E., et al., Cetuximab plus irinotecan, fluorouracil, and leucovorin as first-line treatment for metastatic colorectal cancer: updated analysis of overall survival according to tumor KRAS and BRAF mutation status. J Clin Oncol, 2011. 29(15): p. 2011-9.

1136. Ye, L.C., et al., Randomized controlled trial of cetuximab plus chemotherapy for patients with KRAS wild-type unresectable colorectal liver-limited metastases. J Clin Oncol, 2013. 31(16): p. 1931-8.

1137. Bokemeyer, C., et al., Fluorouracil, leucovorin, and oxaliplatin with and without cetuximab in the first-line treatment of metastatic colorectal cancer. J Clin Oncol, 2009. 27(5): p. 663-71.

1138. Hurwitz, H., et al., Bevacizumab plus irinotecan, fluorouracil, and leucovorin for metastatic colorectal cancer. N Engl J Med, 2004. 350(23): p. 2335-42. 
1139. Stathopoulos, G.P., et al., Treatment of colorectal cancer with and without bevacizumab: $a$ phase III study. Oncology, 2010. 78(5-6): p. 376-81.

1140. Guan, Z.Z., et al., Efficacy and safety of bevacizumab plus chemotherapy in Chinese patients with metastatic colorectal cancer: a randomized phase III ARTIST trial. Chin J Cancer, 2011. 30(10): p. 682-9.

1141. Saltz, L.B., et al., Bevacizumab in combination with oxaliplatin-based chemotherapy as first-line therapy in metastatic colorectal cancer: a randomized phase III study. J Clin Oncol, 2008. 26(12): p. 2013-9.

1142. Passardi, A., et al., Effectiveness of bevacizumab added to standard chemotherapy in metastatic colorectal cancer: final results for first-line treatment from the ITACa randomized clinical trial. Ann Oncol, 2015. 26(6): p. 1201-7.

1143. Kabbinavar, F., et al., Phase II, randomized trial comparing bevacizumab plus fluorouracil (FU)/leucovorin (LV) with FU/LV alone in patients with metastatic colorectal cancer. J Clin Oncol, 2003. 21(1): p. 60-5.

1144. Tebbutt, N.C., et al., Capecitabine, bevacizumab, and mitomycin in first-line treatment of metastatic colorectal cancer: results of the Australasian Gastrointestinal Trials Group Randomized Phase III MAX Study. J Clin Oncol, 2010. 28(19): p. 3191-8.

1145. Tol, J., et al., Chemotherapy, bevacizumab, and cetuximab in metastatic colorectal cancer. N Engl J Med, 2009. 360(6): p. 563-72.

1146. Hecht, J.R., et al., A randomized phase IIIB trial of chemotherapy, bevacizumab, and panitumumab compared with chemotherapy and bevacizumab alone for metastatic colorectal cancer. J Clin Oncol, 2009. 27(5): p. 672-80.

1147. Saltz, L., et al., Phase III trial of cetuximab, bevacizumab, and 5-fluorouracil/leucovorin vs. FOLFOX-bevacizumab in colorectal cancer. Clin Colorectal Cancer, 2012. 11(2): p. 101-11.

1148. Diaz-Rubio, E., et al., Role of Kras status in patients with metastatic colorectal cancer receiving first-line chemotherapy plus bevacizumab: a TTD group cooperative study. PLoS One, 2012. 7(10): p. e47345.

1149. Simkens, L.H., et al., Maintenance treatment in metastatic colorectal cancer. Lancet Oncol, 2015. 16(16): p. e582-3.

1150. Hegewisch-Becker, S., et al., Maintenance strategies after first-line oxaliplatin plus fluoropyrimidine plus bevacizumab for patients with metastatic colorectal cancer (AIO 0207): a randomised, non-inferiority, open-label, phase 3 trial. Lancet Oncol, 2015. 16(13): p. 1355-69.

1151. Zhao, L., et al., Meta-analysis comparing maintenance strategies with continuous therapy and complete chemotherapy-free interval strategies in the treatment of metastatic colorectal cancer. Oncotarget, 2016. 7(22): p. 33418-28.

1152. Pereira, A.A., et al., The impact of complete chemotherapy stop on the overall survival of patients with advanced colorectal cancer in first-line setting: A meta-analysis of randomized trials. Acta Oncol, 2015. 54(10): p. 1737-46.

1153. Berry, S.R., et al., Continuous versus intermittent chemotherapy strategies in metastatic colorectal cancer: a systematic review and meta-analysis. Ann Oncol, 2015. 26(3): p. 477-85.

1154. Esin, E. and S. Yalcin, Maintenance strategy in metastatic colorectal cancer: A systematic review. Cancer Treat Rev, 2016. 42: p. 82-90.

1155. Simkens, L.H., et al., Maintenance treatment with capecitabine and bevacizumab in metastatic colorectal cancer (CAIRO3): a phase 3 randomised controlled trial of the Dutch Colorectal Cancer Group. Lancet, 2015. 385(9980): p. 1843-52.

1156. Quidde, J., et al., Quality of life assessment in patients with metastatic colorectal cancer receiving maintenance therapy after first-line induction treatment: a preplanned analysis of the phase III AIO KRK 0207 trial. Ann Oncol, 2016. 27(12): p. 2203-2210.

1157. Rothenberg, M.L., et al., Superiority of oxaliplatin and fluorouracil-leucovorin compared with either therapy alone in patients with progressive colorectal cancer after irinotecan and fluorouracil-leucovorin: interim results of a phase III trial. J Clin Oncol, 2003. 21(11): p. 205969.

1158. Rougier, P., et al., Randomised trial of irinotecan versus fluorouracil by continuous infusion after fluorouracil failure in patients with metastatic colorectal cancer. Lancet, 1998. 352(9138): p. 1407-12.

1159. Cunningham, D., et al., Randomised trial of irinotecan plus supportive care versus supportive care alone after fluorouracil failure for patients with metastatic colorectal cancer. Lancet, 1998. 352(9138): p. 1413-8.

1160. Tournigand, C., et al., FOLFIRI followed by FOLFOX6 or the reverse sequence in advanced colorectal cancer: a randomized GERCOR study. J Clin Oncol, 2004. 22(2): p. 229-37.

1161. Van Cutsem, E., et al., Addition of aflibercept to fluorouracil, leucovorin, and irinotecan improves survival in a phase III randomized trial in patients with metastatic colorectal cancer previously treated with an oxaliplatin-based regimen. J Clin Oncol, 2012. 30(28): p. 3499-506. 
1162. Tabernero, J., et al., Ramucirumab versus placebo in combination with second-line FOLFIRI in patients with metastatic colorectal carcinoma that progressed during or after first-line therapy with bevacizumab, oxaliplatin, and a fluoropyrimidine (RAISE): a randomised, double-blind, multicentre, phase 3 study. Lancet Oncol, 2015. 16(5): p. 499-508.

1163. Giantonio, B.J., et al., Bevacizumab in combination with oxaliplatin, fluorouracil, and leucovorin (FOLFOX4) for previously treated metastatic colorectal cancer: results from the Eastern Cooperative Oncology Group Study E3200. J Clin Oncol, 2007. 25(12): p. 1539-44.

1164. Bennouna, J., et al., Continuation of bevacizumab after first progression in metastatic colorectal cancer (ML 18147): a randomised phase 3 trial. Lancet Oncol, 2013. 14(1): p. 29-37.

1165. Masi, G., et al., Continuation or reintroduction of bevacizumab beyond progression to first-line therapy in metastatic colorectal cancer: final results of the randomized BEBYP trial. Ann Oncol, 2015. 26(4): p. 724-30.

1166. Cao, R., et al., A multi-center randomized phase II clinical study of bevacizumab plus irinotecan, 5-fluorouracil, and leucovorin (FOLFIRI) compared with FOLFIRI alone as second-line treatment for Chinese patients with metastatic colorectal cancer. Med Oncol, 2015. 32(1): p. 325.

1167. Sobrero AF, F.L., Rivera F, et al. , Phase III trial of Cetuximab plus Irinotecan after Fluoropyrimidine and Oxaliplatin Failure in Patients with Metastatic Colorectal Cancer J Clin Oncol 26: 2311-2319, 2008.

1168. Peeters, M., et al., Randomized phase III study of panitumumab with fluorouracil, leucovorin, and irinotecan (FOLFIRI) compared with FOLFIRI alone as second-line treatment in patients with metastatic colorectal cancer. J Clin Oncol, 2010. 28(31): p. 4706-13.

1169. Ciardiello, F., et al., Cetuximab continuation after first progression in metastatic colorectal cancer (CAPRI-GOIM): a randomized phase II trial of FOLFOX plus cetuximab versus FOLFOX. Ann Oncol, 2016. 27(6): p. 1055-61.

1170. Derangere, V., et al., Does bevacizumab impact anti-EGFR therapy efficacy in metastatic colorectal cancer? Oncotarget, 2016. 7(8): p. 9309-21.

1171. Wainberg, Z.A. and A. Drakaki, The importance of optimal drug sequencing in metastatic colorectal cancer: biological rationales for the observed survival benefit conferred by first-line treatment with EGFR inhibitors. Expert Opin Biol Ther, 2015. 15(8): p. 1205-20.

1172. Zaniboni, A. and V. Formica, The Best. First. Anti-EGFR before anti-VEGF, in the first-line treatment of RAS wild-type metastatic colorectal cancer: from bench to bedside. Cancer Chemother Pharmacol, 2016. 78(2): p. 233-44.

1173. Hecht JR, C.A., Dakhil SR, et al. , SPIRITT (study 20060141): A randomized phase II study of FOLFIRI with either panitumumab (pmab) or bevacizumab (bev) as second-line treatment (tx) in patients (pts) with wild-type (WT) KRAS metastatic colorectal cancer ( $m C R C)$. . Journal of Clinical Oncology, 2013. 31: p. 4_suppl, 454 - 454.

1174. Hiret S, B.C., Bertaut A, et al. , Bevacizumab or cetuximab plus chemotherapy after progression with bevacizumab plus chemotherapy in patients with wtKRAS metastatic colorectal cancer: $A$ randomized phase II study (Prodige 18 -UNICANCER GI). . J Clin Oncol 2016. 34: p. suppl; abstr 3514.

1175. Peeters, M., et al., Analysis of KRAS/NRAS Mutations in a Phase III Study of Panitumumab with FOLFIRI Compared with FOLFIRI Alone as Second-line Treatment for Metastatic Colorectal Cancer. Clin Cancer Res, 2015. 21 (24): p. 5469-79.

1176. Van Cutsem, E., et al., Open-label phase III trial of panitumumab plus best supportive care compared with best supportive care alone in patients with chemotherapy-refractory metastatic colorectal cancer. J Clin Oncol, 2007. 25(13): p. 1658-64.

1177. Jonker D.J. , M.D., Chris J. O'Callaghan, Ph.D., Christos S. Karapetis, M.D., John R. Zalcberg, M.D., Dongsheng Tu, Ph.D., Heather-Jane Au, M.D., Scott R. Berry, M.D., Marianne Krahn, M.D., Timothy Price, M.D., R. John Simes, M.D., Niall C. Tebbutt, M.D., Guy van Hazel, M.D., Rafal Wierzbicki, M.D., Christiane Langer, M.D., and Malcolm J. Moore, M.D., Cetuximab for the Treatment of Colorectal Cancer. N Engl J Med 357:2040, November 15, 2007 Original Article, 2007.

1178. Price, T.J., et al., Panitumumab versus cetuximab in patients with chemotherapy-refractory wild-type KRAS exon 2 metastatic colorectal cancer (ASPECCT): a randomised, multicentre, open-label, non-inferiority phase 3 study. Lancet Oncol, 2014. 15(6): p. 569-79.

1179. Mayer, R.J., et al., Randomized trial of TAS-102 for refractory metastatic colorectal cancer. $\mathrm{N}$ Engl J Med, 2015. 372(20): p. 1909-19.

1180. Yoshino, T., et al., TAS-102 monotherapy for pretreated metastatic colorectal cancer: a doubleblind, randomised, placebo-controlled phase 2 trial. Lancet Oncol, 2012. 13(10): p. 993-1001.

1181. Li, J., et al., Regorafenib plus best supportive care versus placebo plus best supportive care in Asian patients with previously treated metastatic colorectal cancer (CONCUR): a randomised, double-blind, placebo-controlled, phase 3 trial. Lancet Oncol, 2015. 16(6): p. 619-29. 
1182. Grothey, A., et al., Regorafenib monotherapy for previously treated metastatic colorectal cancer (CORRECT): an international, multicentre, randomised, placebo-controlled, phase 3 trial. Lancet, 2013. 381(9863): p. 303-12.

1183. Santini, D., et al., Cetuximab rechallenge in metastatic colorectal cancer patients: how to come away from acquired resistance? Ann Oncol, 2012. 23(9): p. 2313-8.

1184. Agcaoglu, O., et al., Complementary use of resection and radiofrequency ablation for the treatment of colorectal liver metastases: an analysis of 395 patients. World J Surg, 2013. 37(6): p. 1333-9.

1185. Sasaki, K., et al., Combined resection and RFA in colorectal liver metastases: stratification of long-term outcomes. J Surg Res, 2016. 206(1): p. 182-189.

1186. van Amerongen, M.J., et al., Short term and long term results of patients with colorectal liver metastases undergoing surgery with or without radiofrequency ablation. Eur J Surg Oncol, 2016. 42(4): p. 523-30.

1187. Ruers, T., et al., Local Treatment of Unresectable Colorectal Liver Metastases: Results of a Randomized Phase II Trial. J Natl Cancer Inst, 2017. 109(9).

1188. Gillams, A.R. and W.R. Lees, Five-year survival in 309 patients with colorectal liver metastases treated with radiofrequency ablation. Eur Radiol, 2009. 19(5): p. 1206-13.

1189. Wybranski, C.G., A. Ricke, J., Hepatisch metastasiertes kolorektales Karzinom. Der Onkologe, 2015. 21(1): p. $69-82$.

1190. Van Tilborg, A.A., et al., Long-term results of radiofrequency ablation for unresectable colorectal liver metastases: a potentially curative intervention. Br J Radiol, 2011 . 84(1002): p. 556-65.

1191. Otto, G., et al., Radiofrequency ablation as first-line treatment in patients with early colorectal liver metastases amenable to surgery. Ann Surg, 2010. 251(5): p. 796-803.

1192. Saxena, A., et al., Impact of treatment modality and number of lesions on recurrence and survival outcomes after treatment of colorectal cancer liver metastases. J Gastrointest Oncol, 2014. 5(1): p. 46-56.

1193. Gillams, A., et al., Thermal ablation of colorectal liver metastases: a position paper by an international panel of ablation experts, The Interventional Oncology Sans Frontieres meeting 2013. Eur Radiol, 2015. 25(12): p. 3438-54.

1194. Tanis, E., et al., Local recurrence rates after radiofrequency ablation or resection of colorectal liver metastases. Analysis of the European Organisation for Research and Treatment of Cancer \#40004 and \#40983. Eur J Cancer, 2014. 50(5): p. 912-9.

1195. Wang, D.S., Davalos, E.A., Hwang, G.L., Louie , J.D., Kuo, W.T., Shah, R.P. , Hovsepian, D., Hofmann, L.V., Sze, D.Y., Kothary, N., Superselective transhepatic arterial chemoembolization for hepatocellular carcinoma in high risk patients as a bridge towards liver transplantation Abstract No. 30. Journal of Vascular and Interventional Radiology, 2012. 23(3): p. S 16.

1196. Solbiati, L., et al., Small liver colorectal metastases treated with percutaneous radiofrequency ablation: local response rate and long-term survival with up to 10-year follow-up. Radiology, 2012. 265(3): p. 958-68.

1197. Correa-Gallego, C., et al., A retrospective comparison of microwave ablation vs. radiofrequency ablation for colorectal cancer hepatic metastases. Ann Surg Oncol, 2014. 21 (13): p. 4278-83.

1198. Ruers, T., et al., Radiofrequency ablation (RFA) combined with chemotherapy for unresectable colorectal liver metastases (CRC LM): Long-term survival results of a randomized phase II study of the EORTC-NCRI CCSG-ALM Intergroup 40004 (CLOCC). J Clin Oncol, 2015. 33(suppl; abstr 3501).

1199. Petersen, C.G., T., Möglichkeiten der lokal-ablativen Bestrahlung (SBRT) bei metastasiertem kolorektalem Karzinom. Der Onkologe, 2016. 22: p. 115 - 120.

1200. Herfarth, K.K., J. Debus, and M. Wannenmacher, Stereotactic radiation therapy of liver metastases: update of the initial phase-I/II trial. Front Radiat Ther Oncol, 2004. 38: p. 100-5.

1201. van der Pool, A.E., et al., Stereotactic body radiation therapy for colorectal liver metastases. Br J Surg, $2010.97(3)$ : p. 377-82.

1202. Ricke, J., et al., Local response and impact on survival after local ablation of liver metastases from colorectal carcinoma by computed tomography-guided high-dose-rate brachytherapy. Int J Radiat Oncol Biol Phys, 2010. 78(2): p. 479-85.

1203. de Baere, T., et al., Radiofrequency ablation is a valid treatment option for lung metastases: experience in 566 patients with 1037 metastases. Ann Oncol, 2015. 26(5): p. 987-91.

1204. Vogl, T.J., et al., Thermal Ablation of Colorectal Lung Metastases: Retrospective Comparison Among Laser-Induced Thermotherapy, Radiofrequency Ablation, and Microwave Ablation. AJR Am J Roentgenol, 2016. 207(6): p. 1340-1349.

1205. Hendlisz, A., et al., Phase III trial comparing protracted intravenous fluorouracil infusion alone or with yttrium-90 resin microspheres radioembolization for liver-limited metastatic colorectal cancer refractory to standard chemotherapy. J Clin Oncol, 2010. 28(23): p. 3687-94. 
1206. Van Hazel, G., et al., Randomised phase 2 trial of SIR-Spheres plus fluorouracil/leucovorin chemotherapy versus fluorouracil/leucovorin chemotherapy alone in advanced colorectal cancer. J Surg Oncol, 2004. 88(2): p. 78-85.

1207. Sharma, R.A., et al., Radioembolization of liver metastases from colorectal cancer using yttrium-90 microspheres with concomitant systemic oxaliplatin, fluorouracil, and leucovorin chemotherapy. J Clin Oncol, 2007. 25(9): p. 1099-106.

1208. van Hazel, G.A., et al., SIRFLOX: Randomized Phase III Trial Comparing First-Line mFOLFOX6 (Plus or Minus Bevacizumab) Versus mFOLFOX6 (Plus or Minus Bevacizumab) Plus Selective Internal Radiation Therapy in Patients With Metastatic Colorectal Cancer. J Clin Oncol, 2016. 34(15): p. 1723-31.

1209. Sharma RA, W.H., van Hazel GA et al., Overall survival analysis of the FOXFIRE prospective randomized studies of first-line selective internal radiotherapy (SIRT) in patients with liver metastases from colorectal cancer. . J Clin Oncol, 2017. 35(suppl; abstr3507).

1210. Garlipp B, G.P., Van Hazel GA, et al. , REsect: Blinded assessment of amenability to potentially curative treatment of previously unresectable colorectal cancer liver metastases (CRC LM) after chemotherapy 6 RadioEmbolization (SIRT) in the randomized SIRFLOX trial. J Clin Oncol, 2017. 35((suppl. Abstract 3532).).

1211. Kemeny, N.E., The re-birth of hepatic arterial infusion for colorectal liver metastases. J Gastrointest Oncol, 2013. 4(2): p. 118-20.

1212. D'Angelica, M.I., et al., Phase II trial of hepatic artery infusional and systemic chemotherapy for patients with unresectable hepatic metastases from colorectal cancer: conversion to resection and long-term outcomes. Ann Surg, 2015. 261(2): p. 353-60.

1213. Levi, F.A., et al., Conversion to resection of liver metastases from colorectal cancer with hepatic artery infusion of combined chemotherapy and systemic cetuximab in multicenter trial OPTILIV. Ann Oncol, 2016. 27(2): p. 267-74.

1214. Fiorentini, G., et al., Intra-arterial infusion of irinotecan-loaded drug-eluting beads (DEBIRI) versus intravenous therapy (FOLFIRI) for hepatic metastases from colorectal cancer: final results of a phase III study. Anticancer Res, 2012. 32(4): p. 1387-95.

1215. Martin, R.C., 2nd, et al., Randomized controlled trial of irinotecan drug-eluting beads with simultaneous FOLFOX and bevacizumab for patients with unresectable colorectal liver-limited metastasis. Cancer, 2015. 121(20): p. 3649-58.

1216. Turaga, K., et al., Consensus guidelines from The American Society of Peritoneal Surface Malignancies on standardizing the delivery of hyperthermic intraperitoneal chemotherapy (HIPEC) in colorectal cancer patients in the United States. Ann Surg Oncol, 2014. 21(5): p. $1501-5$.

1217. Elias, D., et al., Modified selection criteria for complete cytoreductive surgery plus HIPEC based on peritoneal cancer index and small bowel involvement for peritoneal carcinomatosis of colorectal origin. Eur J Surg Oncol, 2014. 40(11): p. 1467-73.

1218. van Oudheusden, T.R., et al., Incidence and treatment of recurrent disease after cytoreductive surgery and intraperitoneal chemotherapy for peritoneally metastasized colorectal cancer: A systematic review. Eur J Surg Oncol, 2015. 41(10): p. 1269-77.

1219. Petrelli, F., et al., FOLFIRI-bevacizumab as first-line chemotherapy in 3500 patients with advanced colorectal cancer: a pooled analysis of 29 published trials. Clin Colorectal Cancer, 2013. 12(3): p. 145-51.

1220. Wulaningsih, W., et al., Irinotecan chemotherapy combined with fluoropyrimidines versus irinotecan alone for overall survival and progression-free survival in patients with advanced and/or metastatic colorectal cancer. Cochrane Database Syst Rev, 2016. 2: p. CD008593.

1221. Rosmarin, D., et al., Genetic markers of toxicity from capecitabine and other fluorouracil-based regimens: investigation in the QUASAR2 study, systematic review, and meta-analysis. J Clin Oncol, 2014. 32(10): p. 1031-9.

1222. Basch, E., et al., Symptom Monitoring With Patient-Reported Outcomes During Routine Cancer Treatment: A Randomized Controlled Trial. J Clin Oncol, 2016. 34(6): p. 557-65.

1223. Molassiotis, A., et al., Effectiveness of a home care nursing program in the symptom management of patients with colorectal and breast cancer receiving oral chemotherapy: $a$ randomized, controlled trial. J Clin Oncol, 2009. 27(36): p. 6191-8.

1224. Neugut, A.I., et al., Duration of adjuvant chemotherapy for colon cancer and survival among the elderly. J Clin Oncol, 2006. 24(15): p. 2368-75.

1225. de Raaf, P.J., et al., Systematic monitoring and treatment of physical symptoms to alleviate fatigue in patients with advanced cancer: a randomized controlled trial. J Clin Oncol, 2013. 31(6): p. 716-23.

1226. Enblad, P., et al., The risk of subsequent primary malignant diseases after cancers of the colon and rectum. A nationwide cohort study. Cancer, 1990. 65(9): p. 2091-100. 
1227. Cali, R.L., et al., Cumulative incidence of metachronous colorectal cancer. Dis Colon Rectum, 1993. 36(4): p. 388-93.

1228. Hermanek, P., Jr., et al., [Long-term results of surgical therapy of colon cancer. Results of the Colorectal Cancer Study Group]. Chirurg, 1994. 65(4): p. 287-97.

1229. Yamazaki, T., et al., What is the risk factor for metachronous colorectal carcinoma? Dis Colon Rectum, 1997. 40(8): p. 935-8.

1230. Hemminki, K., X. Li, and C. Dong, Second primary cancers after sporadic and familial colorectal cancer. Cancer Epidemiol Biomarkers Prev, 2001. 10(7): p. 793-8.

1231. Green, R.J., et al., Surveillance for second primary colorectal cancer after adjuvant chemotherapy: an analysis of Intergroup 0089. Ann Intern Med, 2002. 136(4): p. 261-9.

1232. Harris, G.J., et al., Factors affecting local recurrence of colonic adenocarcinoma. Dis Colon Rectum, 2002. 45(8): p. 1029-34.

1233. Merkel, S., et al., Late locoregional recurrence in rectal carcinoma. Eur J Surg Oncol, 2002. 28(7): p. 716-22.

1234. Poulin, E.C., et al., Local recurrence and survival after laparoscopic mesorectal resection forrectal adenocarcinoma. Surg Endosc, 2002. 16(6): p. 989-95.

1235. Bulow, S., et al., Recurrence and survival after mesorectal excision for rectal cancer. Br J Surg, 2003. 90(8): p. 974-80.

1236. Secco, G.B., et al., Factors influencing incidence and extension of metachronous liver metastases of colorectal adenocarcinoma. A multivariate analysis. Hepatogastroenterology, 1997. 44(16): p. 1057-62.

1237. Pietra, N., et al., Risk factors of local recurrence of colorectal cancer: a multivariate study. Hepatogastroenterology, 1998. 45(23): p. 1573-8.

1238. Scholmerich, J., [Sense and nonsense in after-care of colorectal carcinoma]. Med Klin (Munich), 1996. 91(6): p. 420-7.

1239. Kievit, J., Colorectal cancer follow-up: a reassessment of empirical evidence on effectiveness. Eur J Surg Oncol, 2000. 26(4): p. 322-8.

1240. Kievit, J., Follow-up of patients with colorectal cancer: numbers needed to test and treat. Eur J Cancer, 2002. 38(7): p. 986-99.

1241. Wichmann, M.W., et al., Results of long-term follow-up after curative resection of Dukes $A$ colorectal cancer. World J Surg, 2002. 26(6): p. 732-6.

1242. Yamamoto, S., et al., Postsurgical surveillance for recurrence of UICC stage I colorectal carcinoma: is follow-up by CEA justified? Hepatogastroenterology, 2005. 52(62): p. 444-9.

1243. Korner, H., et al., Systematic follow-up after curative surgery for colorectal cancer in Norway: a population-based audit of effectiveness, costs, and compliance. J Gastrointest Surg, 2005. 9(3): p. 320-8.

1244. Talbot, I.C., et al., The clinical significance of invasion of veins by rectal cancer. Br J Surg, 1980. 67(6): p. 439-42.

1245. Minsky, B.D., et al., Resectable adenocarcinoma of the rectosigmoid and rectum. II. The influence of blood vessel invasion. Cancer, 1988. 61(7): p. 1417-24.

1246. Krasna, M.J., et al., Vascular and neural invasion in colorectal carcinoma. Incidence and prognostic significance. Cancer, 1988. 61(5): p. 1018-23.

1247. Inoue, T., et al., Vascular invasion of colorectal carcinoma readily visible with certain stains. Dis Colon Rectum, 1992. 35(1): p. 34-9.

1248. Bruinvels, D.J., et al., Follow-up of patients with colorectal cancer. A meta-analysis. Ann Surg, 1994. 219(2): p. 174-82.

1249. Rosen, M., et al., Follow-up of colorectal cancer: a meta-analysis. Dis Colon Rectum, 1998. 41(9): p. $1116-26$.

1250. Jeffery, G.M., B.E. Hickey, and P. Hider, Follow-up strategies for patients treated for nonmetastatic colorectal cancer. Cochrane Database Syst Rev, 2002(1): p. CD002200.

1251. Renehan, A.G., et al., Impact on survival of intensive follow up after curative resection for colorectal cancer: systematic review and meta-analysis of randomised trials. Bmj, 2002. 324(7341): p. 813.

1252. Makela, J.T., S.O. Laitinen, and M.I. Kairaluoma, Five-year follow-up after radical surgery for colorectal cancer. Results of a prospective randomized trial. Arch Surg, 1995. 130(10): p. 1062-7.

1253. Ohlsson, B., et al., Follow-up after curative surgery for colorectal carcinoma. Randomized comparison with no follow-up. Dis Colon Rectum, 1995. 38(6): p. 619-26.

1254. Kjeldsen, B.J., et al., A prospective randomized study of follow-up after radical surgery for colorectal cancer. Br J Surg, 1997. 84(5): p. 666-9.

1255. Pietra, N., et al., Role of follow-up in management of local recurrences of colorectal cancer: a prospective, randomized study. Dis Colon Rectum, 1998. 41(9): p. 1127-33. 
1256. Schoemaker, D., et al., Yearly colonoscopy, liver CT, and chest radiography do not influence 5year survival of colorectal cancer patients. Gastroenterology, 1998. 114(1): p. 7-14.

1257. Secco, G.B., et al., Efficacy and cost of risk-adapted follow-up in patients after colorectal cancer surgery: a prospective, randomized and controlled trial. Eur J Surg Oncol, 2002. 28(4): p. 418 23.

1258. Jeffery, M., B.E. Hickey, and P.N. Hider, Follow-up strategies for patients treated for nonmetastatic colorectal cancer. Cochrane Database Syst Rev, 2007(1): p. CD002200.

1259. Wattchow, D.A., et al., General practice vs surgical-based follow-up for patients with colon cancer: randomised controlled trial. Br J Cancer, 2006. 94(8): p. $1116-21$.

1260. Rodriguez-Moranta, F., et al., Postoperative surveillance in patients with colorectal cancer who have undergone curative resection: a prospective, multicenter, randomized, controlled trial. J Clin Oncol, 2006. 24(3): p. 386-93.

1261. Laubert, T., et al., Intensified surveillance after surgery for colorectal cancer significantly improves survival. Eur J Med Res, 2010. 15(1): p. 25-30.

1262. Papagrigoriadis, S. and B. Heyman, Patients' views on follow up of colorectal cancer: implications for risk communication and decision making. Postgrad Med J, 2003. 79(933): p. 403-7.

1263. Cooper, G.S., T.D. Kou, and H.L. Reynolds, Jr., Receipt of guideline-recommended follow-up in older colorectal cancer survivors : a population-based analysis. Cancer, 2008. 113(8): p. 2029 37.

1264. Rocklin, M.S., C.A. Slomski, and A.L. Watne, Postoperative surveillance of patients with carcinoma of the colon and rectum. Am Surg, 1990. 56(1): p. 22-7.

1265. Benson, A.B., 3rd, et al., 2000 update of American Society of Clinical Oncology colorectal cancer surveillance guidelines. J Clin Oncol, 2000. 18(20): p. 3586-8.

1266. Berman, J.M., R.J. Cheung, and D.S. Weinberg, Surveillance after colorectal cancer resection. Lancet, 2000. 355(9201): p. 395-9.

1267. Desch, C.E., et al., Recommended colorectal cancer surveillance guidelines by the American Society of Clinical Oncology. J Clin Oncol, 1999. 17(4): p. 1312.

1268. Macdonald, J.S., Carcinoembryonic antigen screening: pros and cons. Semin Oncol, 1999. 26(5): p. 556-60.

1269. Duffy, M.J., et al., Clinical utility of biochemical markers in colorectal cancer: European Group on Tumour Markers (EGTM) guidelines. Eur J Cancer, 2003. 39(6): p. 718-27.

1270. Duffy, M.J., Carcinoembryonic antigen as a marker for colorectal cancer: is it clinically useful? Clin Chem, 2001. 47(4): p. 624-30.

1271. Moertel, C.G., et al., An evaluation of the carcinoembryonic antigen (CEA) test for monitoring patients with resected colon cancer. Jama, 1993. 270(8): p. 943-7.

1272. Safi, F. and H.G. Beyer, The value of follow-up after curative surgery of colorectal carcinoma. Cancer Detect Prev, 1993. 17(3): p. 417-24.

1273. Zeng, Z., A.M. Cohen, and C. Urmacher, Usefulness of carcinoembryonic antigen monitoring despite normal preoperative values in node-positive colon cancer patients. Dis Colon Rectum, 1993. 36(11): p. 1063-8.

1274. Huebner, R.H., et al., A meta-analysis of the literature for whole-body FDG PET detection of recurrent colorectal cancer. J Nucl Med, 2000. 41(7): p. 1177-89.

1275. Annovazzi, A., et al., 18-fluorodeoxyglucose positron emission tomography in nonendocrine neoplastic disorders of the gastrointestinal tract. Gastroenterology, 2003. 125(4): p. 1235-45.

1276. Ahlquist, D.A., et al., Accuracy of fecal occult blood screening for colorectal neoplasia. $A$ prospective study using Hemoccult and HemoQuant tests. Jama, 1993. 269(10): p. 1262-7.

1277. Hunerbein, M., et al., The role of transrectal ultrasound-guided biopsy in the postoperative follow-up of patients with rectal cancer. Surgery, 2001. 129(2): p. 164-9.

1278. Mitry, E., et al., Epidemiology, management and prognosis of colorectal cancer with lung metastases: a 30-year population-based study. Gut, 2010. 59(10): p. 1383-8.

1279. Desch, C.E., et al., Colorectal cancer surveillance: 2005 update of an American Society of Clinical Oncology practice guideline. J Clin Oncol, 2005. 23(33): p. 8512-9.

1280. Chau, I., et al., The value of routine serum carcino-embryonic antigen measurement and computed tomography in the surveillance of patients after adjuvant chemotherapy for colorectal cancer. J Clin Oncol, 2004. 22(8): p. 1420-9.

1281. Sugarbaker, P.H., et al., A simplified plan for follow-up of patients with colon and rectal cancer supported by prospective studies of laboratory and radiologic test results. Surgery, 1987. 102(1): p. 79-87.

1282. Wang, T., et al., The role of postoperative colonoscopic surveillance after radical surgery for colorectal cancer: a prospective, randomized clinical study. Gastrointest Endosc, 2009. 69(3 Pt 2): p. 609-15. 
1283. Rulyak, S.J., et al., Outcome of follow-up colon examination among a population-based cohort of colorectal cancer patients. Clin Gastroenterol Hepatol, 2007. 5(4): p. 470-6; quiz 407.

1284. Rex, D.K., et al., Guidelines for colonoscopy surveillance after cancer resection: a consensus update by the American Cancer Society and the US Multi-Society Task Force on Colorectal Cancer. Gastroenterology, 2006. 130(6): p. 1865-71.

1285. Bouvier, A.M., et al., The lifelong risk of metachronous colorectal cancer justifies long-term colonoscopic follow-up. Eur J Cancer, 2008. 44(4): p. 522-7.

1286. Sobhani, I., et al., Early detection of recurrence by 18 FDG-PET in the follow-up of patients with colorectal cancer. Br J Cancer, 2008. 98(5): p. 875-80.

1287. Selvaggi, F., et al., FGD-PET in the follow-up of recurrent colorectal cancer. Colorectal Dis, 2003. 5(5): p. 496-500.

1288. Eckardt, V.F. and G. Bernhard, Nachsorge beim kolorektalen Karzinom. Eine Analyse von Effektivität und Kosten. Deutsches Ärzteblatt, 1997. 94: p. A456-62.

1289. Schwibbe, G., [Changes in quality of life in oncological patients in the course of an inpatient after-care program]. Rehabilitation (Stuttg), 1991. 30(2): p. 55-62.

1290. Gartner, U., et al., [Physical complaints, stress and quality of life of oncologic patients. Effects and patient assessment in inpatient rehabilitation]. Med Klin (Munich), 1996. 91(8): p. 501-8.

1291. Etzioni, D.A., A.B. El-Khoueiry, and R.W. Beart, Jr., Rates and predictors of chemotherapy use for stage III colon cancer: a systematic review. Cancer, 2008. 113(12): p. 3279-89.

1292. Grothey, A., L. Kellermann, and H.J. Schmoll, [Deficits in management of patients with colorectal carcinoma in Germany. Results of multicenter documentation of therapy algorithms]. Med Klin (Munich), 2002. 97(5): p. 270-7.

1293. von der Schulenburg, J.M., A. Prenzler, and W. Schurer, Cancer management and reimbursement aspects in Germany: an overview demonstrated by the case of colorectal cancer. Eur J Health Econ, 2010. 10 Suppl 1: p. S21-6.

1294. Adler, G. and T. Seufferlein, [Hope and disappointment in cancer medicine]. Z Arztl Fortbild Qualitatssich, 2007. 101(5): p. 287-92.

1295. Harrison, J.D., et al., What are the unmet supportive care needs of people with cancer? A systematic review. Support Care Cancer, 2009. 17(8): p. 1117-28.

1296. Lewis, R., et al., Nurse-led vs. conventional physician-led follow-up for patients with cancer: systematic review. J Adv Nurs, 2009. 65(4): p. 706-23.

1297. Howell, D.M., et al., A mixed-method evaluation of nurse-led community-based supportive cancer care. Support Care Cancer, 2008. 16(12): p. 1343-52.

1298. Ouwens, M., et al., Implementation of integrated care for patients with cancer: a systematic review of interventions and effects. Int J Qual Health Care, 2009. 21 (2): p. 137-44.

1299. Jahn, P., et al., Reduction of chemotherapy-induced anorexia, nausea, and emesis through a structured nursing intervention: a cluster-randomized multicenter trial. Support Care Cancer, 2009. 17(12): p. 1543-52.

1300. Jahn, P., et al., Improvement of pain related self management for oncologic patients through a trans institutional modular nursing intervention: protocol of a cluster randomized multicenter trial. Trials, 2010. 11: p. 29.

1301. Chen, R.C., et al., Patient-reported acute gastrointestinal symptoms during concurrent chemoradiation treatment for rectal cancer. Cancer, 2010. 116(8): p. 1879-86.

1302. Meyerhardt, J.A., et al., Impact of body mass index and weight change after treatment on cancer recurrence and survival in patients with stage III colon cancer: findings from Cancer and Leukemia Group B 89803. J Clin Oncol, 2008. 26(25): p. 4109-15.

1303. Meyerhardt, J.A., et al., Physical activity and survival after colorectal cancer diagnosis. J Clin Oncol, 2006. 24(22): p. 3527-34.

1304. Meyerhardt, J.A., et al., Impact of physical activity on cancer recurrence and survival in patients with stage III colon cancer: findings from CALGB 89803. J Clin Oncol, 2006. 24(22): p. 3535-41.

1305. Ng, K., et al., Multivitamin use is not associated with cancer recurrence or survival in patients with stage III colon cancer: findings from CALGB 89803. J Clin Oncol, 2010. 28(28): p. 435463.

1306. Stumpf, C., et al., Retrospektive Untersuchung zur Therapie mit Mistelextrakten bei Patienten mit kolorektalem Karzinom. Dtsch Zschr Onkol 2007. 39: p. 12-22.

1307. Friedel, W., et al., Fermented Europaen mistletoe (Viscum album L.) extract in supportive care in patients with primary non-metastatic colorectal cancer., in Die Mistel in der Tumortherapie, R. Scheer, et al., Editors. 2009, KVC Verlag: Essen. p. 563-576.

1308. Kienle, G.S. and H. Kiene, Review article: Influence of Viscum album L (European mistletoe) extracts on quality of life in cancer patients: a systematic review of controlled clinical studies. Integr Cancer Ther, 2010. 9(2): p. 142-57. 
1309. Shimizu, M., et al., (-)-Epigallocatechin gallate suppresses azoxymethane-induced colonic premalignant lesions in male C57BL/KsJ-db/db mice. Cancer Prev Res (Phila), 2008. 1(4): $p$. 298-304.

1310. Hoensch, H., et al., Prospective cohort comparison of flavonoid treatment in patients with resected colorectal cancer to prevent recurrence. World J Gastroenterol, 2008. 14(14): p. 2187 93.

1311. Ito, K., et al., Long-term effect of 5-fluorouracil enhanced by intermittent administration of polysaccharide $K$ after curative resection of colon cancer. A randomized controlled trial for 7 year follow-up. Int J Colorectal Dis, 2004. 19(2): p. 157-64.

1312. Sakamoto, J., et al., Efficacy of adjuvant immunochemotherapy with polysaccharide $\mathrm{K}$ for patients with curatively resected colorectal cancer: a meta-analysis of centrally randomized controlled clinical trials. Cancer Immunol Immunother, 2006. 55(4): p. 404-11.

1313. Kassab, S., et al., Homeopathic medicines for adverse effects of cancer treatments. Cochrane Database Syst Rev, 2009(2): p. CD004845.

1314. Ärztliches Zentrum für Qualität in der Medizin (ÄZQ), et al., Kompendium Q-M-A. Qualitätsmanagement in der ambulanten Versorgung. 3. ed. 2008: Dt. Ärzte-Verl.

1315. Leitlinienprogramm Onkologie (Deutsche Krebsgesellschaft (DKG), D.K.D., Arbeitsgemeinschaft der Wissenschaftlichen Medizinischen Fachgesellschaften (AWMF)), Entwicklung von Leitlinien basierten Qualitätsindikatoren. Methodenpapier für das Leitlinienprogramm Onkologie. Version 2.0. 2017. 2017, Berlin.

1316. Vasen, H.F., et al., New clinical criteria for hereditary nonpolyposis colorectal cancer (HNPCC, Lynch syndrome) proposed by the International Collaborative group on HNPCC. Gastroenterology, 1999. 116(6): p. 1453-6.

1317. Hermanek, P., Polypectomy in the colorectum: histological and oncological aspects. Endoscopy, 1983. 15: p. 158-161.

1318. Okuyama, T., M. Oya, and H. Ishikawa, Budding as a risk factor for lymph node metastasis in pT1 or PT2 well-differentiated colorectal adenocarcinoma. Dis Colon Rectum, 2002. 45(5): p. 628-34.

1319. Fruhmorgen, P., et al., [Endoscopic Therapy of Early Colorectal Cancer (pT1) - A Prospective Study]. Z Gastroenterol, 2003. 41(8): p. 703-10.

1320. Frühmorgen, P. and e. al, Kolorektale Polypen, in Empfehlungen der Deutschen Gesellschaft für Verdauungs- und Stoffwechselkrankheiten (DGVS) für die Durchführung endoskopischer Untersuchungen. 2000. p. 127-134.

\section{Erstveröffentlichung: \\ $01 / 1999$}

\section{Überarbeitung von:}

Die AWMF erfasst und publiziert die Leitlinien der

Fachgesellschaften mit größtmöglicher Sorgfalt - dennoch kann die AWMF für die Richtigkeit des Inhalts keine

Verantwortung übernehmen. Insbesondere bei Dosierungsangaben sind stets die Angaben der Hersteller zu beachten!

Autorisiert für elektronische Publikation: AWMF online 